\title{
PATTERNING CULTURE: \\ DEVELOPING A SYSTEM FOR THE VISUAL NOTATION OF GREETINGS
}

MARIE THERESE SHORTT

A thesis submitted in partial fulfilment of the requirements of the University of Brighton for the degree of Doctor of Philosophy

May 2015

The University of Brighton 


\section{Abstract}

This practice-based thesis examines ways in which the cultural patterning of greetings can be understood through their visualisation. The graphic design practice uses a range of digital tools and software to develop an animated graphic notation system for analysing nonverbal aspects of greetings both within and across cultures. The thesis explicates the process of this development and outlines its context and significance. Previous systems of visualisation devised by anthropologists for the study of greetings have stopped short of using contemporary digital technology. Further, although intercultural contact has increased, most existing greetings studies still focus on intracultural greetings.

The research centres on devising a method for the visual presentation and analysis of greetings. The method involves a twofold approach: data gathering, and analysis of the data through a visual process. Data gathering was carried out during fieldwork in the multicultural city of Stavanger in Norway. It included 27 participants from six different cultures, and broadly involved: setting up a studio situation for people to greet; filming greetings from multiple angles; and recording interviews. The data was then analysed through a visual process involving creating vector animations of the videos, adding specially created visual codes, and organising the animations into interactive forms.

The final interactive structure allows the viewer to access a range of information fields. A system of graphic coding has been devised to indicate nonverbal features of each greeting, including objective information such as spatial relationships and touch, and subjectively perceived dynamics, such as dominance and subordination, which have been explored through detailed interviews with the participants.

This method of visualisation can potentially provide researchers into human behaviour with a new form of analysis that works alongside current textual methods, and can also be used to enhance multicultural communication.

Chapter one sets out the multicultural context of the research, and the significance of studying greetings in a multicultural city. Chapter two gives a historical overview of greetings studies within anthropology, and discusses the extent to which visual tools and notation systems have already been used within this field. Chapter three presents a survey of existing techniques for visualising body movement, ranging from dance notation to motion tracking, and explores a range of information and communication technologies. Chapter four discusses the methods used in the fieldwork and in the development of the visual system. The final chapter is a reflection on the system and discusses its findings, limitations, potential improvements and uses. 


\section{Contents}

Abstract .................................................................................................... ii

List of Tables.............................................................................................. vii

List of Figures.......................................................................................... vii

Acknowledgements......................................................................... xxii

Declaration .......................................................................................... Xxiv

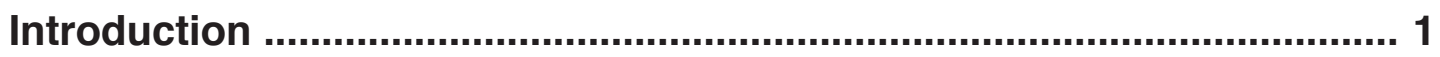

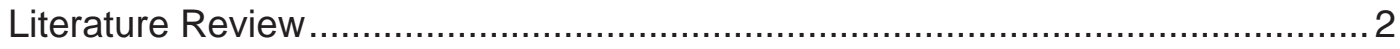

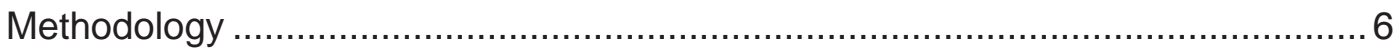

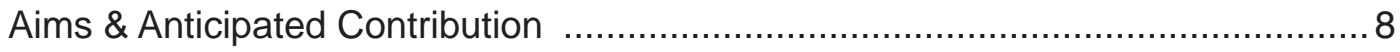

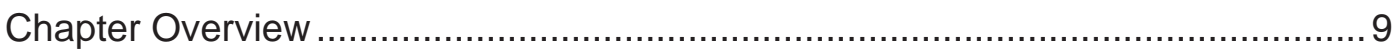

1. The Multicultural Background .................................................. 12

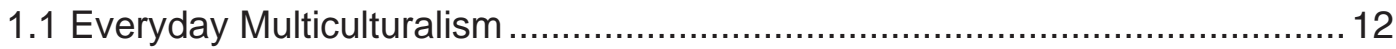

1.2 The Multicultural Situation in Scandinavia ..................................................... 21

1.3 The Immigrant Population of Scandinavia ............................................... 30

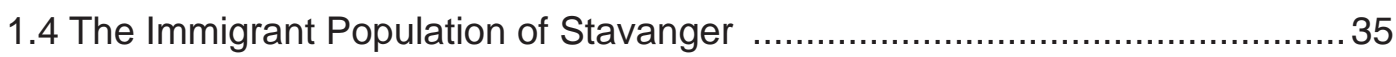

1.5 Everyday Interactions \& Nonverbal Communication in a Multicultural Setting......

2. Greetings: Approaches and Visual Notation within Anthropology. 43

2.1 Functions of the Greeting ................................................................... 43

2.2 Verbal \& Nonverbal Aspects of Greetings................................................. 46

2.3 Visual Representations of Human Interactions Including Greetings............... 48

2.4 An Intercultural Study of Greetings ........................................................... 79 
3. Visualising Movement: Documentation and Notation Systems, and the Potential of Digital Media ................................................................. 83

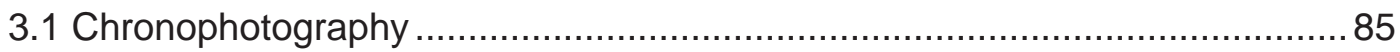

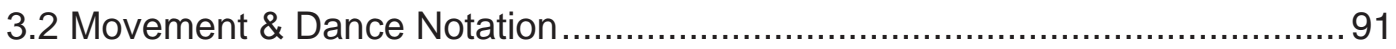

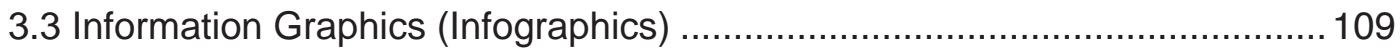

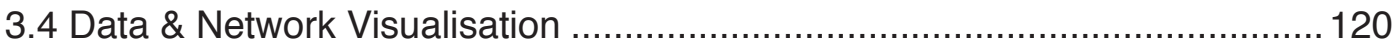

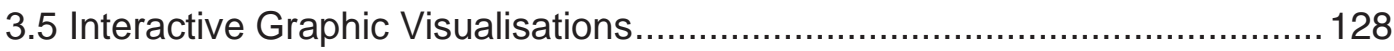

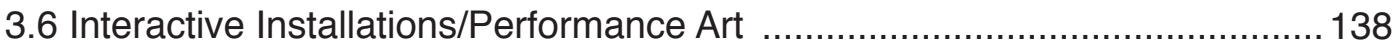

4. Visually Representing Layers of Nonverbal Information in Greetings: Developing a New System of Visual Notation ................... 157

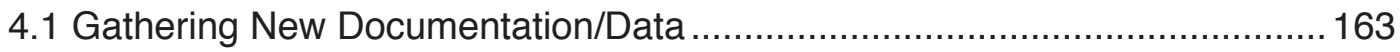

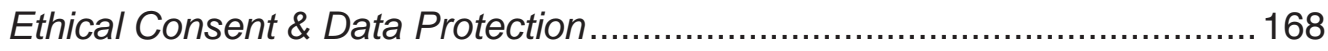

Reactions to Studio Setting \& Performing Greetings ................................. 170

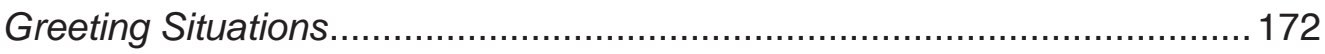

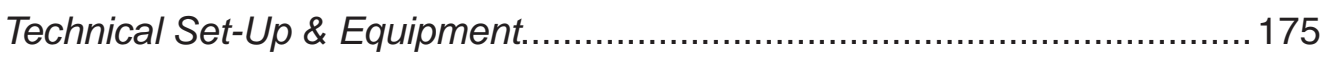

4.2 From Video Documentation to Graphic Representation .............................. 178

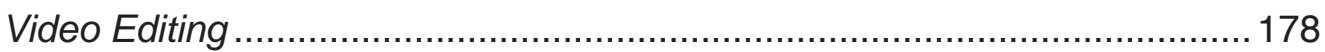

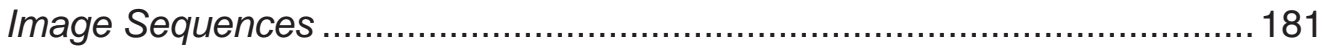

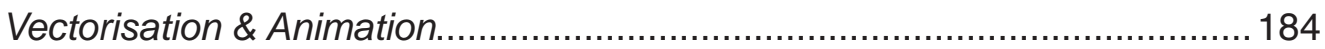

4.3 Visual Notation of Nonverbal Signals ......................................................... 186

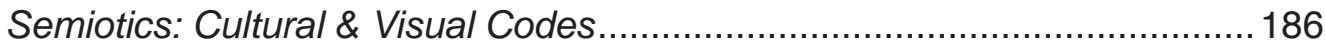

Visualising Personal Space/Intimate Zones............................................. 190

Visualising Touch \& Space In-Between .................................................... 197

Visualising Subjective Experiences \& Perceived Meaning...........................202

4.4 Technical Process and Settings: An Overview............................................210

4.5 The System for the Visual Notation of Greetings: Interactive Interface ........213

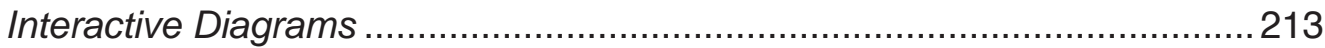

A Visual Analysis of the 'Hillary Clinton Meets the Capps' Greetings............228

Power \& Submission: Obama Greeting World Leaders ...............................2 233

4.6 Other Experiments Using Animation \& Motion Tracking ..............................2. 235

Patterning Moments of Human Interaction - Exhibition Piece ......................235

Interactive Greetings in a Mediated Performance Space - Prototype ......... 238

Interactive Greeting Space - Installation....................................................241 


\section{Findings and Conclusions: A Reflection on the System for the Visual Notation of Greetings .............................................................. 250}

5.1 Cultural Knowledge Through Visual Notation .......................................... 251

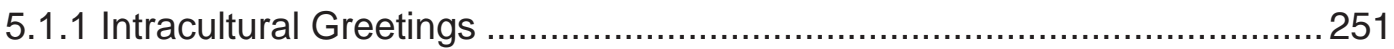

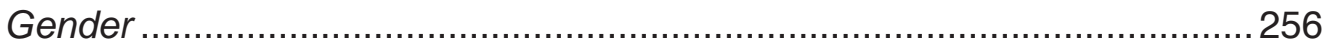

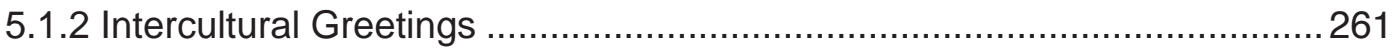

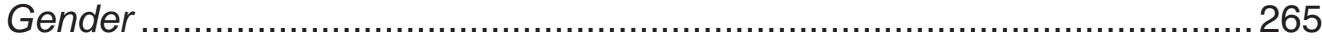

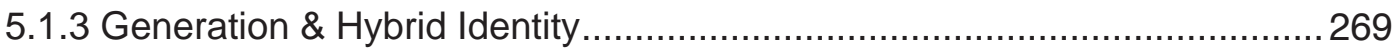

5.2 Present Limitations of the System .................................................... 272

Structural Limitations........................................................................... 272

Technical Limitations ........................................................................ 274

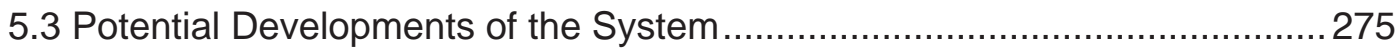

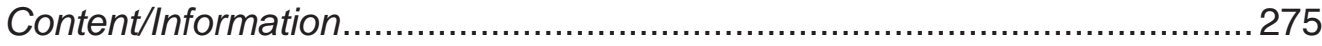

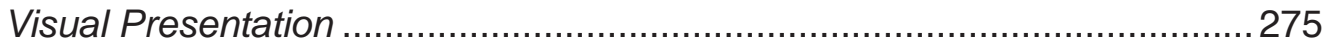

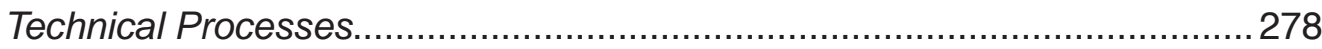

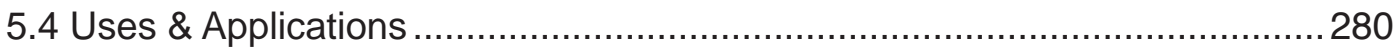

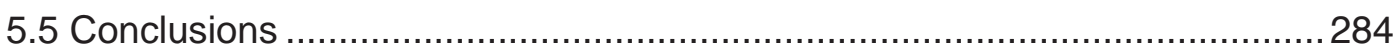

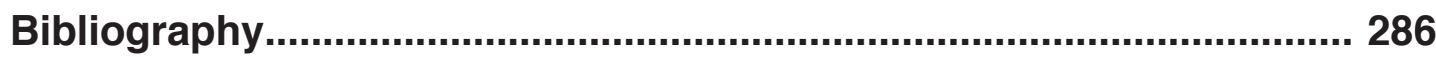

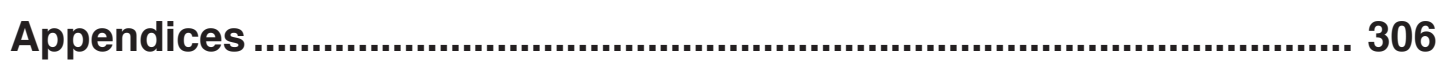

Appendix 1: Kinesics Project (Summary) …............................................... 307

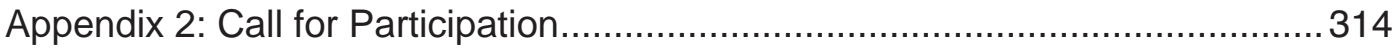

Appendix 3: Conversations During Breakfast with Vietnamese Participants ......316

Appendix 4: Programme for Greeting Documentation ...................................... 320

Appendix 5: Research Information Sheet .................................................... 322

Appendix 6: Participant Consent Form ............................................................. 325

Appendix 7: Research Ethics Approval Application Outcome............................. 328

Appendix 8: Conversations During Video Recording of Greetings ..................... 330

Appendix 9: Report on Greetings Documented During Fieldwork ..................... 336

Appendix 10: Interview questionnaire and transcripts .......................................356

a: Interview Questionnaire.................................................................. 356

b: Interview with Norwegian female participant....................................... 357

c: Interview with Norwegian male participant ............................................365 
d: Interview with Turkish female participant.

e: Interview with Turkish male participant.

$f$ : Interview with Vietnamese female participant 405

g: Interview with Angolan participant.

Appendix 11: Actionscript 3.0 code used to programme greeting diagrams

On DVD:

Appendix 12: Interactive Greeting Diagrams (Flash interface)

Appendix 13: Visualisation Process (video)

Appendix 14: Duranti's \& My Analysis of Hillary Clinton Greetings (video)

Appendix 15: Obama Greeting World Leaders (video)

Appendix 16: Video Experiments

a: Angola/Norway Strangers - Video Collage (video)

b: Vietnam Father/S on - Video Collage (video)

c: Vietnam Father/Daughter - Video Collage (video)

$\mathrm{d}$ : Vietnam Father/S on - Motion Blur (video)

Appendix 17: Installation Experiments

a: Patterning Moments of Human Interaction - Exhibition Piece (video)

b: Interactive G reetings in a Mediated Performance Space - P rototype (video)

c: Interactive Greeting Space - Installation (video)

Appendix 18: Greeting Videos Archive (videos)

Appendix 19: Interviews Archive (audio files)

Appendix 20: Visual Notebook (PDF file of Appendices 21-22)

As a bound visual notebook:

Appendix 21: Visual Development Sketches

Appendix 22: Graphic Development of the System 


\section{List of Tables}

Table 1 - Participants of fieldwork study.

169

\section{List of Figures}

Fig. 1 - Daily greetings of Dutch person with hybrid identity 20

Fig. 2 - 'Should there compared with the present be greater or lesser refugee access?' (Graphic by Shortt, 2015, based on table by Lithman, 2013: 247) 27

Fig. 3 - 'Most immigrants enrich the nation's cultural life.' (Ibid.) ........................................ 27

Fig. 4 - 'Most immigrants are a threat to security.' (Ibid.).................................................. 27

Fig. 5 - 'Most immigrants [in Norway] misuse the social welfare arrangements' (Graphic by Shortt, 2015, based on statistics from Statistics Norway, 2014b)

Fig. 6 - Numbers of foreign nationals who migrated to Norway in 1980, 1994 and 2013

(Graphic by Shortt, 2015, based on numbers from Statistics Norway, 2014c) 31

Fig. 7 - Compositions of populations in Norway, Sweden and Denmark, 2012 (Graphic by Shortt, 2015, based on graph by Pettersen and Østby, 2013).

Fig. 8 - Distribution of the native and the immigrant population of Stavanger in 2014

(Graphic by Shortt, 2015, based on numbers from Stavanger-statistikken, 2014) 36

Fig. 9 - Number of Immigrants (incl. Norwegians with immigrant parents) living in Stavanger 1980, 1994, and 2014 (Graphic by Shortt, 2015, based on numbers from Stavanger-statistikken, 2014)

Fig. 10 - Types of immigration to Norway and Stavanger 1990-2013 (Graphic by

Shortt, 2015, based on numbers from Egge-Hoveid, 2014). 37

Fig. 11 - Norwegian Constitution Day (17th May) international celebrations in Stavanger, 2012 39

Fig. 12 - Flyer distributed by Borgervernet [Fikk nabovarsel om asylmottak i Posten] (Hæreid, 2014) 40

Fig. 13 - Organisations (SOS Rasisme and Motmakt) demonstrating against racism in Stavanger (Photograph by Lars Idar Waage, in Tiller, 2014)... 40 
Fig. 14 - Hall's proxemic notation: sex and basic posture [From: Key to combined proxemic notation system] (Hall, 1963: 1020) 50

Fig. 15 - Hall's proxemic notation: spatial arrangements or orientation (Ibid.) .................... 50

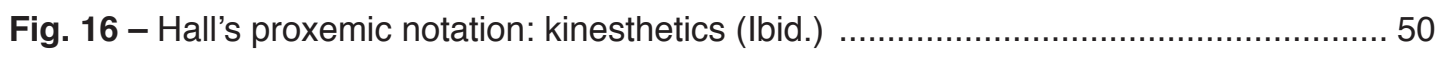

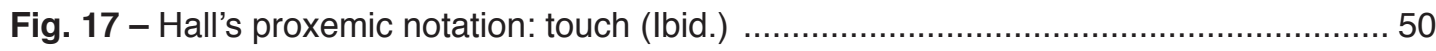

Fig. 18 - Hall's proxemic notation: visual code (Ibid.) .................................................. 50

Fig. 19 - Hall's proxemic notation: thermal code (Ibid.) ……............................................ 50

Fig. 20 - Hall's proxemic notation: olfaction code (lbid.) ……....................................... 50

Fig. 21 - Hall's proxemic notation: scale of voice loudness (Ibid.) .................................... 50

Fig. 22 - Hall's proxemic notation in use: recorded transaction and key [Recorded

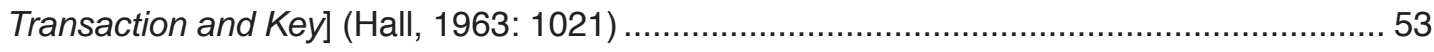

Fig. 23 - Birdwhistell's notation of kinesics: head [Kinegraphs - Total Head] (Birdwhistell, 1970: 259)

Fig. 24 - Birdwhistell's notation of kinesics: face [Kinegraphs - Face] (Birdwhistell, 1970: 260) 56

Fig. 25 - Birdwhistell's notation of kinesics: seated [Kinegraphs - Seated] (Birdwhistell, 1970: 277) 57

Fig. 26 - Birdwhistell's notation of kinesics: hand and finger activity [Kinegraphs - Hand and Finger Activity] (Birdwhistell, 1970: 268).

Fig. 27 - Example of interaction analysis of a dialogue using Birdwhistell's notation of kinesics [Sample Conversation with Description] (Birdwhistell, 1970: 285) 58

Fig. 28 - Kendon's facial notation [Explanation of Facial Notation] (Kendon, 2009: 124) .... 60

Fig. 29 - Kendon's facial notation in use [Periods of Sustained Facial Pattern] (Kendon, 2009: 125)

Fig. 30 - Kendon's map of action-sequence of a face-to-face encounter [A Map of the Action Sequence of the First Segment of C.14.1] (Kendon, 2009: 128) 62

Fig. 31 - Kendon's diagram of a greeting [Diagram of a Greeting] (Kendon, 2009: 162) ..... 63

Fig. 32 - Kendon's diagram of a greeting segment [From: These diagrams show the relationship in time between M's orientation and approach components and F's orientation and approach components and changes in F's face, for the beginning of kissing involvements at 111.1, and 111.2 and the Second Segment] (Kendon, 2009: 134) .. 64 Fig. 33 - Kendon's F-formation system [The F-Formation System] (Kendon, 2009: 217) .... 65 
Fig. 34 - Facial Action Coding System (FACS) by the Paul Ekman Group [Facial Action Coding System - Action Unit 4 - Brow Lowerer] (Ekman, Friesen, Wallace and Hager, 2002)

Fig. 35 - Head nod and head shake illustrated by layering images, featured in Morris' investigation [The Head Nod and the Head Shake] (Morris, 1979: 69) 67

Fig. 36 - Greeting images featured in Morris' investigation (Morris, 1979: 15, 78, 80, 98-99)

Fig. 37 - Taboo zones featured in Morris' investigation [The Taboo Zones of American College Graduates] (Morris, 1979: 204)

Fig. 38 - Eyebrow-flash during greeting featured in Morris' investigation [People all around the world perform a rapid eyebrow-flash daction when greeting...] (Morris, 1979: 13) 69

Fig. 39 - Wex's photographic study on female and male body language: leg and feet positions [Leg and Feet Positions] (Wex, 1979: 12) .... 70

Fig. 40 - Wex's photographic study on female and male body language: postures [Postures: 'Masculine', 'Feminine] (Wex, 1979: 179).....

Fig. 41 - Duranti's illustration of Samoan spatial and sitting arrangement during social gathering [Fono in Sanonu. Route followed by chief Agaiataua when arriving at house full of orators from his subvillage (May 1981)] (1992).

Fig. 42 - Duranti's visualisation of interlocking pattern during greeting exchange [Graphic Representation of Interlocking Greeting Exchange] (Duranti, 1997: 366) 73

Fig. 43 - Duranti's analysis of Clinton and Capps' meeting using audio-visual material [From: Hillary Clinton Meets the Capps] (Duranti, 1996). 75

Fig. 44 - Section 1 of Duranti's transcript/analysis of Clinton and Capps' greeting (Ibid.) )... 76

Fig. 45 - A section of Gail Jefferson's transcript conventions used by Duranti (Ibid.) 76

Fig. 46 - Adjacency pair format found in section 3 of Duranti's analysis of Clinton and Capps greeting (Ibid.) 77

Fig. 47 - Greeting between an Anuta man and a Tikopia man featured in Firth's investigation (Firth, 1954: 11) 80

Fig. 48 - Marey's photographic gun (The Robinson Library, 2014, At: http://www. robinsonlibrary.com/technology/photography/cinematography/marey.htm) 86 Fig. 49 - Muybridge's Zoopraxiscope (Brookman, 2010: 89) 86 
Fig. 50 - Marey's chronophotography from The Human Body in Action, Scientific American, 1914 (Motion Gallery, 2014, At: http://www.experimentsinmotion.com/motiongallery/126/Chronophotographs+from+The+Human+Body+in+Action/\#image)

Fig. 51 - Muybridge's chronophotography: Athletes. Posturing. Plate 115, 1879

(Brookman, 2010: 261).

Fig. 52 - Muybridge's chronophotography: Base-ball; throwing. Plate 286, 1887

(Brookman, 2010: 276). 88

Fig. 53 - Muybridge's chronophotography: The Horse in Motion (Brookman, 2010: 255) .... 89

Fig. 54 - Muybridge's chronophotography: Walking, two models meeting, and partly turning. Plate 45, 1887 (Brookman, 2010: 351).

Fig. 55 - Eshkol-Wachman notation - The body as a system of articulated axes (Eshkol and Harries, 1988: 1) 93

Fig. 56 - Eshkol-Wachman notation - The system of reference is a sphere (Eshkol and Harries, 1988: 2) 93

Fig. 57 - Eshkol Wachman notation - Horizontal plane and vertical planes in the scale $1=45$ degrees (lbid.) 93

Fig. 58 - Eshkol-Wachman notation - Axis of movement, angle of movement and type of movement [Rotary Movement, Conical Movement, Plane Movement] (Eshkol and Harries, 1988: 6)

Fig. 59 - Eshkol-Wachman notation - Plane movement [Horizontal plane, Vertical plane, Intermediate plane] (Eshkol and Harries, 1988) 93

Fig. 60 - Eshkol-Wachman notation - body sections (Eshkol, 1972) ..... 94

Fig. 61 - Eshkol-Wachman notation - structure of staff: each row represents one body section (Eshkol, 1972)

Fig. 62 - Eshkol-Wachman notation - an example of the system in use [An example of writing in Eshkol Wachman notation (redrawn after Hutchinson-guest 1984: 109)] (Farnell, 1996: 157)

Fig. 63 - Benesh movement notation - structure [Direction, Location and Travel for the Solo Dancer and Groups are described in Part 1] (Brown and Parker, 1984: 81) 96

Fig. 64 - Benesh notation system is based on iconic visualisation of the body [Benesh notation system: (a) body parts and staff: (i) matrix representing the performer; (ii) arm 
raised sideways (Redrawn after $J$. and $R$. Benesh 1956: 11); (b) direction symbols for the third dimension] (Farnell, 1996: 154)

Fig. 65 - Benesh movement notation - viewpoint and the three basic signs (Parker, 1996)

Fig. 66 - Benesh movement notation - wrist directions (lbid.) 97

Fig. 67 - Benesh movement notation - direction (Brown and Parker, 1984: 83) 98

Fig. 68 - Benesh movement notation - changing direction (Brown and Parker, 1984: 85) .. 98

Fig. 69 - Benesh movement notation - example of score with dancer's moves above the score (Royal Academy of Dance, 2014) 98

Fig. 70 - Labanotation - staff showing different parts of the body broken into columns (Davies, 2001: 8) 99

Fig. 71 - Labanotation - lines indicating: $A=$ start of staff; $B=$ starting position; $C=$ start of the movement; $D=$ the beat; $E=$ bar line; $F=$ end of the movement; $G=$ large number for the bar; $\mathrm{H}=$ small numbers for the beat (Griesbeck, 1996)

Fig. 72 - Labanotation - direction symbols and levels distinguished by shading (lbid.)

Fig. 73 - Labanotation - direction symbols for different levels of arm and leg movements (lbid.).

Fig. 74 - Labanotation - relationship pins: $L=$ left; $R=$ right; $P=$ place; $F=$ Forward;

$\mathrm{B}=$ backward $; \mathrm{U}=$ up; $\mathrm{M}=$ middle $; \mathrm{D}=$ down (Ibid. .

Fig. 75 - Labanotation - shifting the centre of weight (top) and transference of weight (bottom) (Ibid.)

Fig. 76 - Labanotation - jumps: $A=$ both feet; $B=$ from one foot to the other; $C=$ on the same foot (lbid.)

Fig. 77 - Labanotation - body signs [The basic body signs. (Reproduced with kind permission from 'Labanotation' by Ann Hutchinson, Oxford University Press, 1970)] (Davies, 2001) 103

Fig. 78 - Labanotation - symbols indicating weight on knees/kneeling (Griesbeck, 1996) 103

Fig. 79 - Labanotation - floor plans [From circle into pentagon] (Preston-Dunlop and Sayers, 2010: 113) 
Fig. 80 - Labanotation - example of analysis of Assiniboine storytelling [Assiniboine storytelling with Plains Sign Talk and spoken Nakota: page one of the Labanotated score] (Farnell, 1996: 163)

Fig. 81 - Labanotation - explanation of first part of notation of Assiniboine storytelling ['Long Ago'] (Farnell, 1996: 164) 106

Fig. 82 - Analysis of Dutch 'hip-hop' greeting. Image sequence (top) and analysis using Labanotation: movement score for both people involved in greeting (middle) and floor plan of their movements (bottom)

Fig. 83 - 18th century dance notation by Kellom Tomlinson - The Art of Dancing,

Explained by Reading and Figures (Tufte, 1990: 27,115)

Fig. 84 - Images from a French skiing manual How to Ski by the French Method (Tufte, 2006: 36-37)

Fig. 85 - Megan Jaegerman's Spotting a Hidden Handgun graphic (Tufte, 2006: 116) ..... 112

Fig. 86 - Image mapping of birds by Roger Tory Peterson [Roger Tory Peterson, A Field Guide to the birds (Boston, 1934, 1939, 1947), plate 23, shore-birds] (Tufte, 2006: 45) .... 114

Fig. 87 - How to Defend Yourself From an Attacker (Holmes, 2005: 9) ............................ 115

Fig. 88 - How to Say "Yes" in India (Holmes, 2005: 5) .................................................. 116

Fig. 89 - How to Wave Like a Royal, and How to Curtsy (Holmes, 2005: 3) .................... 116

Fig. 90 - How to Greet Someone in France, Holland, Russia and America (Holmes,

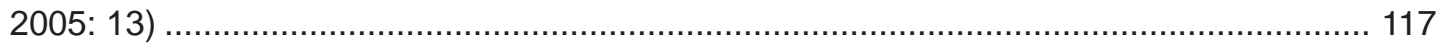

Fig. 91 - Lifestyle: independent vs. dependent (Liu, 2007) ........................................ 118

Fig. 92 - Attitude towards punctuality (Liu, 2007) ……............................................ 118

Fig. 93 - Problem-solving approach (Liu, 2007) ........................................................ 118

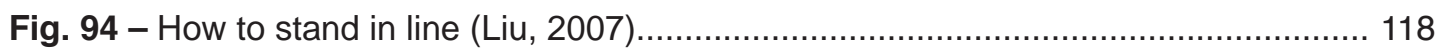

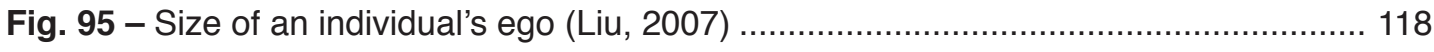

Fig. 96 - Presentation of Self: Cell Trance (Nova et al., 2012: 1) ................................... 119

Fig. 97 - The Classics: Clicker Casting (Nova et al., 2012: 43) ...................................... 120

Fig. 98 - Presentation of Self: The Periscope (Nova et al., 2012: 71) ............................. 120

Fig. 99 - Nightingale's famous coxcomb/rose diagram [Diagram of the Causes of Mortality in the Army in the Easf] (Rogers, 2013: 67) ................................................ 121

Fig. 100 - Types of Information Visualisation by Tufte (McCandless, 2009: 128-129) ...... 122

Fig. 101 - Most Popular Infographics by Alberto Antoniazzi (Rogers, 2013: 295) .............. 123 
Fig. 102 - Jacob Moreno's sociogram of a class of fourth graders from 1933 [Jacob Moreno, one of the first sociograms, published in the New York Times, April 3, 1933, showing the relationships within a class of fourth graders. Boys (triangles) are on the left and girls (circles) are on the right] (Lima, 2011a: 77)

Fig. 103 - Intensity of relationships/relations enhanced by line thickness (Clark, 2009,

At: http://www.visualcomplexity.com/vc/project_details.cfm?id=740\&index=740\&domain= Accessed on 10.06.2013)

Fig. 104 - Colour-coded health condition associations (MIT SENSEable City Lab, 2011, At: http://visualization.geblogs.com/visualization/network/ Accessed on 10.06.2013)

Fig. 105 - Lima's fifteen categories of mapping patterns used for network visualisation (Lima, 2011a: 158)

Fig. 106 - How to use the Gapminder desktop software (Source: Free material from www.gapminder.org)

Fig. 107a - Percentage of broadband subscribers/Internet users in the United Kingdom, Denmark, Sweden, Norway, Russia and India in 2000: chart view (Source: Free material from www.gapminder.org)

Fig. 107b - Percentage of broadband subscribers/Internet users in the United Kingdom, Denmark, Sweden, Norway, Russia and India in 2010: chart view (Ibid.)

Fig. 107c- Percentage of broadband subscribers/Internet users in the United Kingdom,

Denmark, Sweden, Norway, Russia and India in 2010: map view (Ibid.)

Fig. 108 - Filter options for Snake Oil Supplements? Scientific evidence for popular health supplements (McCandless, 2013a) ......

Fig. 109 - Interactive diagram Snake Oil Supplements? Scientific evidence for popular health supplements (McCandless, 2013a).

Fig. 110a - Still from Map Your Moves interactive map (Stefaner, 2010) .....

Fig. 110b - Still from Map Your Moves interactive map (Ibid.)

Fig. 111 - Stills from SoNIA - Streaming Classroom Interactions (McFarland and Bender de-Moll, 2004) 136

Fig. 112 - Chameleon Project - Still image from Prototype 6: Mimicking Emotional Contagion in Social Groups (Gonsalves, 2009)

Fig. 113 - Chameleon Project - Prototype 7: Integration of Mind Reading Technology, installation (lbid.) 
Fig. 114 - Chameleon Project - Prototype 7: Integration of Mind Reading Technology, technology/sensors (Ibid.) 140

Fig. 115 - Tine Beck's light installation Catch Me Now (Beck, 2010) 141

Fig. 116 - Random International's Rain Room installation at Barbican, London (Photograph by Marie Therese Shortt, 2012) 142

Fig. 117 - Set-up of studio and recording equipment for Motion Bank project (Motion Bank, 2013a)

Fig. 118 - Using The Sky: No Time To Fly video overlays (Motion Bank, 2013c)..... 146

Fig. 119 - Using The Sky: travel paths (1) (Ibid.) 146

Fig. 120 - Using The Sky: travel paths (2) (Ibid.) 147

Fig. 121 - Using The Sky: travel paths (3) (Ibid.)..... 148

Fig. 122 - Using The Sky: convex hulls of the travel paths, comparison (Ibid.) 149

Fig. 123 - Using The Sky: convex hulls of the travel paths, combined (Ibid.). 149

Fig. 124 - Using The Sky: Amin Weber's artistic 3D animated representation of the score No Time To Fly (Ibid.) 150

Fig. 125 - Two: Impulse - motion capture of assisted solos (Motion Bank, 2013b) 151

Fig. 126 - Two: Impulse - Attentive Agent, Light Touches (Ibid.) 152

Fig. 127 - Two: Impulse - Attentive Agent, Pressure (1) (Ibid.) 153

Fig. 128 - Two: Impulse - Attentive Agent, Pressure (2) (Ibid.) . 154

Fig. 129 - Two: Impulse - Mixed Roles (Ibid.) 154

Fig. 130 - Images from the Global Gesture Guide showing cultural differences in gestures meaning 'hello' and 'goodbye' in different countries (Grosse, Reker, 2010, In:

Don't Get Me Wrong: The Global Gesture Guide Munich: Bierke Publishing.) 157

Fig. 131 - Kinesics visuals: From video still to vector graphic

Fig. 132 - Kinesics visuals: video angles used, and a graphic visualisation of the video stills. 161

Fig. 133 - Kinesics visuals: colour coded intimate zones and their measurements 162

Fig. 134 - Kinesics visuals: graphic greetings diagrams for comparisons across genders/cultures 162

Fig. 135 - Visual overview of family greetings that were recorded and visualised 173

Fig. 136 - Visual overview of social greetings that were recorded and visualised 174 
Fig. 137 - Visual overview of cross-cultural greetings that were recorded and visualised

Fig. 138 - Greeting documentation: front and overhead perspectives 175

Fig. 139 - Studio and equipment set-up

Fig. 140 - Greeting documentation: the four perspectives/angles that were filmed 177

Fig. 141 - Greeting videos: four videos/perspectives displayed on one screen ... 178

Fig. 142 - Greeting videos: two videos/perspectives displayed on one screen - faces 179

Fig. 143 - Greeting videos: two videos/perspectives displayed on one screen - front and overhead views.

Fig. 144 - Video label: dark purple text indicating a 'true' relationship 181

Fig. 145 - Video label: light purple text indicates an enacted relationship. 181

Fig. 146 - A greeting between a Vietnamese grandfather and granddaughter converted into an image sequence (the images read left to right)

Fig. 147 - A greeting between a Vietnamese grandfather and granddaughter presented as a multi-layered image sequence.

Fig. 148 - Vectorisation: vector drawings were drawn on top of each image in the image sequences

Fig. 149 - Vectorisation: the complete image sequence vectorised onto multiple layers in Adobe Illustrator.

Fig. 150 - Colours in Culture by David McCandless with AlwaysWithHonor.com (2009) ... 191

Fig. 151 - Visualising intimate zones

Fig. 152 - Intimate zones: applied to four different greetings - front and overhead views

Fig. 153 - Animated greeting sequence between two Norwegians, male and female, who are close friends, showing synchronised movement and relation to personal space .. 197 Fig. 154 - Animated greeting sequence between a Norwegian male and an Angolan female who are close friends, showing difference in movement and relation to personal space.

Fig. 155 - Colours initially used to annotate intimate zones (top), touch (middle) and space in-between (bottom).

Fig. 156 - Colour experimentation for touch and space in-between: pink and purple 
Fig. 157 - Colour experimentation for touch and space in-between: dark blue and burgundy. 200

Fig. 158 - Colour experimentation for touch and space in-between: grey and brown..... 200

Fig. 159 - Touch and space in-between 201

Fig. 160 - Image shows four greetings with annotated intimate zones, touch, and space in-between 202

Fig. 161 - Subjective patterns: power \& submission/respect......................................... 206

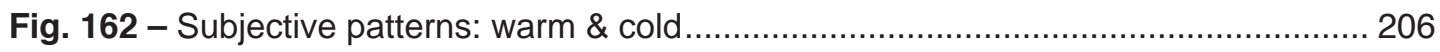

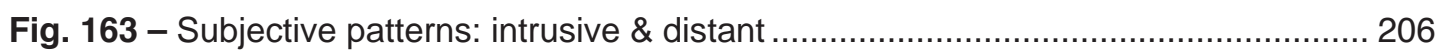

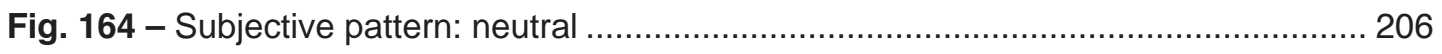

Fig. 165 - Diagram showing intimate zones in combination with patterns of subjective experiences

Fig. 166 - Visualising subjective experiences: the images show how person A (top) and person B (bottom) experience themselves and the other person, during this moment of the greeting......

Fig. 167 - Visualising subjective experiences: the image shows how each person, A and $\mathrm{B}$, experiences the other person during this moment of the greeting..... 209

Fig. 168 - Interactive diagrams interface explained: starting screen.

Fig. 169 - Interactive diagrams interface explained: social greetings diagram

Fig. 170 - Interactive greetings diagram: family greetings

Fig. 171 - Interactive greetings diagram: cross-cultural greetings.

Fig. 172 - Original and new method for visualising a greeting in one still image

Fig. 173 - Interactive greetings diagram: compare greetings within cultures horizontally .. 217

Fig. 174 - Interactive greetings diagram: compare greetings across cultures vertically $\ldots .217$

Fig. 175 - Interactive greetings diagram: Social greetings - highlighted in green are handshake greetings .

Fig. 176 - Interactive greetings diagram: Cross-cultural greetings - highlighted in green are handshake greetings

Fig. 177 - Interactive greetings diagram: Cross-cultural greetings - highlighted in pink are embraces/hugs, and in red are greetings involving kisses on the cheeks

Fig. 178 - Interactive greetings diagram: Family greetings - highlighted in pink are embraces/hugs, and in red are greetings involving kisses on the cheeks 
Fig. 179 - Interactive greetings diagram: Family greetings - highlighted in blue are greetings in which there is no physical contact between the people greeting.....

Fig. 180 - Interactive diagrams interface explained: a specific greeting has been selected

Fig. 181 - Menu for different greeting views

Fig. 182 - Interactive greetings diagram: showing vector animation of a cross-cultural greeting between a Norwegian male and an Angolan female, who are strangers

Fig. 183 - Interactive greetings diagram: showing original greeting footage of a cross-cultural greeting between a Norwegian male and an Angolan female, who are strangers

Fig. 184 - Interactive greetings diagram: showing vector animation, with space in-between highlighted, of a cross-cultural greeting between a Norwegian male and an Angolan female, who are strangers.

Fig. 185 - Interactive greetings diagram: showing vector animation, with touch highlighted, of a cross-cultural greeting between a Norwegian male and an Angolan female, who are strangers

Fig. 186 - Interactive greetings diagram: showing vector animation, with colour coded intimate-zones/personal space, of a cross-cultural greeting between a Norwegian male and an Angolan female, who are strangers

Fig. 187 - Interactive greetings diagram: same as above, only later on in the greeting..... 226

Fig. 188 - Interactive greetings diagram: showing vector animation, with colour coded intimate-zones and patterned subjective meaning, of a cross-cultural greeting between a Norwegian male and an Angolan female, who are strangers 227

Fig. 189 - Interactive greetings diagram: same as above, only later on in the greeting......

Fig. 190 - Opening screen for interface of Duranti's and my analysis of greetings between Hillary Clinton and the Capps 229

Fig. 191 - Duranti's textual analysis (left side) and my visual analysis (right side) of greetings between Hillary Clinton and the Capps - section 1

Fig. 192 - Duranti's textual analysis (left side) and my visual analysis (right side) of greetings between Hillary Clinton and the Capps - section 2

Fig. 193 - Interactive piece of Duranti's textual analysis (left side) and my visual analysis (right side) of greetings between Hillary Clinton and the Capps - section 3 230 
Fig. 194 - Still images from the three greeting animations in which visual annotations have been added to the illustrations

Fig. 195 - A breakdown of all visualisations shown in my three animated analyses of the Hillary Clinton \& Capps' greetings

Fig. 196 - Power and Submission: visual analysis of greetings between Obama and three world leaders, based on images found online (Perry, 2010)

Fig. 197 - Still image from greeting triptych video of Norwegian and Turkish greetings (1)

Fig. 198 - Still image from greeting triptych video of Norwegian and Turkish greetings

Fig. 199 - Work installed at UCA Student Research Conference 2012: Process.

Perception. Phenomena. Visitors interacting with the floor piece.

Fig. 200 - Work installed at UCA Student Research Conference 2012: Process.

Perception. Phenomena. Floor piece

Fig. 201 - Photos from greeting space prototype. Bauhaus inspired costume made by Bahar Sert 239

Fig. 202 - The set-up in Isadora software. A webcam is used for motion tracking 240

Fig. 203 - Testing the Arduino sensor attached to the performer 240

Fig. 204 - Initial sketches of greeting space set-up. 242

Fig. 205 - Initial sketches of greeting space set-up - placement of projector 242

Fig. 206 - Simulation of the greeting space. 243

Fig. 207 - Connects and feeds the Kinect camera into Isadora 244

Fig. 208 - Set-up of motion tracking and columns to project green 244

Fig. 209 - Set-up of motion tracking to project shapes (circles) 244

Fig. 210 - Interactive Greeting space: Set-up of equipment/technology 245

Fig. 211 - Instructions that were printed and attached to the wall of the greeting space ... 245

Fig. 212 - The greeting space in use 246

Fig. 213 - Still images from animations of a greeting between two Vietnamese females who are close friends (Vietnamese - close friends $-\mathrm{f} / \mathrm{f})$ - highlighting the distance between them 
Fig. 214 - Greeting: Vietnamese - close friends - $f / f$. This image shows they are both in the orange intimate zone (1/2 arm's distance to $1 / 4$ arm's distance) at this moment of the greeting......

Fig. 215 - Greeting: Vietnamese - close friends - f/f. This image shows the intimate zones, as in fig. 214, and patterns of subjective experiences show that they are both experiencing this as a warm greeting

Fig. 216 - Greeting: Norwegian - close friends - m/f. Images are stills from animations highlighting space in-between (top), touch (second from top), intimate zones (third from top), and subjective experience (bottom)

Fig. 217 - Greeting: Norwegian - acquaintances - m/f. Images are stills from animations highlighting space in-between (top), touch (second from top), intimate zones (third from top), and subjective experience (bottom)

Fig. 218 - Greeting: Turkish - close friends - m/f. Images are stills from animations highlighting space in-between (top), touch (middle), and intimate zones (bottom) 258

Fig. 219 - Greeting: Turkish - strangers - m/f. Images are stills from animations highlighting space in-between (top), touch (middle), and intimate zones (bottom)

Fig. 220 - Greeting: Turkish - close friends $-\mathrm{f} / f$. This greeting, involving three kisses, is used by Turkish female close friends (Muslims) in Stavanger.

Fig. 221 - Greeting: Turkish - acquaintances - f/f. This greeting, involving two kisses, is generally used among Turkish female close friends (Muslims) outside Stavanger 260

Fig. 222 - Greeting: Norwegian - close friends $-m / m$. Space in-between (top), touch (middle), and intimate zones (bottom).

Fig. 223 - Greeting: Chilean/Norwegian - close friends - m/m. Space in-between (top), touch (middle), and intimate zones (bottom)

Fig. 224 - Greeting: Turkish/Norwegian - close friends - m/m. Space in-between (top), touch (middle), and intimate zones (bottom) 264

Fig. 225 - Turkish social greetings between close friends - m/m (top), $m / f$ (middle), $f / f$ (bottom). The visualisations show levels of touch during a moment of the greetings 266

Fig. 226 - Norwegian social greetings between close friends - m/m (top), $m / f$ (middle), $\mathrm{f} / \mathrm{f}$ (bottom). The visualisations show levels of touch during a moment of the greetings ...... 266 Fig. 227 - Greeting: Norwegian - strangers - m/f. Greeting in the green intimate zone, showing both participants staying within half an arm to an arm's distance 
Fig. 228 - Greeting: Norwegian/Angolan - strangers - m/f. Greeting in the orange and red intimate zones, showing participants' different relations to the closer proximity of the greeting......

Fig. 229 - Images showing inconsistencies in vector drawings such as clothing and hair - vs. no clothing/hair. 276

Fig. 230 - Greeting: Somali/Norwegian - strangers $-\mathrm{m} / \mathrm{m}$. Image shows all layers of notation applied in the same greeting animation

Fig. 231 - Simulation of interface for comparing two greeting animations side by side 278

Fig. 232 - Potential development: using my visual methods to represent other forms/rituals of nonverbal communication such as parting rituals (left) or group interactions (right) 281

Fig. 233 - Potential development: visual diagrams aimed at teaching immigrants about local greeting customs.

Fig. 234 - Potential development: visual diagrams mapping changes in greeting patterns over a period of time (years)

Fig. 235 - Kinesics visuals: From video still to vector graphic 307

Fig. 236 - Kinesics visuals: video angles used, and a graphic visualisation of the video stills

Fig. 237 - Kinesics visuals: colour coded intimate zones and their measurements 308

Fig. 238 - Kinesics visuals: graphic greetings diagrams for comparisons across genders/cultures

Fig. 239 - Kinesics visuals: printed greetings diagrams. Informal greetings (top), greeting the elderly (middle), and formal greetings (bottom)..... 309

Fig. 240 - Kinesics visuals: screenshots of interactive greetings diagrams interface......... 310

Fig. 241 - Kinesics visuals: screenshots of interactive greetings maps interface.

Fig. 242 - Kinesics visuals: two still images from a greeting video in which all greetings were animated

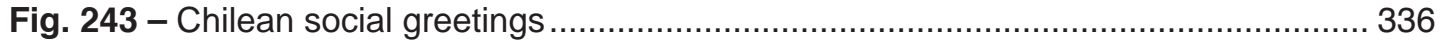

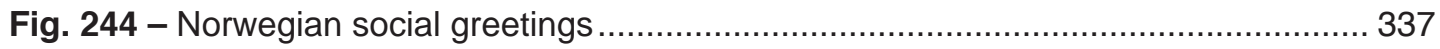

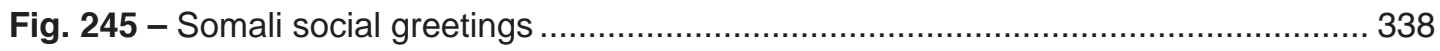

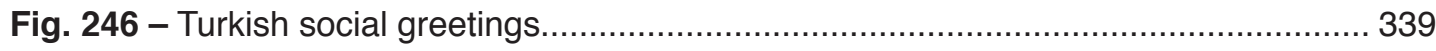

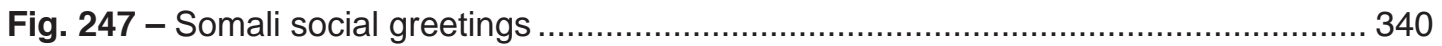




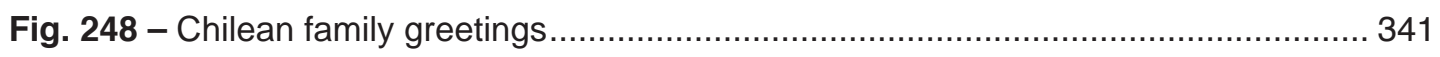

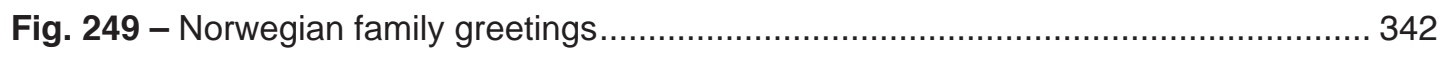

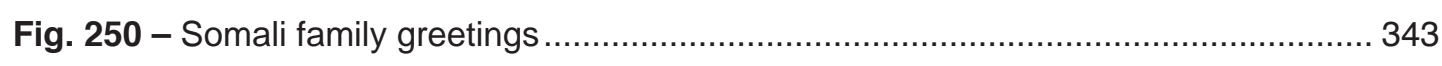

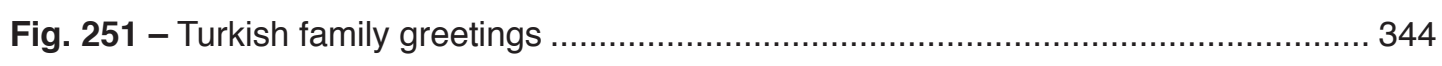

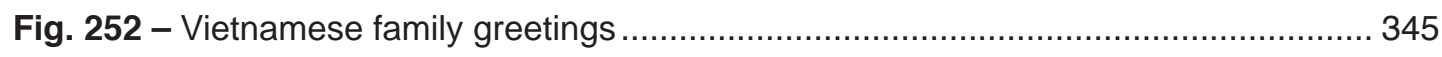

Fig. 253 - Norwegian/Chilean cross-cultural greetings............................................ 347

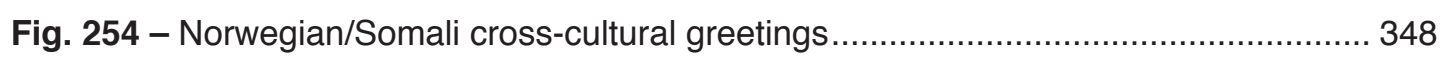

Fig. 255 - Norwegian/Turkish cross-cultural greetings .............................................. 350

Fig. 256 - Norwegian/Vietnamese cross-cultural greetings ........................................... 350

Fig. 257 - Norwegian/Angola cross-cultural greetings................................................. 351

Fig. 258 - Turkish/Angolan cross-cultural greetings ................................................... 352 


\section{Acknowledgements}

I would like to express my special thanks to my supervisors Professor Jean Wainwright and Professor Judith Williamson for such wonderful support and guidance. You have both been so generous with your time and resources, and your dedication to my research has extended far beyond what was required of you. I am so grateful for all your feedback and encouragement: the lively discussions during meetings in London; and all the phone conversations from which I learnt so much about academic writing and structuring my research. I cherish all the special moments we have shared and the friendships we developed over the past few years. I would also like to give a very special thanks to Dr Ricardo Leizaola, my external supervisor, who made the team complete. Ricardo, your expertise and guidance in the fields of anthropology and multiculturalism have been essential in contextualising my research and structuring my fieldwork. Thank you Jean, Judith and Ricardo for your time and enthusiasm - your support has been crucial to the development of my work. I could not have dreamed of a better supervisory team, and am so lucky to have had the chance to work with you all.

A very special thanks to Mum and Dad for all your love and support, it means the world to me. The bicultural Norwegian/lrish home you created is the reason for my love of and curiosity about cultural diversity. Thank you for giving my brothers and I the best of both cultures. Loving thoughts go to my late grandmother Mary for giving us a second home in Ireland, and for everything she taught me about the Irish culture.

Thank you to Internasjonalt Kulturnettverk in Stavanger for helping me in my search for fieldwork participants. To all the Chilean, Norwegian, Somali, Turkish, Vietnamese, and Angolan participants of my fieldwork in Stavanger - thank you for your time and contribution to my research, for sharing your 
experiences, cultures and greetings. You made the development of this system possible and much richer than I had anticipated.

Thank you Aunty Antoinette, my brother Frank and lan Hunt for your comments and feedback, and for proofreading different chapters of the thesis. To Mary O'Hagan and Sian Jones at the UCA Research Office for all your support and for responding to all my emails and questions. To Bahar Sert for the fun and dynamic collaborations creating interactive spaces, and to Phil Marsh at UCA Rochester for helping us with coding for Arduino.

Thank you Aunty Colette, Siri, and Anna for hosting me so well on my London visits. To Grace for all the words of encouragement, and for being such a caring friend, and to the Kings for giving me a 'home away from home'. Thank you to the Cahersiveen girls Grace, Sadhbh, Sarah, Majella, Noretta, Ita and Loreto for always making me feel at home and welcome in Ireland. To my Ålgård girls Jette, Elin, Marie, Rakel Kristin, Brit, Lise and Heidi for continuing to make me feel at home and part of the 'forening' in Norway. To Holly and Tereza for your friendships, yoga and coffee breaks when they were most needed, and to Alessio for printing assistance.

Warm thanks to Mum, Dad, brothers Frank, Patrick and Danny, Aunty Antoinette, Onkel Hans, cousins Heather and Tommy, Aunty Colette, Uncle Sean, Joana and my darling nephews Odin and Akio, and niece Yumi - my small but culturally diverse family from whom I continue to learn so much. And finally, a loving thank you to my partner Guillaume for your continued understanding, support and love, and for teaching me to greet the French way. 


\section{Declaration}

I declare that the research contained in this thesis, unless otherwise formally indicated within the text, is the original work of the author. The thesis has not been previously submitted to this or any other university for a degree, and does not incorporate any material already submitted for a degree.

$$
\text { M.T.Short }
$$

20th May 2015 


\section{Introduction}

This research project develops a graphic system for visualising the nonverbal dimensions of greetings. A greeting is a ritualised form of acknowledgement that is practiced worldwide. It is usually 'performed' as a first introduction in meeting with friends or strangers during social or formal situations: I am using it as an introduction to culturally specific behaviour and as a way into visualising cultural variations of nonverbal interaction patterns.

My system of visualisation is designed as a tool for the comparison and analysis of greetings. When the dynamics of greetings are presented as diagrams, comparisons can be made between specific greeting situations. Within these situations one can compare gender differences and generational differences both intraculturally and cross-culturally. The system I have created employs vector graphics, both still and animated: basic shapes, such as circles, are used to graphically visualise personal space, while patterns visualise personal experiences. Colours and gradients are used to visualise touch, intimacy levels and intermediate space. The use of frame-to-frame animation clarifies various stages and key gestural actions of a greeting.

Anthropologists and scientists of nonverbal communication agree that nonverbal communication plays a central part in human social behaviour. Words, they claim, only make up a part of human communication, and the majority of human communication is actually nonverbal (Birdwhistell, 1970; Argyle, 1988; Hall, 1983, 1990). In a multicultural setting, where nonverbal behaviour is patterned differently by people's cultural backgrounds, this silent language becomes particularly important and sensitive. There are significant cultural distinctions when it comes to greetings, from obvious differences between gestures, such as a nose kiss, kisses on the cheeks, hand kiss, handshake, a hug, a high-five or 
bow and curtsey, to more subtle, but still consequential differences such as variations in people's comfort with proximity, and in touch and gaze. Differences in such subtle signals are harder to acknowledge during the greeting itself, but can often leave greeters with a sense of confusion, invasion, power, frustration, flattery or discomfort. These emotions will be determined largely by social and cultural contexts. While most studies of greetings are intracultural and not intercultural (Duranti, 1992, 1997a; Pillet-Shore, 2008, 2012; Firth, 1954), my research investigates both intracultural and intercultural greetings: it therefore introduces a new element within the field of greetings studies. Intracultural greetings are relatively predictable as they tend to be structured and patterned by social practices and traditions that are familiar to members of the culture, whereas intercultural greetings become unpredictable because they involve the meeting of differing cultural experiences and knowledge. In multicultural contexts confusion arises, as mentioned above, due to cultural differences concerning issues such as personal space, touch, and meaning making. These are precisely the dimensions of the greeting that are visualised within my system.

In devising this system I did not work in the abstract, I gathered all my information and documentation through fieldwork in the Norwegian city of Stavanger. I engaged participants of different cultural backgrounds and conducted studio-based video-recordings of their greetings. I also carried out interviews with participants recording their responses to different greetings. I then used this documentation, and took on board what I learnt from participants, in the creation of the system.

\section{Literature Review}

Three main fields of study inform my research:

(1) Multiculturalism - specifically a) theories of everyday multiculturalism, and b) a social and political survey of the state of immigration and 
multiculturalism in Scandinavia (Olwig, 2011; Samovar, Porter and McDaniel, 2007; Wise and Velayutham, 2009);

(2) Anthropology, from Duranti's studies on human verbal greetings (Duranti, 1992, 1996, 1997a, 1997b) to Hall, Kendon and Birdwhistell's systems for the visual notation of proximity, face-to-face communication, and kinesics (Hall, 1963, 1990; Kendon, 2009; Birdwhistell, 1970);

(3) Visual Studies, from motion capture by Muybridge and Marey (St. George, 2009), dance notation such as Labanotation, Benesh movement notation and Eshkol-Wachman movement notation (Brown and Parker, 1984; Griesbeck, 1996; Parker, 1996; Eshkol and Harries, 1988), information graphics by Tufte (1990, 2006), Lima (2011a) and McCandless $(2009,2014)$, to interactive graphics and performance/ installations projects such as the Cameleon project by Tina Gonsalves (2009) and the Motion Bank project (2013a).

Multiculturalism:

Everyday multiculturalism is, according to Wise and Velayutham (2009), the study of cultural diversity as experienced on the ground in everyday life. It concerns how people live with and relate to people of different cultural backgrounds and differs from the more 'traditional' concepts of multiculturalism, which tend to take a top-down view and concern state policies. Examples of studies on everyday multiculturalism include topics such as neighbourly interaction and exchange in culturally diverse societies, and the study of how the behaviour of youth with hybrid identities are patterned by two or more cultures (Noble, 2009; Radice, 2009; Semi et. al., 2009; Wise and Velayutham, 2009; Wise, 2009). My study of greetings concerns everyday multiculturalism in a culturally diverse Norwegian city (Stavanger) - and visualises ways in which participants' greetings have been patterned by their cultural backgrounds.

The three Scandinavian countries Norway, Sweden and Denmark differ in their immigration policies and in their approaches to multiculturalism. 
While Sweden has very liberal immigration policies, and embraces multiculturalism, Denmark has strict immigration policies and rejects multiculturalism (Olwig, 2011). Norway is somewhere in-between the two. The immigrant population of the Norwegian city of Stavanger is constantly increasing, and today makes up more than 20 per cent of its total population (Stavanger-statistikken, 2014). This makes Stavanger an ideal location for conducting a study on everyday multiculturalism.

Anthropology:

Within the field of anthropology greetings have always attracted attention, as they are formal rituals performed in everyday situations as well as in formal settings. They involve both language and gestures. Alessandro Duranti, a leading figure in the field of greetings studies, was the first to challenge the prevailing view of the 1960s-70s, which was that greetings were phatic forms of communication, i.e. contentfree interaction that functions to create and update social relationships rather than to communicate information (Duranti,1997b). Before Duranti, most anthropological studies on greetings tended to focus on gestures or looked for functionalist explanations of such formalised exchanges. However, Duranti argued that important information exchange does indeed occur during greetings.

In his research Duranti focused on the verbal aspects of the information exchange. My research centres on nonverbal information exchanges and how nonverbal dimensions (such as gestures, proximity and touch) influence how people perceive each other and the interaction. During intracultural greetings the information exchanged is generally understood by members of its culture because of their shared backgrounds and patternings. However, during intercultural greetings (as I will show with examples in chapters 4 and 5) misinformation and miscommunication often occur due to people's cultural backgrounds influencing their 
experiences and understandings of the (nonverbal) information exchange differently.

In the broader field of anthropology, not specifically concerned with greetings, it was researchers such as Edward T. Hall, Adam Kendon and Ray L. Birdwhistell who began to create and use systematic visual notation in the study of human interaction. Their notation systems are intricate and sophisticated and created primarily to be used by researchers to annotate video documentations and textual transcripts. Their publications make limited use of pictures and illustrations from the notation systems: this is because the notation was created, as mentioned above, for analysis purposes, not for presentation of their findings (Hall, 1990; Kendon, 2009; Birdwhistell, 1970). Moving to more contemporary technologies, Duranti made early use of the Internet to integrate visual media with his texts (Duranti, 1996). However, this was done in a very basic way, simply by presenting textual analysis next to video clips.

Duranti's use of digital media and moving image has laid the foundation of my work. As mentioned before, he has also been influential because he challenged previous understandings of greetings as interactions that are free of content. Though he focused his understanding of information exchange in greetings on a study of verbal language, he did so without excluding other aspects of greetings such as nonverbal language. He introduced the key concepts I use in my research - the understanding of greetings as involving important information exchange (with a particular focus on the nonverbal), coupled with making the information accessible through visual language and digital media.

Visual Studies:

Human movement has always been a topic of great curiosity for artists and designers, and many techniques and systems have been created to express and make sense of it. Chronophotography (stop motion 
photography) is one such technique developed in the 19th century by practitioners such as Muybridge and Marey (Braun, 2009; St George, 2009). Another method of visually representing body movement, one which uses abstracted graphic forms, can be found in dance notation systems such as Labanotation, Benesh movement notation, and EshkohlWachman movement notation (Brown and Parker, 1984; Griesbeck, 1996; Parker, 1996; Eshkol and Harries, 1988). These systems make possible the analysis, documentation and preservation of body movement and dance choreography through a graphic language.

Today there are a great many visualisation techniques that could potentially be used to analyse movement, such as information graphics, which is currently used by graphic designers, data journalists and others to communicate data and information in a simple graphic form (Tufte, 1990, 2006; McCandless, 2009, 2014; Lima, 2011a). Current software also makes possible the creation of motion graphics, which can be programmed into advanced interactive interfaces with viewing and filter options that react to users' selections and interests (Rosling, 2006 February; McCandless, 2013a, 2013b). Such technological advancements could potentially be used to create more contemporary and sophisticated interfaces and tools to be used in human motion/interaction studies. Futher, motion tracking technologies, such as those used to create interactive art and performance installations, can also potentially be used in anthropology and studies of human behaviour for tracking of greetings (and any other form of human interaction) (Gonsalves, 2009; Random International, 2014; Motion Bank, 2013a).

\section{Methodology}

The core of my research has been to devise a system for the visual analysis of greetings. My method has involved fieldwork in a multicultural city, in which I video-recorded a variety of greetings; this provided the raw material from which I developed the visual system. My recording 
method involved filming actual greetings in a studio, to provide a uniform backdrop, as the angles, space and perspectives of the greetings needed to be cross-culturally comparable. To document random greetings on the street or other public spaces would pose a range of ethical and legal implications, as well as fail to capture accurate video perspectives that are needed in order to devise a consistent system. Using the raw studio documentation I then looked for ways to represent various dimensions of the greeting: (1) personal space - showing how people's uses of personal space match or differ; (2) the use of touch - what situations, cultures or gender combinations invite either more or less physical contact during greetings; (3) personal experiences and perceived meanings - showing how the same greeting situation can be experienced differently, as cold/ warm, powerful/submissive, intrusive/distant and more. Through my visual system one can begin to grasp these highly intricate patterns of behaviour that co-exist in multicultural settings, patterns that can be either complementary to, or conflicting with, each other.

As part of my working method, I have investigated different perspectives, shapes, colours, structures, frame rates and software in order to visualise all these dimensions. My system has developed over time, by trial and error. The elaborate devising of this system is the central task of the PhD project, and the details of various visual techniques/methods that I have tried, discarded or chosen are presented in sections 4.1-4.4 of Chapter 4.

The second dimension of my method is the contextualising of my practical project by studying the fields outlined in my literature review, with the aim of placing my work in the relevant contexts and showing how it has developed. In chapters 1, 2 and 3 I go more deeply into these contextual fields and show how they have contributed to my method of practice. 


\section{Aims \& Anticipated Contribution}

My aim, as discussed above, is to develop a system that seeks to analyse and make visible nonverbal dimensions of greetings in order to complement and expand on current understandings of greetings. Situated at the intersection between anthropology and information visualisation, this research is anticipated to provide the field of greetings studies, and the broader field of anthropology, with a new visual method for producing understandings in a field previously studied through observation and video documentation without systematic graphic analysis. In employing video, and still and motion graphics to produce and test a methodology for micro-ethnography, I am contributing a new set of tools for future researchers into the nonverbal dimensions of greetings. By using new technologies to reveal and analyse the multidimensional layers of human interaction, I hope to show that it is possible to visualise cultural difference in a contemporary manner, at a time when digital tools are becoming ever more important in academia and social sciences, and we are seeing an important shift from text to visual thinking (Hart, 2004).

There is, however, a wider and non-academic goal to my work. In a constantly globalising world, where the meeting and interaction of widely different cultures happens daily in multicultural cities and societies, it is important to work towards improved intercultural understanding and empathy. The need for both interpersonal skills and self-reflective understanding is essential when it comes to human communication in multicultural societies. People with significantly differing worldviews, and thereby behaviours, are living in close proximity to each other, and members of such societies are having to adjust to each others' diverse backgrounds in order to function as a society.

I aim to provide not only researchers into human behaviour, but the arena of multicultural communication, with new forms of mediation that transcend verbal language barriers. Besides making a contribution to the 
field of anthropology, I aim to increase understanding between people of different cultures in wider social settings. For instance, I have already worked with Internasjonalt Hus (International House) and Internasjonalt Kulturnettverk (International Cultural Network) in Stavanger, and I intend to collaborate further on community projects with such organisations.

My system could potentially be used widely: in immigration centres teaching immigrants about local (and cross-cultural) social behaviour and different greeting customs; for international relations - such as intercultural business meetings as a way of creating a basic cross-cultural understanding of the cultures present (this is highly relevant in Stavanger, a city known as the 'Oil Capital' of Norway); and to create local or regional dynamic mappings over periods of time to illustrate how greetings are changing and merging between traditional and multicultural ones.

The system can also be developed in the future to study other forms of human interactions, such as group interactions, nonverbal communication during conversation, parting rituals or similar. A technical evolution of the system would be to develop software that could track the greeting videos and automatically add the various visual dimensions to them. Its potential for further uses and development is extensive.

\section{Chapter Overview}

Chapter 1, The Multicultural Background, sets out the multicultural context of the research: it frames the project as a study of everyday multiculturalism, referring to my own bicultural background and discussing the wider context of cultural and hybrid identities in multicultural societies. It gives an overview of the state of multiculturalism and immigration in Scandinavia as a whole, and Stavanger in particular, including a discussion of immigration and integration policies and the populations' attitudes towards immigrants. It further lays out the challenges of cross- 
cultural communication, identity, and the importance of nonverbal communication in a multicultural setting.

Chapter Two, Greetings: Approaches and Visual Notation within Anthropology, gives a historical overview of greetings studies within anthropology, and discusses the extent to which visual tools and notation systems have already been used within this area of research. It provides a detailed study of Hall's, Kendon's and Birdwhistell's systems of notation, and of Duranti's interactive greeting analysis.

Chapter Three, Visualising Movement: Documentation and Notation Systems, and the Potential of Digital Media, is a survey and critical analysis of existing visualisation techniques and their success or potential in communicating body movement. This survey ranges from the nineteenth century chronophotographers such as Eadward Muybridge and Étienne-Jules Marey, to dance notation systems (Labanotation, Benesh movement notation, and Eshkol-Wachman movement notation), to more contemporary techniques of information visualisation such as those used in the work of Edward Tufte, David McCandless and Manuel Lima. It further looks at interactive installations and motion studies using advanced motion tracking technologies, such as work by Random International, Tina Gonsalves, and the Motion Bank project.

Chapter Four, Visually Representing Layers of Nonverbal Information in Greetings: Developing a New System of Visual Notation, provides a detailed overview of my visual method, and explains how I use it as an analytical tool in my study of greetings. This chapter describes my fieldwork in Stavanger, and outlines the methods used in the fieldwork and in the development of the visual system - a process that takes the fieldwork material from video to graphic representation, and finally into an interactive system/interface. The chapter explains how the system and 
interface can be used, and also summarises other experiments I have carried out using motion tracking software and sensors.

Chapter Five, Findings and Conclusions: A Reflection on the System for the Visual Notation of Greetings, is a reflection on the notation system and the fieldwork interviews. It shows how the system can be used to generate cultural knowledge about intracultural and intercultural interactions, including gender and generational differences, and uses the findings from the fieldwork in Stavanger as examples. It features examples of interviews and reflects on the everyday multicultural situation in Stavanger. The chapter also discusses limitations and potential future developments of the system and gives examples of its uses and applications. 


\section{The Multicultural Background}

\subsection{Everyday Multiculturalism}

This study addresses greetings in a multicultural context. My own background is that of a bicultural' person (Norwegian and Irish) who grew up in Norway, and is currently living in Ireland. I have always identified with two cultures, spoken two languages and felt at home in two countries. I never viewed myself, nor did others tend to consider me, as fully Norwegian or fully Irish. People often asked if I 'felt' more Irish or Norwegian. I was both. The Irish culture was celebrated in my family home in Norway in the form of food, language, music, cheering for Ireland during sports events such as the World Cup, and (to a lesser degree) the Catholic religion. I experienced it as a great privilege to be part of two cultures, and being slightly 'different' was always positive; perhaps due to the fact that visually, when it came to looks, clothes and skin colour I did not stand out as being different. Fortunately I never experienced any negative reactions due to my being bicultural. My two cultures were similar enough for me not to feel any cultural 'conflict' in identifying with them both. Being bicultural provided me with an awareness of, and interest in cultural difference. I would often, for instance, compare how others viewed me in Norway and in Ireland.

These comparisons were my first experiences where I acknowledged that behaviour and its meaning is context- and culture-specific. I could behave in a similar fashion among Norwegian or Irish friends, but be experienced

\footnotetext{
1 Being bicultural, according to Schwartz and Unger, represents having competence in one's 'heritage culture' as well as the culture one has settled into. This applies to immigrants/refugees as well as to second-generation immigrants who often identify with the culture into which they were born and with the cultural traditions of their parents. Bicultural people are able to interact with people from both of their cultures, and identify with both cultures (Schwartz and Unger, 2010). Eytan (2004) discusses new forms of biculturalism related to globalisation: for example, teenagers may identify with the local culture and a global (sub-)culture. For a critical assessment of biculturalism see Dennis Rutledge's Biculturalism, Self Identity and Societal Transformation (Rutledge, 2008).
} 
in very different ways such as: outgoing in Norway, and calm in Ireland. In retrospect, it is clear that culture-related differences in behaviour, such as how I was greeted in either culture, influenced my experience and view of both cultures. After summers spent in Ireland I would tell my Norwegian friends that Irish people are much more friendly than Norwegians. This view was based on the way I was received and greeted by people in my father's hometown of Cahersiveen in the South-West of Ireland. When walking down the main street of this Irish town, most people I passed, including strangers, would greet me with a 'hi', 'hello', 'hi, how are you?' or 'hi, it is a lovely morning, isn't it?', while in Norway we would only greet people we knew, never strangers. Nonverbally, greetings also displayed some cultural differences. In both countries I found that a handshake was a safe way to greet when meeting someone for the first time, or when greeting in formal situations. I had learnt (in Norway) that a handshake had to be firm and confident, and this seemed to be the same in Ireland during first-time greetings. Daily greetings in Norway were verbal greetings such as 'hi' or 'hello', and I would hug my Norwegian friends upon greeting only if we had not seen each other in a long time. Greetings in Ireland were similar, 'hi' or 'hello' but followed by more small talk, and friends would greet each other with one kiss on the cheek followed by a hug, if they had not met in a while. The kiss on the cheek went unnoticed by me for many years; I used to move straight in for a hug as I had been accustomed to in Norway.

When it came to cross-cultural interaction in Norway, I was introduced to children and teenagers from a wide variety of cultural backgrounds at a much earlier age than my Norwegian peers due to being part Irish, or more specifically, because I was Catholic. During primary school, preparing for my first holy communion, and then later in secondary school, preparing for Confirmation, I attended compulsory Catholic religion classes in Stavanger. Only a few of the pupils in my class were Norwegian. Like me, many of them were bicultural, and we shared the experience of identifying with two individual cultures. Others were first- or second-generation immigrants from 
countries such as Sri Lanka, Vietnam, the Philippines, France and Poland. Most of the children spoke one language at home and with people of the same cultural background, while Norwegian was the common language in our group. Most of the Vietnamese pupils, who were the largest cultural group in our class, had two names: a Vietnamese name that they used when interacting with other Vietnamese people, and a Norwegian name that they used otherwise.

While I was exposed to cultural diversity at an early age through these classes, I did not encounter a great number of foreign cultures during everyday interaction in my hometown. The classes did not reflect the (multicultural) situation outside of the group, where interaction with children from other cultures was much more limited. At that time, during the late 1980s until the mid-1990s, the immigrant population of Stavanger was more than 200 per cent smaller than its current size (Stavanger-statistikken, 2014). The situation in and around Stavanger today is very different, and one can meet and interact with people from a great number of cultures during everyday social interactions. Recently I have met and greeted Spanish, Portuguese, Bulgarian, Turkish, Brazilian, American, Vietnamese, Swedish and Norwegian people, with a range of social and religious backgrounds, all during the same social gathering held in Stavanger. This diversity found during everyday interactions would have been unimaginable in Stavanger 20 years ago. With this change and cultural diversity comes a change in the way we interact. Previously, when interacting with other Norwegians, social situations followed set patterns of interaction. Gradually this new multicultural situation has started to blur such familiar and still dominant patterns and introduced new, unfamiliar ones. Examples of this can be found in greeting encounters, such as a handshake upon meeting someone for the first time. While this is a greeting known worldwide and the most common way of greeting in Norway, it is not always used in the same way, or in the same situations, across cultures. While I have learnt to greet with a firm handshake, I have noticed when meeting with people from different cultures, such as Vietnamese, in 
particular women, that a weak handshake is favoured, or no handshake at all. During first-time meetings with Spanish, Portuguese and Brazilian people, both male and female, my handshake has often been ignored in favour of two kisses on the cheeks.

In this research project I draw on my awareness of first-hand experiences of cultural difference, and the approach I take is one of 'everyday multiculturalism', as defined by Amanda Wise and Selvaraj Velayutham (Wise and Velayutham, 2009). While multiculturalism is often talked about from a top-down perspective, and concerns policies by nation states, as I will discuss in more detail in section 1.2, Wise and Velayuntham define everyday multiculturalism as an approach that draws on the sociology of everyday life. It deals with cultural diversity experienced and negotiated on the ground. Their view is that by focusing on the micro-sociology of everyday multiculturalism, a deeper understanding of wider social structures can be reached (Wise and Velayutham, 2009). These everyday situations are defined as being fluid, in constant change and flux (Semi et al., 2009; Wise, 2005; Wise and Velayutham, 2009). Semi, Colombo, Camozzi and Frisina (2009) divide everyday multiculturalism into two (theoretical) categories: a category of analysis, and a category of practice. As a category of analysis, it uses methods such as listening and participant observation during daily practices to investigate what meanings people attach to their activities and interactions. As a category of practice it refers to urban situations of encounters with difference. It looks at how to live with difference and use it as a tool for interaction and communication (Semi et al., 2009: 73-74).

Wise (2005) argues that survey-based quantitative data and abstract multicultural policies, though useful, do not always reflect the real, fluid and lived experiences of people's everyday lives. On a similar note, Wise and Velayutham (2009) point to state policies as necessary for recognising inequalities and ensuring tolerance, but argue that racial and social tensions that are experienced on the ground are seldom recognised officially. 
Likewise, Wise observes that people on the ground do not live the 'parallel' and segregated lives that are often presented in political debates. She claims that on the ground is where 'non-assimilationist forms of integration' can truly happen (Wise and Velayutham, 2009: 40).

Examples of research using the everyday multiculturalism approach include studies of activities such as fishing, relations at the workplace and in educational institutions, cultural dynamics at the gym, exchanges at ethnically diverse markets, and many more (Wise and Velayutham, 2009). Wise (2005) presents a number of stories of simple acts of what she calls intercultural neighbourly care, such as watering each others' plants, exchanging vegetables from the garden, sharing recipes and other types of daily reciprocity. Goodall, Wearing, Byrne and Cadzow (2009) study everyday multiculturalism through fishing. They discuss how Vietnamese, Lebanese, local Australians and others interact, or don't interact, through this everyday activity. They illustrate how culture is learned through everyday activities and social environments, and how elements of cultural knowledge, such as fishing techniques, are passed on through generations and exchanged between groups (Goodall et al., 2009).

The reality of a multicultural city is that cultures are mixing, and many people have bi- or multicultural identities, meaning they often practise and adapt to customs and traditions from different cultures simultaneously. Secondand third-generation immigrants also tend to have one foot in their parents' culture and the other in the dominant, for example the Norwegian culture, and if from a bi- or multicultural family background they often have more than two cultures that they identify with, so-called hybrid identities (Wise and Velayutham, 2009). Samovar, Porter and McDaniel (2007), in their study on intercultural communication, also discuss this situation. They use an example of a Thai woman who immigrated to the United States as a refugee when she was a child. She grew up identifying with two cultures - Thai culture and American culture. She speaks both languages: Mien at home, and English 
with her friends (Samovar, Porter and McDaniel, 2007: 9). Samovar, Porter and McDaniel conclude that it is common to belong to more than one culture: to adhere to patterns of the dominant culture, as well as those of minority cultures. The dominant culture is defined as the people who 'historically have controlled the institutions within the culture' (Samovar, Porter and McDaniel, 2007: 10-11). Kapuściński's (2008) study of the Other also draws attention to the fact that migration of people from rural areas to big cities of the 'Third World' is causing new hybrid identities to form, which sometimes results in the weakening of more traditional cultural practices (Kapuściński, 2008: 33,47). Many studies of everyday multiculturalism concern this reality of people practising hybrid identities. In his 2009 study on everyday cosmopolitanism Noble (2009) found evidence of hybrid lives and community identities among Australian youth. He claims that the theoretical notion of multiculturalism as different cultures living side by side is incapable of representing the complexity of identities in modern societies because cultural variety has become even more diverse and hybrid, in what he describes as a 'diversification of diversity' (Noble, 2009: 46-47). His study included young second- and third-generation immigrants who did not necessarily identify with just one cultural identity. He found that they often moved between different groups and environments and maintained traditions and practices from their parents' culture as well as adapting to local Australian customs (Noble, 2009: 47-48). Another study, by Semi, Colombo, Camozzi and Frisina (2009), also discusses youth in Italy with hybrid identities, and reveals that young children in secondary school reject being categorised into a single ethnic or national identity. As children of immigrants they tend to identify with multiple groups/ cultures, and do not feel completely one or the other, such as completely Italian or completely foreign - they feel both. The children who were studied expressed a wish to be viewed equally with their peers while at the same time not wanting to give up their other identity (Semi et al., 2009: 77). These examples make clear the fact that young people do not always like to be 'boxed in' to one identity, because they do not always identify with a single ethnic culture (Wise and Velayutham, 2009). 
The observations made in these studies of everyday multiculturalism correspond to my own experiences of being bicultural and identifying with more than one cultural identity. It also relates to what Radice (2009) terms 'identity cosmopolitanism', which deals with how people lay claim to identity through belonging to national, cultural, and social groups. People can have multiple identities within cosmopolitanism, and, as has been illustrated above, this is often the lived reality for immigrants and their offspring, and for children of parents in mixed marriages (Radice, 2009: 146-147). In Olwig's 2011 study of migrants and refugees in the Scandinavian ${ }^{2}$ welfare societies, she found that young refugees or children of refugees find it difficult to deal with adaptation to Norwegian society while at the same time fulfilling obligations towards their families. Living with both can be a challenge, as the different cultures can be conflicting or contrasting. She also found that young Cape Verdeans born and raised in Sweden, where immigrants are encouraged to maintain their cultural traditions, find it frustrating that they are expected to identify with the Cape Verdean identity when in reality they identify more with Swedish culture (Olwig, 2011: 190).

My study is on the everyday activity of greeting: an interaction during which having a hybrid identity can influence the greeting exchange. While my own two cultures practise similar greeting rituals, people of other bi- or multicultural backgrounds have to adapt to different, sometimes contrasting, ways of greeting, despite also sharing the greeting 'template' of the host culture. A Somali girl who is Muslim and living in Norway, for example, could greet other Somali females with hugs and kisses, but when greeting her Norwegian female friends there may be less hugging and kissing. She could perhaps greet Somali men only verbally without any physical contact, as practised within their shared culture and religion, while she may greet Norwegian males with a handshake. Because of her hybrid identity she

2 Scandinavia includes the three kingdoms of Norway, Sweden and Denmark, whereas the Nordic countries also include Finland and Iceland. 
adjusts to different situations in her way of greeting as regards gender and cultural relations.

A similar example of hybridity and adjustment was found in my 2006-7 study Kinesics - Creative intercultural learning, on greetings in the Dutch town of Eindhoven ${ }^{3}$. A group of male hip-hop dancers in their late teens to early twenties were part of the study. They were all second-generation immigrants of mixed cultural backgrounds, such as Turkish/Dutch, Colombian/Dutch and Chilean/Dutch. They explained that when they greet each other (other 'B-boys'4) they use a number of different choreographed handclaps that are usually followed by hugs. When they greet other Dutch friends who are not part of their hip-hop culture they greet them the Dutch way, which is three kisses on the cheeks, or just a handshake. When greeting family members they usually greet the way their parents are accustomed to in their culture. So for one participant that meant greeting some friends with elaborate handclaps, other friends with three kisses on the cheeks, Dutch strangers with a handshake, and his parents the Colombian way of greeting: one kiss on the cheek (see fig. 1).

These examples of hybrid identities both suggest a cosmopolitan view of culture and demonstrate the role of agency 5 in the expression of identity. Hybrid identities, also referred to as cosmopolitan identities by Radice, are part of, and also an outcome of, multicultural societies (Radice, 2009; Noble, 2009: 6). Studies of everyday multiculturalism show that cultural identity can be diverse and complex, whereas traditional concepts and policies of

3 Kinesics - Creative Intercultural Learning was a study I conducted in 2006-7 for my MA, and included participants from nine different cultures living in Eindhoven, Netherlands. The study documented traditional dance and greetings performed by the participants, and the findings were presented graphically. This study is described in more detail in Appendix 1.

4 B-Boy is the name used, since the 1980s, for break dancers and young men that are involved in hip-hop culture (Kipfer and Chapman, 2007; Urban Dictionary, 2006).

5 Agency in anthropology and sociology refers to the ability of the individual to make their own free choices, to control their own behaviour, and act independently of the constraints of culture (Duranti, 2001: 268; Tulane University, 2000). 


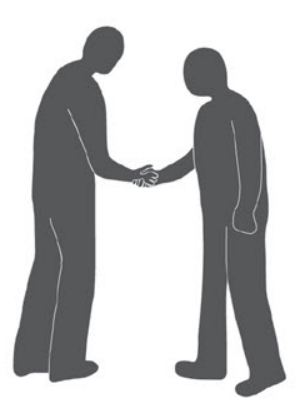

Dutch strangers

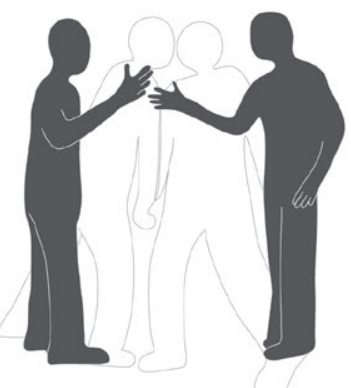

Dutch friends (hip-hop)

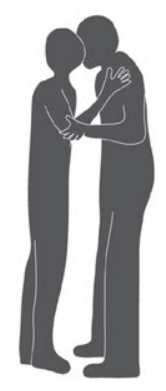

Dutch friends

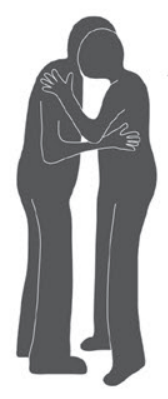

Colombian Family

Fig. 1 - Daily greetings of Dutch person with hybrid identity

multiculturalism have tended to assume that people have only one ethnic identity/affiliation (Radice, 2009: 146-147; Song, 2010; Noble, 2009: 6, 46). Multiculturalism as a descriptive term refers to the plurality of cultures within a society and the policies that promote this diversity. I discuss such policies, and the concepts that underpin them, in the following section. However, the somewhat different approach and understanding of everyday multiculturalism means that a 'theoretical criticism' of multiculturalism can be made on the grounds of its inability to capture the real lived complexities of culturally diverse societies (Noble, 2009: 6).

My own study is concerned with everyday multiculturalism and cultural diversity in the city of Stavanger. While everyday multiculturalism and more traditional concepts and policies of multiculturalism are sometimes in 
opposition, state policies of immigration and multiculturalism do impact on the everyday lives of residents in culturally diverse societies, as I will outline in the next section. Section 1.2 places the present study in its Norwegian and Scandinavian context and gives an outline of the state of immigration to the Scandinavian countries in general, and to the city of Stavanger in particular. It also includes a brief discussion on the differing policies of the Scandinavian countries in regard to integration of immigrants, and whether they embrace or reject multiculturalism.

\subsection{The Multicultural Situation in Scandinavia}

In providing an overview of the state of immigration and multiculturalism in Scandinavia, I shall focus on some key texts on these issues. The texts are primarily those featured in Peter Kivisto and Östen Wahlbeck's book Debating Multiculturalism in the Nordic Welfare State (Kivisto and Wahlbeck, 2013). They include Thomas Faist's study on social inequalities related to multiculturalism (Faist, 2013: 22-47), Hugo Stokke's study on commonalities and differences in Nordic multiculturalism (Stokke, 2013: 74-109), Grete Brochmann and Anne Britt Djuve's study on whether the Norwegian welfare state's approach is multiculturalism or assimilation (Brochmann and Djuve, 2013: 219-245), and Yngve Lithman's comparison of multicultural debates in the different Scandinavian countries (Lithman, 2013: 246-269). Reports published through Statistics Norway, and comparative studies on Scandinavia by Karen Fog Olwig (2011) and others, are also used.

Kivisto and Wahlbeck (2013: 2-3) understand multiculturalism as the principle and the practice of embracing, respecting, and accommodating difference to create multicultural solidarity. In doing so, and allowing cultural difference in society, it helps to foster integration, and increase social equalities (Kivisto and Wahlbeck, 2013: 2-3; Faist, 2013: 23; Stokke, 2013: 75). It is not merely a descriptive term (i.e. - multiple cultures). As Faist discusses, multiculturalism has multiple meanings, being a description, an ideology and a set of policies, and they all have in common the emphasis on 
the protection of immigrants and cultural minorities, as well as on advancing their sense of belonging in society (Faist, 2013: 24-25).

According to Horsti (2009: 4-7), in her study of multiculturalism in European broadcasting, multiculturalism developed as a criticism of two key things: assimilation policies and racism. Kivisto and Wahlbeck (2013) debate the difference between multiculturalism and assimilation policies in achieving 'societal solidarity'. The two policies display clear differences: the assimilation approach is that difference must be overcome, while the multiculturalism perspective is that celebration of difference is the way to reach such solidarity. Horsti interestingly points out that multiculturalism has historically been connected to anti-racism debates and movements. However, she also notes that more recently there has been a shift, and anti-racist movements tend to oppose multiculturalism's focus on cultural difference. Critics of multiculturalism from the anti-racist camp are sceptical of its emphasis on culture and cultural traditions from the past, and of the notion that cultures exist in some kind of static, segregated form (Horsti, 2009: 4-7).

Supporters of multiculturalism consider cultural diversity as being of positive value and a contribution to enriching society. Their stance is that members of minority and immigrant cultures receive recognition and respect through multiculturalism. Critics of multiculturalism see it as the opposite: rather than integrating minorities and immigrants, they argue that it limits relations and connections with other cultures in society, promotes self-segregation, fails to create a unifying national culture, alienates minority cultures, and in extreme cases leads to the growth of terrorism (Stokke, 2013: 75-76; Kivisto and Wahlbeck, 2013).

In Scandinavia multiculturalism is embraced and rejected to varying degrees. Olwig has discussed this in her study of the three countries Denmark, Sweden and Norway. These countries have strong political, social and cultural similarities but display clear differences in policies relating to the 
social inclusion of immigrants. Denmark, with a very nationalistic society, has a restrictive, assimilation-oriented policy, which rejects multiculturalism. Sweden has a multicultural policy, which celebrates cultural diversity: it admits refugees and allows immigrant family reunifications to a much higher degree. The policy of Norway is somewhere between the two, but leans more towards the restrictive policies of Denmark (Olwig, 2011). Brochmann and Djuve (2013) discuss multiculturalism in the Norwegian context, and claim that in Norway it functions more as a description of ethnically plural societies, and as relating to integration policies, rather than as an '-ism' (Brochmann and Djuve, 2013) ${ }^{6}$. When it comes to the 'layout' of the different Nordic nation states, Sweden and Norway both consist of one dominant ethno-cultural group, Finland is a multi-ethnic state and Denmark is a state with overseas territories: Greenland and the Faroe Islands (Stokke, 2013: 104). Stokke outlines different uses of multiculturalism in different Northern countries: he considers Denmark to apply multiculturalism towards its indigenous populations, and Finland to apply it towards cultural minorities, while Sweden is the only Nordic country that he considers multicultural towards its immigrants. He also notes that policymakers in the Nordic countries embrace the international human rights agenda to a higher level than in other democratic countries. However, he argues further that the human rights framework is too general to be used for policymaking, because it will not be able to target specific issues that minority cultures are concerned with (Kymlicka, 1995, cited in Stokke, 2013: 78).

Kivisto and Wahlbeck argue an important point, that multiculturalism must not be considered as a set of predefined policies and objectives ready to be implemented. Multiculturalism policies are not static, they must be shaped for specific contexts and nations, and tested and re-evaluated over time alongside changes in society (Kivisto and Wahlbeck, 2013). The extent to

6 The definition of -ism according to Oxford Dictionaries: Denoting a system, principle, or ideological movement: 'Anglicanism', 'feminism', 'hedonism' (Oxford Dictionaries, 2014). 
which policies of multiculturalism are implemented in European countries has been analysed and evaluated through various studies. Two tools used for this type of study are the Multiculturalism Policy Index (MPI) and the Migrant Integration Policy Index (MIPEX). A study using the MPI to compare multiculturalism in 21 different countries reveals that the Nordic countries Sweden, Norway and Finland are considered high scoring when it comes to their implementation of multicultural policies. Sweden is the highest scoring country in Europe, while Norway is in the middle compared to other Western European Countries (Kivisto and Wahlbeck, 2013; Lithman, 2013: 255).

In 2010 a number of world leaders, starting with the German chancellor Angela Merkel, followed by British prime minister David Cameron, and former French president Nicolas Sarkozy, among others, declared that multiculturalism had failed. They claimed that multiculturalism had led to the segregation of migrants, and that it 'has been a divisive force in coherent national societies' (Faist, 2013: 22-23). In Scandinavia, Denmark stood out as the country that clearly denounced multiculturalism, whereas Norway was for cultural diversity but started putting more emphasis on the duties of immigrants. Sweden did not see the problems of segregation as being caused by immigration, but rather by bad city planning, bad management of schools, or similar failings (Lithman, 2013: 259). Interestingly Lithman argues that this apparent European backlash against multiculturalism has not had any influence on actual policies and practices that are considered multicultural in these countries (Lithman, 2013: 260). On a similar note, Kivisto and Wahlbeck (2013) claim that the backlash has been in the area of multicultural discourse and not in abandoning multicultural policies (Kivisto and Wahlbeck, 2013). Bowen (2011) criticises national leaders for blaming everything on multiculturalism, instead of re-evaluating their foreign policies, racism, schools etc. to find out what has gone wrong.

Horsti discusses Denmark's 'End of Tolerance' strategy which explicitly declared immigrants and ethnic minorities to be 'incompatible with Danish 
values', and viewed multiculturalism as unacceptable. While not as extreme as in Denmark, signs of this shift can also be seen in the other Nordic countries (Horsti, 2009: 2). Kivisto and Wahlbeck (2013) explain that in Scandinavia, as anti-immigration sentiments and nationalism increased, multiculturalism started to be perceived negatively. Rath (2011: 1) too notes that recently, in differing degrees, the Scandinavian and a number of other European governments have moved towards more tightly controlled immigration and integration policies, in an attempt to introduce stricter entry criteria and an increased emphasis on the norms of the national culture. This move is seen as a reaction to discontent among native populations and increasing support for anti-immigrant parties (Rath, 2011: 1). While Sweden has had no populist right-wing party in parliament since 1994, Denmark and Norway have both had far-right parties in their recent parliaments. Dansk Folkeparti (The Danish People's Party) and Fremskrittspartiet (the Norwegian Progress Party) are sister parties which have continually been putting pressure on their governments to implement more restrictive refugee policies (Hervik, 2012). Kivisto and Wahlbeck group together Nordic populist parties such as the Danish People's Party, the True Finns, the Norwegian Progress Party and the Sweden Democrats, owing to their shared characteristics of being EU-sceptical, having anti-immigration policies and being against multiculturalism (Kivisto and Wahlbeck, 2013). The Norwegian Progress Party has an anti-immigrant agenda, with a stance that only those willing to become Norwegian should be admitted to the country.

Denmark has introduced strict policies for family reunification, so that it has become very difficult for immigrants to marry someone from their own country through family unification. However, because Sweden has a much more liberal policy on transnational marriages, many immigrants in Denmark move to Sweden to settle with their bride or groom from their own country (Olwig, 2011: 184). Issues relating to the anti-immigration and anti-multiculturalism policies of Denmark have received a lot of media coverage in recent years. One example was in 2005 when the Danish national newspaper Jyllands- 
Posten published 12 cartoons depicting the prophet Muhammad. This caused uproar from Muslims in Denmark and elsewhere, but despite the media coverage it received worldwide, the Danish government refused to offer any apologies or to have any dialogue about the publication, and the prime minister refused to meet with ambassadors from Muslim countries who wished to discuss the matter (Hervik, 2012: 217).

In 1973 the Swedish government introduced a key Government White Paper which aimed to improve the relationship between their national citizens and immigrants through the following goals: equal opportunities, freedom of choice and collaboration. Norway followed with a similar new political focus, and adopted Sweden's integration ideology statement with the aim of enabling social equality for all residents. These new policies considered cultural identity an individual matter (Lithman, 2013; Brochmann and Djuve, 2013). More recently the 2004 Stortingsmelding, which is a formal Norwegian governmental report, highlighted that immigrants also have individual social responsibilities, and supported the idea that it is fully possible to be a 'good Norwegian' while simultaneously identifying with and belonging to a minority culture (Lithman, 2013: 258). Norway was the first government with a majority of female ministers, and a key focus in Norwegian integration debates is the well-being of women, particularly that of Muslim women (Lithman, 2013).

While immigration policies are one matter, the daily life of, encounters with, and attitudes towards immigrants are another. Lithman (2013) discusses a Norwegian survey carried out yearly from 2002-6, which found that two thirds of the population in each of the Scandinavian countries responded with a positive attitude towards immigrants (see figs. 2-4). The survey indicates that the general population does not agree with inflammatory political statements and those fronted in mass media that tend to be negative. However, when asked how they perceived the size of the immigrant population in their country, the study reveals that they all overestimated the actual size by 60 per cent or more. 

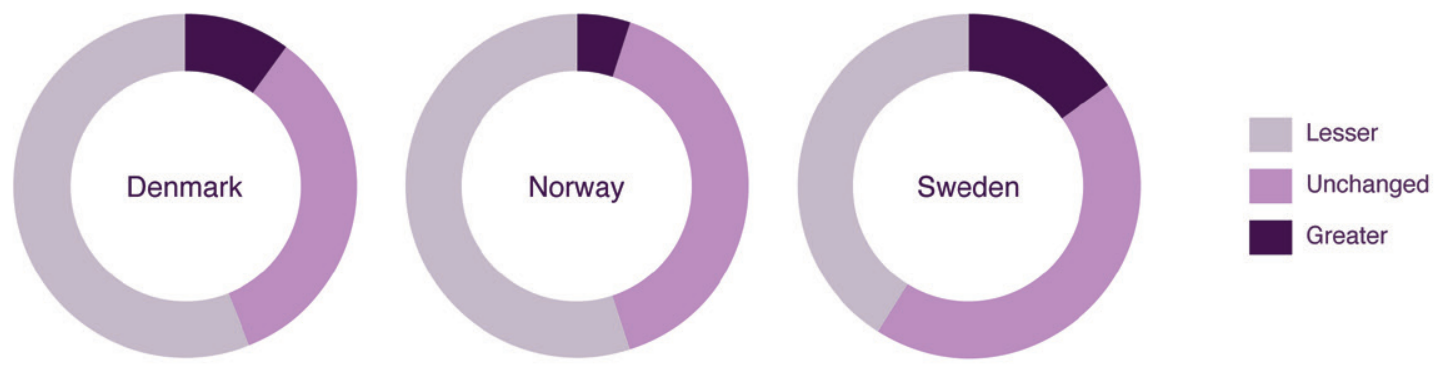

Fig. 2 - 'Should there compared with the present be greater or lesser refugee access?' (Graphic by Shortt, 2015, based on table by Lithman, 2013: 247)
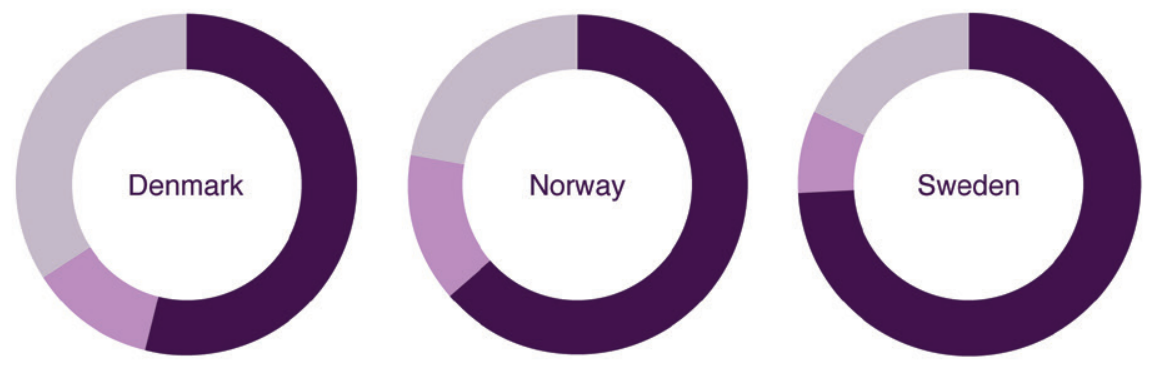

Disagree

Don't know

Agree

Fig. 3 - 'Most immigrants enrich the nation's cultural life.' (Ibid.)
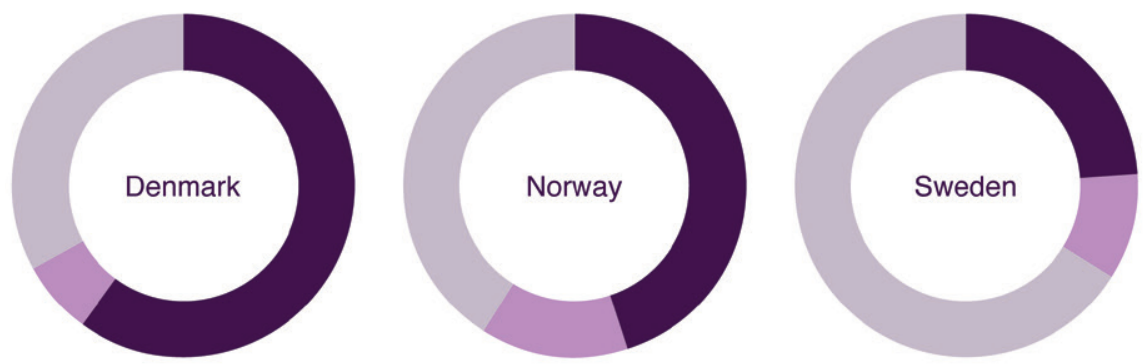

Disagree

Don't know

Agree

Fig. 4 - 'Most immigrants are a threat to security.' (Ibid.) 
In Norway, the view of the population on immigrants and their contribution to society is broadly positive. However, Olwig (2011) argues that although Norway strives to portray an image of supporting human rights and is active in leading international peace negotiations, in reality many Norwegians view foreigners with suspicion. Surveys carried out by Statistics Norway (2014b) reveal that the population's agreement as to whether immigrants make a valuable contribution to employment fell from 80 per cent in 2012 to 77 per cent in 2014. Furthermore, one third of the population believes that immigrants abuse the social welfare system. However, the majority of people still believe that immigrants contribute towards the enrichment of the cultural life of Norway, and 88-89 per cent believe that immigrants should have the same employment opportunities as Norwegians. The survey also shows that older people are more hostile towards immigrants and immigration than are other age groups, and that acceptance of immigrants is clearly highest amongst those with higher levels of education (see fig. 5) (Statistics Norway, 2014b).
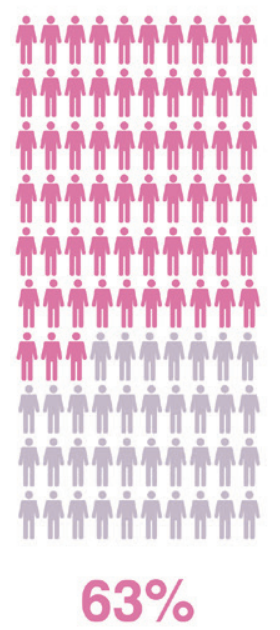

16-24 yrs

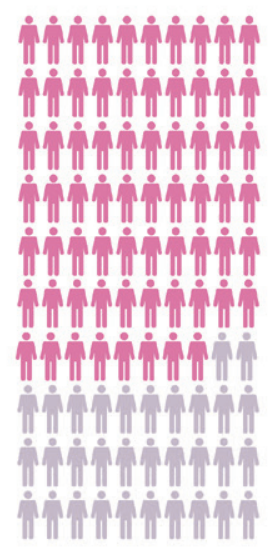

$68 \%$

$25-44$ yrs

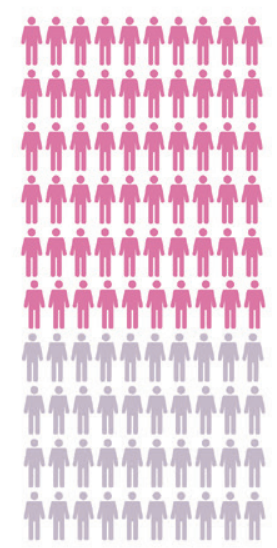

$60 \%$

$45-66$ yrs

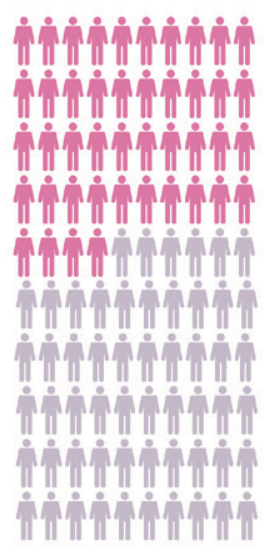

$44 \%$

$67-79$ yrs

Fig. 5 - 'Most immigrants [in Norway] misuse the social welfare arrangements' (Graphic by Shortt, 2015, based on statistics from Statistics Norway, 2014b) 
Despite this seemingly positive outlook on immigration and immigrants, Norway has experienced some serious and brutal attacks aimed at its multicultural society. The most recent, and by far the most violent, was the terror attack by Norwegian Anders Behring Breivik on the 22nd July 2011, which killed 77 people. Breivik set off a car bomb outside a government building in Oslo, which killed eight people. His intention was to demolish the Parliament's administrative building and in so doing kill the entire government working therein. He then targeted the island Utøya where the governing Labour party were organising a youth camp, and carried out a massacre there killing 69 more people, most of them teenagers. He claimed that the purpose of the attacks was to provoke a wider civil war against Islam and multiculturalism (Lister, 2012). During his trial, Breivik stated that he did not recognise the Norwegian courts, because he said the courts had received their mandates from Labour and political parties that support multiculturalism. Although he did acknowledge his acts, he did not acknowledge guilt, because he claimed that they were acts of self-defence against Norway and Europe's embrace of multiculturalism (Carlsen, 2012). Kivisto and Wahlbeck (2013: 3) draw attention to Breivik's ideology as an extreme example of how multiculturalism is often misunderstood. In the Norwegian election following the Breivik massacre, support for political parties with anti-immigration related policies, such as the Progress Party, fell dramatically. The Progress Party dropped from its usual 20 per cent of the votes to only 11.5 per cent in 2011 (Lithman, 2013). The attacks triggered a public debate on multiculturalism and immigration in Norway, and also started a debate on the possible spread-effect of Breivik's ideology (Wernersen and Sandvik, 2012). However, despite the immediate effects of the Breivik case in generating support for multiculturalism, it is striking that since 2011, the Norwegian government and policies have moved rightwards. The current government, elected in September 2013, is a centre-right coalition between the Conservative Party and the Progress Party. The previous government was a red-green coalition between the Labour Party, the Socialist Left Party and the Centre Party who had been in power since 2005. The new coalition government 
marks a historical change for Norway, as it is the Progress Party's first time in government since its establishment in 1973. The Progress Party, as mentioned, is known for pushing towards stricter immigration policies.

It is clear that multiculturalism is an important and live issue in Scandinavia. In a European context, Scandinavia has scored high when it comes to implementing multicultural policies. However, recent years have seen an increase in nationalism, more negative attitudes towards multiculturalism, and the implementation of more restrictive immigration policies. Despite surveys showing that there are currently broadly positive attitudes towards immigrants, recent years have seen a growing sentiment of negativity and hostility towards immigrants. Changes at governmental level influence the lives of immigrants in terms of their rights and what is expected of them when it comes to integration and their contribution to society. However, as I have discussed in the previous section, studies of everyday experiences of living with cultural diversity on the ground often paint a different picture of the real situation than do policies and legislations. I shall return to examples of everyday multiculturalism in my own study of cultural diversity in Stavanger. To provide a more detailed context for this study, I will next give an overview of the immigration populations in Scandinavia and Stavanger.

\subsection{The Immigrant Population of Scandinavia}

The fact that the three Scandinavian countries have considerably different policies on immigration and political asylum for refugees has affected the size of the immigrant population in each country. Denmark has a relatively low level of immigration, owing to their restrictive immigration policy, while Sweden has a higher level of immigration, mainly because they receive a large number of refugees in need of protection. The number of immigrants in Norway has risen dramatically since 1980 (see fig. 6). Sweden has around three times as many immigrants as Norway and Denmark (see fig. 7) (Pettersen and Østby, 2013; Stokke, 2013). 


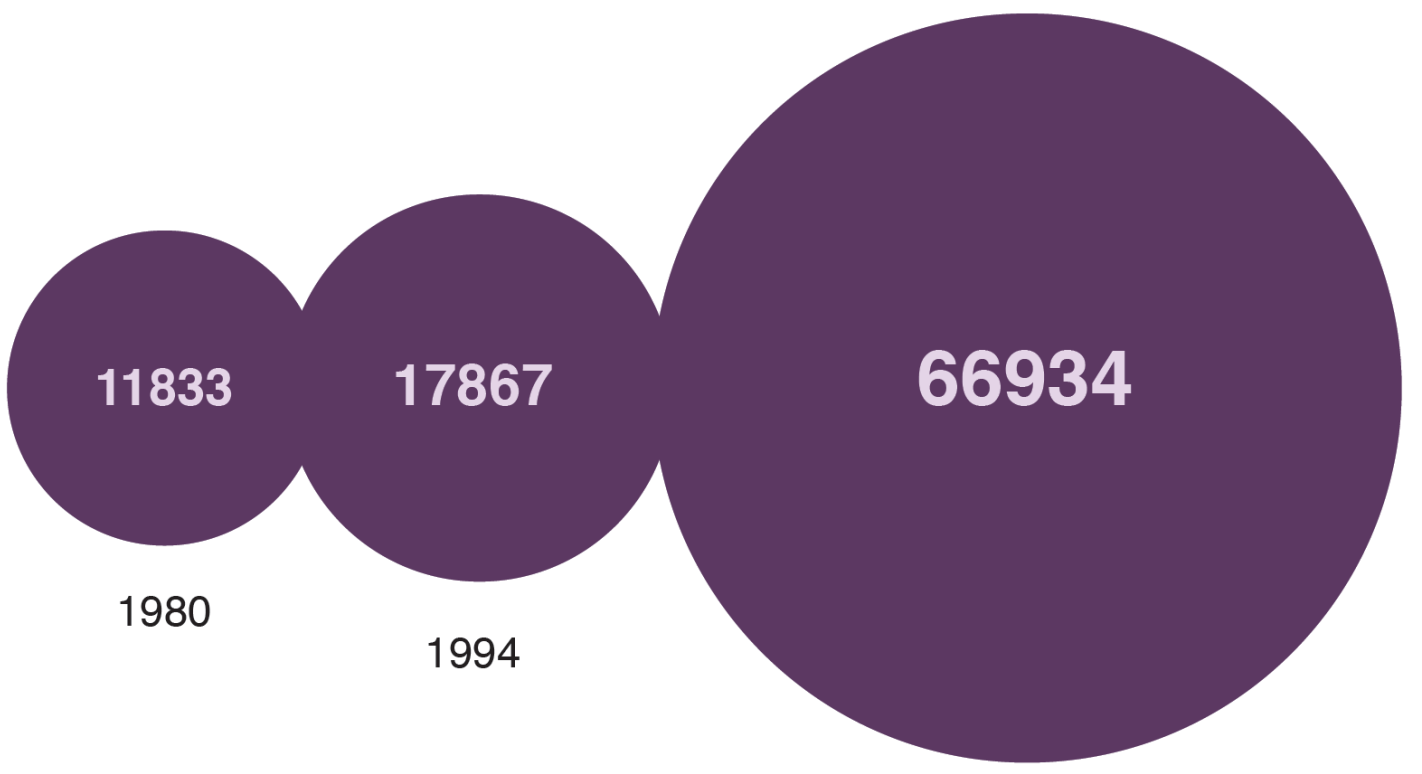

2013

Fig. 6 - Numbers of foreign nationals who migrated to Norway in 1980, 1994 and 2013 (Graphic by Shortt, 2015, based on numbers from Statistics Norway, 2014c)

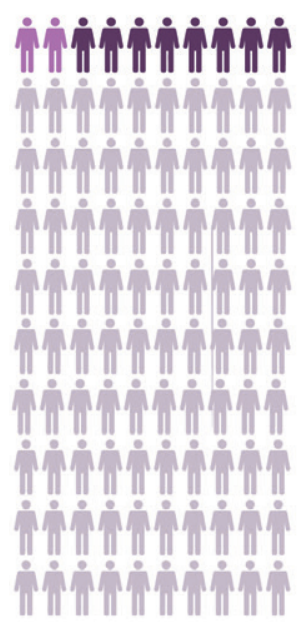

Denmark

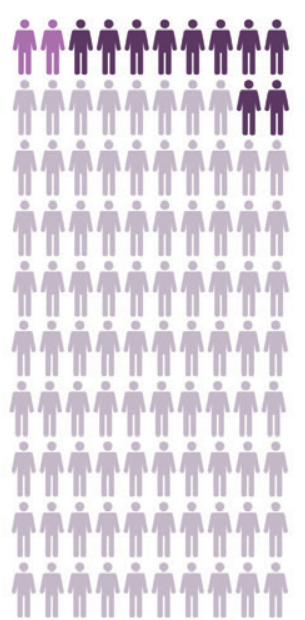

Norway

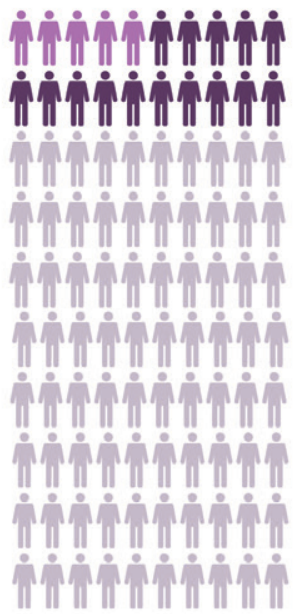

Sweden

Fig. 7 - Compositions of populations in Norway, Sweden and Denmark, 2012 (Graphic by Shortt, 2015, based on graph by Pettersen and Østby, 2013) 
Until 1973, the main driving force of immigration to the Scandinavian countries was people arriving for employment. However, the oil crisis ${ }^{7}$ resulted in restrictions on labour immigration, and after this point, the reasons for immigration to Scandinavia changed from seeking labour to, primarily, family reunifications and fleeing war. In this period from the early 1970s to mid 1990s, immigrants came mainly from Chile, Vietnam, Iran, the former Yugoslavia, Iraq and Somalia. More recently, since 1994, the Nordic countries have been open to the European labour market, and the EUextension in 2004 opened Norway, even though it is not a member of the European Union, for labour immigration yet again. Since 2004, immigration has mainly consisted of workers from Poland and the Baltic States, as well as people arriving for family reunifications, and war refugees. Almost half of immigrants in Scandinavia are from Asian, African or Latin-American countries (Pettersen and Østby, 2013).

Norway has had a higher number of labour immigrants from the EU than its neighbouring countries. The country has become attractive abroad owing to its economic development in the past years. As employment opportunities are many and salaries are high ${ }^{8}$, immigrants tend to get work in Norway more easily and benefit more quickly from it, than they do in the other two Scandinavian countries. While the number of immigrants has increased, the duration of their stay in Norway has shortened. Labour immigrants who came from Turkey and Pakistan to Norway and Denmark in the 1970s tended to settle down and bring up their children in Scandinavia. Today the majority of immigrants tend to stay in Norway for a few years only (Pettersen and $\varnothing$ stby, 2013).

7 The oil crisis of the 1970s developed after Arab oil producers imposed an oil embargo to punish the US and the west for their support of Israel in the Arab-Israeli war. This caused a sharp increase in oil prices, and industrial countries suffered economic downturn as a result. In Scandinavia it led to restrictions on immigration from countries outside the Nordic region (Macalister, 2011; Kemp and Harkavy, 1997; Pettersen and Østby, 2013).

8 The average monthly wage of people with full-time employment in Norway was NOK 42500 (approximately £ 4261) in 2013 (Statistics Norway, 2014a). 
When it comes to the integration of immigrants, the Scandinavian welfare systems have implemented extensive social services and health programmes. In all three countries, refugees are expected to complete an elaborate introduction programme before they seek employment. This programme involves learning the local language, and the social values, customs - including greetings - and traditions of the country (Olwig, 2011). The visual system developed in my research project could potentially make a valuable contribution to such introduction programmes, helping to raise awareness among newcomers about the different local greeting customs. It could also be valuable in teaching Norwegian residents about the different greeting customs of immigrants and newcomers to the country.

Welfare and induction programmes are examples of points where government systems and everyday experiences intersect. Kivisto and Wahlbeck (2013) note that welfare states are 'viewed pragmatically', meaning policies are revised and changed when called for by changing societies and conditions - they are not static sets of policies (Lithman, 2013). The Norwegian welfare state is central in both controlling immigration, and integrating new arrivals into working life and society in general. It has committed to creating equal treatment for foreign nationals and Norwegian citizens (Brochmann and Djuve, 2013: 222). The Introductory Act brought in new measures in 2002, giving immigrants the right to participate in a full-time qualification programme. This programme is the most financially generous integration programme of its kind. Participants of the full-time programme receive benefits of NOK 175,000 yearly. The goal is for newcomers to be prepared for the labour market and to integrate better into society (Brochmann and Djuve, 2013). However, critics of the welfare system view it as having ineffective integration policies, and have raised questions regarding the quality of training efforts, and concerning welfare dependency, claiming that the system undermines immigrants and does not encourage them to be self-reliant. 
Valenta and Bunar (2010) carried out a comparative study of refugee integration policies in Sweden and Norway. They argue that the policies have been unsuccessful in reducing inequalities between refugees and the rest of the population, and claim that such policies are based on outdated theories and concepts. Both countries have extensive state-run introduction programmes, but have two very different ways of approaching these programmes. In Norway the authorities impose more demands on the refugees, such as the penalty of losing their cash benefits if they do not attend the compulsory introduction programme. In Sweden it is up to the individual municipality to decide what to do, and whether to penalise dropouts from their programmes. Owing to its strict immigration policy, Norway has a much higher participation rate in its programmes than Sweden. In both countries, the authorities are responsible for providing housing for refugees and also decide the location where they will be settled. In Norway, if the refugees wish to settle elsewhere, they will not receive governmental support and are responsible for paying their own way. Valenta and Bunar conclude that although Sweden and Norway have very well developed integration programmes and housing assistance for immigrants, the policies have limited effect, and are in themselves not enough to successfully integrate refugees (Valenta and Bunar 2010). Olwig argues that the welfare systems have gone too far and adopted many of the responsibilities that other countries consider to be responsibilities of the family. She claims that integration policies and programmes are partly attempts at removing refugees from their tight-knit family groupings, treating them as individuals who should integrate into the Scandinavian culture and society (Olwig, 2011: 179-180).

The backlash against multiculturalism in Scandinavia and Europe does not mean that assimilation is, in reality, taking its place as state policy and social practice. As Wise points out, changes in policies and discourse do not necessarily reflect everyday experience (Wise, 2005). People have to negotiate their interpersonal and cross-cultural interactions whether their governments embrace or reject multiculturalism. While there is a link between 
policies and everyday multiculturalism, the latter has a 'dynamic of its own', and the study of everyday interactions offers the greatest understanding of how people interact cross-culturally (Kivisto and Wahlbeck, 2013: 336).

I have already discussed studies that deal with Scandinavian populations' attitudes towards minorities, but interestingly, as noted by Kivisto and Wahlbeck (2013), there is a lack of studies focusing on the interaction between majority and minority groups. Part of my research deals with such interactions, and looks at greetings across a number of cultural groups. While the state-run introduction programmes educate immigrants in Norwegian traditions, language, behaviour and greeting customs, this knowledge of the host culture does not eliminate the complexity of cross-cultural interactions and greetings that exist in daily life. Cultural difference persists, as can be seen during both majority/minority and minority/minority interactions. In terms of greetings such differences can relate to what type of greeting is used or, as mentioned earlier, to more subtle nuances such as the way and with whom people perform a specific greeting. These are exactly the types of interactions my research seeks to document and analyse.

\subsection{The Immigrant Population of Stavanger}

My research (discussed in detail in chapter 4) focuses on interactions within and between different cultures in the multicultural city of Stavanger. Stavanger has seen a steady rise in immigration in the past 30-40 years, and the various types of immigrants from all over the world make it an interesting source for the study of cultural difference. This section aims to give a picture of the immigrant population of Stavanger in terms of growth, changes in types of immigration, and the organisations and events promoting and celebrating this diversity, as well as those opposing it.

The immigrant population of Stavanger has increased by over 14 per cent since 1980, and now makes up more than 20 per cent of the total population (see figs. 8-9). The steady growth of the immigrant population in Stavanger 


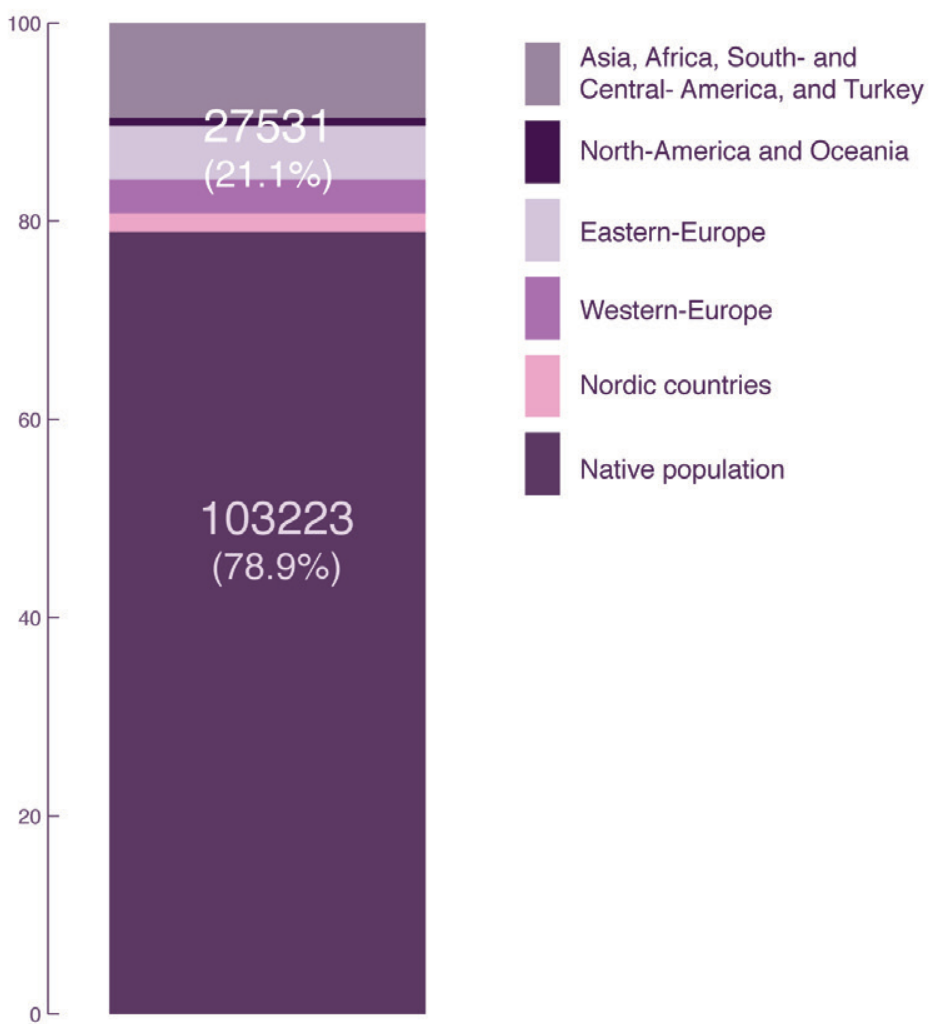

Fig. 8 - Distribution of the native and the immigrant population of Stavanger in 2014 (Graphic by Shortt, 2015, based on numbers from Stavanger-statistikken, 2014)

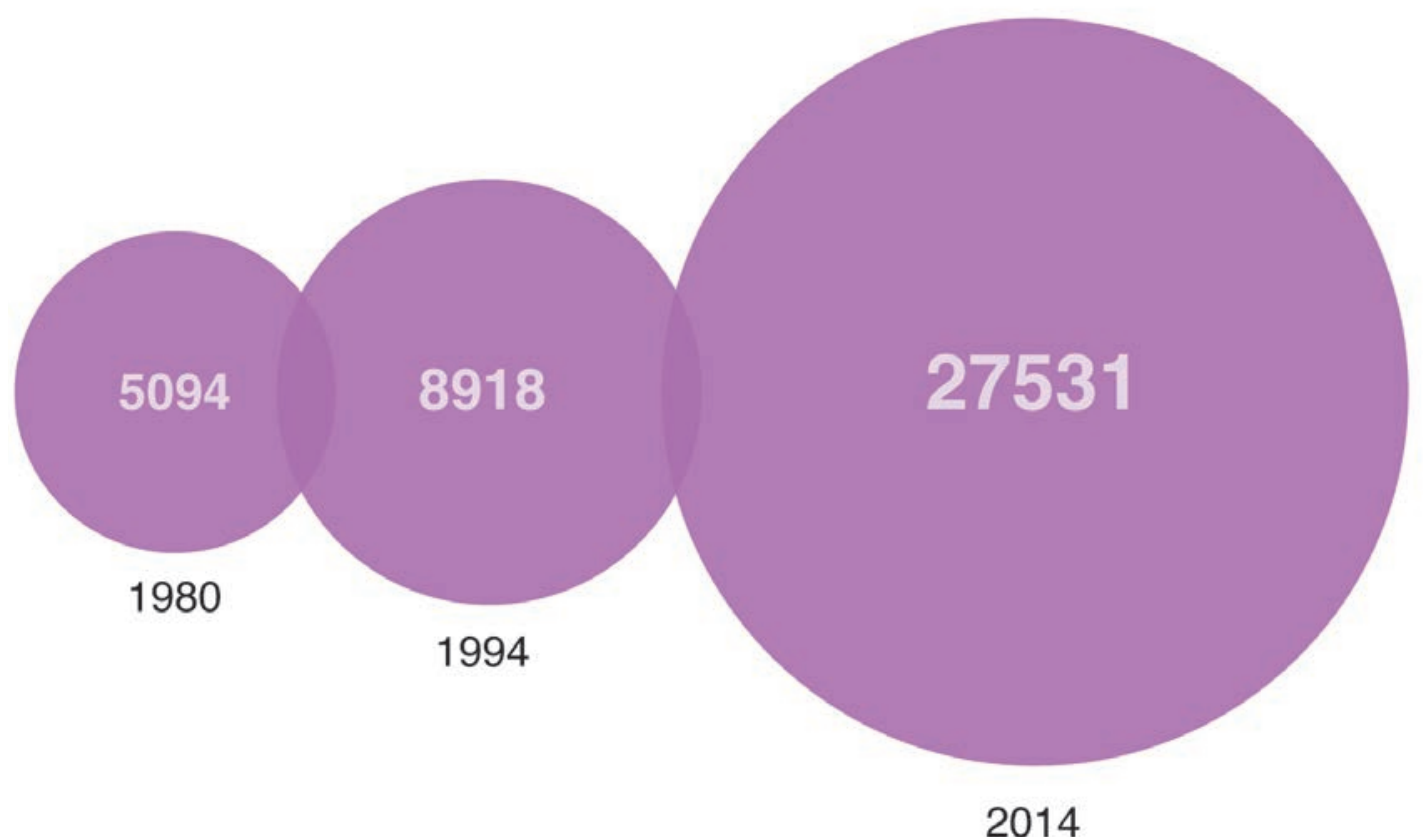

Fig. 9 - Number of Immigrants (incl. Norwegians with immigrant parents) living in Stavanger 1980, 1994, and 2014 (Graphic by Shortt, 2015, based on numbers from Stavanger-statistikken, 2014) 
has turned Norway's fourth most populated city into a culturally diverse society. The immigrant population includes over 170 different nationalities (Stavanger-statistikken, 2014). People from other Nordic countries and Europe make up around one half of the immigrant population in Stavanger, while immigrants from Asia, Africa, South- and Central- America, and Turkey make up the other half (see fig. 8).

From 1990 to 2012, while the majority of immigration to Norway as a whole was for family unifications, the predominant form of migration to Stavanger in particular was labour immigration, and then family unifications (see fig. 10). The growing oil industry, which has its heart in Stavanger, also named the 'Oil Capital of Norway', is part of the reason for the high number of labour immigrants.
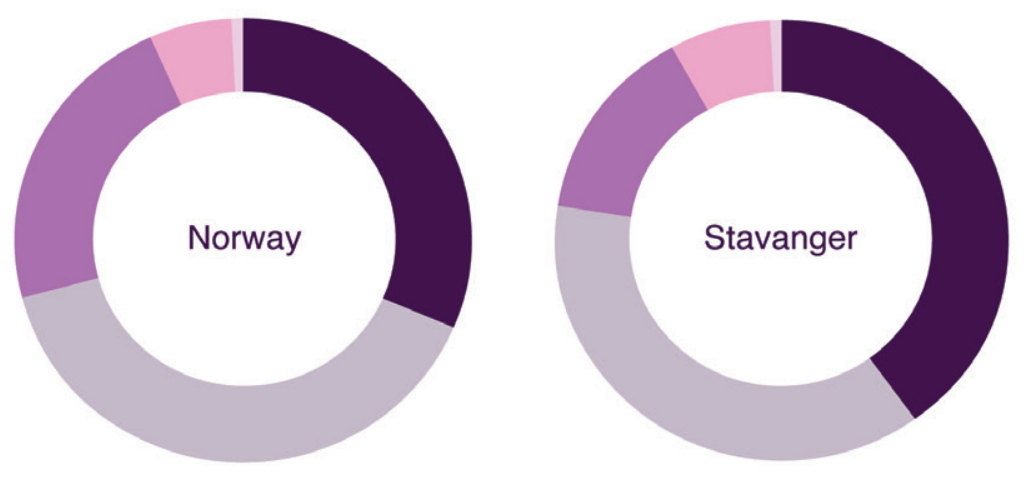

Fig. 10 - Types of immigration to Norway and Stavanger 1990-2013 (Graphic by Shortt, 2015, based on numbers from Egge-Hoveid, 2014)

There are many events organised regularly in Stavanger celebrating its ethnic and cultural diversity. Every Saturday afternoon an 'international cultural café' is organised in a location in the city centre, and is run by people from a different culture each week - such as Pakistani, Filipino, Somali, Turkish, Greek, and others - who cook and sell traditional food from their country. Meals are cheap and there is often entertainment, such as 
music or dance, during the events. Another event called 'Global Morning' is arranged monthly at Sølvberget, the city's cultural arena and library. This event combines breakfast with presentations by researchers and influential people working on topics of multiculturalism, culture and humanitarian aid (Sølvberget, 2014). However, the largest celebration of Stavanger's cultural diversity takes place annually on the national Constitution Day on the 17th May. On a day dedicated nationally to celebrating the Norwegian constitution, when patriotism and Norwegian flags are flying high, an event celebrating Stavanger's cultural diversity is held in a park close to the city centre. The event includes music, dance and entertainment from different cultural organisations based in the region. The organisations, and some ethnic restaurants, also have food stalls in the park (see fig. 11). The event draws large crowds of people and has been extremely successful in recent years.

In contrast to such events aimed at celebrating diversity is an on-going current of racism. A group named Borgervernet Rogaland ('Vigilante Committee Rogaland') recently distributed 1500 flyers in the Stavanger region and that of its neighbouring city, Sandnes (see fig. 12). Two different flyers were distributed: one was a warning to all neighbours that they should oppose an asylum centre that is planned to be built in the area, and the other was a flyer promoting Nordfront, a Norwegian resistance organisation. The organisation, which originated in Sweden, promotes a united people and opposes immigration and a multicultural society, and is criticised for being racist and fascist (Hæreid, 2014; Frafjord, Selmer-Anderssen and Topdahl, 2014). Their action received huge coverage and criticism in local and national media. As a response to the flyer distribution, a socialist NGO, Motmakt ('Counterpoise'), organised a demonstration against racism, opposing organisations such as Borgervernet Rogaland and Nordfront (Wilkins, 2012). The demonstration was held outside Sølvberget in Stavanger and attracted large crowds (see fig. 13). 

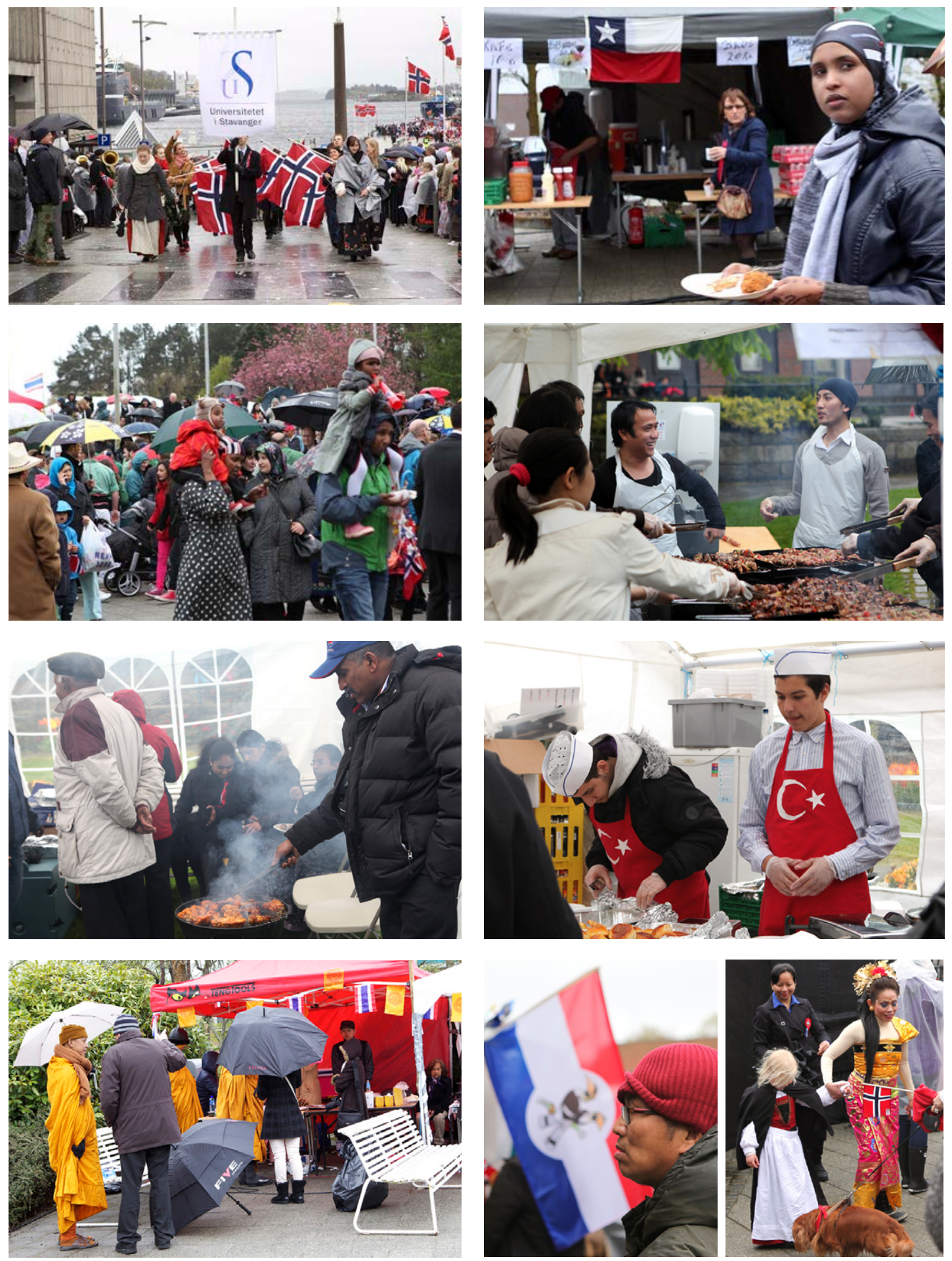

Fig. 11 - Norwegian Constitution Day (17th May) international celebrations in Stavanger, 2012 


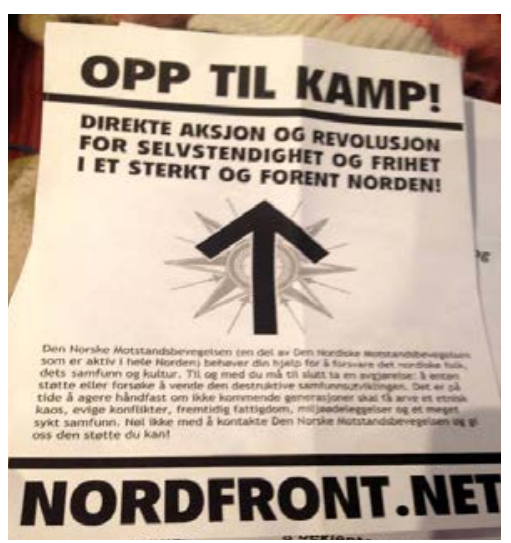

Fig. 12 - Flyer distributed by Borgervernet (Hæreid, 2014)

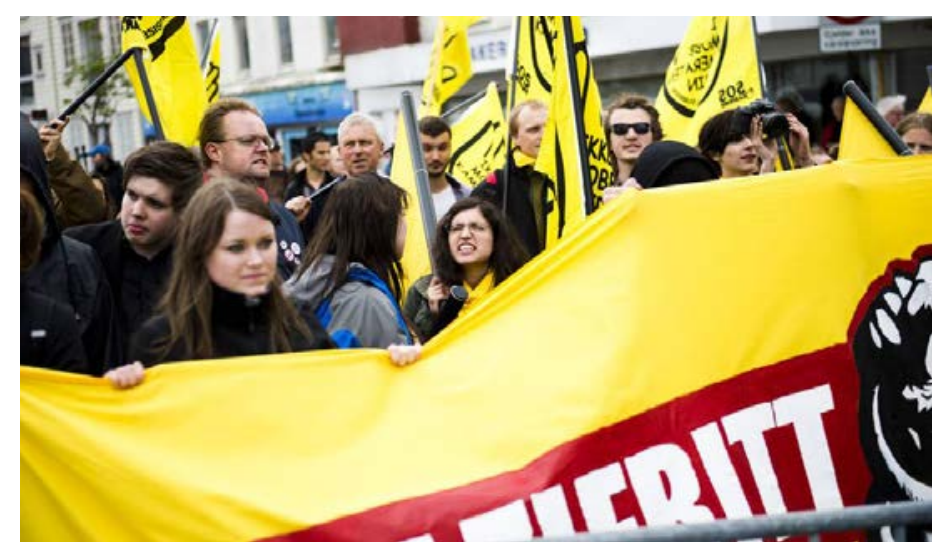

Fig. 13 - Organisations (SOS Rasisme and Motmakt) demonstrating against racism in Stavanger (Waage, 2014)

Demonstrators also criticised the management of Sølvberget, who were preparing to host a debate on racism a few days later. The debate included representatives of regional asylum centres, political parties, organisations fighting racism and also, to the annoyance of the demonstrators, the leader of the vigilante committee. Demonstrators claimed that this would give him and his organisation undeserved media coverage, even a form of validity, while organisers were adamant that the debate must include all parties and opinions, and went ahead with the debate as planned (Tiller, 2014; Aalen, 2014). So, while Stavanger is a highly multicultural city and hosts a number of events and organisations focused on celebrating its diversity, there is also a vocal strand of public sentiment and various pressure groups that oppose immigration and cultural diversity. This is the context in which my present study takes place.

\subsection{Everyday Interactions \& Nonverbal Communication in a}

\section{Multicultural Setting}

It is clear that whether the goal for integration is assimilation or

multiculturalism, and whether we discuss everyday multiculturalism, cultural diversity or hybrid identities, successful interaction and communication between cultures is crucial in any society. The most obvious challenge to communication in any multicultural society is verbal language barriers. 
While second-generation immigrants tend to speak the local language, their parents sometimes do not. Likewise, newly arrived refugees and immigrants also need time to learn the local language properly. Communication across cultures can be challenging without a shared language in which people can express themselves fully. In her study of multicultural interactions in an Australian suburb, Wise (2005) found that immigrants often feel shy and embarrassed about not being able to speak the (English) language. This can lead to conflict and misunderstanding: existing residents feel cut off, lonely and angry in a neighbourhood of strangers with whom they have no interaction, and so view immigrants as antisocial and unfriendly, when they are merely separated by language (Wise, 2005). The same issue was found to be central in Hudson, Phillips and Ray's study, in which participating immigrants from Somalia expressed that it was frustrating not to be able to speak English, and therefore hard for them to approach and interact with the local English people. The locals, on the other hand, often experienced this as the Somalis not wanting to mix (Hudson, Phillips and Ray, 2009).

These communication challenges are the reasons why my study focuses on the nonverbal dimensions of greeting. Because such language barriers exist in multicultural societies, I think it is increasingly important to create visual systems and tools for analysis, rather than relying on textual analysis. A visual system can cross language barriers: it opens up the possibility to communicate across languages, to potentially impact wider audiences. The aim with my visual system of presenting greetings is to create awareness and understanding of cultural differences within multicultural societies. The system has a number of potential social applications. If installed in a public location, for example, it could be used by local residents, in this case the residents of Stavanger, to view the different dimensions of their own greeting behaviour compared to that of people from other cultural backgrounds within their society. Due to its visual nature it would be accessible to everyone involved in the study and beyond it, regardless of whether they speak the local language or not. 
I have chosen to study the greeting as a form of interaction because it is a daily ritual that is practised within and across most cultures. However, as I discussed at the start of this chapter, the 'performance' of a greeting, its nonverbal dimensions, can be as culturally different as the verbal greetings. During a greeting we project an abundance of nonverbal cues and information: about personal space, physical contact, posture and facial expressions. The universality of greetings lends itself superbly to a study like this. In a multicultural context the greeting can be studied as a symbol and a projection of cultural identities. It reminds us of difference, and functions as an identity marker through which difference can be studied. The example previously given (see fig. 1), of the Dutch person who was bicultural and also part of a subculture, is a good illustration of this. His different identities influenced his behaviour and greeting interactions considerably.

My study aims to cast light on what Wise and Velayutham (2009) termed the micro-sociology of everyday interactions, which also reflect wider social structures and processes. In doing so I wish to approach the situation on the ground to reveal aspects of how people live with and experience the complexities of cultural difference, and of how they adapt and relate to each other on an everyday basis, through the seemingly straightforward ritual of greeting. 


\section{Greetings: Approaches and Visual Notation within Anthropology}

\subsection{Functions of the Greeting}

As discussed in the previous chapter, the aim of the visual system developed in my research is to advance a wider understanding of nonverbal, culturerelated dimensions of greetings. As well as potentially playing a role in multicultural communications, my system contributes new visual research methods to the field of anthropology and studies of human behaviour. My system builds on other systems developed within anthropology, dance, and information visualisation, and uses digital technologies to create new pathways to explore the visual notation of human interaction.

In this chapter I will discuss three key areas concerning the anthropological study of greetings: first, I will outline the function and meaning of greetings, and chart how the understanding of greetings has changed during the past 40-50 years; second, I will explore the extent to which visual methods have been used in the study of greetings and human encounters, mainly in the 1960s and 1970s, and how such methods could potentially be advanced using contemporary technologies and software; third, I suggest that there is a lack of intercultural greetings studies, and that today's multicultural world needs studies concerning cross-cultural greetings.

The greeting is a universal daily ritual involving both verbal and nonverbal content such as gestures and facial expressions. Studies of greetings have evolved significantly over the past 40-50 years and this period has seen a major transformation within anthropology in the perception of both their function and their wider cultural meanings. Definitions have gradually shifted from greetings being considered rituals of phatic communication in the 1960s-70s to their being understood, since the early 1990s, as exchanges of important social information. My research project approaches the study 
of greetings by investigating the cultural information conveyed by their nonverbal signals.

In the 1960s-70s key researchers in the field, such as Eibl-Eibesfeldt and Kendon \& Ferber, considered greetings as rituals of appeasing and bonding with the function of avoiding aggressive behaviour in human encounters (Duranti, 1997b: 64). Greetings were also defined in this period as accessrituals (for example, by Firth \& Goffman) because they were seen as gateways allowing increased access to interaction. A minimal and non-hostile form of interaction, the greeting was believed to function as an introduction to further social interaction and relationships. Greetings marked the transition from a state of no contact to a state of increased communication between human beings. This interaction was considered important when it came to ensuring the continuity of social relationships (Ameka, 2009: 127; Duranti, 1997b: 65). Further studies in the mid-1970s interpreted greetings as acts of recognition, politeness and solidarity affirmation (Youssouf, Grimshaw and Bird 1976: 811). Common to all these understandings was that greetings were considered as actions either preventing harm or actions inviting to further forms of communication. However, greetings were not considered as meaningful message exchanges in themselves, and the general view at the time was that they were functional but contentless, phatic forms of communication.

This view was challenged in the 1990s when the deeper sociocultural dimensions of greetings, which Duranti $(1992,1997 \mathrm{~b})$ termed their 'contextual and propositional content', became the prevailing focus of greetings studies. Professor of anthropology Alessandro Duranti has published extensively on the role of verbal and visual communication in human greetings since the early 1990s. His focus is on agency, intentionality and intersubjectivity, and his research methods combine participant observation and audiovisual recordings of spontaneous interactions. Duranti, a seminal figure in the field of greetings studies and linguistic anthropology, 
views language as a cultural practice that gains meaning through its temporal organisation. He studies different types of speech acts, such as the greeting act, to analyse the role of verbal and visual communication in political and everyday situations. Though Duranti (1997b) agreed with Firth \& Goffman that greetings are formulaic expressions, he argued that greetings also adapt to and create new contexts, and involve important information exchange. He found that owing to the predictable nature of greetings, researchers tended to ignore the value of what is said during the greeting. Duranti's criticism of the greeting being considered a phatic form of communication was that this view did not consider variations in what is said as relevant to the meaning of the greeting exchange. Although greetings are ritualistic and conventional, he claims that both what is said and how it is said are important and can communicate new information. Duranti saw the need for an accurate definition of what qualifies as a greeting, so he created six criteria that would enable a universal identification of what a greeting is. In broad terms these criteria cover three important areas: what is said during the greeting; definitions of greetings that are based on re-formulations of other researchers' definitions; and the temporal and spatial organisation of the greeting (Duranti, 1997b: 67-68).

Duranti's view was that the key to understanding the greeting is found in the culture that performs it. He noted that greetings can reflect social hierarchies, as the act of greeting often distinguishes between beings worth recognising and those that are not. Such a hierarchy is investigated in his studies on Samoan ceremonial greetings. During these ceremonial greetings children and servants, for instance, are not recognised and greeted, while chiefs and higher rank people are greeted. Further he illustrates that participants in the ceremonial greetings interact as part of a group and hierarchy, while simultaneously maintaining their individual identities. Through Duranti's analysis of the internal organisation of the ceremonial greetings subtle individual differences in status and verbal performance are revealed. His studies prove that greetings are more than phatic communication: they can 
reveal layers of social and individual information if documented and analysed carefully (Duranti, 1992, 1997a, 1997b).

This key shift in greetings studies, which moves away from the idea of phatic communication, has brought with it the development of important cultural knowledge and an increasing number of studies on the sociocultural dimensions of greetings. Some key examples of these studies focus on: the initiation and social order of greetings (Kendon, 2009; Arendsen, 2008; Schleicher 1997); the hierarchy of salutation and submission (Walter, 2009); the religious dimensions of greetings (Ameka, 2009; Duranti, 1997b; Schleicher, 1997); and rituals that visualise inequality (Ameka, 2009). Studies such as these show that greetings are rich sources of cultural knowledge, and can reveal culture-related behaviour patterns. Each greeting has a 'cultural-situational frame' (Ameka, 2009: 131) and valuable cultural knowledge can be extracted from, and visualised through, systematic studies of greetings. It is the context of the interaction that determines what greeting is performed and how the relationship between the people who greet is affirmed (Kendon, 2009). History, religion, social hierarchy and gender roles are all reflected and intertwined in the greeting ritual. According to the late anthropologist Edward T. Hall (1990), one can study any aspect of a culture and come up with the same result in terms of the cultural knowledge produced. If by studying one part the whole can be understood, then studying the specific act of greeting can provide insights into the broader patterns of interaction and behaviour in society.

\subsection{Verbal \& Nonverbal Aspects of Greetings}

Greetings studies vary in the extent to which they focus on the verbal or the nonverbal dimensions of the interaction. Duranti's studies, and those following his, focus on the sociolinguistic aspects of greetings - analysing the meaning of what is said during the encounter. However, while his own fieldwork focuses on the spoken dimension, the definitions and criteria for studying greetings that Duranti developed also emphasise the importance 
of nonverbal features (Duranti, 1992; 1997b: 68). These nonverbal features include posture, gesture and movement, which are all important bearers of meaning that can give us information about each participant in the greeting exchange. Although Duranti's own research focused on verbal exchanges, interestingly he claimed that information is exchanged whether there is talk present or not.

A fundamentally nonverbal approach to the study of face-to-face communication, including greetings, is found in Adam Kendon's work. Kendon, who is considered a world-leading researcher on gesture, has since the late 1980s published extensively on the topics of communication during face-to-face interaction, and on the relationship between gestures and spoken language. In his book Conducting Interaction Patterns of Behavior in Focused Encounters, first published in 1990, he analyses the nonverbal dimensions of face-to-face encounters including: sequential organisation of greetings, movement coordination, facial patterns, male/female differences, duration and gaze (Kendon, 2009 [1990]). Kendon's and other studies of the nonverbal aspects of greetings tend to discuss such observable patterns and their functions without going further to examine the cultural meanings of these nonverbal signals and patterns.

Before Duranti's studies in the early to mid-1990s, greetings studies generally tended to focus on the nonverbal (Kendon 2009 [1990], Birdwhistell, 1970). However, after Duranti, when the idea of information exchange came into focus, attention shifted from the nonverbal to the verbal. My attention is on the nonverbal, and I would argue that a study of both the nonverbal signals and their cultural meanings is necessary in order to understand greetings. This is what my research aims to make possible by means of visual representation and analysis. 


\subsection{Visual Representations of Human Interactions Including Greetings}

Despite the varied themes and focuses within greetings studies, the research methods used until now by researchers in this area have remained predominantly textual. Though most studies involve ethnographic methods and the use of photography or video as documentation, the analysis of greetings consists primarily of textual transcripts and explanations. There are a few exceptions, in which anthropologists have used or created visual tools or notation systems to analyse human interaction. Some such examples are Hall's notation of proxemic behaviour (Hall, 1963), Birdwhistell's notation of kinesics (Birdwhistell, 1970) and Kendon's notation of face-to-face interaction (Kendon, 2009 [1990]). I will provide a detailed description and analysis of selected elements of these different visual systems in order to chart how each researcher has used graphic symbols to communicate intricate aspects of nonverbal communication relating to personal space, body movement and facial expressions. While technology has developed considerably since the creation of these systems, they are still of significant value and an inspiration to the development of my own system. My aim is to convey human interactions, or greetings, in a new way through a clear, visual language. I am able to build on the knowledge and methods provided by researchers such as Duranti, Hall, Birdwhistell and Kendon while also being able to utilise today's tools and technologies for creating a digital interface for visualisation.

The late American anthropologist Edward T. Hall (1914-2009) published his most influential work on nonverbal communication and international relations between 1955 and 1977. Hall's theories of intercultural communication, in particular relating to the use of personal space, have been invaluable in my research. He coined the term proxemics, which means people's use of space and its relation to interpersonal communication. Proxemics can be separated into two main studies: personal space, and territory. Personal space relates to the immediate space surrounding a person, and territory refers to (physical) areas a person may lay claim to, such as a room or a car. Hall's view was that much of behaviour is learned and patterned by culture, 
and that once learned, proxemic behaviour becomes a form of unconscious behaviour. His theories are still highly relevant today and are referenced by many authors in the field of nonverbal behaviour. He developed a system for the notation of proxemics in 1963 on the grounds that people need to be able to read each other's behaviour, and because he saw a need for a method that could accurately record and communicate what goes on during human-to-human interaction (Hall, 1963). The notation system was primarily developed to create some guidelines for the research on proxemics and to enable fieldworkers to document their observations in a visual way, allowing them to record and compare similar events across time and space. Therefore the main purpose of Hall's system was for use in documentation, rather than for public presentation of analyses - he used the visual notation in his own private recordings, but they were not featured in his publications for readers to see, perhaps because he felt the system needed further development.

Hall's notation system is a rigorous one that covers numerous possible combinations of distance and kinesthetic relationships, of contact/non-contact situations, and sixteen combinations of the ways in which people look at each other. While today, digital technologies such as computer visualisation software make it possible to carry out very precise visual representations more easily, his system was created at a time when the available technology was much more basic and costly. His system includes notation of: sex and basic posture (see fig. 14); spatial arrangements or orientations (see fig. 15); kinesthetics (see fig. 16); touch (see fig. 17); vision (see fig. 18); thermal factors (see fig. 19); olfaction (see fig. 20); and the scale of voice loudness (see fig. 21).

The sex and basic posture of the two people interacting are the first key factors determined graphically within Hall's proxemic notation. His notation includes three symbols for male, and three for female, to illustrate whether they are lying down, sitting or squatting, or standing (see fig. 14). 
1) Postural-sex identifier

male

Fig. 14 - Hall's proxemic notation: sex and basic posture (1963)

3) Kinesthetic factors
$11 \|$
$33 \square$
$101 \|$
14
$12 \mathrm{~K}$

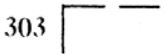
$102 \mid<$

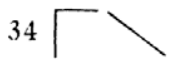
$22 P$
44

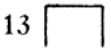
404
$103 \square$

Fig. 16 - Hall's proxemic notation: kinesthetics (1963)

5) Retinal combinations (Visual code)

$\begin{array}{lll}\text { foveal } & \mathrm{f} & 1 \\ \text { macular (clear) } & \mathrm{m} & 2 \\ \text { peripheral } & \mathrm{p} & 3 \\ \text { no contact } & \mathrm{nc} & 8\end{array}$

Fig. 18 - Hall's proxemic notation: visual code (1963)

\section{7) Olfaction code}

differentiated body odors detectable do 1 undifferentiated body odors detectable ubo 2 breath detectable olfaction probably present olfaction not present

Fig. 20 - Hall's proxemic notation: olfaction code (1963)
2) Orientation of bodies (SFP axis)

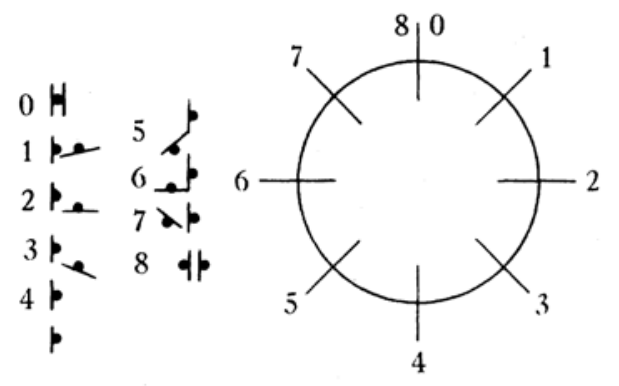

Fig. 15 - Hall's proxemic notation: spatial arrangements or orientation (1963)

4) Touch code

caressing \& holding feeling or caressing prolonged holding holding or pressing against spot touching accidental brushing no contact

0
1
2
3
4
5
6

Fig. 17 - Hall's proxemic notation: touch (1963)

6) Thermal code

$\begin{array}{lll}\text { contact heat } & \text { thc } & 1 \\ \text { radiant heat } & \text { thr } & 2 \\ \text { probable heat } & \text { th } & 3 \\ \text { no heat } & \text { th } & 8\end{array}$

Fig. 19 - Hall's proxemic notation: thermal code (1963)

8) Voice loudness scale

$\begin{array}{lll}\text { silence } & \text { si } & 0 \\ \text { very soft } & \text { vs } & 1 \\ \text { soft } & \mathrm{s} & 2 \\ \text { normal } & \mathrm{n} & 3 \\ \text { normal } & \mathrm{n} \text { t } & 4 \\ \text { loud } & \mathrm{l} & 5 \\ \text { very loud } & \mathrm{vl} & 6\end{array}$

Fig. 21 - Hall's proxemic notation: scale of voice loudness (1963) 
These symbols, which look like simplified stick figures, illustrate the posture they represent. They consist of lines and a half circle for the head, with subtle variations of the head, with fill or without fill, to distinguish between male and female.

In his notation of spatial arrangements and the orientation of bodies in relation to each other (see fig. 15), Hall determined eight positions for his model that he felt were 'operationally relevant to the participant' (Hall, 1963: 1008). Though he did experiment with a more extensive and elaborate range of positions, he considered his eight-point compass the most appropriate one. The notation of orientation of bodies ranges from zero - symbolising two people face to face, to eight - symbolising two people back to back. Position four is two people standing side by side, shoulder to shoulder. These positions are illustrated effectively with each person graphically notated as if from an overhead view, using a line with a half circle for the head. With these simple shapes, he gives a clear and instant picture of eight key spatial positionings of two bodies.

The third and last of the graphic sections within his system of notation records kinesthetic factors (see fig. 16). The kinesthetic factors include how people move their legs and arms, and the distance between the bodies that are interacting. For this section of the system he uses straight lines to illustrate the shapes and angles of the bodies, and defined four ways of relating with the body: (1) touching with the head or trunk; (2) touching with forearms, elbows or knees; (3) touching with arm fully extended; and (4) with the arm and the leg extended and body leaning. Hall also included the option to add some space to each of these four distances. Physical space is added visually with some space between the two symbols, or numerically by adding a 0 . As seen in fig. 16, the symbol II (or number 11) is a combination of the symbols I and I (or numbers 1 and 1), meaning that both people are touching with the head or trunk. Extra space is symbolised in this instance with space between the symbols: I I (or number 101), meaning that they are 
just outside body contact distance (but within touching distance). Although Hall was obviously concerned with using visual symbols as part of his system, he stated that the quickest way to 'read' the codes is by referring to the graphic symbols with numbers. The notation of thermal, voice, odour, and visual factors were made up of letter codes, for instance thc $=$ thermal heat contact (see fig. 19), and do = differentiated body odour detectable (see fig. 20), and these codes can also be referred to by a number, i.e. thc $=1$ and do $=1$. Referring to the codes by numbers is problematic in the sense that one number can have multiple meanings depending on what the number refers to. Without further explanation the number combination 33, for instance, could refer to any of these meanings: both pairs are males and sitting down; both pairs are 'holding or pressing against'; or 'probable heat' is detected in both pairs. To clarify what the number signifies one would need to refer to it with additional information such as: Postural - sex identifier $=33$; Thermal code $=33$; or Touch code $=33$. This use of numbers accompanied with textual explanation makes the visual secondary and somewhat superfluous in his notation system. However, even though it is not a complete visual system, Hall's work was groundbreaking in that it did create a clear and organised way of analysing aspects of human interaction using few words.

Fig. 22 shows a detailed analysis of an interaction using his notation system ${ }^{1}$. Here he uses numbers and titles rather than symbols, again diminishing the importance of the visual within the system. However, the figure does provide us with a very clear and elaborate recording of the proxemics and nonverbal factors that take place in this interaction between two men. Aware of its shortcomings and of his visual system being incomplete, Hall (1963: 1022) stated that 'Currently the visual dimension stands out, out of all those described, as the one requiring additional treatment', and he encouraged ethnographers to use and further develop it. Despite its imperfections, his system was groundbreaking in providing this area of research with a

1 Touch code (4) seems as if it should be 6 rather than 0 , this appears to be a mistake in the original figure in Hall's article (1963: 1021). 


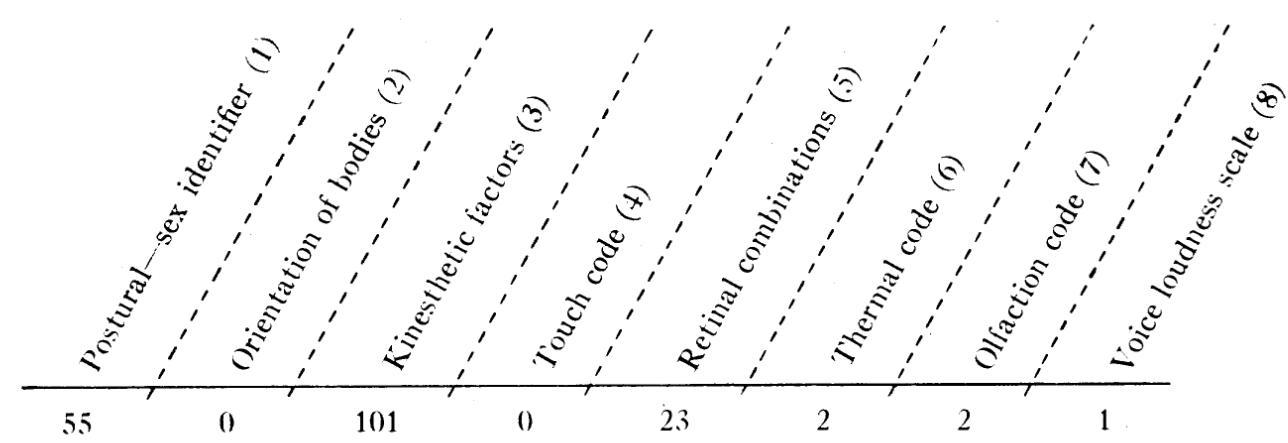

1) Two men standing;

2) facing each other directly;

3) close enough so that hands can reach almost any part of the trunk;

4) touch does not play any part;

5) man speaking looking at, but not in the eye, partner only viewing speaker peripherally;

6) close enough so that radiant heat would have been detected;

7) body odor but not breath detectable;

8) voice very soft.

Fig. 6. Recorded transaction and key.

Fig. 22 - Hall's proxemic notation in use: recorded transaction and key (1963)

crucial starting point for visualising proxemics behaviour. If it were to be developed further, as suggested by Hall, it could indeed become a fully visual system with its own unique visual language, which could be used for visual presentation as well as documentation.

Visualisation of spatial movements was also explored by the late anthropologist Ray L. Birdwhistell (1918-1994). His studies in the 1950s-70s were influenced by Hall's observations on human spatial arrangements and pioneering work in proxemics (Birdwhistell, 1970: xii). Birdwhistell maintained that a high percentage of communication during conversation is nonverbal, kinetic communication (Birdwhistell, 1970). Often referred to as a pioneer in the study of human body motion, Birdwhistell coined the term kinesics in the 1950s, to define the ways in which gestures and body movements function as nonverbal communication. In his studies on kinesics he applied research techniques used by structural linguists, because he considered structural linguistics the field with the most appropriate models for his analysis. His 
methods also included the use of observation, still images, interviews, movie cameras and slow-motion projectors which he used to analyse motion in great detail. His studies were mainly of Americans, and his belief was that interactional behaviour was learned and patterned by culture, and therefore it could be broken down systematically into isolated elements to be analysed. Birdwhistell created a system for the notation of kinesics, the first version of which was published in 1952, in order to be able to analyse and record the dimension of kinesic behaviour during human interactions. As with Hall's, the aim with Birdwhistell's system was to develop a way to write down nonverbal behaviour - in his case, kinesics. Although his notation was created for documentation purposes, sample conversations with visual descriptions are also presented in some of his publications. This application of a notation system as presentation, as well as for documentation, introduces another dimension to the use of notation systems within the field.

It was the physiological structure of body motion behaviour that Birdwhistell was concerned with and visualised. His notation system does not aim to include the communicative aspects, or the meaning, of behaviour, because he claimed that it is the social situation and not the gesture itself that determines the meaning of the behaviour (Birdwhistell, 1970: 173). He used the smile as one example to illustrate this point, because although it may be a cross-cultural act or expression, the meaning of the smile can change from one culture or situation to the next (Birdwhistell, 1970: 34). Though obviously aware of, and sensitive to the importance of the meaning of behaviour, this was not something he explored or visualised in his own system. For his system he created a number of symbols for recording body movements and developed kinesic codes such as: kinesic stress; distal and proximal movements; motion qualifiers - intensity, duration and range of behaviour; patterned speech and movement; tentative kinemes of juncture; and body sections. His studies on kinesics were predominantly concerned with the latter, the motion of different body sections. 
In his system Birdwhistell divided the body into eight sections: head and neck, face, shoulders and trunk, right arm, left arm, pelvic region, right leg, and left leg. His notation system, or Kinegraphs ${ }^{2}$, represents each of the eight sections in great detail and with multiple variations of movement. They were originally hand-drawn by Birdwhistell, and make up an extensive resource of notation of posture, gesture and facial expressions (Birdwhistell, 1970: 257). His system consists of both symbols and numbers, and each section of the body has a range of different symbols, depending on their nonverbal expression. Fig. 23 shows a small section of head movement Kines. The head is symbolised by $H$ and $h$, and nodding of the head is added to the $H / h$ using a bent line, circle and arrows.

\section{Total Head}

\section{"Norm" Stress Oversoft Variants

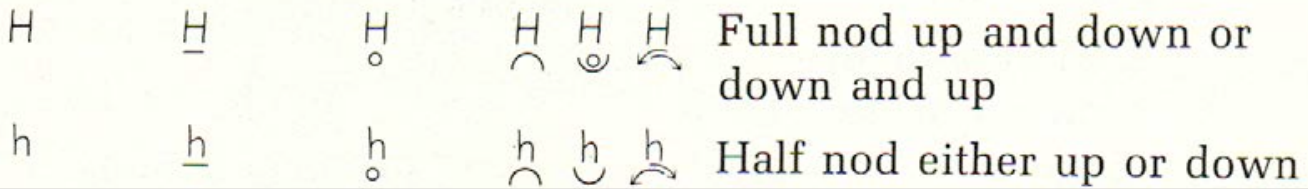

Fig. 23 - Birdwhistell's notation of kinesics: head (1970)

Perhaps more recognisable symbols can be seen in the second section of his system, symbols for face notation (see fig. 24). In this section Birdwhistell has used simple lines, circles and dots to illustrate eyebrow movements such as 'single raised brow' and 'medial brow contraction': these symbols are more familiar as they are similar to expressions used for cartoon characters. The same goes for notation of the mouth, with the most basic movements such as a smile, a 'droopy mouth', or a 'toothy smile' being quite familiar while

2 Kinegraphs is the term Birdwhistell used for the symbols in his eight-sectioned notation system. Kine is the term he used for body positions (Birdwhistell, 1970: 287). 


\begin{tabular}{|c|c|c|c|}
\hline \multicolumn{2}{|c|}{ 2. Face } & $\infty$ & $\begin{array}{l}\text { Out of the side } \\
\text { of the mouth (left) }\end{array}$ \\
\hline$-\frown$ & $\begin{array}{l}\text { Single raised brow } \frown \\
\text { indicates brow raised }\end{array}$ & $\bigcirc$ & $\begin{array}{l}\text { Out of the side } \\
\text { of the mouth (right) }\end{array}$ \\
\hline$-\sim$ & Lowered brow & $\sim$ & Set jaw \\
\hline ' & Medial brow contraction & $\cup$ & Smile \\
\hline$\because \because$ & Medial brow nods & & tight - loose o \\
\hline$\frown \frown$ & Raised brows & $\longmapsto$ & Mouth in repose \\
\hline 00 & Wide eyed & & lax o tense - \\
\hline-0 & Wink & $r$ & Droopy mouth \\
\hline$=<$ & Lateral squint & ᄀ) & Tongue in cheek \\
\hline ン ン & Full squint & $q$ & Pout \\
\hline & Shut eyes (with & \# & Clenched teeth \\
\hline A & A-closed pause 2 count & 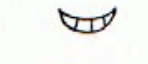 & Toothy smile \\
\hline$\pi \pi$ or & Blink -1 & 世卉 & Square smile \\
\hline & B-closed pause 5 plus count & (2) & Open mouth \\
\hline$\infty \theta$ & Sidewise look & $\mathrm{s} \bigcirc \mathrm{L}$ & Slow lick-lips \\
\hline 앙 & Focus on auditor & $\mathrm{Q} \bigcirc \mathrm{L}$ & Quick lick-lips \\
\hline$\otimes \otimes$ & Stare & $\infty$ & Moistening lips \\
\hline () () & Rolled eyes & $\infty$ & Lip biting \\
\hline$\oint \oint$ & Slitted eyes & i & Whistle \\
\hline$\theta \theta$ & Eyes upward & $-, 0,-$ & Pursed lips \\
\hline
\end{tabular}

Fig. 24 - Birdwhistell's notation of kinesics: face (1970)

he develops these familiar symbols in more complex movements such as the 'slow lick - lips' for which he combines graphic shapes with the letters $S$ and L. Birdwhistell used graphic symbols in quite a sophisticated manner, combining simple shapes, letters and symbols to illustrate very specific postures and movements of the body. His notation of seated postures, for instance, communicates complex arrangements of the legs and positioning of the feet, using line drawings (see fig. 25). He also allows for complex notation of hand and finger movement by visually dividing the hand, fingers and joints into numbered sections (see fig. 26). 

$\begin{array}{ll}\wedge \wedge \cdot \mathrm{X} & \text { Reverse } \mathrm{X} \text {. Lower legs crossed, feet posterior to } \\ \text { knee point. }\end{array}$

$\lambda 4$

Tight 4: Legs crossed: total femoral contact, knee behind knee.

내 Loose 4: Legs crossed: ankle or foot rests on op-

ᄂ十 posing lower extremity of femur.

Over 4: Kneecap over kneecap.

Fig. 25 - Birdwhistell's notation of kinesics: seated (1970)

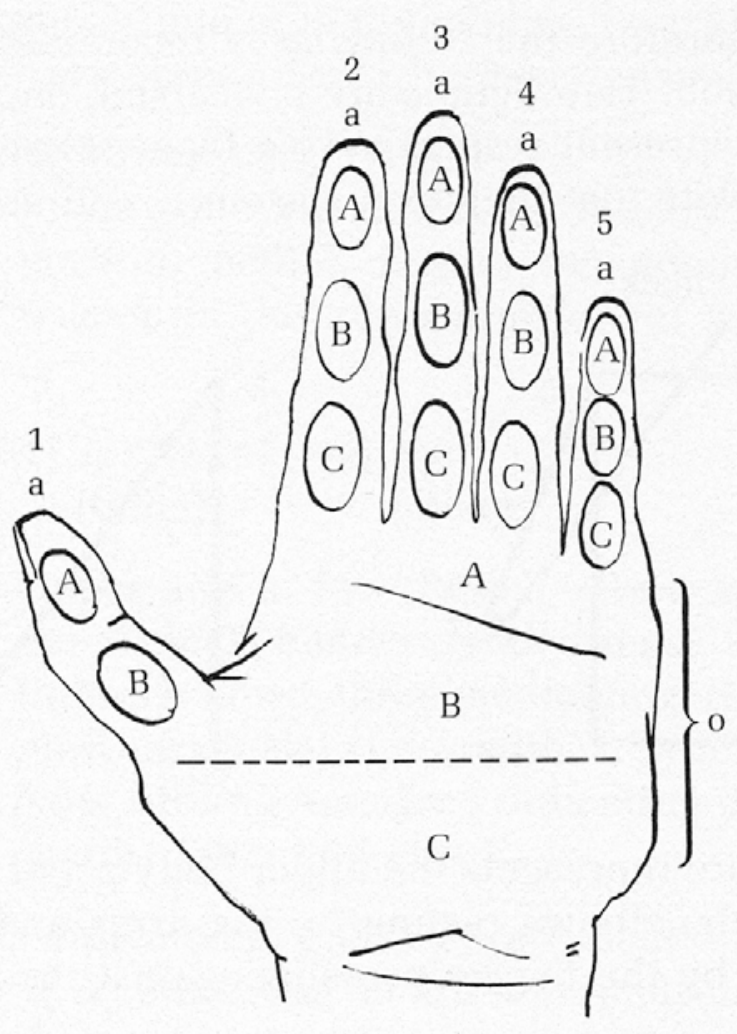

5. Hand and Finger Activity

A. Ball of finger.

a. Tip of finger.

(A), (B), (C), and (O) indicates back of finger or hand.

In notating, when particular finger is under discussion, list in the following order:

Hand: (R) or (L)

Fig. 26 - Birdwhistell's notation of kinesics: hand and finger activity (1970) 
While some of the symbols, like those for mouth and eyes, are instantly recognisable, most of his symbols are not, and require users to learn the system or have textual descriptions explaining the symbols at hand during their analysis.

Fig. 27 shows part of a conversation that Birdwhistell transcribed using his notation system. The conversation is between a mother and her child during a bus ride. The notation gives information about the mother's nonverbal behaviour such as her frowning, speaking through her teeth, mouth being pursed, her forcing a square smile and squeezing the boy's arm. In combination with the text, the notation, once interpreted, gives us a clear picture of the mood, emotions and overall unspoken aspects of the interaction.

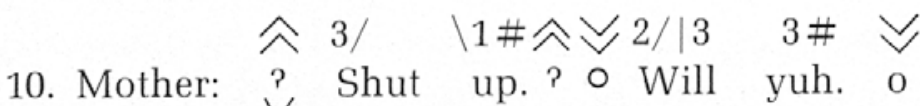

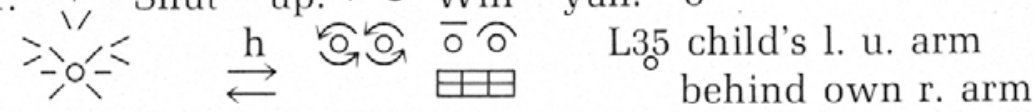

Fig. 27 - Example of interaction analysis of a dialogue using Birdwhistell's notation of kinesics (1970)

Birdwhistell's notation with its structure of the body into these eight sections proved to work well for his analysis of American behaviour; however, through observation he did conclude that this structure would not work crossculturally owing to different uses of posture and movement patterns found across different cultures. As for his facial notation, he claimed a face can make more than 250,000 different expressions and that the fifteen placement symbols plus eleven special markers that he created were sufficient to record all the significant facial expressions he had come across in his studies. In total, he claimed that his less than one hundred symbols would be able to deal with any kinesic subject (Birdwhistell, 1970: 8). 
Birdwhistell's notation system can be used to analyse nonverbal behaviour using symbols rather than having to refer to the symbols with numbers, as is necessary with Hall's system. Birdwhistell acknowledged that his notation operated as a 'relatively static system' and he encouraged future researchers to make it more dynamic. He created a 'model' which was intended for others to use. Birdwhistell recognised that ongoing improvements in technology, for example in photography, film and projection techniques, offered the possibility of a 'phenomenological revolution in the study of human behaviour' (Birdwhistell, 1970: 50). He envisaged the potential that technology and media could have in his field - both for future documentation and analysis of human behaviour.

Another principally visual system for documenting human interaction is that devised by Adam Kendon. In 1964, together with Jacques Ex, he created a notation system for face-to-face communication (Kendon, 2009). Fig. 28 shows a later version of this system, which was modified by Kendon. Their aim with the system was to create a way of writing down the expressions of the face during analysis of videos of human interaction, so that the expressions could later be reconstructed. Like Hall's and Birdwhistell's systems, Kendon's system was developed mainly for documentation and analysis purposes. However, Kendon did feature the notation in different formats in his publications: as visual transcripts of conversation sequences, as action-sequence maps, and as visual greeting diagrams. Kendon's notation of facial expressions (see fig. 28) consists of symbols that signify different eyebrow, eye, and mouth signals. Each symbol is unique for one type of expression of the eyes, eyebrows or mouth, and a combination of three symbols can provide us with a clear picture of a facial expression. Fig. 28 shows the symbols and their explanations used in his facial notation system. Eyebrows are illustrated in a realistic way using lines and the letters $n, m$ and $w$ to symbolise the different shapes the eyebrows exhibit for various expressions. He used different variations of lines and circles to illustrate 


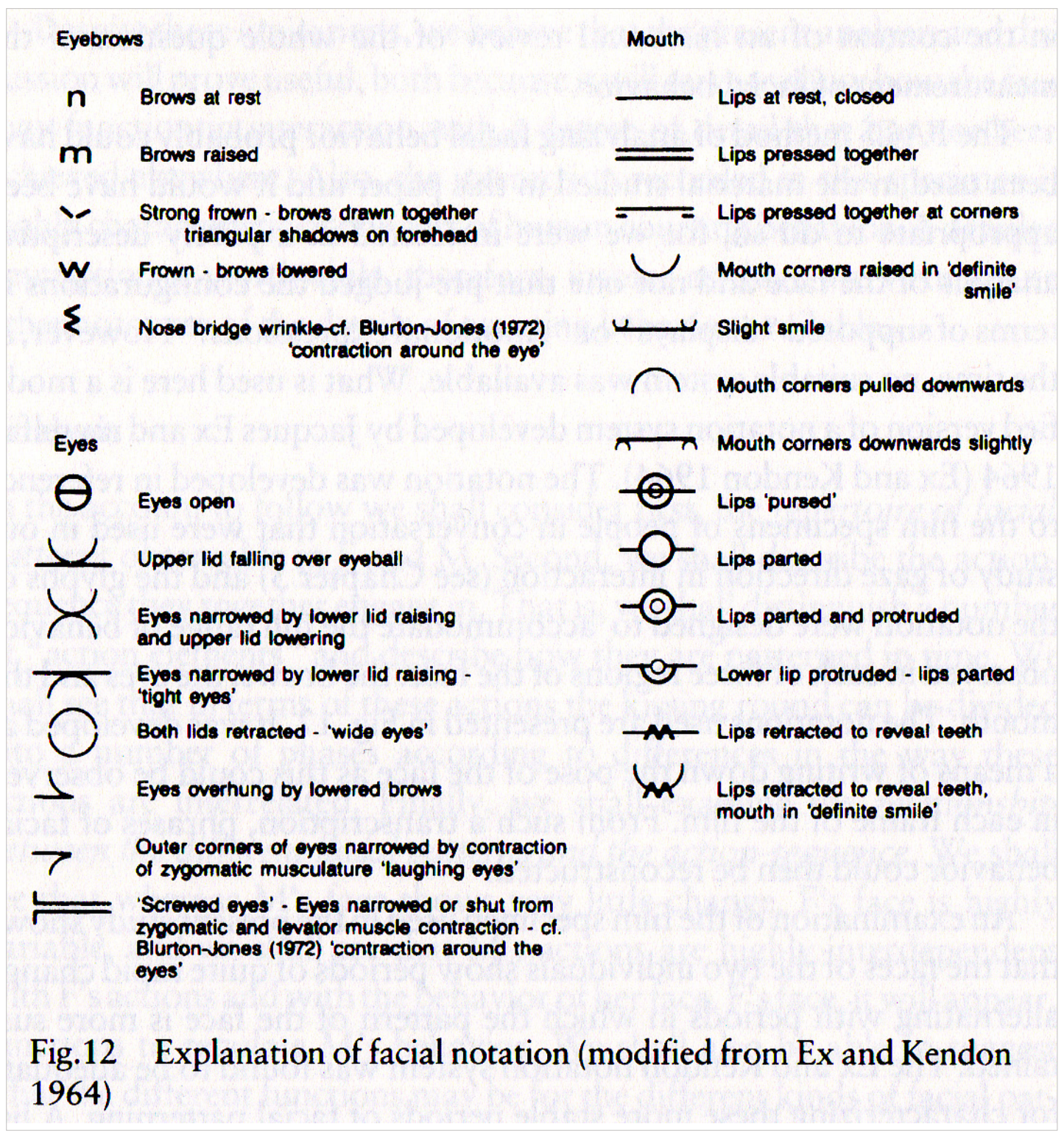

Fig. 28 - Kendon's facial notation (2009)

eye and mouth expressions. They are graphic representations of the actual expressions: 'lips at rest, closed', for example, is illustrated by a straight horizontal line, while 'mouth corners pulled downwards' is illustrated by a line bending downwards. By notating the expressions in such a way, Kendon and Ex were able to reconstruct and analyse patterns of facial behaviour over a time period, and they found that the system was sufficient for noting more 'stable periods of facial patterning' (Kendon, 2009: 123). 


\section{F's FACE}

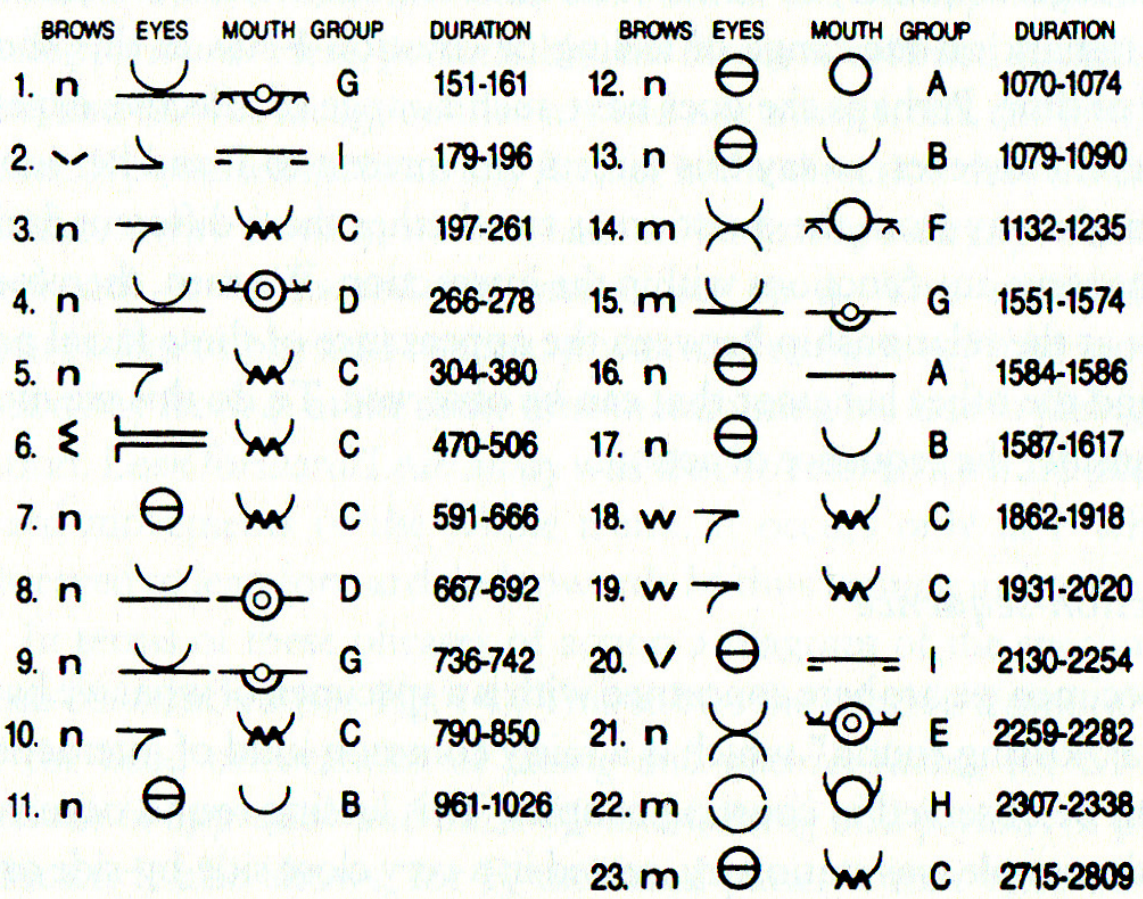

\section{M'S FACE}

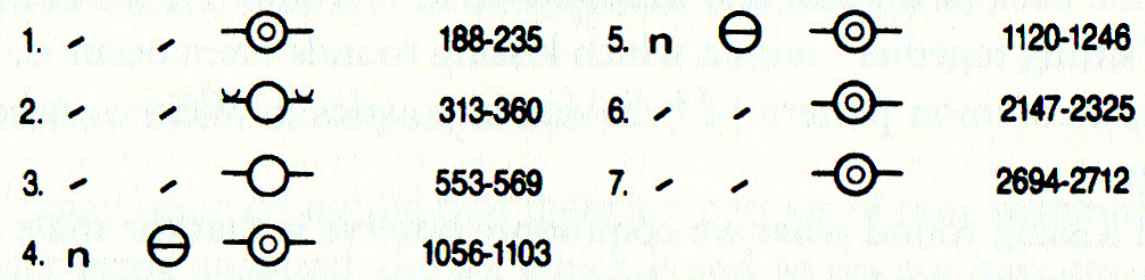

Fig. 13 Periods of sustained facial pattern. Notations for each of the periods of sustained facial patterns for $M$ and $F$ in the First Segment of C.14.1. The duration of each pattern, as it could be seen in the film, is given in terms of numbers of frames, counted from the zero frame. Zero frame is explained in the text

Fig. 29 - Kendon's facial notation in use (2009)

Fig. 29 shows the system in use, and presents a list of facial patterns that were recorded during an interaction between ' $F$ ' and ' $M$ ', showing different segments of the encounter. The first segment, for instance, shows that ' $F$ 's' brows are at rest, the upper lids of the eyes are falling over the eyeballs, the mouth corners are pulled downwards, the lower lip is protruded and the lips 
are parted, while 'M's' lips are parted and protruded. With simple symbols each segment of the interaction is recorded and provides us with a detailed picture of the facial patterns without the need for lengthy textual explanations. Kendon also created other visual techniques to represent greeting sequences. His maps of action sequences in face-to-face encounters visualise facial notation and different sequences of approach, positioning and initiation during these encounters. Fig. 30 is part of an illustration of an action sequence during the kissing round between $\mathrm{F}$ and $\mathrm{M}$. The map gives a very detailed analysis of different phases of the kissing round, of how $F$ and $M$ are positioned in relation to each other, of physical contact, and of F's facial patterns during any second, or video frame, of the sequence.

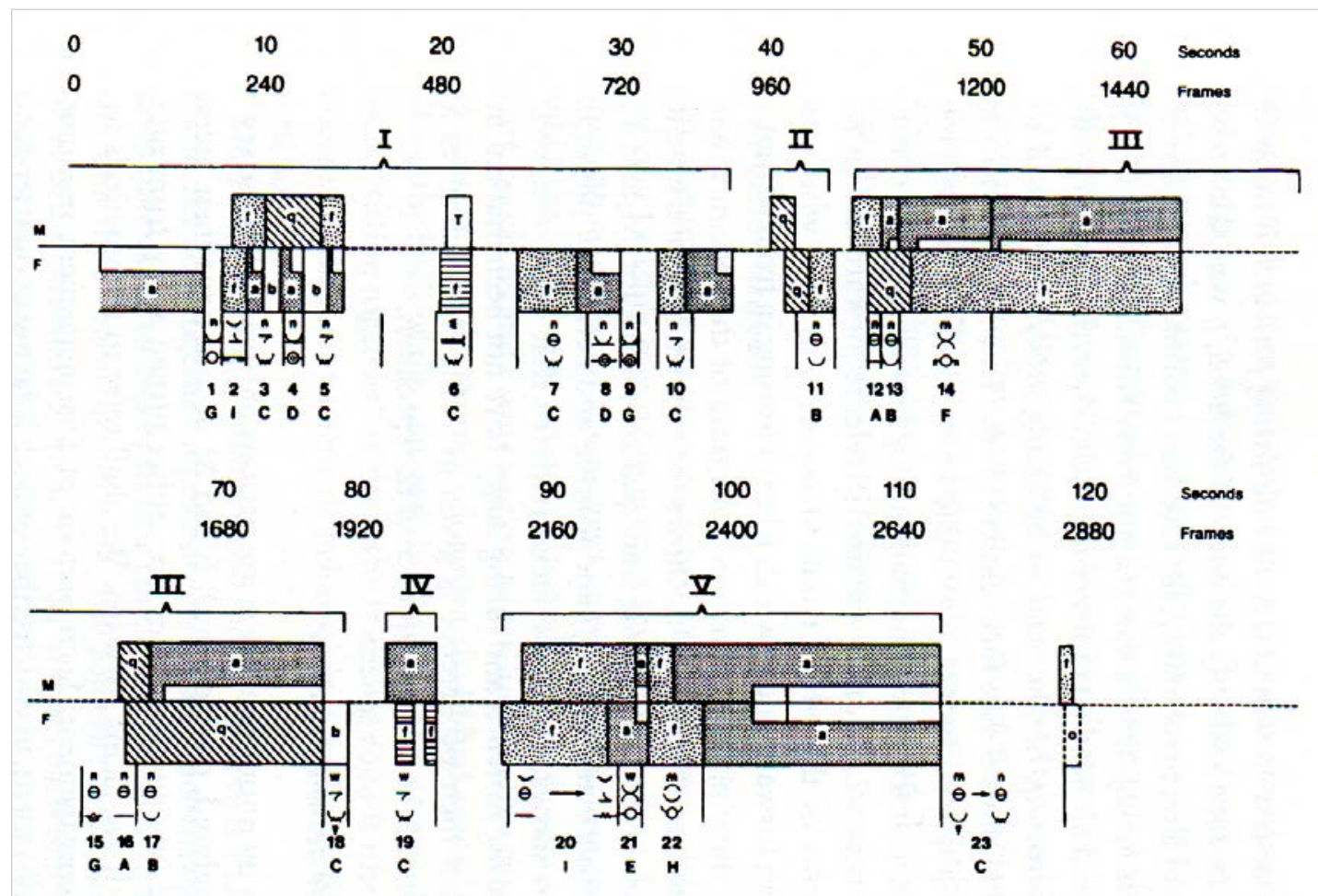

Fig. 14 This presents a map of the action-sequence of the first segment of C.14.1, together with the locations of all of F's facial patterns. The map has been plotted to the nearest eight frames (1/3 of a second). In the interests of clarity of presentation only facing (f), quarter-facing (q) and approach (a) head orientation units have been plotted. 1 = lean-forward; $b=$ back-off; $o$ = turnaway; white blocks on center line represent periods of physical contact. Exact time relations between $\mathrm{M}$ and F's actions are given in Table 9.

Fig. 30 - Kendon's map of action-sequence of a face-to-face encounter (2009) 
In his diagram of a greeting (see fig. 31) Kendon visualises the structure of a typical greeting: it shows the movement towards each other of the two people greeting, the orientation of their bodies, and their salutations. Similarly, his diagram of greeting segments also visualises the orientation and approach components, as well as changes in the face during the encounter (see fig. 32). By including facial notation in this diagram he makes it possible for one to read the facial expressions and how they change during the different parts of the greeting sequence. By combining the two, the movement diagram with the facial notation, Kendon creates a more complete picture of the greeting and its emotional expressions, and the picture becomes a visual timeline of the interaction. Nevertheless, although they are all visual representations of facial expressions and interaction sequences, most of these diagrams and maps need additional textual explanations to make sense. They are difficult, and time-consuming, to interpret. However, if, for instance, the timeline and

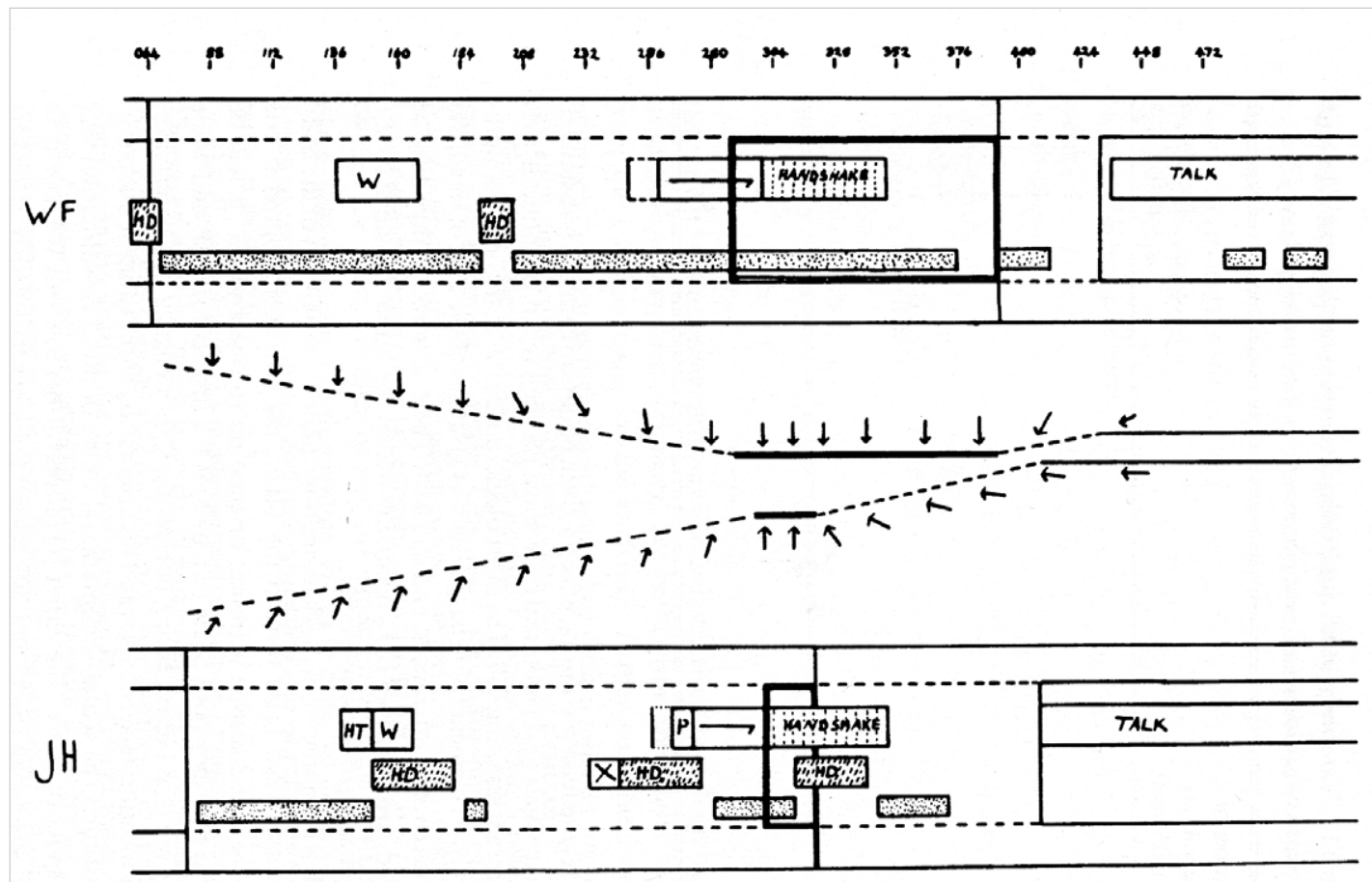

Fig. 17 Diagram of a greeting (for explanation see text)

Fig. 31 - Kendon's diagram of a greeting (2009) 


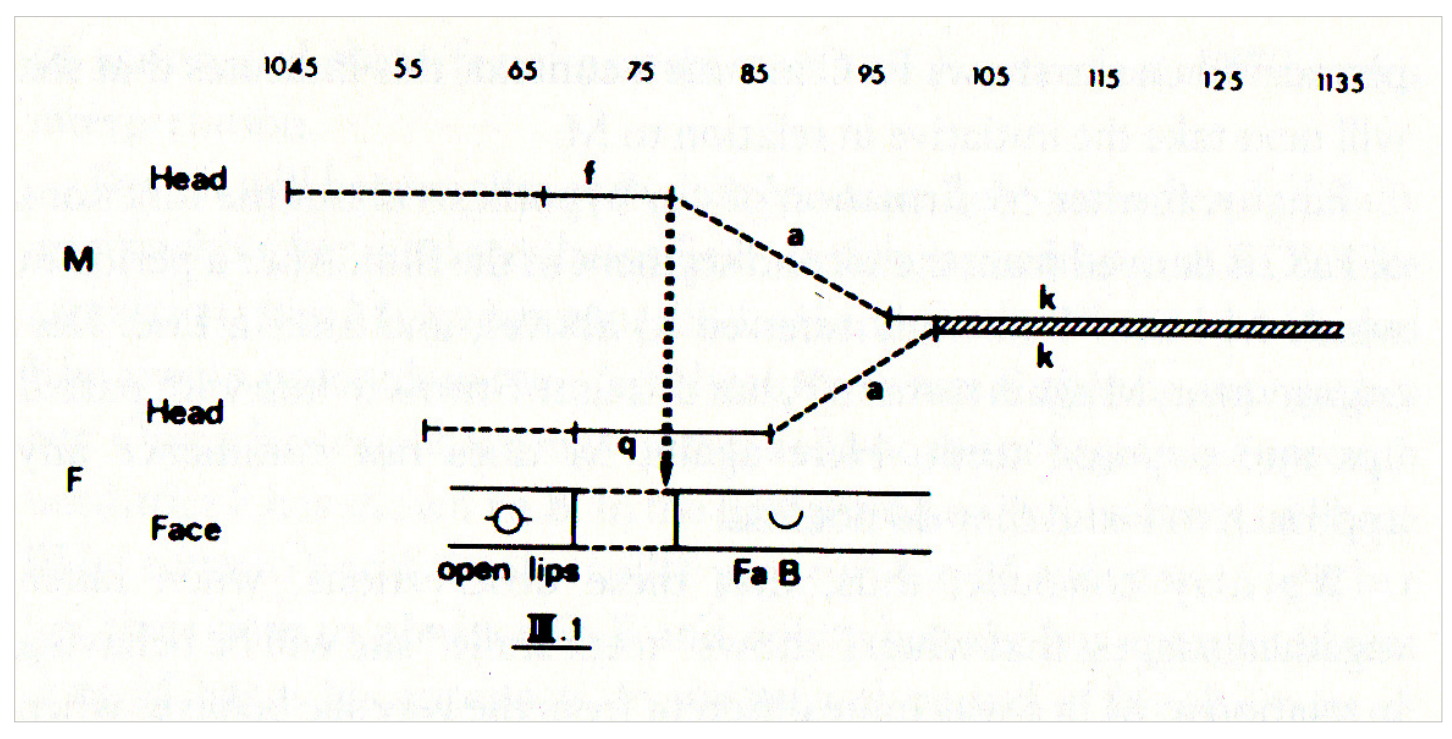

Fig. 32 - Kendon's diagram of a greeting segment (2009)

the annotations were animated, the analysis of the timeline could be viewed more clearly and would be made much easier to understand.

Finally, Kendon created visualisations of spatial organisation, also called the F-formation system (see fig. 33) in which he used simple oval shapes, lines and arrows to visualise movement and spatial arrangements of the interactions between a group of people. The visuals also reveal how the group co-operates to maintain 'spatial and orientational relationships' (Kendon, 2009: 216). The illustrations in the F-formation system give us a clear overhead view of the spatial interactions. This overhead view, a view also used in Hall's notation system, is ideal for visualising movement in space, the organisation of space and aspects of personal space. Again, if this piece was made into an animation, it could show how the group dynamics change and evolve over time. I adapt this overhead view in my own documentation and visualisation of greetings, in order to visually analyse the movement and use of space by the two people greeting. As I will discuss and illustrate in detail in chapter 4 , this perspective gives a clearer view of the greeters' relation to personal space, or proxemics, than a front or side view does. 


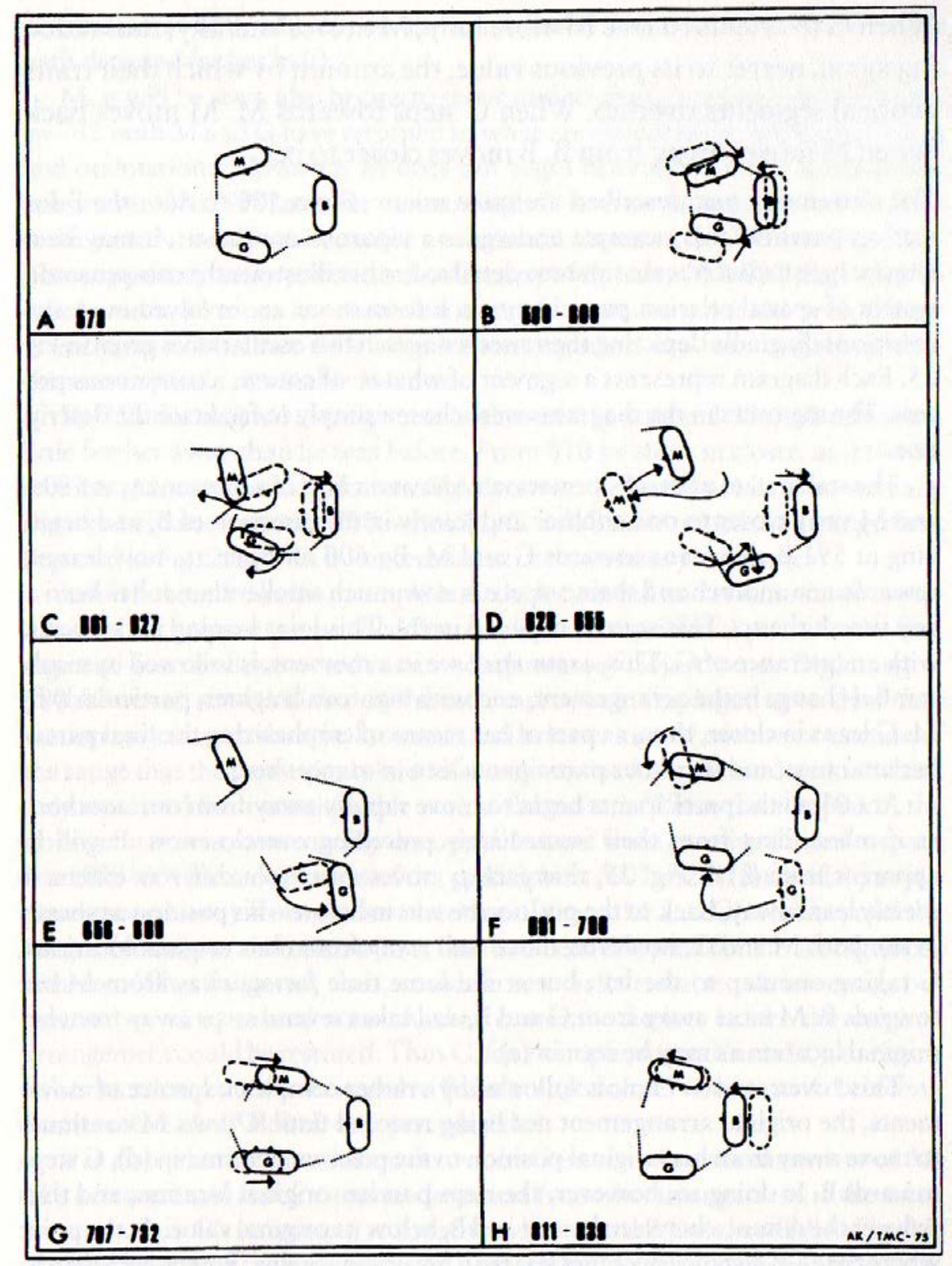

Fig. 33 - Kendon's F-formation system (2009)

In all three notation systems I have discussed, the use of graphic symbols provides researchers with a quick method of notating and reading valuable information about interaction and movement in space. There are other notation systems that are more advanced, in terms of technology and detail, than the three mentioned, for example the Facial Action Coding System by 
the Paul Ekman Group (Ekman, Friesen and Hager, 2002) (see fig. 34), but this system focuses solely on facial expressions and is based on number and letter codes rather than on graphic symbols. As my investigation focuses on visual systems using graphic symbols and graphic representations, systems based solely on letter and number codes will not be discussed.

\begin{tabular}{|l|l|l|l|l|l|}
\hline & &
\end{tabular}

Fig. 34 - Facial Action Coding System (FACS) by the Paul Ekman Group (2002)

Hall, Birdwhistell and Kendon, all pioneers in their own area of human behaviour studies, explored visual language for research purposes, but the visual aspect was never the main focus of their studies. They created notation systems for data collection rather than for presentation of their findings. The three notation systems discussed here cover relatively different characteristics of nonverbal interaction, though there are some overlapping areas to be found. The three systems have in common a desire to make sense of nonverbal aspects of human interaction through visual, rather than textual means. Their aims were to develop ways to record and annotate transcripts of nonverbal aspects of communication and to be able to recheck/ revisit the observations. While Kendon's system is easier to 'read' because it focuses solely on the face, Birdwhistell's system is more elaborate in that it includes notation of the entire body, and of its different sections. Though Birdwhistell's and Kendon's systems are more advanced in terms of the visual, Hall's system is built on a very comprehensive theoretical foundation that would benefit from further visual exploration. Both Hall and Kendon expressed a hope that others would develop their systems to become even more visual and more dynamic. With subsequent revolutions in technology, 
which has moved far beyond what was available to Hall, Kendon and Birdwhistell, the visualisation of nonverbal interactions can be explored further. Today's digital media hold enormous potential for the presentation and analysis of human behaviour as my study sets out to show.

Many other anthropologists and researchers besides Hall, Kendon and Birdwhistell have used visual means, such as video recording and photography, for documenting and analysing their findings. However, few have used any visual means in the presentation of their investigations. Zoologist and ethologist Desmond Morris occupies a position somewhere between these two approaches, using the visual both to gather documentation and in the presentation of his findings. His work is dedicated to the study of human behaviour and ethology, and includes an influential and substantial investigation of human nonverbal communication focused on gestures, postures and greetings. He uses photography as his method for data collection and for presentation of the research (Morris, 1979). The photographs and illustrations featured in his work were collected from a wide variety of sources and include cross-cultural variations of behaviour. Though he did not create a notation system, he presented his data in a new and visual way. Photography is the most prominent medium of his visual material (see figs. 35-36).
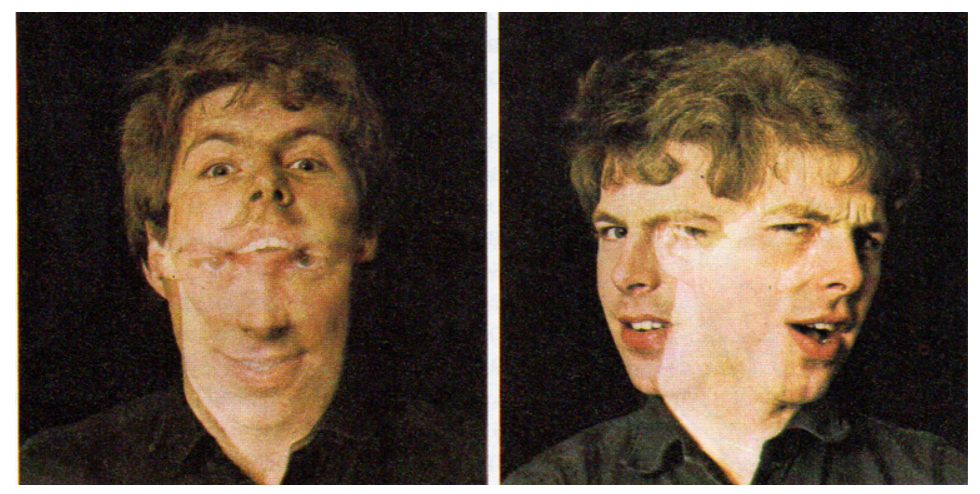

Fig. 35 - Head nod and head shake illustrated by layering images, featured in Morris' investigation (1979) 

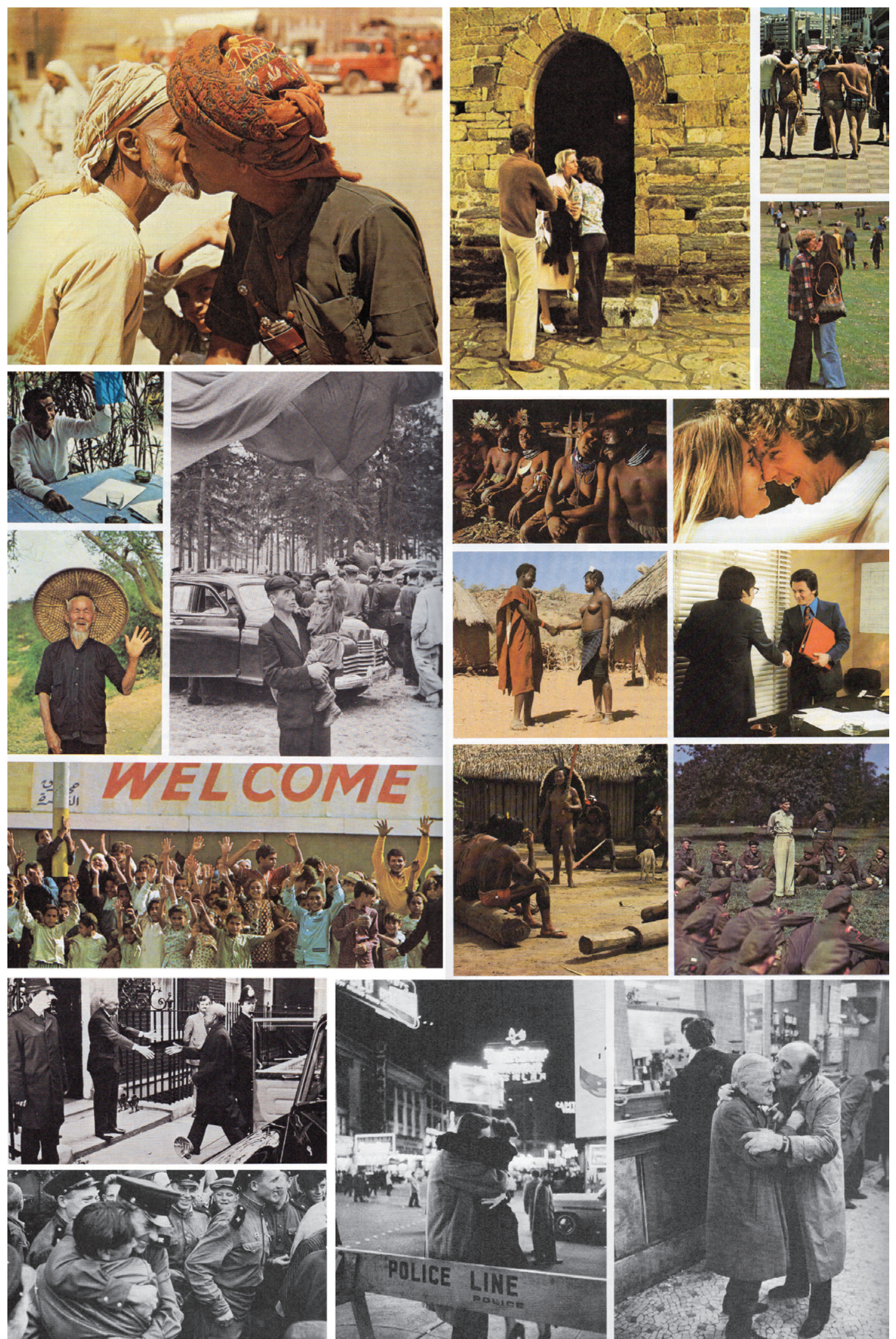

Fig. 36 - Greeting images featured in Morris' investigation (1979) 
Fig. 36 is a collection of greeting images from around the world, used in various parts of his investigation. Morris also included illustrations to illuminate important points about his findings, such as the 'taboo zones' areas of the body which other people should avoid touching (see fig. 37), and eye-brow movements during greetings (see fig. 38). Morris' use of visuals is

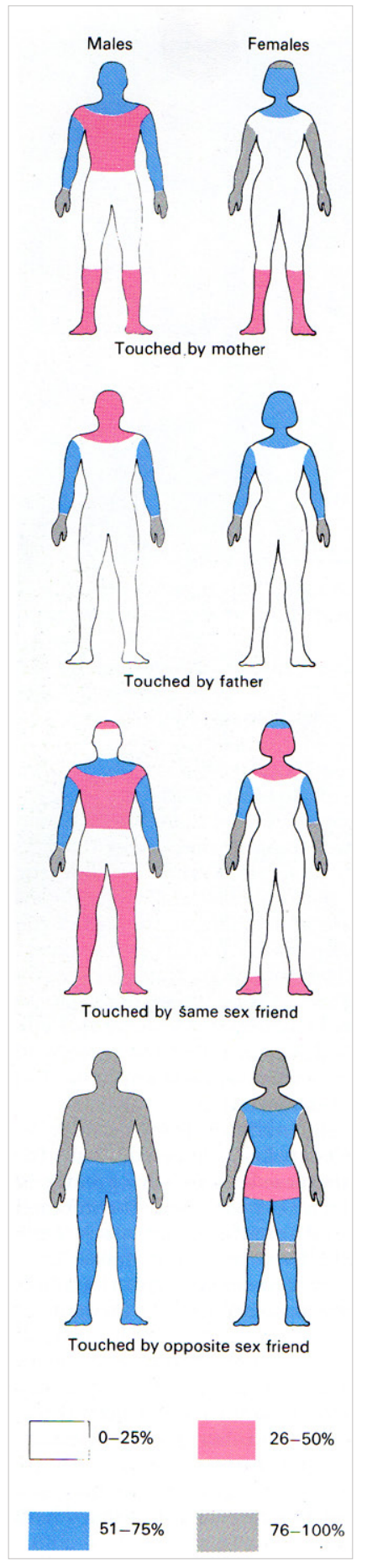

Fig. 37 - Taboo zones featured in Morris' investigation (1979) more illustrative than systematic. He used existing visuals and did not attempt to create an analytical tool as Hall, Birdwhistell and Kendon did with their notation systems. However, he did add a more visual focus to a field that had traditionally tended to be heavily textual.

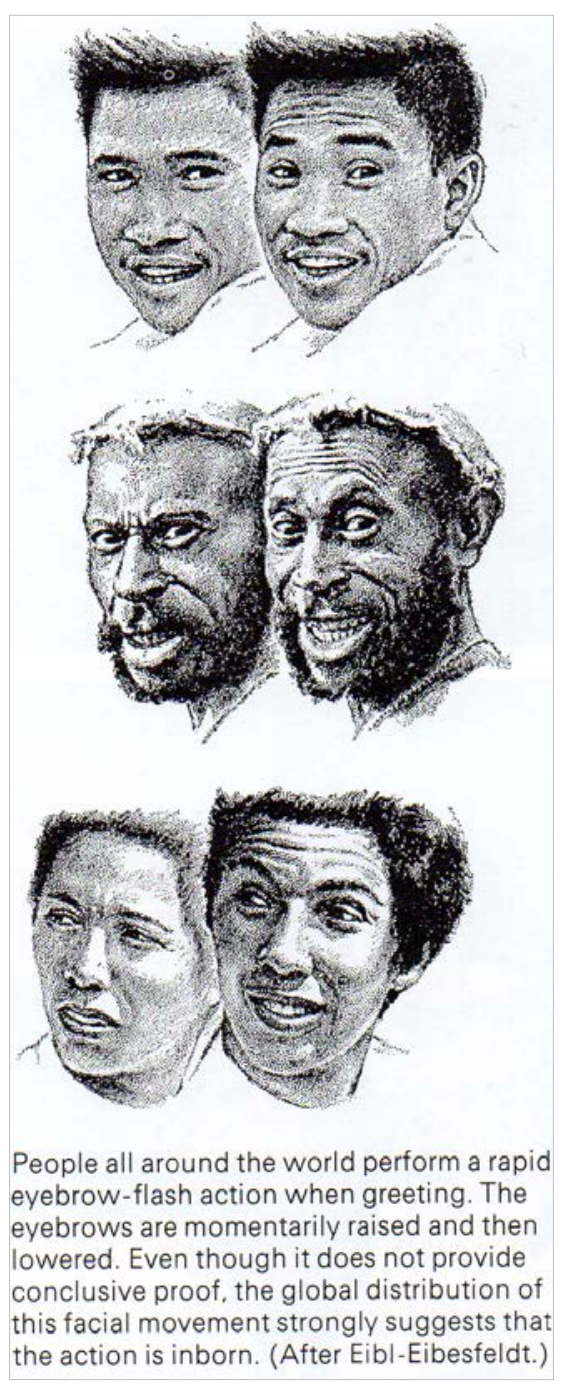

Fig. 38 - Eyebrow-flash during greeting featured in Morris' investigation (1979) 
A similar approach to Morris' is seen in Marianne Wex's (1979) photographic research and presentation of the difference between female and male body language. Wex's book Let's Take Back Our Space: 'Female' and 'Male' Body Language as a Result of Patriarchal Structures is a visual catalogue of gender-related postures and gestures. Fig. 39 shows one page from the book which visualises leg and feet positions of men (above) and women (below), showing clear differences in what were found to be typical feminine or masculine ways of sitting. Fig. 40 shows another page presenting postures that are considered to be masculine (above) and feminine (below).

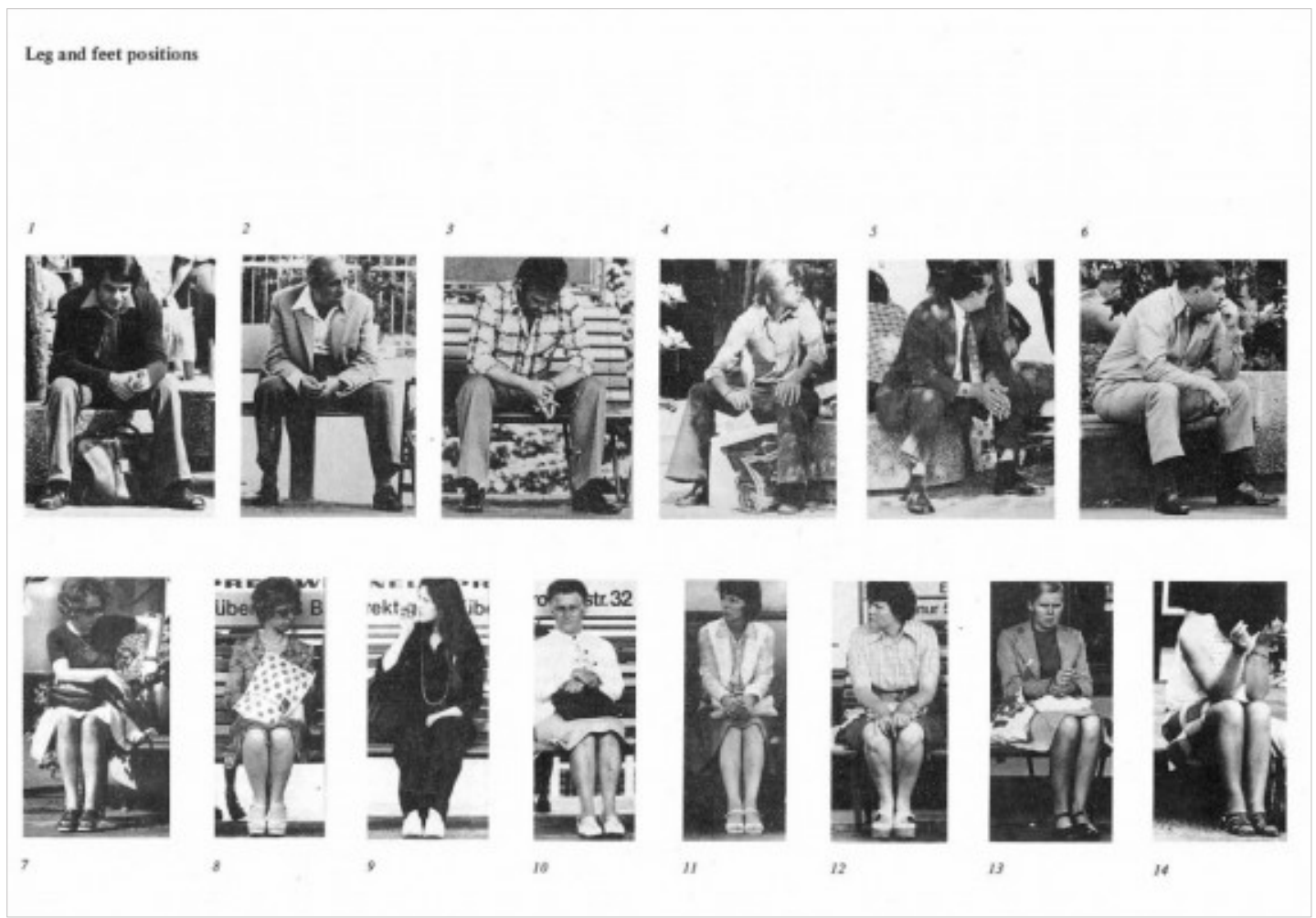

Fig. 39 - Wex's photographic study on female and male body language: leg and feet positions (1979) 

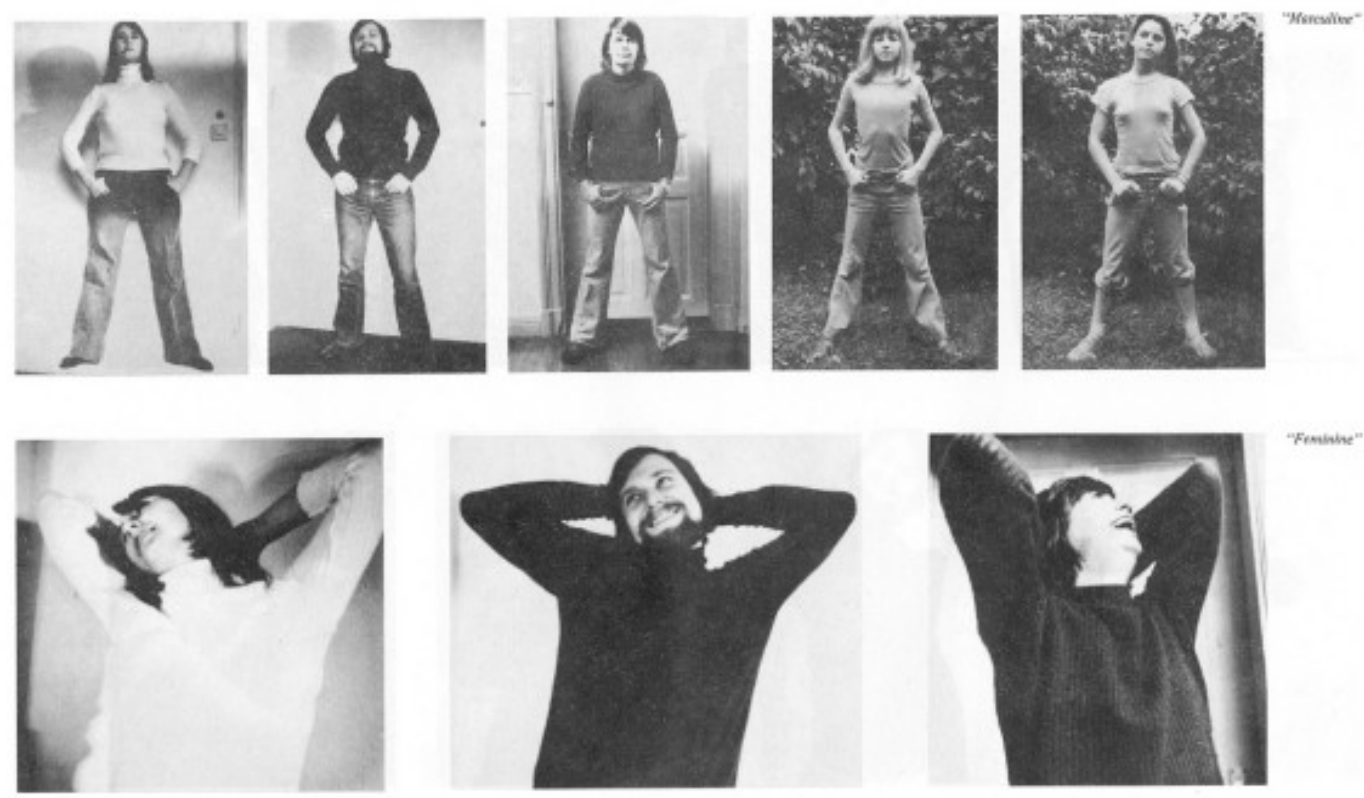

Fig. 40 - Wex's photographic study on female and male body language: postures (1979)

More recent greetings studies using visual means for collection and analysis of data have been developed by Alessandro Duranti (1992, 1996, 1997a, 1997b). According to Duranti, greetings are ubiquitous, repetitive and easy to recognise, but they are also complex interactions, and he reveals some of their complexity in his analysis of their verbal patterns. His view in his text 'Universal and Culture-Specific Properties of Greetings' (1997b) was that greetings studies must include (1) ethnography, (2) the recording of what is said, and (3) a definition of what is identifiable as a greeting (Duranti, 1997b: 67). He argued that too many studies lack one or more of these three aspects, either using improper recordings, or being based on field notes or observations alone. Duranti believed that the documentation, at very least the transcripts of the greetings, should always be included in the analysis and be made available to the reader to open up the possibility for independent analysis (Duranti, 1992). This was not common practice in the early 1990s, when analysis tended to be based on the researcher's field notes. Duranti 
questioned the reliability of such studies because the absence of transcripts meant there was no room for re-examination of the material.

Duranti did include transcripts in his texts, and in some instances he also included visual diagrams to highlight points about movement, hierarchy and overlapping during greeting rituals. Fig. 41, from a 1992 study of Samoan ceremonial greetings, shows the sitting arrangements and spatial positioning inside a house during a public gathering in a Samoan community. The orators of higher rank sit in the front of the room; they are the decision makers during the gathering. The chiefs sit in the 'tala', and the back is the place for the junior members or lower-class people. The people sitting in the front and in the tala are usually ceremonially greeted, as they are recognised as important and of higher rank. The illustration shows the route taken by chief Agaiatua when he arrives at the house. Duranti utilised illustrations such as

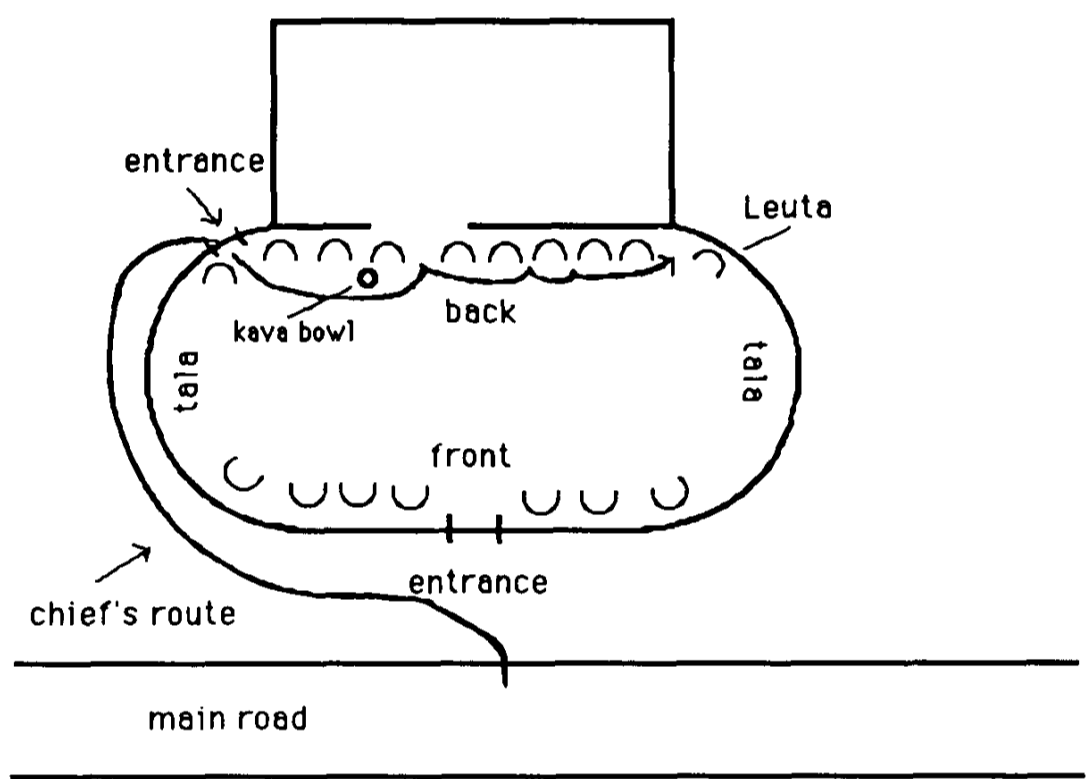

Figure 6

Fono in Sanonu. Route followed by chief Agaiataua when arriving at house full of orators from his subvillage (May 1981).

Fig. 41 - Duranti's illustration of Samoan spatial and sitting arrangement during social gathering (1992) 
this one, created with simple graphic shapes, to give a visual overhead view of complex hierarchical organisations of status and space in Samoan societies.

These illustrations help to clarify important points and observations in his text, but they do not communicate this knowledge as stand-alone graphic images. Another visualisation he uses in a slightly later study is that of an interlocking pattern (see fig. 42) (1997a). This illustration shows that there is overlapping in the verbal welcoming and response of a Samoan ceremonial greeting. The overlapping does not happen at a transition-relevant place in the greeting, as shown very clearly in the graphic. The welcoming by one person is overlapped by a response by the other person before the welcoming is completed. Duranti's studies reveal that this type of overlapping was very common in Samoan ceremonial greetings.

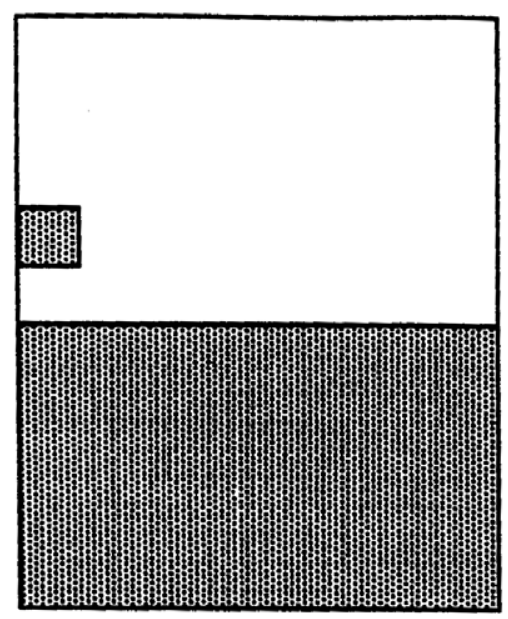

Key:

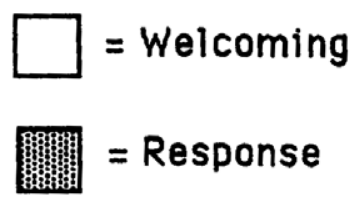

Figure 1. Graphic representation of interlocking greeting exchange

Fig. 42 - Duranti's visualisation of interlocking pattern during greeting exchange (1997) 
In the mid-1990s Duranti's use of visuals as part of his analysis developed from including static images only, to using more dynamic media. He saw the potential of multimedia and the Internet when it came to presenting his studies and analysis. His website features several audio-visual resources such as videos, sound-clips and photographs taken in different communities worldwide by different video-ethnographers (Duranti, 1996). These resources were put online to give his students an interactive experience when learning to analyse human encounters. This transition from using static diagrams and static textual transcripts to creating interactive multimedia presentations and analyses of his work marked an important change to the discipline. Duranti has developed the visual and interactive elements of greeting analysis more than anyone else.

One section of the audio-visual material featured on Duranti's website deals specifically with greetings; he also gives examples and instructions on how to study and analyse the greeting videos. The aim was to teach his students about the complexities and the social organisation of greetings, and to show how to analyse them to reveal some of these properties. One of the greeting encounters featured as a resource to study on his website, presented with a video, sound clips and textual analysis, is a greeting between Hillary Clinton and the Capps family, during the late Walter Capps' 1995-96 political campaign when he ran as a Democrat for the US Congress. During that year, Walter Capps gave Duranti access to record his speeches, interviews and conversations in both political and private contexts, which resulted in almost 60 hours of video footage (Duranti, 2006).

Duranti's audio-visual resource titled Hillary Clinton Meets the Capps is presented as an interactive multi-layered transcript with video and audio clips next to the different sections of the greeting transcripts (see fig. 43). The video features a multiple interaction where Hillary Clinton greets Walter Capps, his wife, and his son. The analysis is divided into three sections, 


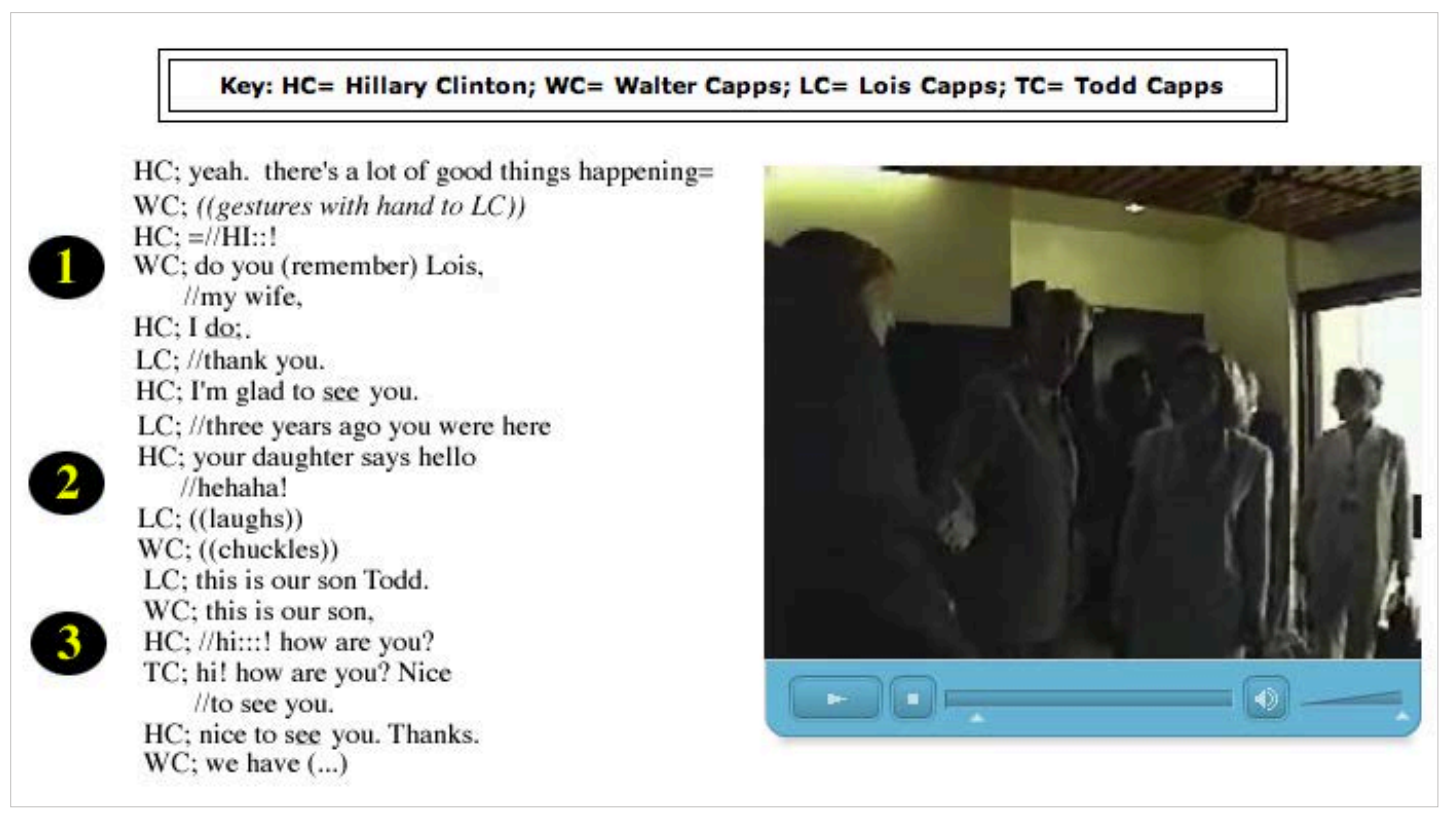

Fig. 43 - Duranti's analysis of Clinton and Capps' meeting using audio-visual material (1996)

one for each greeting, and the format is interactive in that you can click into different sections of the greetings transcripts to view their analysis. The analysis includes an annotated transcript with speech and gestures recorded in the left column, and a textual analysis of these in the right column (see fig. 44). In his analysis Duranti makes use of transcript conventions originally developed by Gail Jefferson for use in English conversation analysis (Sacks, Schegloff, Jefferson, 1974, cited in Duranti, 1996). These conventions include notation of aspects such as: who is speaking; lengths of sounds; pauses; overlapping talk; and unclear speech (see fig. 45). His analyses of the verbal exchanges reveal some of the history of the participants' friendships, such as the length of time since they last saw each other, and the way their comembership ${ }^{3}$ is made known through the mentioning of the Capps' daughter.

3 'Doing co-membership' is a term used in sociology when a piece of common history between people is established during conversation (Duranti, 1996). 


\section{Hillary Clinton Meets the Capps (Section 1)}

The following transcript is an example of what linguistic anthropologists call an annotated transcript -- in addition to talk and gestures (in the left column), it contains some background information and a preliminary analysis of the occasion (in the right column). As we become interested in various aspects of the interaction, our transcript will be revised to fit new analytical ends.

To Listen to the Sound clip, click on the play button

Transcript, section 1, version a

$1 \mathrm{HC}$; yeah. there's a lot of good 2 things happening=

3 WC; ((gestures with hand to LC))

列

$4 \mathrm{HC} ;=\mathrm{H} / / \mathrm{I}::$ !

5 WC; do you (remember)=

$6 \mathrm{LC}$; (? ? )

7 WC; =Lois, //my wife,

$8 \mathrm{HC}$; I do;

9 LC; //thank you.

$10 \mathrm{HC}$; I'm glad to see you.
Ethnographic notes and preliminary analysis

This is the end of a brief exchange between Hillary Clinton and Walter Capps regarding the panel she just participated in.

Walter starts to gesture towards his wife Lois before verbally introducing her. (see Gestures for more on this)

Hillary starts to greet Lois Capps before Walter has verbally introduced her. Hillary's higher volume and emphatic "hi" overlaps with Walter's conventional introduction "do you remember Lois, my wife?" which, in turn, overlpas with Lois' first words to Hillary. Routine expressions (e.g. I'm glad to see you) can reveal important details about participants' history and relationships (see Remembering) while the order of greetings suggests that a protocol may be at work.

Fig. 44 - Section 1 of Duranti's transcript/analysis of Clinton and Capps' greeting (1996)

?Walter; a question mark before the name of the speaker stands for a probable but not safe guess regarding the identity of the speaker.

(1.5) Numbers between parentheses indicate length of pauses in seconds and tenths of seconds.

... Three dots indicate an untimed pause.

[...] Three dots between square brackets indicate that some material of the original transcript or example has been omitted or that the transcript starts or ends in the middle of further talk.

= Equal signs indicate 'latching,' that is, two utterances that follow one another without any perceptible pause.

[ A square bracket between turns indicates the point at which overlap by another speaker starts.

// Double obliques indicate the point at which overlap by the next speaker begins.

(don't) Words between parentheses in the transcripts represents the best guess of a stretch of talk which was difficult to hear.

( ? ? ) Blank spaces inside parentheses with occasional question marks indicate uncertain or unclear talk of approximately the length of the blank spaces between parentheses.

Fig. 45 - A section of Gail Jefferson's transcript conventions used by Duranti (1996) 
The analyses also illustrate how greetings are formulaic and follow certain formats, such as the adjacency pair ${ }^{4}$ format: two adjacent turns by two speakers. An example is when Hillary Clinton greets Todd Capps with 'Hi, how are you?' and he responds with 'Hi, how are you. Nice to see you.' (see fig. 46): this is an adjacency pair and is a typical greeting format. Through these analyses, in which he uses traditional as well as contemporary methods of representation, Duranti illustrates the complexities of greetings, showing that they are rituals that involve important information exchange, while at the same time being formulaic.

\section{Hillary Clinton Meets the Capps (Section 3)}

Here is an annotated transcript of the second segment of the greeting exchange:

\begin{tabular}{|l|l|}
\hline $\begin{array}{c}\text { To Listen to the Sound clip, } \\
\text { click on the play button }\end{array}$ \\
\hline \\
\hline
\end{tabular}

The greeting exchange in lines 3-5 conforms to the format described by conversation analysts as typical of greetings: two adjacent turns by two speakers:

Speaker A: \{greeting\}

Speaker B: \{greeting\}

This format is called adiacency bair.

Fig. 46 - Adjacency pair format found in section 3 of Duranti's analysis of Clinton and Capps greeting (1996)

4 Duranti discusses the adjacency pair format in Universal and Culture-Specific Properties of Greetings (Duranti, 1997b). 
Although documentation through photography and video is a fairly widespread method of gathering anthropological data (Kendon, 2009; Pillet-Shore, 2008), it was Duranti's treatment of the photographic material that brought him, and this research field, another step forward in realising the analytic potential of the visual. Before his work, ethnographic video material was used simply for data collection and not for presentation. With the development of using video and interactive text in his transcripts and analyses he advanced the visual research of greetings from relying on static images and videos to utilising more dynamic tools of presentation, and in doing so opened up newer ways of viewing, studying and representing greetings. Duranti made use of the Internet and its capability of displaying multimedia content to present his audio and video files alongside his textual analysis. The use of the Internet also meant that the way Duranti shared his research, both with his students and with a wider global research community, changed immensely. As discussed by Hart (2004), technological advancements since the introduction of the World Wide Web in the early 1990s have shifted the ways messages are exchanged - from being text and number based to becoming more visual. Duranti embraced this change, but despite his move towards using more advanced contemporary technologies such as the Internet, his transcripts and analysis remain textual. However, considering that his studies focus on the spoken characteristics of greetings, heavy reliance on text may be seen as unavoidable.

In chapter 4 I will return to the Hillary Clinton Meets the Capps multiple greeting and use my own system of visual notation to analyse the nonverbal aspects of the greetings. My method will illustrate how digital technologies could contribute, alongside more traditional techniques such as those used by Duranti, to the study and representation of greetings.

It is clear that the anthropological understanding of greetings has developed enormously over the past 60 years, but as I have discussed, the visual research aspect of this field of enquiry is still underdeveloped and marginal. 
Various researchers have used visual methods to collect data, but despite the development of new technologies, the use of visual methods in the study of greetings has remained ancillary - secondary and subordinated to textual explanations. The systems of notation I have discussed illustrate the desire to establish visual notation systems for use in anthropology and behavioural studies, yet this desire has not been fulfilled as such systems have not been further developed visually and dynamically to meet their potential use.

Recent advances in digital technologies offer unprecedented potential to achieve such development, and there have been major changes in the realm of visual language since Hall, Birdwhistell and Kendon created their notation systems. They used the tools and visual language available at the time, to produce notations that relied on static symbols to signify movement. Today, infographics and data visualisations of all kinds of information are changing how we see the world. My research aims to develop a more contemporary notation system which reflects a current and dynamic visual language using available multimedia technologies. A historical and multidisciplinary overview of methods and technologies involved in graphic visualisation will be discussed in detail in chapter 3.

\subsection{An Intercultural Study of Greetings}

Although the precise focus within greetings studies has varied, as discussed above, such studies almost all share the aim of investigating intracultural interactions: greetings within a specific society, culture or sub-culture (Ameka, 2009; Caton, 1986; Duranti, 1992; Walter, 2009). Samovar, Porter and McDaniel (2007) compare how gendered greeting interactions work in different cultures. They investigate gender relations and differences in behaviour between cultures such as Saudi, Finnish and Mexican. However, though they compare cultures, their study does not include greetings performed across cultural borders, between people with different ethnicities - encounters that are increasingly common in multicultural communities. Though Duranti carried out a number of studies of class within cultures, very 


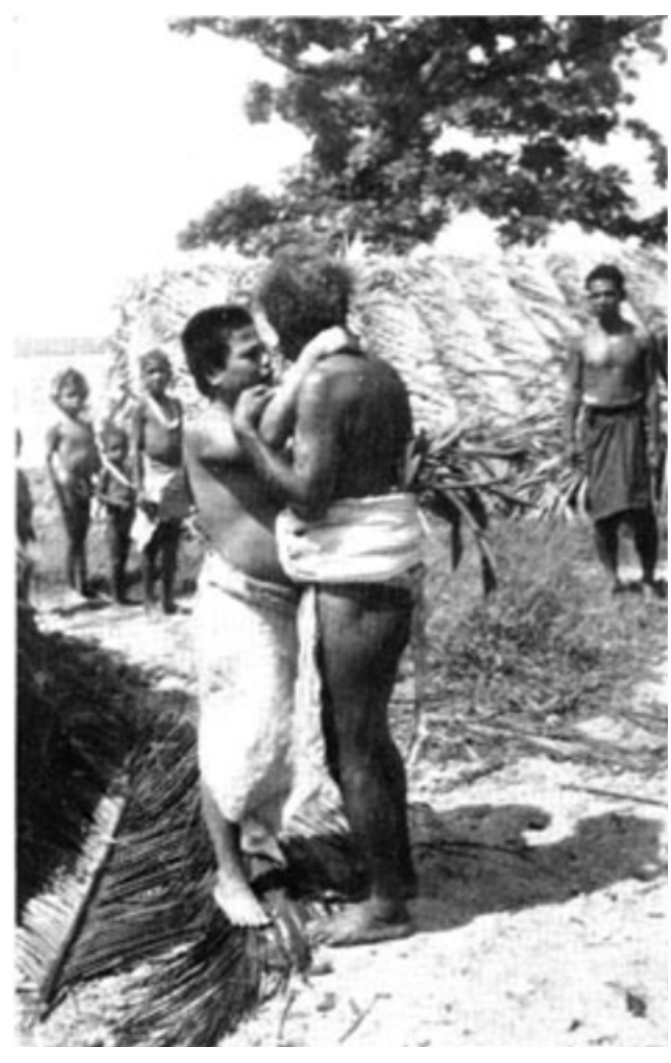

Fig. 47 - Greeting between an Anuta man and a Tikopia man featured in Firth's investigation (1954) few attempts have been made at investigating intercultural greetings, across classes or cultures.

An exception here is Raymond Firth's (1954) intercultural study on the communication between the Anuta and Tikopia societies in the Western Pacific. He examines the social relations between these two islands and their people in great depth, describing their relationship as a symbiotic one. These two societies, though they rely on each other and have many similar cultural structures, also have some very different ones, such as the initiation rite and speech/

dialects. Firth (1954) describes the greetings between the two societies during exchange trips as sometimes being 'affectionate scenes of greeting', indicating that there is positive communication between them despite their apparent cultural differences. His study also involved the use of photography as documentation, with one image capturing a greeting between an Anuta man and a Tikopia man (see fig. 47). Today, intercultural greetings are highly frequent in multicultural societies. Intercultural contact has increased due to a number of different factors, including technological developments and the Internet, immigration resulting from globalisation, and more opportunities to travel (Samovar, Porter and McDaniel, 2007; Kapuściński, 2008). This presents us with a new context in which to understand greetings: they can be understood not only as rituals of shared interaction patterns but also, increasingly, as meetings of different patternings. These patternings and symbol systems that now meet daily are so distinct that they 'alter the communication event' (Samovar, Porter and McDaniel, 2007: 9) and we 
are faced with a new and often unfamiliar type of encounter. During such moments of intercultural contact, verbal language barriers are obvious obstacles for smooth communication. The sometimes more subtle nonverbal differences, which are the focus of this study, can often be even greater barriers to interaction because people are less aware of them. These differences in behaviour can teach us a great deal about cultural variation and patterning. Studying the interaction between people with different cultural backgrounds presents us with a range of 'issues' that in intracultural studies are either taken for granted or are nonexistent. These nonverbal cues relate to differing movement patterns, gesturing, use of space and personal space, touch/non-touch situations and, as mentioned earlier, differences in the perceived meanings of the interactions. All these aspects of the nonverbal greeting become more apparent and relevant in intercultural settings, as the comfort of familiar and predictable behaviour during greetings is replaced by unpredictable, sometimes uncomfortable greeting situations.

As discussed at the start of this chapter, greetings are formulaic expressions, systematically patterned by culture. How to greet is usually the first thing one learns when going to a new country or place and when learning a new language. In Norway greetings, and other forms of local social behaviour, are taught to immigrants during state-run induction programmes. Greetings provide a valuable focal point for studying cross-cultural behavioural differences in a multicultural society. In this multicultural setting, greetings involve interactions both between co-cultures ${ }^{5}$, and between co-cultures and the host culture. For immigrant communities, the national greeting becomes a symbol of an introduction to the new culture and of adapting to the host society. At the same time their own greetings become tokens of their identities and as such get entangled in the politics or identity of the

5 Samovar, Porter and McDaniel (2007: 11) define co-cultures as 'groups or social communities exhibiting communication characteristics, perceptions, values, beliefs, and practices that are sufficiently different to distinguish them from the other groups, communities, and the dominant culture'. 
multicultural society. This study is intended as a contribution to the dialogue between cultures, and aims to create an awareness of different cultures through increasing our understanding of greetings and their varied cultural patternings. It seems timely to carry out an intercultural study of greetings in order to bring this area of anthropology into the contemporary multicultural context, where dynamic and unpredictable interaction patterns occur every day in multicultural societies worldwide.

In this chapter, three important observations about the study of greetings have been made. First, it has been determined that greetings are formulaic expressions that involve important information exchange and cultural knowledge. It has also been established that an important part of the message exchange is nonverbal. Second, it has been shown that the use of visual tools for studying human encounters has so far been marginal and secondary to text. Though important developments of systems for notation were made in the 1960s-70s, these systems have not been successfully followed up and developed in a way that keeps pace with technological advancements. Third, it has been found that most existing studies of greetings focus on intracultural encounters, and do not address the multicultural world that we live and interact in today. These observations outline the anthropological context for my research, and show the starting point within this field for the development of my system. In chapter 3 I give an overview of existing visual notation systems and methods for analysing movement, and discuss contemporary visual languages and technological techniques that could potentially advance the study and analysis of greeting interactions. 


\section{Visualising Movement: Documentation and Notation Systems, and the Potential of Digital Media}

As discussed in chapter 2, visual techniques such as photography, video recording and graphic notation have been used to some extent in the study of greetings, but mainly for documentation purposes. When it comes to the analysis of greetings, visual techniques have remained secondary to text. Notation systems created for the purpose of documentation and analysis of human interaction by Hall, Kendon and Birdwhistell in the 1960s-70s have not been further developed in terms of concept, visual expression, or technology. However, while anthropological research on greetings and human interactions has made limited use of visual systems, this has not been the case in all fields of human study. Visual notation and analysis have had longstanding and important functions in the understanding of human movement - and dance in particular. In this chapter I will give an overview of some of the visual techniques and technologies that have changed how we study, analyse, and communicate about human movement and how we convey social information. I will also discuss visual techniques that display great potential for use in the visualisation of movement. More specifically, I am looking at five different aspects of visualisation: chronophotography, movement and dance notation, information graphics, data and network visualisation, and interactive technologies.

The first two sections of this chapter concern methods that have been used in the past to document movement. Historically these techniques have been of great importance to the field of movement analysis. The first section discusses techniques of 'capturing' motion through chronophotography, which was developed in the late 1800s. Chronophotography was used by photographers such as Eadweard Muybridge and Étienne-Jules Marey to document objects in motion, and to study and reveal aspects of movement that were not obvious to the naked eye. The essence of their techniques is still relevant and broadly used today, often through formats such as 
stopmotion video and time-lapse photography. The second section discusses techniques for visualising dance choreography with systems such as Labanotation and Benesh movement notation, used from the early 1900s. Notation systems for dance were created to enable the reading, analysis, writing, and reconstruction of movement using graphic symbols. They are accurate recordings of human movement that have been used in many areas of movement analysis beyond the field of dance, but they cannot be utilised or understood without specialised knowledge. Labanotation is of course still in use today but it was devised nearly a century ago in a different technological age. While the visual language and technology used by Muybridge and in the creation of Labanotation have developed considerably since, it is the concept of these techniques that is relevant to my study: the concept of documenting, distilling and preserving movement in the forms of photography and graphic notation.

The third and fourth sections of this chapter concern present-day visualisation techniques that have great potential to communicate movement and greetings. In the third section, which relates closely to my own practice, I show how contemporary information graphics, and digital media techniques and applications, have much to offer to the field of greetings studies and visual anthropology. The fields of information graphics (infographics), data journalism and network visualisations, with leading practitioners such as Edward Tufte, David McCandless, Manuel Lima, Hans Rosling, and others, offer useful methods for image mapping, colour coding, shape scaling and the visualisation of changes over time. I discuss some of these techniques and technologies and how they apply to my own process of developing a system for the notation of greetings. In the fourth and final section I discuss contemporary artworks and installations that use sophisticated technologies such as facial emotion analysers and motion tracking. Works by Random International, the Motion Bank project, and others, utilise sophisticated software and technology to create, document, and analyse human interaction/movement. The Motion Bank projects in particular, which focus on 
the documentation and visual preservation of dance choreography/scores, show the huge impact that digital media and technologies have had on the field of dance. While these projects do not seek to replace more traditional and static systems like Labanotation, they illustrate how digital media are moving the notation and preservation of dance forward from the static graphic symbols used in Labanotation to dynamic and interactive visualisations displaying movement in time and space. Such technological advancements are promising when it comes to the visual analysis of greetings in particular, and for studies of human interaction in general.

\subsection{Chronophotography}

Chronophotography is the term French scientist and physiologist ÉtienneJules Marey used to define a technique of taking sequential photographs, which he and others developed in the late 19th century. At that time photography was seen as an important means to study and solve scientific problems (Braun, 2009: 32). Chronophotography developed from the desire to analyse movement in space, and scientists needed a method of photography that would show movement in a sequence of images (Robertson, 2009: 170; Canguilhem, 2009: 70; Braun, 2009: 22). While Étienne-Jules Marey and others came to photography through science, Eadweard Muybridge came to science through photography (Braun, 2009: 32). Marey, Muybridge and others who worked at the intersection between photography and science built instruments and equipment to make the recording of image sequences possible: Marey's photographic gun and Muybridge's Zoopraxiscope (see figs. 48-49) were two such instruments, among many others (St George, 2009: vii-viii).

These pioneering devices were designed to take a series of still photographs from the same viewpoint at specific and continuous intervals (Georges, 2009: 178). When played in sequence, these photographs appear as a continuous image because each frame interacts with the next one. The 'gap' 


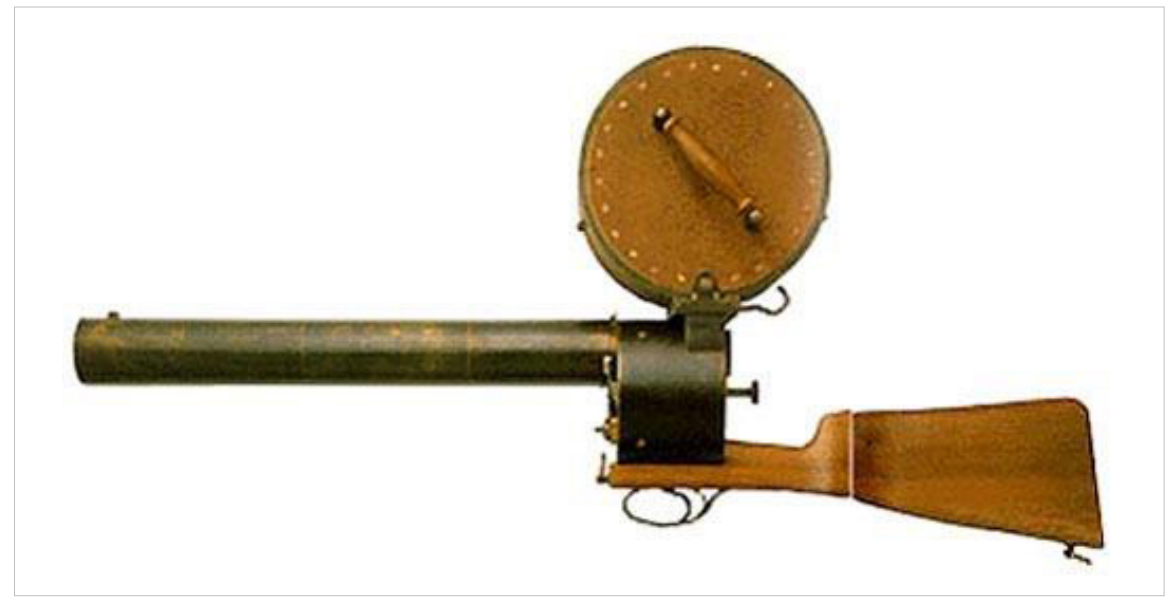

Fig. 48 - Marey's photographic gun (The Robinson Library, 2014)

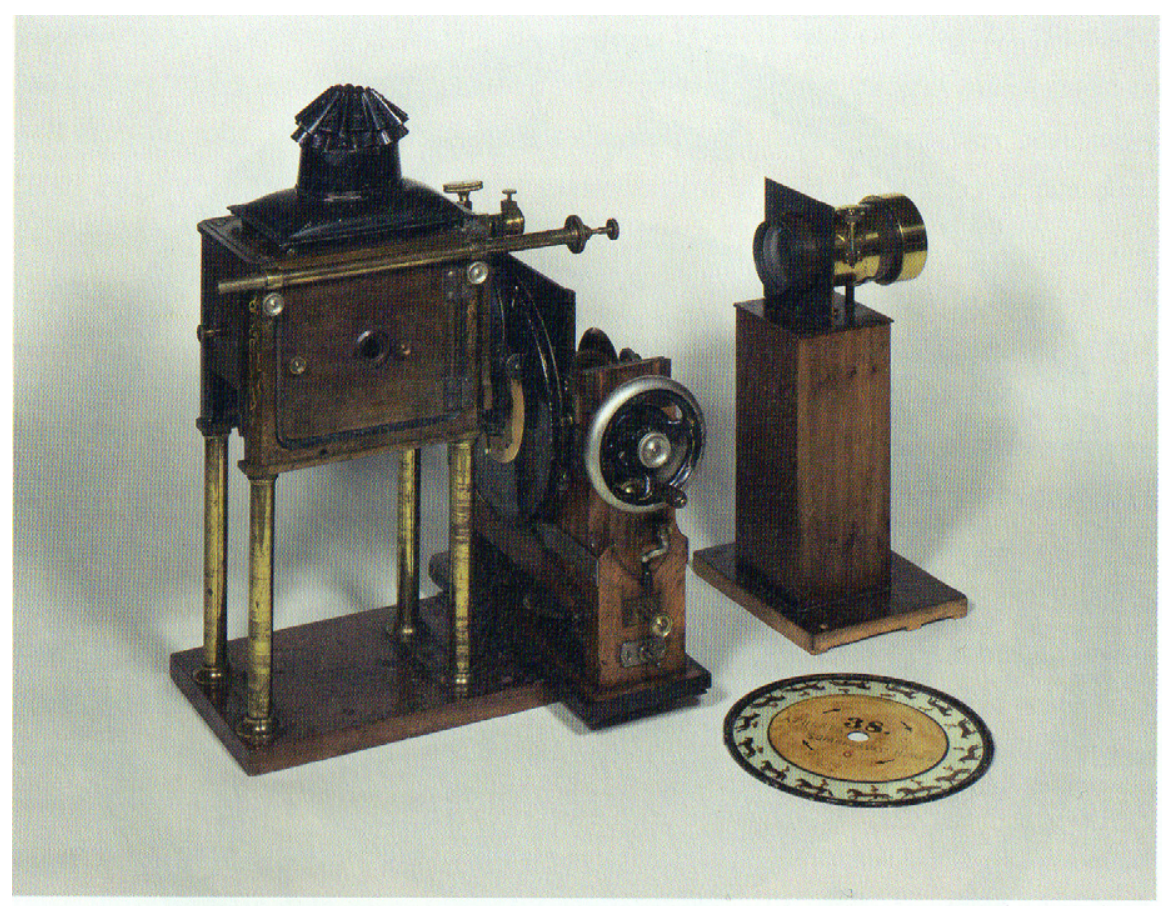

Fig. 77. Zoopraxiscope, 1879-85

Fig. 49 - Muybridge's Zoopraxiscope (Brookman, 2010:89) 
and change from one image to the next is small enough for us to understand what happened between them (St George, 2009: 13; Monaghan, 2009: 128). This technique enabled a 'crystallization of time' in which subjects that were moving could be seen and studied (Pisano, 2009: 63-64). While still photography lacks the ability to capture movement over time, chronophotography allows for the study of 'micro-moments' of bodies in motion (Cloninger, 2009: 114-116).

Chronophotographic sequences can reveal aspects of movement that we cannot see by live observation, for example the movement of animals' legs in motion (St George, 2009: 2; Askham, 2009: 123). Marey and Muybridge both used chronophotography to explore elements of animal and human movement that were usually unseen, concealed or private (see figs. 50-52) (Braun, 2009: 44).
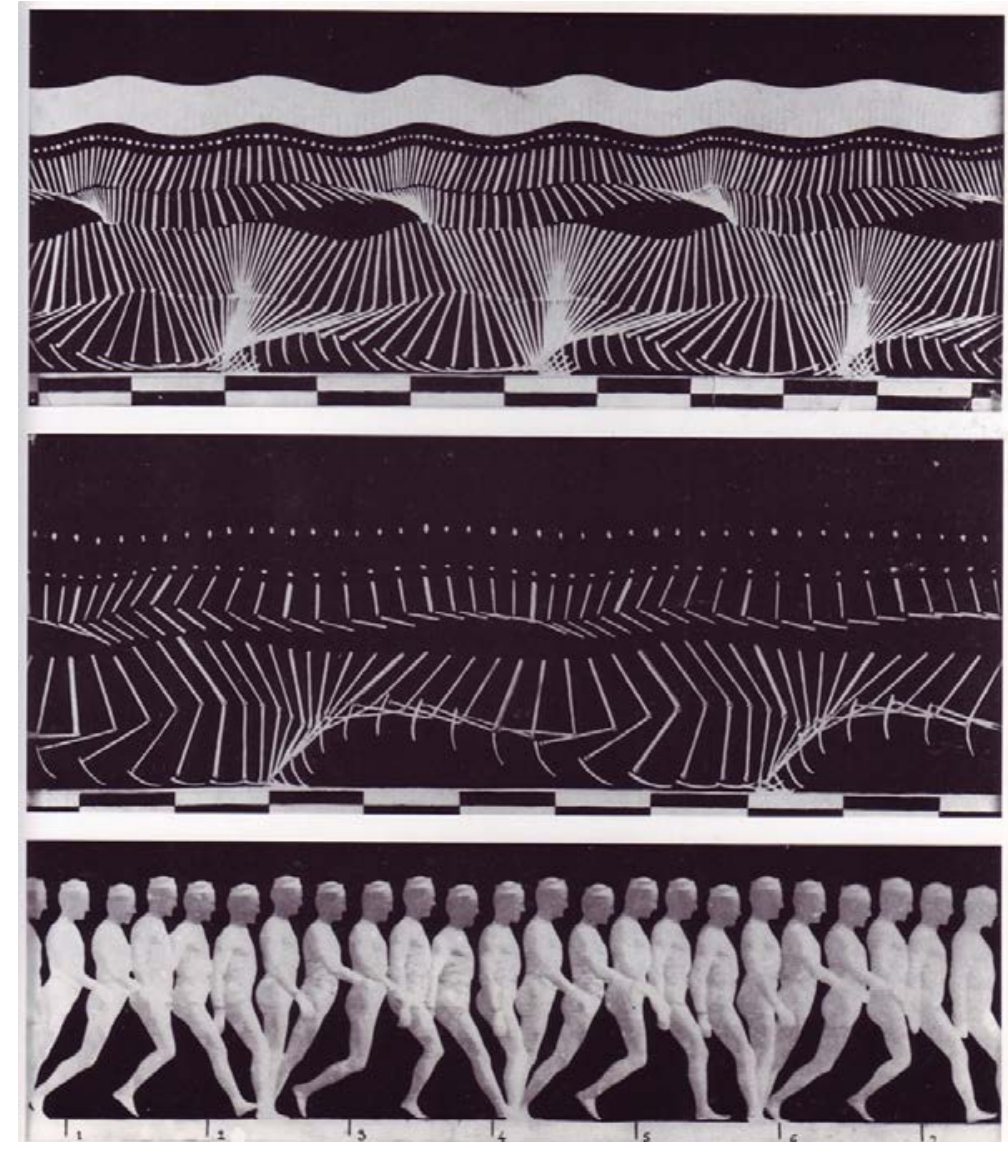

Fig. 50 - Marey's chronophotography from The Human Body in Action, Scientific American, 1914 (Motion Gallery, 2014) 


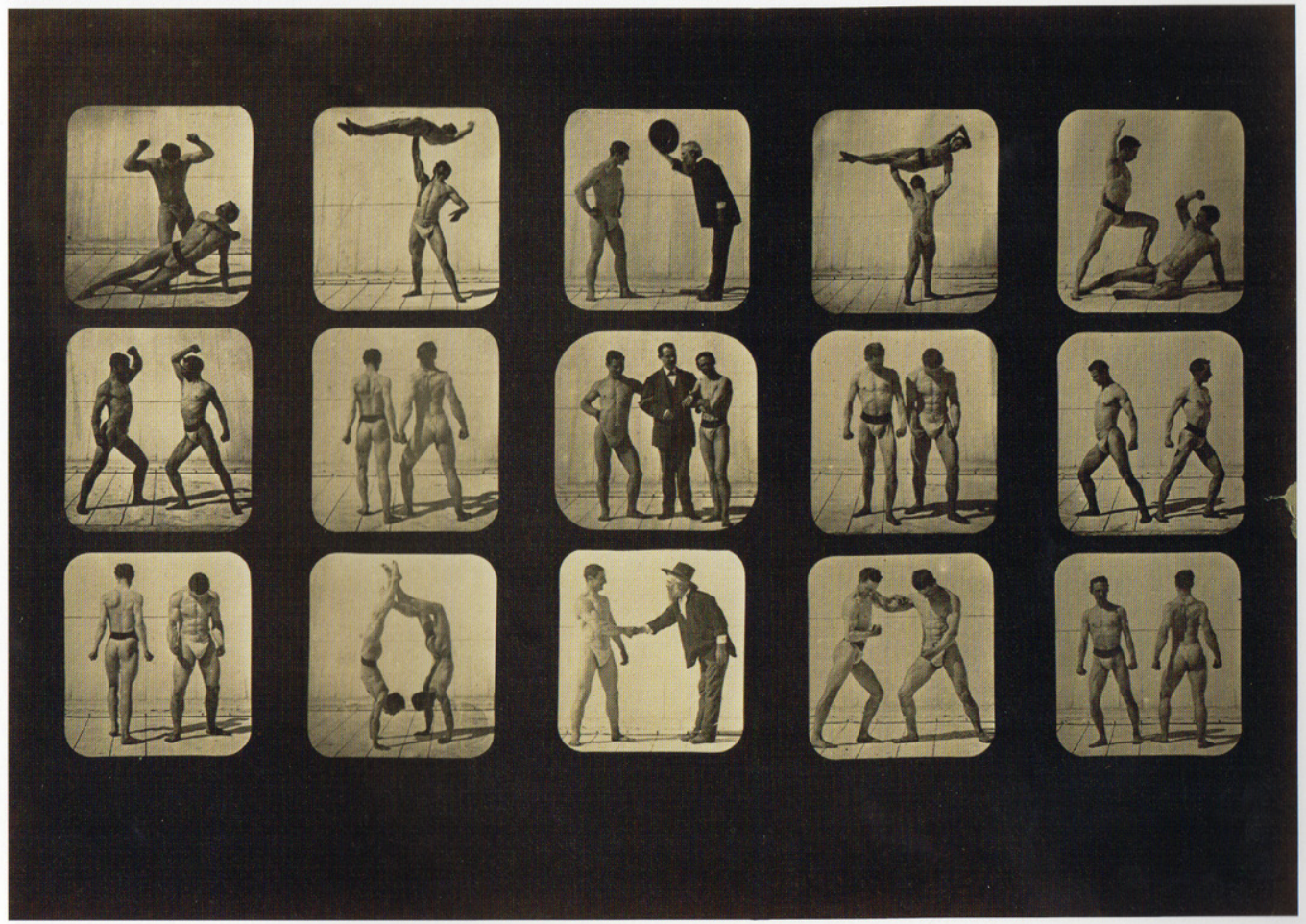

135. Athletes. Pasturing. Plate 115, 1879, from The Attitudes of Animals in Motion, 1881

Fig. 51 - Muybridge's chronophotography: Athletes. Posturing. Plate 115, 1879 (Brookman, 2010: 261)

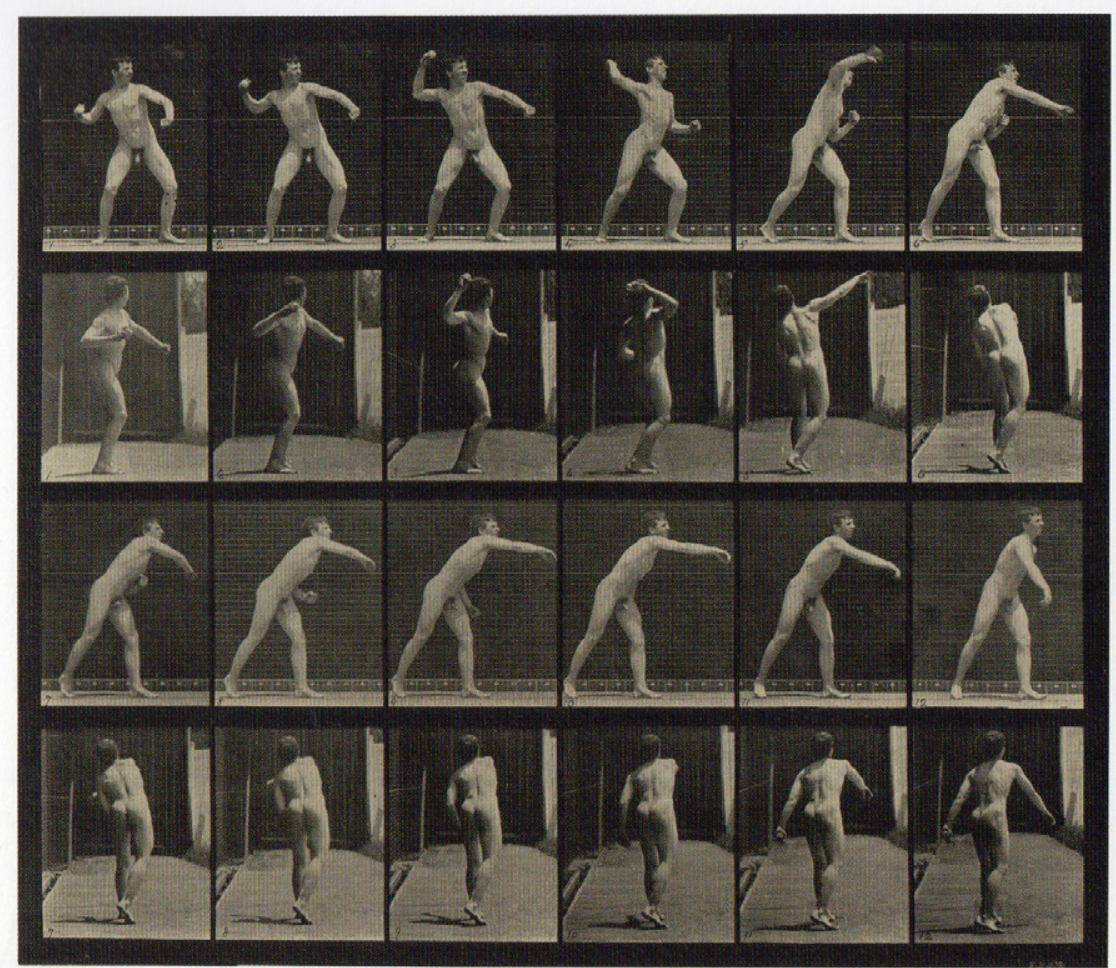

Fig. 116. Base-ball; throwing. Plate 286, 1887

Fig. 52 - Muybridge's chronophotography: Base-ball; throwing. Plate 286, 1887 (Brookman, 2010: 276) 
In 1878 Muybridge had produced his first successful sequence of photographs of a horse in motion (see fig. 53) (Cloninger, 2009: 118). A year later he used up to twenty-four cameras to photograph sequences of horses that were running, trotting, walking and moving in a range of ways (Brookman, 2010: 85). Muybridge was always experimenting with creating new equipment and testing new techniques to improve his sequential photography. In 1884 he photographed subjects carrying out one moment of action, for example an athlete throwing a ball (see fig. 52), and he did so using multiple cameras at different angles, with a grid of lines and numbers as the background. He also photographed two female models passing/greeting (see fig. 54). This sequence captured how the two women approached each other, gave a slight greeting (turning), and continued in opposite directions.

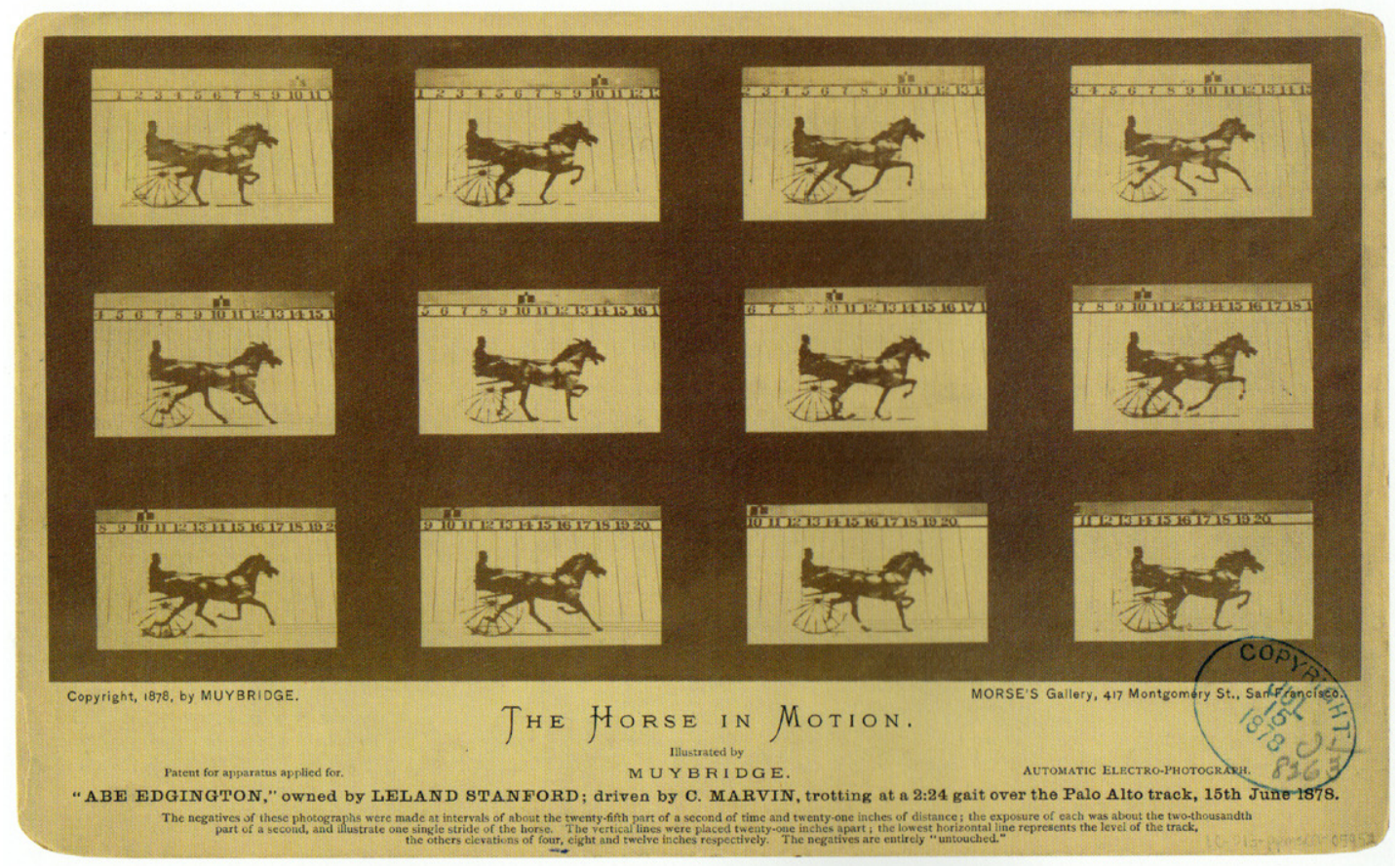

129. The Horse in Motion. "Abe Edgington," owned by Leland Stanford; driven by C. Marvin, trotting at a 2:24 gait over the Palo Alto track, $15^{\text {th }}$ fune 1878,1878

Fig. 53 - Muybridge's chronophotography: The Horse in Motion (Brookman, 2010: 255) 


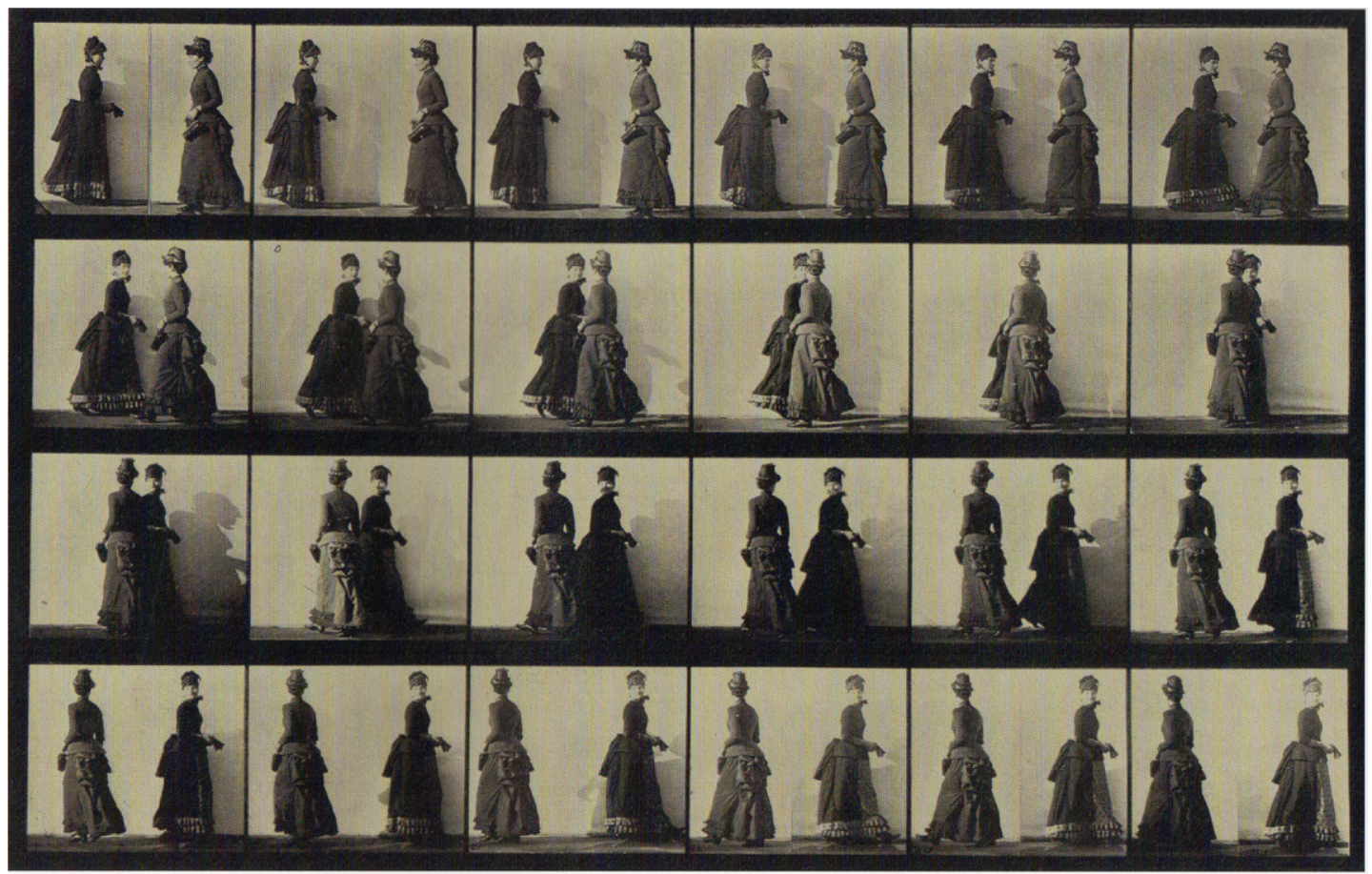

174. Walking, two models meeting, and partly turning. Plate 45,1887

Fig. 54 - Muybridge's chronophotography: Walking, two models meeting, and partly turning. Plate 45, 1887 (Brookman, 2010: 351)

This way of documenting, or distilling, an interaction draws attention to the space around, and between, the two women, and to their posture. Their postures reveal moments of engagement between them, and when that engagement starts and ends.

Muybridge's ambition with his chronophotography went beyond just scientific research and analysis. His work also had a commercial purpose and appeal, and he started selling prints from his collection Animal Locomotion in 1887. He hoped his prints, which were organised as an atlas of images about movement in space and time, would appeal to painters, sculptors, anatomists, physiologists, the general public and horse owners. A number of artists, such as the painter and sculptor Edgar Degas, were indeed inspired by his work, as the images helped them with the modelling for their work of humans and animals in movement (Brookman, 2010: 93-95). Today artists and visual practitioners in many fields still utilise the essential concept of chronophotography, but using more contemporary techniques 
such as stop motion/time-lapse photography and video (St George, 2009: ix; Georges, 2009: 178). In my documentation of greetings I use a 'reverse' chronophotography method: first filming greetings in the studio, and then extracting image sequences from the video footage, from which I analyse (micro-) moments of the greetings. This method enables me to study particular moments of the greeting in a series of individual images, as well as viewing the greeting sequence as a continuous interaction.

Muybridge's work has had a huge impact on the representation and understanding of movement (Pisano, 2009: 58). However, it was subject to some criticism in the late 19th century for being staged and constructed. The main critique was that Muybridge's recordings of fluid movement were constructed narratives for which he made decisions about the duration, angles and which moments to record. Muybridge used models to re-enact movements and postures, and his critics accused him of having staged the actions in the images, arguing that his recordings were not logical or representative of the 'truth' (Monaghan, 2009: 128). I will not go into depth concerning the different debates about Muybridge's and Marey's work, as I am simply interested in the visual techniques used and how movement was recorded. However, like Muybridge's photographic studies, my greeting documentations are carried out in a studio setting, and the debate about whether the 'truth' is represented when the narrative is constructed is relevant to my method of documenting greetings, as I will discuss further in chapter 4.

\subsection{Movement \& Dance Notation}

Another established method for documenting and visualising movement is the visualisation of dance choreography through movement notation systems. These systems make possible the reading, analysis, writing and reconstruction of movement using graphic symbols. Since the fifteenth century at least 87 movement writing systems have been created and used in Europe and North America (Farnell, 1996). Farnell, in her text 'Movement 
Notation Systems', introduces some of the earlier systems created in the fifteenth century; however these systems were not used or developed further because they were created for specific research or dance styles at the time.

The challenge for creators of dance notation has been to create systems that make it possible to notate all anatomical actions of the body. Today there are three systems in use which do that, all created in the twentieth century: Labanotation, Benesh movement notation, and Eshkol-Wachman notation (Farnell, 1996).

The Eshkol-Wachman notation system was published in 1958 by modern dance choreographer Noa Eshkol and architect Abraham Wachman, two Israelis who shared an interest in movement in space. Their system has been used for contemporary choreography, but is not limited to use in dance or human movement. It has also been used for architectural design, animal behaviour studies and for Israeli sign language (Farnell, 1996).

The notation expresses relations between different parts of the body. Limbs are imagined as straight lines, and each limb lies between two joints or has one joint and one free side (see fig. 55). The system of reference for the notation is a sphere. The sphere has a horizontal plane and a vertical plane, and different coordinates are ascribed to different sections (lines) of the sphere (see figs. 56-57). When a limb rotates around itself (0 degrees), it is called a rotary movement, when the angle of the movement is 90 degrees and the limb creates a large circle, the movement is called plane movement. Any movement in between these two, creating circles of different sizes, is called conical movement (see fig. 58). These three types of movements are represented with different symbols in the notation system: $\frown=$ rotary movement; $\Lambda$ = conical movement; and $\uparrow=$ plane movement (Eshkol, Harries, 1988). There is one horizontal plane, eight vertical planes, and 32 intermediate planes (see fig. 59) through which one can move (Eshkol, Harries, 1988). 


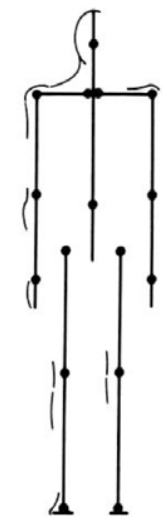

Fig. 55 - EshkolWachman notation The body as a system of articulated axes (Eshkol and Harries, 1988)

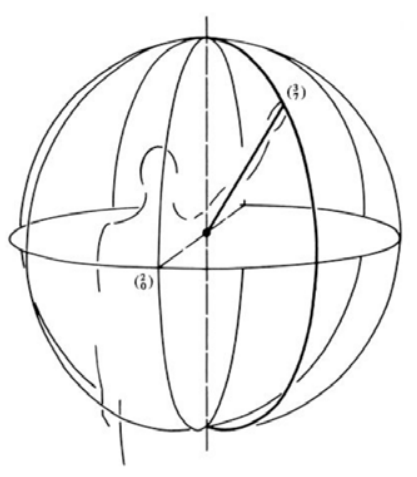

Fig. 56 - Eshkol-Wachman notation - The system of reference is a sphere (lbid.)

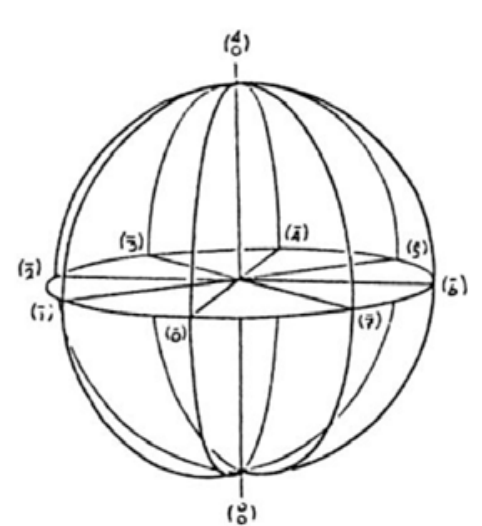

Fig. 57 - Eshkol Wachman notation - Horizontal plane and vertical planes in the scale $1=45$ degrees (lbid.)

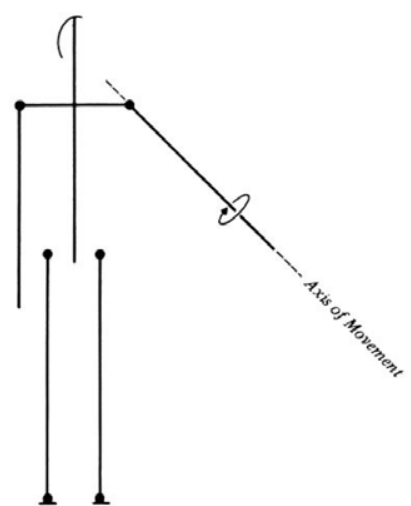

Fig. 8. Rotatory movement

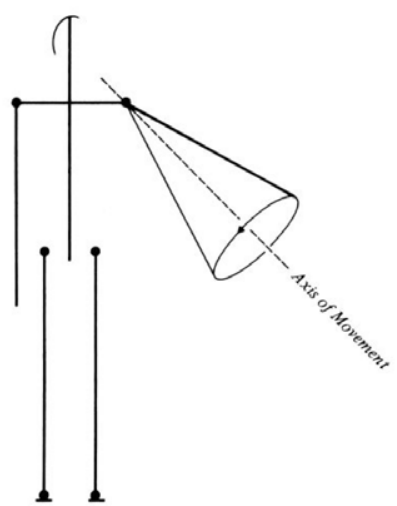

Fig. 9. Conical movement

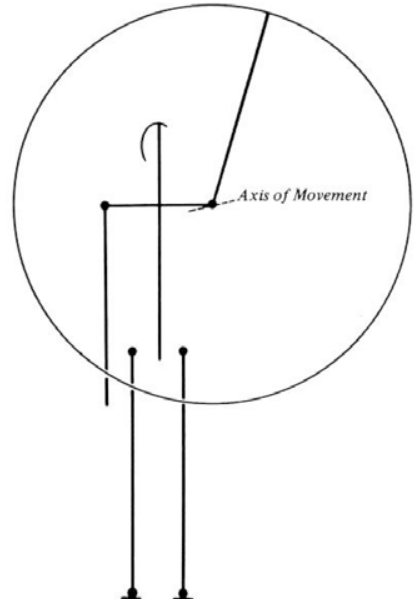

Fig/ 10. Plane movement

Fig. 58 - Eshkol-Wachman notation - Axis of movement, angle of movement and type of movement (Ibid.)

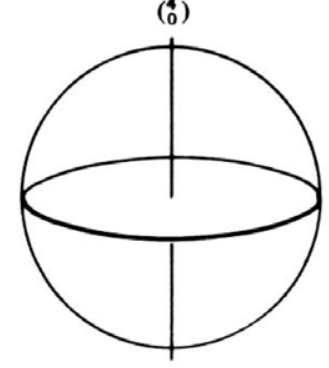

$\left(\begin{array}{l}0 \\ 0\end{array}\right)$

(i) Horizontal plane

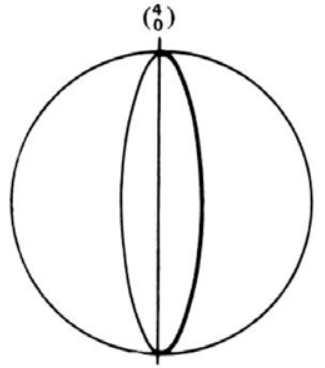

$\left(\begin{array}{l}0 \\ 0\end{array}\right)$

(ii) Vertical plane

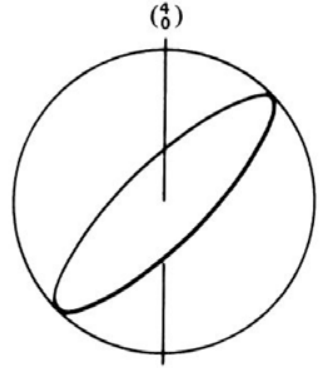

$\left(\begin{array}{l}0 \\ 0\end{array}\right)$

(iii) Intermidiate plane

Fig. 11.

Fig. 59 - Eshkol-Wachman notation - Plane movement (Ibid.) 
When it comes to writing down the movement notation using the EshkolWachman method, the body is divided into eighteen sections/numbers on a staff, and one row of the staff represents one body section (see figs. 60-61). Body parts are numbered from three to twenty. Symbols representing movements and numbers representing planes are written on these horizontal columns, and the score is read horizontally from left to right as a timeline of movement (see figs. 61-62).

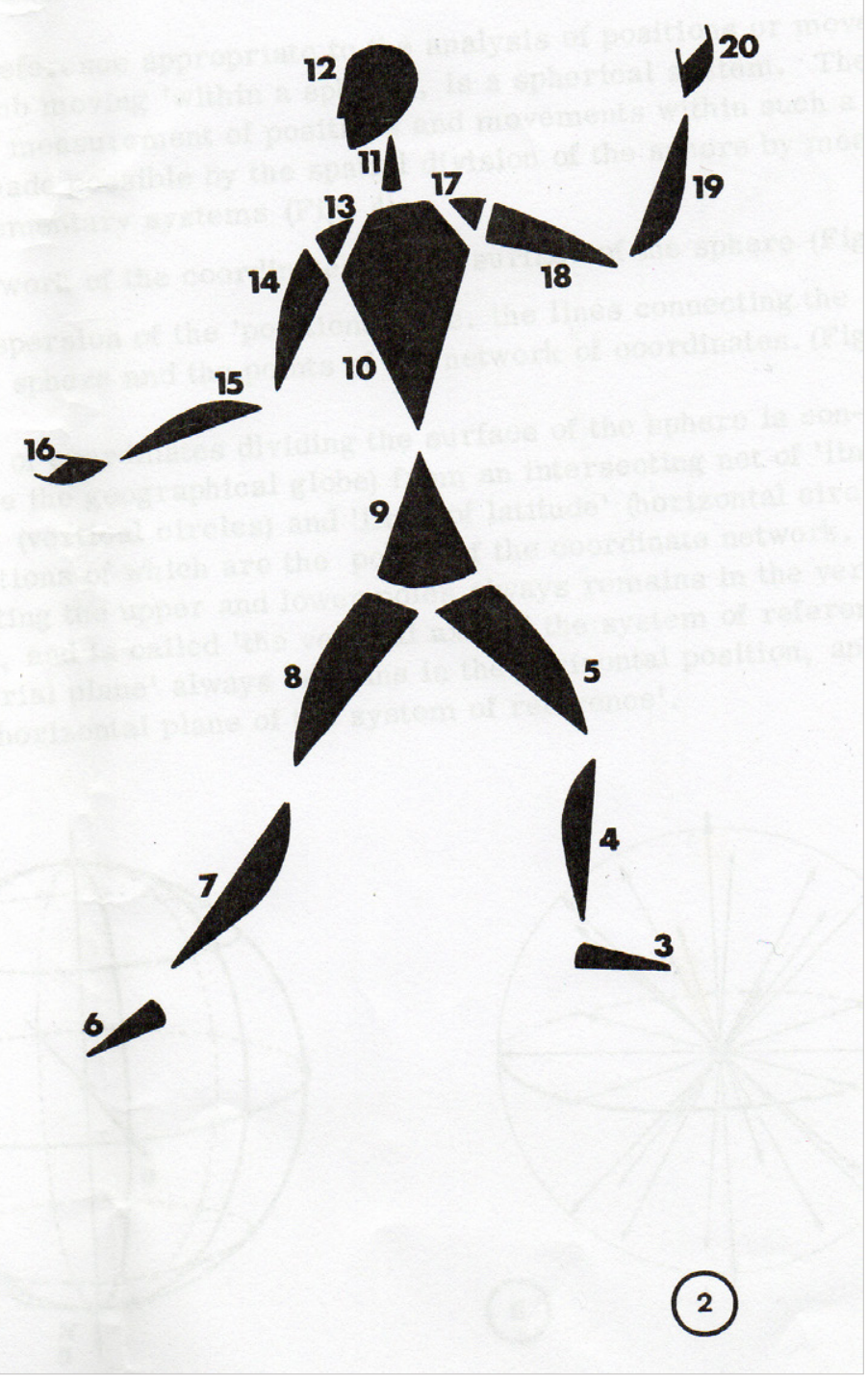

Fig. 60 - Eshkol-Wachman notation - body sections (Eshkol, 1972) 


\begin{tabular}{||l|l|l|l|l|l|l|l|l|l|l|l|}
\hline & & Hand & & & & & & & & 20 \\
\hline & & Forearm & & & & & & & 19 \\
\hline \\
\hline
\end{tabular}

\begin{tabular}{||c|c|c|c|c|c|c|c|c|c|c|}
\hline & & Hand & & & & & & & & 16 \\
\hline & \\
\hline & Right Forearm & & & & & & & & 15 \\
\hline \\
\hline
\end{tabular}

\begin{tabular}{|l|l|l|l|l|l|l|l|l|l|l|l|l|}
\hline & Head & & & & & & & & & & $\mathbf{1 2}$ \\
\hline & Neck & & & & & & & & & & 11 \\
\hline & Teck t \\
\hline
\end{tabular}

\begin{tabular}{|l|l|l|l|l|l|l|l|l|l|l|l|}
\hline & & Thigh & & & & & & & & & 8 \\
\hline \\
\hline
\end{tabular}

\begin{tabular}{||l|l|l|l|l|l|l|l|l|l|l|l|}
\hline & & Thigh & & & & & & & & 5 & \\
\hline & Left & Lower Leg & & & & & & & 4 & \\
\hline & \\
\hline & \\
\hline
\end{tabular}

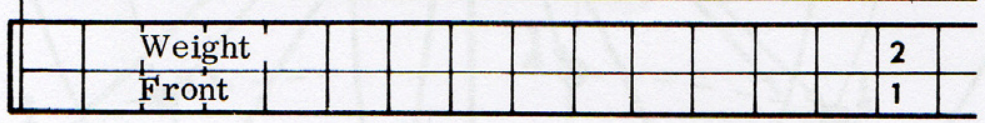

$\stackrel{\text { Time }}{\longrightarrow}$

Fig. 61 - Eshkol-Wachman notation - structure of staff: each row represents one body section (Eshkol, 1972)

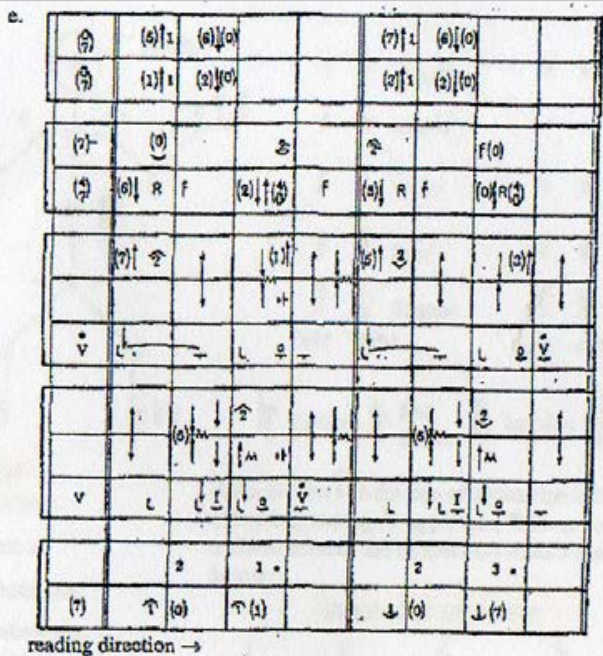

Figure 8b. An example of writing in Eshkol Wachman notation (redrawn after Hutchinson-guest 1984; 109).

Fig. 62 - Eshkol-Wachman notation - an example of the system in use (Farnell, 1996: 157) 
Eshkol-Wachman notation is a sophisticated system that allows for detailed representation of the movement of bodies through space and time. The score as a whole reflects movement of all the body parts in relation to each other. It shows spatial relations, and changes thereof, throughout the movement sequence. The organisation of the staff into different rows also makes it easy to follow the movements of specific parts of the body throughout the sequence/choreography.

Another system designed for a similar purpose is Benesh movement notation, which was created in 1955 by English ballet dancer Joan Benesh and her husband Rudolf Benesh. Joan Benesh was interested in

Structure

Benesh Movement Notation is written from left to right on a five-line stave. Floor patterns are written below the stave.

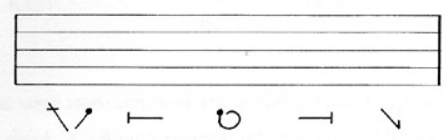

Direction, Location and Travel for the Solo Dancer and Groups are described in Part I.

Fig. 63 - Benesh movement notation structure (Brown and Parker, 1984: 81)

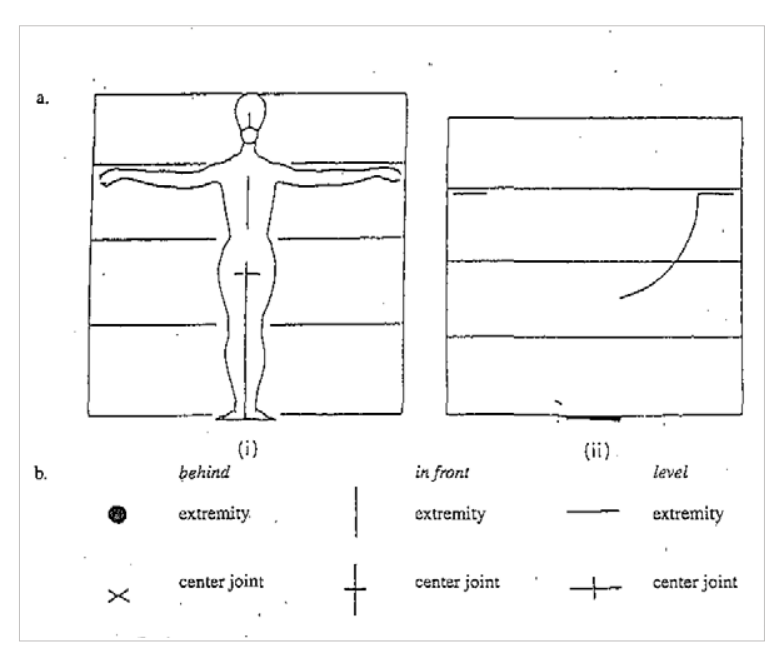

Fig. 64 - Benesh notation system is based on iconic visualisation of the body (Farnell, 1996: 154) choreography and the initial purpose of the system was for the recording of ballet. However, the system has also been used in other forms of dance and movement therapy. It uses an iconic representation of the body within a five-line stave, which is read horizontally (see fig. 63).

Fig. 64 shows how the different body sections fit into the stave: the top line represents the height of top of the head; the second line the height of the shoulders; the third line the height of the waist; the fourth line the height of the knees; and the fifth and bottom line the floor. However, in the notation the body is not represented as a full figure: the 

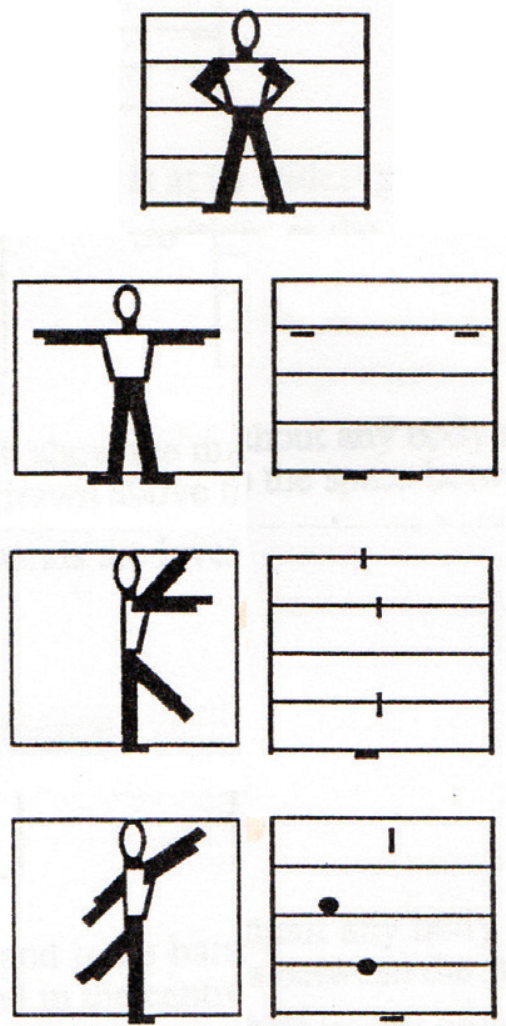

Fig. 65 - Benesh movement notation - viewpoint and the three basic signs (Parker, 1996) hands, elbows, feet, knees, head and body parts are represented using different signs.

The signs used vary depending on the positioning and placement of the body parts. The viewpoint in Benesh movement notation is from directly behind the performer.

Different signs are used depending on whether the hands and feet are at level with the body (second row from top of fig. 65 ), in front of the body (third row from top of fig. 65), or behind the body (bottom row of fig. 65). Fig. 66 shows that the different signs are also used to show whether wrists are facing in, out, forward, backward, up or down (Brown, Parker, 1984; Parker, 1996).

\begin{tabular}{|c|c|c|c|c|c|}
\hline In & Out & Front & Back & Up & Down \\
\hline C $\supset$ & כ c & $u v$ & $\cdot n n$ & し J & r 1 \\
\hline
\end{tabular}

- arms in front at shoulder height

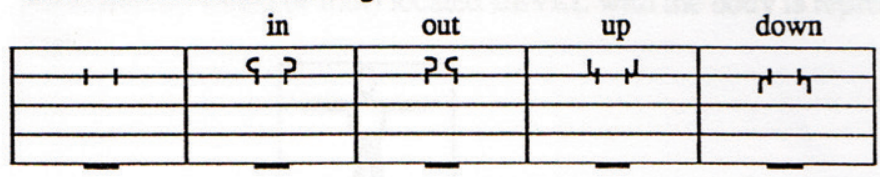

Wrists cannot face front or back in this position.

- arms to the side at shoulder height

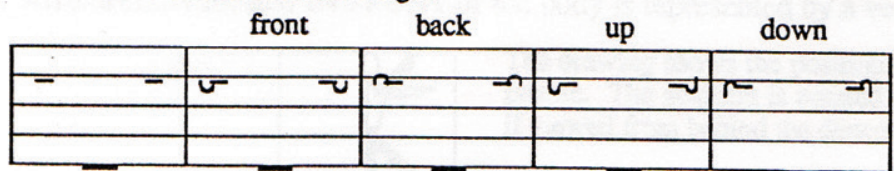

Wrists cannot face in or out in this position.

- arms behind the body at thigh height

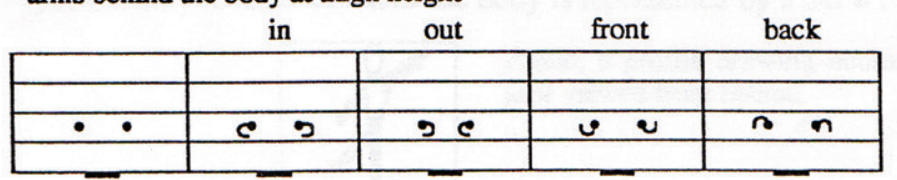

Wrists cannot face up or down in this position

Fig. 66 - Benesh movement notation - wrist directions (Ibid.) 
The direction of movement is illustrated using a 'pin'. The round part of the pin indicates the head, and the orientation of the pin correlates to the direction that the dancer faces (see fig. 67). Bent pins indicate changes in movement direction or turns (see fig. 68). To show more than one full turn in one sign, a number is added inside the pin (Brown, Parker, 1984).

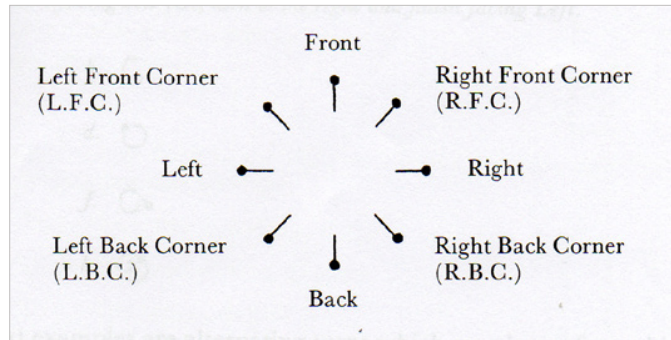

Fig. 67 - Benesh movement notation direction (Brown and Parker, 1984: 83)

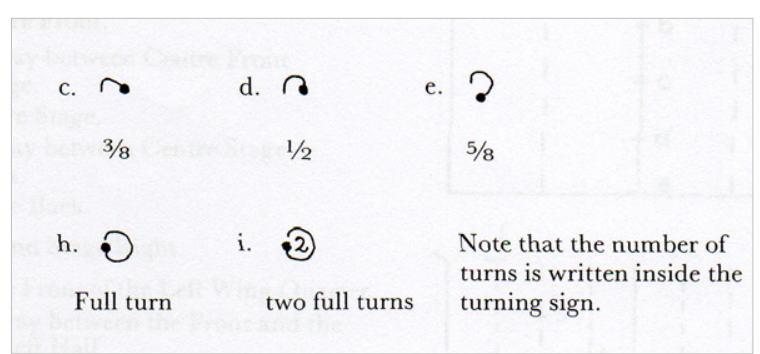

Fig. 68 - Benesh movement notation changing direction (Brown and Parker, 1984: 85)

I have only introduced the structure and most basic signs of the Benesh movement notation system (there are further sets of signs, such as those for illustrating location on the stage with floor plans). Fig. 69 shows the system in use: how a dancer's movements are translated into a Benesh movement

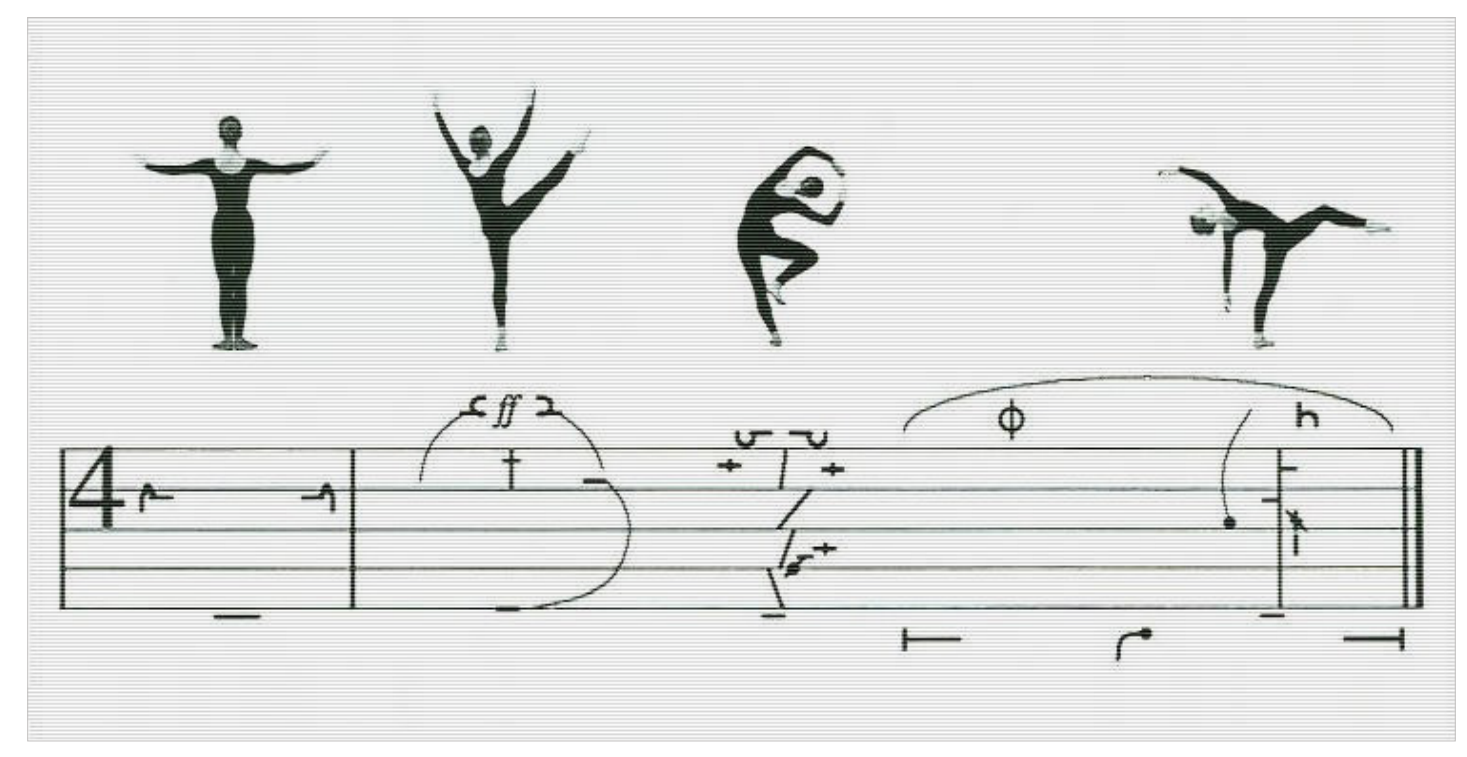

Fig. 69 - Benesh movement notation - example of score with dancer's moves above the score (Royal Academy of Dance, 2014) 
score. The images of the dancer are not part of the score; they are added to show how the notation corresponds to 'live' movement.

The third system relevant to my study which I will outline here is Labanotation. This is a standardised system for recording and analysing any form of human motion, invented by the Austrian-Hungarian Rudolf von Laban in 1928. Laban studied movement in many different situations, from dance and mime to manual labour. His first publication of the notation was called Kinetographie, and from this initial publication several people, such as Ann Hutchinson Guest, continued the development of the notation into what we today know as Labanotation (Farnell, 1996; Griesbeck, 1996). Creating a notation system is a complex task, and according to Farnell the graphic signs in a notation system must represent the following: all parts and surfaces

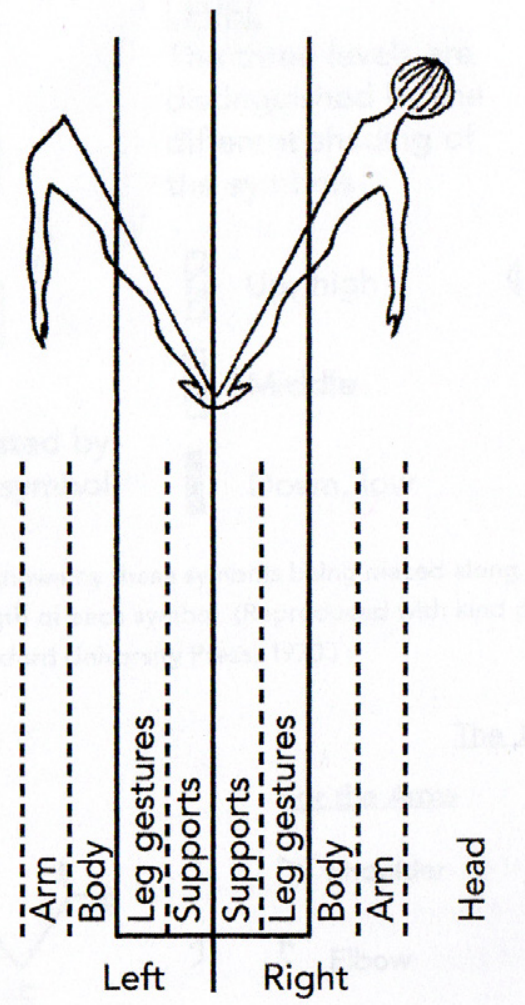

Center Line

Fig. 70 - Labanotation - staff showing different parts of the body broken into columns (Davies, 2001: 8) of the body, three dimensional space, time, dynamics, relationships between body parts and relationships to other persons or objects. Labanotation does this, and Griesbeck (1996) argues that it is possible to record all kinds of human movement using this system.

In Labanotation different parts of the body are represented in a staff consisting of three lines that run vertically (see fig. 70). Columns named 'supports' record the change in weight, such as if the weight is on the right foot, left foot or both. These columns will be empty when a jump occurs because then there is no weight on the ground. All the other columns record gestures. The staff can 


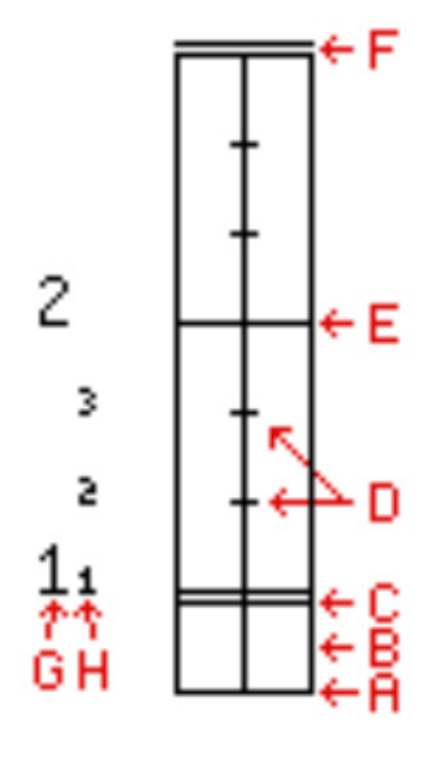

Fig. 71 - Labanotation lines indicating: $A=$ start of staff; $B$ = starting position; $\mathrm{C}=$ start of the movement; $\mathrm{D}=$ the beat; $\mathrm{E}=$ bar line; $\mathrm{F}=$ end of the movement; $\mathrm{G}$ = large number for the bar; $\mathrm{H}=$ small numbers for the beat (Griesbeck, 1996) be adapted, and endless additional columns can be added for more complex combinations of movement. Different types of lines are used in the staff to indicate the starting position, the start of the movement, the beat, and the end of the movement (see fig. 71). The benefit of the scores being read from the bottom up is that they are written from the dancer's point of view. Symbols for the left side of the body are notated on the left and symbols for the right side of the body are notated on the right, making it easy for dancers to read the score if it is put on the floor in front of them. Benesh notation, Eshkol-Wachman notation, and other notation systems are read horizontally, from left to right (Griesbeck, 1996).

Direction symbols indicate the horizontal direction a person moves in, and the vertical position of the body is illustrated with three different types of shading for high/up, middle, and down/low (see fig. 72) (Farnell, 1996). Arm and leg gestures are indicated using these direction symbols; fig. 73 shows how the symbols are adapted for arm and leg gestures. Relationship pins (see fig. 74) illustrate relations in space: the orientations of the pins show the horizontal directions while the heads of the pins show the vertical directions. These pins can be used in different ways, to show direction, relation between two parts of the body, and intermediate position.

Examples of the shifting of the centre of weight, and transference of weight/ steps are illustrated in the scores in fig. 75. Jumps are illustrated in fig. 76 where A shows jumps (the gaps) with both feet, B shows leaps from one foot to the other and $\mathrm{C}$ shows hops on the right leg. 


\section{DIRECTION}<smiles></smiles>

Direction is indicated by the shape of the symbol.
LEVEL

The three levels are distinguished by the different shading of the symbols

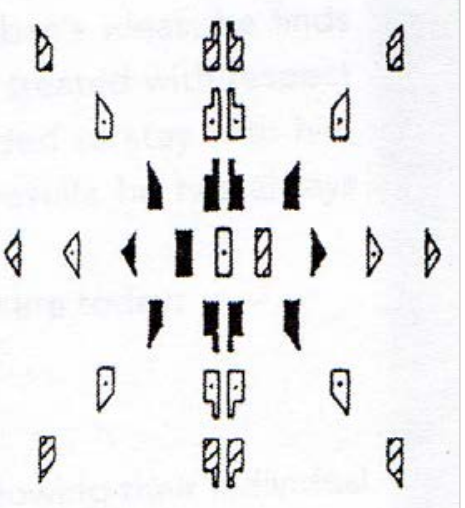

Fig. 2. Direction was shown by these symbols being placed along the relevant part of the staff, and timing by the length of each symbol. (Reproduced with kind permission from Labanotation by Ann Hutchinson, Oxford University Press, 1970.)

Fig. 72 - Labanotation - direction symbols and levels distinguished by shading (Griesbeck, 1996)
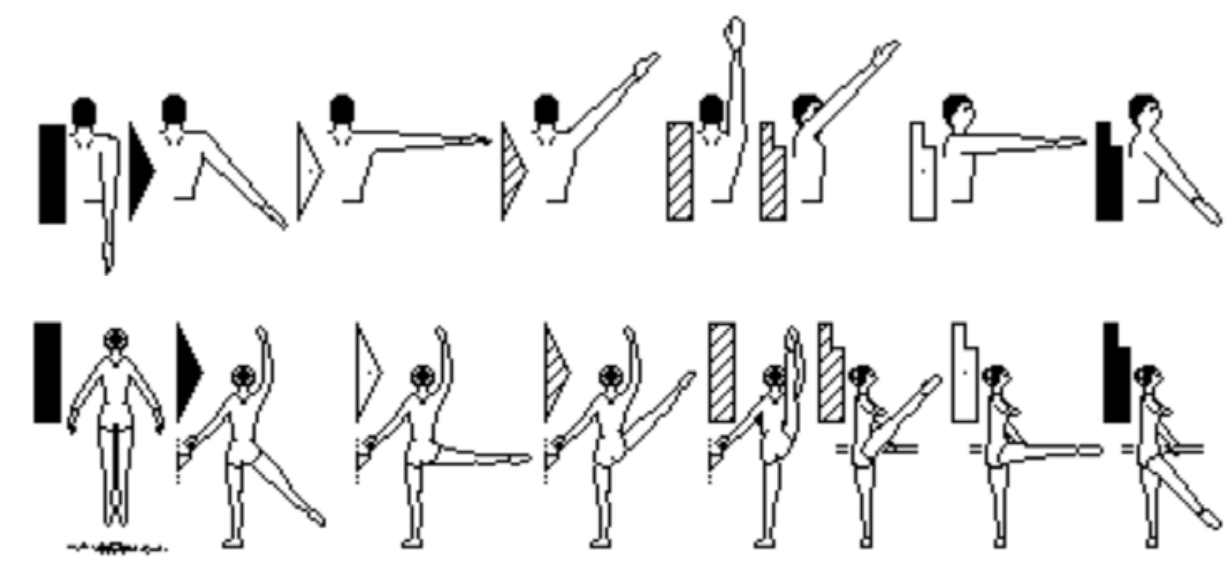

Fig. 73 - Labanotation - direction symbols for different levels of arm and leg movements (lbid.) 

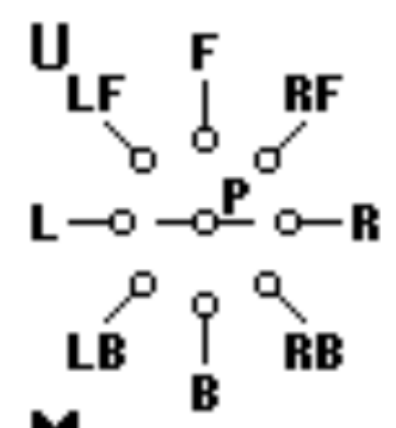

$M$

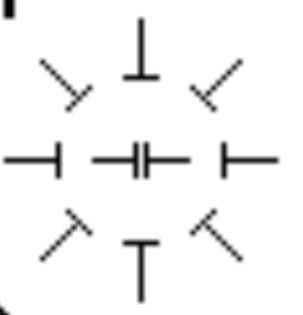

D
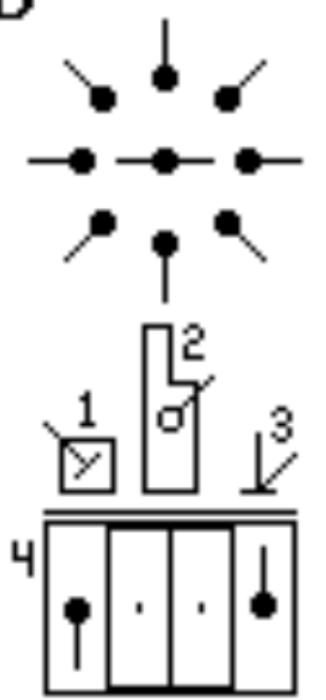

Fig. 74 - Labanotation relationship pins: $L=$ left; $\mathrm{R}=$ right; $\mathrm{P}=$ place; $\mathrm{F}=$ Forward; $\mathrm{B}=$ backward; $\mathrm{U}=$ up; $\mathrm{M}=$ middle; $\mathrm{D}=$ down (lbid.)
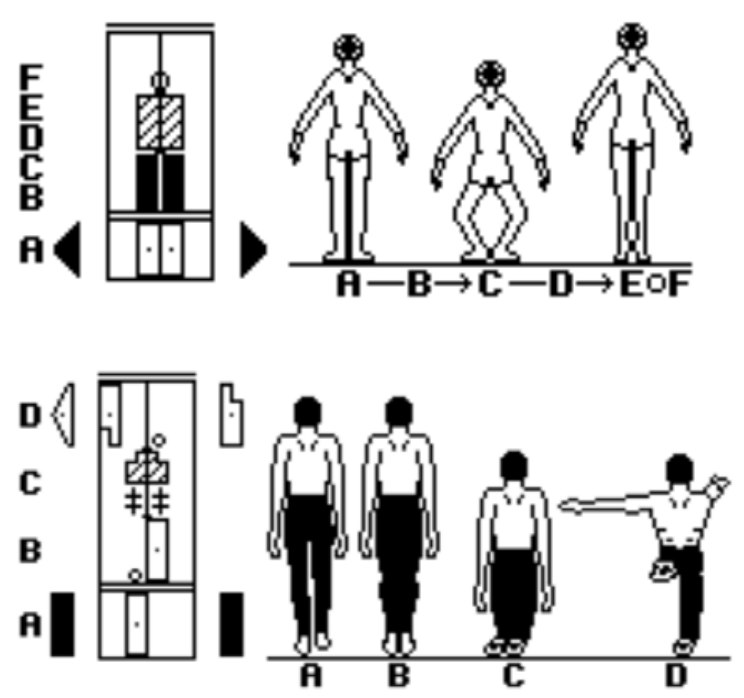

Fig. 75 - Labanotation - shifting the centre of weight (top) and transference of weight (bottom) (lbid.)
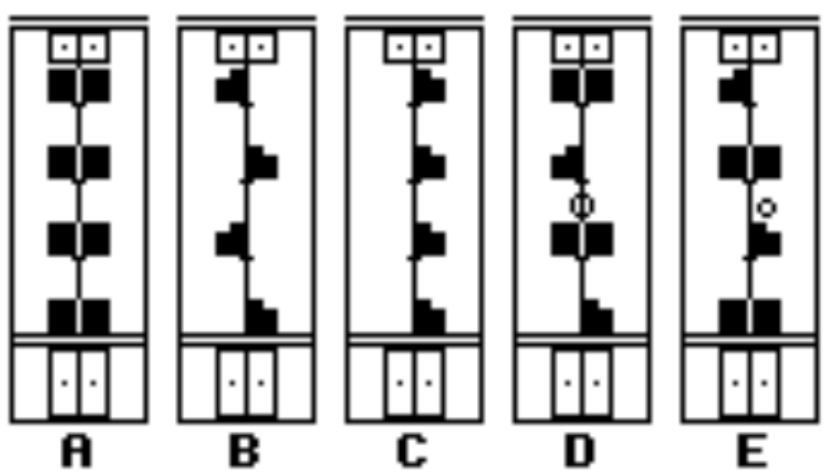

Fig. 76 - Labanotation - jumps: $A=$ both feet; $B=$ from one foot to the other; $C=$ on the same foot (lbid.) 


\section{THE BODY SIGNS}

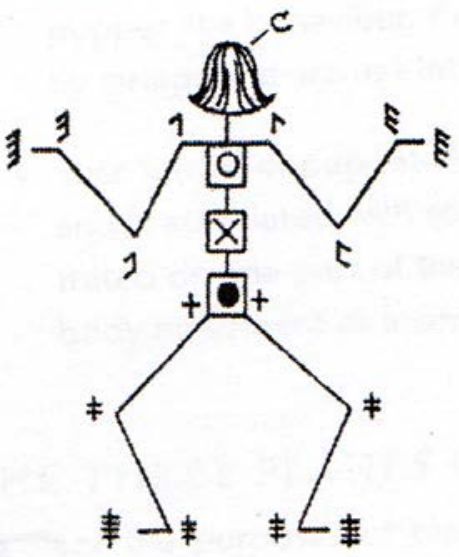

Body Areas

\section{The Joint Signs}

\section{For the Arms}

For the Legs

$\uparrow$ Shoulder

$+\quad t$

$\vdash$ Hip

3

E Elbow

$\neq \quad \neq \quad F$ Knee

3

E Wrist

羊 $\quad$ E Ankle

3 \& Hand

夆手年Foot

$\exists$ Fingers

青青 意 Toes

left right

\section{Basic sign for an area}

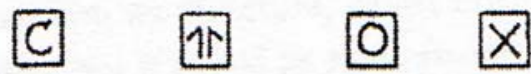

Area of Shoulder Chest Waist head section (rib cage)
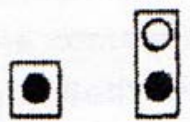

Pelvis Whole torso

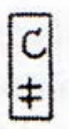

国 etc.

Unit of knee Unit of foot to head to chest

Fig. 3. The basic body signs. (Reproduced with kind permission from Labanotation by Ann Hutchinson, Oxford University Press, 1970.)

Fig. 77 - Labanotation - body signs (Davies, 2001)

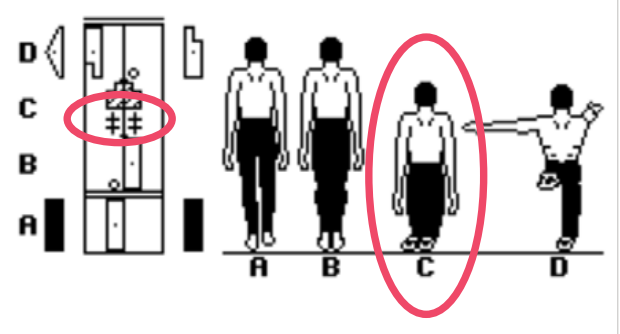

Fig. 78 - Labanotation - symbols indicating weight on knees/kneeling (Griesbeck, 1996)
All body parts are labelled using different signs (see fig. 77). These signs are added to the score to indicate what particular parts of the body are doing. In fig. 78, for example, the knee sign is used in the supports column, which instructs the dancer to kneel down (put weight on the knees).

As in musical notation, Labanotation allows for the representation of relationships between the mover's body parts, or between people/objects, by using arc lines. Vertical arc lines are used for actions that are done at the 
same time, while horizontal arc lines show connections in space (Farnell, 1996; Griesbeck, 1996).

What I have explained so far is the use of symbols in a staff to create a score of the movements of an individual within dance choreography. Labanotation also uses floor plans to notate complex movement patterns involving many people. While scores show detailed movements of different parts of the body, floor plans show how the whole body moves in space and in relation to other dancers. Floor plans are usually written below or beside the staff (Griesbeck, 1996). Fig. 79 shows how dancers' movements in space are translated into floor plans.

\section{Triangle}
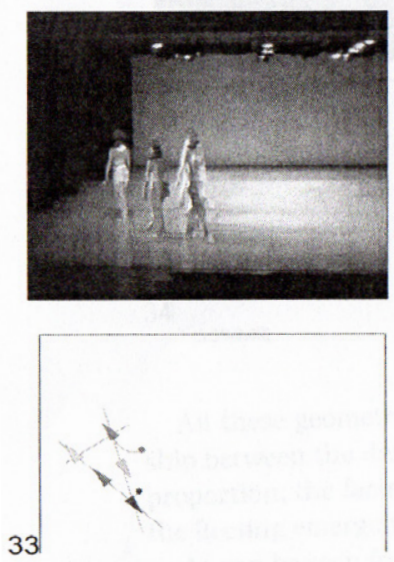

[3:37-3:38]

From circle ind pentagon
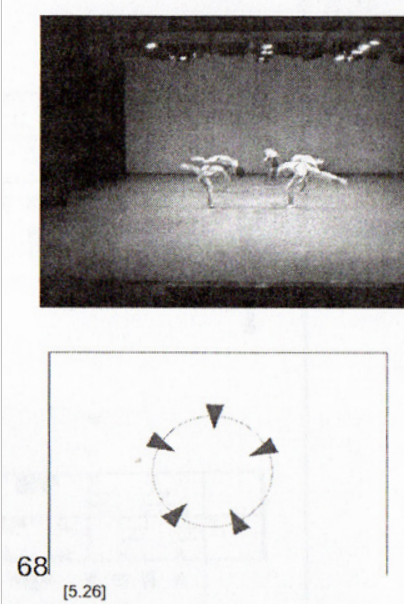

Fig. 79 - Labanotation - floor plans (Preston-Dunlop and Sayers, 2010)

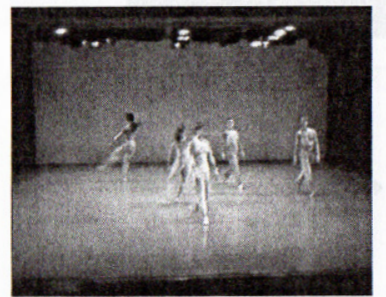

11
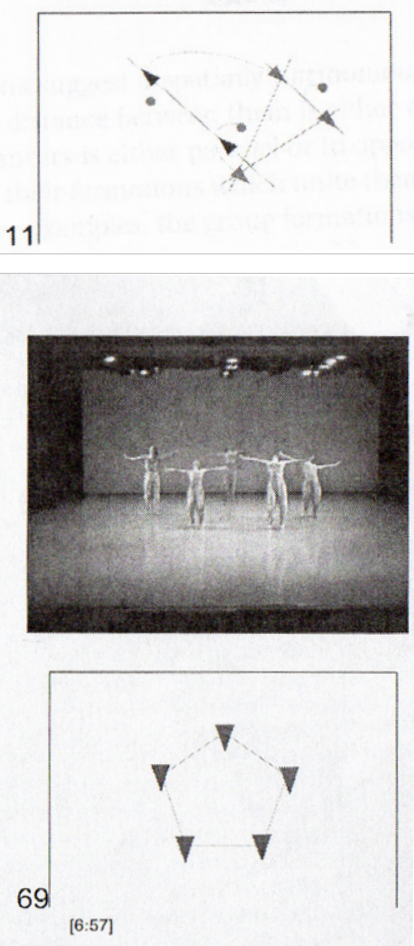
There are many more symbols in the Labanotation system that I have not discussed, such as notation of whether different parts of the feet are in contact with the floor. Other parts of the system such as hold weight, turns, quantity signs, key signs, and accent signs have also not been discussed (Griesbeck, 1996). Rather than discussing the system in any more technical detail, I will show a couple of examples of analysis using Labanotation that are not dance-related.

One such example is Brenda Farnell's use of Labanotation for anthropological analysis. Farnell, who is a professor of anthropology at the University of Illinois at Urbana-Champaign, and who has a background in modern dance, utilised Labanotation in her analysis of Assiniboine storytelling which involved Plains Sign Talk and spoken Nakota (see fig. 80).

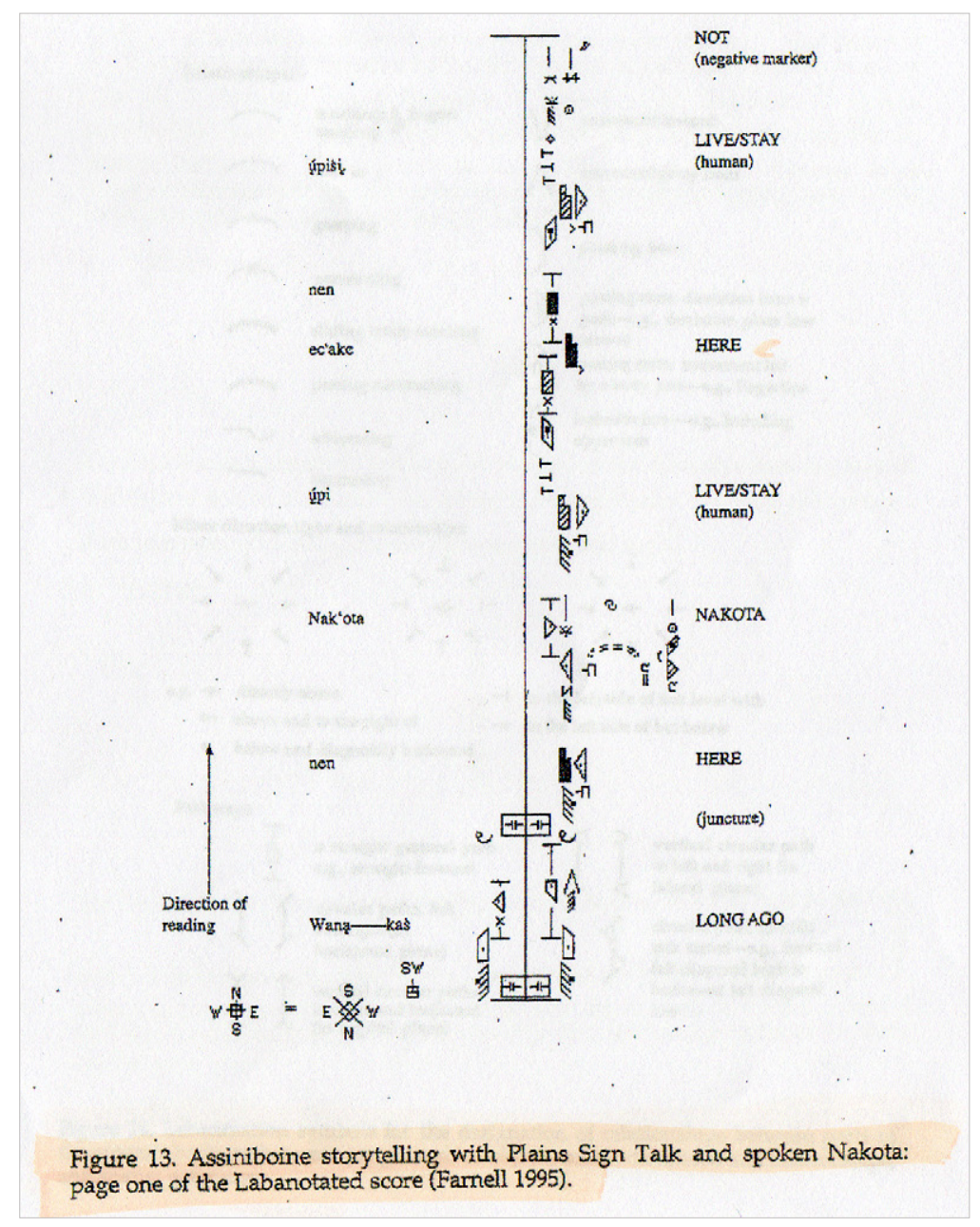

Fig. 80 - Labanotation - example of analysis of Assiniboine storytelling (Farnell, 1996) 
Farnell's aim was to show that a notation system 'creates concepts specific to the medium' and in her example she illustrates that graphic signs can be used to record nonverbal language or “ "talk" from the body' (Farnell, 1996: 168). Fig. 81 shows an annotation of the first section of Farnell's analysis. The notation gives an elaborate visual analysis of how hand and finger gestures are used during the storytelling, as well as of movement paths and orientation of the legs and body. Farnell shows here that the notation system can be used to study and represent any kind of movement, even detailed gestures and body language used in communication during storytelling. Her use of Labanotation for the analysis of anthropological data illustrates that the significance of the system extends beyond the field of dance.

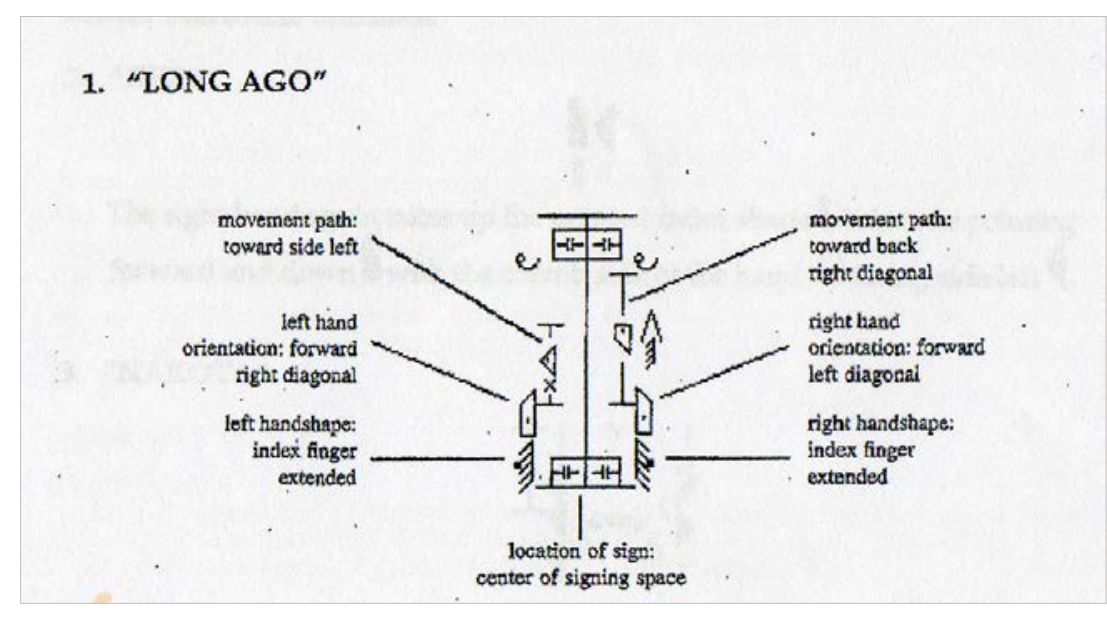

Fig. 81 - Labanotation - explanation of first part of notation of Assiniboine storytelling (Farnell, 1996)

To test the use of Labanotation for the analysis of greetings, I have applied it to a greeting I mentioned in chapter 1, that of two Dutch males who are friends doing a greeting used in their hip-hop dance group. For this I used LabanWriter 4.7.2, which is a software using Labanotation for movement analysis. The top part of fig. 82 shows the image sequence from my recording of the greeting. The analysis/score of the greeting has been annotated in red and is featured in the middle. 


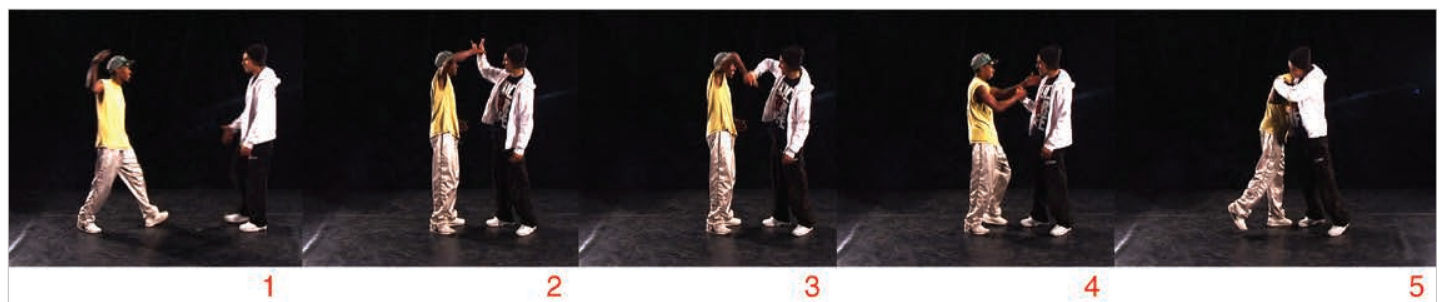

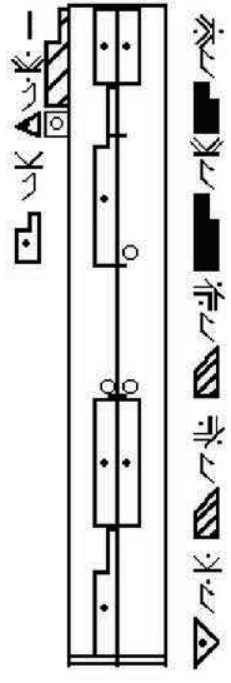

点
5: Arms folded into embrace. Body leaning forward. Weight from left leg to both legs.

4: Right arm slightly angeled forward. Left arm facing downward folding at elbow. Left leg forward, weight on both.

3: Weight still on both legs. Right arm facing up and forward and folded downwards at elbow.

2: Weight on both legs. Right arm facing up, and folded at elbow. Lower arm pointing left.

1: Moving forward. Weight on left leg. Right arm facing straight out to the right, elbow folded at 90 degrees upwards.

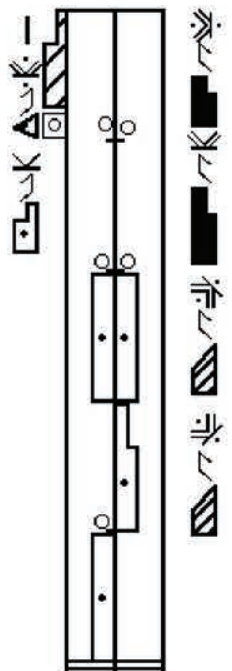

Person on the left (yellow t-shirt)

Person on the right (white hoodie)

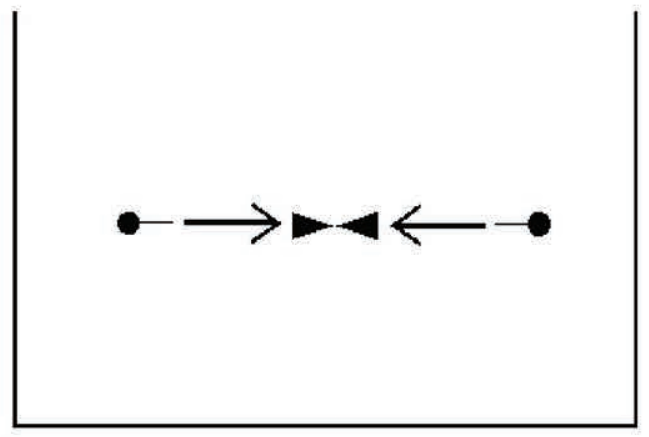

Floor plan

Fig. 82 - Analysis of Dutch 'hip-hop' greeting. Image sequence (top) and analysis using Labanotation: movement score for both people involved in greeting (middle) and floor plan of their movements (bottom) 
A floor plan of the interaction can be seen below the scores (also using Labanotation). In my movement scores I have not included notation of details such as hand and finger movements, or of foot or toe movements beyond those concerning weight and travelling. I have focused on larger movements such as arm angles and folding, weight of the legs and in the last section (5) of the score, the forward movement of the chest during the embrace. The floor plan shows that one person moves from left to right in a straight line, and the other from right to left in a straight line, and they reach their ending points (black rectangles) in the middle where the interaction occurs.

It is clear that Labanotation, Benesh movement notation and EshkolWachman notation enable very accurate recording of body movement. They are complex systems and can all be used beyond the field of dance notation. All three systems have been used in many kinds of movement analysis: in the fields of architecture, body therapy, anthropology, nonverbal communication and many more. While these systems enable the visualisation of most types of anatomical movement, and of different dynamics of movement, they are relatively difficult to learn and memorise. Reading the scores requires the reader to be trained in the systems. I have shown that Labanotation can be applied to the visual notation and analysis of greeting. However, fig. 82 reveals that while the photographic image sequence represents the movements of the greeting very clearly so that they are instantly recognisable, the Laban score does not. This shows that a more accessible and iconic visual language for greeting analysis would be desirable; one which does not require specific training in order to understand it. The three systems discussed are static and focus mainly on anatomical movements and gestures, as well as movement in space. While these are important aspects in greetings studies, my primary interests relate to the use of personal space, physical contact between the people greeting, and their subjective experiences of the encounter. These are dimensions of greetings that may be better portrayed by more dynamic visual representations. In the following sections I will discuss contemporary visual techniques that 
are currently being employed in other fields of research, and that show great potential for use in creating more dynamic visual representations of greetings.

\subsection{Information Graphics (Infographics)}

Information graphics, or infographics, is the use of graphics to explain facts, data or networks. An important figure in this field has been Edward Tufte. His book Beautiful Evidence is a key study of how observations are turned into visual evidence (Tufte, 2006). Tufte, a retired Yale professor in public affairs and political science, and lecturer in statistical graphics and information design, is also an artist and the founder of Graphics Press LLC ${ }^{1}$. He has worked with and advised IBM, NASA and other companies on data analysis and display design (Aston, 2009), and has been nicknamed 'the da Vinci of data' and 'graphics guru' (Shermer, 2005; Cookson, 2013). Tufte has had a significant impact on the field of visual information and data analysis and his extensive publications critique the way information is usually presented to us, such as in charts and cluttered, misleading design. He suggests that 'clear and precise seeing becomes as one with clear and precise thinking', and shows how to successfully visualise data with a focus on simplicity, clarity and elegance (Rosenberg, 1997: 2; Tufte, 2006; Cookson, 2013; Shermer, 2005)

Tufte was fascinated by images of dance notation created in the 18th century (see fig. 83), in particular concerning the visualisation of music and threedimensional space over time. He saw dance notations as beautiful and complex systems of visualisation that enable the preservation of the 'visual instant' (Tufte, 1990: 114). However, he also questioned the success of such systems. While in a few systems the signs used to translate human movement reflect the actual movements and lines found in the dance, in most systems they do not. This he found to be particularly true for contemporary dance notation, such as Labanotation, in which the symbols

1 LLC - Limited Liability Company 

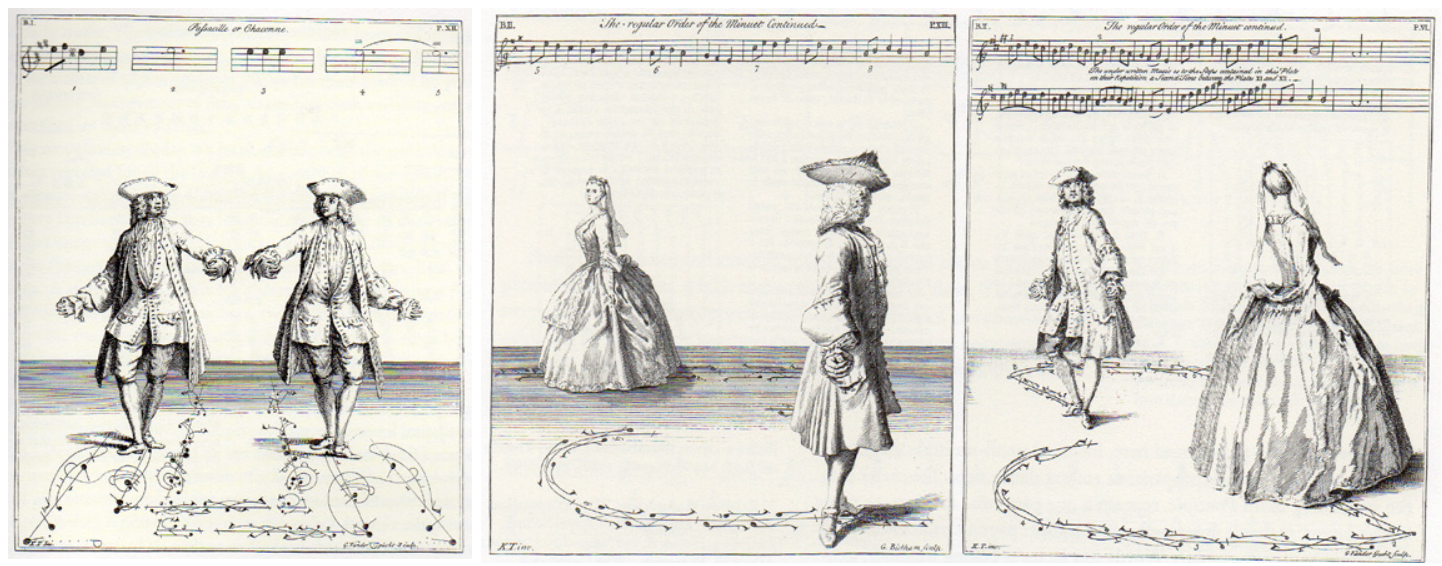

Fig. 83 - 18th century dance notation by Kellom Tomlinson - The Art of Dancing, Explained by Reading and Figures (Tufte, 1990: 27,115)

used are abstracted and microscopic encodings of the actual movements. Tufte further found that in many notation systems, text is used to explain graphics, so the reader must jump back and forth to interpret the notation. The only connection between the text and graphics is sequential alphabetical labels. He found that this reduction of the four-dimensional reality of time and space to a set of simple marks on paper was often fussy and the effect was clumsy compared to what the marks were supposed to represent (Tufte, 1990: 115-119). However, like Hall and Birdwhistell, Tufte (1990) did envisage the potential of future technology for improving such systems of notation: 'Perhaps one day high-resolution computer visualizations, which combine slightly abstracted representations along with a dynamic and animated flatland, will lighten the laborious complexity of encodings - and yet still capture some worthwhile part of the subtlety of the human itinerary' (Tufte, 1990: 119).

Tufte also discussed other visualisations that illustrate movement, such as a skiing manual How to Ski by the French Method which features a sequence of 200 annotated photographs of experts skiing (see fig. 84). The images are read left to right, showing changes over time such as how the posture of the skier changes according to the different grade of slope: gentle slope, medium slope, and steep slope. Tufte defined the style of the design as 'Classic 

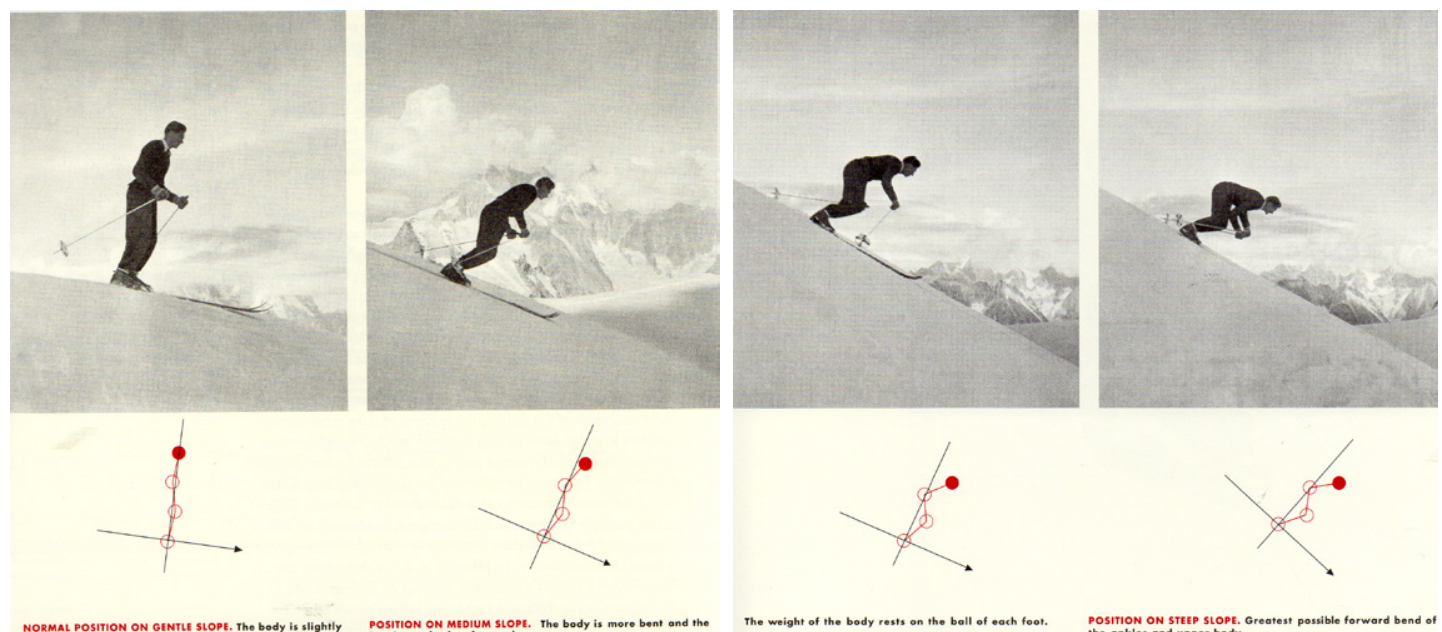

Fig. 84 - Images from a French skiing manual How to Ski by the French Method (Tufte, 2006: 36-37)

Modern' because it uses simple geometric diagrams and images rather than words. The images successfully illustrate movement and posture in space and time. The graphic representation of the image sequence simplifies the shape of the human body, and draws attention to key positions of the body and the four body parts that are relevant to this activity: the head, bottom, knees and ankles. An arrow is used to indicate the angle of the hill and the direction of the athlete's movement (Tufte, 2006: 36-38).

Another design discussed by Tufte, which he likened to dance notation, is the Spotting a Hidden Handgun designed by Megan Jaegerman in 2004-52. The similarity between them, he stated, is that they show the choreography of movement in three-dimensional space by using motion arrows, mappings and sequences. Words and images work together to explain the sequences. Jaegerman's infographic shows how a person's walk is influenced by carrying a gun in their pocket: how the arm swings, the steps become shorter, and what direction the person will turn when passing another person. It also shows hand positions and adjustments made to the gun in different circumstances (see fig. 85).

2 Spotting a Hidden Handgun was an adaptation of a graphic that Megan Jaegerman originally created for the New York Times in 1992. 


\section{Spotting a hidden handgun}

\section{ASYMMETRICAL GAIT}

A gun in a right-hand pocket or tucked into the right side of a waistband may hinder leg movement on that side, making the right stride shorter than the left.

A slightly clipped arm swing may also signal a hidden gun: the forearm on the gun side will tend to stay close to the body, instinctively guarding the weapon.

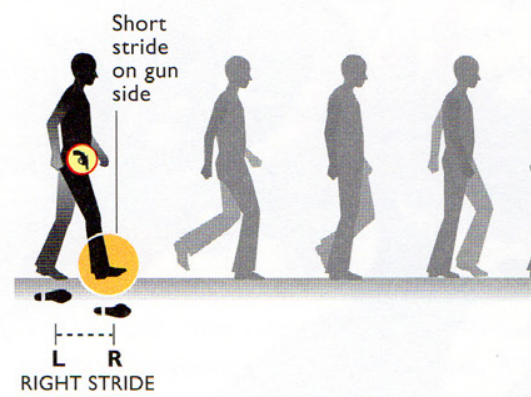

\section{A QUICK ADJUSTMENT}

A gun's weight is distributed unevenly, with more of its weight in the grip than in the barrel.

Vertical motion - like descending stairs or stepping onto a curb tends to shift the barrel upward.

A quick, circular movement of the hand or forearm adjusts the gun's position.
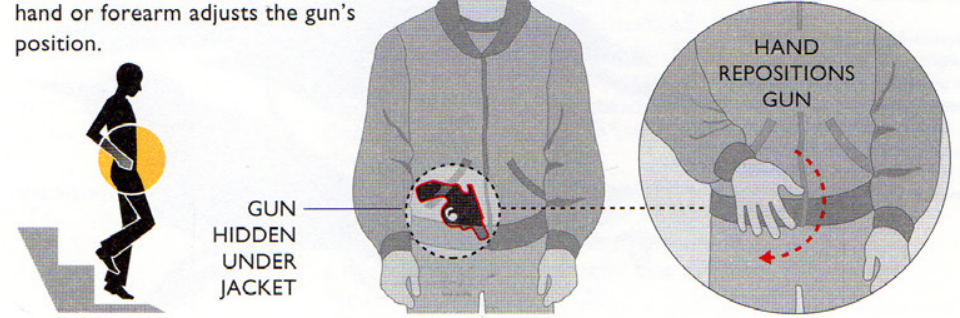

\section{UPPER BODY SHIFT}

When approached from the front, a person with a hidden gun will instinctively turn the gun side of his body away from the person approaching, and may veer to that side to avoid a face-to-face encounter. He is also likely to pull his arm in toward his body on the gun side.

\section{CONSPICUOUS CLOTHING}

Garments worn to conceal weapons often appear odd, mismatched, or out of season, and can actually draw attention to a person trying to avoid scrutiny. A closer look may reveal movements or other characteristic signs of a hidden handgun.

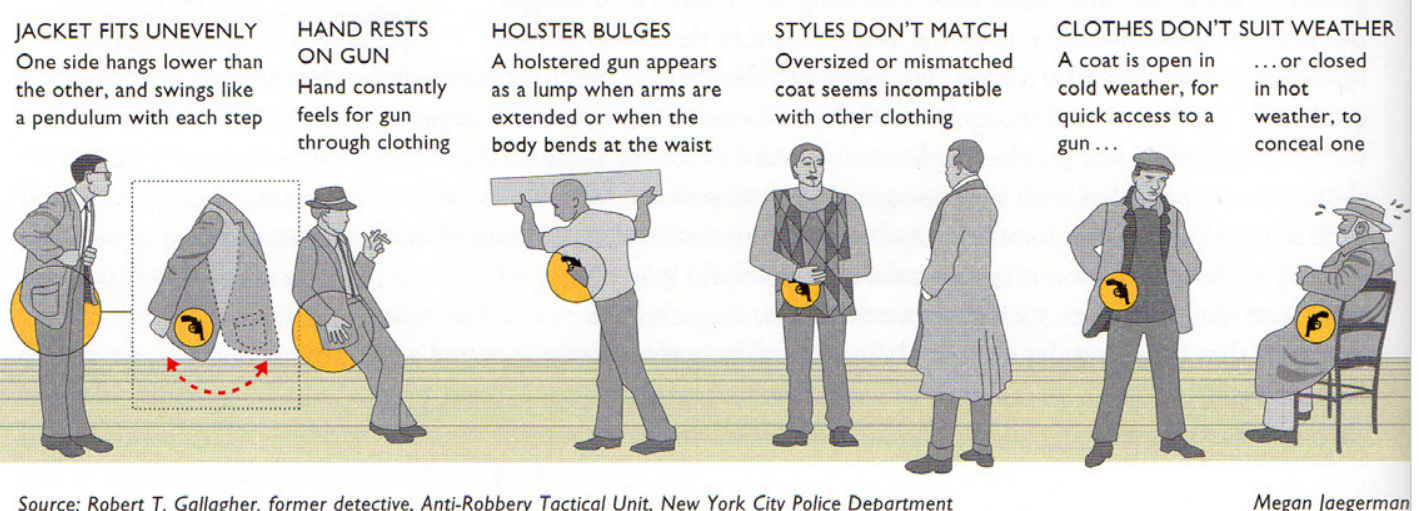

RUNNING FROM THE RAIN

When running toward shelter from rain, or across a busy street, a person concealing a gun is likely to brace the weapon with an arm or hand.

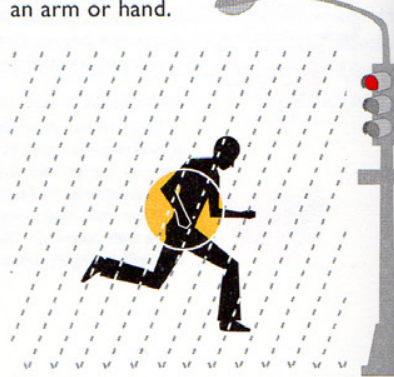

Fig. 85 - Megan Jaegerman's Spotting a Hidden Handgun graphic (Tufte, 2006: 116) 
The bottom part of the figure illustrates how to tell that a person is carrying a gun by noticing what they are wearing - for example, unevenly fitted jackets, and clothes that do not match. With my own study in mind, fig. 85 is interesting in the way it clearly visualises movement, walking, turning and gestures. Subtle adjustments of the hidden gun are also illustrated clearly. For visualising and analysing greetings all these dimensions of movement, posture and gesture are important. Drawing attention to hidden layers of nonverbal communication is a key element in my project. Jaegerman's work is inspiring in the way it makes the hidden visible: the concealed gun is clearly presented, by use of yellow coloured circles, as something which is not visible to the naked eye.

In analysing the success of this infographic, Tufte draws attention to the fact that Jaegerman did both the research and the design herself: in her study for the work, she interviewed Robert T. Gallagher of the New York City Police Department and took part in role-playing and demonstrations for handling the gun. Tufte noted that too often the process of research and the creation of the design are separate, and argues that being involved in both improved Jaegerman's understanding of the content and thereby also her design, which he observes 'amplifies' the content (Tufte, 2006: 116-117). My research involves a similar hands-on approach, in which I gather the content for the design through fieldwork and personal interaction with the participants in my study. This connection to the content is important in my understanding of it because my design, like Jaegerman's, seeks to 'amplify the content' (Tufte, 2006: 117).

Also relevant to my own work is Tufte's discussion of the mapping of images, because it relates somewhat to my 'mappings', or vectorisations, of greeting sequences. Tufte explains mapped images as being realistic image evidence combined with focused and explanatory diagrams. The purpose of mappings is to clarify 'why the image matters' (Tufte, 2006: 45). He uses an image from Roger Tory Peterson's publication A Field Guide to the Birds as an example 


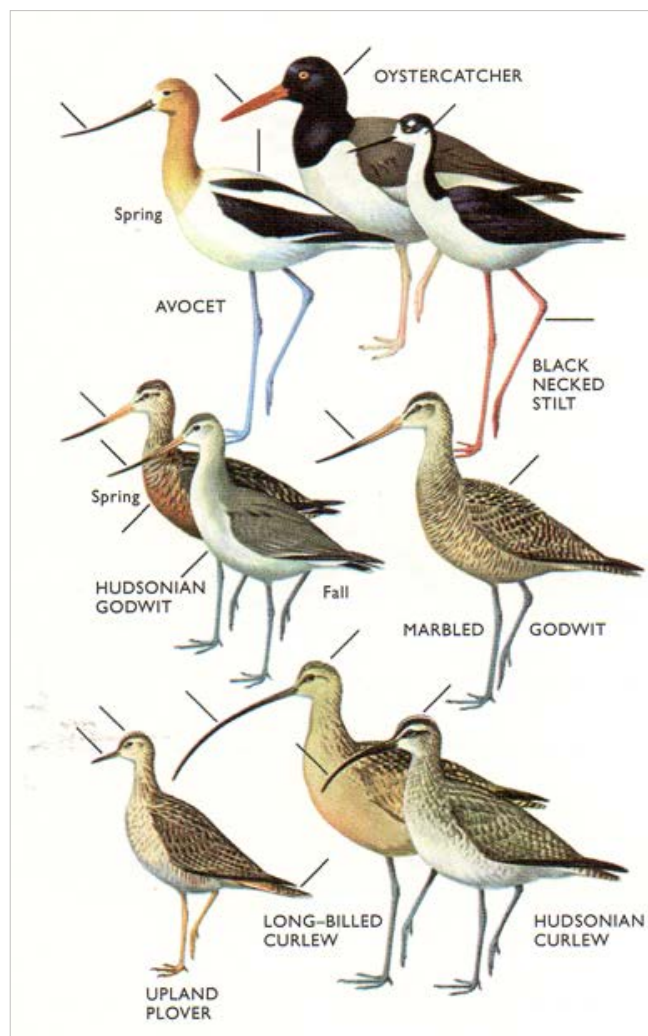

Roger Tory Peterson, $A$ Field Guide to the Birds (Boston, 1934, 1939, 1947), plate 23, shore-birds.

Fig. 86 - Image mapping of birds by Roger Tory Peterson (Tufte, 2006: 45) of a mapped image (see fig. 86) (Tufte, 2006: 45). The pointer lines were added to the image to highlight distinctive features of the different birds. While such a visualisation serves a clear purpose, which is to highlight existing aspects of the images, Tufte stressed that it is often useful to give the viewer the option of seeing the unmapped version of the image, to distinguish between the manipulated and nonmanipulated views. Doing so shows more clearly what has been added to the picture, and also gives future analysts the possibility to re-analyse the image themselves (Tufte, 2006: 45). Similarly, in the presentation of my research on greetings, I give the viewer the option of viewing both the manipulated, vectorised greeting animations, and the non-manipulated greeting footage - so that the sources of my animations are available for others to view or to carry out their own analysis.

While Tufte may be considered a pioneer in the field of information visualisation, many others have developed the field in a number of interesting directions. One such direction, which is of particular interest to my own study, concerns using infographics for communicating body movement, posture and cultural differences. A number of designers have created works which do just that. Nigel Holmes, an award winning British illustrator and graphic designer, shows the power of illustration, or what he calls 'explanation graphics', in his book Wordless Diagrams (Holmes, 2005). The book features 100 visual 
diagrams depicting everyday actions, from how to defend yourself from an attacker (see fig. 87), to how to blow a bubble gum bubble, how to say "yes" in India (see fig. 88), and how to fold a t-shirt. His diagrams successfully communicate posture, order of movement, direction of movement and repetition of movement, in clear, and at times witty, ways. In the 'How to Say "Yes" in India' diagram, subtle angles and movements of the head are communicated using arrows and lines. Some of his diagrams feature greeting gestures, such as the graphic explaining how to wave like a royal, how to curtsy (see fig. 89), and how to greet someone in France, Holland, Russia and America (see fig. 90). The 'How to Wave Like a Royal' and 'How to Curtsy' graphics illustrate movement using three sequential graphics. They visualise different steps of the movements and the order of kissing when greeting. In these illustrations a national symbol representing each of the countries - the French Eiffel tower, a Dutch windmill, the Russian hammer and sickle, and an American city - is placed above each face. Indicators of nationalities are also found in the different headwear added to each figure.

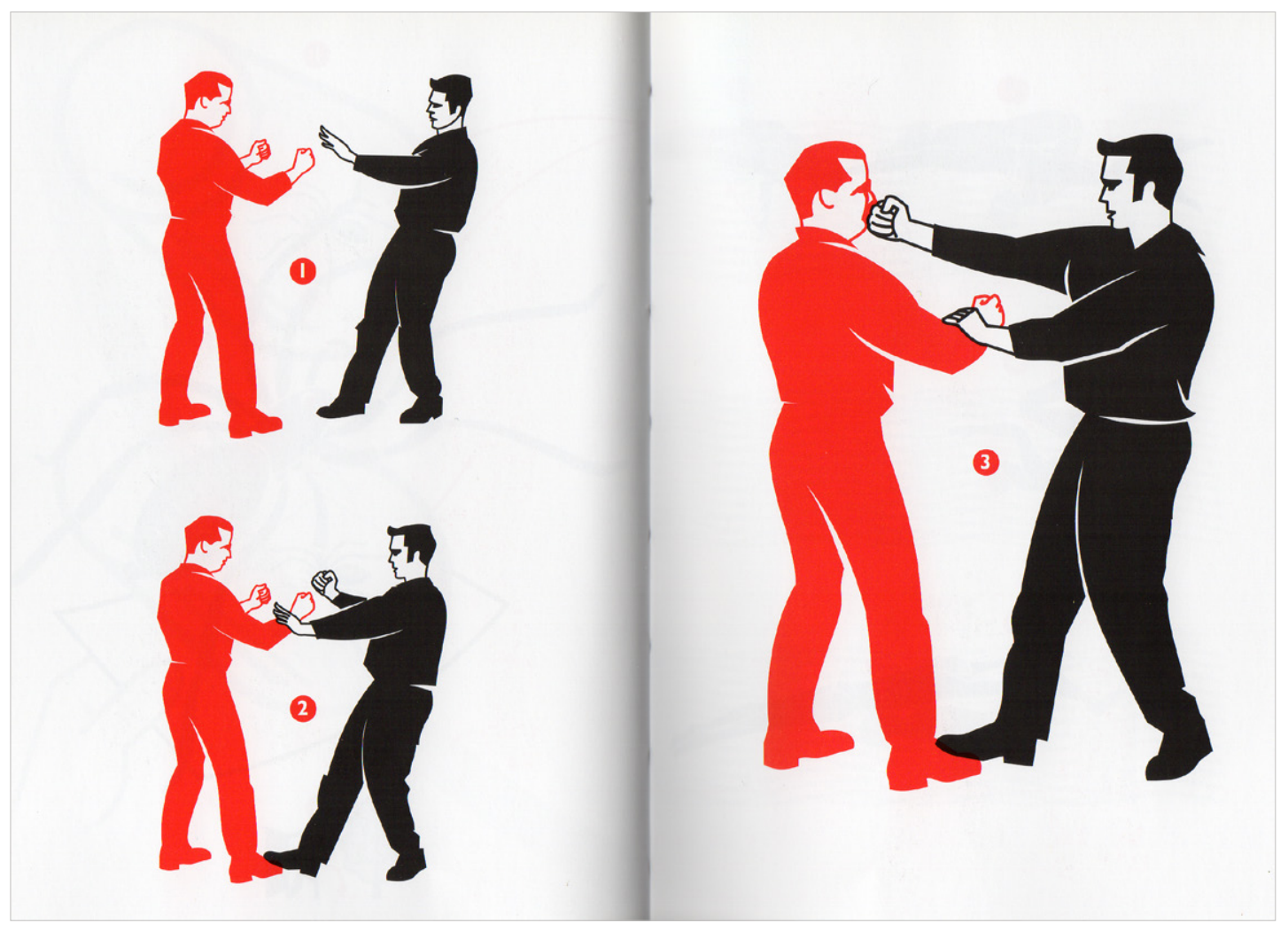

Fig. 87 - How to Defend Yourself From an Attacker (Holmes, 2005) 


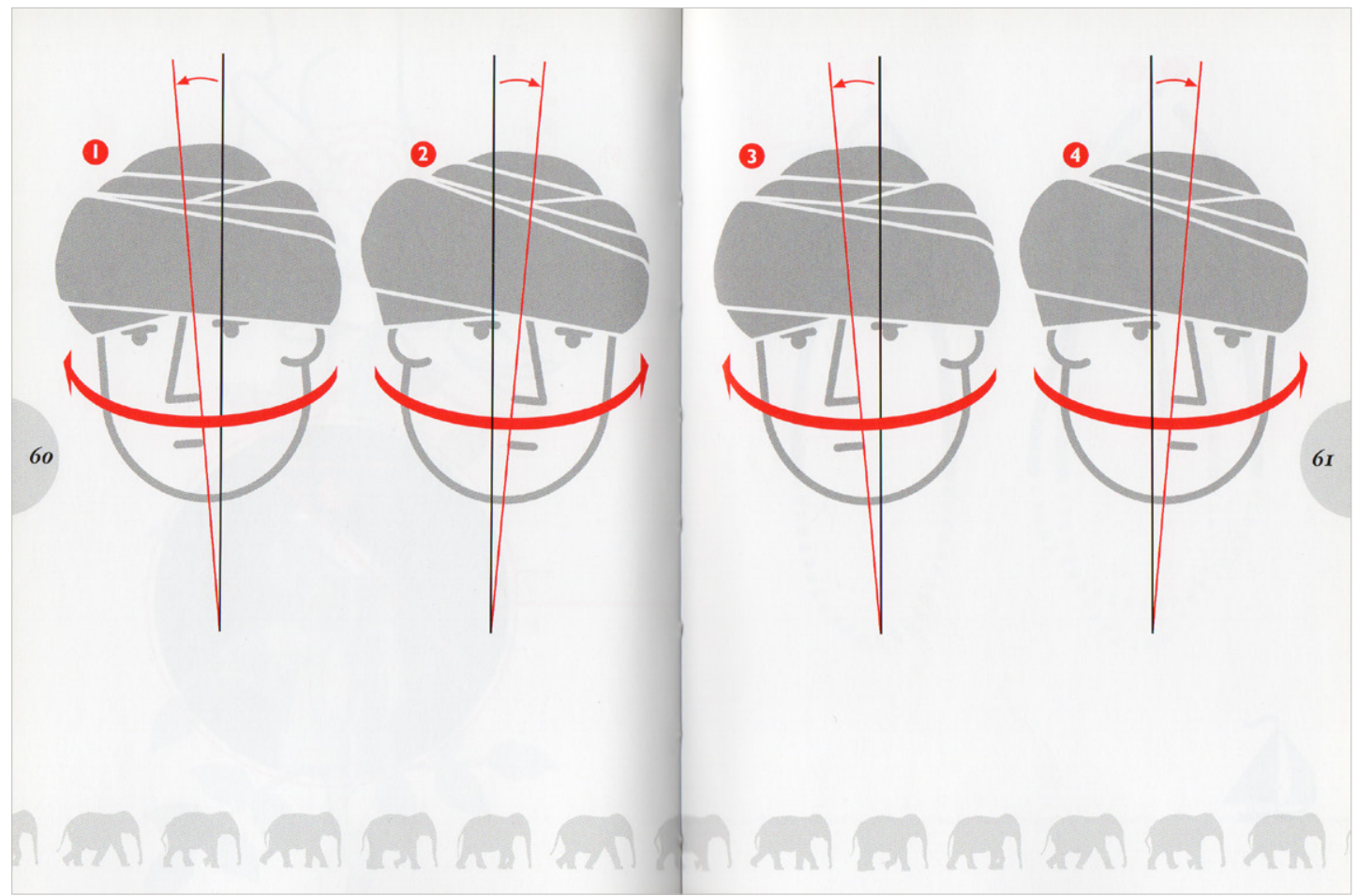

Fig. 88 - How to Say "Yes" in India (Holmes, 2005)

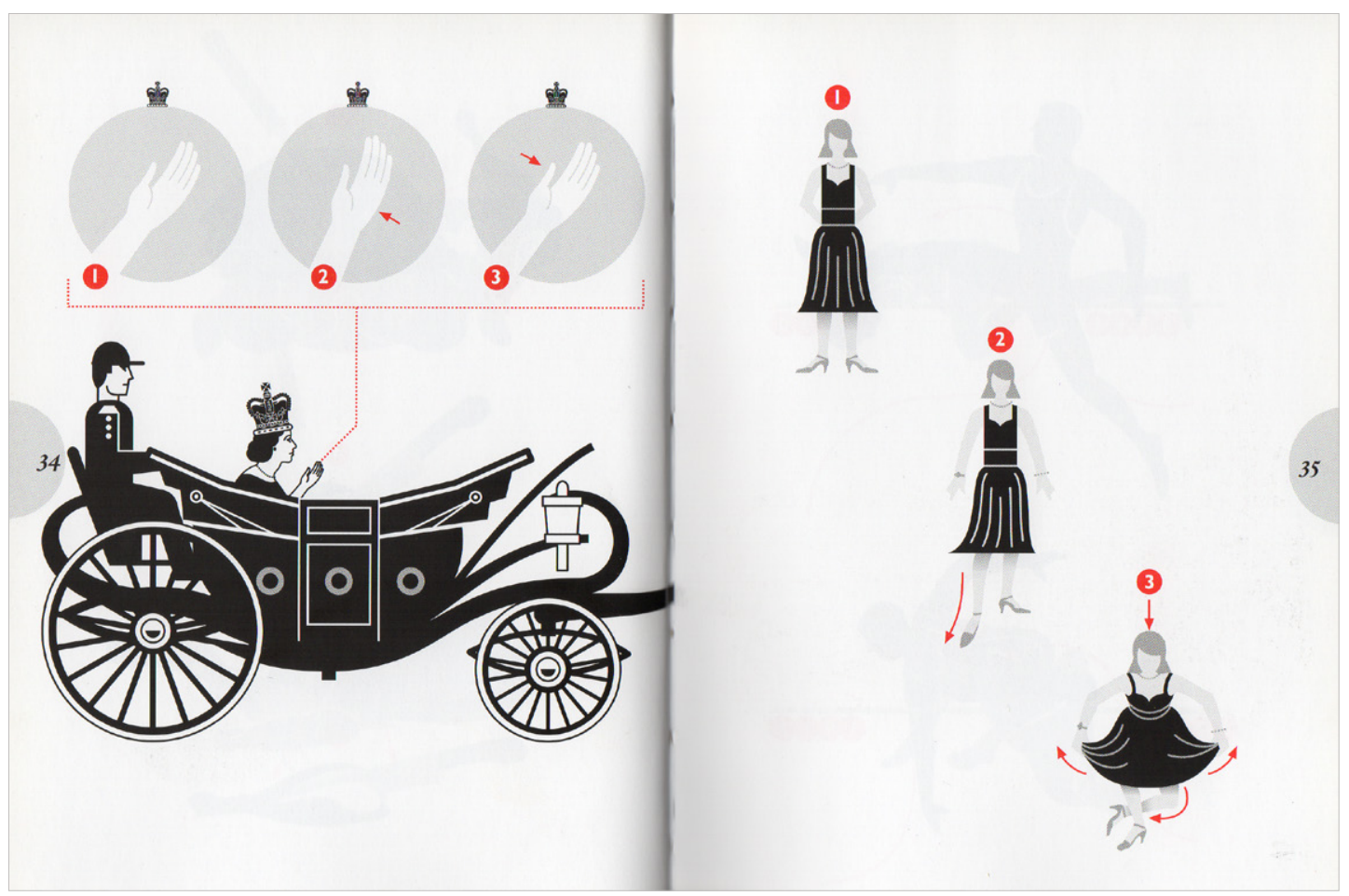

Fig. 89 - How to Wave Like a Royal, and How to Curtsy (Holmes, 2005) 


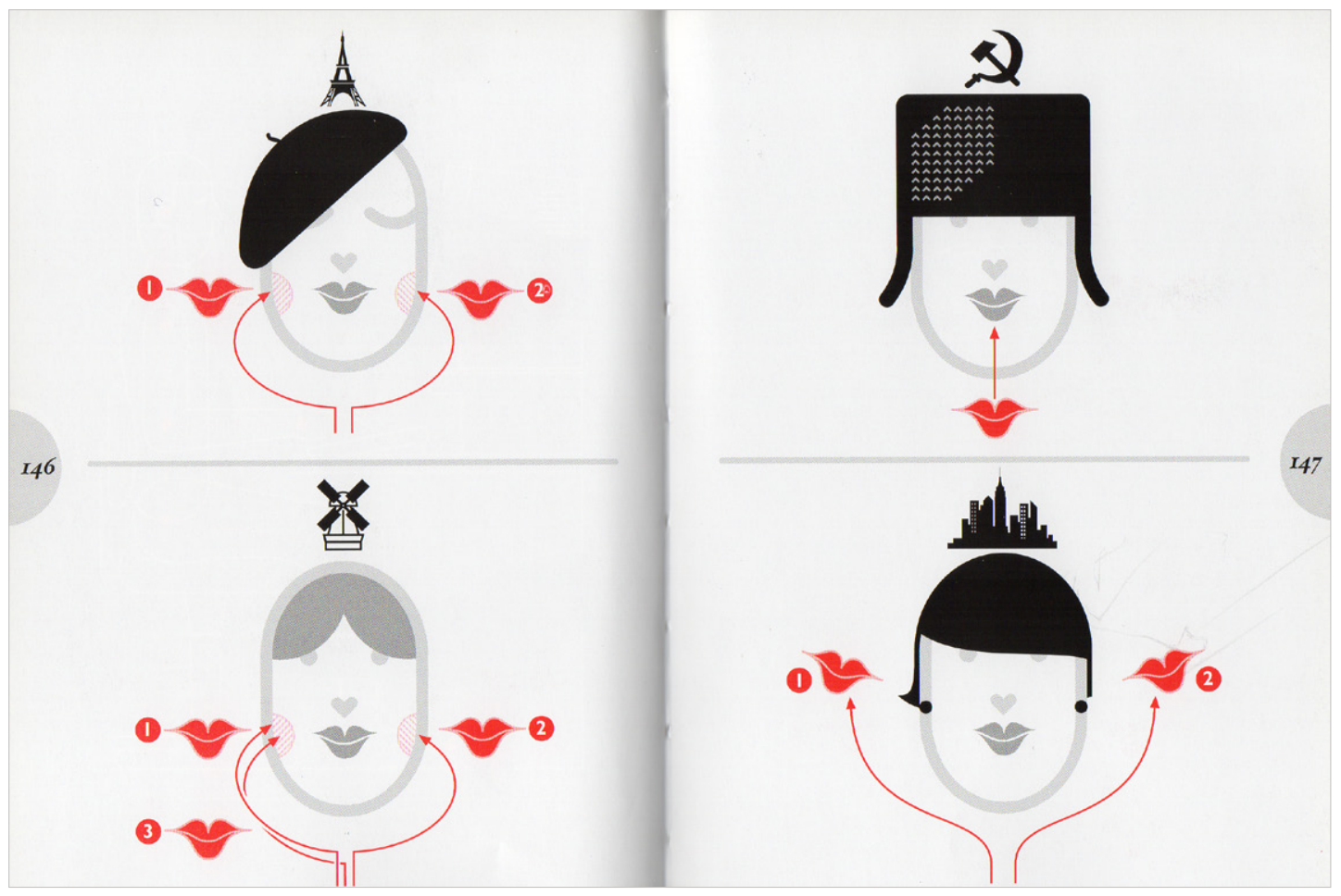

Fig. 90 - How to Greet Someone in France, Holland, Russia and America (Holmes, 2005)

These illustrations show the difference in how many kisses are used when greeting in the different countries: France - two kisses; Holland - three kisses; Russia - one kiss, and America - two kisses. Numbers indicate the order of the kisses and which side to kiss first, second, and third.

A similar graphic style can be seen in Yang Liu's book East Meets West and in her more recent book Man Meets Woman (Liu, 2007, 2014). Liu is a graphic designer born in China who moved to Germany when she was 14. Of particular relevance to this research is the East Meets West project which is an iconographic documentation of her first-hand experiences of belonging to two cultures - an Asian culture and a European culture. The blue side of her graphic pieces represents Germany and the red side represents China. Some of the cultural differences she has visualised include: independent vs. dependent lifestyles (see fig. 91); attitudes towards punctuality (see fig. 92); problem solving approaches (see fig. 93); how to stand in line (see fig. 94); and size of the individual's ego (see fig. 95). 


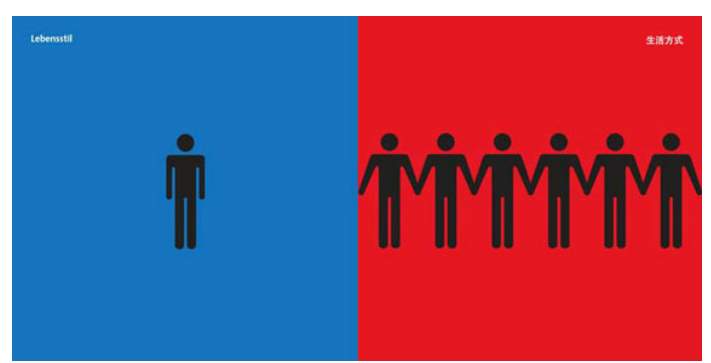

Fig. 91 - Lifestyle: independent vs. dependent (Liu, 2007)

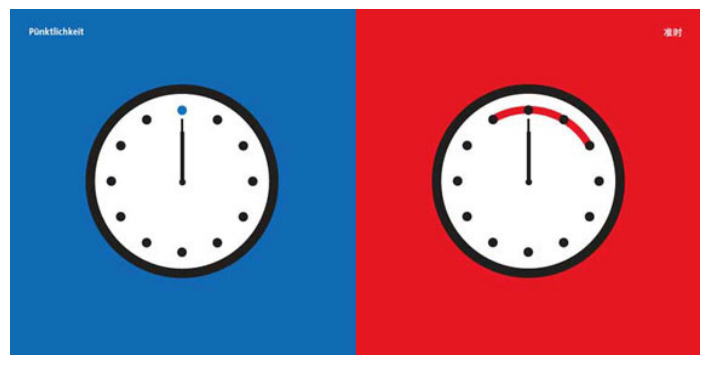

Fig. 92 - Attitude towards punctuality (Liu, 2007)

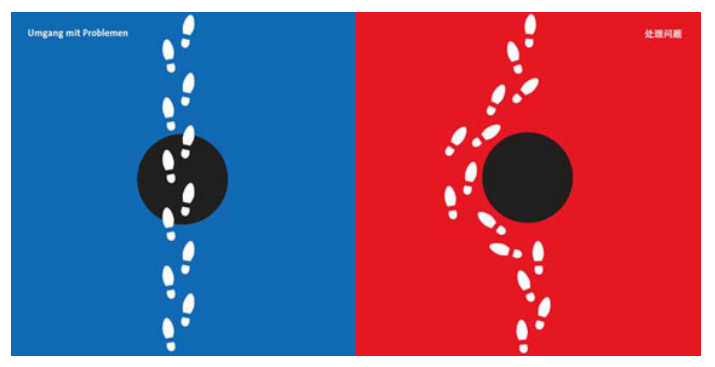

Fig. 93 - Problem-solving approach (Liu, 2007)

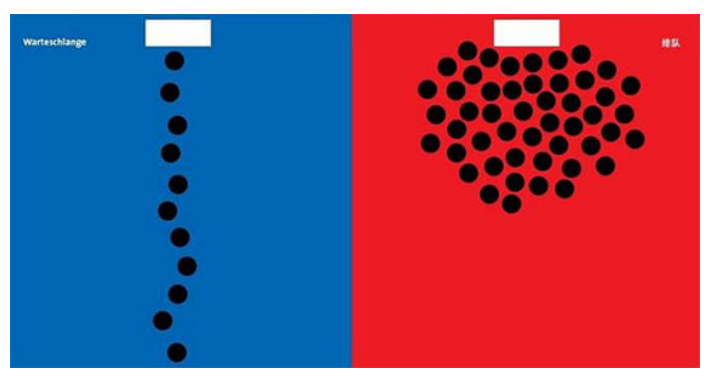

Fig. $94-$ How to stand in line (Liu, 2007)

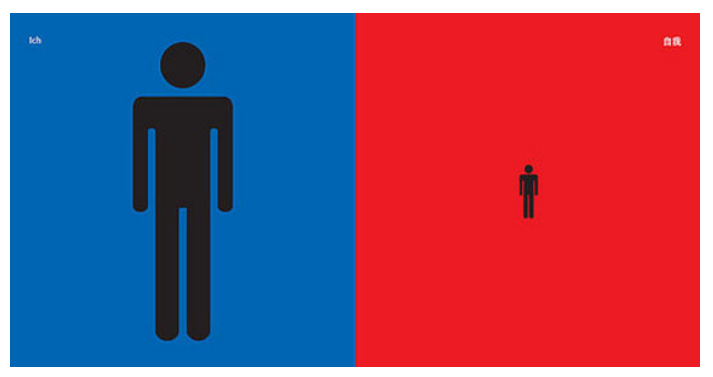

Fig. 95 - Size of an individual's ego (Liu, 2007)
The pieces communicate a variety of issues of cultural difference, and visualise why communication across cultures may encounter confusion and difficulties, for example the different attitudes towards punctuality could potentially cause irritation and impatience if a punctual German is meeting with a Chinese person who considers being ten minutes late as perfectly fine. Liu's graphics reveal interesting aspects of cultural information and offer explanations for situations that could potentially involve cultural confusion. This dimension of her work relates closely to mine in that she is expressing subjective experiences through a graphic language. Although some of the images may appear culturally problematic, stereotypical or even racist, such as fig. 95, nevertheless, given that Liu has grown up in both China and Germany, her work represents an attempt to visualise subjective experience, and this is an important exploration in my own work.

Another project using infographics and focused on human posture is titled Curious Rituals, and was 
carried out at the Art Center College of Design in Pasadena by researchers Nicolas Nova, Katherine Miyake, Walton Chiu and Nancy Kwon (2012). The project investigates gestures, postures and rituals that humans have come to inhabit as a result of digital technologies. The book Curious Rituals documents how technology is influencing the way we move and gesture, and how possible future technological development may further affect our gestures. The book features illustrations accompanied by textual explanations and background information. Colour is used to draw attention to particular movements such as walking while talking on the phone - highlighted in orange/red - and arm movements while using the remote control, tablets or phones - highlighted in light orange/brown (see figs. 96-98). The project uses graphics and illustration in ingenious ways to communicate how these new gestures have become part of our daily routines (Nova et al., 2012).

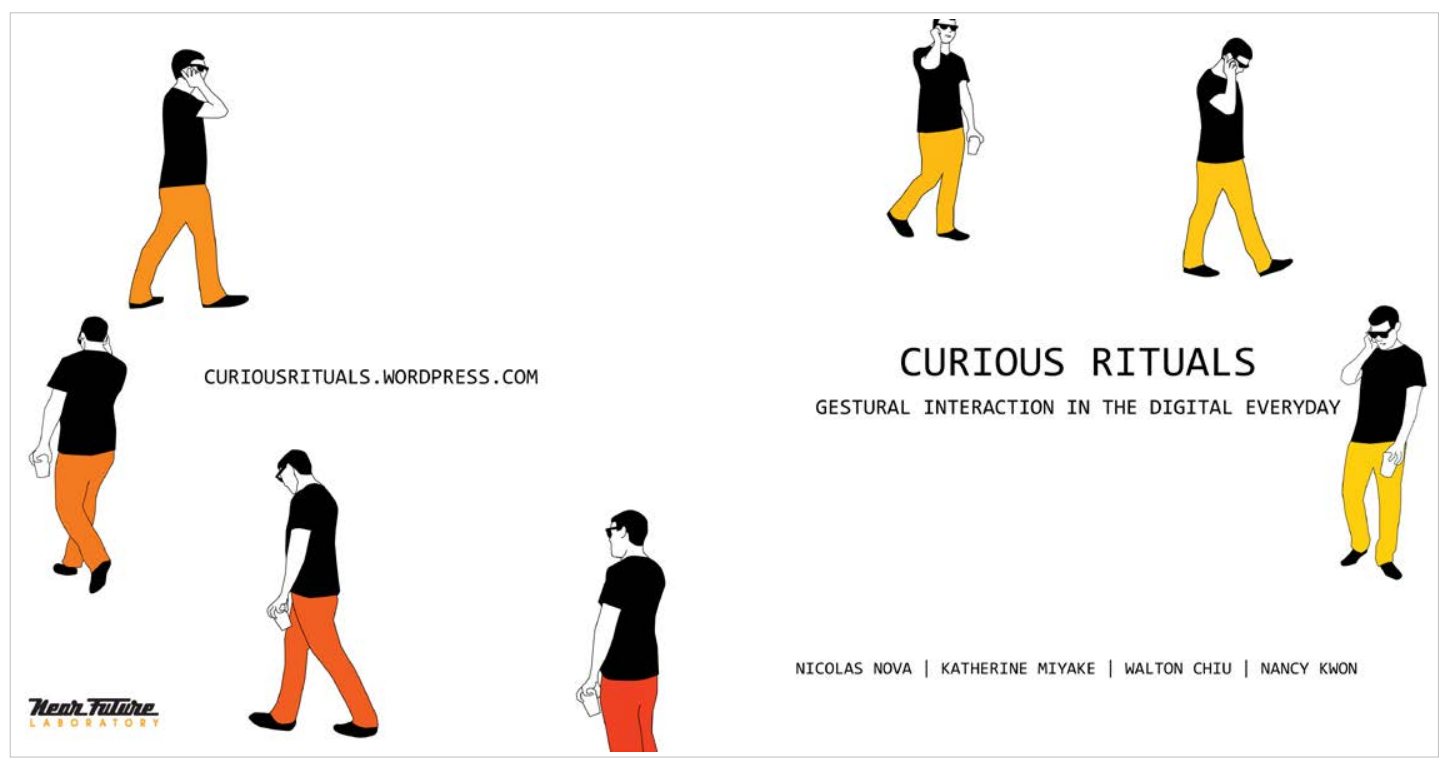

Fig. 96 - Presentation of Self: Cell Trance (Nova et al., 2012) 


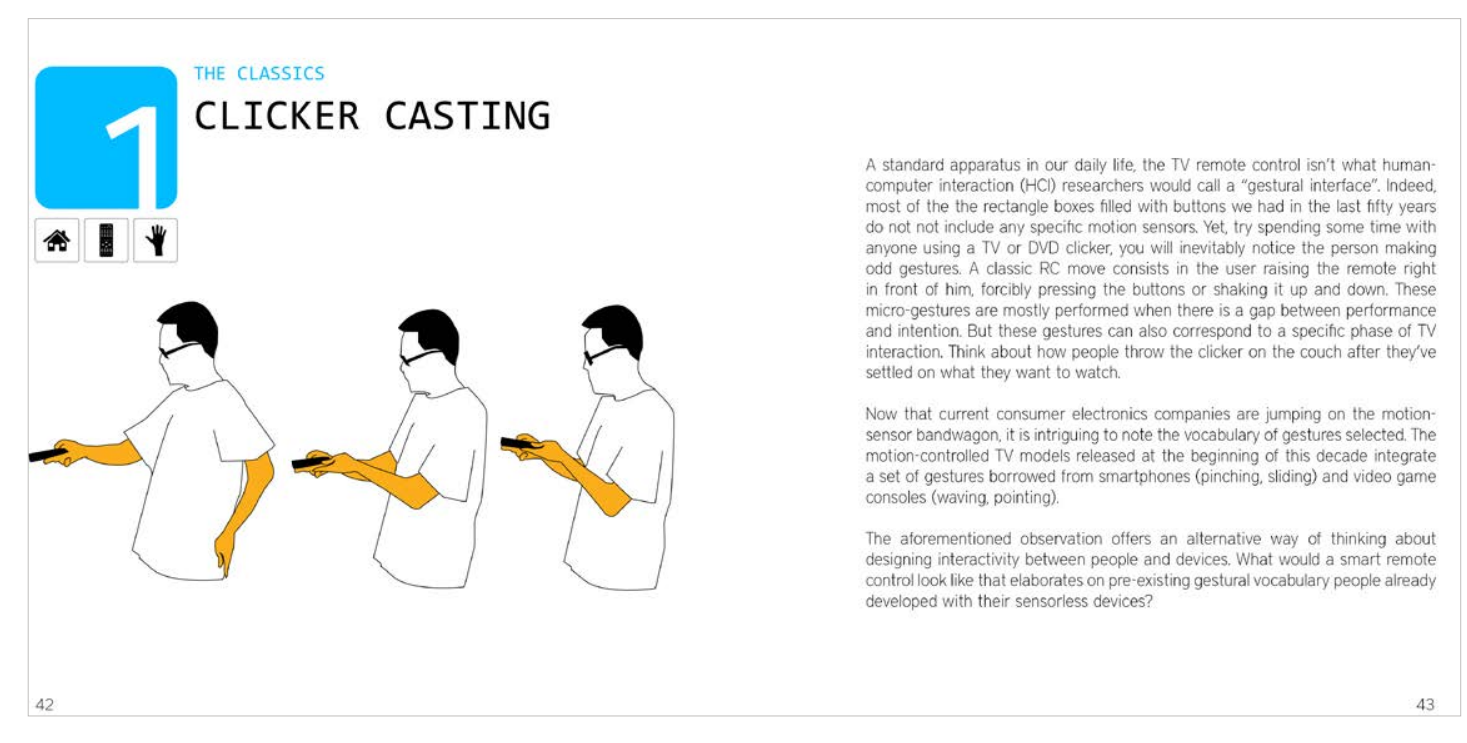

Fig. 97 - The Classics: Clicker Casting (Ibid.)

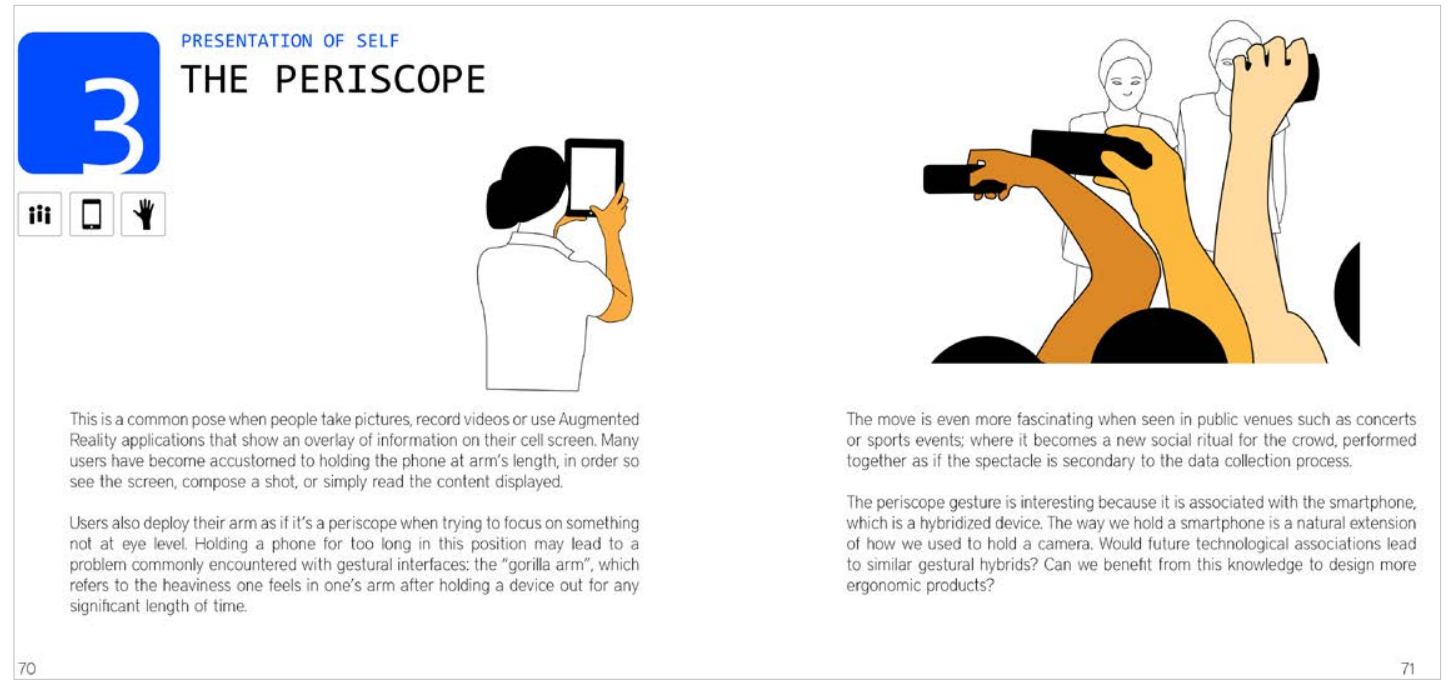

Fig. 98 - Presentation of Self: The Periscope (Ibid.)

\subsection{Data \& Network Visualisation}

Infographics are also used for the presentation of data and complex networks. While the examples of infographics mentioned above focus on communicating specific observations, movement and postures, the focus of data visualisation and network visualisation is on communicating large amounts of data, or complex networks, in visual form. Data visualisation and network visualisation are rapidly growing forms of information representation, and are currently seen as very cutting-edge, but they are not new fields. In 
1858, the nurse, statistician and social campaigner Florence Nightingale designed what has become a famous graphic illustrating the medical conditions faced by British soldiers during the Crimean War (see fig. 99). Her statistical graphics were among the first to popularise and improve the visualisation of information (Rogers, 2013: 65). At that time, and until quite recently, data and graphics that were published in books were referred to as figures that accompanied text. Today, visualisation of information often replaces lengthy textual explanations and analyses, and has become a much more digital process.

\section{The coxcomb, or rose diagram}

Nightingale's most famous chart

$$
\text { APRIL } 1855 \text { ro MARCH } 1856
$$

DLAERXM OP THS CAUSBS OR MORTAJTITY
IN THE ARMY IN THE EAST.

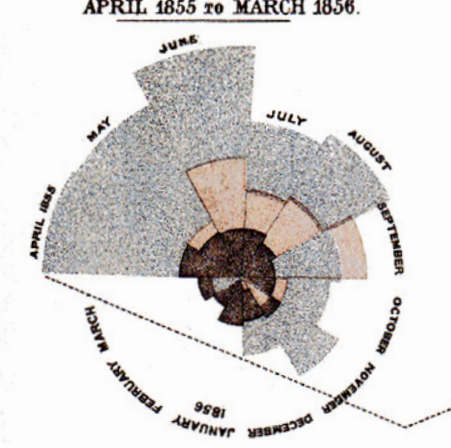

The Areas of the blue, red, \& black vedges are cach measured from the centre as the common vertex.

The blue wedges measured from the centre of the circle represent area for area the deaths from Prepentible or Mitigable Zymoluc diseases, the red wedges measured from the centre the deaths from wounds, \& the black wedges measured from the centre the deaths from all other causes. The black line acrass thered triangle in Nov. 1854 marks the boundary of the death from all other couses during the morith

In October 1854, \& April 1855, the black area coincides woth the red,

in January \& Pebruary 1856, the bluse coincides with the blach

The entire areas may be compared by following the blue, the red \& the black lines enclosing than

\section{APRIL 1854 TOMARCH 1855}


A leading practitioner in this field is independent data journalist and information designer David McCandless, author of the books Information is Beautiful (2009) and Knowledge is Beautiful (2014) and founder of the blog with the same name (informationisbeautiful.net). McCandless creates and collects infographics, diagrams and visualisations aimed at creating and enhancing understanding of the world. He discusses the way that today people absorb a huge amount of information online, and the fact that an increasing proportion of this information is visual. His principle is to make information not merely visual, but 'beautiful', and he believes that by distilling the world's data into visual diagrams and graphics the information becomes more approachable (McCandless, 2009; McCandless, 2010, July; BBC Newsnight: Information Graphics, 2010, August 9). In Information is Beautiful he presents a visual by Tufte, an index of forty different diagram types used for information visualisation (see fig. 100). A similar graphic created by

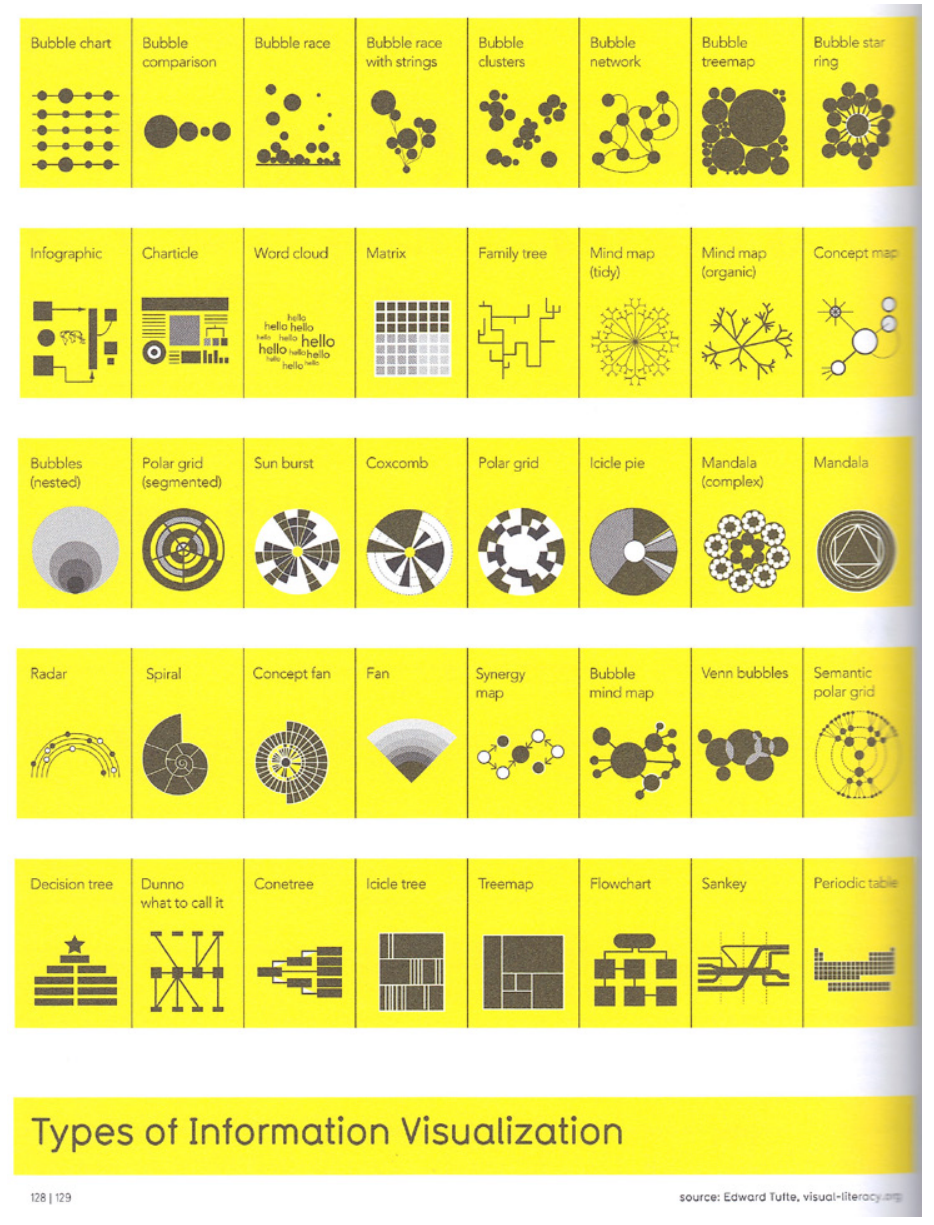

Fig. 100 - Types of Information Visualisation by Tufte (McCandless, 2009) 
Alberto Antoniazzi presents the most popular and widely used infographics, and is featured in the book Facts are Sacred by Simon Rogers (see fig. 101). These two graphics are indicative of the current trend and popularity of infographics and information visualisation. McCandless' work illustrates how graphic communication, employing techniques such as colour coding and the scaling of shapes, can visualise important comparisons and relationships.

Scaled and colour-coded shapes are not new techniques of visualisation, but data journalism has changed the way they are presented, not simply as pie charts or diagrams, but in more aesthetically pleasing ways. More attention is now given to the visual layout and colour combinations that are used. Geometric shapes, traditionally used in graphs and stats, are increasingly being combined with or replaced with icons that relate more directly to the topics or issue they represent.

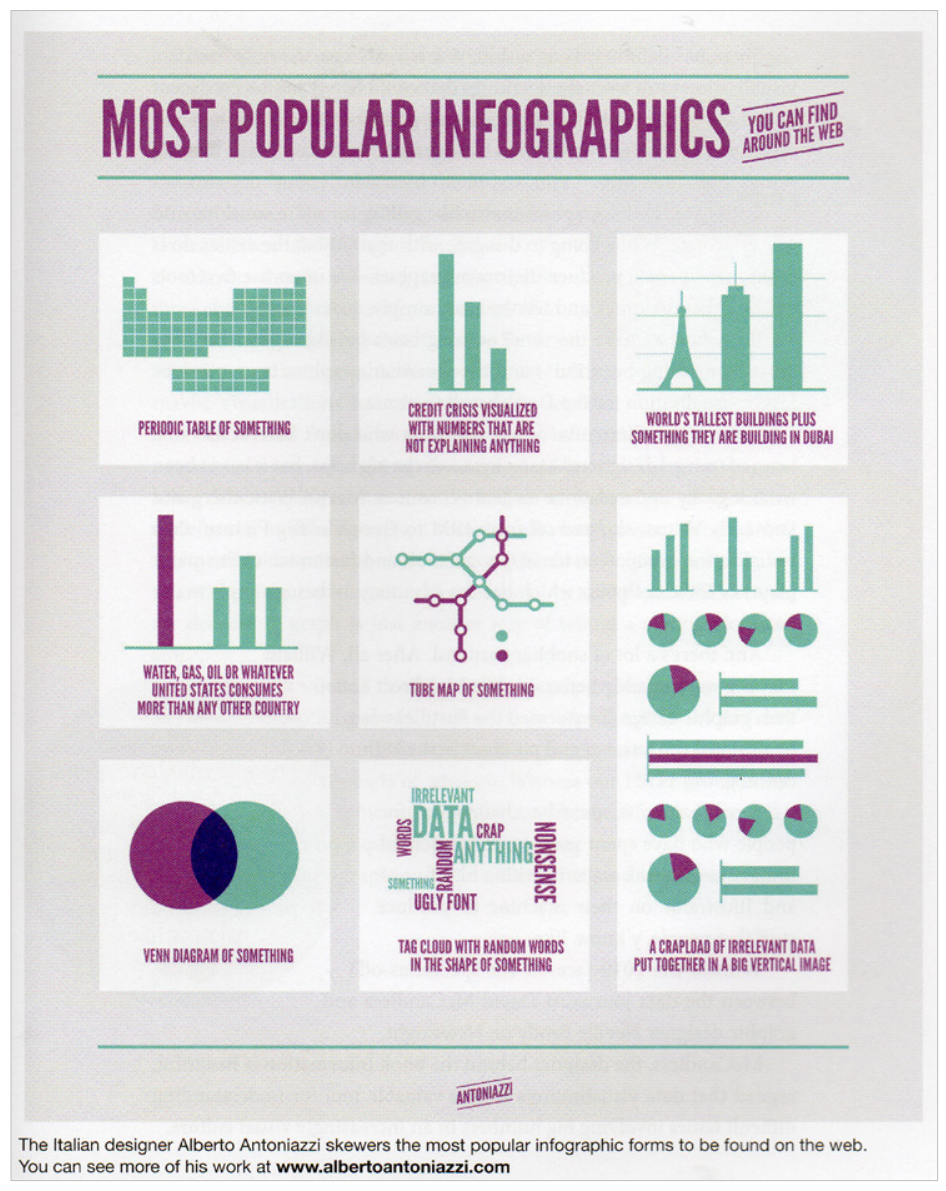

Fig. 101 - Most Popular Infographics by Alberto Antoniazzi (Rogers, 2013) 
In Facts are Sacred, Rogers states that while in the 19th century photography and photojournalism were seen as creating accurate, objective pictures documenting the world, today photography seems to have lost its status as a means of representing the truth. This is mainly owing to raised awareness of image manipulation and enhancement (Rogers, 2013: 272). Rogers notes that while data doesn't give information that perfectly represents the world, data produced by public bodies is probably the best source of information available to us (Rogers, 2013: 273-275). Rogers' book presents visuals that communicate a range of different datasets and facts about the world, from statistics about women in media and use of social media, to medals won at the Olympics and immigration statistics. As in McCandless' work, colour coding is essential in these visuals, and enables the viewer to get quick overviews of world facts. Theo van Leeuwen, in his study on social semiotics, also discusses the role and status of the visual and how it has changed enormously in recent times. While language used to hold the highest status in print media, he argues, today the role of the visual is becoming increasingly important in both print and digital media. The use of visual language is also more systematic and 'more strictly controlled' than before, because it is no longer simply an addition/attachment to the written language - the visual is a language in itself (Leeuwen, 2006: 52).

Manuel Lima confirms van Leeuwen's point about the visual becoming more strictly controlled and now being its own language, in his investigations of systematic mappings of networks. Lima is a designer, researcher, teacher and founder of visualcomplexity.com, an online research space dedicated to those interested in the visualisation of complex networks (Lima, 2011a). Lima's study illustrates the role the visual plays in the representation of intricate networks. Visualisations presented on the website and in his book Visual Complexity: Mapping Patterns of Information (2011a) are mainly network visualisations presenting systems such as business networks, knowledge networks, political networks, semantic networks, web networks, transportation networks, and social networks. In the book Lima discusses 
Romanian psychologist Jacob Moreno's visualisations of social structures from the 1920s-30s. Moreno's research focused on interpersonal relations and he invented the sociogram - a way of graphically representing social structures (Lima, 2011a: 75-76). Fig. 102 shows one of Moreno's first sociograms which is a visual representation of social connections between girls and boys in a primary school: it shows the relationships between boys (on the left) and girls (on the right) in fourth grade. Boys are represented using triangles and girls using circles. The sociograms were created to analyse and present choices or preferences that take place during group interaction.

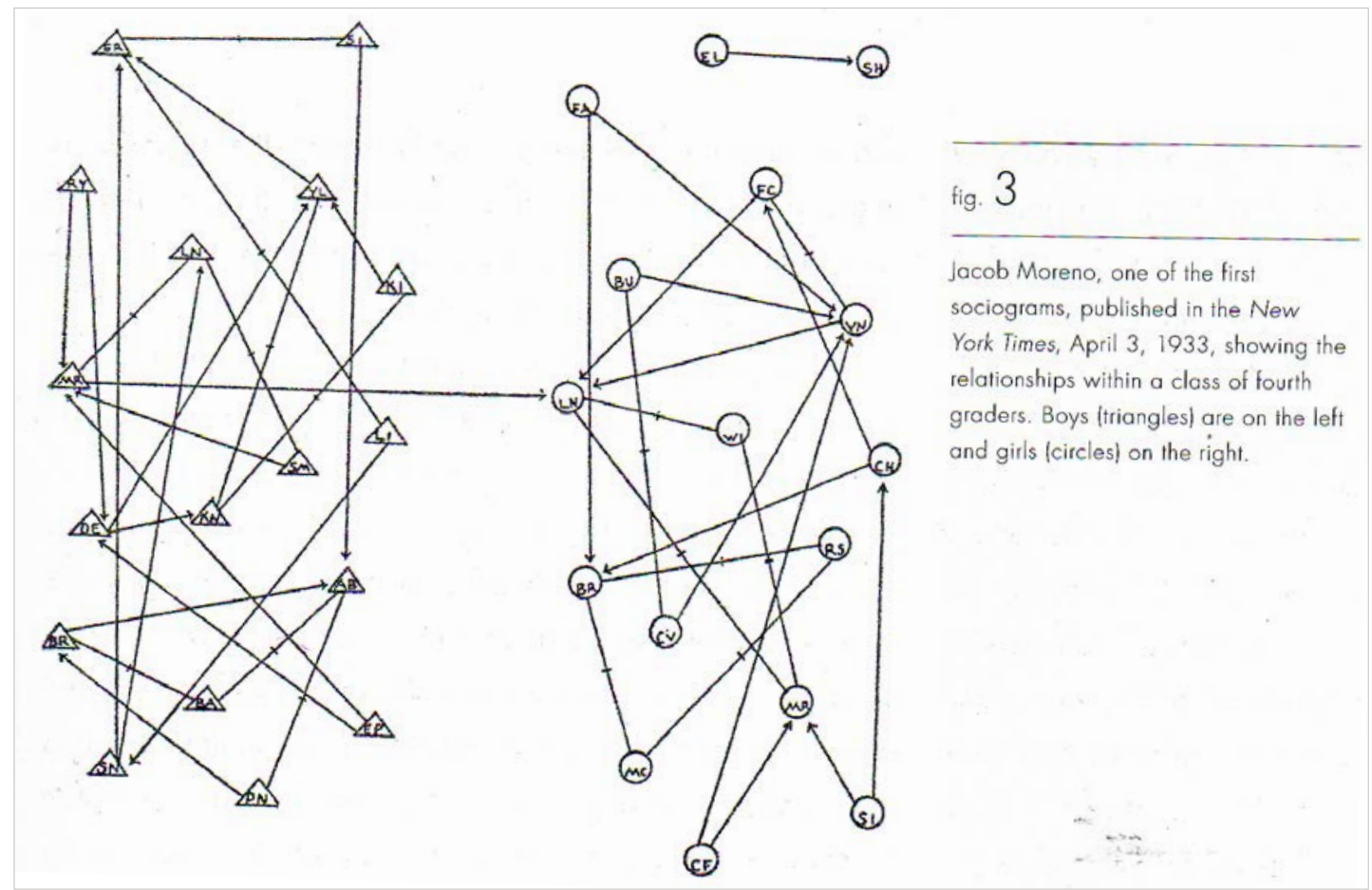

Fig. 102 - Jacob Moreno's sociogram of a class of fourth graders from 1933 (Lima, 2011a: 77)

Manuel Lima defines Moreno's sociogram as one of the earliest graphic representations of social interaction: his work represents a turning point in the development and power of network visualisations. It marked the start of using mapping in the analysis and measuring of human relationships in sociology - which has later become known as social network analysis (Lima, 
2011a: 75-76). Moreno showed a keen interest in the power of visualisation, and his method was not simply to present his findings visually; rather, the visual process was a 'method of exploration' (Lima, 2011a: 76). While my own work focuses on the interaction between two people rather than group interactions, I too use the visual process as a method of exploration.

It is clear that data and network visualisation are useful in communicating large amounts of data, but network visualisations can also, as illustrated above, communicate complex human relationships. They enable us to perceive structural complexity through visual means (Lima, 2011a: 79). The intensity of a relationship can be communicated by changing the thickness/ weight of a line (see fig. 103) and colours are used to differentiate between different categories and groups (see fig. 104). The same dataset can be visualised in many different ways, and Lima is building a taxonomy, or what he calls 'The Syntax of a New Language', which maps out fifteen main categories that people use across different areas and sciences to visualise networks (see fig. 105) (Lima, 2011, March; Lima, 2011, April; Lima, 2011a; Lima, 2011b). The visualisation of networks is no longer limited to static visual representations. Today technology and digital media allow for the creation of interactive visualisations of data, information, and networks.

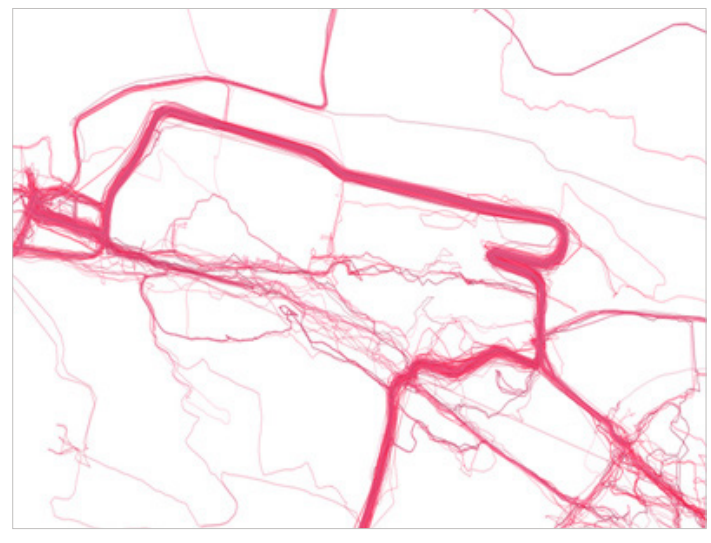

Fig. 103 - Intensity of relationships/ relations enhanced by line thickness (Clark, 2009)

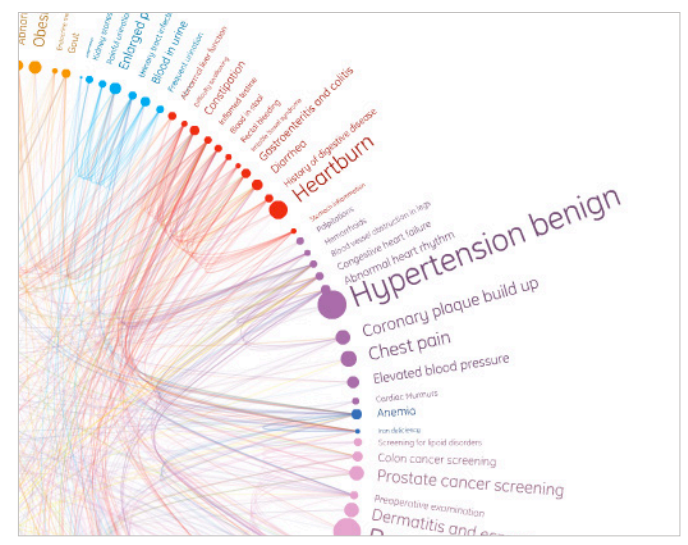

Fig. 104 - Colour-coded health condition associations (MIT SENSEable City Lab, 2011) 

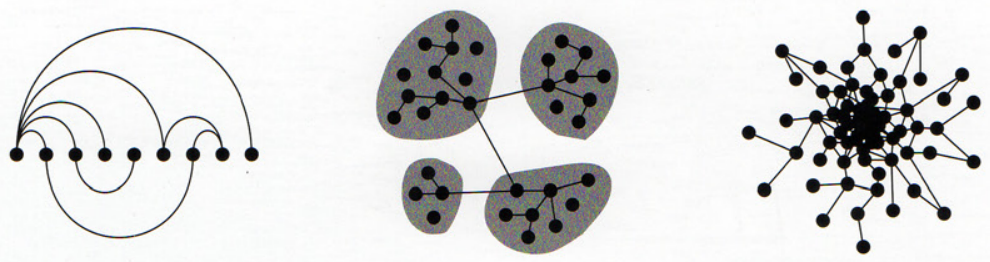

Arc Diagram

Area Grouping
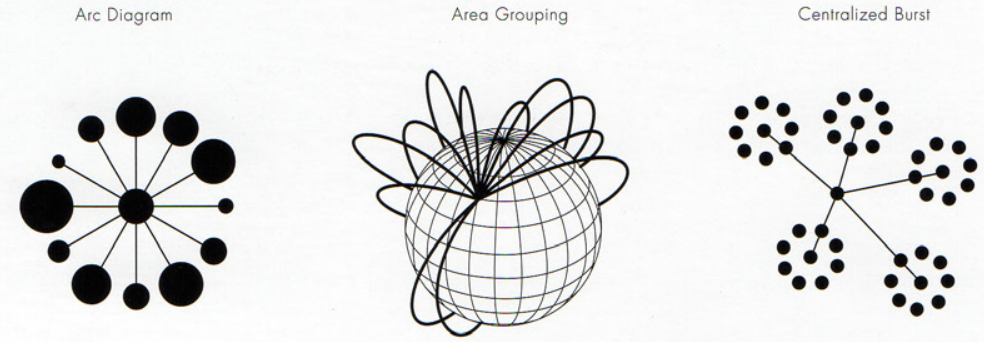

Centralized Ring
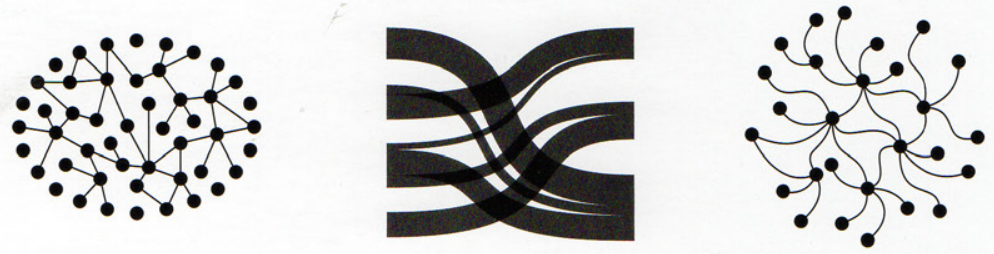

Elliptical Implosion

Flow Chart

Organic Rhizome
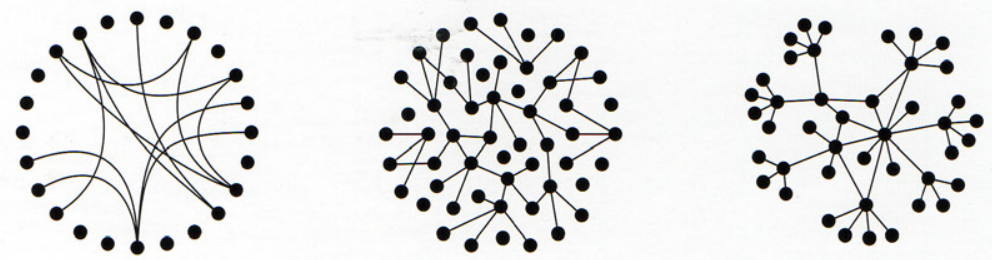

Radial Convergence

Radial Implosion

Ramification
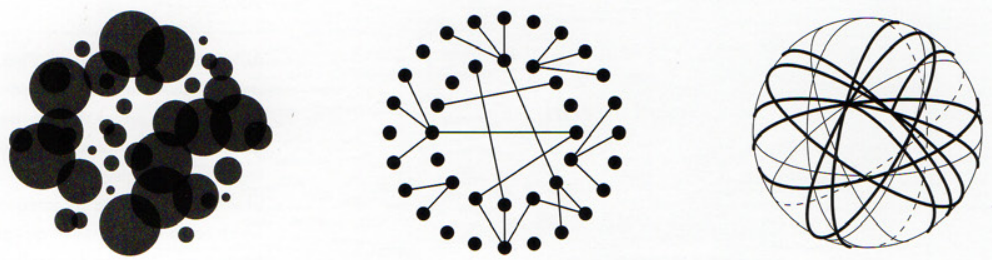

Scaling Circles

Segmented Radial Convergence

Sphere

Fig. 105 - Lima's fifteen categories of mapping patterns used for network visualisation (Lima, 2011a: 158) 


\subsection{Interactive Graphic Visualisations}

Methods of graphic representation that were once limited to print can now be made interactive and more dynamic, and communicate multi-layered messages and information. (This is the direction my own practice has taken.) Hans Rosling, a Swedish medical doctor, academic and statistician, is seen as something of a pioneer in the field of data visualisation, particularly concerning presenting data interactively (Rosling, 2006, February). With his son and daughter in-law he co-founded the Gapminder Foundation in 2005, a foundation that works to promote the use and understanding of statistics (www.gapminder.org). They developed the Trendalyzer software, which visualises data into interactive, animated statistics. Trendalyzer was acquired by Google Inc. in 2007.

The Gapminder website and software enables the user to explore a range of statistics about different countries' development in areas such as poverty, HIV, technology, climate, natural disasters, economy, and child health, according to year, country and animated growth/decline. The user is presented with a number of customisation options and selection filters (see fig. 106). Information is presented in the form of interactive bubble charts, showing, for example: percentage of broadband subscribers/Internet users by country and year; in what countries HIV is increasing or decreasing; average income; and life expectancy age. The user presses play to view an animated visualisation of growth or decline by year. The software also enables the user to select certain countries in order to compare them. Fig. 107 shows my customised selection in which I compared the percentage of broadband subscribers/Internet users in the United Kingdom, Denmark, Sweden, Norway, Russia and India over a decade. The three images are screenshots of the results and show the percentage of broadband subscribers/Internet users in those countries for 2000 and 2010. It also shows the two views available: as a chart (figs. 107a-b) or as a world map (fig. 107c). In this interactive application, Rosling and his team present the user with the option 
of viewing changes over time in the form of animated diagrams, and they have used colour coding for different parts of the world, so that one can also see patterns of technological development for the different continents, for example: the image for 2010 shows that European countries (orange circles) have more broadband subscribers and Internet users than other countries - the UK, Sweden, Norway and Denmark have $70 \%$ or more - while African countries and India (blue circles) all have less than 30\%. The Scandinavian countries have been among the countries with the highest proportion of broadband subscribers and Internet users since the early 2000s.

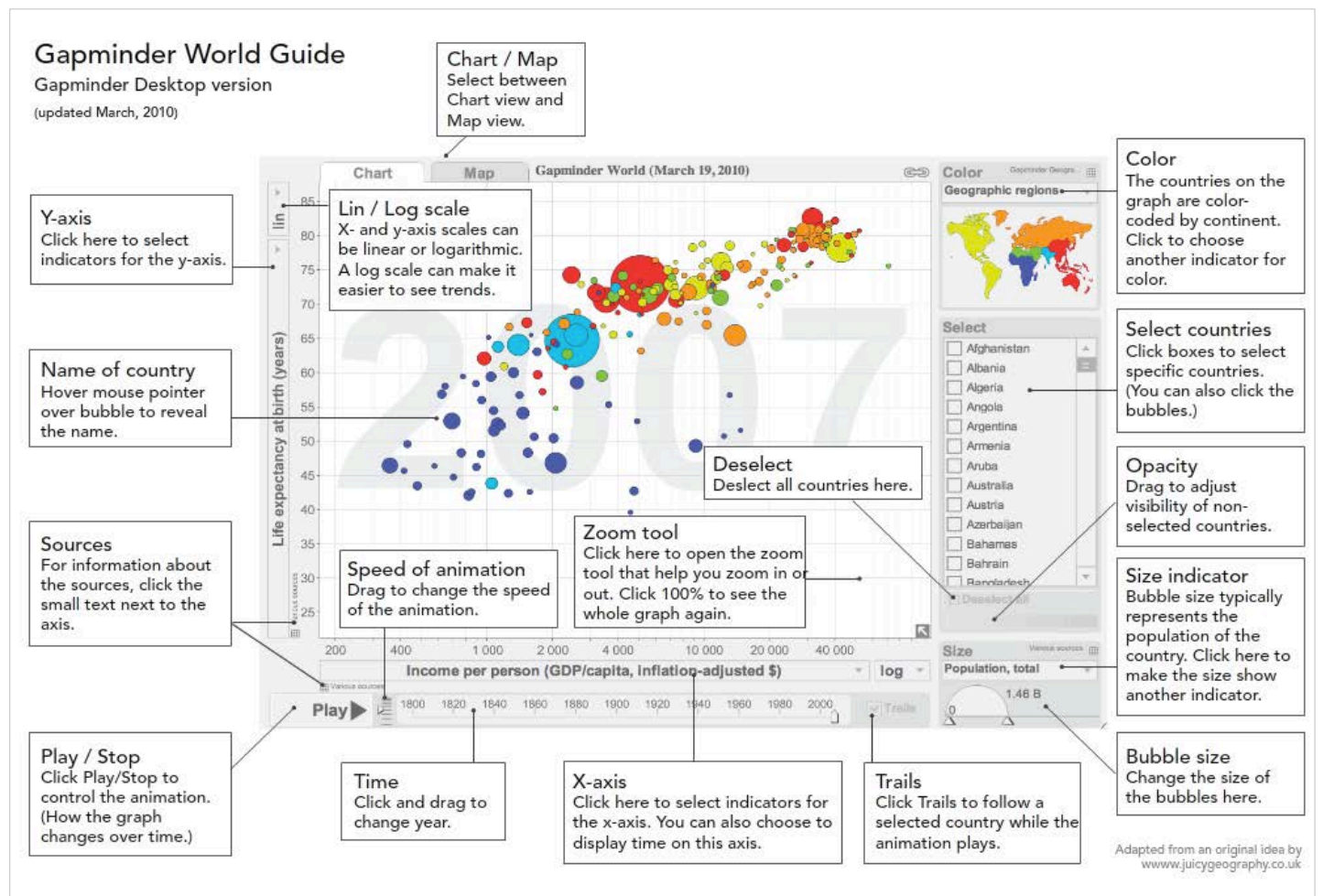

Fig. 106 - How to use the Gapminder desktop software (Source: Free material from www.gapminder.org) 


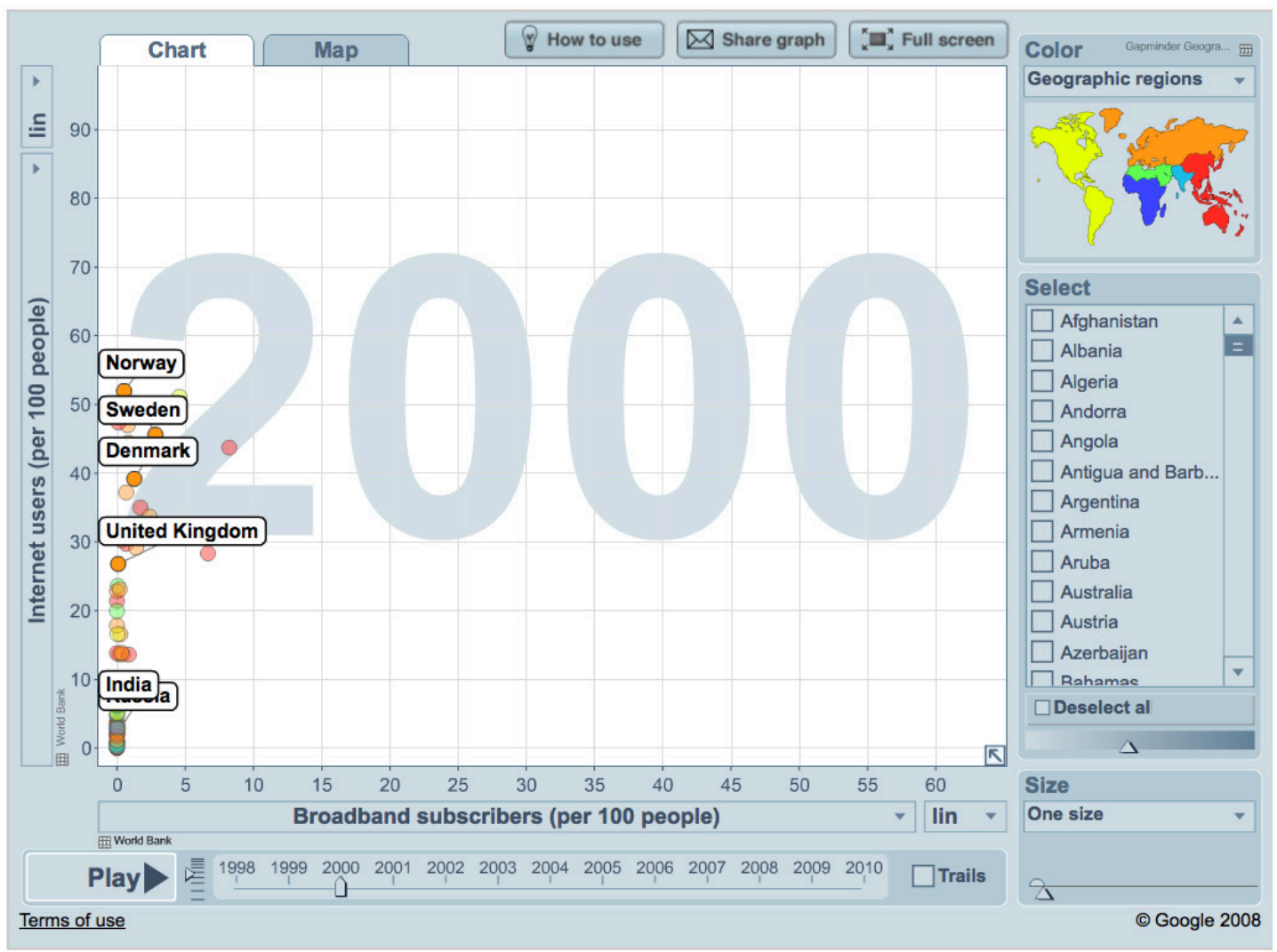

Fig. 107a - Percentage of broadband subscribers/Internet users in the United Kingdom, Denmark, Sweden, Norway, Russia and India in 2000: chart view (Source: Free material from www.gapminder.org)

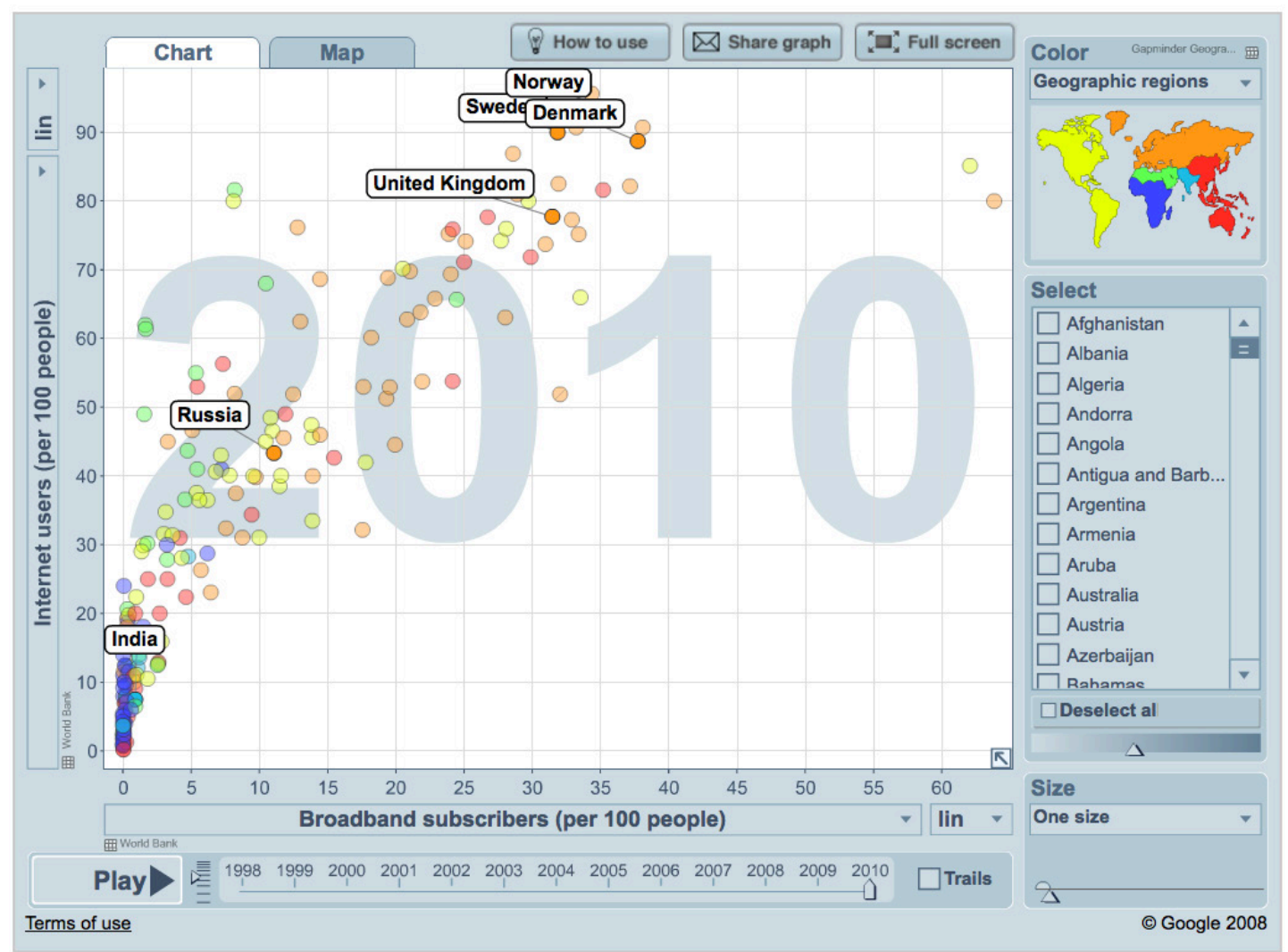

Fig. 107b - Percentage of broadband subscribers/Internet users in the United Kingdom, Denmark, Sweden, Norway, Russia and India in 2010: chart view (Ibid.) 


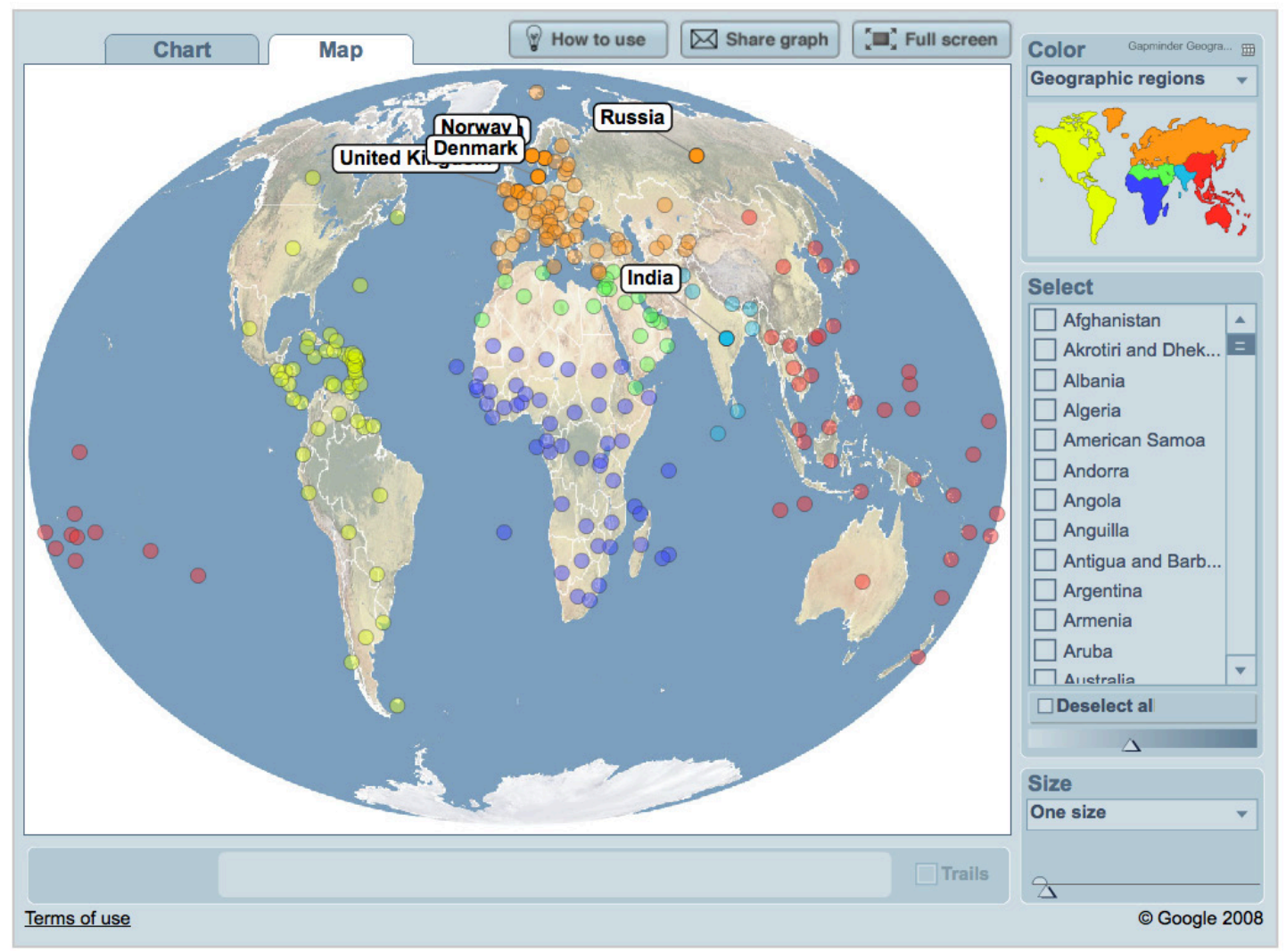

Fig. 107c - Percentage of broadband subscribers/Internet users in the United Kingdom, Denmark, Sweden, Norway, Russia and India in 2010: map view (Ibid.)

Rosling's work and software illustrate the important contribution that digital media have made to the field of information visualisation: enabling the user to view statistics customised to their own interests and selection criteria, and to view changes that have occurred over time. Presenting big datasets of world statistics in this way makes the information easier to 'read' and absorb, and a dimension of interactive engagement or 'play' becomes part of the search for information.

Today computer software is used by an increasing number of designers and data journalists to create interactive visualisations. On the Information is Beautiful website, for instance, David McCandless presents mainly static information visualisations, but he also presents some interactive diagrams. One such diagram is the Snake Oil Supplements? Scientific evidence for popular health supplements (McCandless, 2013a). As in Rosling's 
Gapminder charts, information is presented in the form of an interactive bubble chart. The interface differs from Gapminder in that it presents the user with more selection criteria and filter options to view the information that is of most interest to them. The application allows the user to filter the view according to health conditions, such as: cancer; cardio; diabetes; infections; and so on, and according to type, such as: mineral; plant/herb; vitamin; and others. The interactive diagram reveals whether there is found to be strong, good, promising, inconclusive, slight, or no evidence of the health benefits of the supplement (see figs. 108-109). The diagram uses datasets, and is regularly updated with new data so that it keeps up with current scientific research and evidence.

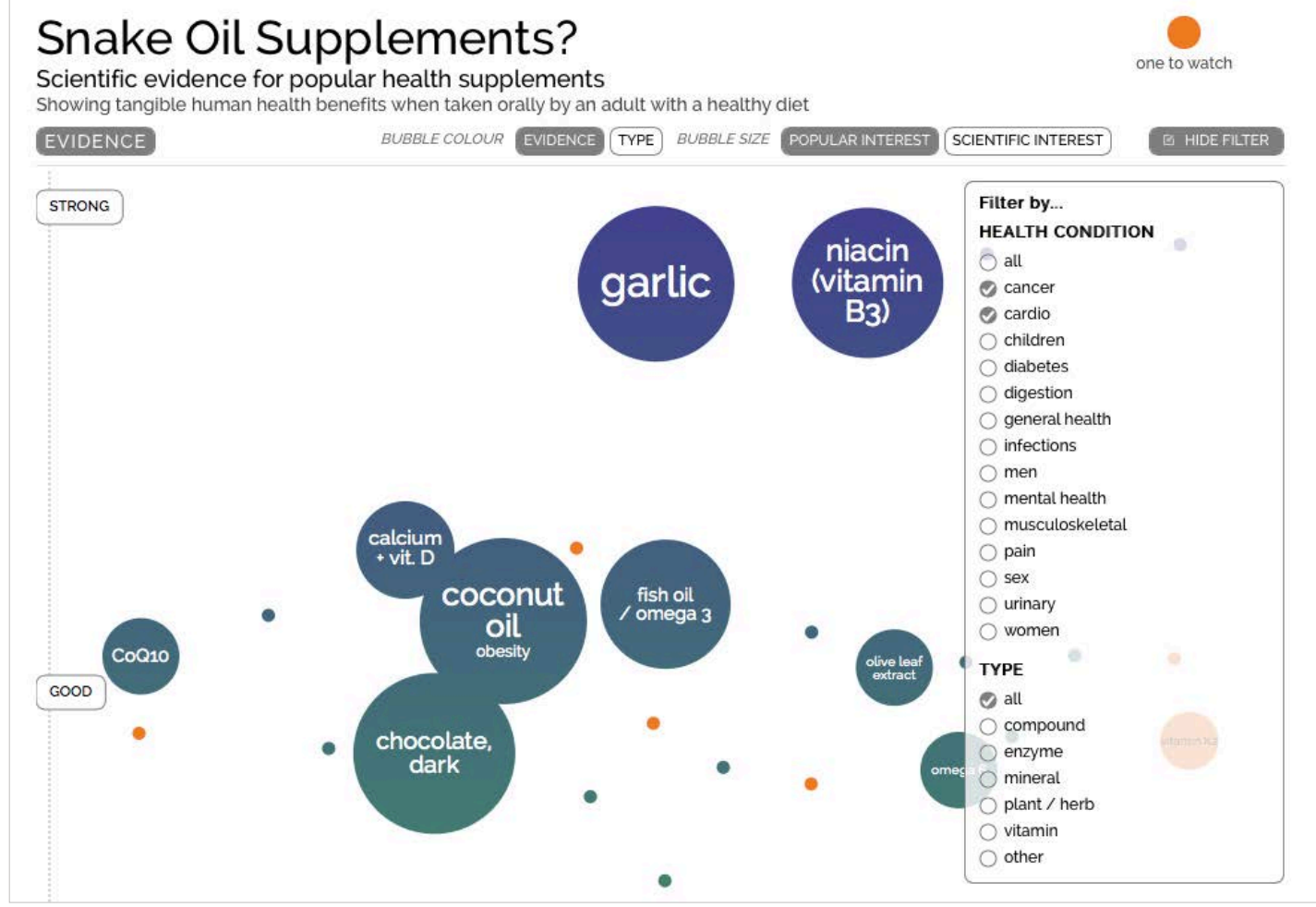

Fig. 108 - Filter options for Snake Oil Supplements? Scientific evidence for popular health supplements (McCandless, 2013a) 


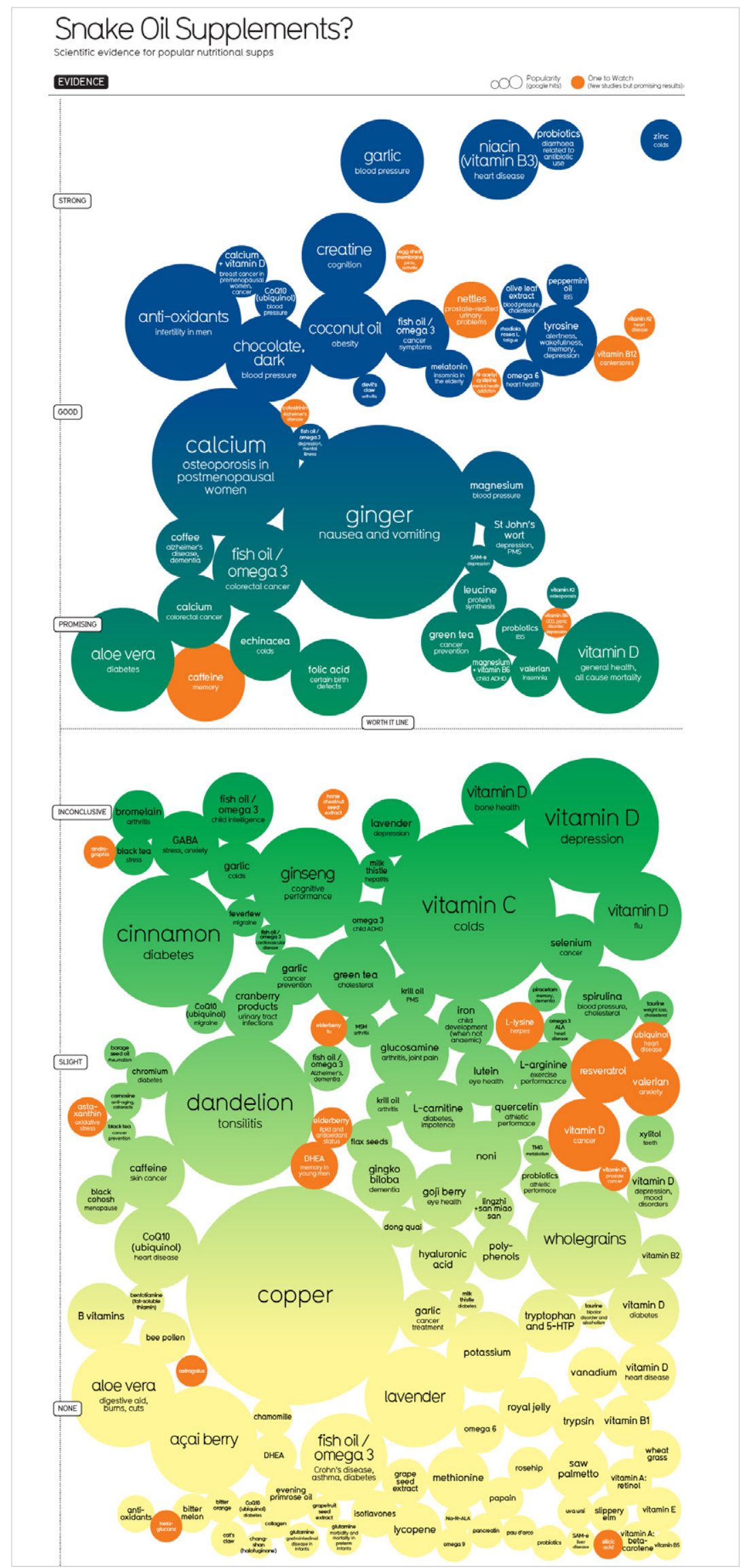

Fig. 109 - Interactive diagram Snake Oil Supplements? Scientific evidence for popular health supplements (McCandless, 2013a) 
Other diagrams, with different research focuses, such as the World's Biggest Data Breaches, have been made using the same visual layout and technology. McCandless and his team are currently developing software to generate these interactive bubble charts. The software, named VizSweet, which is in beta version at the time of writing, can be used to generate interactive visualisations using any dataset (McCandless, 2013b). In McCandless' newly released book Knowledge is Beautiful (2014) many more of his data visualisations have an online interactive version as well as the printed version. This indicates that there is an increasing trend towards representing data and infographics interactively.

Manuel Lima, too, has collected a number of works in which interactivity is key for the visualisation and communication of data and networks. One example, featured on visualcomplexity.com, is an interactive map created by Moritz Stefaner, called Map Your Moves (see fig. 110a and b). It maps where people from New York have moved to and from. The interactive nature of the map allows the user to navigate around to reveal information about specific areas of New York (Stefaner, 2010). The user can click on different circles, which represent postcodes, to reveal the specific numbers, statistics and destinations for immigration/emigration for that area. The colour blue

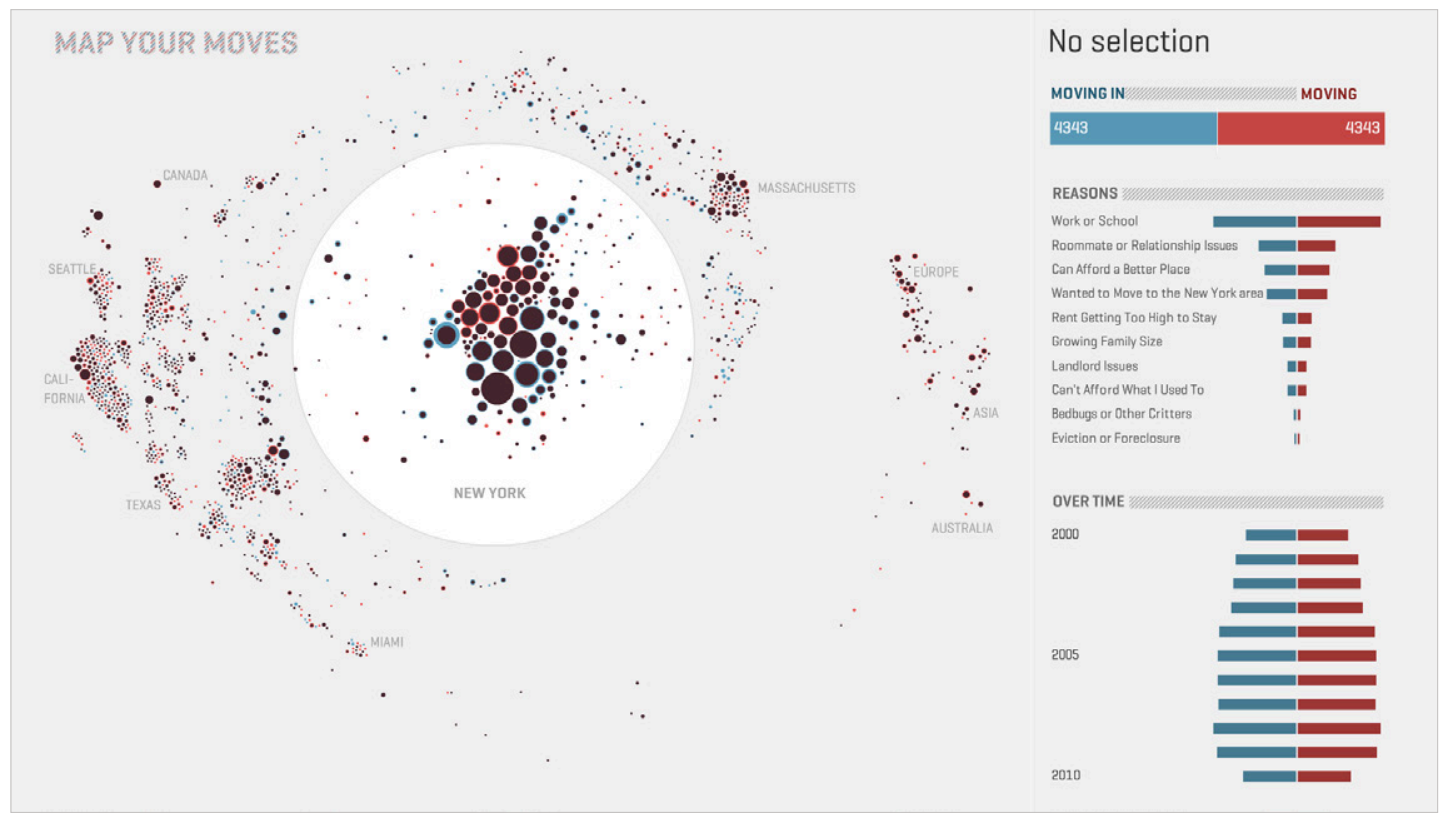

Fig. 110a - Still from Map Your Moves interactive map (Stefaner, 2010) 

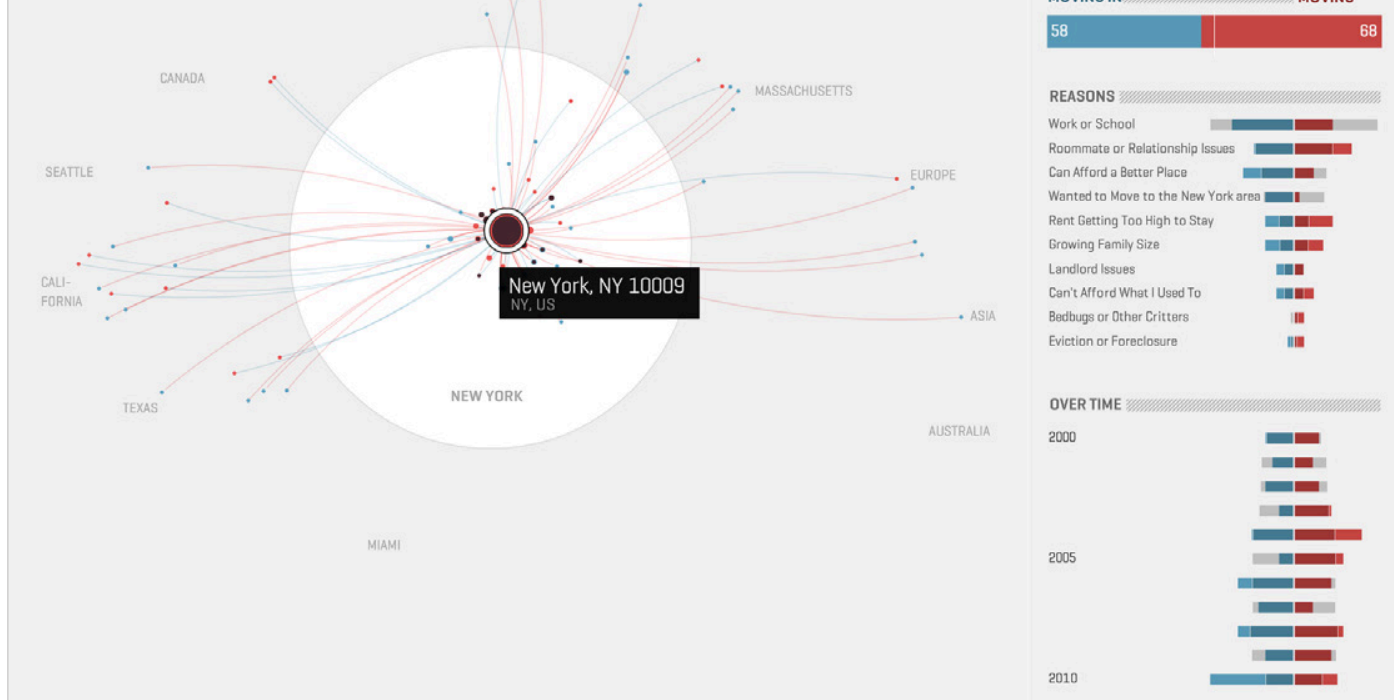

Fig. 110b - Still from Map Your Moves interactive map (Ibid.)

represents people moving in and red represents people moving out. The sizes of the circles represent the number of people moving. On the right side of the graphic map are statistics showing the reasons for moving in and out and how that has changed over time. The use of colour coding and simple graphic circles makes the map easy to read and navigate through.

When it comes to methodologies of visualisation, Lima is of the opinion that there is not one correct method, but the best visual method depends on each project and its context. He stresses that it is important to embrace time in mappings of social connections because many of our connections change and evolve over time. Time is also an important element in my study and its representations of greetings. Lima presents an example in which time is crucial to the visualisation process, in Daniel McFarland's video Streaming Classroom Interactions, which portrays the interactions between students and a teacher in a classroom over a certain time period (see fig. 111) (McFarland and Bender-deMoll, 2004; Lima, 2011a: 84-85). The network movie is created using the java package SoNIA (Social Network Image Animator) and visualises conversation turns in the classroom. The movie, 


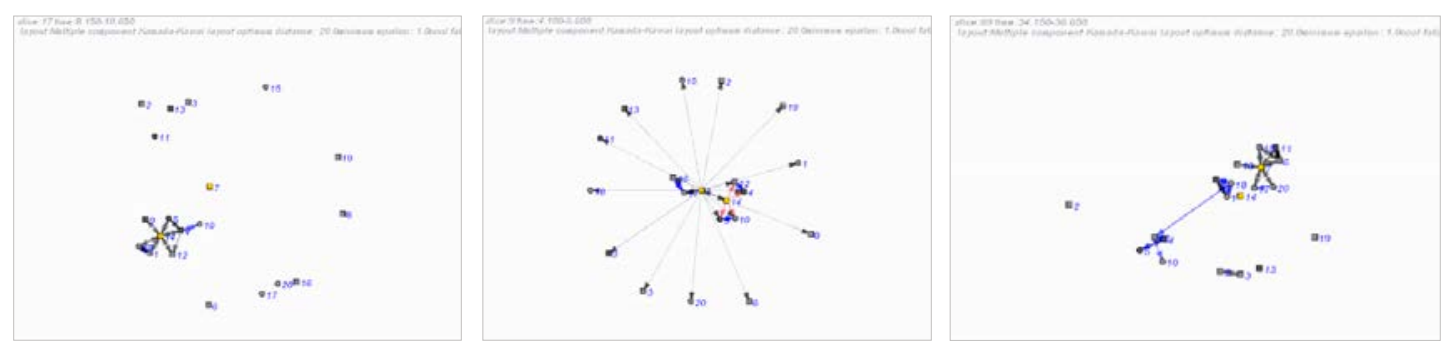

Fig. 111 - Stills from SoNIA - Streaming Classroom Interactions (McFarland and Bender de-Moll, 2004)

which bears a visual resemblance to Moreno's sociograms, visualises two distinct types of interaction: task interactions, which relate to teacher-given tasks; and sociable interactions, which are to do with everyday lives and social events. At the start of the movie the teacher assigns the students a task; when the students become distracted and overly social, the teacher comes in and breaks them up to focus them back onto the classroom task. Fig. 111 shows changes over time: how the interactions shift from involving a few people to most of the class. The key purpose for this visualisation is to map the changes that occur over time, and it does that very clearly by using basic graphic shapes: circles, lines and squares, to represent the teacher, students and different types of interactions. This visualisation illustrates how technology has moved the visualisation of social networks forward since Jacob Moreno's first sociogram, from being static graphic representations to becoming interactive ones that visualise changes over time. Although my research does not directly relate to network visualisations, the principles discussed by Lima, such as colour coding, change over time, and technologies for dynamic mapping, are highly relevant to my visualisation of greetings.

The examples of data visualisation and information graphics that I have discussed show the enormous communicative potential of this field. However, as both Rogers and McCandless point out, data never tells the whole truth. Data is filtered, analysed and often incomplete, and sometimes out- 
dated. However, data is useful in giving us an indication of the true situation (Rogers, 2013: 273-274). The usefulness of visualised information - whether the works are simply decorative, or whether they actually enable people to pay attention to the information and retain it - was discussed in a public debate by David McCandless and Neville Brody, on an episode of BBC Newsnight in August, 2010. McCandless' opinion was that infographics can help us to understand the world better, and help aid democracy. Brody, who is a graphic designer, typographer and art director (former art director for The Face magazine), argued that by making information pretty, one often hides the core message and misses important points, or the story in the information. McCandless agreed that people too often take the infographics at face value, and that these often contain mistakes. However, McCandless argued that the public demand for information is growing so rapidly that the visualisation of complex data plays a key role today in helping to clarify and communicate information that is important (BBC Newsnight: Information Graphics, 2010, August).

Brody's argument is a valid one, particularly when considering the popularity information visualisation has reached in recent years. The primary focus of any piece of information visualisation should not be on the sheer beauty of the visualisation, but rather on the communicative power of the visual. The fact is that the world's data is becoming increasingly digital and visual. Rogers stated that in $2007,94 \%$ of the world's data was stored in digital form (Rogers, 2013: 144). With this in mind, visualisation of complex data and of changes over time, as seen in examples discussed by Tufte, Lima and in Rosling's Gapminder project, are important in communicating some of that data in order for 'ordinary people' to be able to make sense of them.

I have discussed a number of examples of information graphics and data visualisation. While I have only included a few practitioners in a constantly expanding field, they were chosen because of their techniques and applications, and their relevance to my own project. From visual instruction 
manuals, image mappings, complex network mappings, and information visualisation, through to more interactive projects such as Gapminder, and the diagrams Snake Oil Supplements? and Map Your Moves, the fields of information visualisation and data journalism have a great deal to offer in terms of techniques for information representation and distillation. Techniques such as colour coding and scaling, and the infographic representation of changes over time, could potentially be used in the analysis of anthropological information and data. That is what my practice aims to do. I apply some of these techniques in my visualisation of nonverbal body movement and cultural differences in greetings.

\subsection{Interactive Installations/Performance Art}

I have so far discussed how interactive technologies are used for information visualisation and for creating visual statistics and diagrams. These technologies are also used, arguably more creatively and experimentally, for creating interactive installations and performance art. There are also many examples of performance art in which greeting is a central theme ${ }^{3}$; however, my study here is not a thematic survey but is focused on the means of visualising movement through the use of interactive technologies. The works I will discuss are all examples of interactive installations or performances that have been selected for their creative and ground-breaking uses of technology, and which relate to my research in terms of their focus on nonverbal communication and body movement.

One example of a project using interactive technologies is the Chameleon Project by Tina Gonsalves. The Chameleon project is an interactive art installation which reacts to the emotions of its visitors (Gonsalves, 2009).

3 Some examples of performance and video art in which images of human greetings are portrayed include Bill Viola's video installation The Greeting (Viola, 1995), Mark Wallinger's video installation Threshold to the Kingdom (Wallinger, 2000), and Martin Parr's photographs of Haute Couture kisses (Parr, 2005). Greetings also play a less direct but more symbolic role in works such as: Marina Abramovic's 700 hour long performance The Artist is Present (Abramovic, 2010), Breda Beban's Arte Vivo (2008-11), and Mark Lewis' The Pitch (1998). 
It uses technology that reads the facial emotion expressions of the visitors, and its algorithms respond to these emotions by playing different video clips. The videos are recordings of faces expressing a range of emotions. The aim with the project was to leave the participants with a sense and understanding of how their own emotions/nonverbal signals impact and influence the emotions of the group. The project is a collaboration between artist Tina Gonsalves, neuroscientists Chris Frith and Hugo Critchley, and computer scientists Rosalind Picard and Rana El Kaliouby. The work consists of nine prototypes that lead up to the final version of the installation. Prototype $6-$ Mimicking Emotional Contagion in Social Groups, is a single channel video of 12 people's faces, and the emotions of each person continuously change the harmony and emotions of the entire group (see fig. 112). Prototype 7 - Integration of Mind Reading Technology, reacts to the visitors' facial expressions when they enter the room and triggers video portraits that empathise and identify with their emotions (see fig. 113). The project utilises a highly sensitive facial emotion analyser (see fig. 114), which analyses different axes of facial expressions.

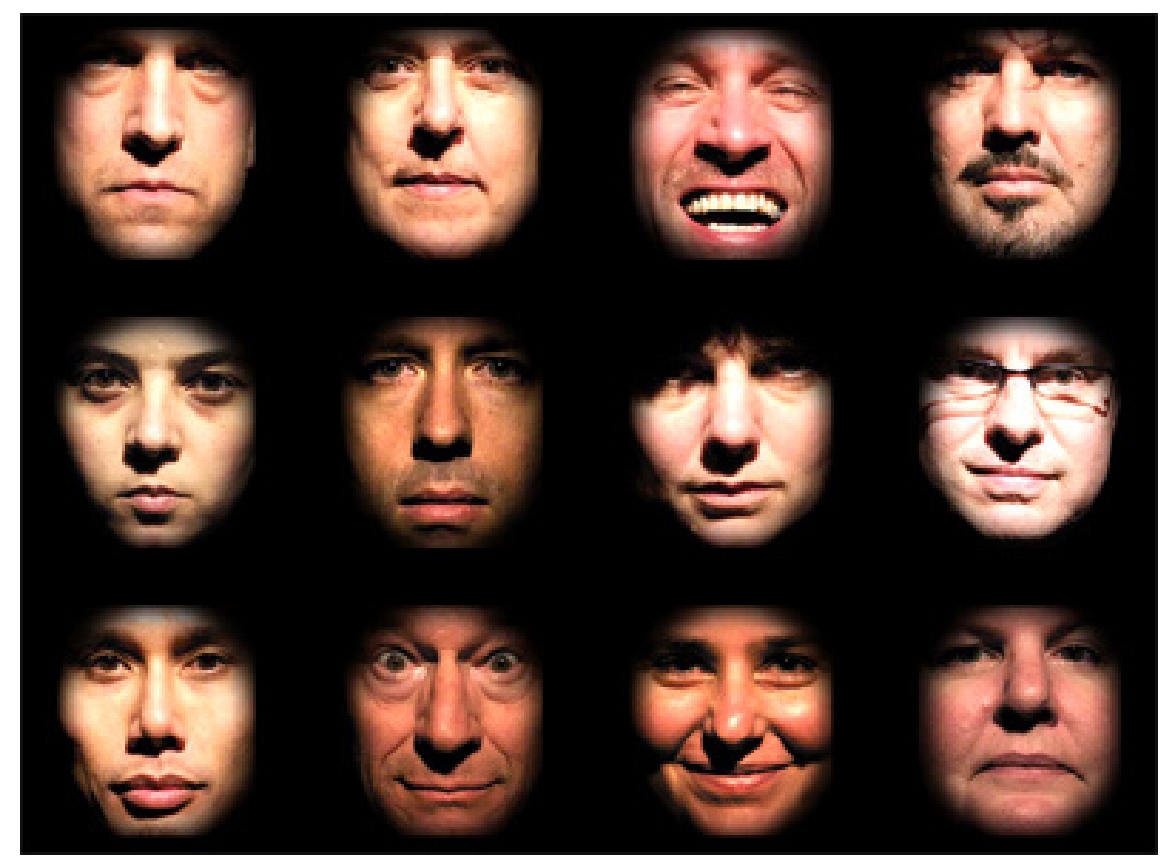

Fig. 112 - Chameleon Project - Still image from Prototype 6: Mimicking Emotional Contagion in Social Groups (Gonsalves, 2009) 


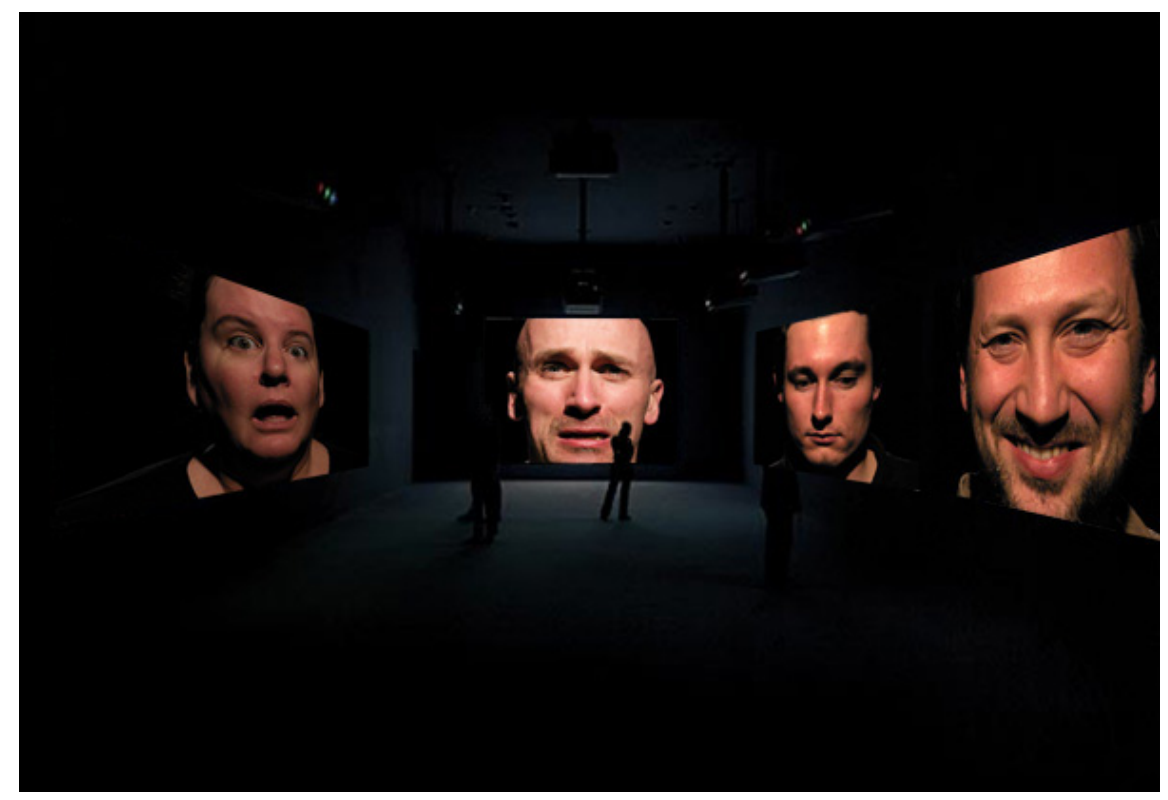

Fig. 113 - Chameleon Project - Prototype 7: Integration of Mind Reading Technology, installation (Ibid.)
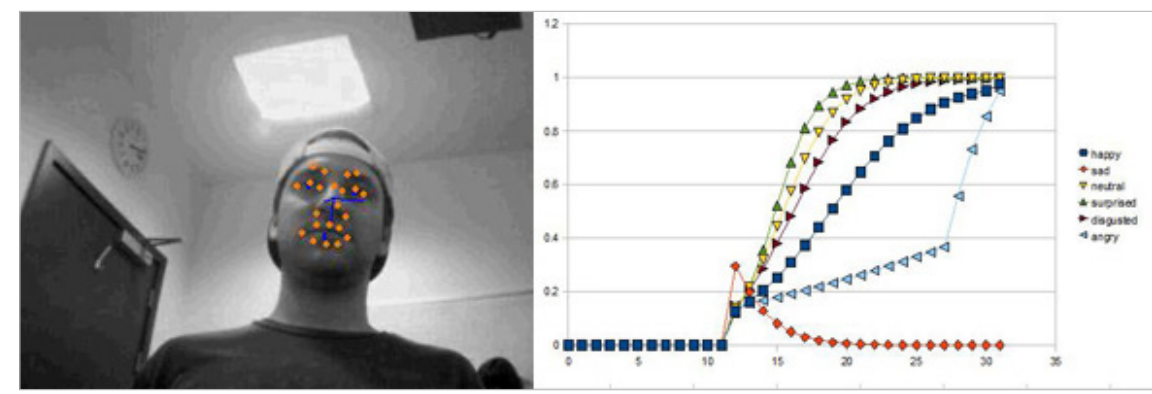

Fig. 114 - Chameleon Project - Prototype 7: Integration of Mind Reading Technology, technology/sensors (Ibid.)

The analyser was developed in collaboration with researchers at MIT Media Lab in Cambridge, Massachusetts. The installation becomes interactive when an audience member enters the room. The audience members are first faced with a screen featuring a bored looking face. The face starts to talk once the audience member looks at the screen. The visitors' different emotions are measured with the facial emotion analyser, and video sequences responding to those emotions are triggered. The use of sensor technologies, and the employment of tools of biomedical science and computer science, enable the recording of and responding to subtle facial expressions. 
In documenting greetings, sensor technologies could be useful for mapping out what emotions and expressions are present during the different greeting situations. While the scope of my own project does not include a specific focus on facial expressions, the use of sensor technologies for this aspect of greetings studies holds great potential, and could be utilised in the near future when such technologies become more readily available and affordable. Since my main project has been to devise and design a system, I have only briefly experimented with sensor technologies for tracking movement during greetings, and I will discuss these experiments in chapter 4.

There are other projects that have utilised motion tracking to create more interactive and playful installations, such as Catch Me Now and Rain Room. Danish artist and researcher Tine Beck's interactive installation Catch Me Now, created in 2010, is a playful piece in which coloured spotlights project circles of light that move around on the floor. When a visitor steps on the light it starts following them, and the circle grows (see fig. 115). The project
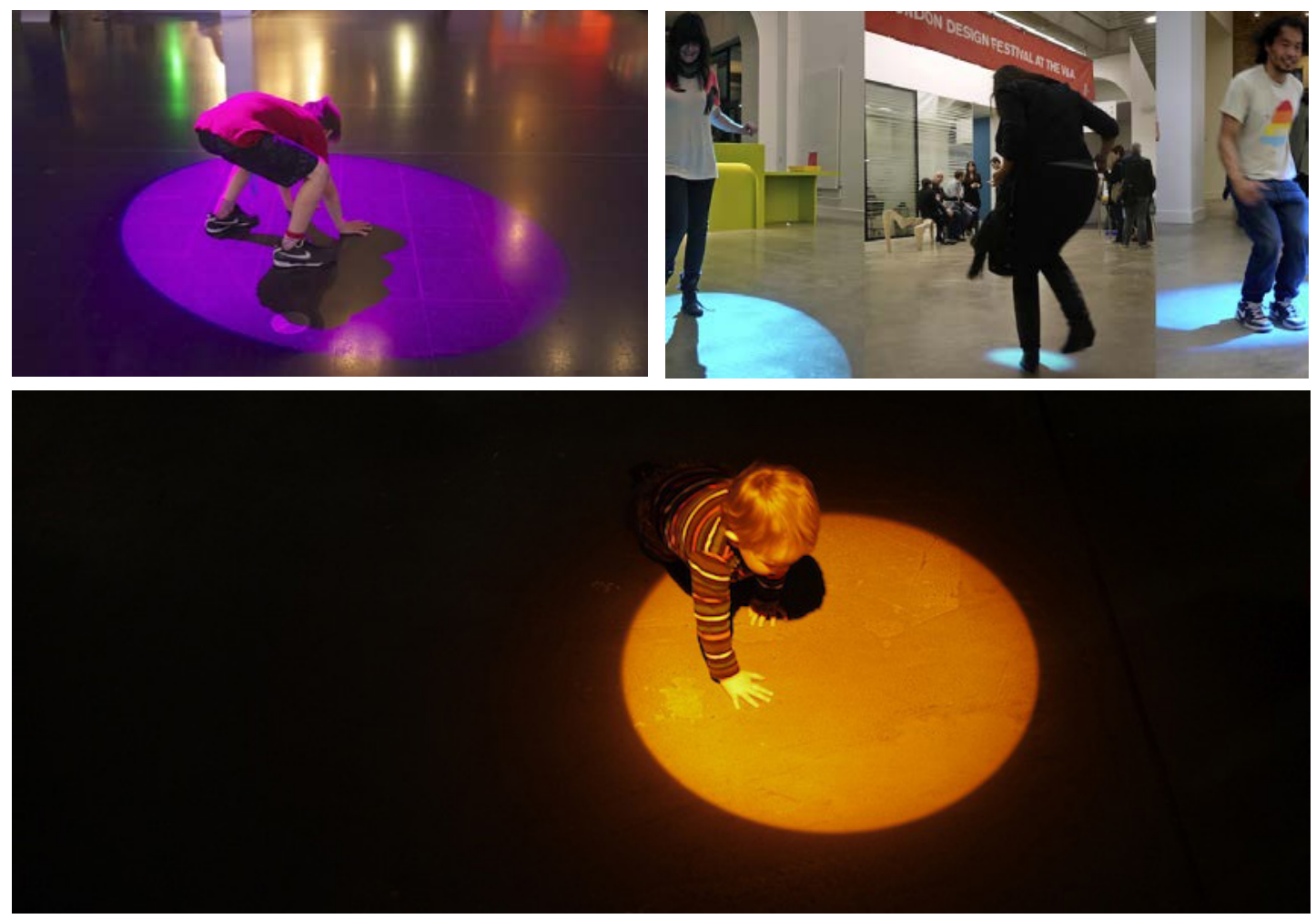

Fig. 115 - Tine Beck's light installation Catch Me Now (Beck, 2010) 
uses sensors to track the user's movements, and is programmed to perform different motion cues (Beck, 2010). The focus of Catch Me Now is on the play between the visitor and technology.

Another playful, yet much more sophisticated installation is Random International's Rain Room (see fig. 116). Random International is a London based studio that creates artworks and installations in which the body and interactivity are at the centre. Fundamental to their work is user participation and human behaviour, and they utilise cutting-edge technology and science to encourage interaction (Random International, 2014). Rain Room, installed at the Barbican in London in 2012-13, was a hundred square metre space with falling rain. However, visitors walking through the room did not get wet. A path opened up around the visitor, or visitors, and the area around the visitor(s) remained dry. Random International used custom software and 3D tracking cameras to track the visitors, and a complex water management
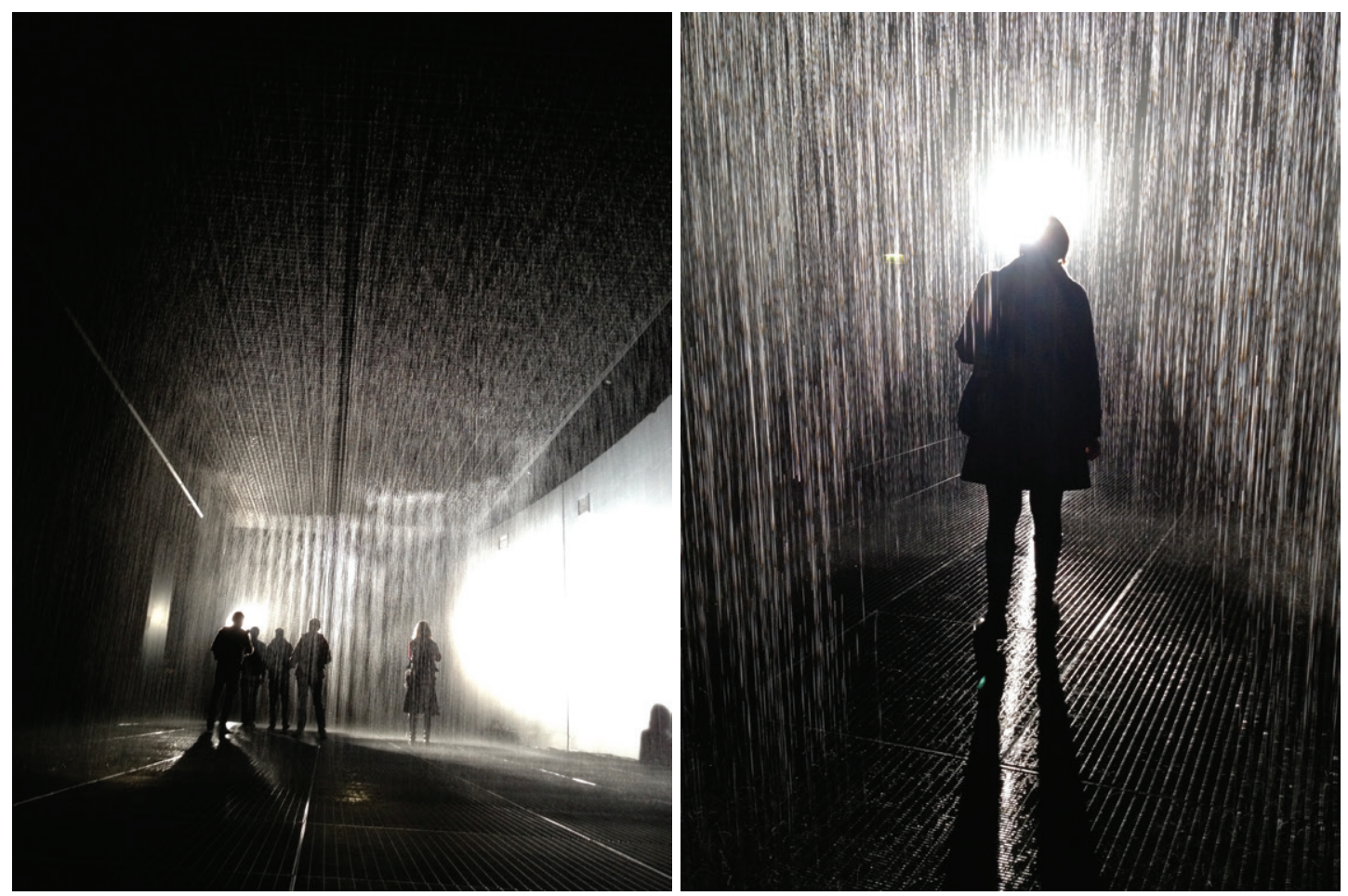

Fig. 116 - Random International's Rain Room installation at Barbican, London 
system that was regulated through the software. The visitors controlled the rain simply by being in the space. Random International were interested in the audience's reactions and interaction within the space - how people interact with strangers within such a space, and in the relationship between people and intelligent technologies. While Rain Room was not a greeting space or a study on greetings specifically, it did enable Random International to observe how people interact with each other: how they meet, greet, behave and move according to others in the room.

The main project I will discuss relating to interactivity and performance is the Motion Bank project, which uses sophisticated motion recording and visualisation (Motion Bank, 2013a). This project relates more directly to my research topic of visualising movement. Motion Bank was a four-year research project by German-based The Forsythe Company, who produce work in performance, installation, film and educational media. The project's director was Scott deLaHunta, who is a senior research fellow at the Centre of Dance at Coventry University. Motion Bank is a research into choreographic dance practice, and is a collaboration between The Forsythe Company and internationally renowned research institutes, dancers, and choreographers. Through the project the research team developed online digital dance scores focused on making available the choreographic approaches by the different choreographers Debora Hay, Jonathan Burrows and Matteo Fargion, Thomas Hauert, and Bebe Miller. The researchers observed that existing notation systems for dance, such as the systems discussed earlier in this chapter, are not widely known and not used universally. The Motion Bank project does not use any of these systems as a basis for their experiments, but instead brings the practice of dance notation into a contemporary and technological frame. The aim with the project was to document and preserve dance scores digitally and to create understanding of the language of dance among the public (Motion Bank, 2013a). 
Researchers from one of the key technical partners of the project, the Department of Virtual and Augmented Reality at the Fraunhofer Institute for Computer Graphics Research in Darmstadt, developed sophisticated visualisation techniques to record dancers' movements and to visualise digital scores. The computer scientists, mathematicians and interaction designers from the Institute collaborated to develop these approaches. The production phase involved digital designers and programmers who worked together with the choreographer and designers from the Offenbach Academy of Art and Design. They video-recorded dancers' performances from different angles simultaneously, and in the production phase used these recordings to create unique ways of visualising movement. The results were shared online.

One central project within the Motion Bank project, titled Using the Sky, was created by the technical team in collaboration with American choreographer Debora Hay (Motion Bank, 2013c). The project uses her written dance score No Time to Fly and focuses on her work from different perspectives: analytical, artistic and conceptual. The score was given to three solo dancers who made their own adaptation of the piece, and each dancer's adaptation was performed and recorded seven times. Computer vision technology was used to estimate the 3D positions and pathways of the dancers on stage and to track their movements. Usually such estimations are done using a motiontracking suite or by attaching sensors to the dancers, but the Motion Bank researchers found that such interventions can influence the movement of the dancers. Instead they used a large stage and four cameras, positioned at high angles to capture images from several points of view (see fig. 117). They were then able to calculate the three positions of the dancer on stage. The recordings were used to study patterns that could be drawn out in the design process to create different visualisations of the choreography. They also used Piecemaker - a software in development by Motion Bank for annotating video in real-time - to mark up changes in the dance. 


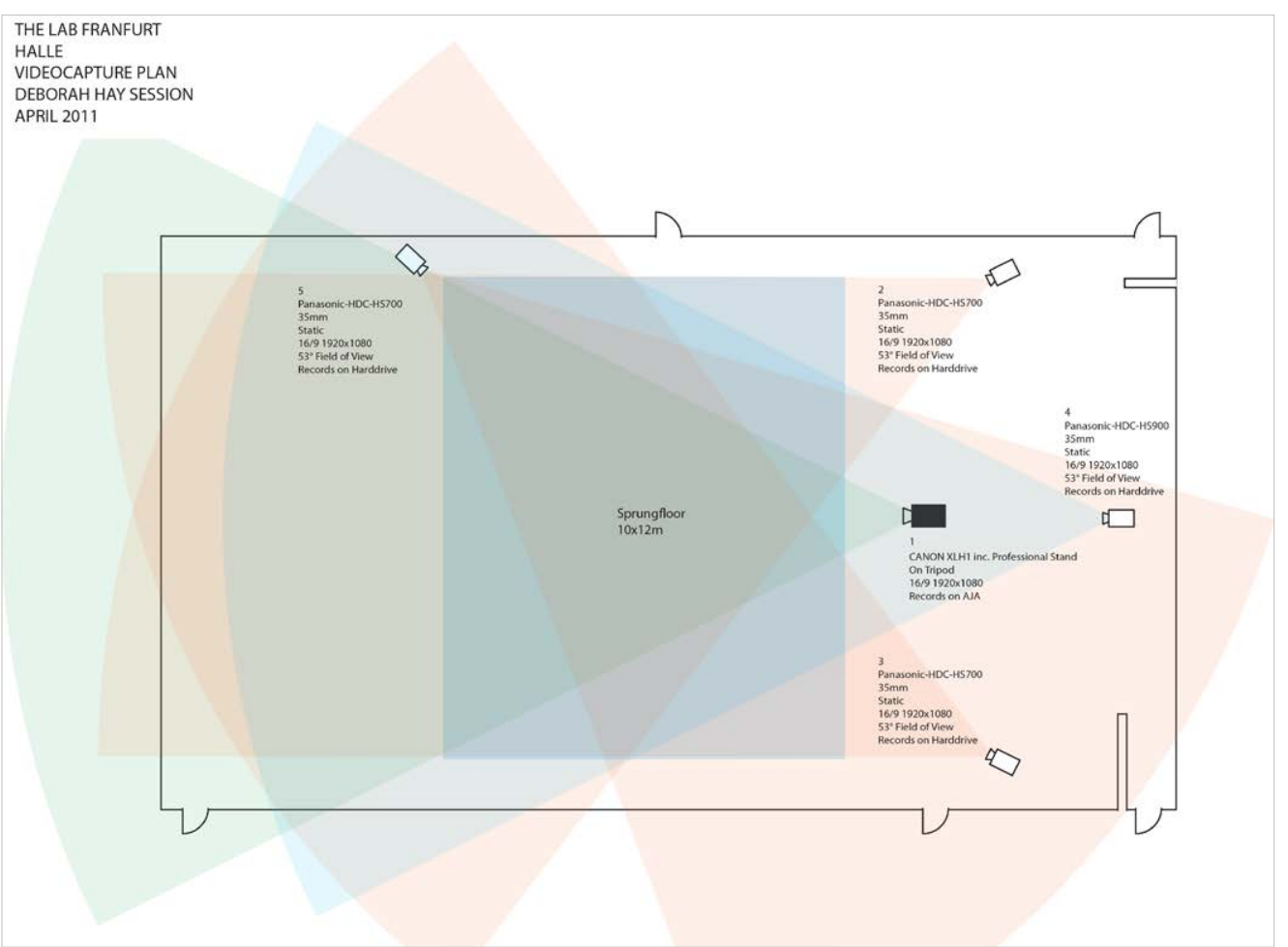

Fig. 117 - Set-up of studio and recording equipment for Motion Bank project (Motion Bank, 2013a)

One of the visualisation approaches they employed in Using the Sky involved video overlays: all seven of each of the three dancers' recordings, 21 in total, were aligned and layered into one video. The overlays make it possible to see both the variety and the regularity of the dancers' interpretation of space and time across the seven times they performed the piece. The overlay video is presented next to Deborah Hay's written score, and shows the dancers' interpretations of different parts of the score. The user can navigate through the score's 25 different sections by clicking on the numbered tabs to the right of the score text (see fig. 118).

Another dimension of movement explored by this project was movement 'character': it created visual scores of the travel paths used by the three dancers. Fig. 119 shows the travel paths presented to the left of the dance footage, as lines visualised in a different colour for each of the three dancers. 


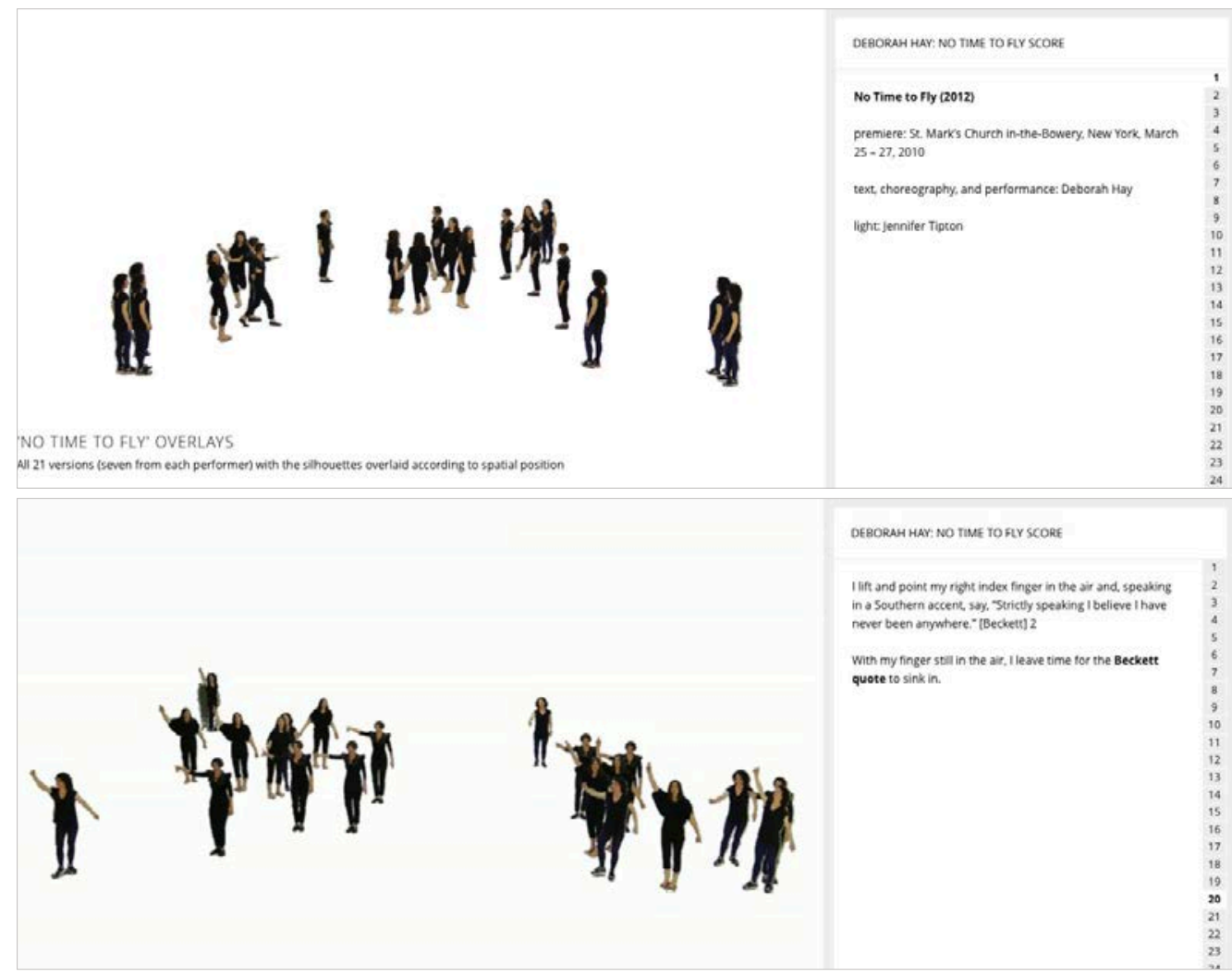

Fig. 118 - Using The Sky: No Time To Fly video overlays (Motion Bank, 2013c)

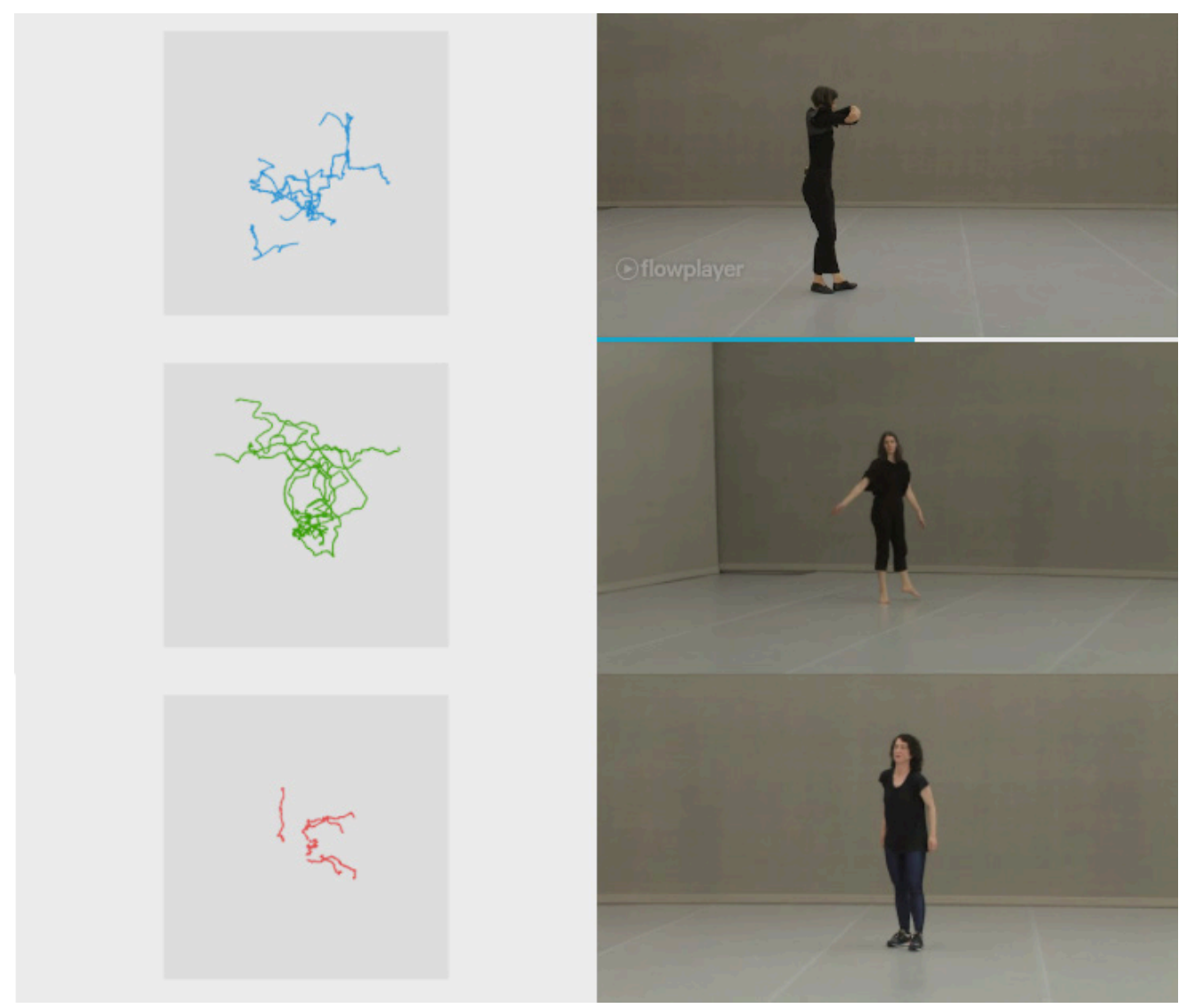

Fig. 119 - Using The Sky: travel paths (1) (Ibid.) 
The travel paths are static images that give an overhead view of where the dancer moves on stage. Each travel path in fig. 119 combines all of the seven recordings into one visualisation, illustrating the consistency, or lack of it, between each of their performances. As with the overlay videos, it is possible to navigate through the 25 sections of the score and view how the travel paths change. Figs. 119-120 compare the three dancers' travel paths at two different points of their performances. In fig. 121 all of the travel paths for one dancer, including all of her seven recordings, are combined into one illustration. This image shows how she has moved across most parts of the stage during the performances.

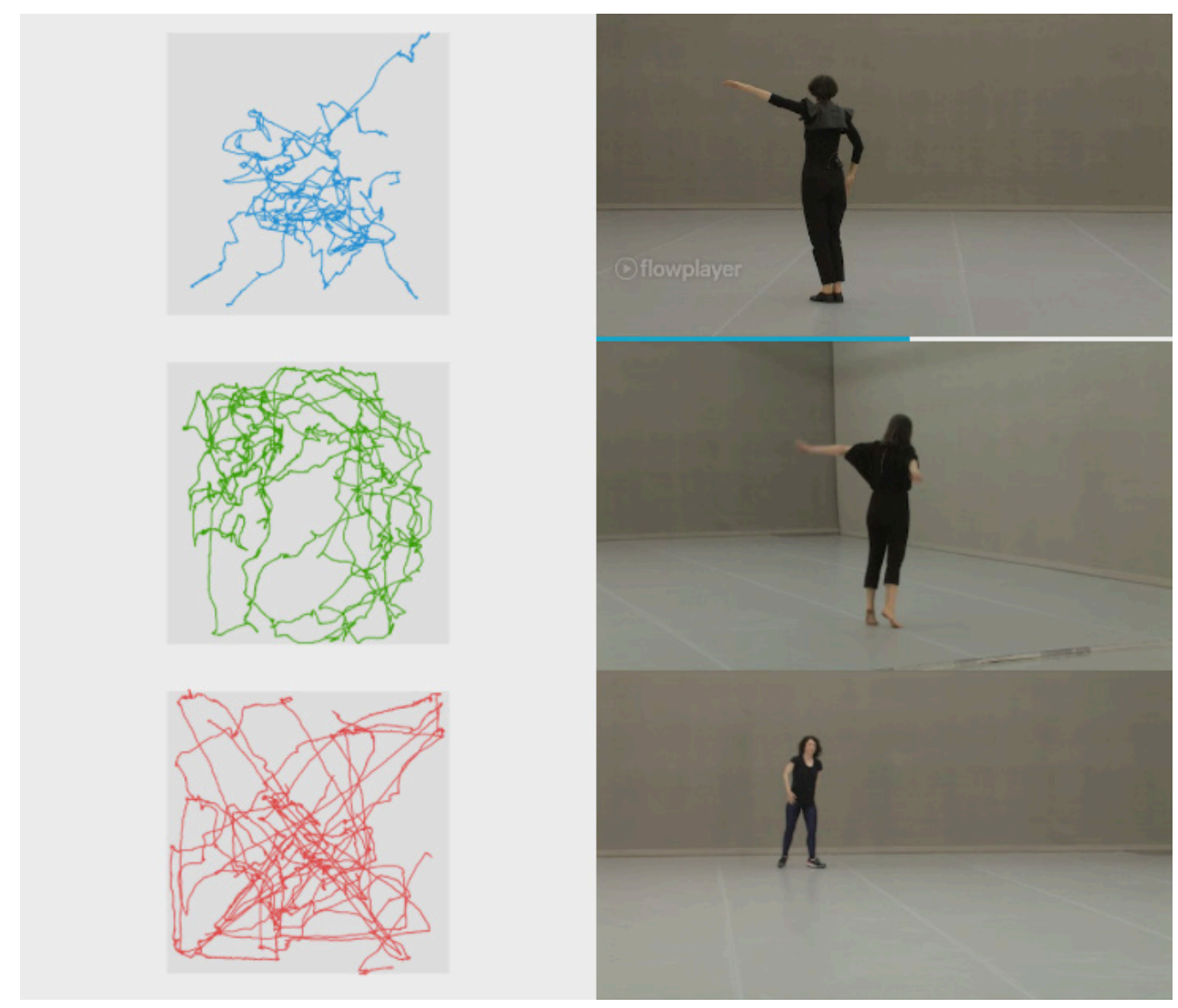

Fig. 120 - Using The Sky: travel paths (2) (Ibid.) 


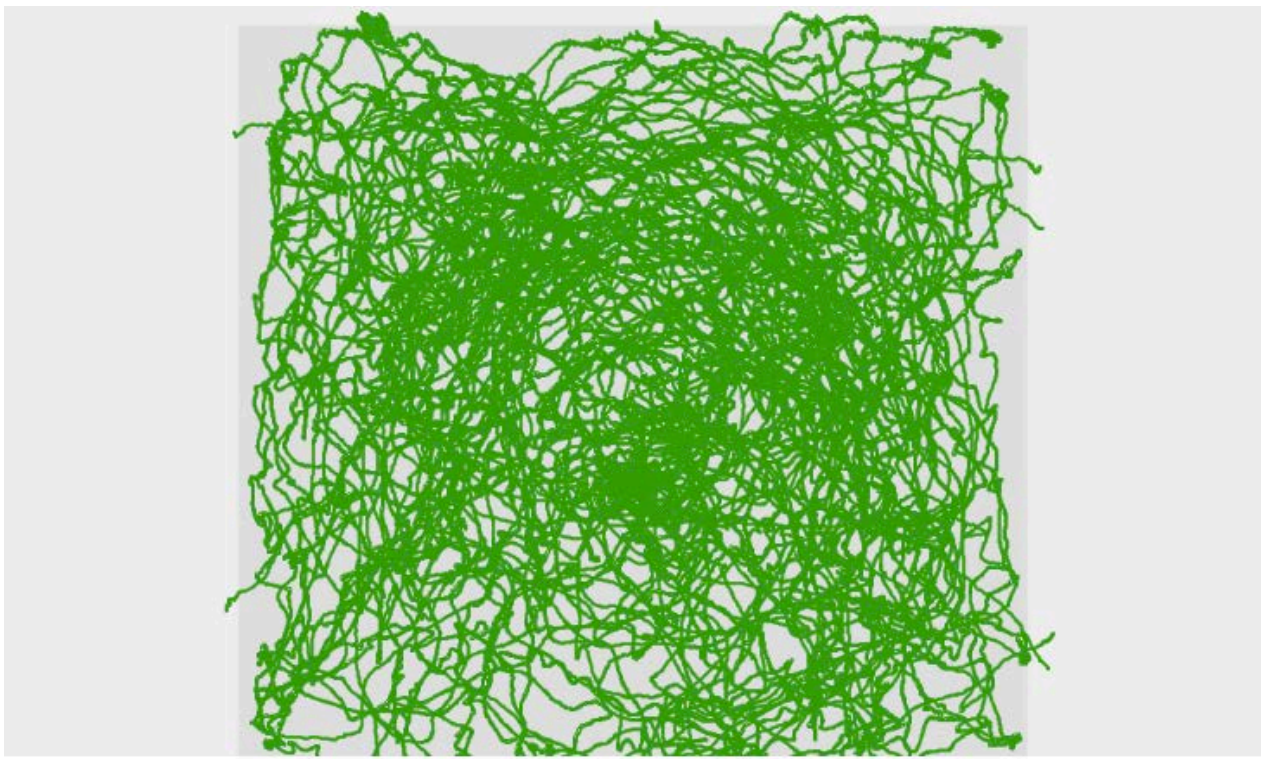

FULL TRAVEL PATH JULIETTE MAPP ALL 7 RECORDINGS

This image shows the travel path of 7 performances of No Time To Fly (April 2011) at once.

Fig. 121 - Using The Sky: travel paths (3) (Ibid.)

Motion Bank explores the visualisation of travel paths further through transforming the line illustrations into convex hulls ${ }^{4}$. The convex hulls are shapes made by connecting the outer points of the travel paths (see figs. 122-123). This form of visualisation focuses on what areas the dancer moves through on stage, while the travel paths simply show the actual path of movement. Fig. 122 compares the three dancers' movement areas at one part of their performance, and shows that the top dancer, illustrated using blue for the convex hulls, uses much less of the stage in her interpretation of that particular section of the score than the other two dancers who are illustrated in green and red. The convex hulls are presented in the same way as the other travel paths, with the 25 tabs. Both versions of the travel path visualisations make possible the comparison of the three dancers' use of space/stage and movement paths.

4 Convex is a term used in science and technology and is defined as 'having a curved form which bulges outward, resembling the exterior of a sphere or cylinder or a section of these bodies.' A convex hull is a term borrowed from mathematics and is defined as 'the smallest convex set containing a given collection of points in a real linear space' (McGraw-Hill Dictionary of Scientific \& Technical Terms, 6E., 2003a; 2003b). 


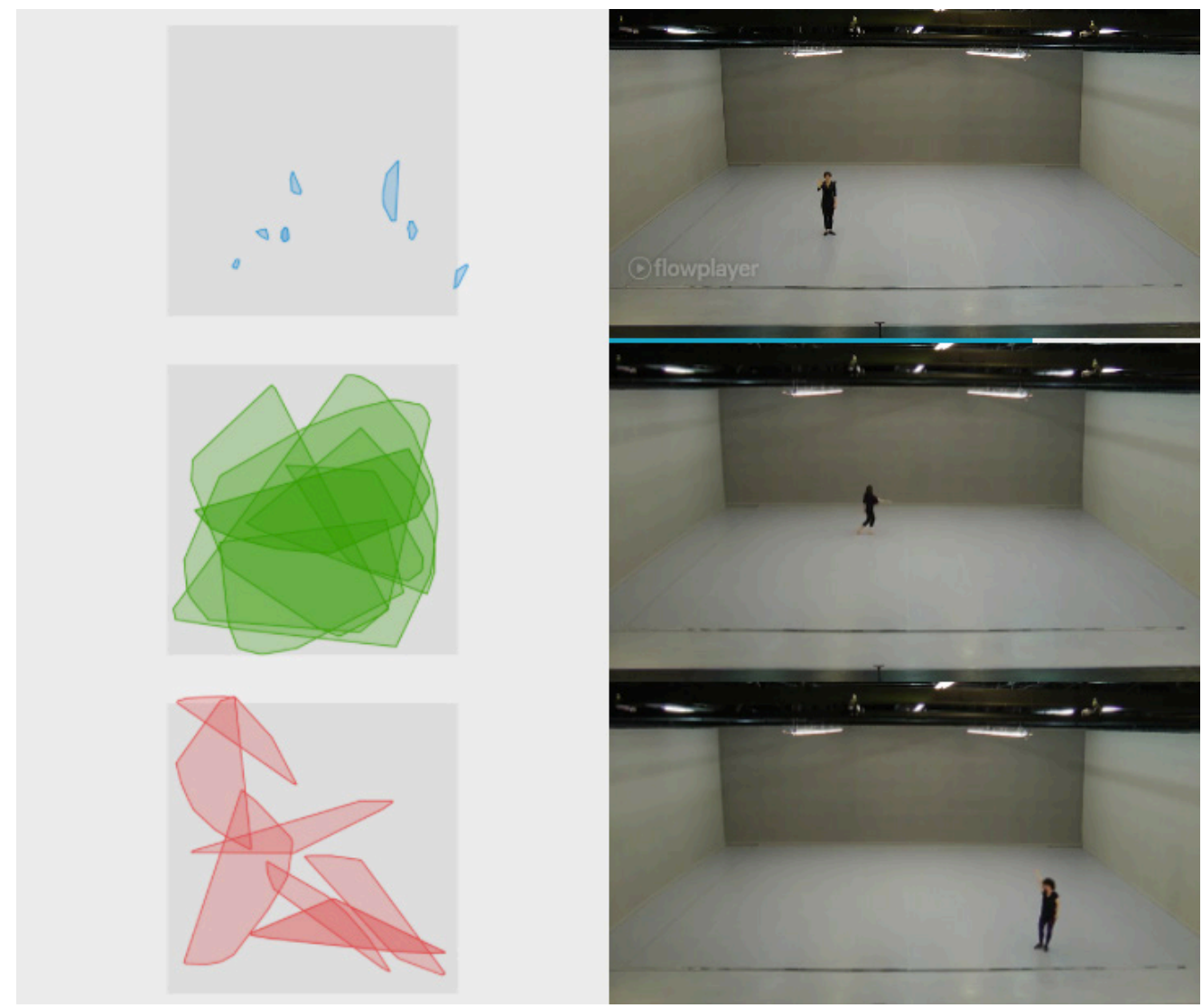

Fig. 122 - Using The Sky: convex hulls of the travel paths, comparison (Ibid.)

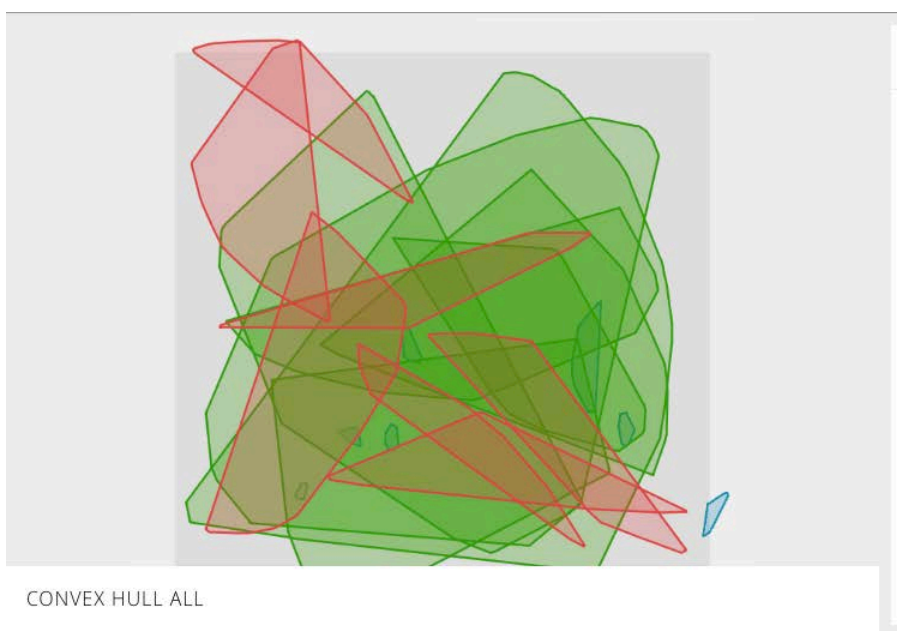

CONVEX HULL

The visualization above shows "convex hulls" of the travel paths in relation to the score. A convex hull is a shape formed by connecting the outmost points of a dataset like a rubber band that is wrapped around it.

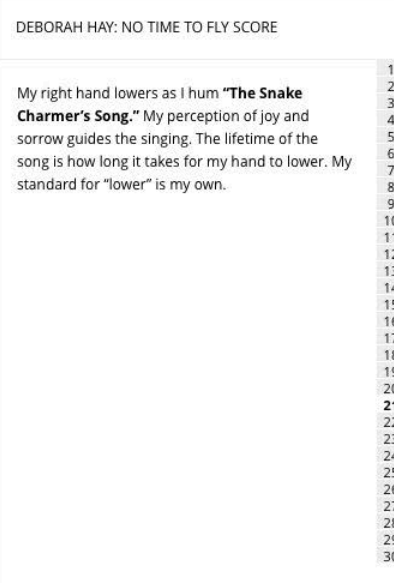

USE OF SPACE

Try tab numbers: $7,11,14,18,21,23,25,26$ and compare location and use of space in the visualizations to the right

Fig. 123 - Using The Sky: convex hulls of the travel paths, combined (Ibid.) 
The use of the colours blue, green, and red distinguishes the dancers from each other and makes differences and similarities between their movements visible and easy to follow.

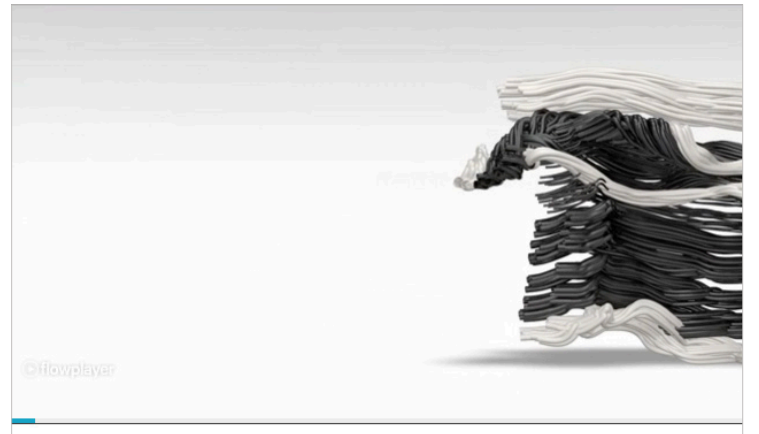

"NO TIME TO FLY" DIGITAL ADAPTATION BY AMIN WEBER This artistic response to Deborah Hay's invitation to develop a digital adaptation is linked to the sequence of the written score to the left

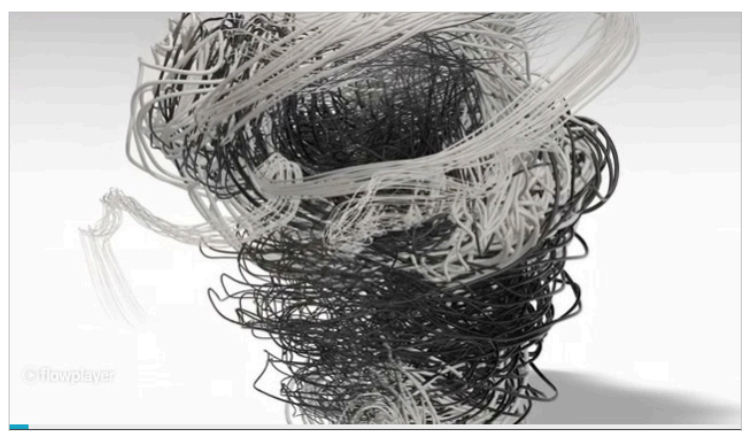

"NO TIME TO FLY" DIGITAL ADAPTATION BY AMIN WEBER This artistic response to Deborah Hay's invitation to develop a digital adaptation is linked to the sequence of the written score to the left.

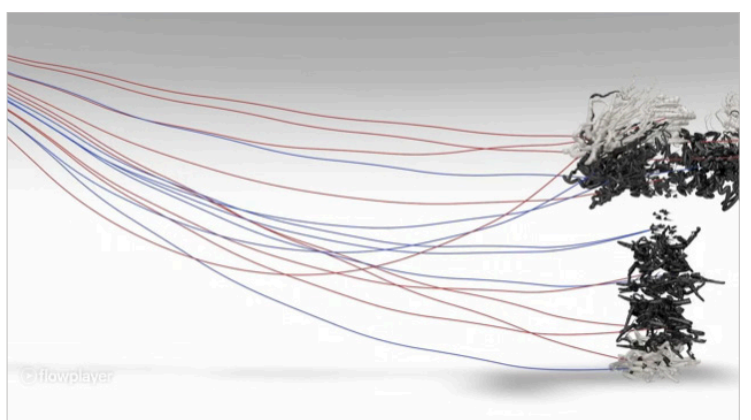

"NO TIME TO FLY" DIGITAL ADAPTATION BY AMIN WEBER

This artistic response to Deborah Hay's invitation to develop a digital adaptation is linked to the sequence of the written score to the left.

Fig. 124 - Using The Sky: Amin Weber's artistic 3D animated representation of the score No Time To Fly (lbid.)
The score No Time To Fly was also interpreted into a more artistic piece by designer Amin Weber. His digital adaptation shows the movements of the dancer as an abstract body in a 3D animation (see fig. 124). The designer reproduced the dance by setting parameters based on key movements and gestures, which were then animated computationally (Motion Bank, 2013c).

Another project of Motion Bank is named Two, and is a collaboration between the Motion Bank team and two independent choreographers: Belgian/ Czech Thomas Hauert and American Bebe Miller. Fig. 125 shows the motion capture data of the performers. The motion capture data can be viewed as independent animations, but the purpose of this data is for documentation. 


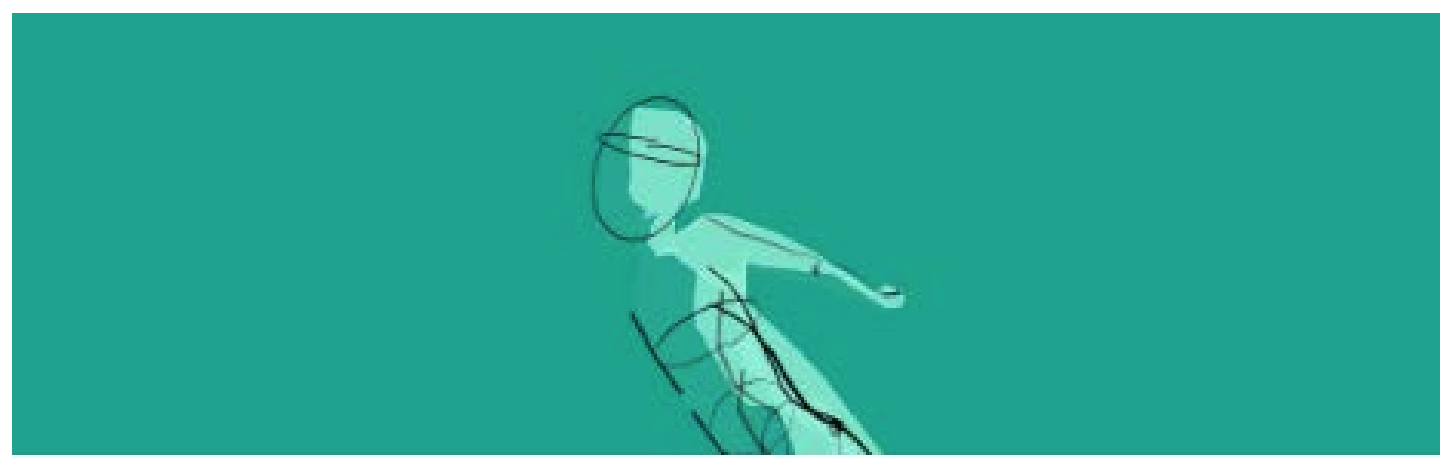

\section{ASSISTED, SOLO PRESSURE (MOTION CAPTURE)}

In this motion capture data, you are able to see the soloist alone and then watch the results of the input from assistants on the soloist's actions.

Assisted Solos are a creative tool and training exercise Zoo Company uses where partners or assistants determine the chain of places where motion can happen in the actions of a soloist. Dancer: Sara Ludi
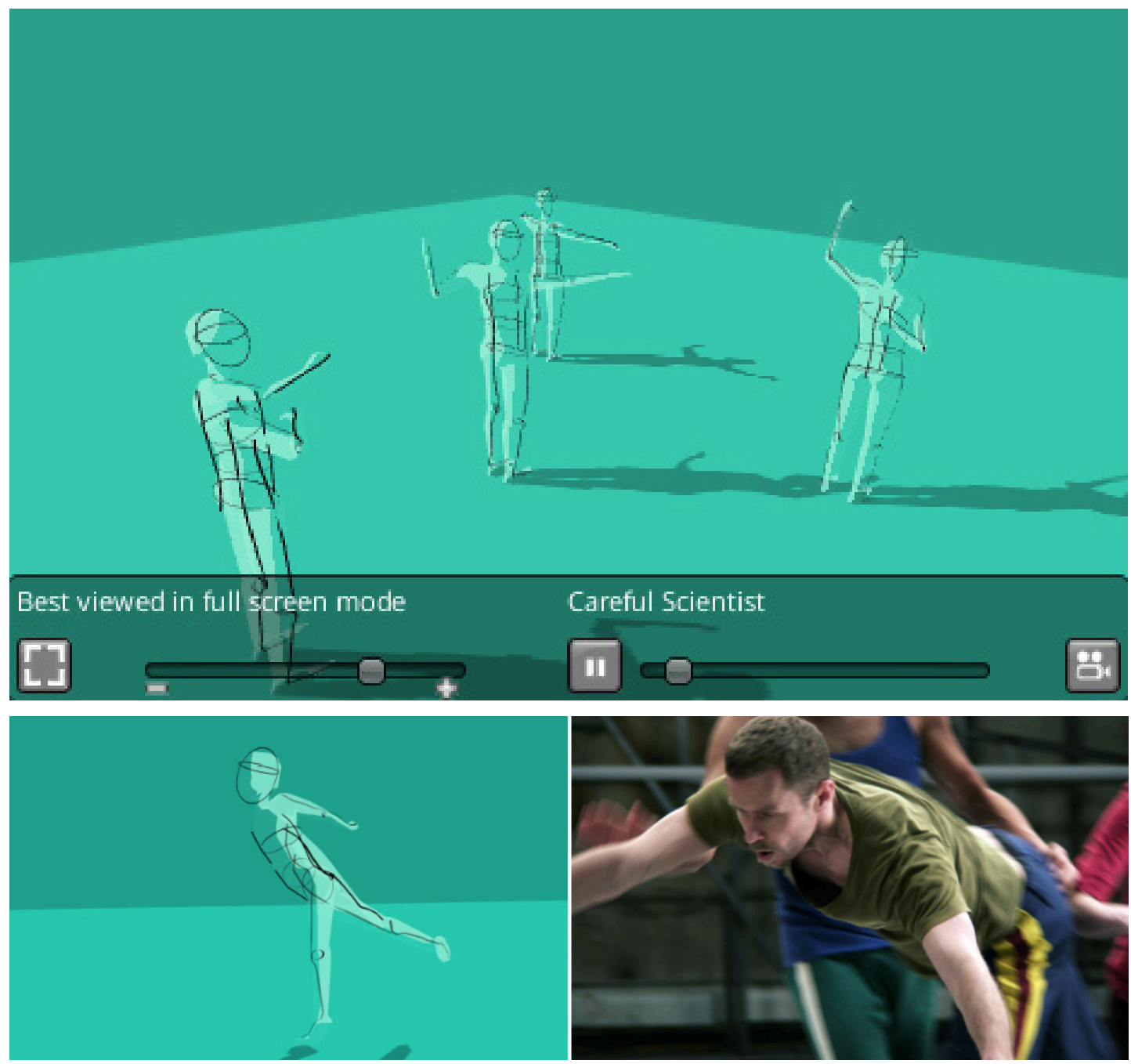

Fig. 125 - Two: Impulse - motion capture of assisted solos (Motion Bank, 2013b) 
Two focuses on four separate categories: habits, tendencies, impulses and memories in action. Some visually pleasing and interactive score pieces have been created as part of the investigation, in particular for Impulse (Motion Bank, 2013b). Two: Impulse focuses on a dance technique called assisted solos, which Thomas Hauert and his dance company have used frequently. In assisted solos, dance assistants or partners give impulses to the soloist, such as light touches to his or her joints, which then 'trigger' what part of the body the soloist should move or modify next (Motion Bank, 2013b).

The scores of assisted solos have been visualised dynamically using coloured 'blobs' that have seven contact points represented by grey circles (see fig. 126). The animations give an overhead view of the movement

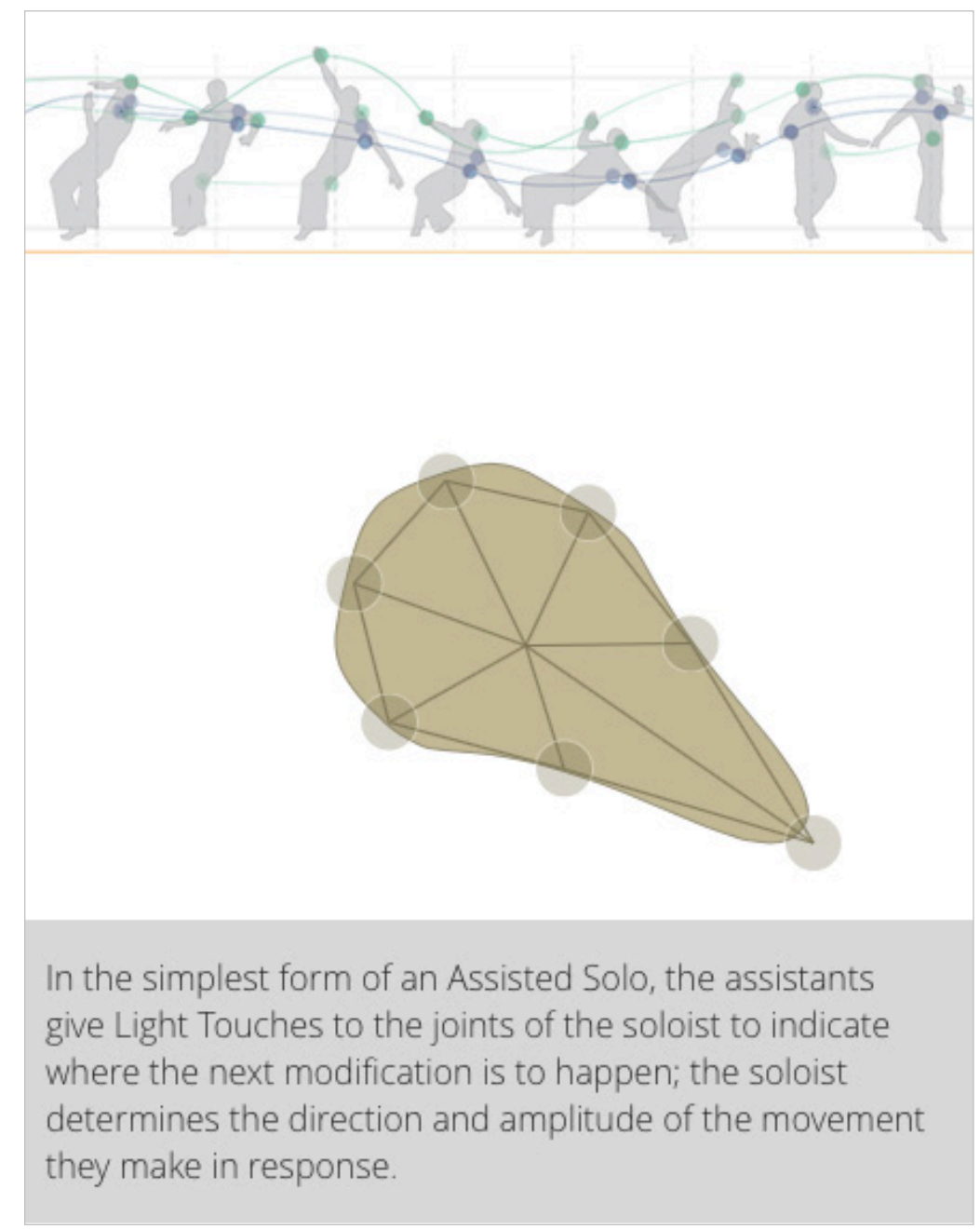

Fig. 126 - Two: Impulse - Attentive Agent, Light Touches (Ibid.) 
of the dancers. The blobs, which are abstracted shapes of the dancers, are coloured differently to show clearly how the touches of the assistants influence the movement of the dancer. The blobs change shape according to the movements of the dancers. The animations are fluid and aesthetically pleasing. Three different versions of the animations illustrate different aspects of the relations between the dancers and the assisted solo technique. The Attentive Agent, Light Touches version (see fig. 126) features one blob the user can interact with: its shape can be changed by clicking on and dragging the grey circles, or pulling the lines.

In a different version, Attentive Agent, Pressure (see figs. 127-128), the viewer can either watch the performance represented as an animation in which three blobs interact - based on the movement of the three dancers (left side of fig. 127), or the viewer can influence the way the blobs interact by pulling and clicking on the circles or lines. The third version, Attentive Agent, Mixed Roles, enables the viewer to click on the animation to add as many blobs as they wish, and then interact with them by pulling the shapes around (see fig. 129). This version makes it possible to view the visualisation of more complex relationships between as many dancers as the viewer chooses.
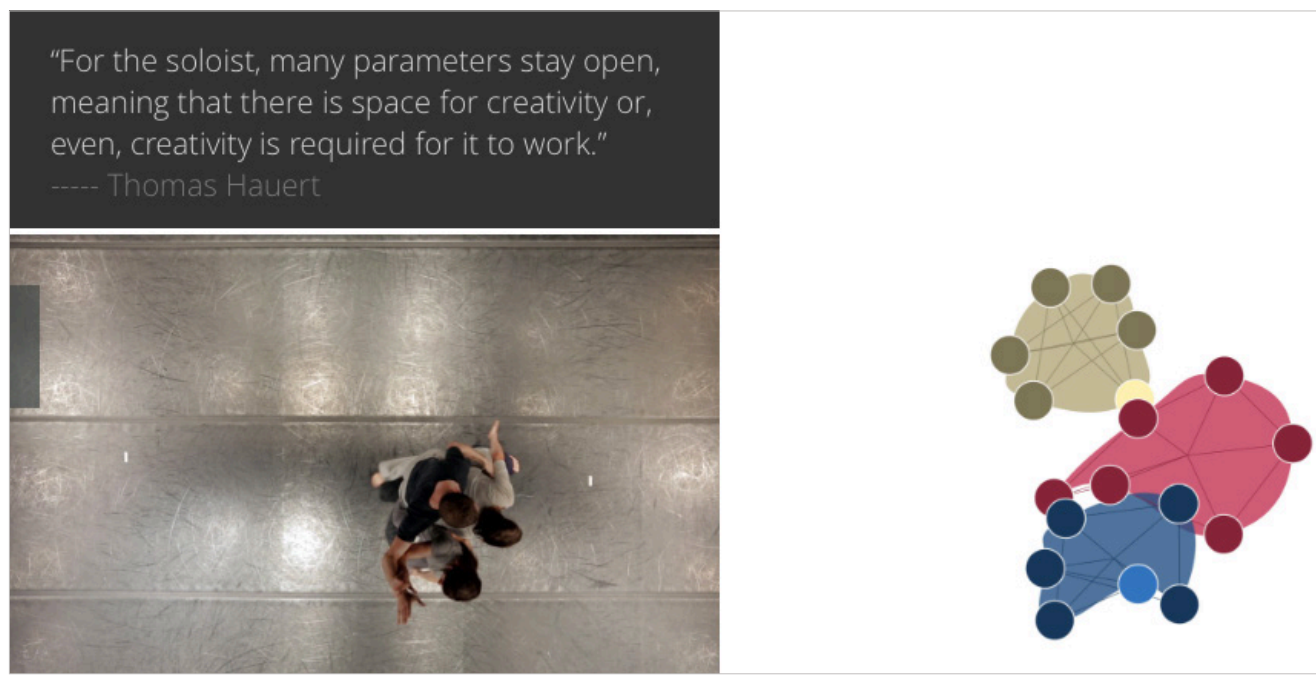

Fig. 127 - Two: Impulse - Attentive Agent, Pressure (1) (lbid.) 

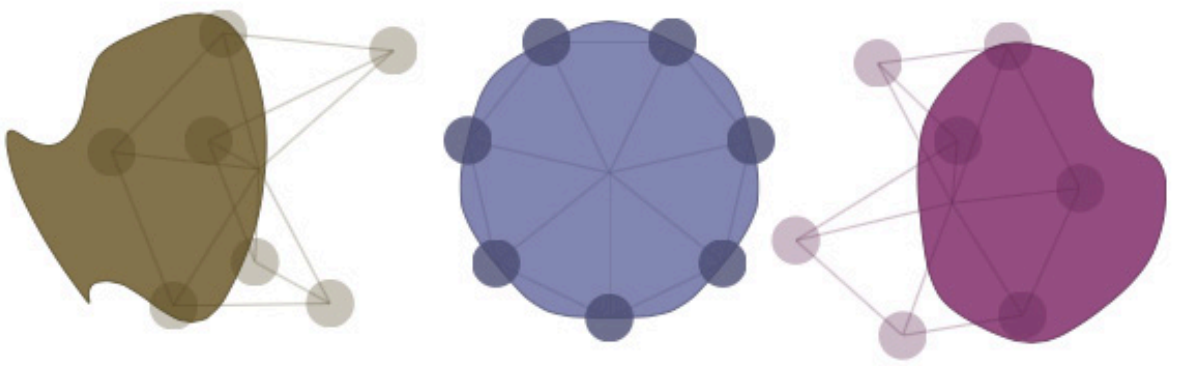

Fig. 128 - Two: Impulse - Attentive Agent, Pressure (2) (Ibid.)

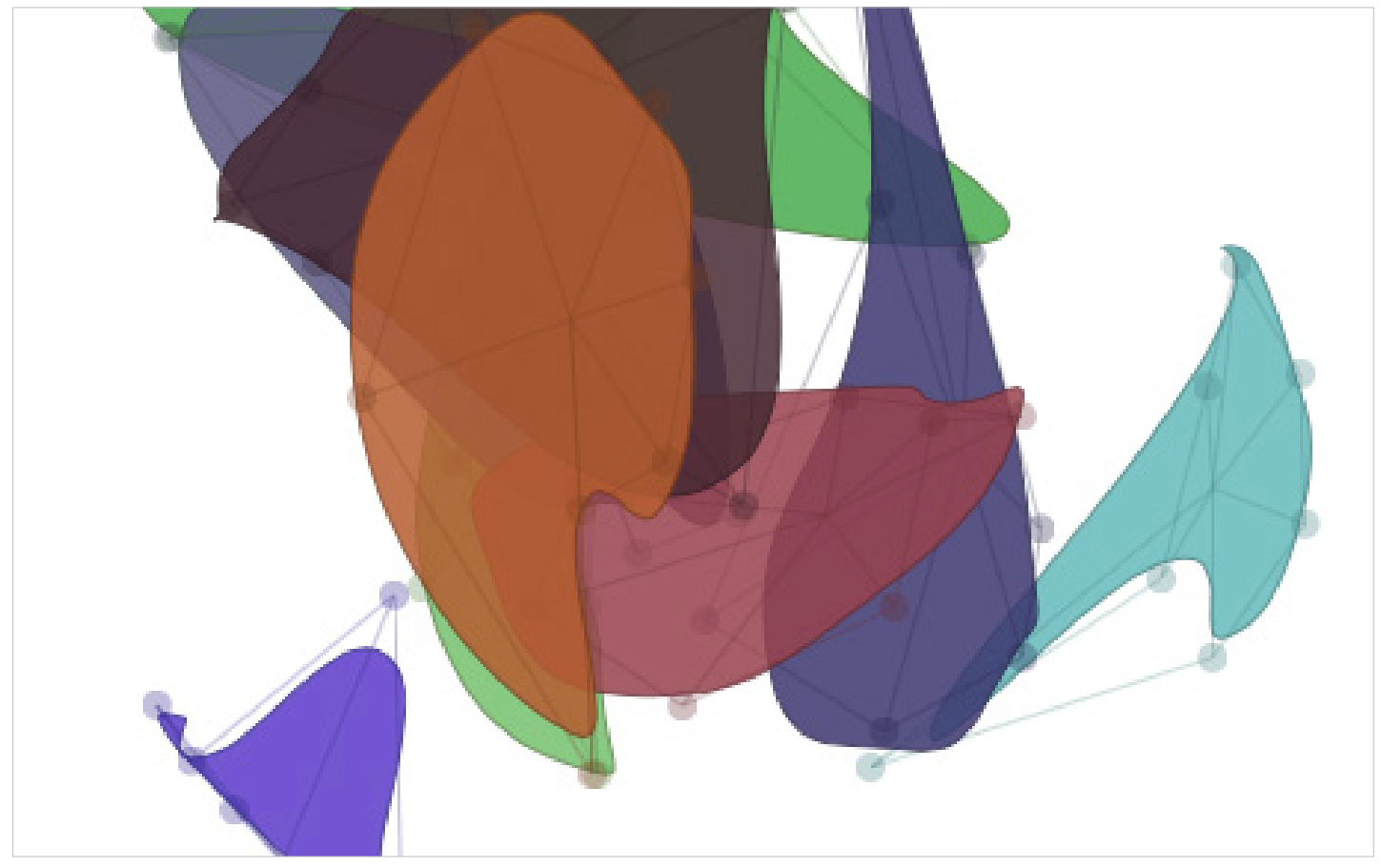

Fig. 129 - Two: Impulse - Mixed Roles (Ibid.) 
The different versions of the Impulses exploration illustrate complex relationships between human interaction and movement. The animations are not static representations of the movement, but sophisticated motion graphics that can be explored like an interactive game.

Common to all the projects of Motion Bank is the collaboration between programmers, designers, choreographers and dancers, and the use of sophisticated technologies and techniques for recording and visualising movement. The examples mentioned here are just a few of many explorations that make up the complete Motion Bank project. I have only presented the projects that are particularly relevant to my own interests in terms of their aesthetic and technical/interactive outcomes, and which could potentially inspire further development of my system for visualising greetings. While dance notation systems from the early to mid-1900s such as Labanotation and Benesh Movement Notation preserved dance scores in a static graphic form, the Motion Bank projects show some of the huge influence and potential that technology can have on the documentation and preservation of dance scores. The project engages with the digital age in which we live, and with how information is shared today - via the Internet and all the scores have been made available online. While the Motion Bank projects that I have presented use more abstracted forms of visualisations than I use in my own system, their use and combination of technology, programming and equipment is very innovative (and could inspire future developments of my own system).

In this chapter I have presented the visual context for my study and system. I have given an overview of visualisation techniques that have changed how we study, analyse and communicate about human movement and interaction. From chronophotography and dance notation to contemporary information/data visualisation, interactive technologies and motion tracking, the potential of visual language and digital media for use in anthropology 
and greetings studies is extensive. Digital media enable interactivity and the visualisation of multiple layers of information in a way that was not possible in the past when traditional notation systems were created. The visualisation of posture, movement, gesture and cultural difference can now be transformed into interactive multi-layered interfaces communicating further dynamics of movement and cultural information - which is what my research project seeks to do. I utilise some of these multi-layered approaches in the presentation of my system for the visual notation of greetings. In chapter 4 I describe and illustrate the process of creating that system. 


\section{Visually Representing Layers of Nonverbal Information in Greetings: Developing a New System of Visual Notation}

In my first three chapters I have discussed: the multicultural context in which my study takes place; the function of the greeting, and visual notation systems used and developed within anthropology; and a range of existing and potential techniques and systems for visualising movement. In this chapter I describe in detail the process of developing my notation system for greetings. The system is developed with the aim of creating understanding of everyday multiculturalism in terms of greetings. While existing books such as travel guides (see fig. 130) approach this issue pragmatically in their use of visual language to illustrate cultural meaning, my practice moves beyond simply creating instructional graphics. It concerns devising a systematic method for documenting, analysing, and presenting greeting interactions and is premised on Duranti's theory that greetings involve important information exchange. My focus is on the nonverbal dimensions of such exchanges (Duranti, 1992, 1997b). In particular I investigate the dimensions of personal space, proximity and touch in greeting interactions, and I have devised ways not only to visualise the physical patterns of greeting but also to indicate the subjective experience or interpretation of the encounter on the part of those
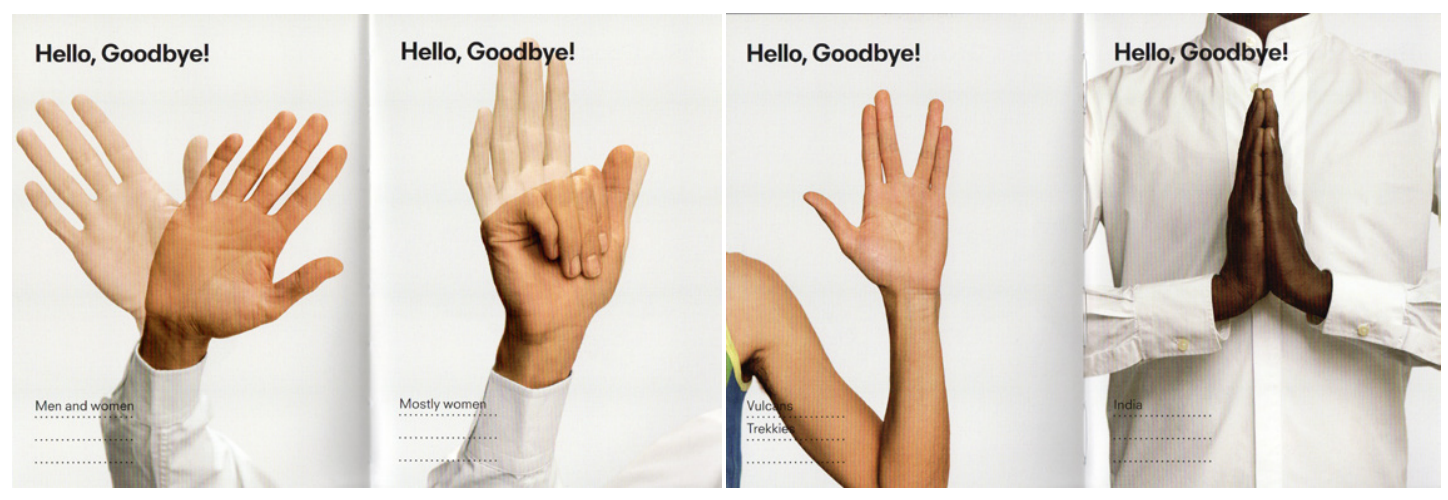

Fig. 130 - Images from the Global Gesture Guide showing cultural differences in gestures meaning 'hello' and 'goodbye' in different countries (Grosse, Reker, 2010) 
greeting. To achieve this, I have employed a range of methods, many of which draw on or develop the principles and techniques discussed in chapter 3.

The purpose of my visualisation process was to create a format that could be used both to document and to analyse a greeting, and that could also work within a method of presentation that would enable a large number of greetings to be viewed and compared. The first aim was to find a way to document greetings clearly in an accessible way. Notation systems such as those discussed in chapters 2 and 3 are complex to read and cannot be understood at all without specialist knowledge. However the medium of film/ video makes it possible to record greetings so that their documentation can take an animated and easily readable form. I decided not merely to record each greeting, but to abstract the central movements of the encounter (in way reminiscent of Muybridge and Marey), using digital technology to transform the live recordings into a schematic but still accurate document of the greeting in real time. This process is described in detail in section 4.2 below.

A certain level of abstraction is needed to facilitate comparison: I needed to document the greetings in a systematised way that would make similarities and differences in greetings between different cultures immediately apparent. Such comparisons are not easy to make with other notation systems, where you cannot directly 'see' the greeting. My goal was to devise a graphic form that is still a record of the greeting, at the same time as being abstracted enough to make points of comparison more noticeable. The format I chose was vector ${ }^{1}$ graphics, both for aesthetic reasons - to achieve a clean,

\footnotetext{
1 Vector graphics are made up of paths that are calculated by mathematical formulas. The paths are made up of points, and vector graphics can be rescaled endlessly without compromising or losing quality. Vector graphics are commonly used to create 2D graphics. Raster images, on the other hand, are constructed by a fixed number of pixels - or 'individual building blocks' - and become pixelated (lose quality) when scaled up. JPEG, GIF and PNG are typical raster image formats (MODassic Marketing, 2015).
} 
contemporary look - and for the flexibility this format offers for screen and print graphics, compared to that of raster images. Vector graphics are flexible in terms of allowing resizing and recolouring, and can be animated in Adobe Flash (as I will discuss in section 4.2).

The second aim was to reveal key information about nonverbal aspects of the greeting in an accurate and accessible way. I was most interested in the use of space and touch - how people inhabit personal space, how they share space, and how or whether they make physical contact. I devised colour coding techniques to make these aspects of greetings immediately apparent in my documentation, and therefore available for comparison and analysis. I discuss the use of colour and other forms of coding in more detail in section 4.3 below. One reason for using a schematised, graphic form of documentation, as outlined above, is that the addition of codes for a range of observable aspects of greetings is facilitated by this visual technique. In the present study I have chosen to focus, as mentioned, on codes for people's personal space, space between greeters, and touch, but others could hypothetically be added.

While these nonverbal elements of a greeting can be perceived externally, by careful observation of the greeting and/or its record, the aspect of greeting that seemed to me most relevant to the issues arising in cross-cultural encounters was the meaning of the nonverbal elements of greeting, as perceived by the participants themselves. This is clearly not a dimension that is externally observable, and researching it involves careful interviewing and discussion with the individuals greeting. This is a dimension of my research that I believe adds a new level to any potential analysis or comparison of greetings. The most significant aspect of a nonverbal exchange between people is what it means to each of them. Meanings are, of course, subjective, but they are also culturally coded. So when people from different cultures greet, their differently patterned nonverbal behaviour, and their systems of meaning of this behaviour, are likely to create mis-communication. In 
attempting to visualise, and therefore communicate, how different greeting encounters are experienced by different people, I hope to contribute to understanding and communication between individuals and cultures. Visualising this dimension of a greeting has been a central part of my project.

The final key aim of my practical research was to create and devise an interactive platform in which to present the system. It was important that the navigation of this interactive platform, or interface, would allow for comparison of the visual material - for example, to make comparisons between genders and between cultures. This meant I needed to design an interface that would allow users to view a range of material simultaneously in the form of still diagrams - to be able to make such comparisons at a glance - while making each diagram an entry point for a more detailed view and analysis of each individual greeting. It was essential that the interface design would respond to selections made by users, and give them some control of what information is presented, so it could cater to specific interests. Such a focus on the user experience is a common feature in interactive interface design, as seen in Rosling's bubble charts and McCandless' interactive diagrams, discussed in chapter 3. Other systems of graphic notation, discussed in chapter 2, were developed so that researchers could carry out anthropological research, and were not focused on presentation of the analysis. My system, on the other hand, has been developed to be presented as an interactive interface which is accessible both to specialised researchers and to the general public.

The context for this research in terms of my own practice is my background as a graphic designer. My initial explorations in applying principles of visual and information design to the documentation and analysis of greetings took place in my 2007 MA project Kinesics - Creative Intercultural Learning. This study involved nine different cultures in Eindhoven, Netherlands, where I was living at the time. The main purpose of the graphic system I devised for the project was to enable the comparison of greetings across different 
gender combinations - comparisons within, and between those cultures. I documented three broad greeting situations: informal greetings, formal greetings and greeting the elderly. I filmed the greetings from two different angles: front view, and an overhead view, and I then converted stills from the video material into vector graphics (see figs. 131-132).
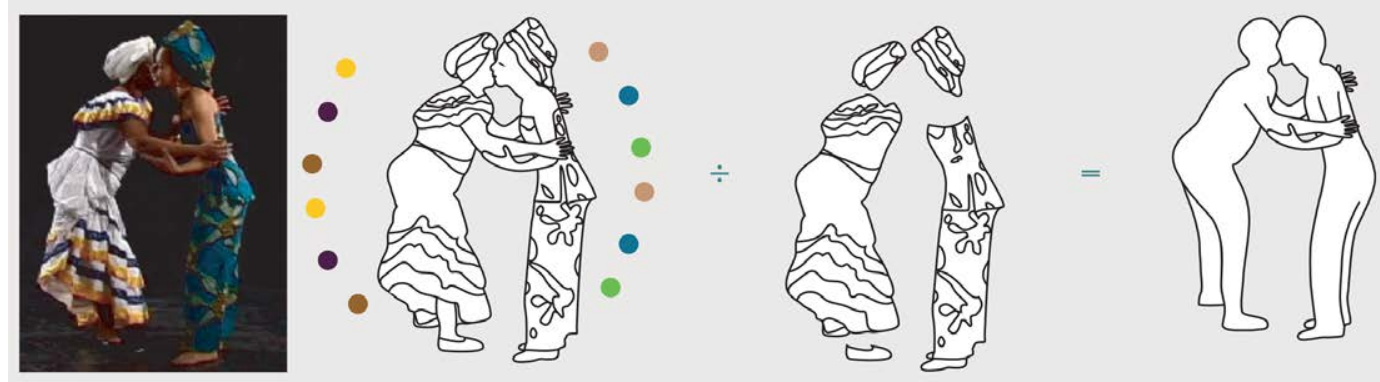

Fig. 131 - Kinesics visuals: From video still to vector graphic
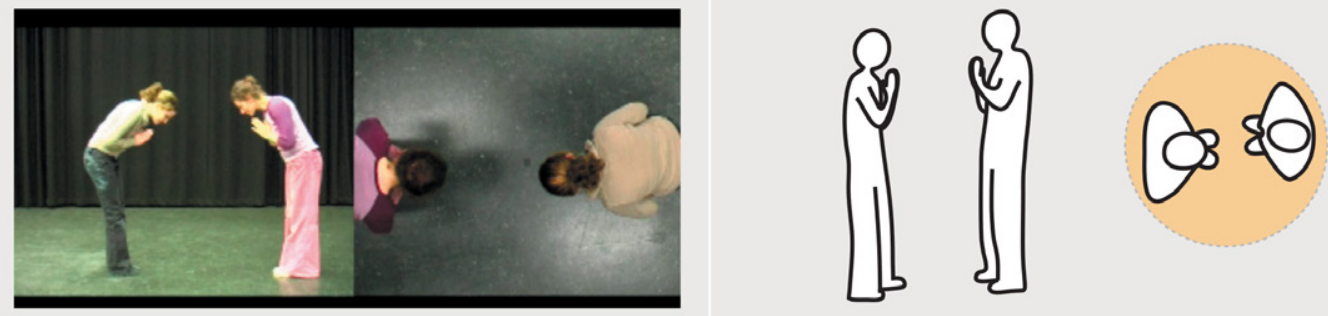

Fig. 132 - Kinesics visuals: video angles used, and a graphic visualisation of the video stills

The key focus of the visualisations was to highlight the use of personal space. So I devised a way of indicating four different 'intimate' zones, using circles that were scaled and colour coded, to visualise personal space. I then created graphic diagrams using the still vector images of the greetings and added the notation of the coded circle to each one (see fig. 133). When these annotated diagrams were presented together as charts they enabled visual comparisons of the greetings and use of personal space across cultures and gender (see fig. 134). 


\section{$200 \cdot$ \\ BLUE ZONE: One arm's distance or more. Little or no physical touch involved. \\ GREEN ZONE: Half an arm's distance or more. Some physical contact is likely. \\ ORANGE ZONE: $15-25 \mathrm{~cm}$ distance. Physical touch is usual. \\ RED ZONE: No distance. Much physical contact.}

Fig. 133 - Kinesics visuals: colour coded intimate zones and their measurements

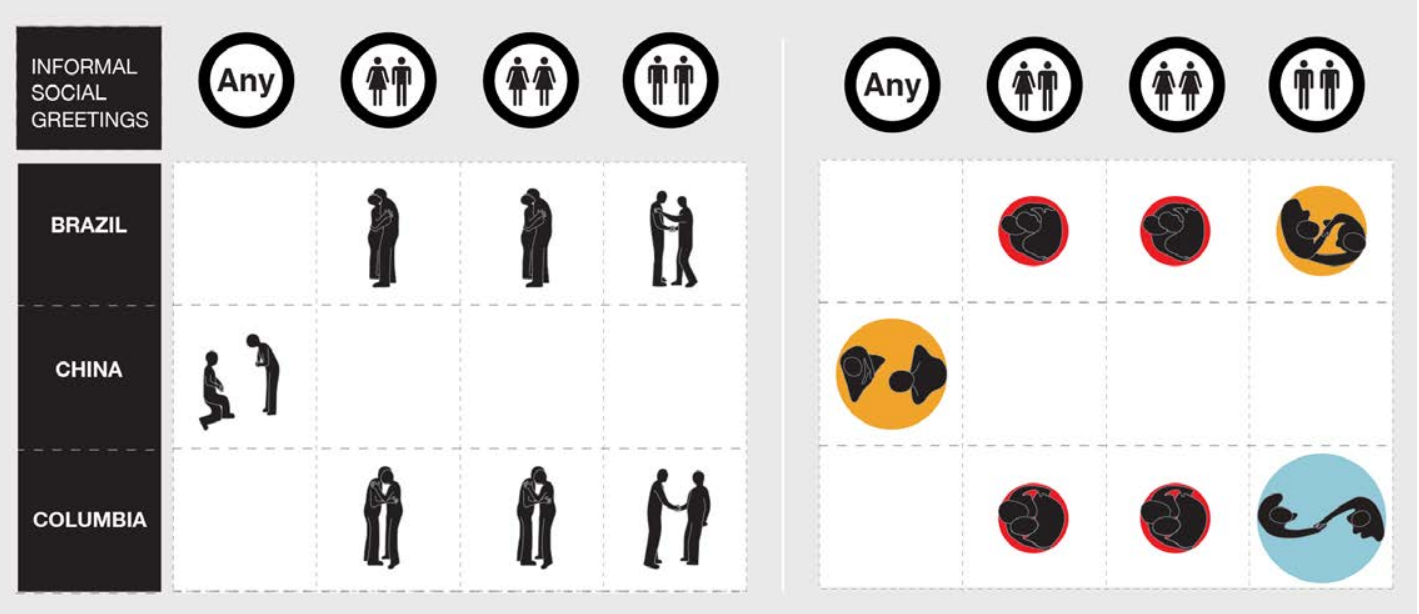

Fig. 134 - Kinesics visuals: graphic greetings diagrams for comparisons across genders/cultures

Kinesics represented the first steps towards my visual system in the form presented here; however, it was far more limited in terms of its visual design, the aspects of greeting it portrayed, and the categories of greeting interactions that were studied. (Further illustrations from Kinesics, and a summary of its process, can be found in Appendix 1.)

In the following sections I describe in detail the practical, technical and creative process of how I have developed on from the earlier project. I will start by explaining the fieldwork undertaken, including the technical equipment and set up of the greeting documentations, then move on to describe technical and visual explorations done with the recorded material - from video documentation to annotated animations - and conclude by 
explaining the interface and navigation of the interactive diagrams. The final section of this chapter summarises other experiments I have done as part of my PhD practice, including video and motion tracking installations. These projects are separate from the greetings diagrams, but are attempts at translating the system and colour codes into performative physical spaces.

\subsection{Gathering New Documentation/Data}

In my Kinesics project, all the greetings I recorded took place within cultures. While this type of intracultural encounter is still included and documented in my current project, a key development has been also to document intercultural greetings to show the meeting of different communication patterns. As argued in chapter 1, this is important when dealing with greetings in a multicultural city - where people meet and greet across cultures on a daily basis.

In order to expand and refine the categories of greeting situations to be researched, I needed to gather new documentation through conducting fieldwork in a multicultural society. I decided to carry out my fieldwork in Stavanger, Norway, because of its growing diversity, and because of my personal connection to the city (discussed in chapter 1). To make sure that the documentation would represent the diversity of the cultural patterns that co-exist in the city, I decided to include five cultures that were wide ranging both in terms of geographical origin, and also in terms of religion. As I intended to include both intercultural and intracultural greetings, I wanted to study cultures that were likely to display different behaviour patterns. So in order to choose which specific cultures to include, I studied the Stavanger statistics and selected some of the largest cultural groups from different parts of the world (numbers indicate each country's total number of inhabitants in Stavanger in 2012 (Stavanger-statistikken, 2014)):

- An African country: Somalia (806)

- An Asian country: Vietnam (534) 
- A South-American country: Chile (252)

- A Eurasian country: Turkey (1210)

- The 'host'/European country: Norway (103137)

I would need a minimum of two male and two female adult participants from each of the above cultures, aged 20 and over, to be able to make crosscultural comparisons of gender differences, with all the gender combinations. I had managed to find some Vietnamese and Norwegian participants, and one Turkish participant, through email ahead of arriving in Norway to carry out my fieldwork. Upon arriving in Norway I had a timeframe of 5-6 weeks to gather participants, and to plan, structure and complete the video documentation. Gathering participants involved a rigorous selection process. While I did not manage to find as many participants per culture as I had hoped for, the number of participants I did find enabled me to gather enough documentation to substantially develop my research and my system of notation.

In Norway, my first and main approach to making contact with potential participants was to meet with Internasjonalt Hus² - the largest organisation in Stavanger that works on promoting cultural diversity in the area. Internasjonalt Hus had formerly received government funding, but since losing this funding it has become an independent, servicebased organisation. So at Internationalt Hus they put me in contact with Internasjonalt Kulturnettverk ${ }^{3}$ instead, an organisation/network working on projects aimed at developing Stavanger into an inclusive city for everyone across cultural, ethnic, generational and social divisions. I met with the managers of Internasjonalt Kulturnettverk, who were very positive towards my project: they provided me with a list of contacts for all the cultural/ethnic organisations and groups in Stavanger, and advised me who to contact. They also suggested I use a central location in the city centre of Stavanger

2 Internasjonalt Hus website: http://www.inthus.no

3 Internasjonalt Kulturnettverk Facebook Page: https://www.facebook.com/ internasjonaltkulturnetterk 
to do the recordings of greetings, one that would be easily accessible for all participants, and offered me a good deal for renting a space in the cultural house, Sølvberget ${ }^{4}$, where I could set up a recording studio.

In the search for participants, I started the process of contacting groups and organisations from the list given to me by Internasjonalt Kulturnettverk (see the Call for Participation in Appendix 2). Before arriving in Norway I had, as mentioned, received a quick reply to my email to the leader of Vietnamesisk Flyktningforening i Stavanger, the organisation for Vietnamese immigrants, saying she would provide me with five Vietnamese participants. She invited me to join four of them for breakfast on a Sunday morning so that I could get to know them and learn more about their culture, because, they said, greetings are deeply rooted in their culture and would need further explanation. I had a very pleasant meeting with these Vietnamese participants, who were very welcoming, and aged between 46-82. They explained that they had selected older people from their community to participate, all first generation immigrants, because they claimed that they were the most knowledgeable about the Vietnamese culture. They said that the younger generations, born in Norway, tend to be less interested in preserving their cultural backgrounds than they were. (See Appendix 3 for transcripts of our conversations during the breakfast meeting.)

While the Vietnamese person I contacted responded promptly to my enquiry, the process of gathering participants from the remaining cultures was more time-consuming and involved a variety of different approaches. I contacted a number of organisations, including: a Turkish women's organisation - Tyrkisk kvinneforening i Stavanger; a Turkish mosque - Mevlana moské; a Somali women's organisation - Somalisk kvinneforening i Stavanger ${ }^{5}$; Rogaland

\footnotetext{
4 Website for Sølvberget: http://www.stavanger-kulturhus.no

5 Somalisk Kvinneforening i Stavanger website: http://www.fokuskvinner.no/en/ About-FOKUS/Member-organizations/Somali-Womens-Association/
} 
Muslim Society6; two Chilean organisations - Rayuela Chile Amigos and the Knadela Salsa Club; another mosque - Eyup Sultan Moské7; an Islamic women's organisation - Islamsk Kvinneforening; ; and the Islamic education centre - Islamsk Opplæringssenter i Rogaland. I also contacted IRIS International Research Institute of Stavanger ${ }^{9}$ to ask for advice on where and how to get participants for my study, because they had conducted a variety of research projects about immigrants and multicultural Stavanger. They suggested contacting mosques, churches and immigrant organisations, as they had successfully done so in the past, but they warned me that it was likely to be a very lengthy task.

I found one Chilean participant through my personal network; he offered to participate, and tried, without luck, to find more participants among his own friends. Because Chile is a Catholic country, I attended mass in the Catholic Church on a Sunday in the hope of meeting more Chilean people, again without any luck. I then visited Johannes Læringssenter ${ }^{10}$ in Stavanger - a centre where immigrants and refugees attend Norwegian language courses as part of induction programmes for immigrants. There the staff were highly enthusiastic about the project and suggested I do all the documentation there at their centre. However, as I had already planned a date and location with other participants, and booked the space, I asked if they could kindly spread the word that I needed participants. This brought me one more female participant from Chile. She had other friends who wanted to attend, but the date and time was not suitable for them, so I only got one male and one female participant from Chile. The female participant brought along her Chilean/Norwegian daughter and Norwegian husband, which allowed for

6 Rogaland Muslim Society website: http://www.moskestavanger.no

7 Eyup Sultan Moské website: http://www.eyupsultan.no

8 Islamsk Kvinneforening, Rogaland Facebook page: https://www.facebook.com/ pages/lslamsk-Kvinneforening-Rogaland/180029732020722

9 International Research Institute of Stavanger: http://www.iris.no/home

10 Johannes Læringssenter website: http://www.velkommentiljohannes.no/vtjportal/ index.html 
some un-planned documentation of cross-cultural family greetings: hence the family diagram which was intended for intracultural greetings, also includes Chilean/Norwegian greetings.

Finding participants from Turkey was an even slower process. I had not received any response from Mevlana Moské, Rogaland Muslim Society or Islamsk opplæringssenter i Rogaland so I contacted a Turkish acquaintance, who had already enthusiastically agreed to participate, through Facebook. He posted my event on a Facebook group he was a member of called Turkish Stavanger, a group which had 29 members at the time. His posting on the group brought me one more male participant. No females had responded to his post, so I had to find alternative solutions, and contacted organisations for women, such as the Islamsk Kvinneforening (Islamic women's organisation) and Tyrkisk Kvinneforening i Rogaland (Turkish women's organisation of Rogaland). A Turkish woman from Islamsk Kvinneforening put me in contact with a young Turkish woman, born in Norway, who was happy to participate. She said she considered it her duty to represent the Turkish culture in Norway. She also brought along another female friend, and her nine-year-old daughter.

Getting in touch with people from Somalia was a challenge, as I did not have any connections in the Somali community. Again, a woman at Islamsk Kvinneforening put me in contact with a young Somali woman who was happy to participate and would gather some of her Somali friends to join too. Unfortunately, they could not make it on the day scheduled to record the greetings, so we had to arrange a separate day to film the Somali greetings.

For finding Norwegian participants I had used my own network in Stavanger. Out of the Norwegian participants, three of them, two females and one male, were friends of mine, and the other male was a friend of a friend. They were all from different cities and towns in Norway: two were from the Stavanger area, one from the West of Norway, and one from further North, Trondheim. 
It was crucial to speak with all my participants prior to the filming, to discuss their thoughts and perceptions about the greetings to be performed. Their comments played an important part in shaping the categories and definitions that I used. Due to a very limited timeframe, I only had the opportunity to meet in person with my Vietnamese participants ahead of the greeting documentation day. This meeting brought to my attention the issue of age and hierarchy. With the other groups of participants, I was able to have a number of phone conversations, which were valuable in the process of determining what greeting types to include in the study. One of the Turkish women, for instance, raised the issue of family greetings - for her, she said, the way she greets a male family member depends on the age of the other person, the closeness of their relationship, how religious he is, and on whether he is an uncle, a cousin, or a grandfather. So rather than categorising the family greeting simply as 'Family - male greeting' as I did for my Kinesics project, I had to define the categories more clearly.

Once all participants were confirmed, I finalised the logistics: I had booked a room in the library of Sølvberget to use as a studio (from here on referred to as the recording studio), and scheduled one hour of documentation per culture. I also invited participants who had time to stay longer to participate in the documentation of cross-cultural greetings. All participants were sent the schedule with a list of the types of greetings we would film, and a research information sheet, a week in advance (see Appendix 4 and Appendix 5).

\section{Ethical Consent \& Data Protection}

All participants signed an ethical consent form, which followed the UCA guidelines, and had been approved by the UCA Research Ethics Committee (see Appendix 6 and Appendix 7). Because my fieldwork took place in Norway, I also consulted Datatilsynet ${ }^{11}$ - the Norwegian Data Protection Authority - to check if my project needed their evaluation for approval. They

11 Datatilsynet website: http://www.datatilsynet.no 
told me that my project would not need formal scrutiny from them, as long as I kept participants' original data (such as consent forms) as physical documents, not stored digitally, because there are strict laws in Norway about the digital storage of personal data. With this condition they advised me to register my project on their website, so I did. This registration needs to be updated every 3 years if I am to use the data after that period.

Table 1 gives an overview of all the participants who took part in my fieldwork, showing: nationality, gender, age (where given), and how long they have lived in Norway. I had originally also included information about

\begin{tabular}{|c|c|c|c|}
\hline Nationality & Gender & Age & Years in Norway \\
\hline \multirow[t]{3}{*}{ Chilean } & Male & $40+$ & 22 \\
\hline & Female & 32 & 7 \\
\hline & Female & 7 & 7 \\
\hline \multirow[t]{5}{*}{ Norwegian } & Male & 41 & - \\
\hline & Male & 34 & - \\
\hline & Male (Chile) & $35+$ & - \\
\hline & Female & 30 & - \\
\hline & Female & 29 & - \\
\hline \multirow[t]{5}{*}{ Somali } & Female & 23 & 23 \\
\hline & Female & 22 & 15 \\
\hline & Female & $20+$ & $20+$ \\
\hline & Male & $20+$ & - \\
\hline & Male & $20+$ & - \\
\hline \multirow[t]{5}{*}{ Turkish } & Male & $35+$ & 4 \\
\hline & Male & $35+$ & - \\
\hline & Female & 20 & 20 \\
\hline & Female & 29 & 29 \\
\hline & Female & $7+$ & $7+$ \\
\hline \multirow[t]{5}{*}{ Vietnamese } & Male & 82 & 21 \\
\hline & Male & 60 & Lives in Canada \\
\hline & Female & 46 & 21 \\
\hline & Female & 80 & 21 \\
\hline & Female & 65 & 30 \\
\hline Angolan & Female & 35 & 9 \\
\hline
\end{tabular}

Table 1 - Participants of fieldwork study 
participants' religious backgrounds (Catholic, Lutheran, Muslim, Buddhist and atheist) but I removed it after further consultation with Datatilsynet. They advised me not to include religion if it could make possible the matching of information in the table with people in the greeting videos, which could risk unintentionally revealing sensitive personal information about participants. Although I do discuss religion in my findings, when participants themselves refer to it in explaining greetings, I decided on the basis of Datatilsynet's advice to leave it off my data overview.

\section{Reactions to Studio Setting \& Performing Greetings}

On the day of the recording of greetings, most participants arrived prepared and had reflected on each of the greetings that we were going to record. A common statement was 'I had to think about how I greet' and 'I talked to my friends about it, and we discussed what are the most usual greetings'. This indicates that a greeting is so familiarly patterned in our behaviour that we do not think about how we greet, and confirms Duranti's and Firth \& Goffman's definition of greetings as being formulaic expressions (Duranti, 1997b).

For the scope of this fieldwork I was unable to gather enough participants with all the necessary categories of relationship to each other (i.e. people from each culture who were strangers, close friends, acquaintances, parents and grandparents). So for some greetings, participants had to enact different roles, and in such cases I recorded their enactment of the greeting. For example, the two Turkish males were friends in real life, but they also demonstrated how they would greet a male stranger, their father, and their grandfather. When performing the greeting between grandfather and grandson, one participant enacted the role of the grandfather, whereas the other showed how he greets his own grandfather. This staged situation brought about conversations about whether the greeting felt false, or if it was simply the setting in which it was performed that was false. All participants agreed that the actual greeting would remain the same: the act of hugging, kissing, handshake or curtsey was so set, or formulaic, that if the greetings 
were done in real life, they would look the same. However, they just did not feel the same because they were enacted. The fact that greetings were recorded in a studio setting, rather than in a real-life situation, also contributed to some of this feeling of participants experiencing the greetings as 'unnatural' or 'strange'.

While the recording of 'natural' and spontaneous greetings in real-life settings might have provided me with more 'real' documentation, the limitations of conducting such a study were too great, owing to the need to secure participants' ethical approval ahead of filming. So whether greetings were recorded in the street or in a studio, participants would have to be aware of, and have consented to, the recording before it happened. This meant that recording random or spontaneous greetings in real time was not an option. Further, I needed all the documentation to be recorded from the same distance, and from the same angles, in order to be able to make accurate comparisons of greetings, so a studio setting seemed the most appropriate, if not only, option. Also, when considering the formulaic nature of greetings, the studio setting seemed justifiable, in the sense that the set patternings of the greetings would not be compromised too much by this staged setting.

As expected, minor problems were experienced with the studio setting, such as participants being (overly) aware of the presence of the camera. During recording, the physical act of doing the greeting went very smoothly. However, in the moments just after the greeting finished, the participants' awareness of the camera and studio setting became apparent in their body language: they would start to look awkward; or start looking at the cameras, or at me for signs of when to stop their interaction. The video documentation also shows, in particular when the participants did not know each other, that many of them would start to move away from each other as soon as the formalised greeting was finished. While the studio setting brought on such reactions, they were also helpful indicators of when the actual greeting was over. On a number of occasions, the participants would focus their gaze 
towards the camera equipment rather than towards the person they were interacting with - in such cases I asked them to show the greeting again.

\section{Greeting Situations}

In deciding which greeting situations to include, I drew on understandings gained both from my theoretical research and from conversations with my fieldwork participants. The extension and refinement of greeting categories represented a significant development from my previous study. In Kinesics, I had categorised three greeting situations:

1) Formal (female/female (f/f), male/male $(\mathrm{m} / \mathrm{m})$, female/male $(\mathrm{f} / \mathrm{m}))$ - How to greet in a formal situation, for instance a business meeting;

2) Informal (f/f, $\mathrm{m} / \mathrm{m}, \mathrm{f} / \mathrm{m})$ - How to greet in an informal situation, when meeting friends or acquaintances in an informal setting;

3) Elderly (f/f, m/m, f/m) - How to greet elderly people (because great cultural differences exist in how one is expected to behave towards the older generation).

It was clear from my fieldwork that these categories were too broad and imprecise. There are, for example, vital differences, both within a culture and between cultures, in how a formal greeting is performed. These differences are determined by who is meeting in this formal situation, and what kind of formal situation it is. The same can be said about 'informal situations' and 'greeting the elderly' categories. These three categories are too vague, and the greeting the elderly category is not a situation but rather a relation. It should not be a stand-alone category, but rather a greeting within a category, for instance how one greets an elderly family member in a formal or informal situation. With all these issues considered, I chose to include the following types of greetings in my new study:

SOCIAL GREETINGS (on mutual ground)

Close Friends (f/f, m/m, f/m)

Acquaintances (f/f, $\mathrm{m} / \mathrm{m}, \mathrm{f} / \mathrm{m})$

Strangers (f/f, m/m, f/m) 


\section{FAMILY GREETINGS}

Mother ( $f / m$ - mother/son, $f / f-$ mother/daughter)

Father $(\mathrm{m} / \mathrm{m}$ - father/son, $\mathrm{m} / \mathrm{f}$ - father/daughter)

Grandmother (f/f - grandmother/granddaughter, $f / m$ - grandmother/ grandson)

Grandfather (f/m - grandfather/granddaughter, m/m - grandfather/ grandson)

\section{GREETINGS ACROSS CULTURES}

Strangers (f/f, $m / m, f / m)$

Close Friends (f/f, m/m, f/m)

These categories could of course be narrowed down to even more specific greeting situations: how one greets a close friend, for instance, could depend on how close a friend he or she is. However, some general parameters must be made in order to compare situations that are found across all cultures.

Figs. 135-137 illustrate the different types of greetings, gender combinations, and what will be visualised from each greeting - as I will discuss in more detail below. The coloured lines indicate the number of recordings and visualisations that were carried out per culture.

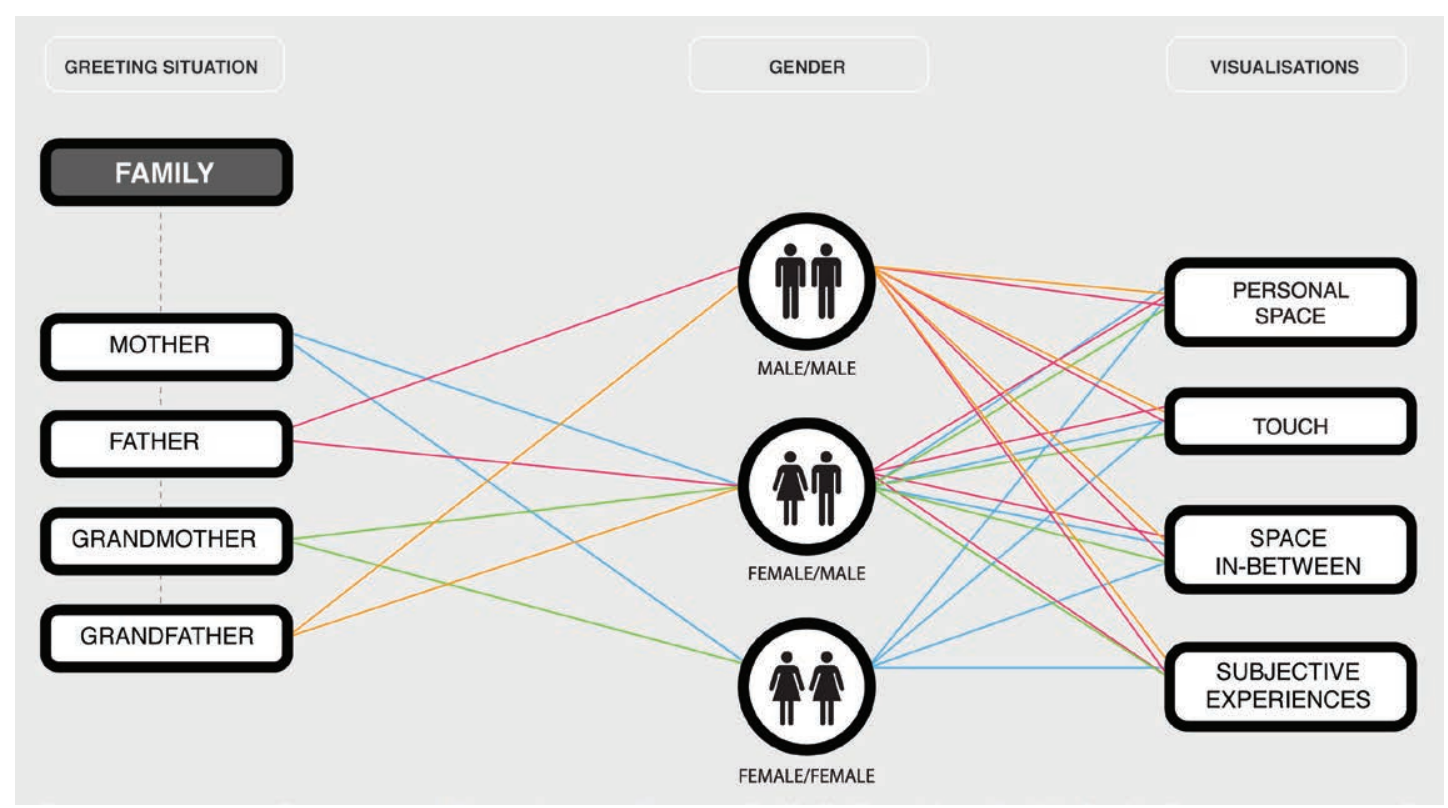

Fig. 135 - Visual overview of family greetings that were recorded and visualised 


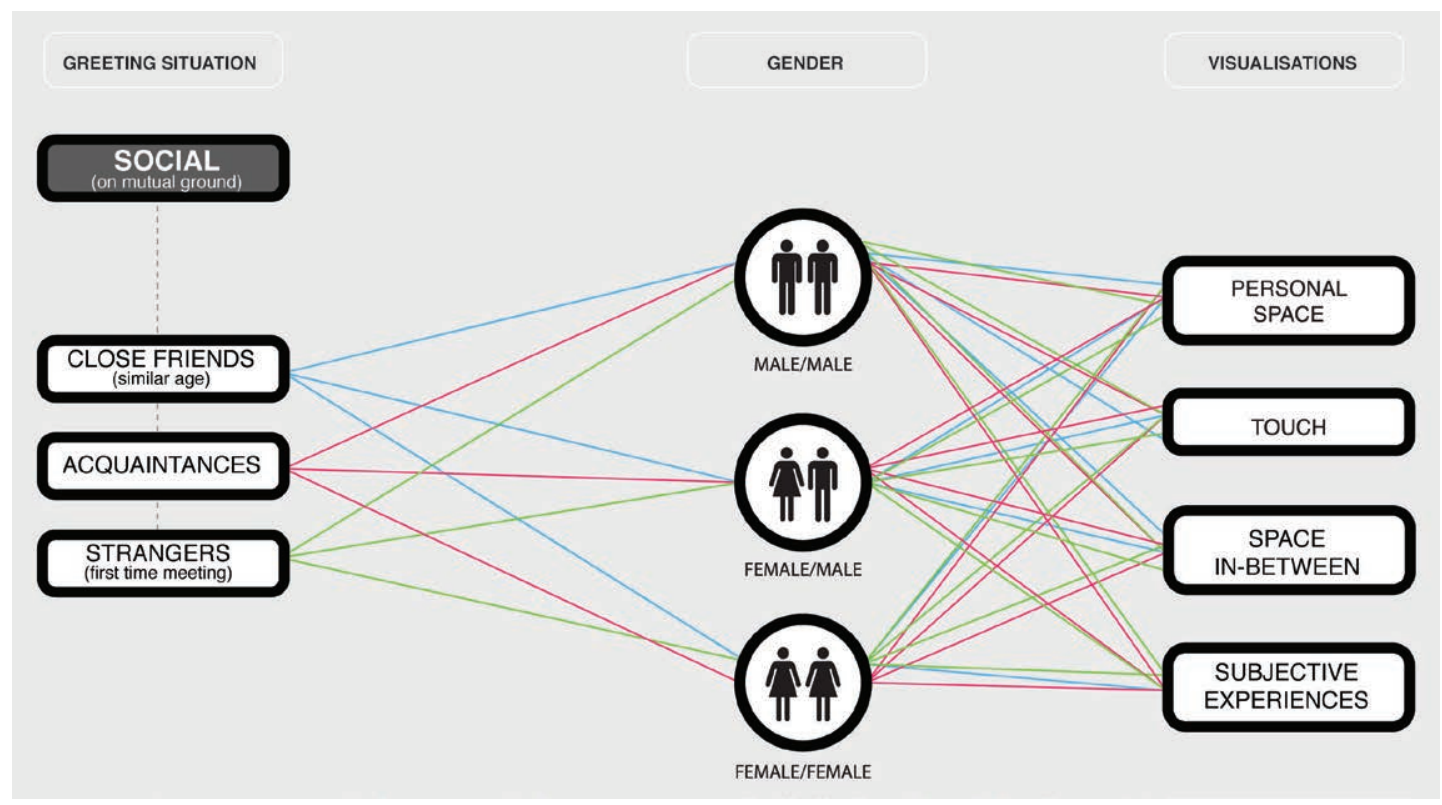

Fig. 136 - Visual overview of social greetings that were recorded and visualised

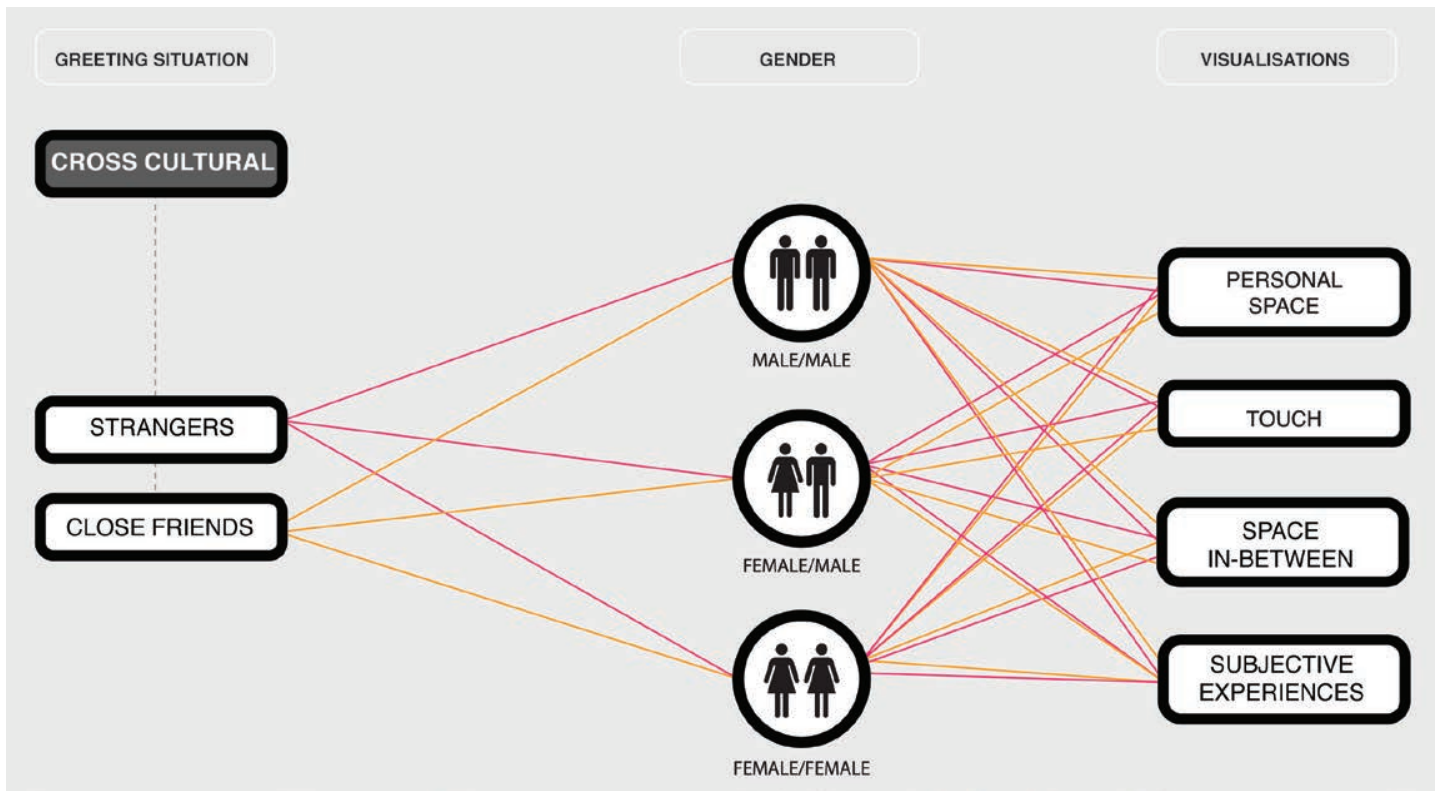

Fig. 137 - Visual overview of cross-cultural greetings that were recorded and visualised 


\section{Technical Set-Up \& Equipment}

In order to increase the amount of information documented for this research project, compared with my earlier one, I had to develop and extend the means of filming the greetings. I still considered the two perspectives used for the Kinesics project - front and overhead - to be the most essential ones. The front view captures the greeting, gesture, posture, touch and distance between the two people greeting, and the overhead view highlights the personal space/proxemics of the greeting (see fig. 138). I decided to keep these two perspectives, and add two more in order also to capture the facial expressions and gaze of the two people interacting. This would enable me to study whether eye contact and other facial expressions play important roles in the information exchange during the greetings.

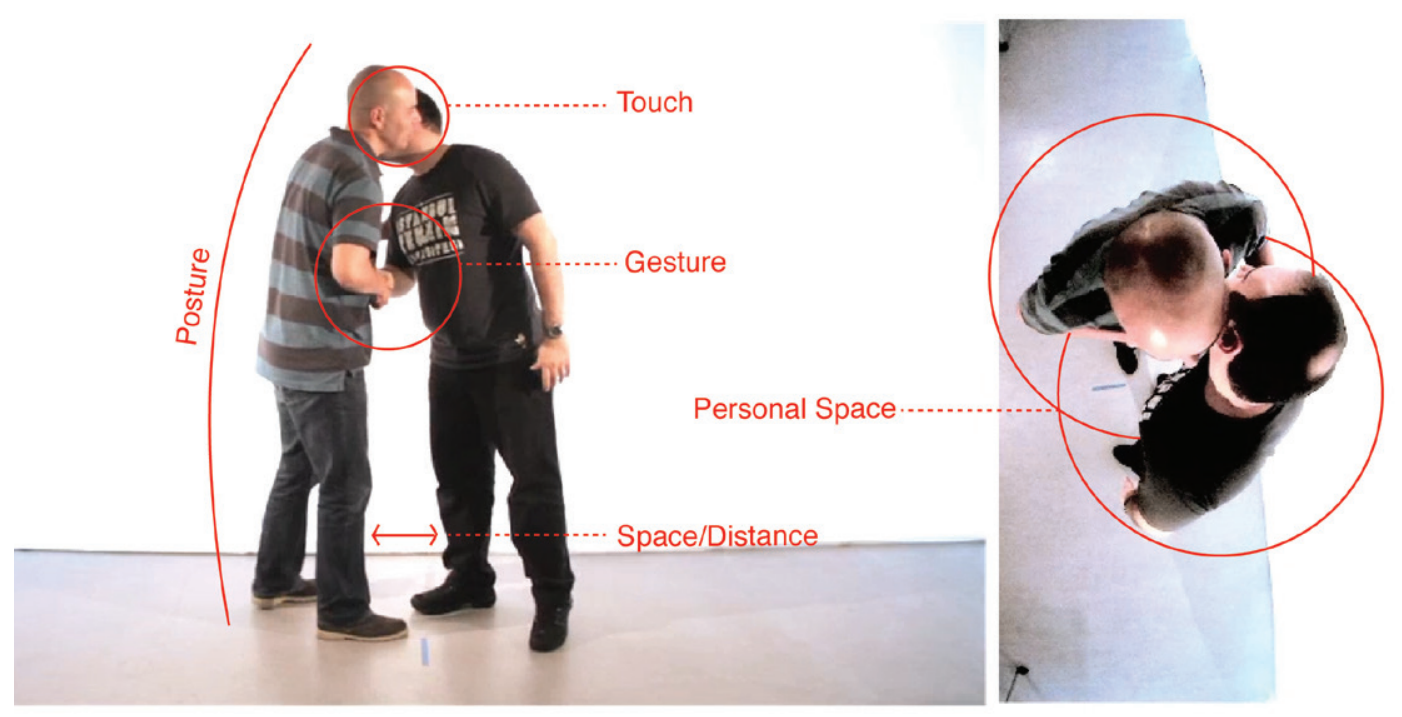

Fig. 138 - Greeting documentation: front and overhead perspectives

I consulted with a filmmaker and technician about the studio and equipment set-up, which resulted in the following layout (see fig. 139):

- A white backdrop behind the greeting area.

- Two studio lights - placed by each top corner of the backdrop 


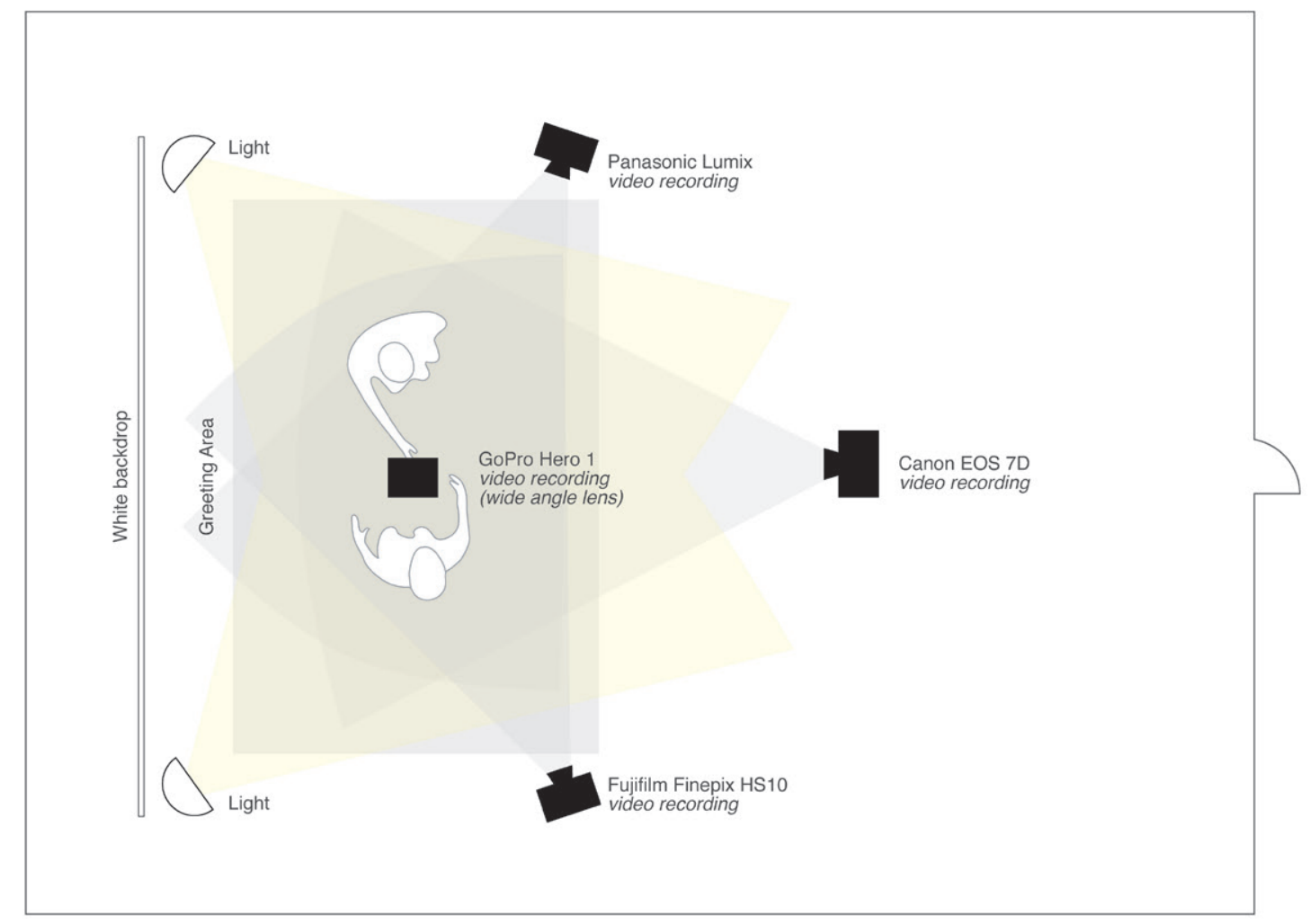

Fig. 139 - Studio and equipment set-up

- A GoPro camera attached to the ceiling - this type of camera was used because the room had a very low ceiling so it was the only equipment available to me that would make it possible to capture the entire floor area of the greeting space, owing to its wide-angled lens.

- A Canon EOS 7D camera placed on a tripod directly in front of the greeting area.

- Two digital cameras (Panasonic LUMIX and Fujifilm Finepix HS10) placed on either front corner of the greeting area, adjusted on a tripod to capture both people's faces and upper bodies during the greetings. These two cameras had to be adjusted according to the height of each couple greeting.

Fig. 140 shows the four perspectives that were recorded; all four cameras were used to capture video. I had the option of using time-lapse photography, but I chose to use video, which I would convert into image sequences in 

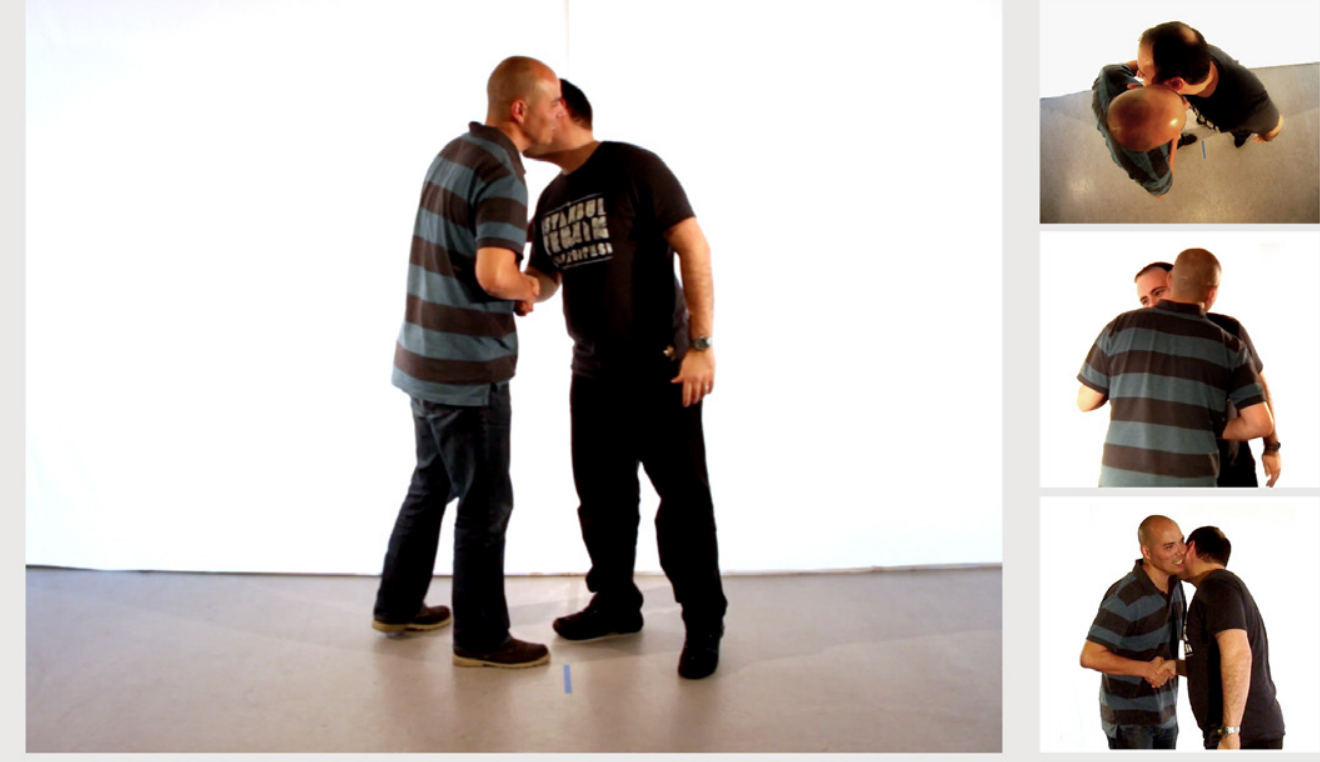

Fig. 140 - Greeting documentation: the four perspectives/angles that were filmed

post-production. By using the video function I was able to capture the audible/verbal aspects of the greetings as well as the visual. During video editing the audio proved very useful for syncing the four video files of the different greeting angles, using the sound graphs to align the different clips.

In the recording studio I also arranged a seating area so that participants could socialise, as I anticipated there would be some waiting between the greeting recordings. Soft drinks and refreshments were made available here and this became a social space where many participants stayed to socialise with other participants after their own session finished. These unplanned social gatherings brought up some interesting conversations about greetings and cultural differences that were recorded using the sound recorder on my iPhone 4s (see Appendix 8 for transcripts of these conversations). For a full report on the greetings documented during the fieldwork, see Appendix 9. 


\subsection{From Video Documentation to Graphic Representation}

\section{Video Editing}

Having documented four different perspectives of each greeting, I experimented with showing different perspectives in combination on the screen. All video editing was done using Final Cut Pro. These experiments were made in order to see whether different layouts would change the view and understanding of the greetings. I also experimented with using different speeds for the video clips, such as slowing them down and speeding them up. The following experiments were made:

1) 4 screens - Normal Speed

In this version, all four perspectives are displayed on the screen, and play at normal speed. This combination gives a unique view of the greeting, showing posture, position, gesture, facial expressions and use of space all at the same time (see fig. 141).

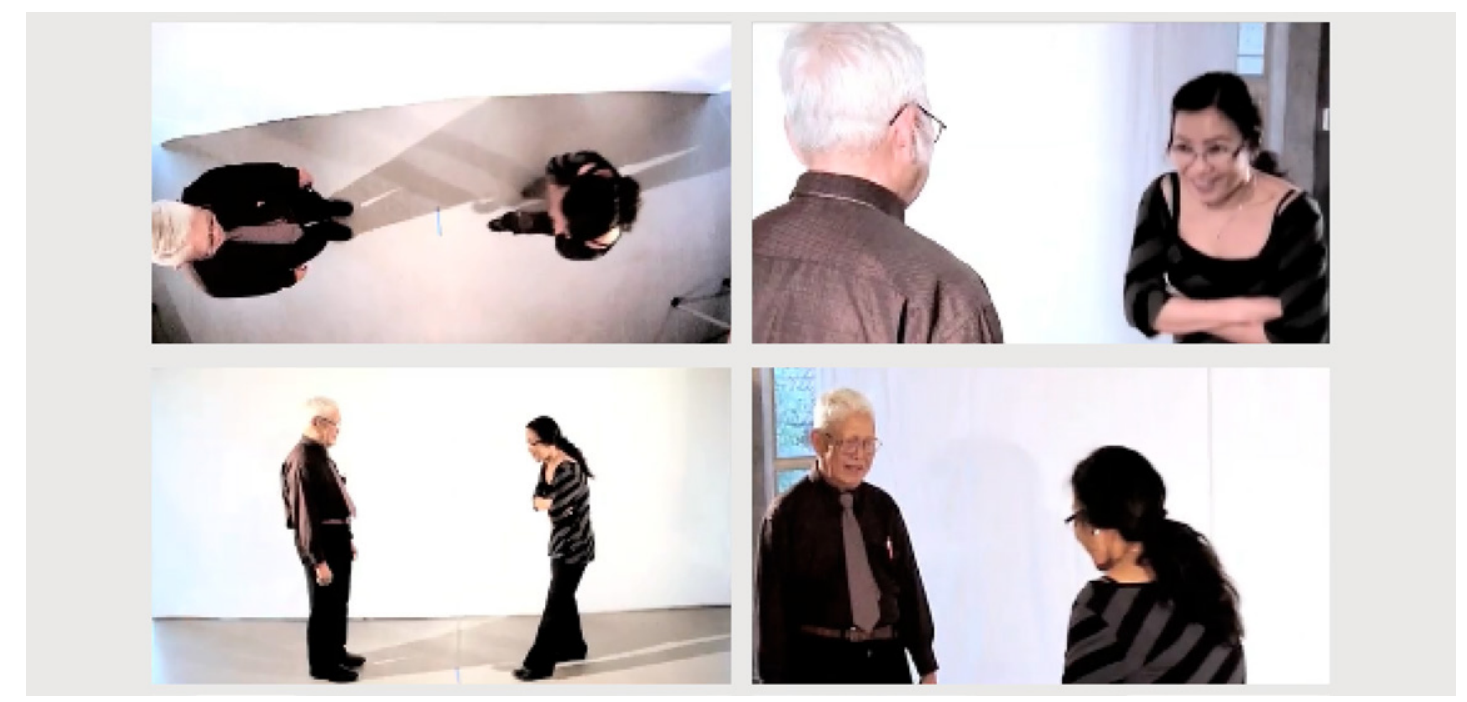

Fig. 141 - Greeting videos: four videos/perspectives displayed on one screen 
2) 4 Screens - Screen by Screen - Slow Motion (50\%)

Similar to the above, except in this version one clip plays at any time, allowing us to view the same greeting four times, drawing attention to different aspects of the greeting, depending on which perspective is playing. The clips play in slow motion, at half of the normal speed, which draws more attention to details in movements and expressions.

\section{3) 2 Screens - Faces - Slow Motion (50\%)}

This version shows the facial expressions of the two people greeting at half of the original speed. Again, slowing the video down makes subtle expressions of the face, such as gaze and smiles/mouth movements, easier to observe (see fig. 142).

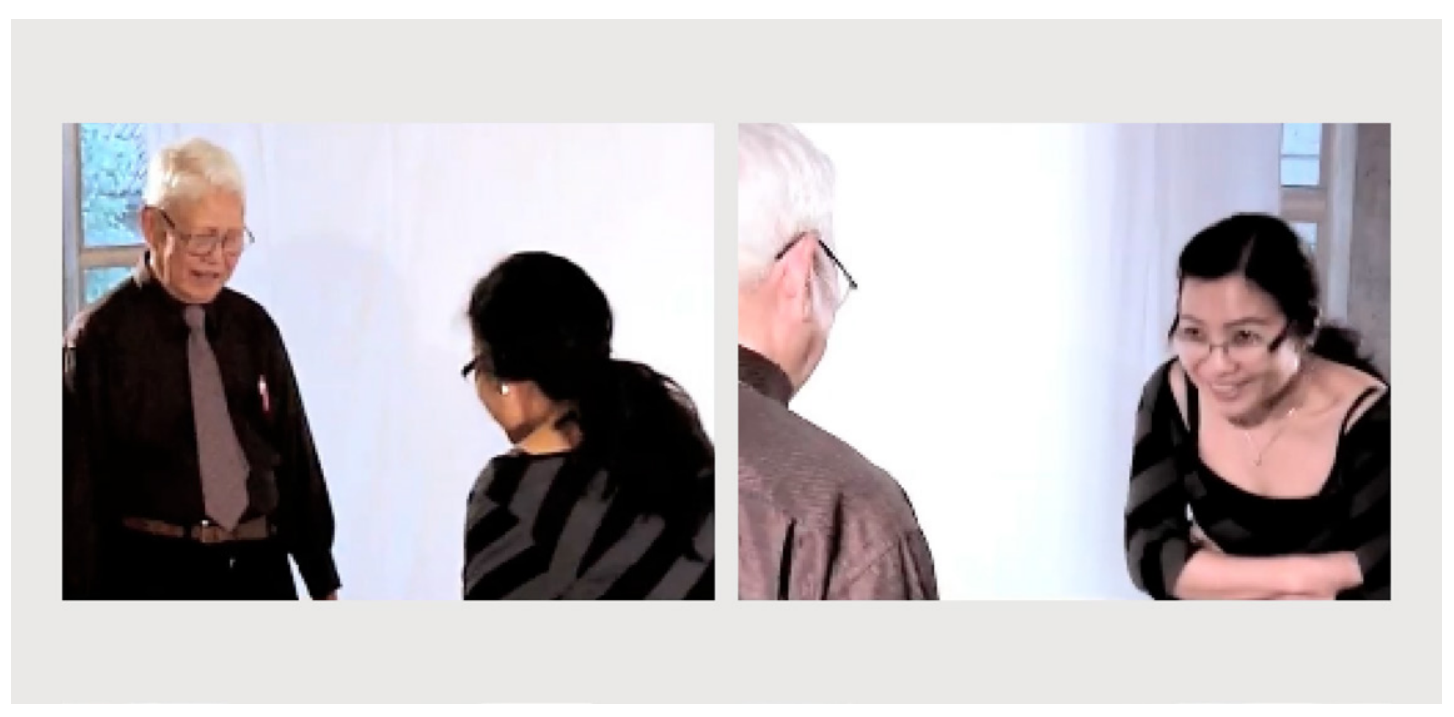

Fig. 142 - Greeting videos: two videos/perspectives displayed on one screen faces

4) 2 Screens - Bodies \& Space - Slow Motion (50\%)

The fourth version shows the two most important views of my current study: front view and overhead view played at half of their original speed, to get a clearer view of the postures, gestures, and movements in the greeting (see fig. 143). 

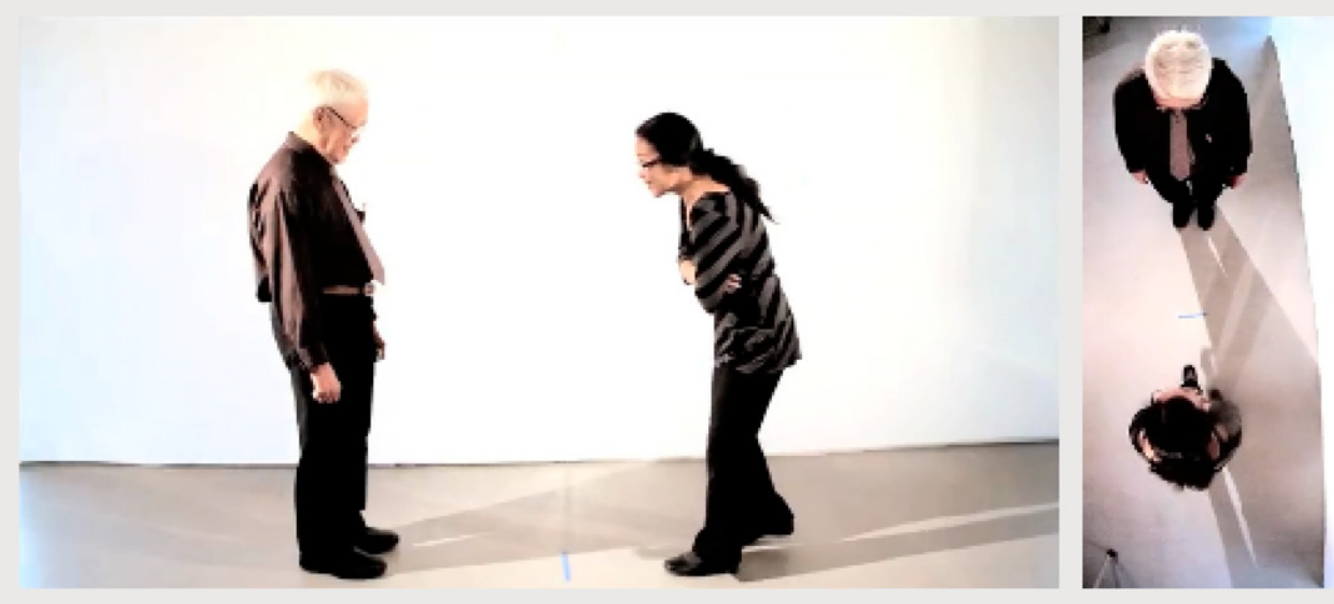

Fig. 143 - Greeting videos: two videos/perspectives displayed on one screen - front and overhead views

\section{5) 2 Screens - Bodies \& Space - Normal Speed}

The last version is the same as the previous one, only shown at normal speed. This is the version I chose to use as my main source: it gives the two main views I have focused on in my visualisations, and at the speed that the greeting actually happened. The only modifications that have been done to these videos are colour corrections, and cropping the frames so that they could fit on the same screen. Due to time constraints, I decided to focus on these two views for the current research, and not on facial expressions, which I had recorded and which can be investigated further in future work (see chapter 5).

To the videos that are presented in my dynamic diagrams, I have added text labeling to give the viewer information about: what type of greeting they are watching; who is greeting; and whether this is their real relationship. This was done, as already mentioned, to clarify the nature of some of the relationships being enacted. The text labels are colour coded with two shades of purple 
(see figs. 144-145). Dark purple indicates that the relationship between the two people greeting in the video is real - i.e. if the text saying 'strangers' is dark purple it means that the two people greeting are strangers in real life. If the relationship between the two people greeting is enacted, that will be clear both in the text, such as 'stand-in grandfather' or 'enacting strangers', but also by the use of a lighter shade of purple. The colour coding makes it clearer and quicker to determine whether the greeting is an enactment or a true relation. The labeling adds a transparency to the documentation and to my system.

Norwegian Man

Strangers

Angolan Woman

Fig. 144 - Video label: dark purple text indicating a 'true' relationship

Chilean Man

Norwegian Woman

Fig. 145 - Video label: light purple text indicates an enacted relationship

Image Sequences

Each greeting video was converted into an image sequence. Each frame included in the sequence shows how the movement evolves over time. Inspired by chronophotography techniques and works developed by Muybridge and Marey, the conversion into image sequences was done in order to analyse key movements and moments of the greeting gestures. This process was done with the 'export as image sequence' function in the software QuickTime 7. 

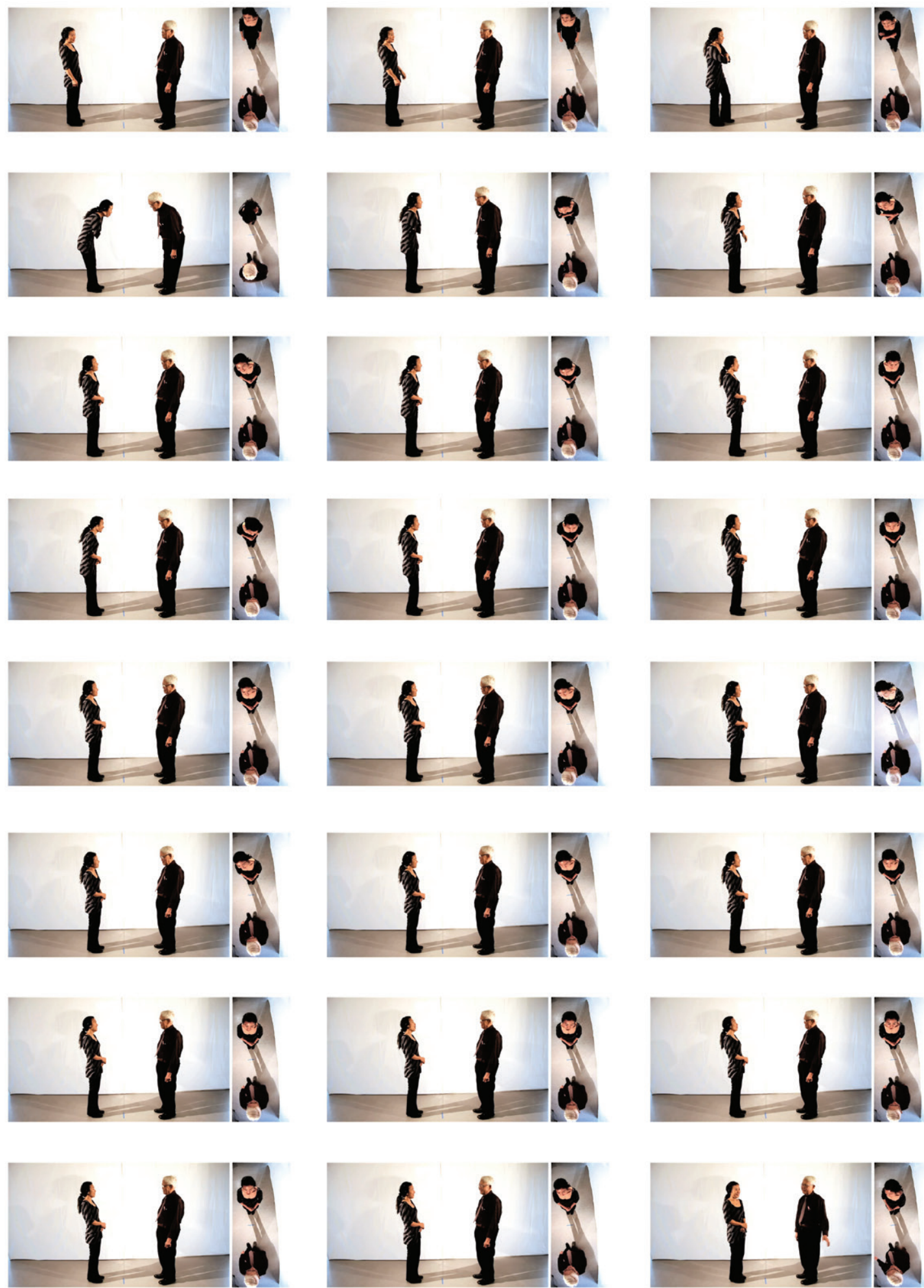

Fig. 146 - A greeting between a Vietnamese grandfather and granddaughter converted into an image sequence (the images read left to right) 
To find the best number of frames to include in each greeting, I experimented with different settings for frames per second (fps): I tried with $6 \mathrm{fps}, 5 \mathrm{fps}, 4$ fps, and 2 fps. Six and five frames per second resulted in too many images, giving too little movement or change from one image to the next. Two frames per second eliminated some important moments of the greetings, three frames per second made calculations complicated, so I settled with four frames per second, which gave me 240 images per minute (4x60). This meant that each image should last for $(1 / 4=) 0.25$ seconds when animated - and 6 frames in Adobe Flash because the frame rate was set to 24fps $(24 / 4=6)$. Four frames per second provided me with more images than I needed for greetings in which there was less range of movement, such as a handshake, so further selection and elimination of images were done in the next step - vectorisation. Fig. 146 shows one greeting, between a Vietnamese grandfather and granddaughter, as an image sequence. Fig. 147 shows the same greeting sequence as a multi-layered still image - an image overlay that works in the same way as Motion Bank's video overlay discussed in chapter 3.
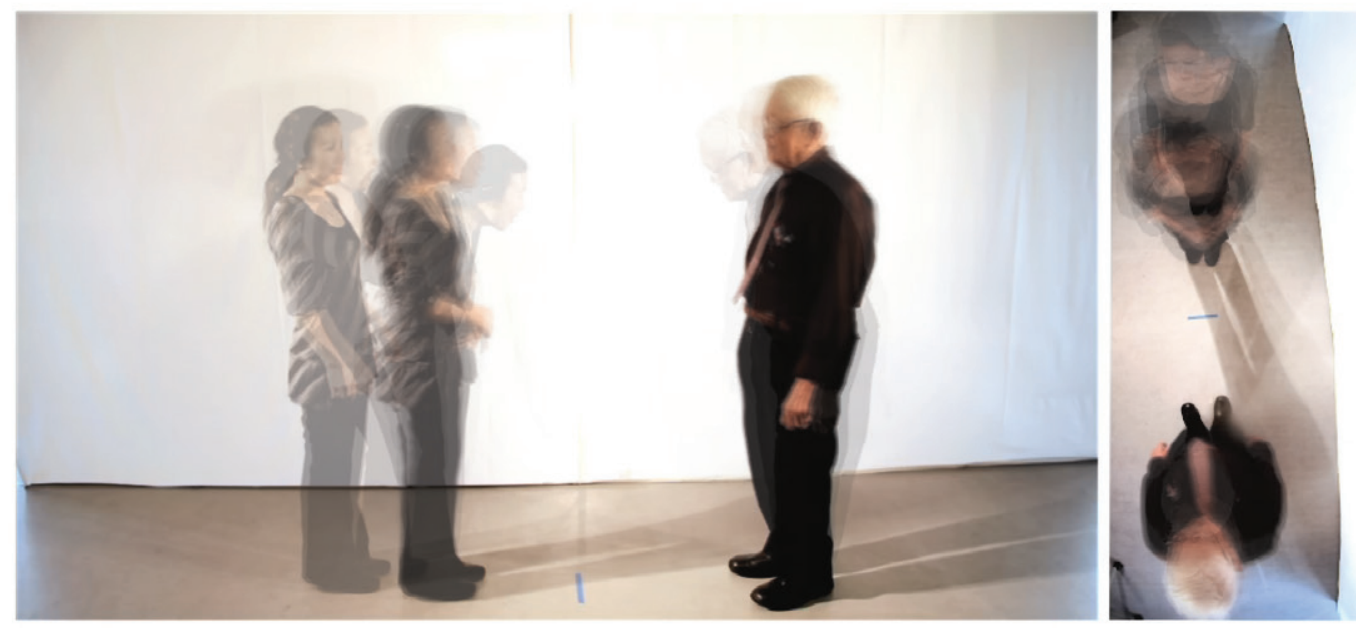

Fig. 147 - A greeting between a Vietnamese grandfather and granddaughter presented as a multi-layered image sequence 


\section{Vectorisation \& Animation}

To vectorise the greetings, the image sequences were imported into separate layers in Adobe Illustrator. All layers were named sequentially, and images that were too similar in which there was little or no change in movement were deleted. Initially, my aim was to preserve the original duration of the greetings. For most image sequences every other image was deleted, in which case each image would have to last for 12 frames instead of 6 frames, to maintain the original timings when animated in Adobe Flash. If I deleted more than one image, layers ' 2 ' and ' 3 ', for example, then layer ' 1 ' would have to last for three times as many frames (18 frames) when it came to animating the greeting in Adobe Flash, to replace the two missing layers. In such an instance I renamed layer '1' to '1-3', to be able to calculate the duration each vector drawing would need to have when it came to animating the greetings. These calculations were important in order to maintain both the 'real' duration of each greeting, and the exact temporal relationships within it, in the animations.

When I had experimented with a few greeting animations in this way, I decided to re-evaluate the speed at which they were shown. I had carefully calculated them to correspond to the real duration of the greetings as they had been filmed, to stay true to the timings of the actual action sequences. However, the speed of the animations seemed too fast considering their purpose which was to be used for visual analysis. I decided to slow down the animations to $50 \%$ of the real speed, in order to be able to study changes in annotations and actions more easily. This change meant that each image would last for 12 frames, and if an image represented two sequential ones, it would last for 24 frames, and so on. With this change the animations no longer represent the actual duration of the greetings, but still maintain accurate temporal relationships within them, at $50 \%$ of real speed.

After the process of eliminating frames, each image remaining was turned into a vector graphic by using the trace and pen tools in Adobe Illustrator 

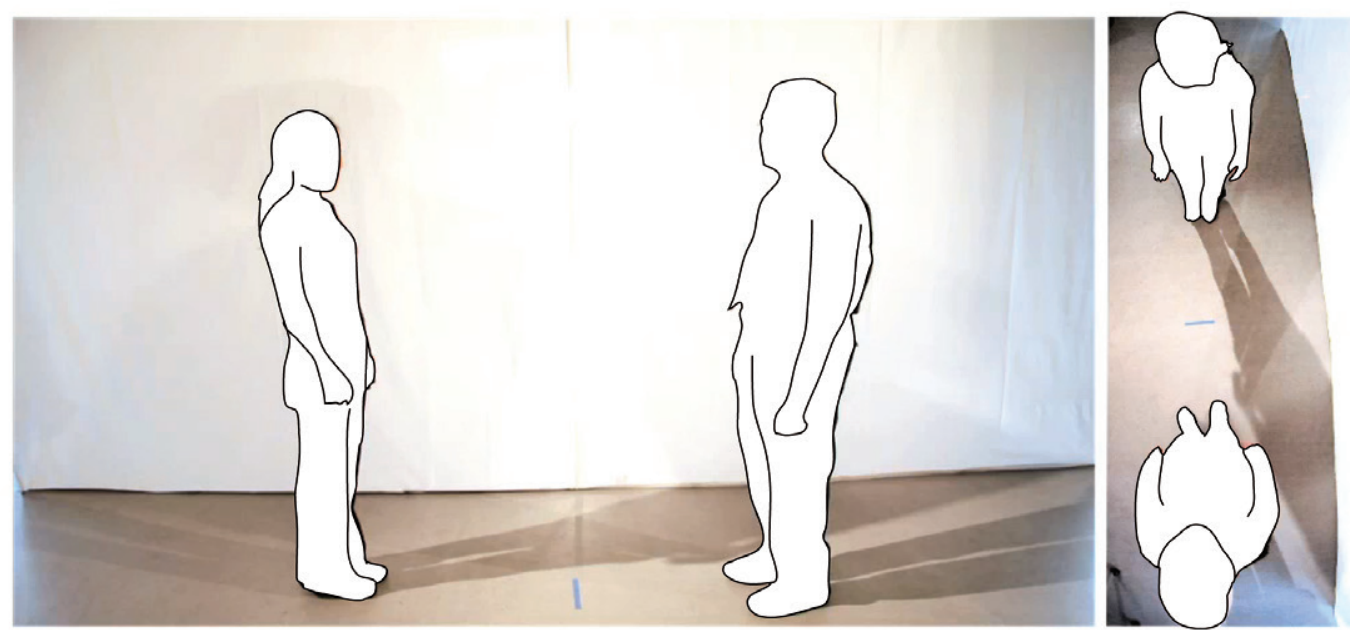

Fig. 148 - Vectorisation: vector drawings were drawn on top of each image in the image sequences

(see fig. 148). I experimented with a great number of different trace settings with the aim of making this step a more speedy and automated one; however there are a number of reasons why it has remained such a manual process. One reason concerned the colours of the studio background and the clothing of the participants. As I did not have access to a professional photography studio, I used a white backdrop, and the floor in the room was grey.

Participants were not asked to wear anything specific on the day of recording, as I did not want to influence their appearance or make the sessions too formal. So, some participants wore white or lightly coloured clothing, which made the contrast to the backdrop too subtle when it came to tracing the images. Some participants had grey or blonde hair, which also created the same problem of carrying out smooth tracing. Had I had the opportunity to film in a green-screen studio, or asked all participants to wear dark clothes (and hats), this step could perhaps have been made a quicker one. However, a green-screen studio was not available to me in Stavanger, and I also think that such a technical studio environment could have influenced participants' behaviour more than my more informal studio setting did. Small changes though, such as suggesting dark clothing, and extending the white backdrop onto the floor to get rid of the grey surface, would have been beneficial. However, while such changes might have helped speed up the process 

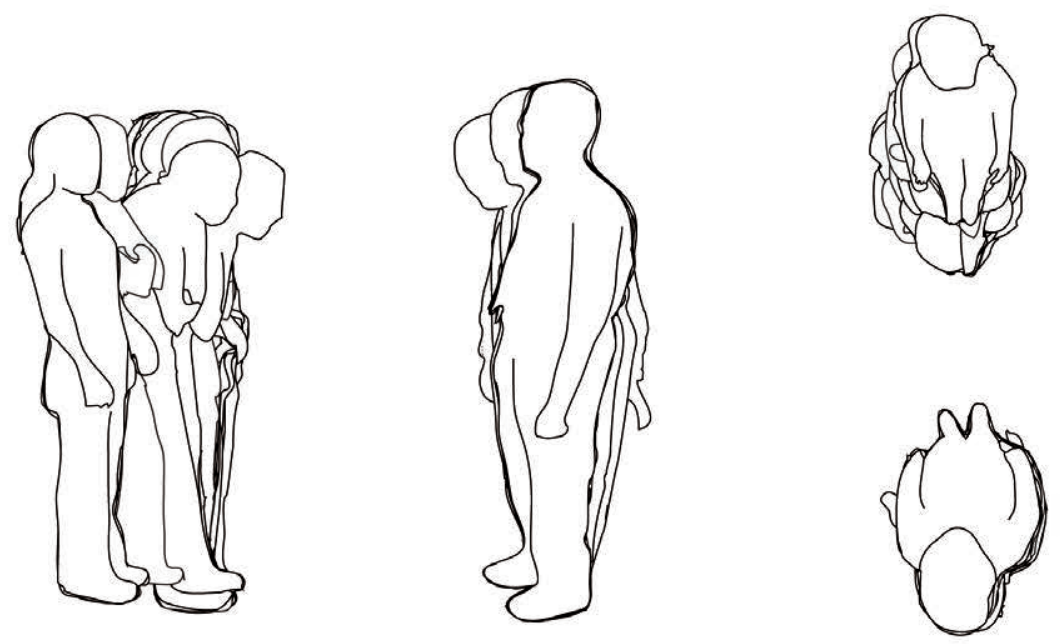

Fig. 149 - Vectorisation: the complete image sequence vectorised onto multiple layers in Adobe Illustrator

somewhat, the graphic style that I wanted for the animations would still have had to involve some manual drawing in Illustrator. I wanted details of hands, arms and legs to be included, rather than showing just a silhouette of the pairs greeting.

Once all layers were vectorised (see fig. 149), they were imported, as layers, into Adobe Flash, where I animated them using stop motion animation. The resulting stop motion animations were graphic representations of the original greeting videos. They were then used as clean 'canvases' to create different versions of the animation; each featuring visual notation of a different nonverbal dimension. The devising of codes for these visual notations was a central part of my project, discussed in the next section.

\subsection{Visual Notation of Nonverbal Signals}

\section{Semiotics: Cultural \& Visual Codes}

The process of visually annotating the greeting animations involved exploring semiotic codes - both in terms of nonverbal signals, which involve social codes, and also how to visualise these signals using colours and other forms of graphic code. While I am not approaching this study as a theoretical 
semiotician, a brief discussion of semiotics seems necessary, to show how my material relates to this field and how it could potentially be theorised from a semiotic viewpoint. I will limit this discussion of semiotics to some very basic concepts and how they relate to nonverbal signaling and to colour coding.

Semiotics or semiology is the study of signs and the process of signification - how signs generate meaning (Hérbert, 2005). Roland Barthes (1915-80), a French philosopher, was a key figure in the study of systems of signification. Barthes explained that the sign is composed of a signifier - the perceivable part of the sign, and a signified - what we understand as its meaning (Barthes, 1964: 48-50). According to Barthes the signifier functions as a 'material mediator' of the signified: and together they make up the sign (Barthes, 1964: 48-50). Signs transmit meaning between members of a culture, group, or society (or within a language) because they are part of a code. Being a member of a culture involves understanding the meanings of different codes and understanding when they are appropriate to use (Chandler, 2007: 148).

A very basic example of a semiotic code, one made up of colours, is the case of traffic lights as discussed by Stuart Hall (1997). For traffic lights the colours red, amber and green are used to represent 'Stop', 'Wait'/'Get Ready', and 'Go'. However, it is not the colours in themselves that produce this meaning, but their order and their relation to each other as part of a code. In theory any colours could be used to convey the same meanings as long as the code was culturally understood. So as Hall (1997: 27) says, the signs are 'arbitrary': it is not each colour itself, but the distinction between the colours that generates the meaning. The red, amber, and green make up a simple system of three colours that are different from one another, and they carry meaning in this very simply coded system of stop, wait and go. 
There are of course far more complex systems of codes, such as languages, music, gestures, and many others (Bullock and Trombley, 1999: 783).

Semiotics includes the study of systems of meaning in all modes of human communicative behaviour. Some of the modes are: audio/vocal, visual, and tactile. The audio/vocal mode includes linguistics, and the visual mode is often called kinesics, a term coined by Birdwhistell - as discussed in chapter 2 - and includes gestures and facial expressions. The tactile mode is often referred to as proxemics - and includes touch and interpersonal movement (Bullock and Trombley, 1999: 784). The term proxemics was coined by E.T. Hall, as also discussed in chapter 2.

It is important to consider that codes often overlap, and one should study the different relations the codes have to each other, rather than study them in isolation, to get a more complete picture of their meaning (Chandler, 2007: 149). When different cultures meet in a greeting, different social codes may collide. Social codes include bodily codes and behavioural codes. Bodily codes involve areas such as: bodily contact, proximity, facial expression, gestures, posture and more. Behavioural codes include, among other codes, rituals (Chandler, 2007: 149).

When dealing with cross-cultural communication, nonverbal codes become increasingly important and it is necessary to understand how cultural semiotics may influence interpersonal communication. Edward Hall and English social psychologist Michael Argyle (1925-2002) dedicated much of their time to studying such codes and how different cultures attach different meanings to nonverbal gestures and behaviours. Hall, in his studies of proxemics, illustrates how different cultures use space. He notes, for example, that the acceptable distance of interaction, or personal space, is much smaller in Latin America than in the United States. During interaction between a Latin American and an American person the assumption of small personal space by the Latin American can cause the American to step back to increase the distance between them. To the Latin American such 
behaviour could make him interpret the American as being cold, and distant, while the American could feel that the Latin American was crowding him (Hall, 1990: 180). Michael Argyle focused on a different nonverbal code, and discussed the meaning of touch in different cultures. He called South America and Arab countries 'the real touch areas' owing to their use of touch during human interaction (Argyle, 1988: 61). He gave an example of a greeting between two Arab men, in which they embrace and kiss each other. To them, this is experienced as a friendly greeting. In Britain or the USA, on the other hand, such a greeting between friends of the same gender would be seen as carrying 'sexual overtones' (Argyle, 1988: 61).

These two examples by Hall and Argyle indicate that cultural values, assumptions and worldviews are communicated through nonverbal codes (Chandler, 2007: 156). It is also obvious from these examples that nonverbal codes such as those relating to personal space and touch are semiotic codes - the same gesture can signify different things in different cultures. As mentioned in chapter 2, Alessandro Duranti too viewed greetings as valuable semiotic resources. Hall's study provides a clear illustration of this, as he shows that to Latin Americans leaving minimal personal space in an encounter signifies warmth, while to Americans it can signify being intrusive. Argyle's study also draws attention to the way that different gender relations operate in different cultures, and how such relations can be encoded in touch patterns during a greeting. It is precisely the nonverbal signals of personal space and touch that my system sets out to visualise, and by systematically visualising them it enables an understanding of, and comparison between, the cultural codes in which they convey meaning.

My visualisation process, in turn, involves the use of colour and pattern to make up visual coding systems for conveying information about the greetings. Just as proximity and touch carry culturally coded meaning, so does colour: colours have multiple meanings and connotations both within and across cultures. Returning to the example of traffic lights: while 
the code for conveying 'stop', 'wait' and 'go' may in theory be arbitrary, it is nevertheless the case that red signifies 'danger' in many cultures, and the traffic light code itself has, over time, affected the connotations of the three colours it employs, though these meanings are not consistent across cultures.

David McCandless with AlwaysWithHonor.com (2009) present a study of the meaning of different colours in different cultures in their visual diagram Colours in Culture (see fig. 150). Their study reveals that the same colour can mean very different, sometimes contrasting things from one culture to the next. In my system colours are primarily used to signify and highlight specific dimensions of nonverbal communication and signaling: in choosing which colours to use for these purposes, I had to consider both the visual distinctions between colours (see the discussion below on Visualising Touch \& Space In-Between), and the potential cultural connotations of each colour.

\section{Visualising Personal Space/Intimate Zones}

In my system, people's ways of inhabiting personal space - the intimate zones, as I have named them - are colour coded to represent different degrees of proximity or distance. I use colours for the graphic purpose of distinguishing different zones from each other, so the varying amounts of space can be easily seen, but the choice of colours was to some extent also based on their cultural connotations. The colour red, used for the smallest intimate zone, is widely known to signify heat (see fig. 150). This meaning is familiar to Western/American, Hindu, Eastern European and Muslim cultures. Red also signifies passion and love. On the other end it signifies danger in Western/American, Japanese and Southern American cultures, and anger in Western/American, Japanese, Eastern European and African cultures (see fig. 150). 


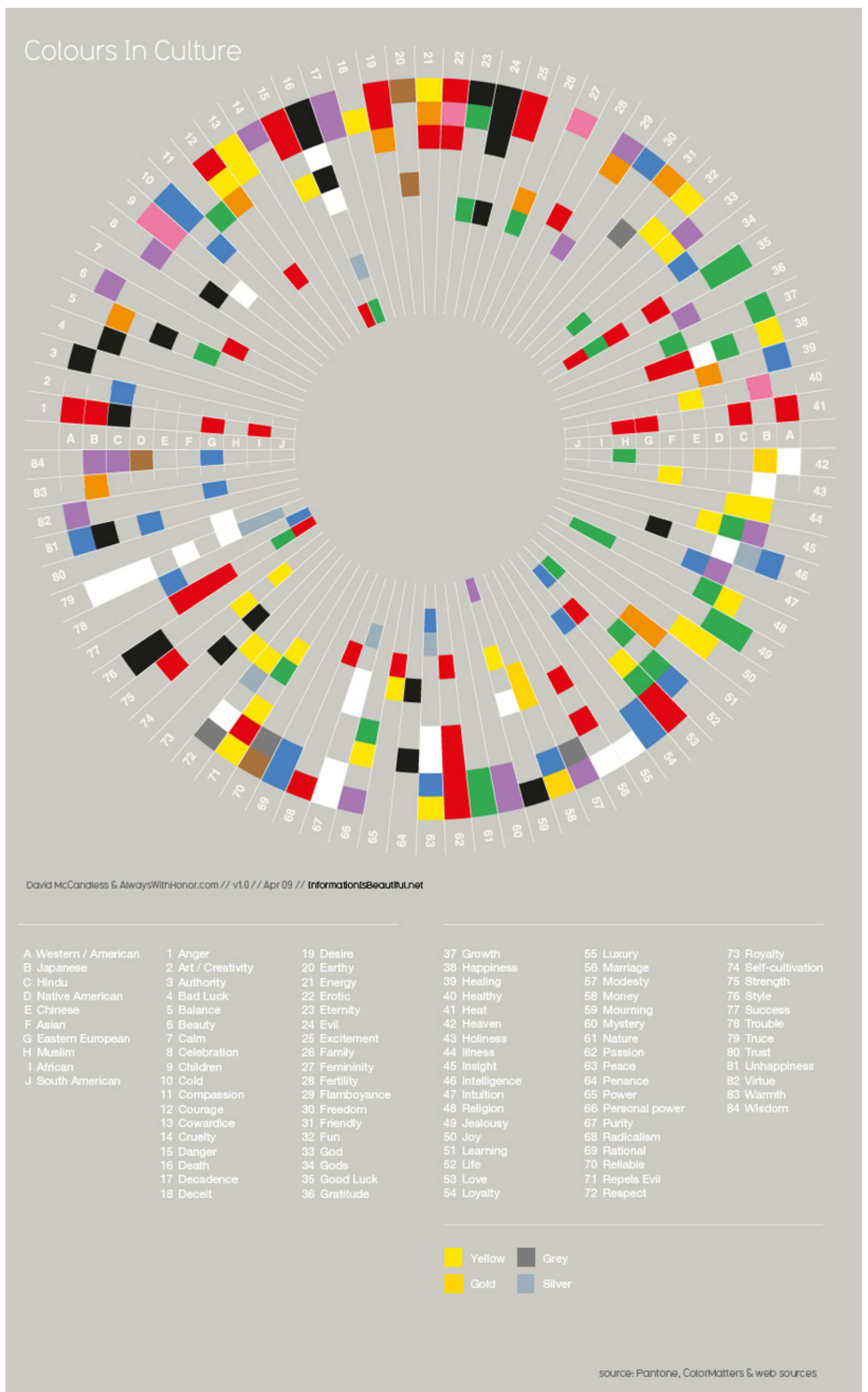

Fig. 150 - Colours in Culture by David McCandless with AlwaysWithHonor.com (2009) 
In my visualisation of intimate zones, red is used to represent the smallest personal space, the intimate zone in which there is hardly any space between the two people greeting. When greeting in this intimate zone there is a lot of physical contact, and red seemed appropriate because of its connotation of heat.

Blue is used to visualise the other end of the scale of intimate zones, the largest distance between the two people greeting, because it is broadly known to signify cold or cool in Western/American, Japanese, and Native American cultures. Blue also signifies unhappiness, rationality, loyalty, intelligence, and freedom within various codes of Western/American cultures. However, in Japanese and African cultures blue signifies peace and life, among other connotations, and in Eastern European cultures it signifies wisdom, virtue and unhappiness (see fig. 150). While blue has many contrasting connotations worldwide, it is used in my system for its widely understood meaning - cold. This seems suitable when considering the physical distance of people greeting in the blue intimate zone and the lack of (body) heat or physical intimacy between them because of that distance.

I chose the colours blue and red to highlight two opposite aspects of personal space: large distance - cold/blue; and small distance - heat/red. For the two intimate zones situated in-between red and blue, I chose the colours orange and green, mainly for the graphic purpose of distinguishing them from the blue and red zones, but also with their potential symbolic meanings in mind. Orange is a 'warm' colour and is both close to red in colour, and in the physical proximity it represents to the red zone. Green is known as a 'cool' colour (on the border to warm), and is close in colour and proximity to the blue zone. Orange and green have a number of cultural connotations, but these are not as specific or generally shared as are some of the connotations of red and blue. Orange symbolises warmth, flamboyance, energy, and balance in Japan, and friendliness in Western/American cultures. Green signifies love, life, religion, insight, compassion, and happiness in 
Hindu cultures. It also signifies nature, good luck and jealousy in Western/ American and Japanese cultures, and success in African cultures (see fig. 150). However, these connotations are not relevant to my visualisations: the key point for my purpose is that graphically the four colours work together so that there is a gradual change in colour from red to orange, from orange to green, and from green to blue, and there is enough contrast between the four colours to tell them apart when presented together ${ }^{12}$. The four selected colours were used for circles illustrating personal space, and were applied to the overhead view of the greeting animations. The colour-coded circles are scaled in proportion to the distances they represent.

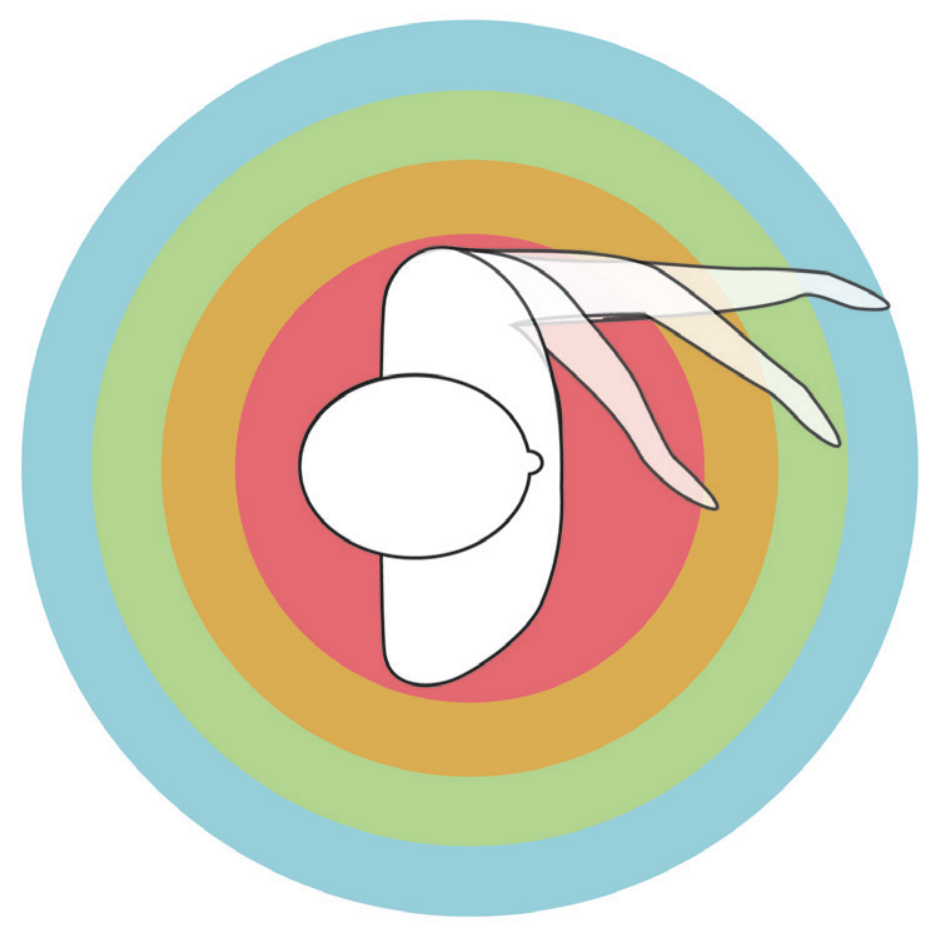

Fig. 151 - Visualising intimate zones

12 I have not, at this stage of developing my system, tested, or considered in the colour selection process, how my system and the colours used would be perceived by people with colour blindness. However, this would be a natural development in the future if the system were to be used by a general public. 
While the use of these specific colours was developed in my Kinesics project, the dimensions/distances that they refer to have changed in my development of the current project. The Kinesics system's intimate zones were defined as following:

Blue: Arm's length or more. Little/no physical contact.

Green: Half an arm's distance or more. Some physical contact.

Orange: $15-25 \mathrm{~cm}$ distance. Physical contact is usual.

Red: No distance. Much physical contact.

These distances use a mix of visual/relative distances (blue and green) and metrically measured distances (orange). The new definitions move away from distances measured metrically, in order to allow for the fact that the people greeting are of different shapes and sizes, so the distance needs to be understood relative to the particular sizes and bodily distances of the two people greeting (see figs. 151 and 152). The new system also defines the zones - for green, orange and red - more accurately than the previous system by specifying range of distance such as half an arm's distance to an arm's distance (green).

The new definitions used are:

Blue: Arm's length or more. Little/no physical contact.

Green: Half an arm to an arm's distance. Some physical contact is usual. Orange: Half an arm's distance to $1 / 4$ arm's distance. Physical contact is usual.

Red: $1 / 4$ arm - to no distance. Much physical contact is usual.

Fig. 152 shows intimate zones applied to both the front and overhead views for four different greetings. While in this example I have visualised intimate zones on both views, in my actual system I only use the overhead view of the intimate zone animations, because it gives the clearest view of use of space. However, when determining which intimate zones a person moves through, I consider both views due to the fact that the overhead footage was shot with a camera with a wide-angle lens. The wide-angle lens does give some degree 


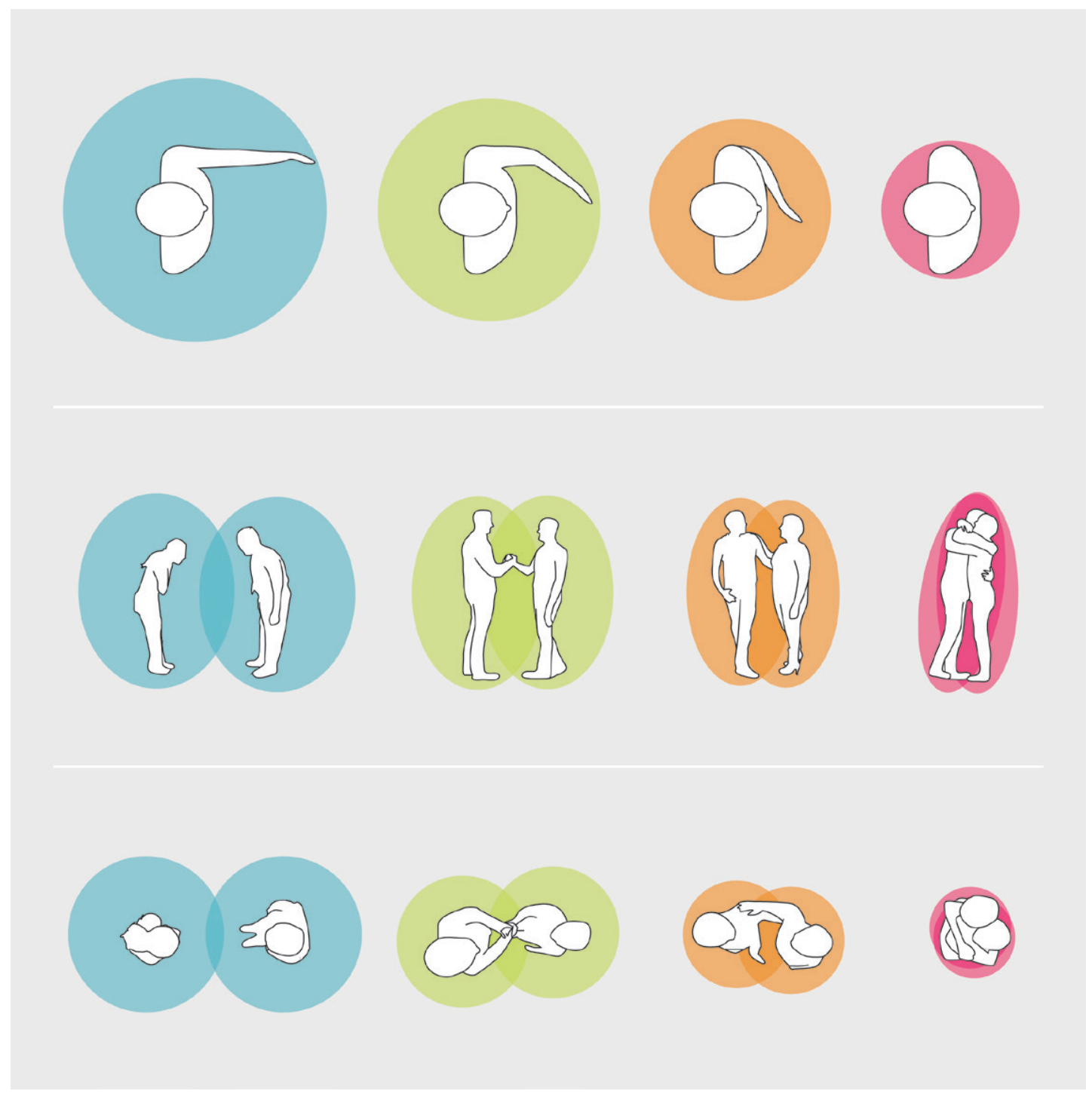

Fig. 152 - Intimate zones: applied to four different greetings - front and overhead views

of distortion to the footage, such as exaggerating depth and relative sizes in the image. In my recordings this level of distortion was very apparent in greetings with tall people - because they were much closer to the camera, so the image seemed to become more distorted than for shorter people. The level of distortion is not very great, but when applying the intimate zones to my vectorised animations, I was aware of the fact that in some instances the two people in the greeting might seem closer in proximity to each other than they actually were. Therefore I made sure to consider both views - the front view and the overhead view - to measure and determine which intimate zones to apply. 
It was clear from my recordings that sometimes in a greeting each person's relation to or use of personal space is different. This was particularly observed in greetings performed across cultures. While in most cases, during intracultural greetings, there was a mutual understanding of how much distance was accepted, during intercultural greetings this type of shared 'prescribed' behaviour was not always present. To put it in semiotic terms, there was a mis-match of spatial codes.

During an intercultural greeting, a person would, for example, approach the other person with the intention of greeting with a kiss or a hug, while the other person would respond with a handshake. This showed that their ideas of and uses of personal space were completely different. In the visualisations this meeting of two different intimate zones should clearly not be represented as a shared space as in my system for Kinesics, where only intracultural greetings were studied and a single colour zone was ascribed to the greeting as a whole. The present research has introduced a radical improvement to the earlier system by visualising each person's intimate zone separately, which is more accurate as it allows for the visualisation of different relationships to space within one greeting. This development makes two kinds of information available: it shows how personal space changes during a greeting, in terms of movement of the two people towards each other; and it reveals the individual's distinct use of personal space. Such an example is illustrated in figs. 153-154. Fig. 153 shows synchronised movement and changes in personal space in a greeting between two Norwegians - a male and a female. Fig. 154, on the other hand, shows difference in movement and relation to personal space in a greeting between a Norwegian male and an Angolan female. This visualisation of people's varying and diverse relations to personal space is a key development of the system. 


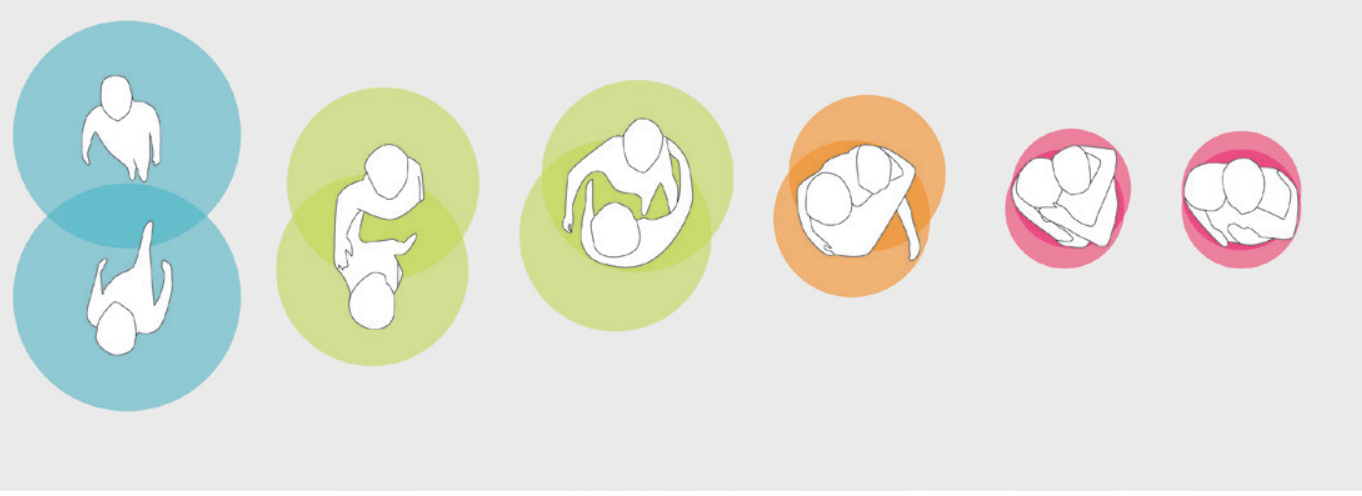

Fig. 153 - Animated greeting sequence between two Norwegians, male and female, who are close friends, showing synchronised movement and relation to personal space

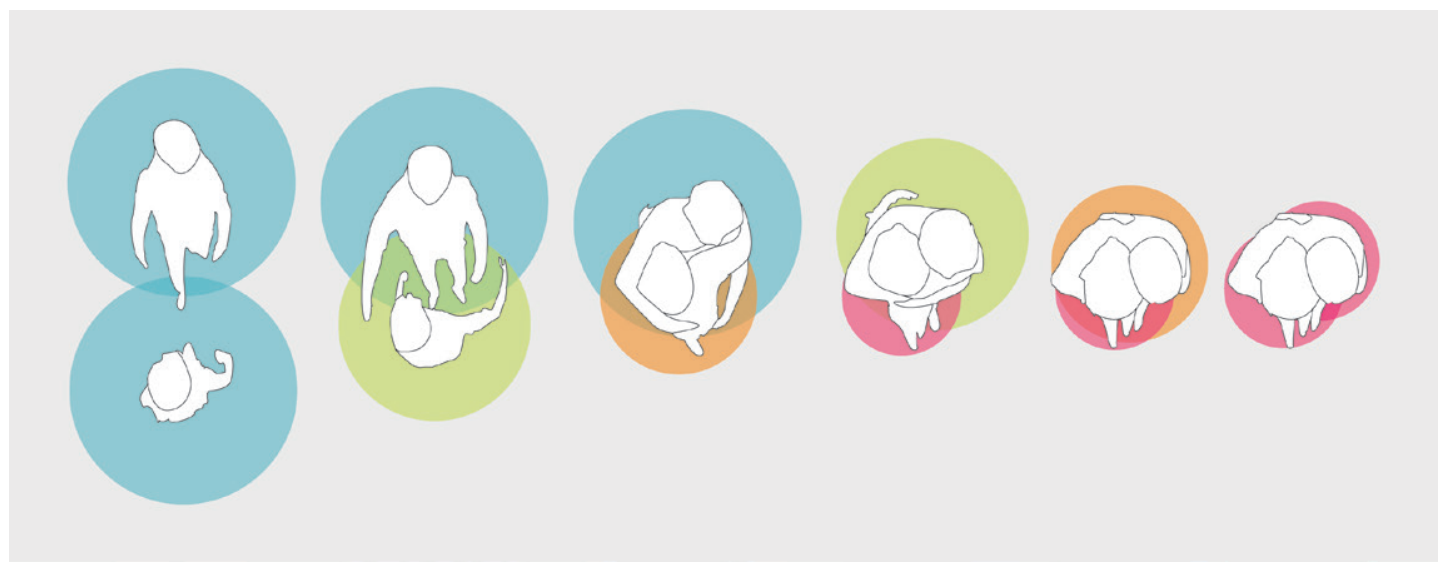

Fig. 154 - Animated greeting sequence between a Norwegian male and an Angolan female who are close friends, showing difference in movement and relation to personal space

\section{Visualising Touch \& Space In-Between}

Another important new development in the present research has been the introduction of ways to visualise touch, and space in-between greeters.

These important elements of nonverbal communication were not investigated in Kinesics and in order to represent them I needed to design further graphic codes that would work alongside, but not overlap with, the code for intimate zones. The process of deciding on the visual codings for touch and space in-between was lengthy and involved a series of experiments. Initially I used a turquoise-blue colour to visualise touch, and a dark pink gradient to visualise space in-between. Aesthetically these colours worked well when the animated greetings were viewed individually. However, when I printed screenshots of all the visual dimensions (touch, space in-between, intimate 

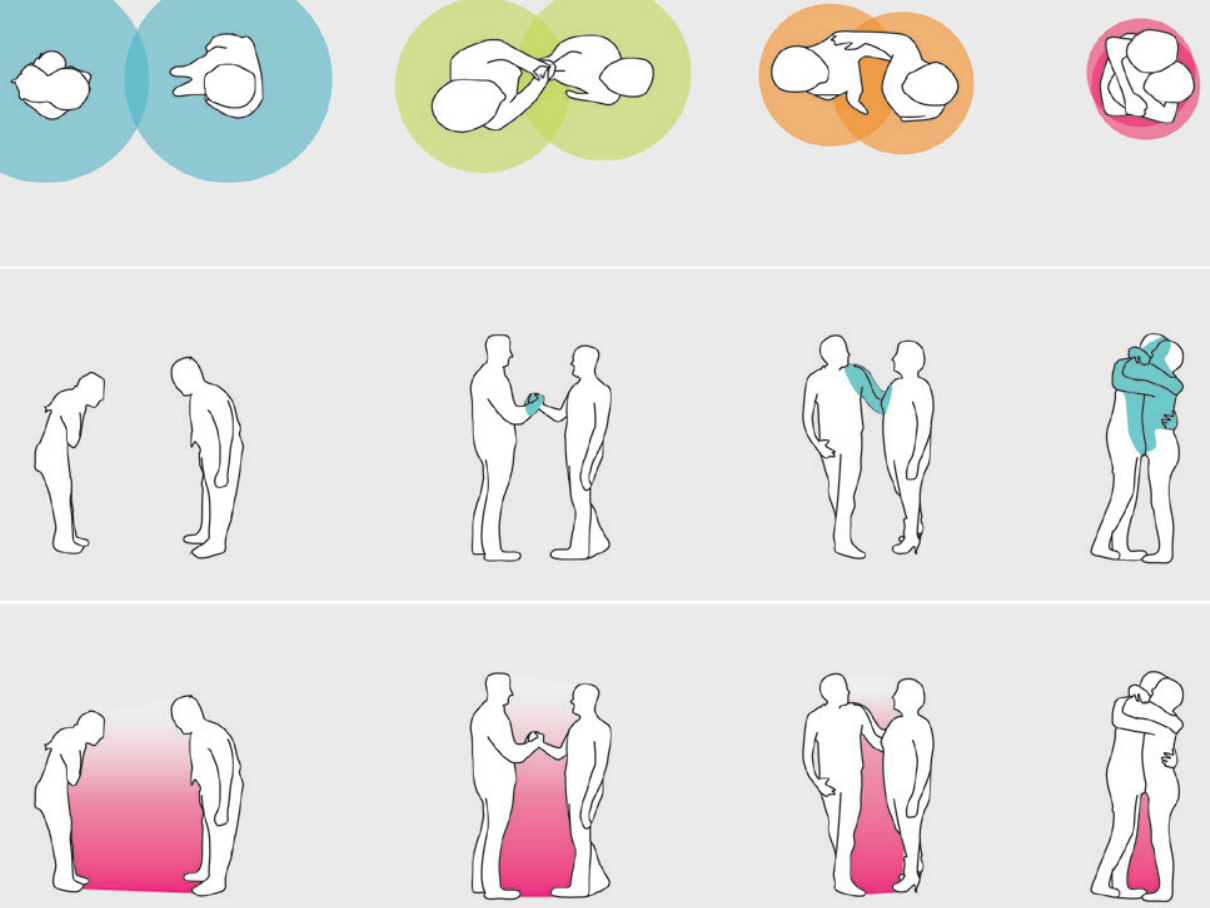

Fig. 155 - Colours initially used to annotate intimate zones (top), touch (middle) and space in-between (bottom)

zones, and subjective experiences) and presented them together, these colours posed some confusion (see fig. 155). While the turquoise was a different shade of blue (and arguably a different colour) from the blue I used for the large intimate zone, and the pink gradient was more pink than the smallest red intimate zone, they were nevertheless too close in colour. It could appear as though the intention with the pink gradient was to signify heat as with the red intimate zone, which it was not. For example, during a greeting where there was a big distance between the two people greeting, such as in the bottom left illustration of fig. 155, the visualisation of space inbetween seemed to suggest heat and intimacy rather than simply illustrating the physical distance between them. Similarly the turquoise colour for touch could suggest cold, just as the blue intimate zone was meant to do. This did not make sense because touch involves heat and some level of intimacy, so the turquoise could possibly give the wrong connotations of cold or distance, such as in the middle-right illustration in fig. 155. 
On the basis of these reflections I started experimenting with colours that could replace the turquoise and the pink gradient. I decided to colour-code each of the nonverbal dimensions with a different colour so that when presented next to each other in print, it would be clear that each colour symbolises a different dimension of nonverbal communication. The colours blue, green, orange and red were already used to visualise intimate zones, so I experimented with purples, browns, yellows, greys and many other colours for touch and space in-between. Colours that were too light-toned were unsuccessful in highlighting touch because they were not visually obvious enough, especially when printed (see fig. 156). Different shades of blue and turquoise did not work either because, as already mentioned, they could be confused with the blue intimate zones (see fig. 157). Greys and browns seemed too neutral when compared to the other colour coded visualisations, and tended to blend too much into the background (see fig. 158).
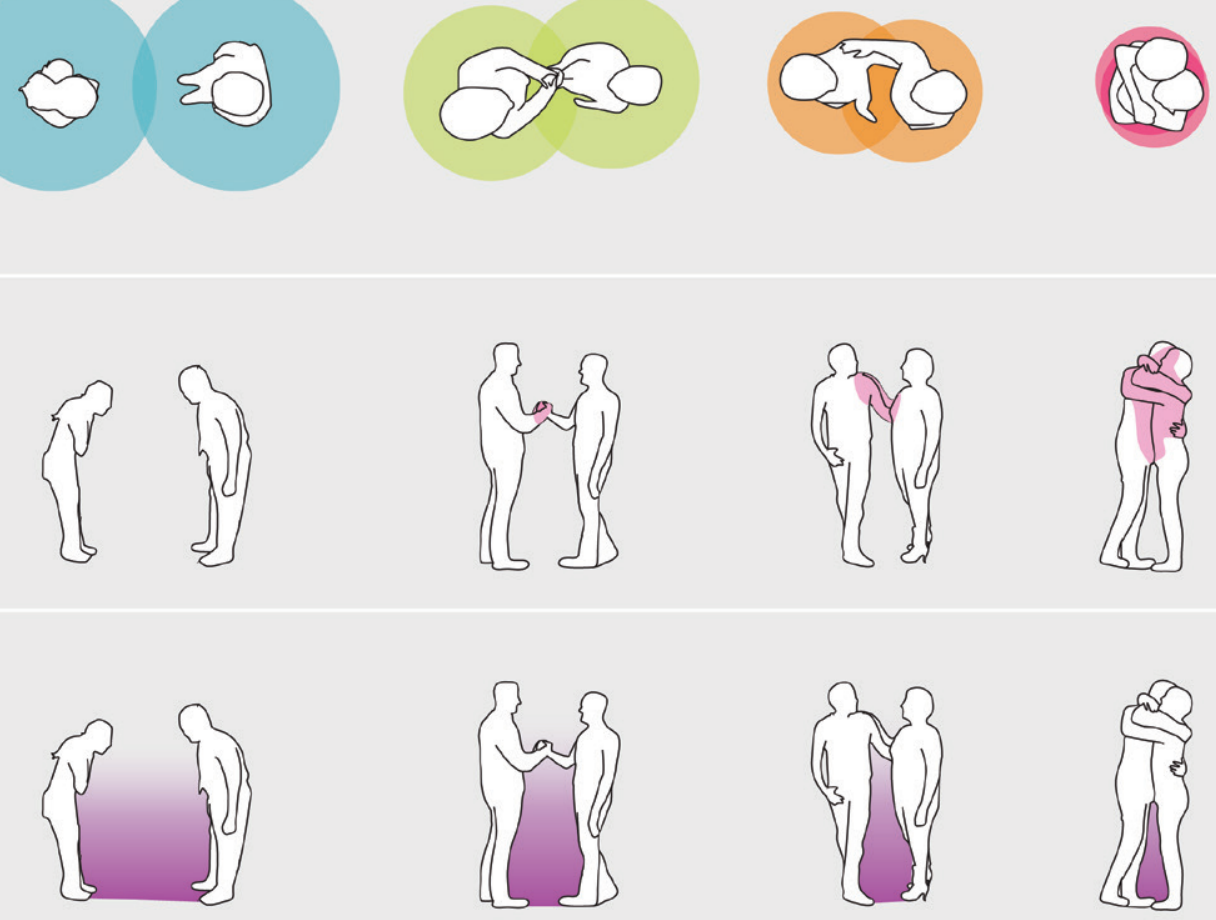

Fig. 156 - Colour experimentation for touch and space in-between: pink and purple 


\section{$\theta=0$}
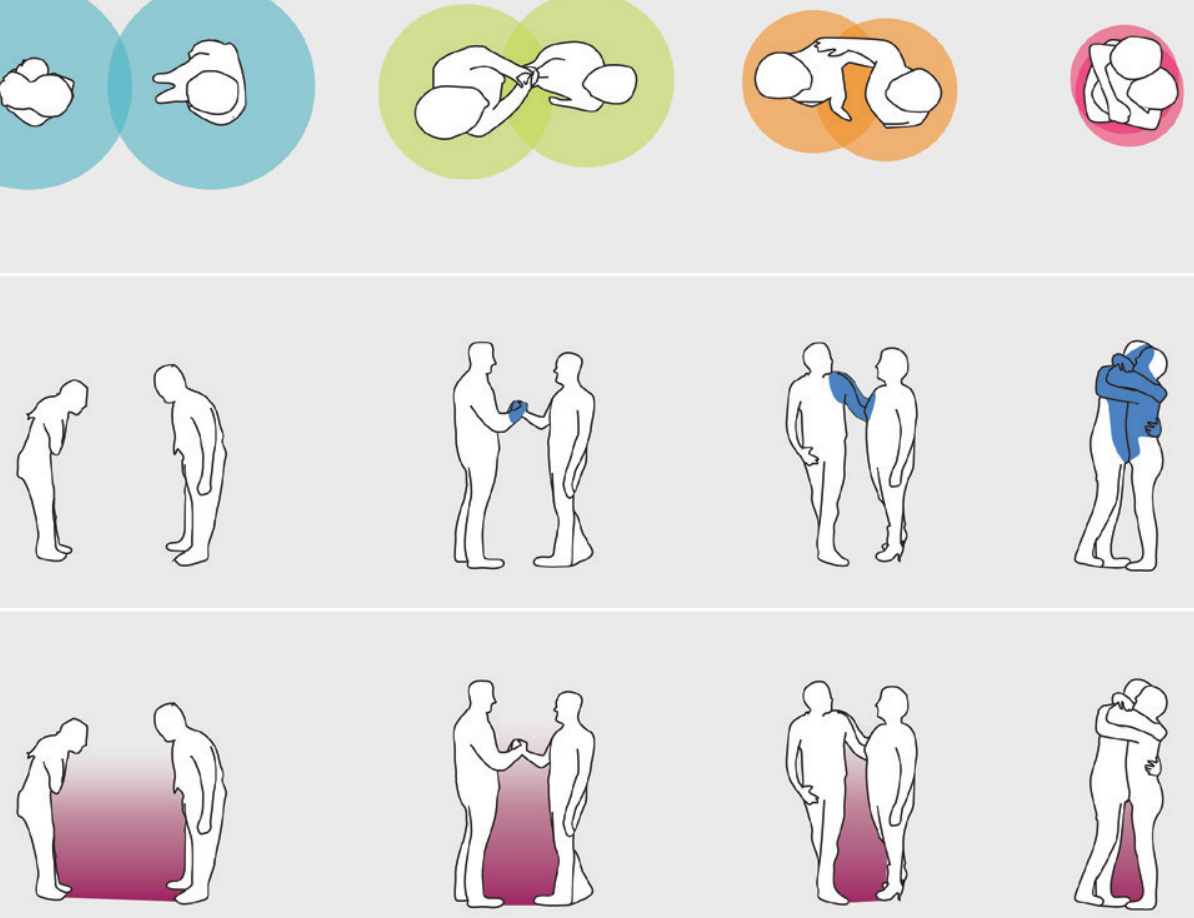

Fig. 157 - Colour experimentation for touch and space in-between: dark blue and burgundy
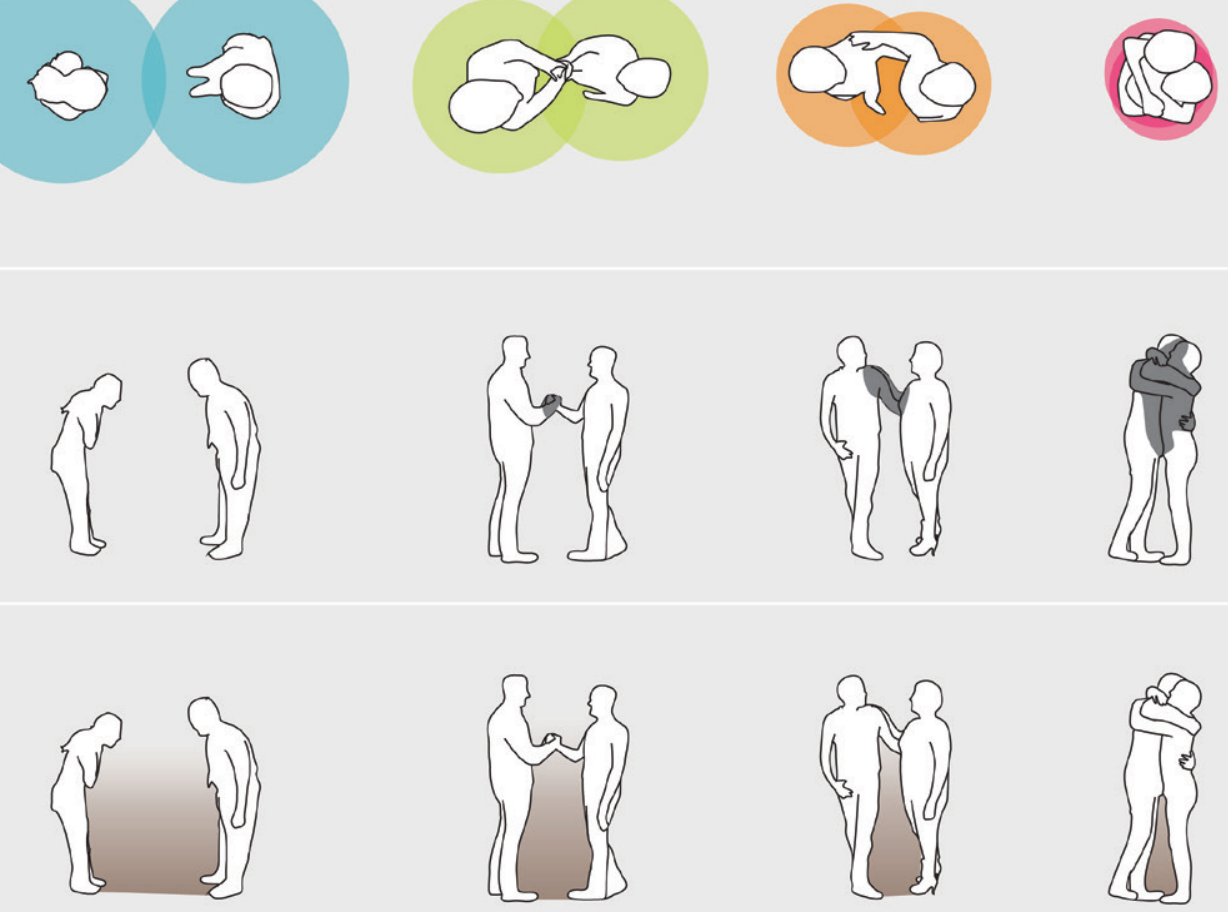

Fig. 158 - Colour experimentation for touch and space in-between: grey and brown 

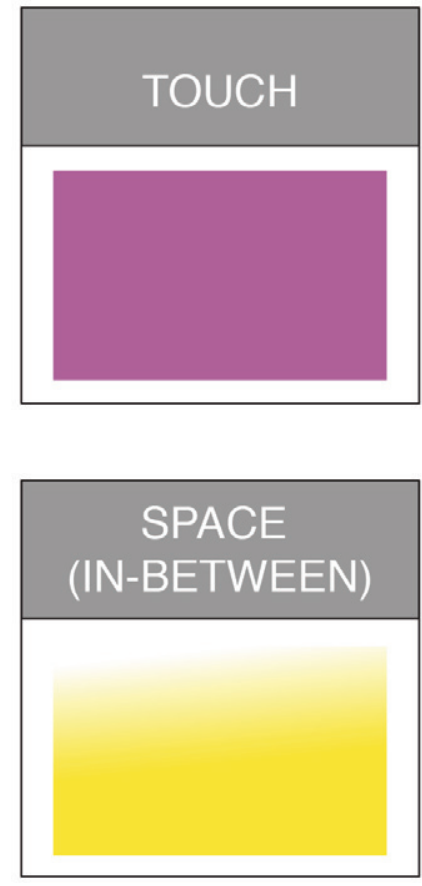

Fig. 159 - Touch and space in-between
After much experimentation with colour, and of considering how the colours work together as a system of colour coding, I chose the following colours: a yellow gradient to visualise space inbetween; and purple to visualise touch (see fig. 159). (All colour experimentations can be viewed in Appendix 22.)

Touch - Shows what areas of the bodies are touched or in contact during the greeting. Space in-between - Visualises the space inbetween the two people greeting, and when animated it shows the increase and decrease of this space during different stages of the greeting.

As with all colours, these colours, too, have a number of cultural connotations. Yellow is used to signify peace in Western/American countries, deceit in Japan, danger in Native American cultures, and is believed to repel evil in Western/American, Hindu and Asian cultures. Purple has a range of meanings in Western/American cultures: virtue, personal power, modesty, mystery, cruelty, and beauty. It also signifies mourning in South American cultures and wisdom in Japanese and Hindu cultures (McCandless and AlwaysWithHonor.com, 2009). However, I did not choose these colours because of their cultural connotations, they were chosen for graphic reasons, to distinguish them from the colours used for intimate zones, ensuring that each dimension of nonverbal communication is represented by its own colour.

Fig. 160 shows the chosen colour codes in use for the visualisation of intimate zones, touch, and space in-between for four different greetings (left to right): a Vietnamese greeting between a grandfather and granddaughter; a greeting between two close Norwegian male friends; a greeting between 

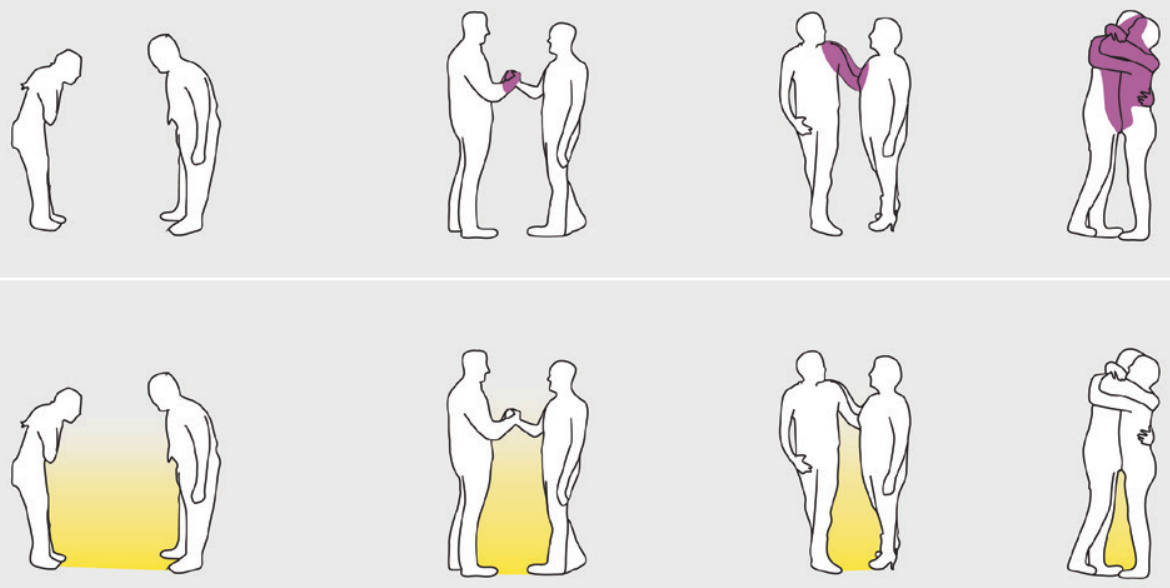

Fig. 160 - Image shows four greetings with annotated intimate zones, touch, and space in-between

a Chilean male and female who are close friends; and a greeting between a Norwegian male and female who are close friends.

\section{Visualising Subjective Experiences \& Perceived Meaning}

Before starting this research project I had considered my graphic visualisations as an objective, almost scientific, study of greetings, in which the gestures, postures, personal space and the observable aspects of this ritual were revealed. However, a deeper study into greetings, into issues such as information exchange and intersubjectivity ${ }^{13}$, which were prevalent in Duranti's studies of greetings, led me to expand upon another dimension of information in my system: subjective experience and perceived world (Bullock and Trombley, 1999: 422). 
meaning of the greeting. Issues concerning subjective experiences arose during numerous discussions with supervisors, and during discussions with fieldwork participants. Some of these issues had to do with: power and submission; warm and cold greetings; intrusive and distant behaviour; and masculinity and femininity. So I decided to start with these few topics and explore how to visualise them. Aspects of masculinity and femininity could already be addressed by comparing the visual analysis of the different male/ male, female/female, and female/male greetings, so were not included in the visualisation of subjective meaning.

For my visualisations to reflect the subjective experience of, and meaning perceived by, the people greeting, I interviewed my participants about their greeting experiences. They were asked, among other questions, what in their view and culture is considered: a respectful greeting; a powerful greeting; a submissive greeting; a warm greeting; a cold greeting; a masculine greeting; and a feminine greeting. When participants were asked what they considered to be a 'warm' greeting in their culture, some of the answers were:

In a warm greeting you are more physical I think, and you use more body language. In a cold greeting you use less facial expressions. - Turkish female

It is to have eye contact when you greet! You have to look at the other person. In our culture we do not embrace or have very near physical contact like they do in other cultures.

- Vietnamese female

To me a hug is a warm greeting. It is friendly, you feel welcome.

- Norwegian male 
A warm greeting is a hug, a good hug, a strong hug. And sometimes if you can use both your hands, and hold around the elbows or the shoulders of the other person, it is kind of respectful and warm at the same time.

- Norwegian female

A warm greeting is the best... I can imagine it is a person laughing actually loud, making noise, and hugging you and really, really warm and tight and with laughs.

- Angolan female

It is the same if you are acquaintances or close friends. Some people hug their close (male) friends, but personally I do not. If I do not know the person very well, especially if it is the opposite sex, I usually fold or hold my own hands and just do a verbal greeting without touching the other person.

- Somali female

Due to time constraints during the days of studio recording, I had to schedule interviews for separate days from the filming. Unfortunately it was not possible to organise meetings with all participants to carry out the interviews, so some of the animations of subjective experiences are based on informal conversations that took place (some recorded, and some not) during the days of video documentation. In a few visualisations, my own interpretation of some of these conversations was used to make judgments on what pattern was suitable. The full questionnaire and transcripts of the interviews can be found in Appendices 10a-g.

Having used colour-coded visualisations for the objective dimensions of nonverbal communication, I chose to explore the use of symbols and patterns for visualising subjective meaning. The patterns are used to visually represent participants' subjective experiences of nonverbal signals during 
greetings. My initial idea was to use one symbol for each of these meanings, but when I considered how to use symbols in combination with the rest of my notation system, I decided that it would make more sense to work with patterns. Doing so would enable me to visually combine the patterns with the colours showing the intimate zones and thereby present layers of information within one visualisation, as I will illustrate below. After much exploration I opted to use patterns made up of simple shapes such as dots, lines and triangles rather than some of the more complex symbols I had explored. The patterns I have used do not in themselves carry very specific cultural connotations. However, I developed patterns based on the visual relationships between pairs of patterns to signify opposing experiences, such as: warm and cold, intrusive and distant. When used together there is a visual contrast between such opposing patterns. While I have focused on only a few aspects of subjective experiences for the scope of this $\mathrm{PhD}$, more patterns can be added in the future to visualise other subjective experiences. The creation of patterns and symbols involved the following explorations and results:

Power/submission - I explored symbols such as triangles facing upwards for power and downwards for submission, and I experimented with combining circles of different sizes. The final patterns use bold circles to signify power, and small dots to signify submission, so that in combination they visualise contrasting feelings (see fig. 161). The bold circles are dominating compared to the small dots. I use the words 'power' and 'submission' here in terms of status/hierarchy - showing respect vs. the respected one - not in terms of control. An example is a greeting between a father and a son: in some cultures the son must show respect to the father, and in such an instance the son shows submission/respect, and the father shows 'power' (or that he is respected).

Warm/cold - I explored symbols such as half circles and swirls to symbolise warmth, and squared swirls and stars (crystal-like) to symbolise cold. 


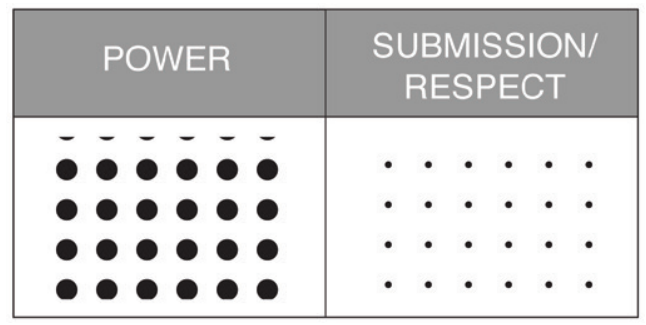

Fig. 161 - Subjective patterns: power \& submission/respect

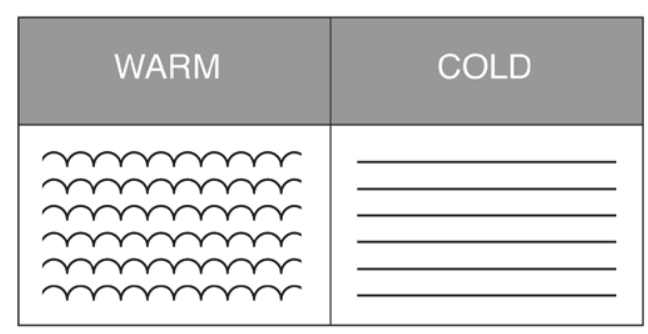

Fig. 162 - Subjective patterns: warm \& cold

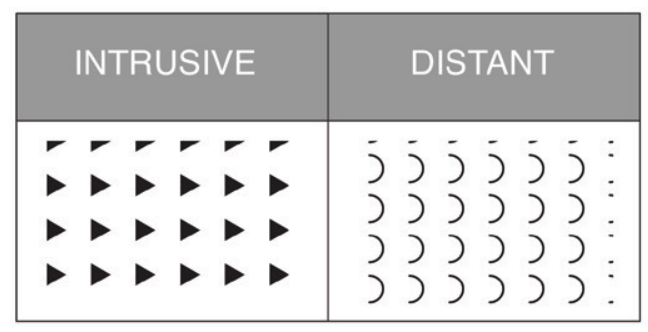

Fig. 163 - Subjective patterns: intrusive \& distant

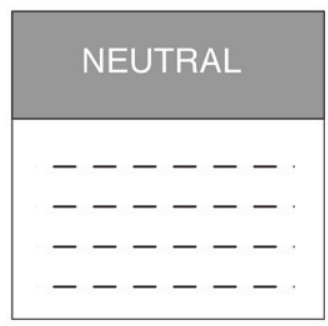

Fig. 164 - Subjective pattern: neutral
However, my final patterns are made up of a wave-like pattern to signify warmth and a straight parallel lined pattern to signify cold. The rounded shapes in the warm pattern makes it seem 'friendly' and in motion whereas the cold pattern is still, motionless (see fig. 162).

Intrusive/distant - I explored different symbol combinations using triangles that were facing each other and shying away from each other. I also experimented with using combinations of different square shapes. However, the chosen designs are: a pattern consisting of triangles facing forward to signify intrusiveness; and a pattern made up of small bent lines, that look as if they are shying away from the intrusive pattern, to signify distant (see fig. 163).

Neutral - To signify neutral I explored different arrangements of squares, and symbols that were made up of two equal halves, but the final and chosen pattern is made up of small, straight and equal lines, signifying a neutral state or emotion (see fig. 164). 


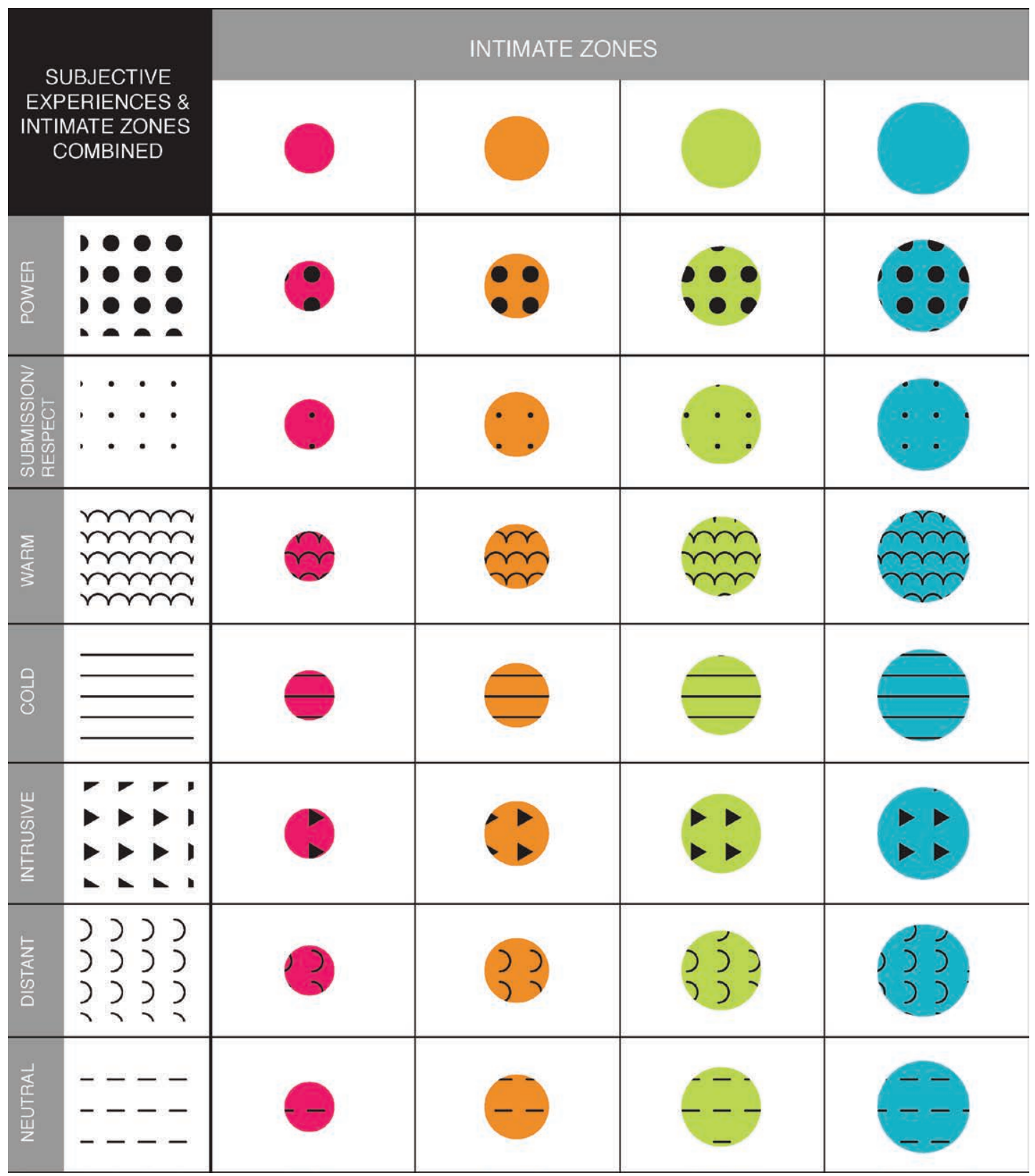

Fig. 165 - Diagram showing intimate zones in combination with patterns of subjective experiences 
Fig. 165 shows the patterns for subjective experience in combination with intimate zones. By combining them (two semiotic codes) in the same circle, the visualisation indicates how the use of personal space may influence how the greeting is experienced, or vice versa.

A challenge with the visualisation of subjective experiences is that the meaning of a greeting changes depending on whose perspective one visualises. I have explored three such perspectives: an outsider's view how, for instance, I as a researcher interpret a greeting between two people; how either of the two people greeting experience the behaviour of the other person and him/herself compared to the other (see fig. 166); and how each

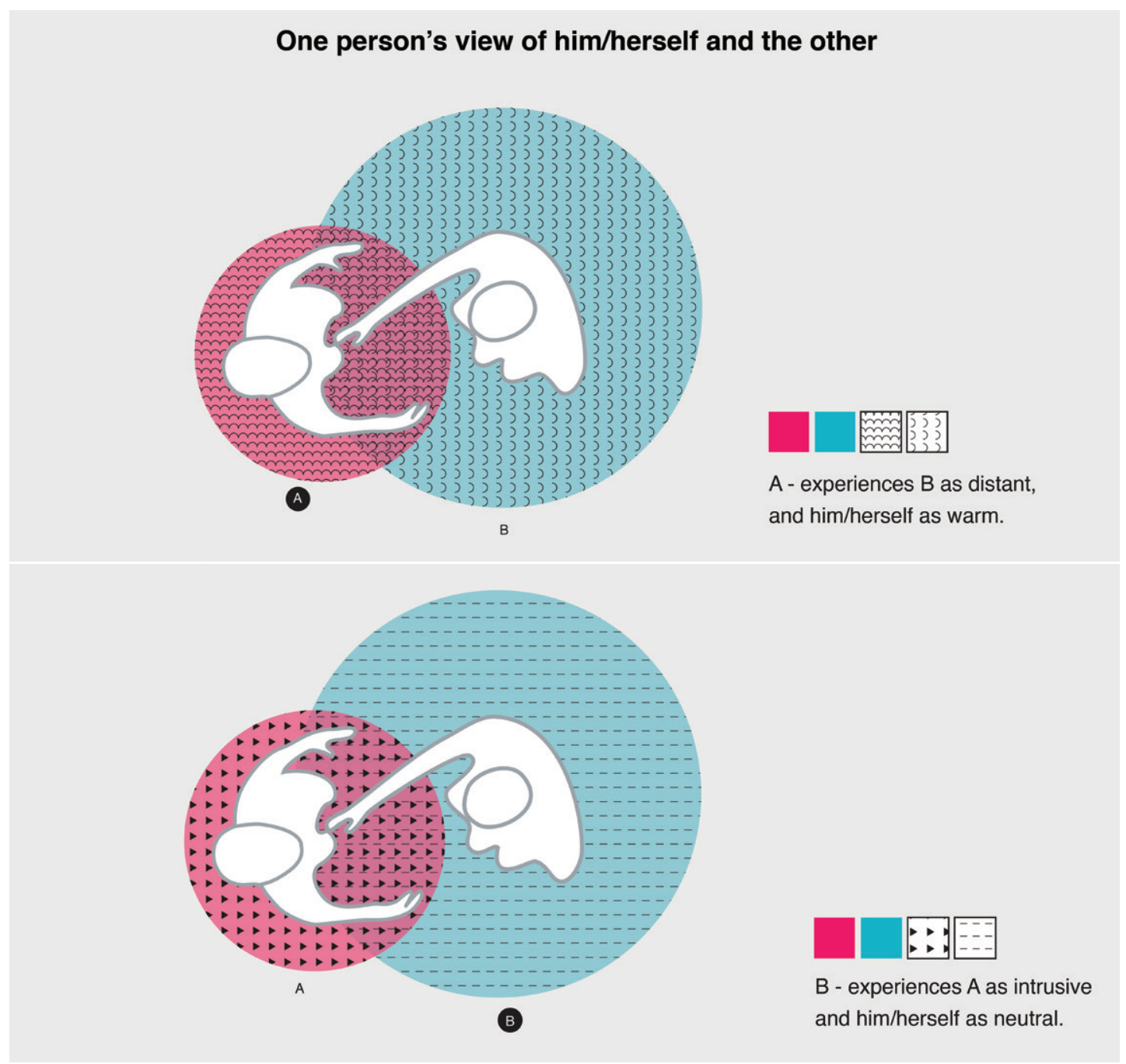

Fig. 166 - Visualising subjective experiences: the images show how person A (top) and person $B$ (bottom) experience themselves and the other person, during this moment of the greeting 


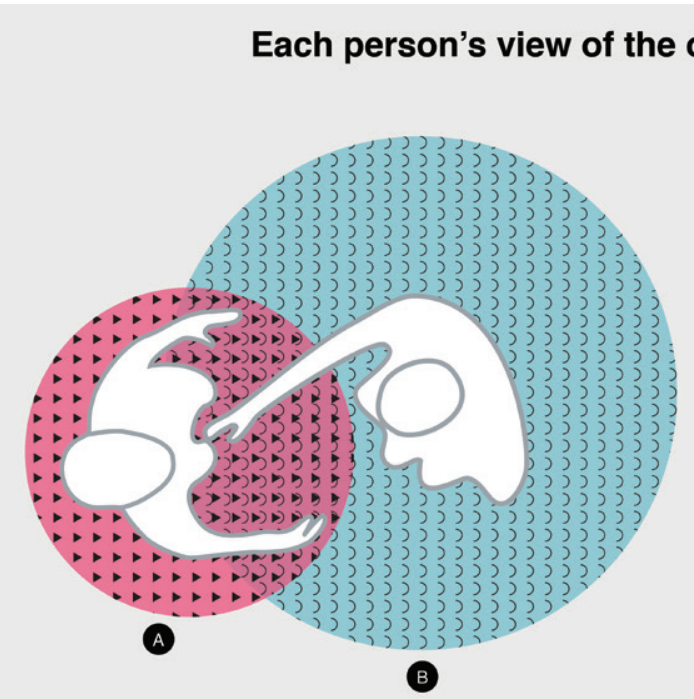

Each person's view of the other

в

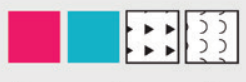

A - experiences $B$ as distant. $B$ - experiences $A$ as intrusive.

Fig. 167 - Visualising subjective experiences: the image shows how each person, $A$ and $\mathrm{B}$, experiences the other person during this moment of the greeting

person greeting experiences the other person (and not him/herself) during the greeting (see fig. 167). I use the third perspective in my visual system, because I wanted to highlight how people experience the behaviour of the person they interact with, and reveal how they each feel about the other's behaviour. This is particularly interesting in intercultural greetings because it can reveal reasons for cultural clashes such as 'invasions' of someone's personal space. It can illustrate how the lack of touch can be interpreted as cold and distant by some cultures, or bring attention to the fact that what is considered 'warm' and friendly in one culture may have the opposite connotation in another, and what is polite in one culture may be impolite in another, and so on.

In this chapter so far I have described in detail: the process of gathering participants; carrying out my fieldwork; the process of converting the video documentation into vectorised graphic representation; and, finally, the visual notation of nonverbal signals using colour codes and patterns. The technical settings and visualisation methods used in this process have been 
summarised in more technical detail in section 4.4 below. This process of visualisation can also be viewed in the video 'Process of Visualisation' in Appendix 13. In the later section 4.5 I will explain how all these elements and visualisations have been organised into interactive greetings diagrams. I will also explain the navigation of and use of the system's interface.

\subsection{Technical Process and Settings: An Overview}

Summary of Stages and Techniques of Visualisation:

1. FILMING - Greetings are filmed from the front using a Canon 7D camera (capturing the gesture, postures and two bodies in relation to each other), from an overhead view using a GoPro HERO (Original) camera (capturing the surrounding space of the two people greeting), from the right using a Panasonic Lumix DMC-FZ28 camera and from the left using a Fujifilm Finepix HS10 camera (capturing the faces and upper bodies of the two people greeting). Final Cut Pro 7.0.3 is used for video editing.

Video archiving: Original greeting footage is trimmed and archived without changing the original settings or frame rates.

Video editing: Videos are edited - the clips are trimmed and colour corrected - and clips with different video angles, primarily overhead and front views, are combined in one screen, using Final Cut Pro. Colour coded text labels are also added to the videos to indicate whether the relationship between the two people greeting is real or enacted. 
The following settings were used for exporting the videos:

Compression: H.264

Quality: High

Frame rate: 24

Key frame rate: 24

Frame reordering: yes

Encoding mode: multi-pass

Size: $1280 \times 720$

\section{SELECTION OF KEY MOMENTS - The greeting videos are} converted into sequences of still images - like chronophotography in order to select and analyse key movements and moments of the greeting gestures.

Technical settings:

Each greeting video is exported as an Image Sequence from QuickTime 7, with the following export settings:

Format: PNG

Frames per second: 4

3. VECTORISATION - The image sequences are then vectorised using the live trace and pencil tools in Adobe Illustrator CC. For the purpose of time-saving, unless there is particularly rapid movement in the original, only every other frame ( 2 frames per second) is vectorised. Each animated greeting is shown at $50 \%$ of its original speed, to make the viewing of the visual analysis easier and clearer: this means that each image should last for 12 frames ( 0.5 seconds) when animated (rather than 6 frames at full speed), and that for greetings in which every other image is deleted, each image should last for 24 frames (1 second). 
4. ANIMATION - The vector graphics are then animated in Adobe Flash CC using frame-to-frame animation. In some instances, if there is no movement, or no relevant movement between two vector images, one image could last for 24 frames, with the other one left out, or, if there is no movement of relevance between 3 frames, two of them can be left out and one frame can last for 36 frames, and so on. This could be the case if there is a long gap where the two people greeting engage in conversation as part of the greeting, or if the two people are performing say a hug that lasts several seconds, or similar.

Intimate zones, touch, space in-between \& subjective experiences: Colour coded intimate zones are animated onto a layer sitting below the greeting animation, using Flash animation. Space in-between is also animated onto layers sitting below the greeting animation using a masking layer. Touch is animated directly onto the greeting animation, using the brush tool, set to the function 'brush inside'. The patterns for subjective experiences are animated onto a layer above the intimate zones, and below the greeting animations. Each greeting is animated manually as there is of yet no function to have them created through a more automated process using Adobe Flash.

\section{INTERACTIVE DIAGRAMS - While all the visual aspects of} the diagrams are created as graphic symbols and Flash timeline animations, the interactive elements and navigation of the system is programmed using ActionScript 3.0 (see Appendix 11 for code). Using ActionScript programming has enabled me to present the animations in dynamic, interactive diagrams, in which the user can select culture, greeting category, and gender combination, and then click to select what aspect of that particular greeting they want to view - i.e. the video, intimate zones, touch, space in-between, or subjective experiences. 


\subsection{The System for the Visual Notation of Greetings: Interactive Interface}

Interactive Diagrams

Once all the visualisations/animations of the greetings documented during the fieldwork were completed, I needed to find a way to present them. It was important to design an interface that would enable them to be easily viewed and compared. To do this, I organised miniature still vector images of the greetings into three different diagrams: social greetings; family greetings; and cross-cultural greetings. Each still greeting image in these diagrams is a clickable button that represents and generates access to a specific greeting and all the animated visual analyses of that particular greeting, as I will show in more detail below. The diagrams were made interactive using ActionScript 3.0 (programming language) in Adobe Flash. Creating interactive diagrams which provided access to the animated analyses of each greeting was an important development in my current project: the interface for Kinesics was only interactive in so far as it allowed the user to choose between different diagrams, and between front and overhead views in the still images they contained.

The current interface is organised systematically through many levels of interactive choices. The first choice the user is presented with is between three buttons - one for each of the three greeting categories: social greetings, family greetings, or cross-cultural greetings (see fig. 168). Once the category is selected, the miniature stills of each greeting within that category become visible and are organised by culture, greeting situation, and gender. For example, social greetings include three greeting situations: greeting close friends, acquaintances, and strangers. Next they are organised by gender combination within each of these three situations: female-to-female, male-to-female, and male-to-male. Fig. 169 shows the social greetings diagram, with explanations of how to navigate it, fig. 170 shows the family greetings diagram, and fig. 171 the cross-cultural greetings diagram. 


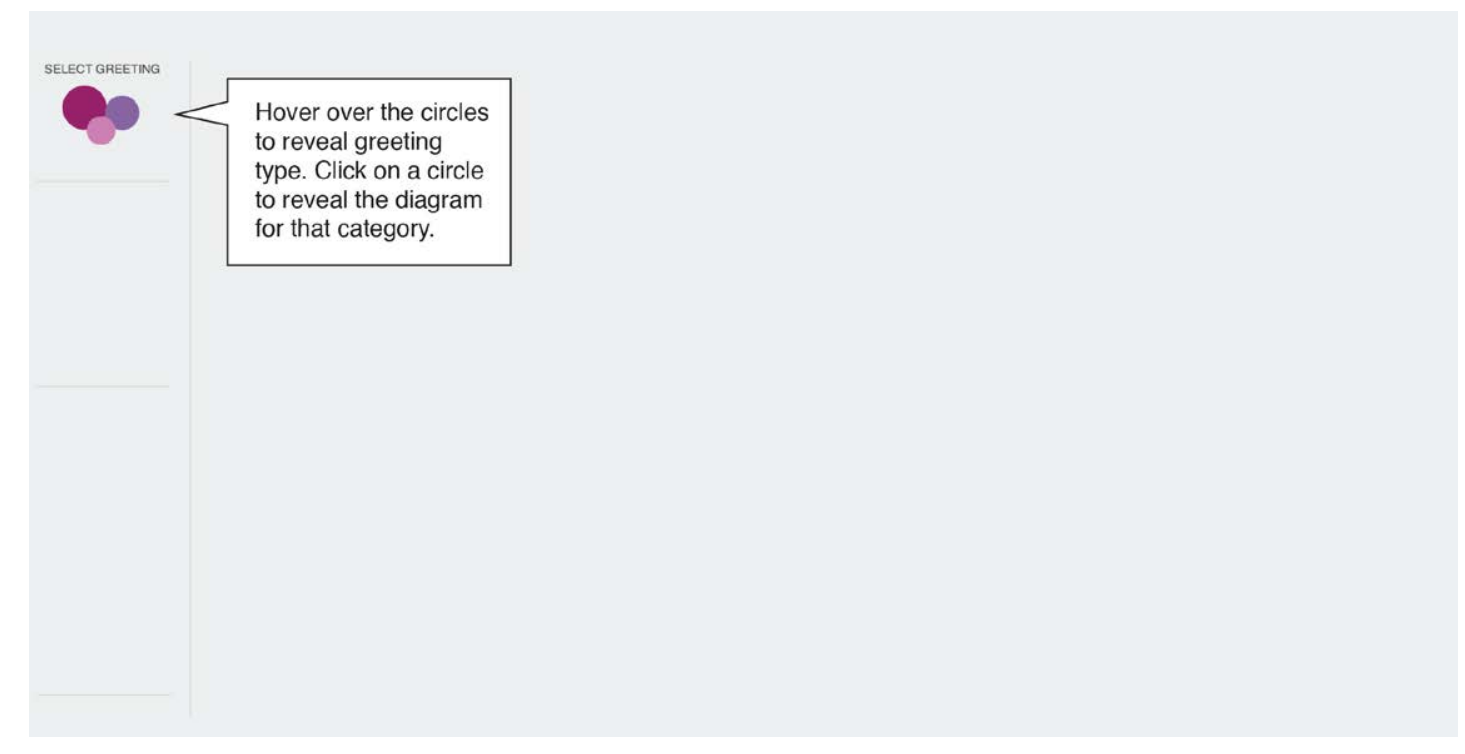

Fig. 168 - Interactive diagrams interface explained: starting screen

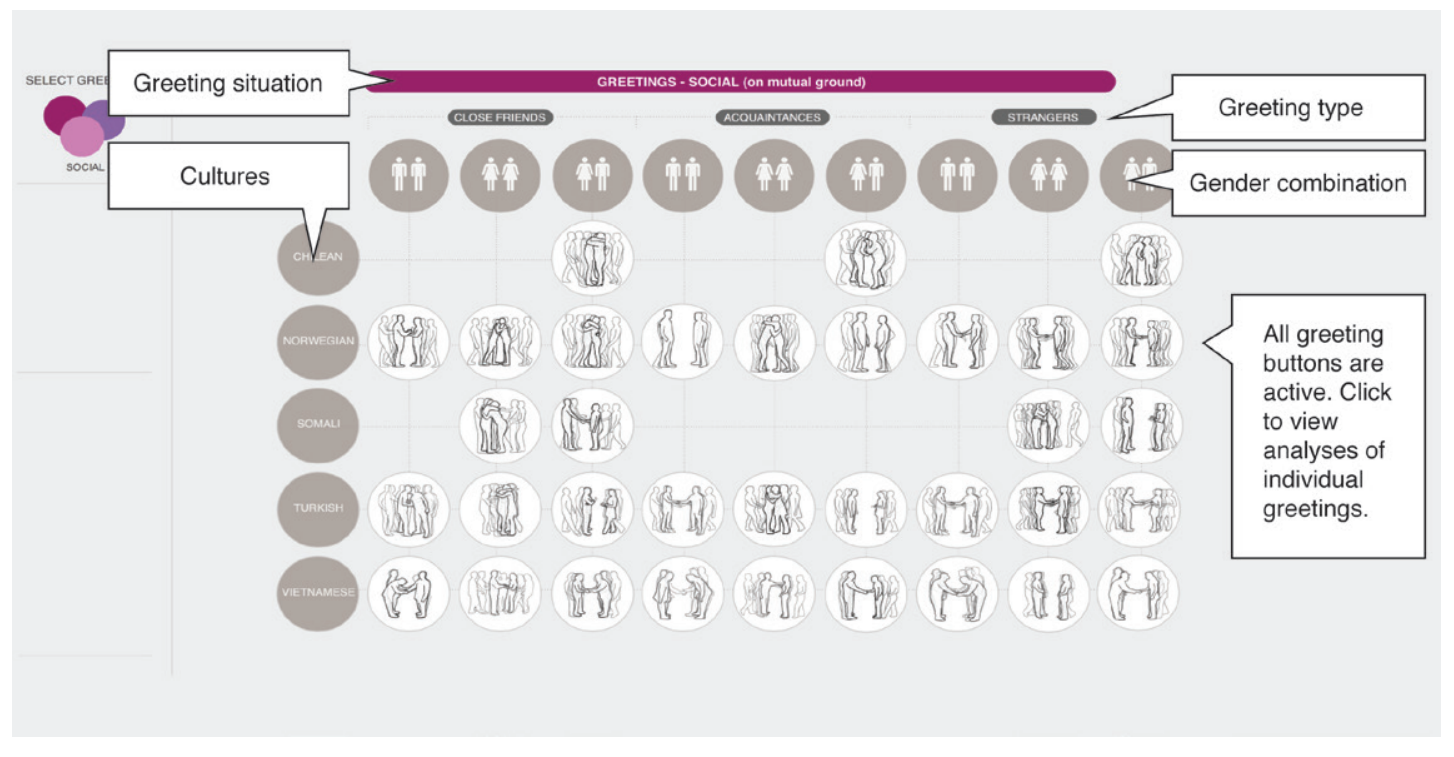

Fig. 169 - Interactive diagrams interface explained: social greetings diagram 


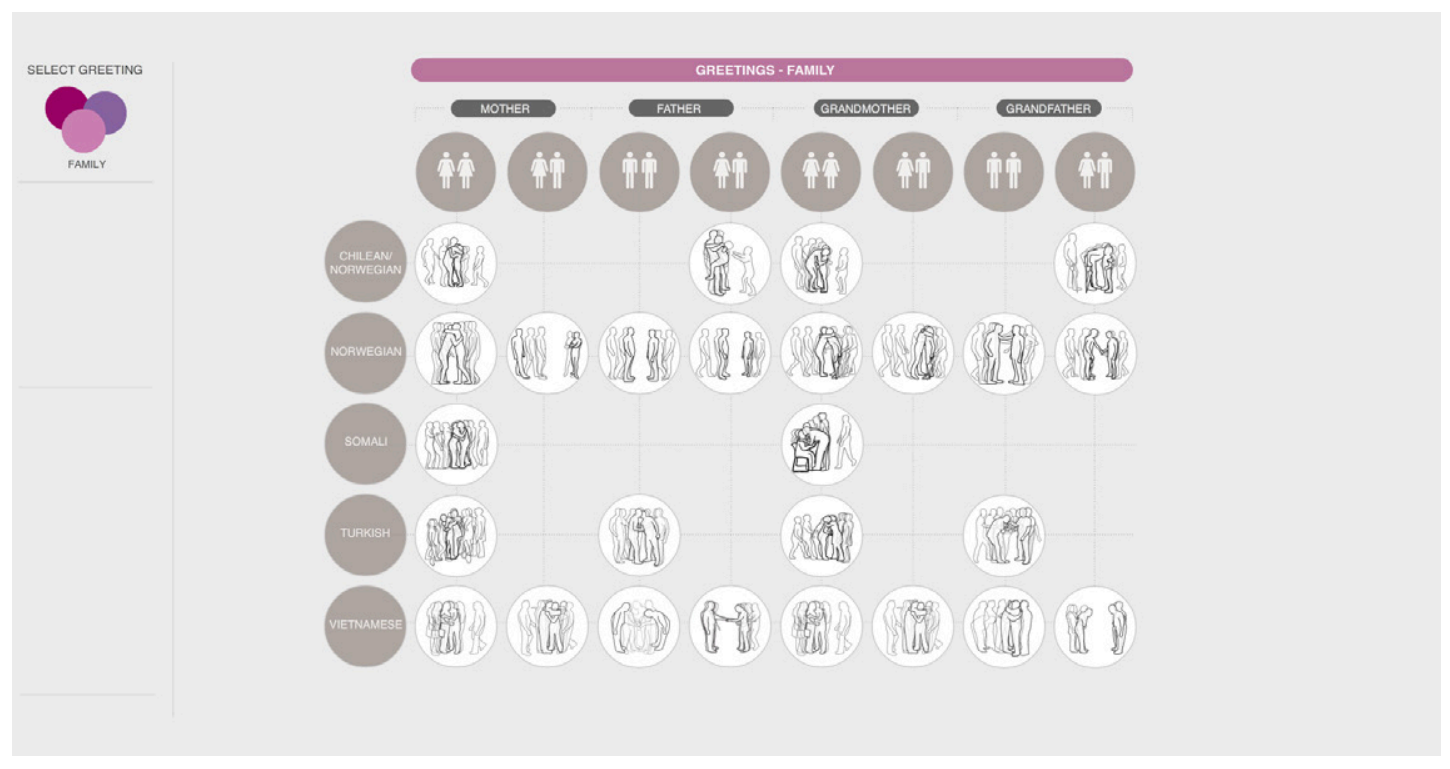

Fig. 170 - Interactive greetings diagram: family greetings

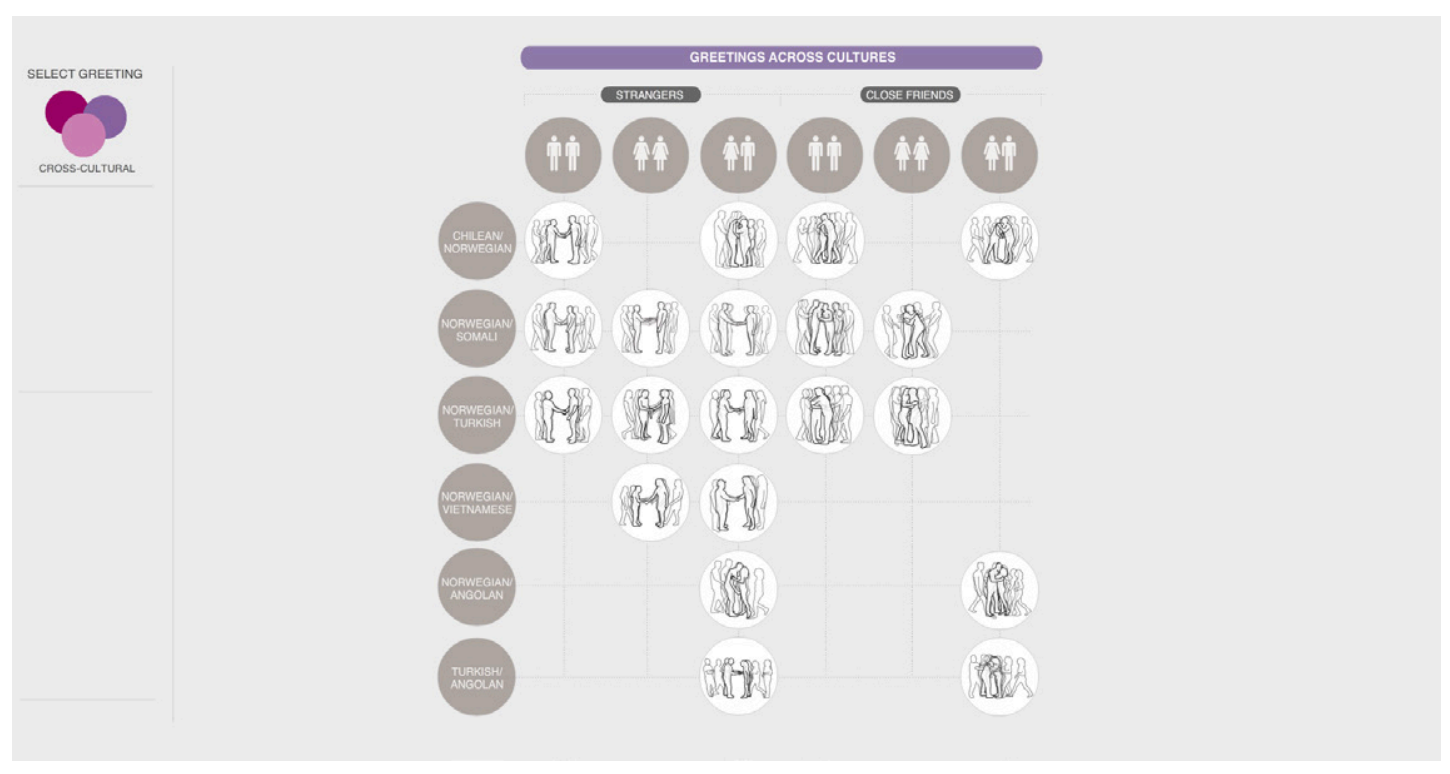

Fig. 171 - Interactive greetings diagram: cross-cultural greetings 
In my previous system each greeting in the diagram was represented by a still vector image referencing one moment of the greeting. However, further consideration led to the realisation that not all greetings that were documented can be represented by one key moment. A greeting often consists of a number of key gestures, for example: a handshake followed by a hug, or a kiss on the hand followed by a kiss on the forehead. In such cases it would not be right to select just one of these moments as the most important of the greeting - for example the hug rather than the kiss. So I explored how to show movement in a still image by layering vectors from the complete sequences. The revised method is a multi-layered still vector image, which visualises the key moments of the greeting (see fig. 172). This new method represents the greeting as a more fluid and dynamic sequence.

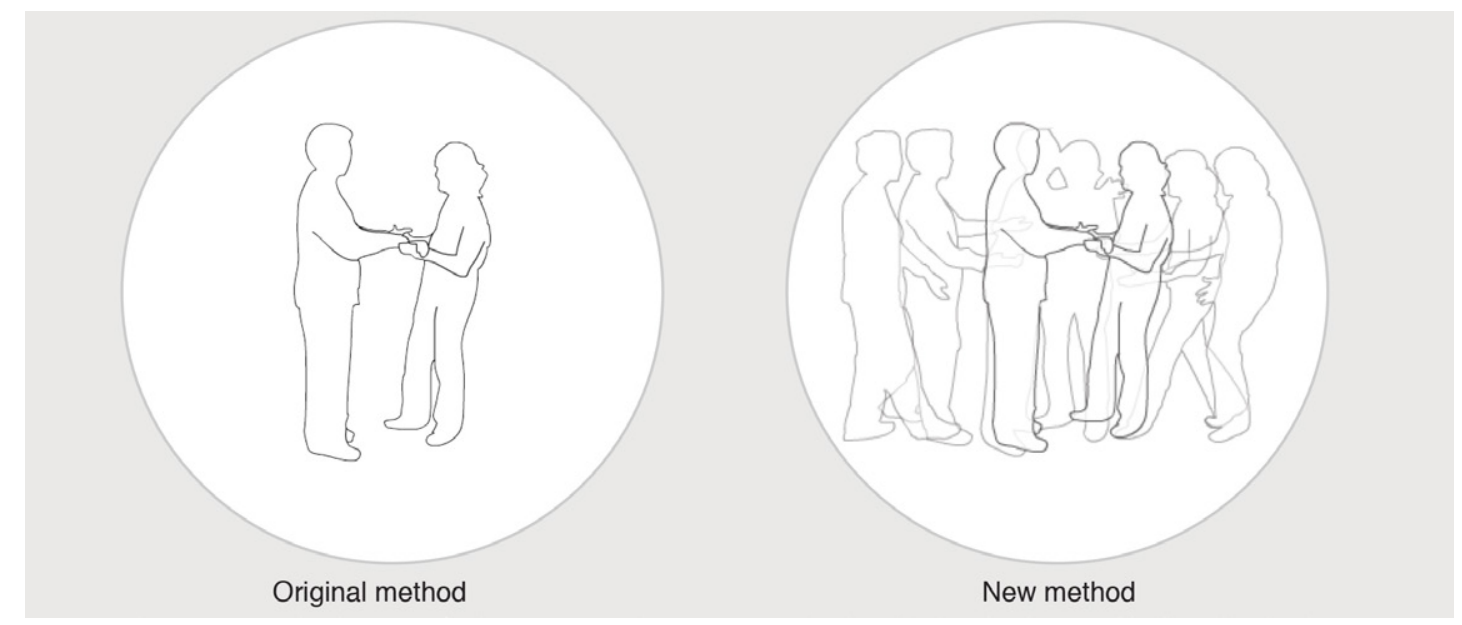

Fig. 172 - Original and new method for visualising a greeting in one still image

The interactive diagrams are set up in a way that enables the user both to study broader tendencies of, and differences between, greetings within and across cultures and genders, and to study individual greetings in the form of annotated animations. Once a greeting situation is selected, the layout of the diagram enables the viewer to compare greetings, in static form, within cultures across different greeting situations and gender combinations (see fig. 173), and across cultures within greeting situations and specific gender combinations (see fig. 174). 


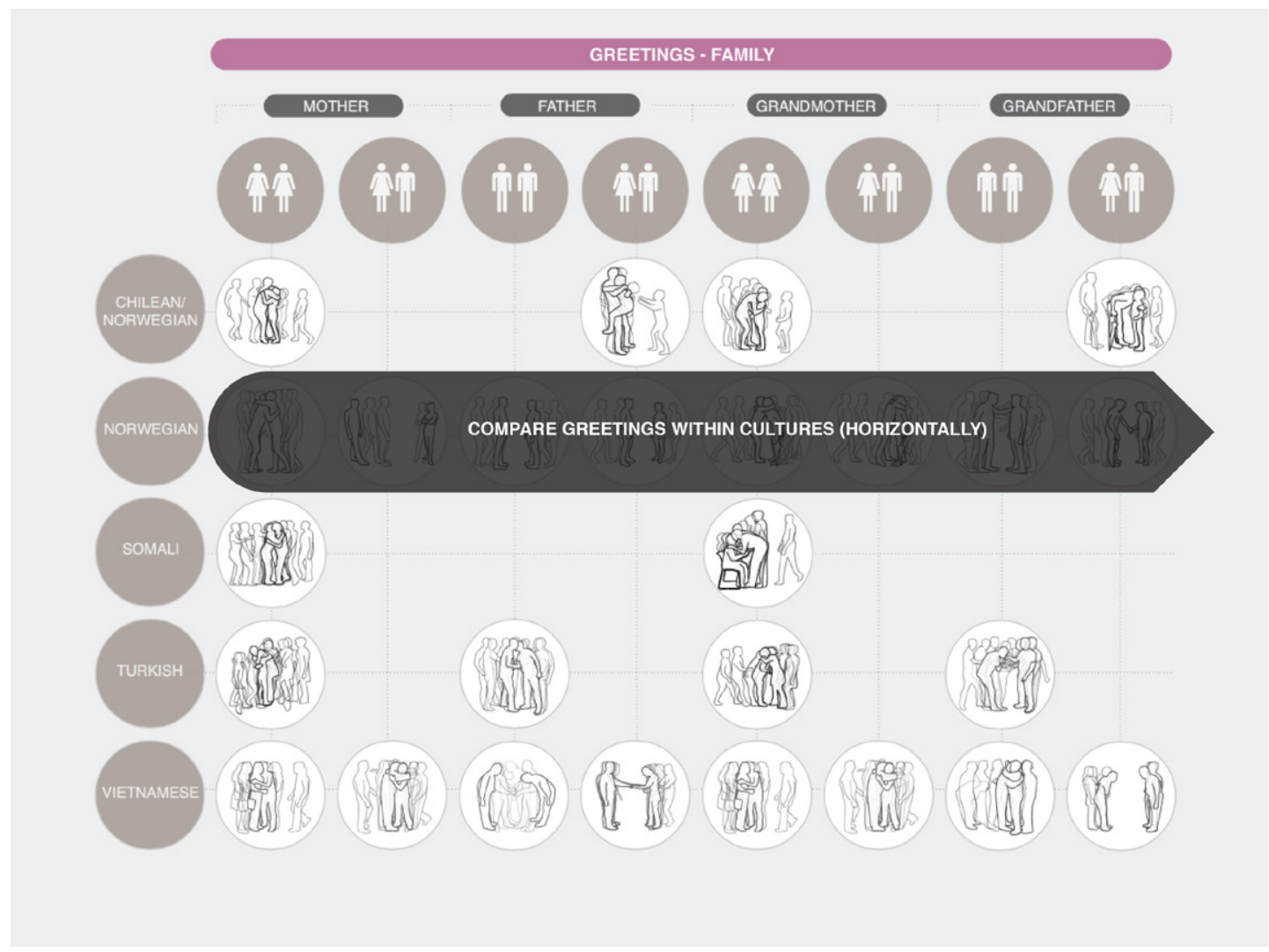

Fig. 173 - Interactive greetings diagram: compare greetings within cultures horizontally

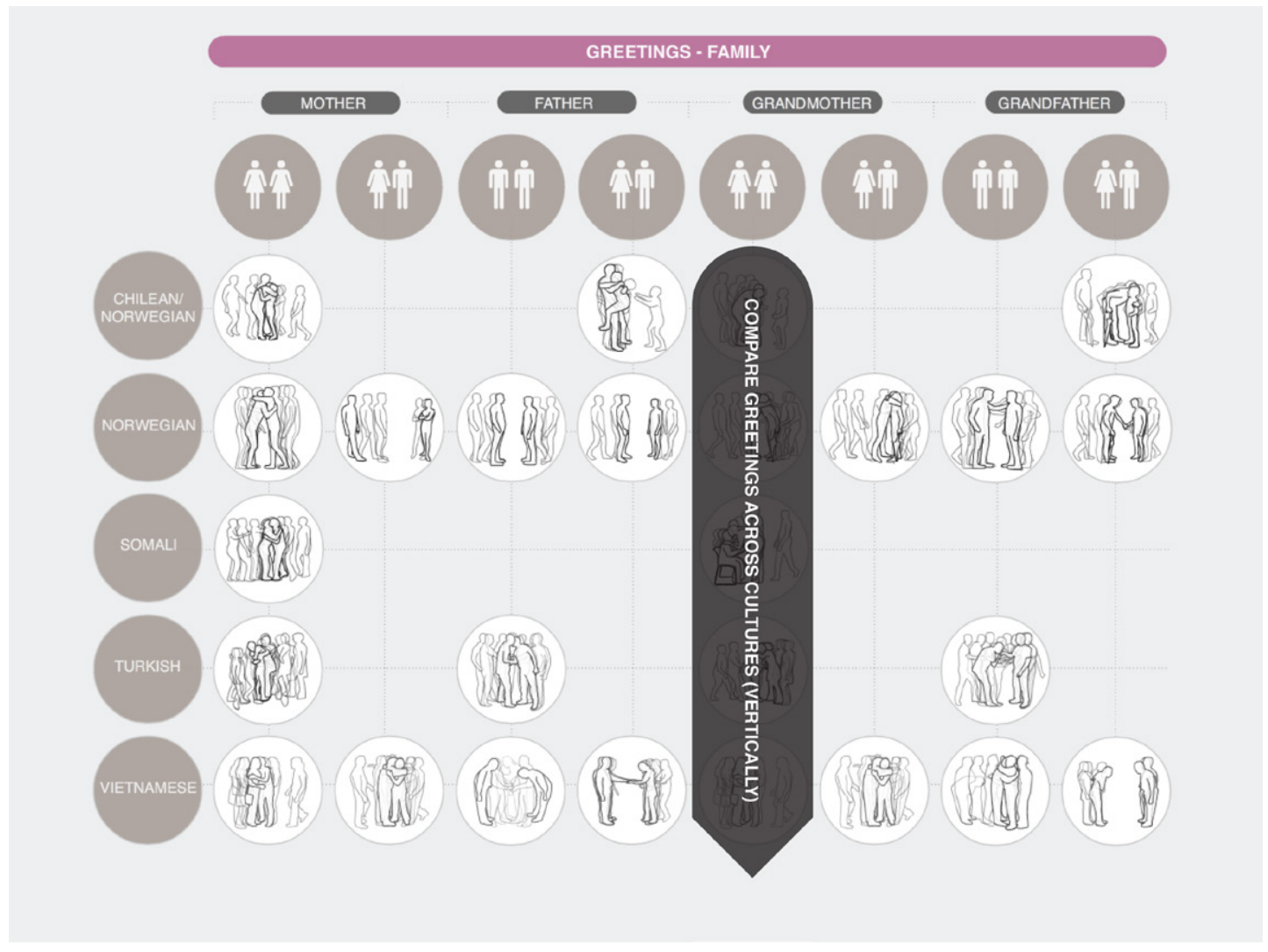

Fig. 174 - Interactive greetings diagram: compare greetings across cultures vertically 
The user can also explore and identify whether there are greeting types that are more common across cultures and situations, or greetings that stand out as being very different from the others. To illustrate this point I have highlighted some of the patterns that I have found across cultures and across the genders in figs. 175-179. Fig. 175 shows that the majority of intracultural social greetings between strangers that were recorded, involved a handshake. The same is seen in cross-cultural greetings between strangers, across the genders (see fig. 176). However, in cross-cultural greetings between close friends, some form of kissing or embrace was done in all greetings that were recorded (see fig. 177). The same greeting type was common in family greetings across cultures (see fig. 178). In contrast to this are a few recorded greetings that do not involve any touch at all (see fig. 179).

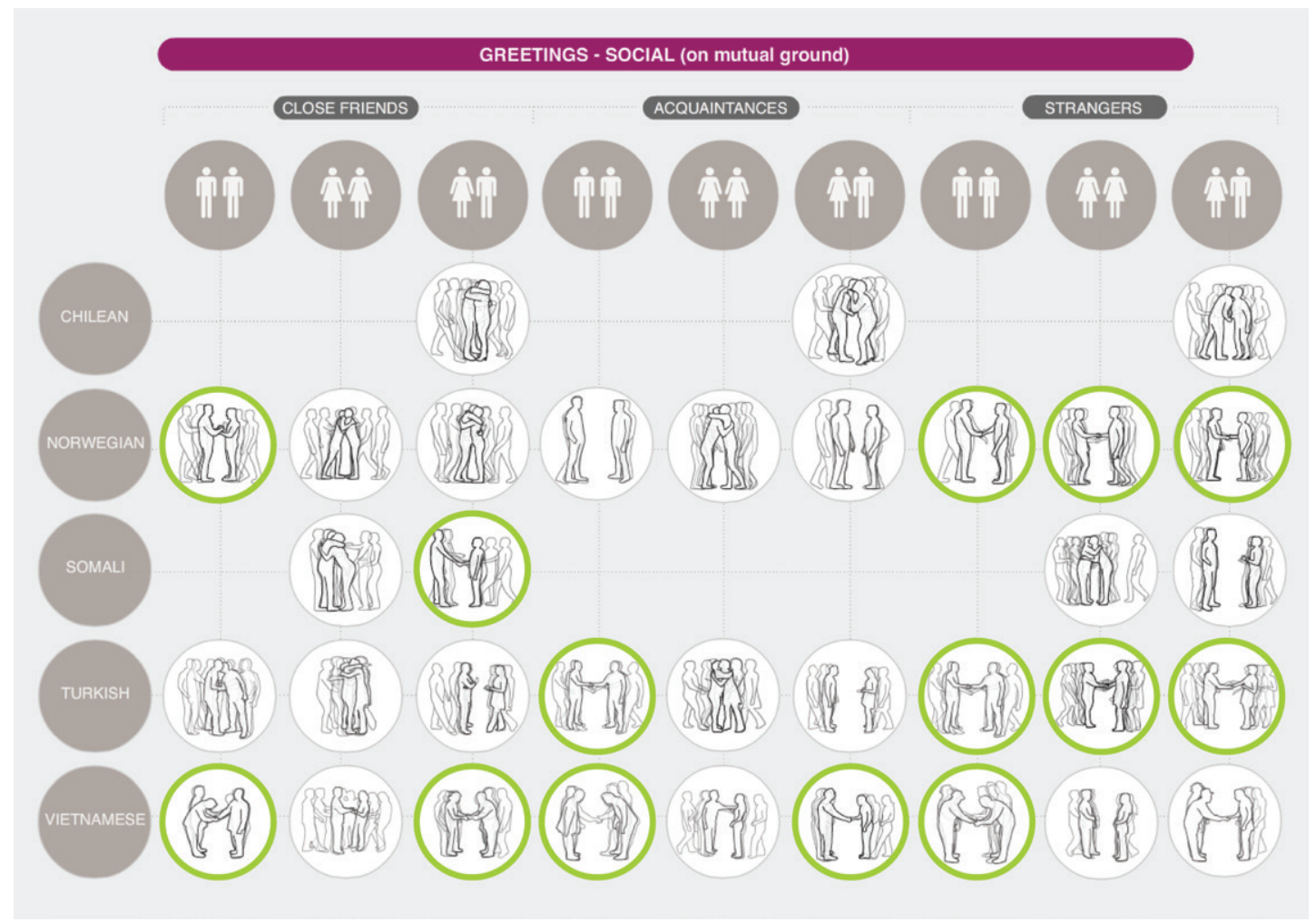

Fig. 175 - Interactive greetings diagram: Social greetings - highlighted in green are handshake greetings 


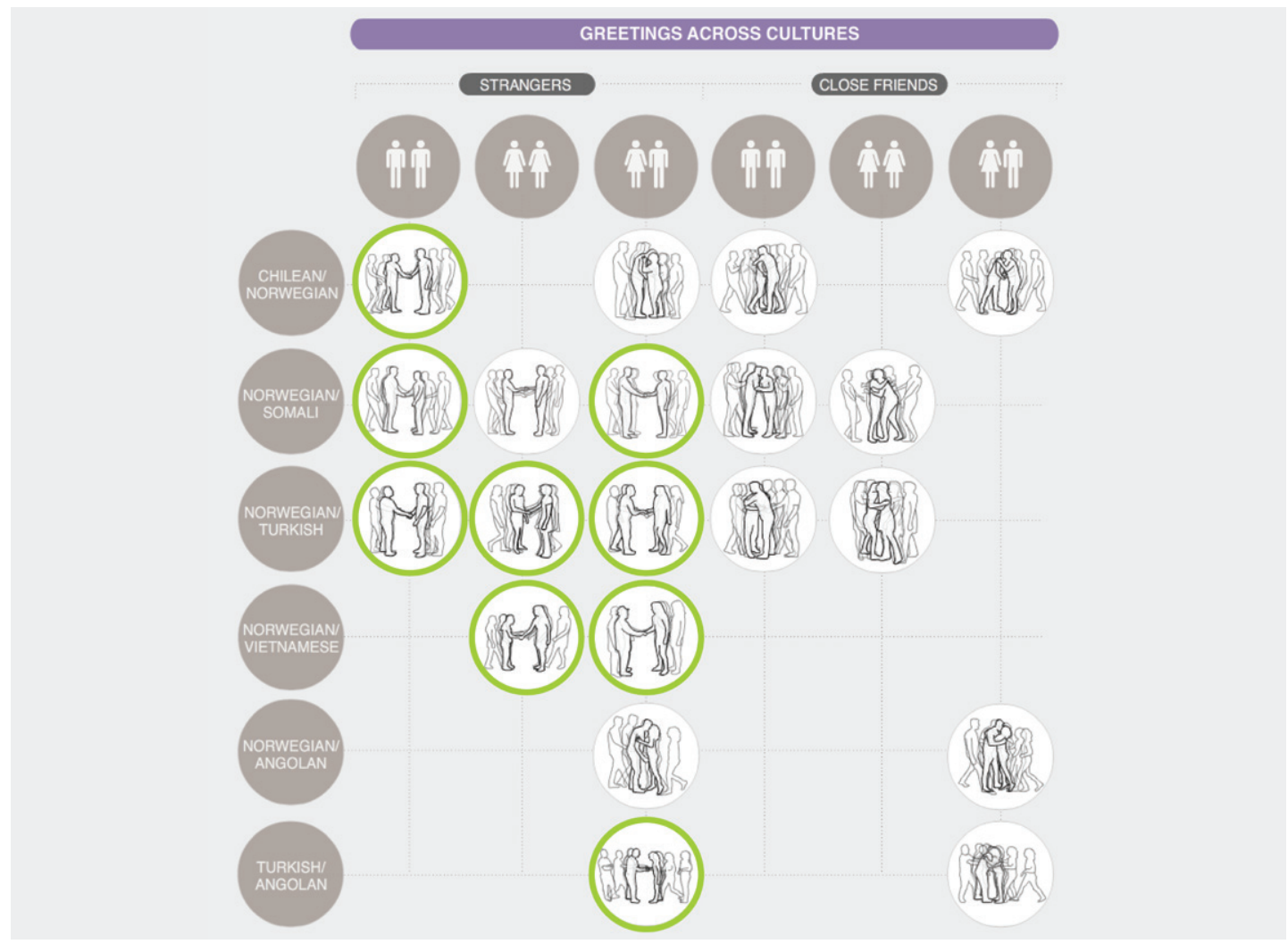

Fig. 176 - Interactive greetings diagram: Cross-cultural greetings - highlighted in green are handshake greetings

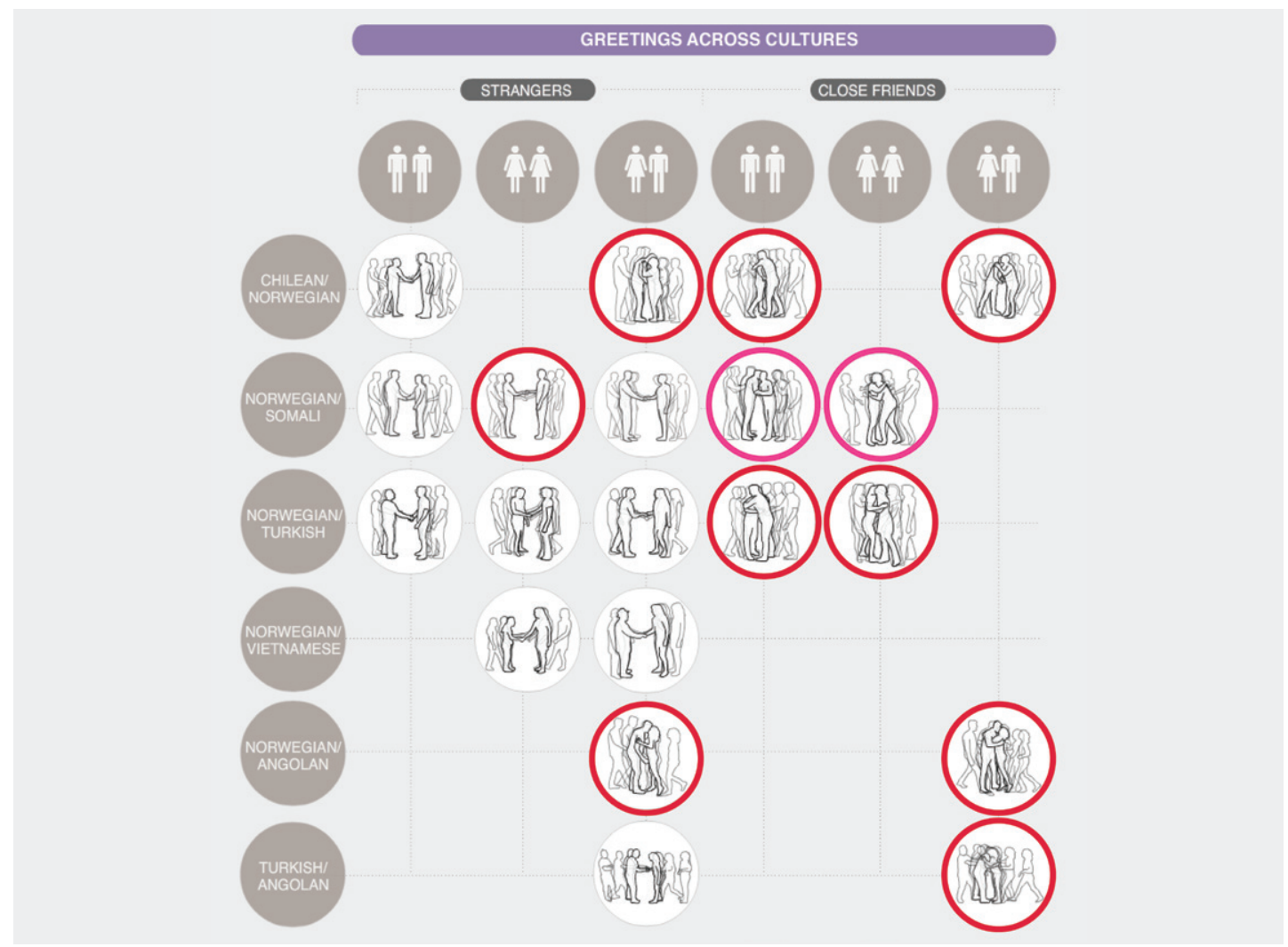

Fig. 177 - Interactive greetings diagram: Cross-cultural greetings - highlighted in pink are embraces/hugs, and in red are greetings involving kisses on the cheeks 


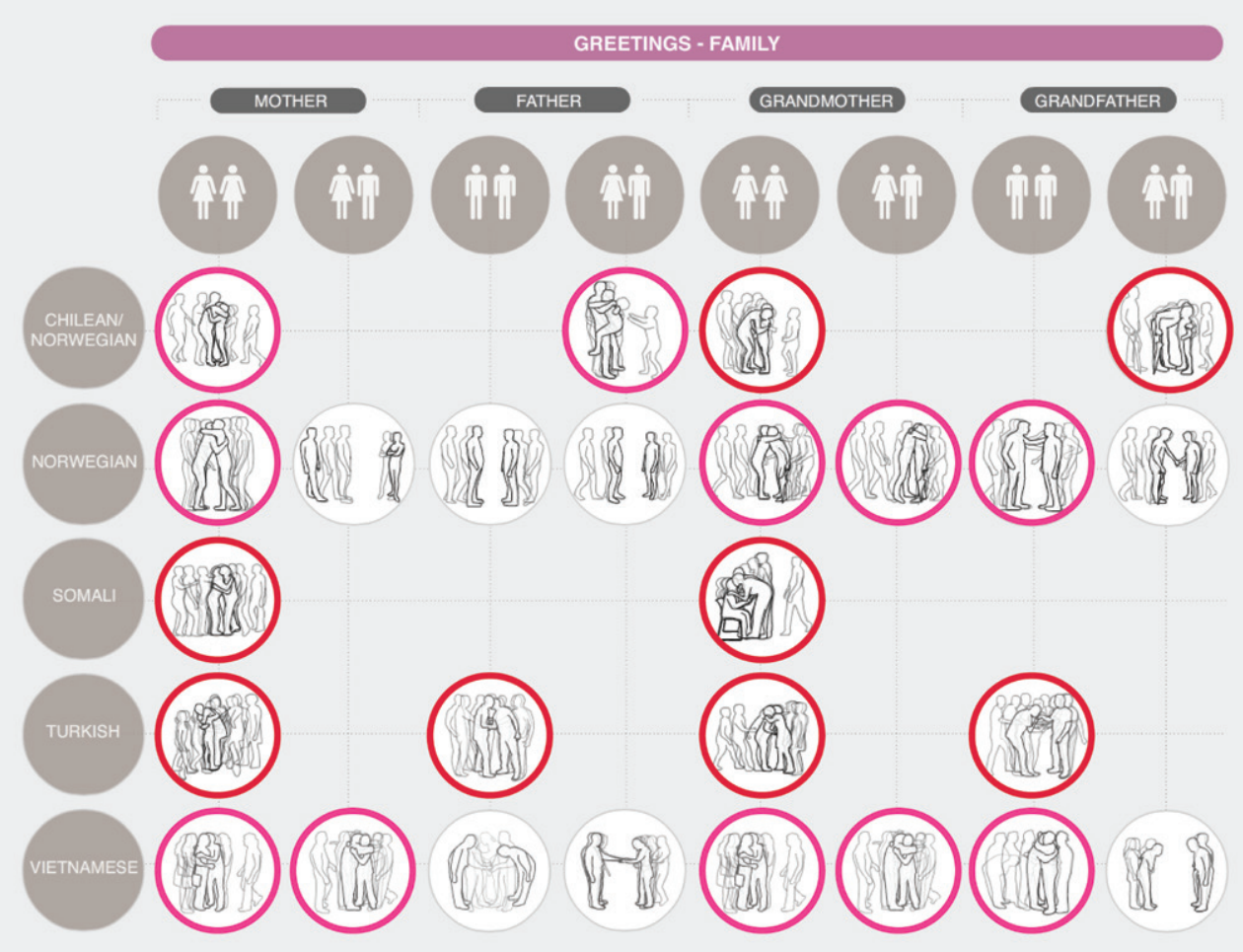

Fig. 178 - Interactive greetings diagram: Family greetings - highlighted in pink are embraces/hugs, and in red are greetings involving kisses on the cheeks

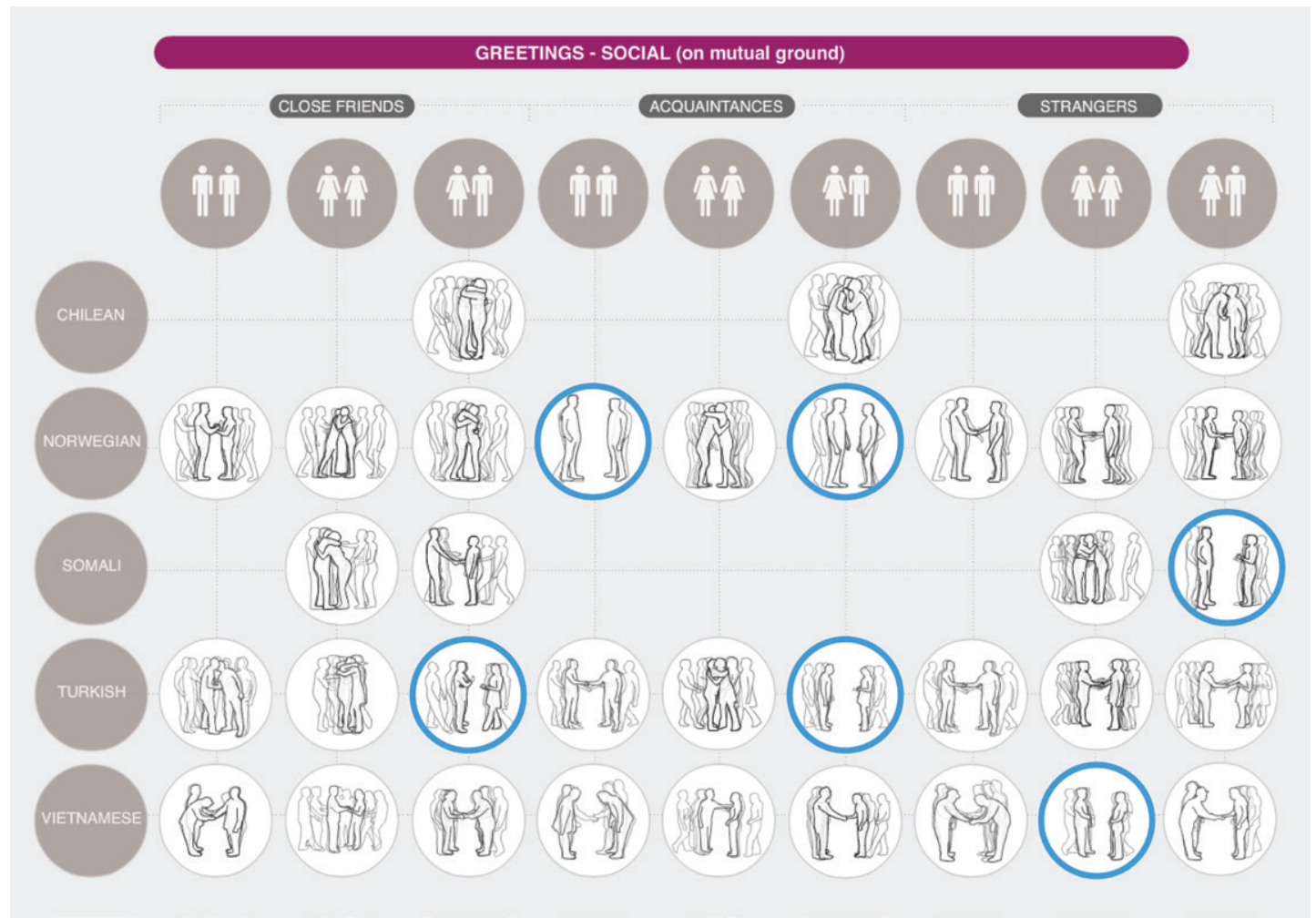

Fig. 179 - Interactive greetings diagram: Family greetings - highlighted in blue are greetings in which there is no physical contact between the people greeting 
The diagrams and examples shown above give an initial overview of the variety of greetings and cultural diversity that exists in Stavanger. The design of the interface also makes it possible to view specific greetings and their visual analyses. Each greeting in the diagram can be clicked on, and once a specific greeting is selected, the diagram area fades out and a vector animation of the selected greeting fades in on the 'stage' area of the interface (see fig. 180). A menu and information window also appear on the left side of the screen. The three greeting category buttons at the top left corner remain active and bring the user back to the three diagrams if clicked on. Just below these three buttons is the information window. This window displays text describing the greeting situation, culture(s), and gender combination currently being viewed. Below the information window is a 'select view' window with buttons and one text field (see fig. 181). The text field is empty until a view is selected.

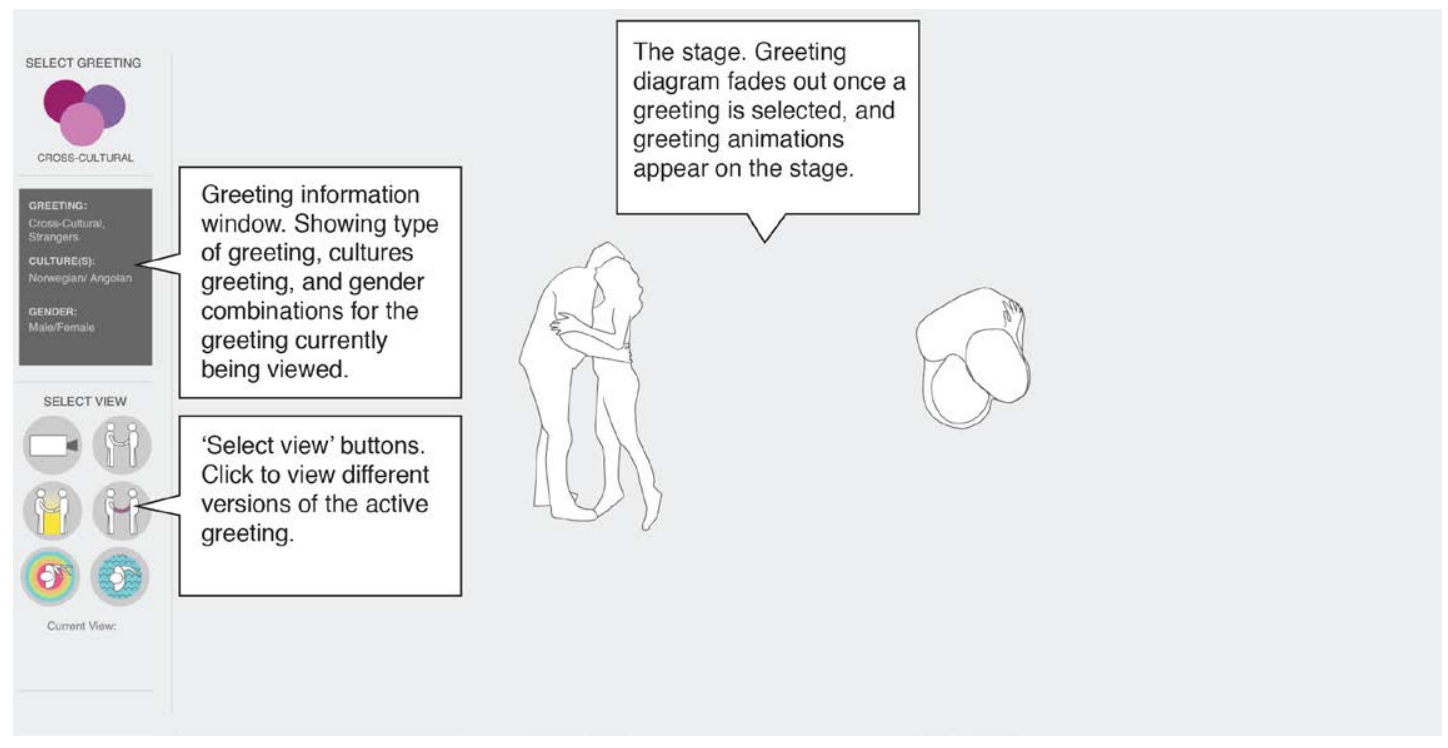

Fig. 180 - Interactive diagrams interface explained: a specific greeting has been selected 


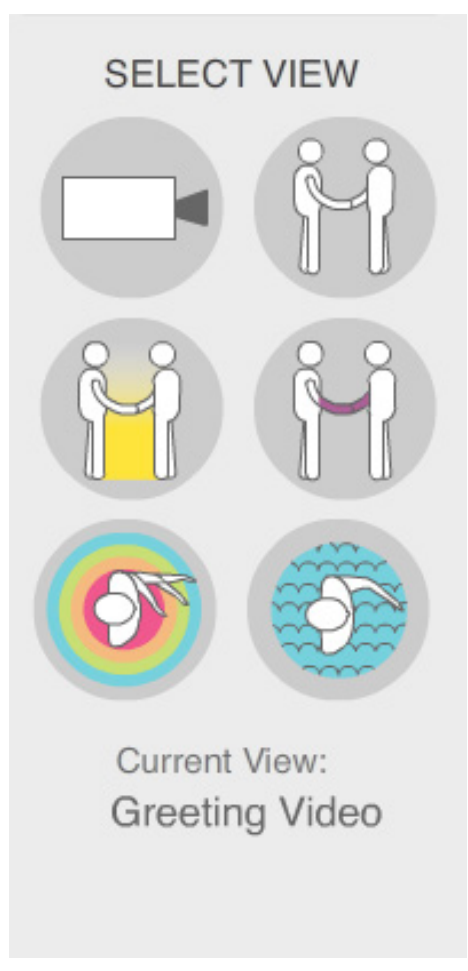

Fig. 181 - Menu for different greeting views
The 'select view' menu presents the user with the following six choices of how to view that specific greeting (starting top-left to right):

1. The original greeting video

2. The greeting as a vector animation

(also the starting view of each greeting)

3. Space in-between

4. Touch

5. Intimate zones

6. Subjective experiences of the greeting

Below I use a greeting between two strangers - a Norwegian male and an Angolan female, to show examples of each of the six views available for each greeting. All videos and animations show two perspectives of the greetings: front and overhead. The opening view of the greeting is a vector animation (see fig. 182). Button number one opens the original greeting video of the

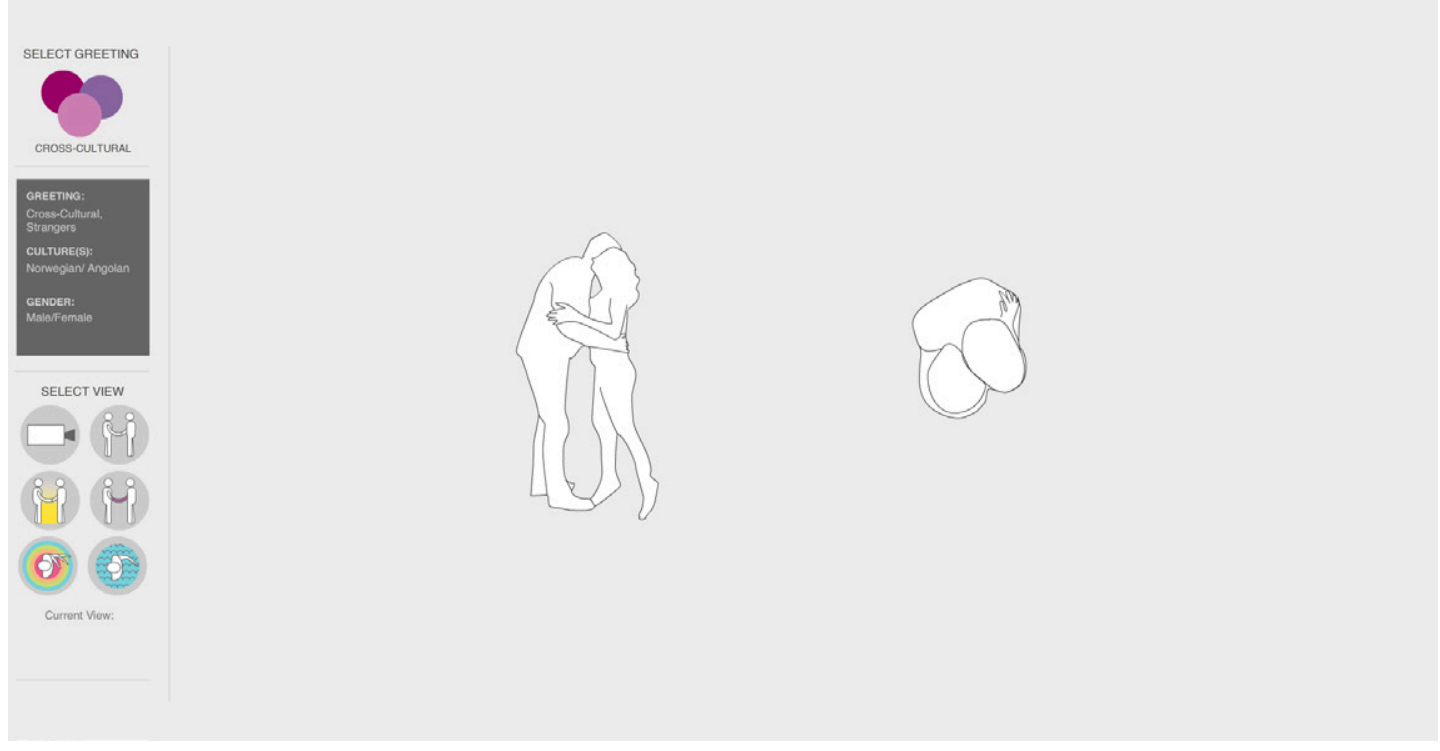

Fig. 182 - Interactive greetings diagram: showing vector animation of a crosscultural greeting between a Norwegian male and an Angolan female, who are strangers 
two participants greeting in the studio (see fig. 183). Sound is available in this version of the video. The video also includes colour-coded text labels discussed above. The second button brings back the initial view, the vector animation (see fig. 182).

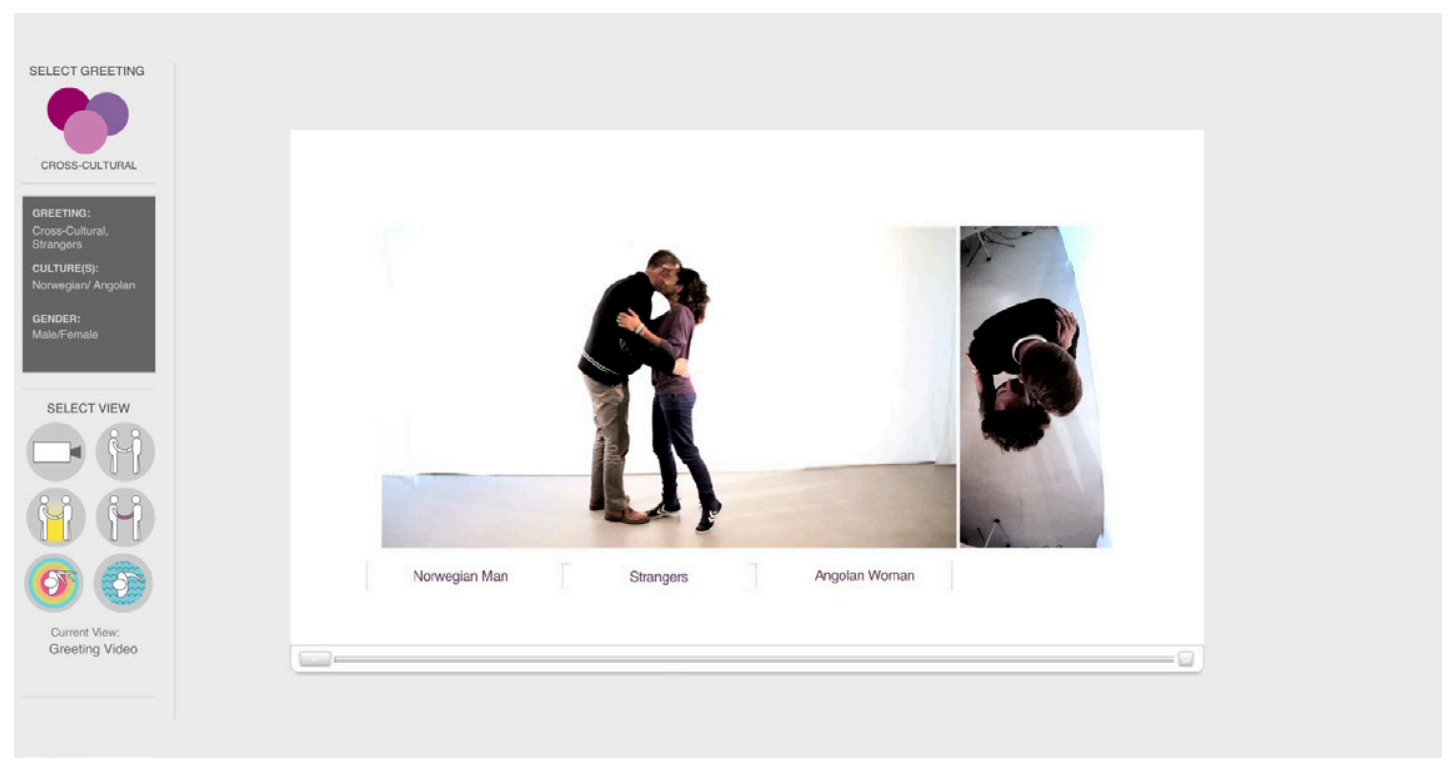

Fig. 183 - Interactive greetings diagram: showing original greeting footage of a cross-cultural greeting between a Norwegian male and an Angolan female, who are strangers

The third button reveals a version of the vector animation, which brings attention to the space in-between the two people greeting (see fig. 184). Highlighted in a yellow gradient, this illustrates the fluctuating amount of space between them during the greeting. The fourth button displays a version of the vector animation annotating levels of touch/physical contact between the two people greeting (see fig. 185). The touch view lets the user follow how the amount of touch, highlighted in purple, increases and decreases during the greeting sequence, and reveals the level of physical contact the greeters engage in during this greeting. It also brings attention to which parts of the bodies are in contact, usually the top half of the bodies. 

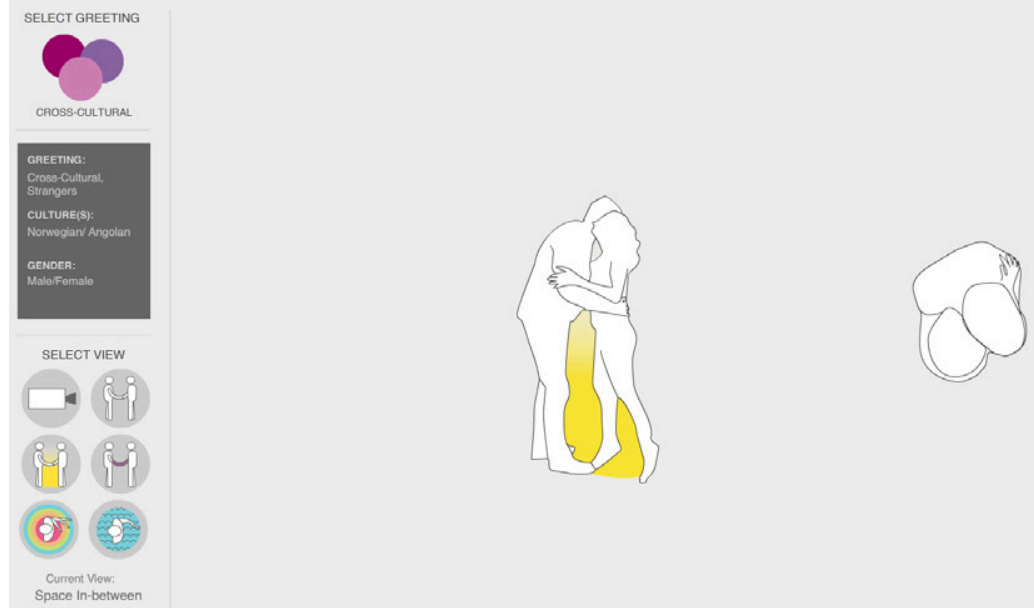

Fig. 184 - Interactive greetings diagram: showing vector animation, with space inbetween highlighted, of a cross-cultural greeting between a Norwegian male and an Angolan female, who are strangers

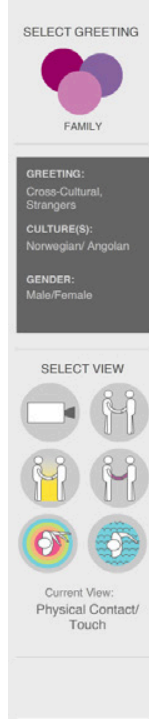

Fig. 185 - Interactive greetings diagram: showing vector animation, with touch highlighted, of a cross-cultural greeting between a Norwegian male and an Angolan female, who are strangers 
The fifth button reveals the animation showing the intimate zones (see figs. 186-187). As the two people approach each other to greet, both the size and the colour of the circles change, depending on how close the greeters are to each other. Fig. 186, for instance, shows the Norwegian man approaching with a handshake, intending to keep a lot of distance, in the blue zone, while the Angolan woman continues to move towards him to give two kisses on his cheeks, keeping a much smaller personal space. The animations show that during the greeting sequence sometimes (as in the images below) greeters move through a range of non-synchronised intimate zones. In other greetings documented, the two people that are greeting move in a more synchronised way, both maintaining the same relation to personal space at the same times of the greeting. When comparing greetings these intimate zones also show cultural, situational and gender differences in how much personal space is attained during the different greetings.

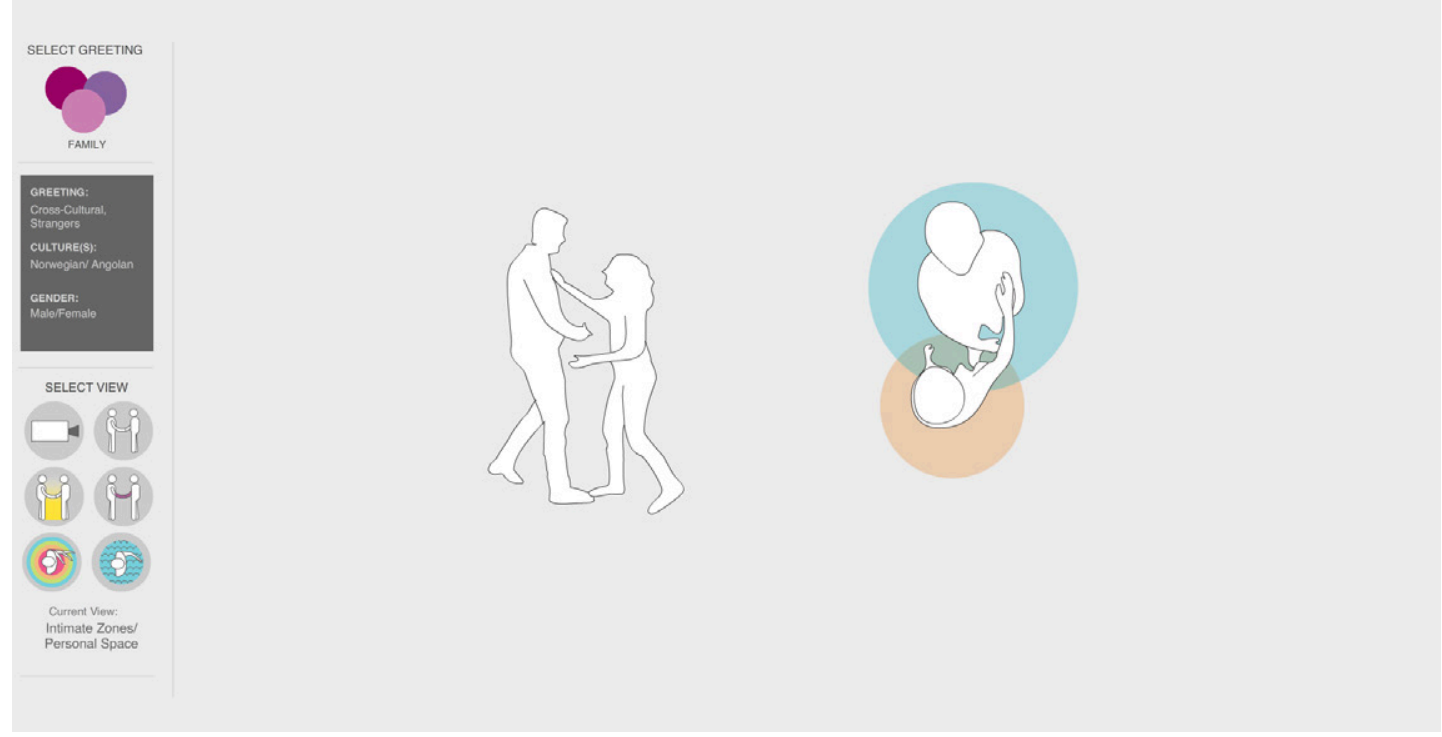

Fig. 186 - Interactive greetings diagram: showing vector animation, with colour coded intimate-zones/personal space, of a cross-cultural greeting between a Norwegian male and an Angolan female, who are strangers 


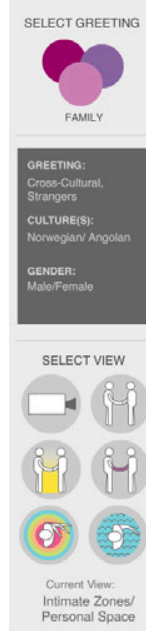

Fig. 187 - Interactive greetings diagram: same as above, only later on in the greeting

The sixth button reveals a vector animation illustrating the subjective experiences and perceived meanings of the greeting (see figs. 188-189). This version shows the intimate zones in combination with subjective experiences illustrated using graphic patterns, again applied to the overhead view. The tables on the right-hand side are animated to highlight how the two people experience the greeting. This subjective element in combination with the visualisation of personal space (intimate zones) indicates how the use of personal space can influence the experience of the greeting situation (as discussed in section 4.3 above). The images below show that the Norwegian man experiences the greeting as neutral when he thinks he is going to greet with a handshake. At that point of the greeting the Angolan female experiences him as being cold. When they are embracing and kissing on the cheek, he experiences the greeting as being intrusive, while she experiences it as distant, because he is not engaged in the embrace and is uncomfortable with such a small personal space. 


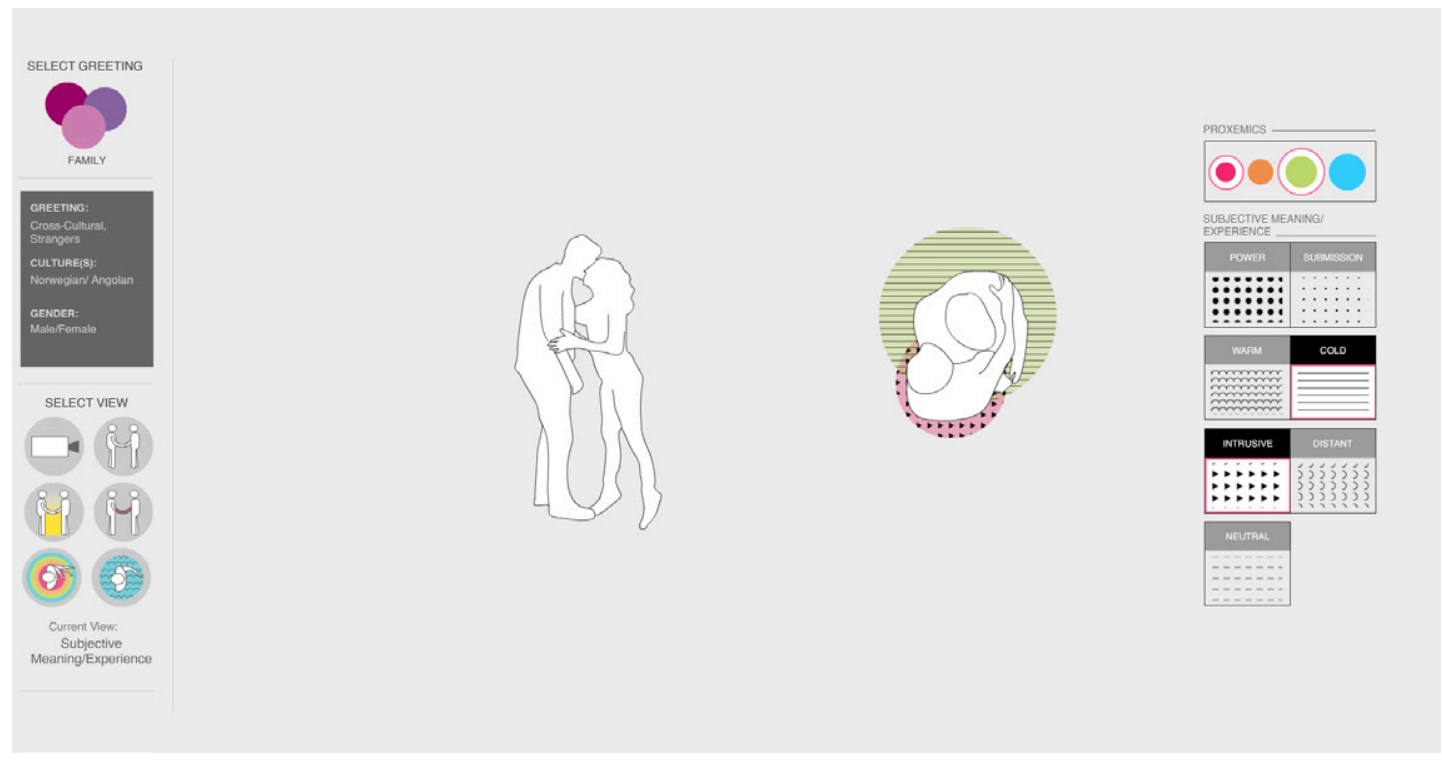

Fig. 188 - Interactive greetings diagram: showing vector animation, with colour coded intimate-zones and patterned subjective meaning, of a cross-cultural greeting between a Norwegian male and an Angolan female, who are strangers

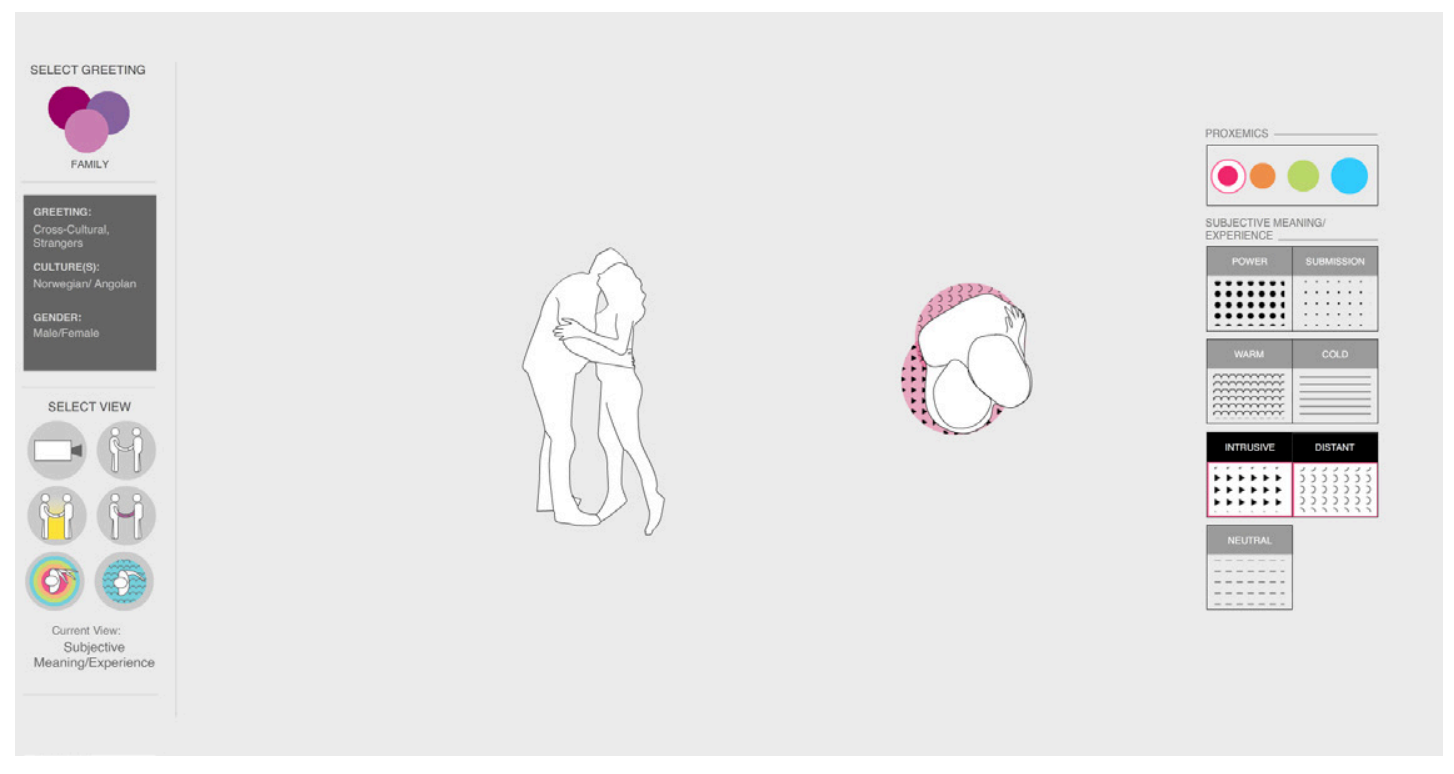

Fig. 189 - Interactive greetings diagram: same as above, only later on in the greeting 
Each of the 82 greetings documented during my fieldwork in Stavanger can be viewed and explored through these six different views. All three diagrams can be viewed in their interactive form in Appendix 12. While the process of visualisation is slow, and is currently excessively manual, I hope that in the future it can be further developed to become more automated. However, while it may be a time-consuming process, this way of visualising greetings does provide a new, dynamic and interactive form of analysis, which helps bring to light some of the complexities and sensitivities of nonverbal communication in a multicultural context. The system has been developed through my own study in Stavanger, but can be used to analyse other greeting sequences too, as I will illustrate below.

\section{A Visual Analysis of the 'Hillary Clinton Meets the Capps' Greetings} In order to show that my system has potential usability and purpose beyond my own fieldwork, I applied my visual notation to the video sequences analysed by Duranti, discussed in chapter 2, of the greetings between Hillary Clinton and the Capps (Duranti, 1996). The aim was to show how my system could contribute as a tool for analysis, alongside and extending current methods for analysis (which are mainly textual). Duranti's analysis focuses on the verbal aspects of the greetings, and he uses textual means, whereas I focus on the nonverbal aspects using visual means. The two types of analysis could complement each other and potentially extend the understanding of greetings.

Figs. 190-192 show how I presented Duranti's and my own analysis in a combined interactive interface. The images of his textual analysis are taken from his website and concern the verbal interactions (Duranti, 1996). I divided the presentation of our combined analyses into three numbered sections, as Duranti had done on his own website. The numbers 1, 2, 3 at the bottom of the interface are active buttons that I have programmed to each reveal one different section of Duranti's and my analyses. Fig. 190 shows Duranti's textual transcript on the left, and a still image from the greeting 


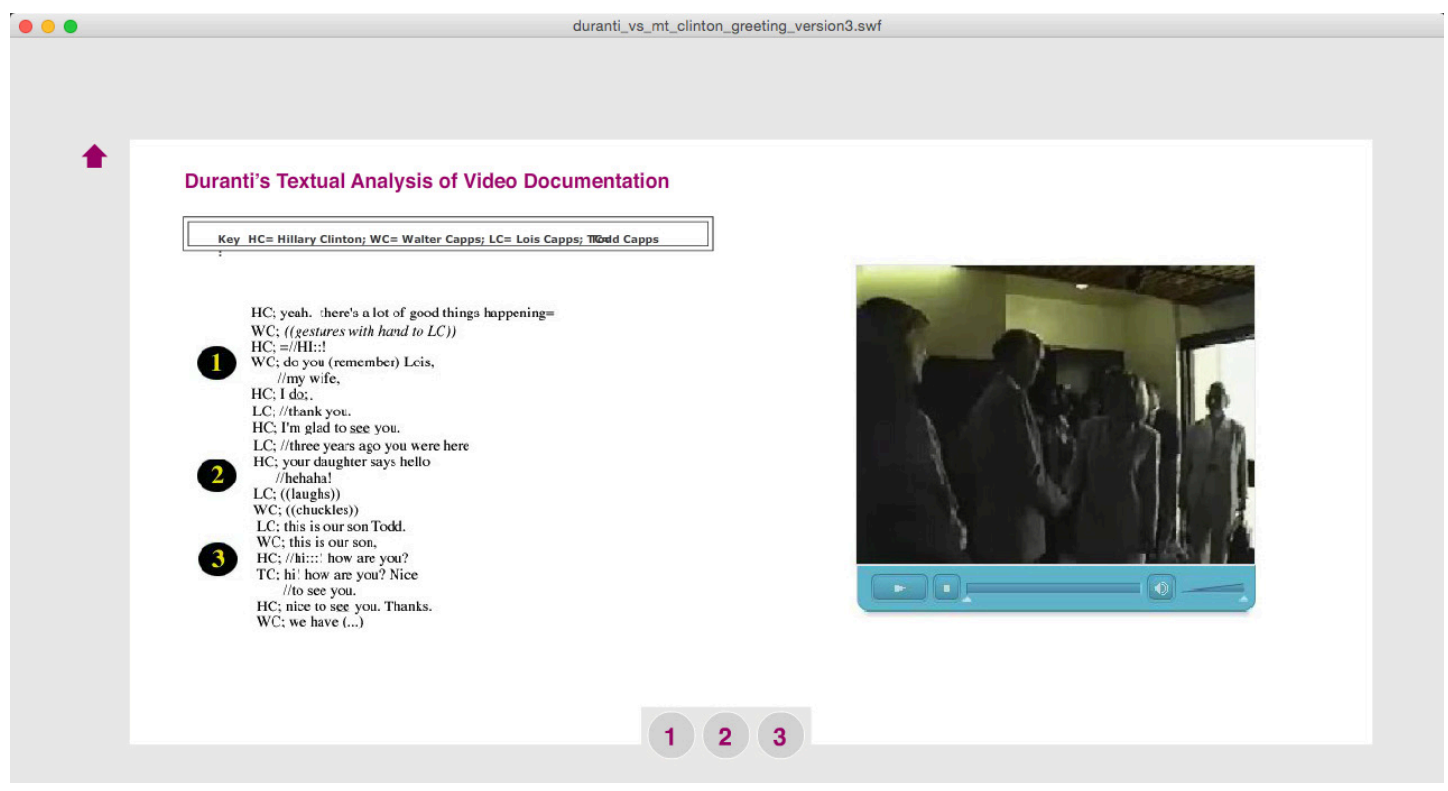

Fig. 190 - Opening screen for interface of Duranti's and my analysis of greetings between Hillary Clinton and the Capps

video he analysed, on the right. Once a numbered button is clicked, the text on the left side is replaced by Duranti's textual analyses, and the right side image is replaced by my animated visual analyses corresponding to the selected section of the greeting. In figs. 191-193 the left side of each image shows Duranti's textual analysis, and the right side shows my visual analysis, which appears when the play button is clicked on.

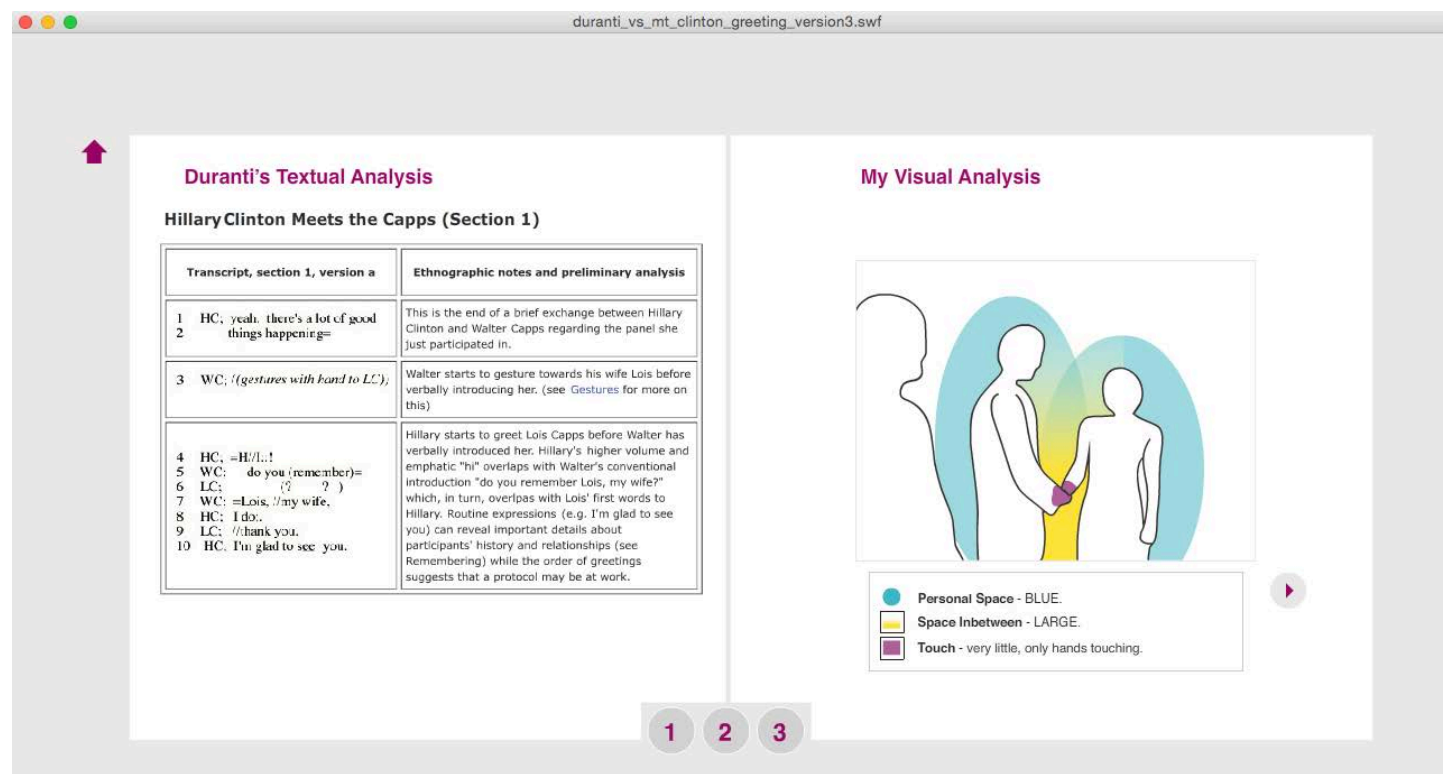

Fig. 191 - Duranti's textual analysis (left side) and my visual analysis (right side) of greetings between Hillary Clinton and the Capps - section 1 


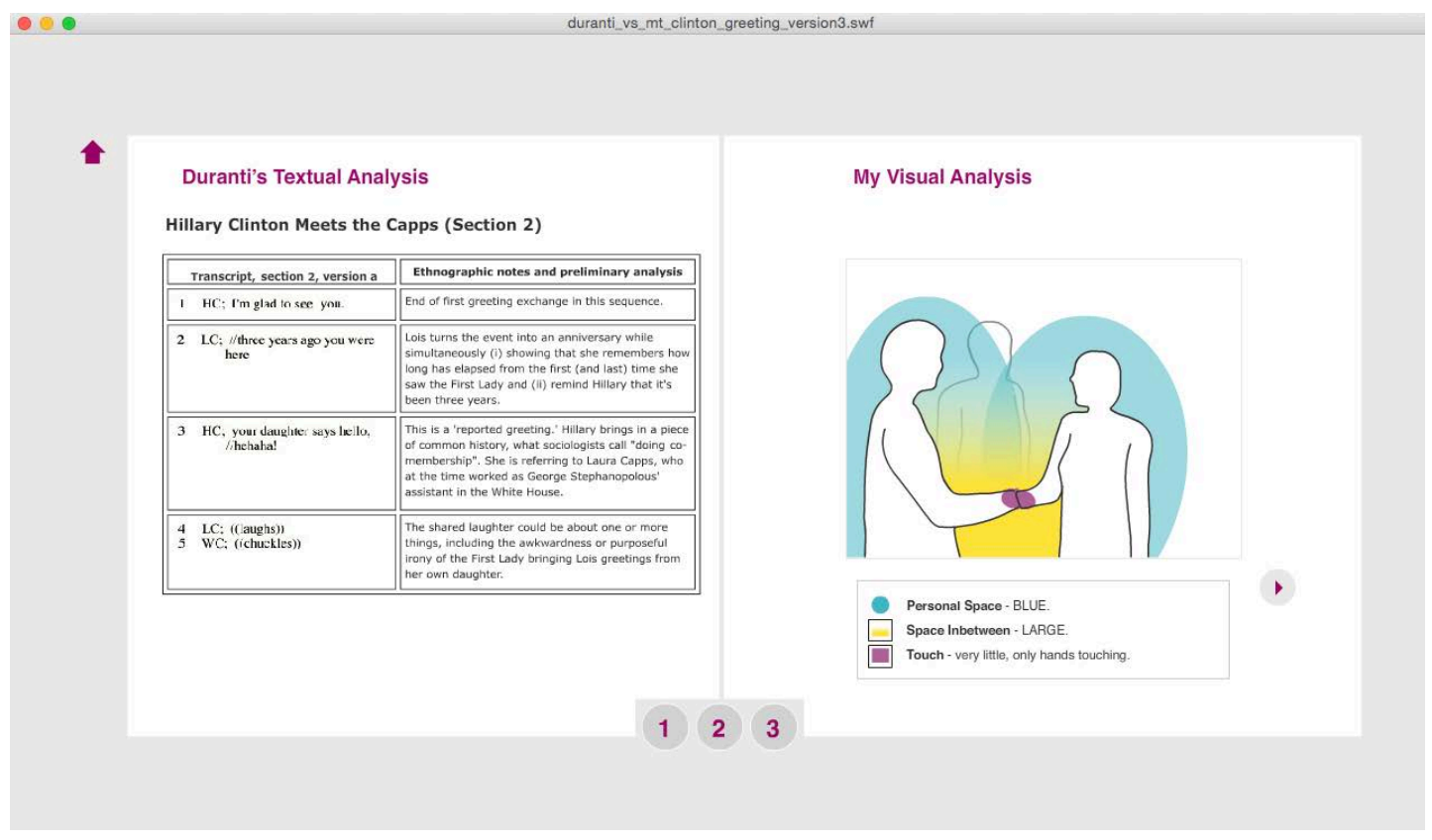

Fig. 192 - Duranti's textual analysis (left side) and my visual analysis (right side) of greetings between Hillary Clinton and the Capps - section 2

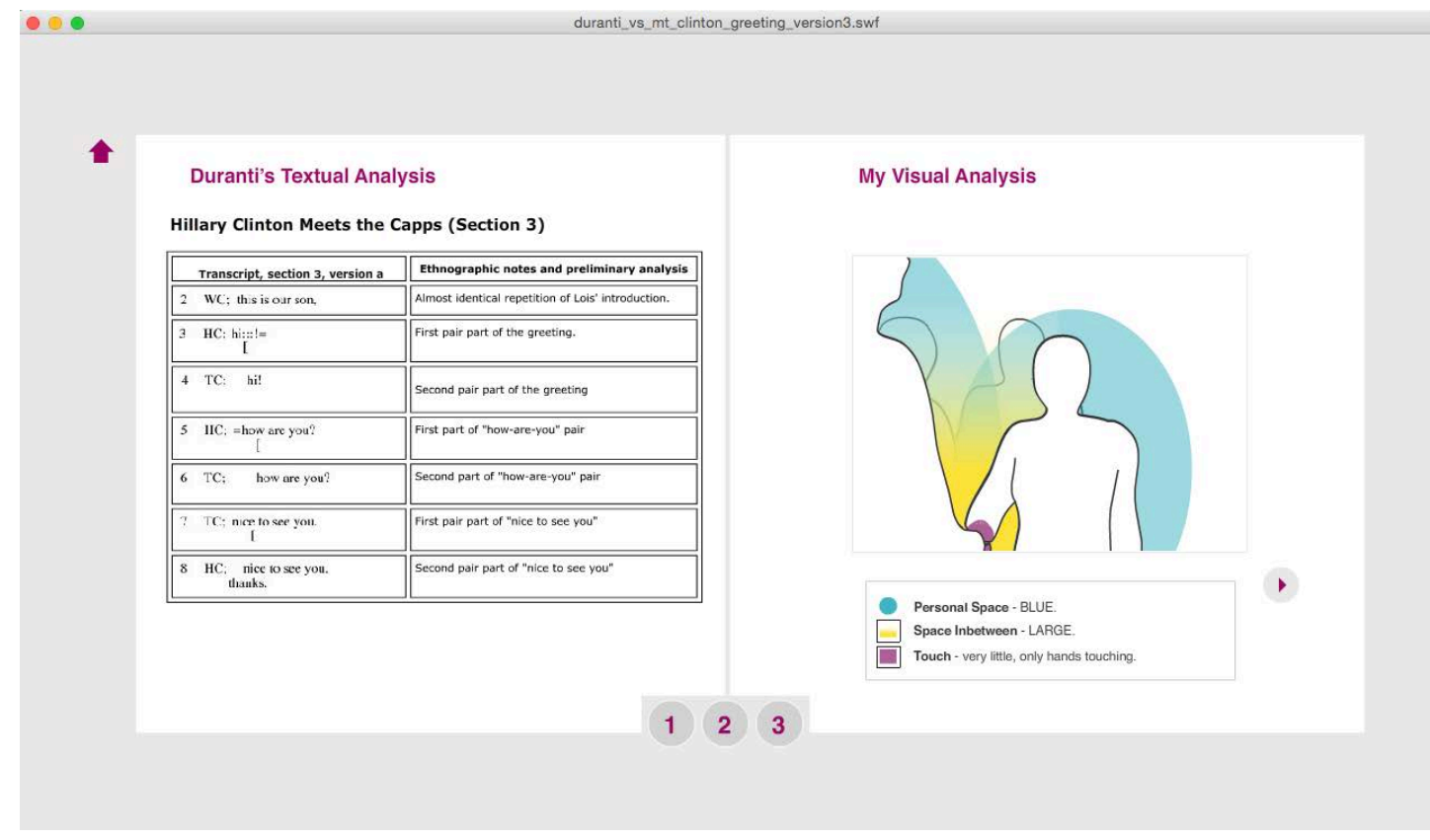

Fig. 193 - Interactive piece of Duranti's textual analysis (left side) and my visual analysis (right side) of greetings between Hillary Clinton and the Capps - section 3 
Fig. 191 shows the first section of the greeting analysis. The left side shows Duranti's greeting transcript and his comments, including the order of the verbal greetings, and also the tonal qualities of the conversation. The left side of fig. 192 shows the second section of the greeting analysis. Here Duranti's greeting transcript includes analysis of how Lois turns the event into an anniversary by talking about Hilary's daughter, and how laughter can represent awkwardness about an ironic statement. The left side of fig. 193 shows the third part of the greeting analysis. In this section Duranti explores different formats of adjacency pairs. (I have not included all of Duranti's textual analysis, as my aim was not to go into detail about what he analyses, but to illustrate how our two methods differ.)

In my visual analyses I followed each of Duranti's three sections, to visualise the three encounters shown in his videos. My analysis includes animated illustrations of the three greeting moments, with annotated notations beneath each animation explaining the meaning of the different colour codes used (see fig. 194). In each greeting we see a blue personal space, signifying a large personal space, at arm's length or more, with little or no touch.
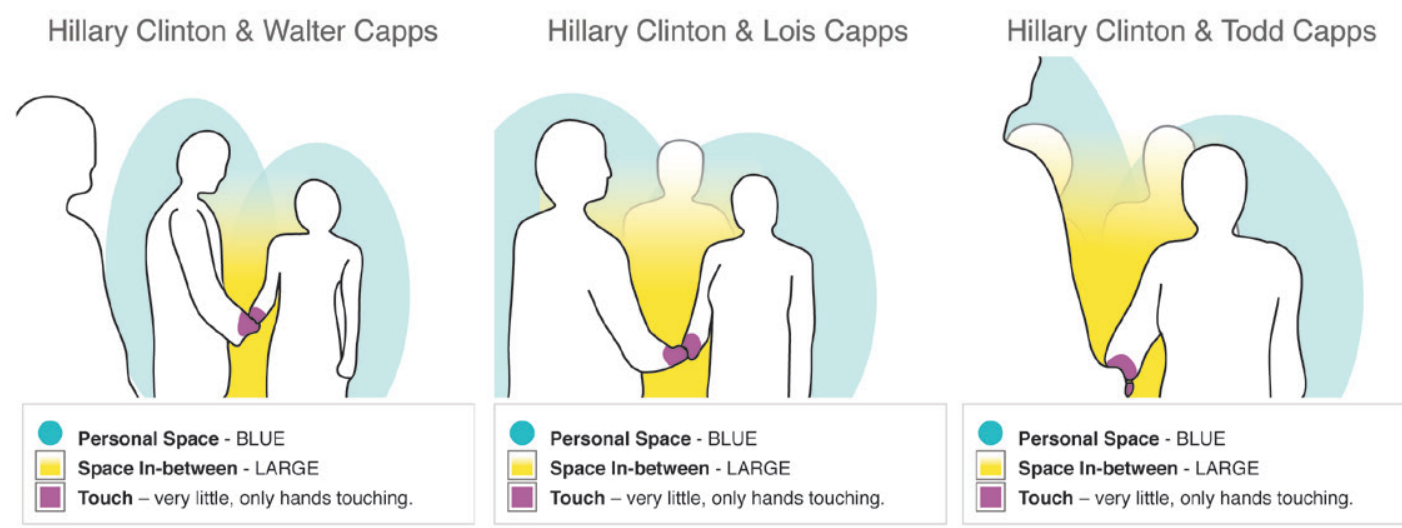

Fig. 194 - Still images from the three greeting animations in which visual annotations have been added to the illustrations 


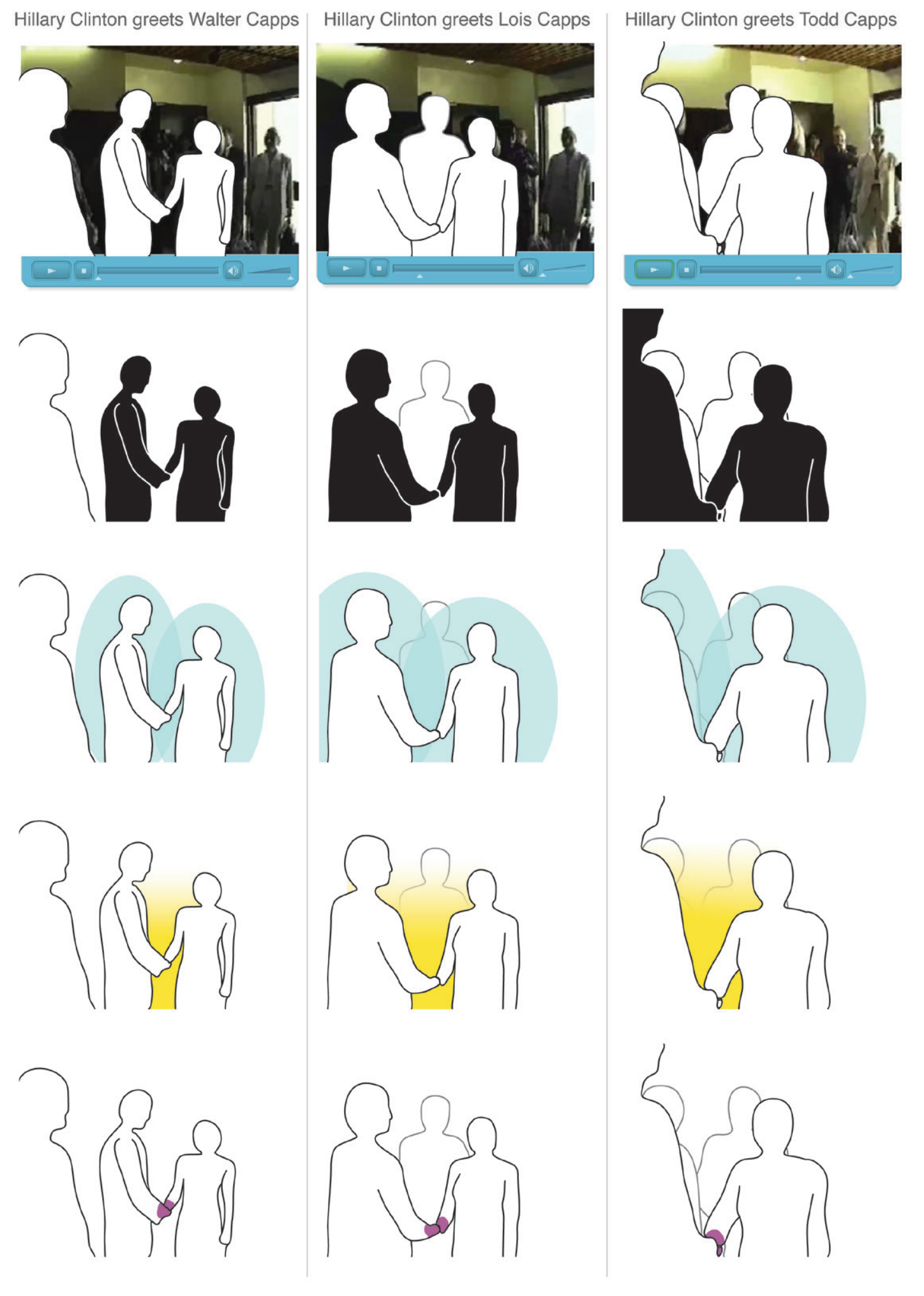

Fig. 195 - A breakdown of all visualisations shown in my three animated analyses of the Hillary Clinton \& Capps' greetings 
Proxemics is illustrated with the yellow gradient showing a large space between Clinton and each of the Capps. In all greetings the physical contact between them is minimal, only their hands are touching - this is highlighted in purple. Comparing the three greeting visualisations reveals further knowledge about these greetings. It shows that in this greeting situation between three Americans during the (slightly) formalised situation of the political campaign, there is little or no difference in their use of personal space, proximity or touch across both gender and generations. Each greeting follows the same nonverbal patterns whether Clinton greets Mr. Capps, Mrs. Capps, or their son Todd Capps. While Duranti reveals different information about the greeting formats and the relationships between Clinton and the Capps through what is said during the greeting, my visual analysis of the nonverbal reveals information about gender and generational behaviour, and in this instance there is very little variation between the three greetings presented. Fig. 195 shows a breakdown of the different visual annotations used in my animations. For a video version of this work, see Appendix 14.

\section{Power \& Submission: Obama Greeting World Leaders}

I also used my visual system to analyse photographs of greetings between American president Barack Obama and three world leaders at the 2010 Nuclear Security Summit in Washington. I was interested in the topic of power and submission among world leaders and in politics. I wanted to find out: who adapts to whom in the greeting, and could a visual analysis help clarify how Obama greets different world leaders in different ways?

Fig. 196 shows my visual analysis of greetings between: Obama and former Chinese leader Hu Jintao; Obama and German chancellor Angela Merkel; and Obama and former Brazilian president Luiz Inacio Lula da Silva. The visualisations show clear differences between the three greetings in all areas of nonverbal communication that have been highlighted: touch, space inbetween, and intimate zones. Obama, who hosted the event (in the host country) seems to attempt to adapt to each of the other leaders' ways of 

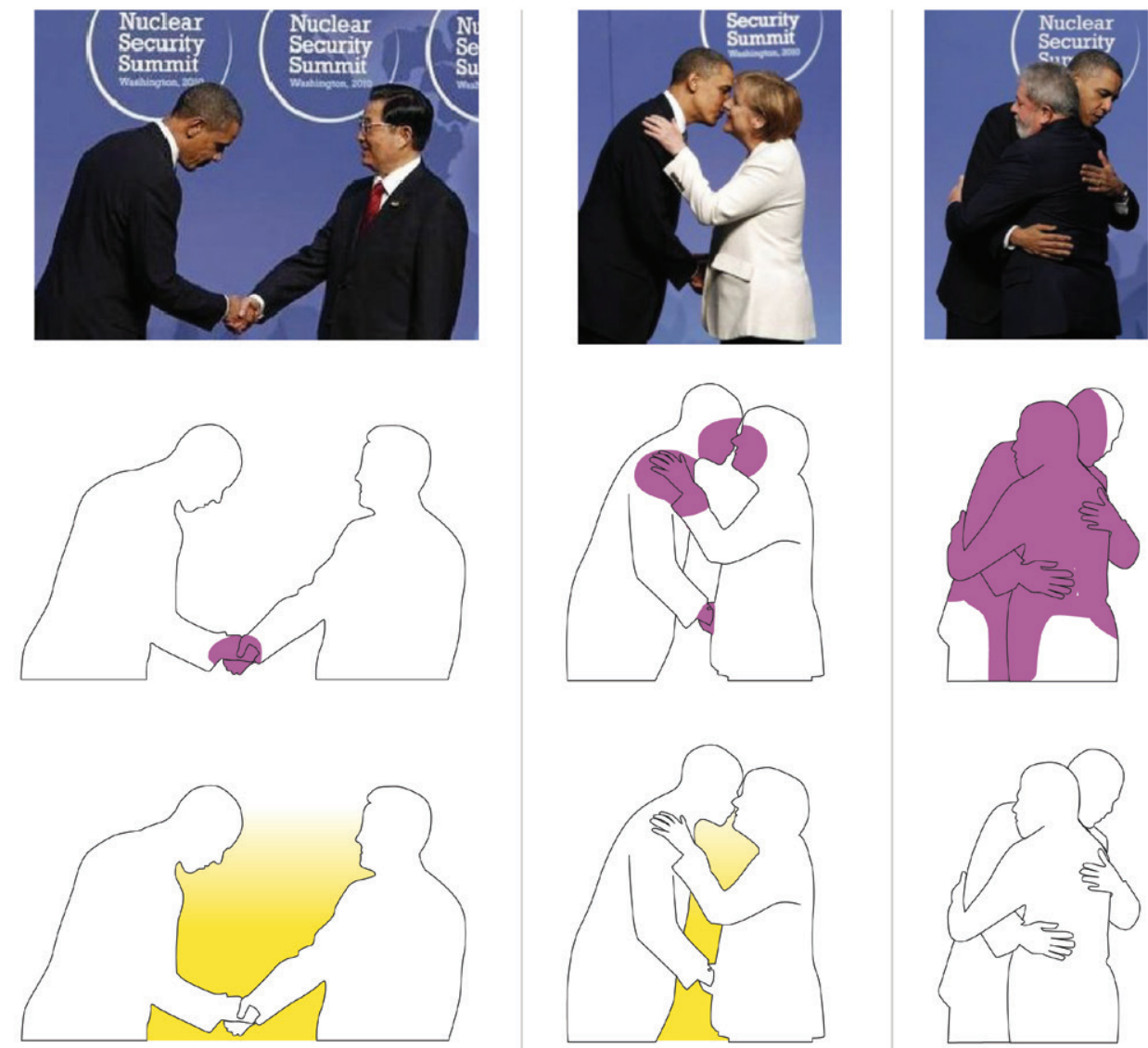

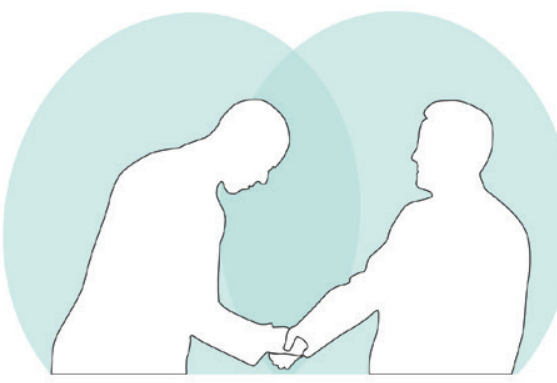

Obama greeting former Chinese leader $\mathrm{Hu}$ Jintao

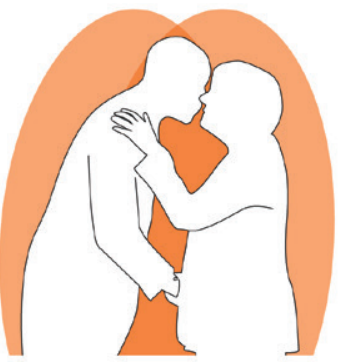

Obama greeting German Chancellor Angela Merkel

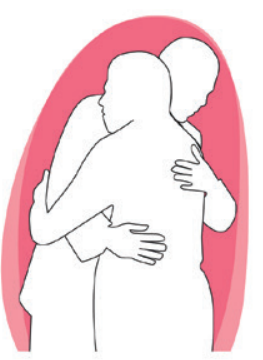

Obama greeting former Braziian President Luiz I. Lula da Silva

Fig. 196 - Power and Submission: visual analysis of greetings between Obama and three world leaders, based on images found online (Perry, 2010)

greeting. He greets $\mathrm{Hu}$ Jintao with a handshake and a slight bow (perhaps assuming that this is how one greets with respect in China), and Jintao responds with a handshake. He greets Angela Merkel with kisses on the cheeks. Finally, Obama and Lula da Silva greet with a full embrace. I will not discuss whether Obama greets each leader in a culturally 'correct' way, as I 
would only be speculating because none of these three cultures were part of my study. I am much more interested in the differences that are visualised, and in the extent to which Obama, the host, adapts (or attempts to adapt) to his visitors' ways of greeting. Rather than expecting them to adapt to his way of greeting, he does the opposite. This could be due to Obama wishing to come across as respectful towards his visitors, but could also, in some cultures, be interpreted as a lack of authority and power.

The visualisations show clear differences in how much touch is involved in the greetings. While his greeting with Jintao on the one hand involves hardly any touch, his greeting with da Silva on the other hand involves a lot of physical contact. The greeting with Merkel is somewhere between the other two. The same can be seen in how much space is in-between them. All three greetings are also 'performed' in different intimate zones. While the photographs show clear differences in types of greetings that are performed, the graphic visualisations help highlight some more distinct aspects of the nonverbal communication that takes place. For an animated version of this work see Appendix 15.

\subsection{Other Experiments Using Animation \& Motion Tracking}

As well as devising the system for notation, I conducted a number of experiments in creating interactive exhibition and installation pieces. This was done with the aim of creating work that would enable visitors to understand the physical dimensions of my study, and to explore technologies such as motion tracking for the study of greetings. Below I discuss four of these experiments.

\section{Patterning Moments of Human Interaction - Exhibition Piece}

The exhibition piece Patterning Moments of Human Interaction was an experimental video exploring various greetings across two cultures: Norwegian and Turkish. It was made up of an animated video and two floor graphics, and it was presented at the UCA Student Research Conference 
2012: Process. Perception. Phenomena. The animated video, which was 2:40 minutes long (see Appendix 17a), is based on the idea that different social contexts influence and alter our way of greeting. Greeting a grandparent, for example, can be very different to how we greet a stranger or someone during a business meeting. Cultural differences were also a focus in this piece, and to draw attention to this I used motion graphics in a more experimental manner than in my notation system. The aim with the graphics was to highlight culture-sensitive aspects of human communication, revealing similarities and differences in relation to personal space, gender, intimacy, touch and message intended/received (see figs. 197-198). During the exhibition, the video was projected on the wall, and the two floor graphics were mounted below it. The aim with the floor pieces, which were made using clear sticky film decorated with pink and white stickers, was to invite the viewers to test their own personal comfort zones during a greeting (see figs. 199-200). The floor graphics were circles containing shapes of two people greeting from an overhead view.
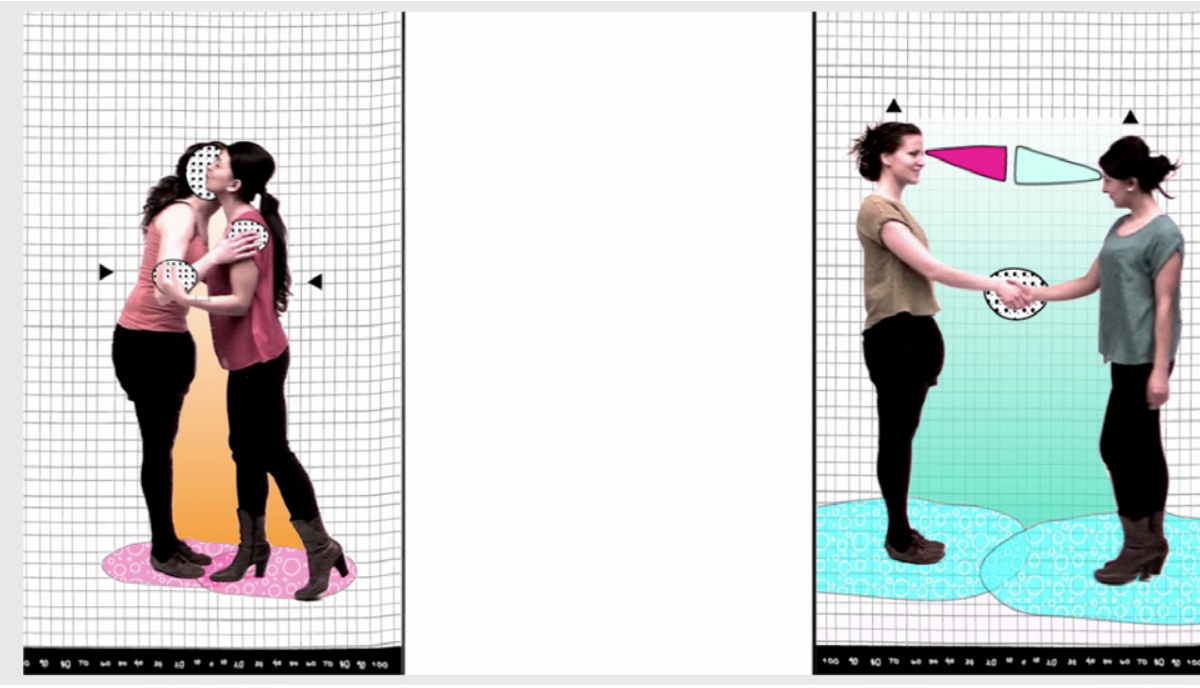

Fig. 197 - Still image from greeting triptych video of Norwegian and Turkish greetings (1) 


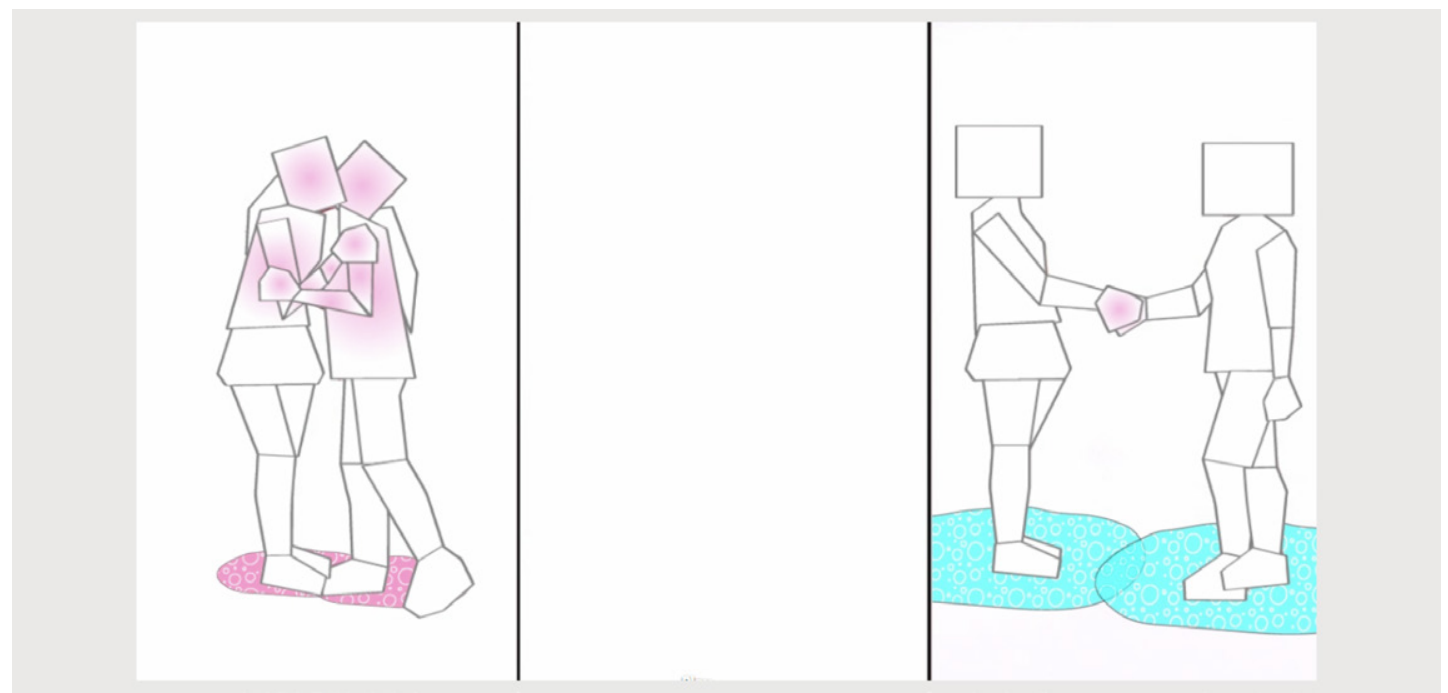

Fig. 198 - Still image from greeting triptych video of Norwegian and Turkish greetings (2)
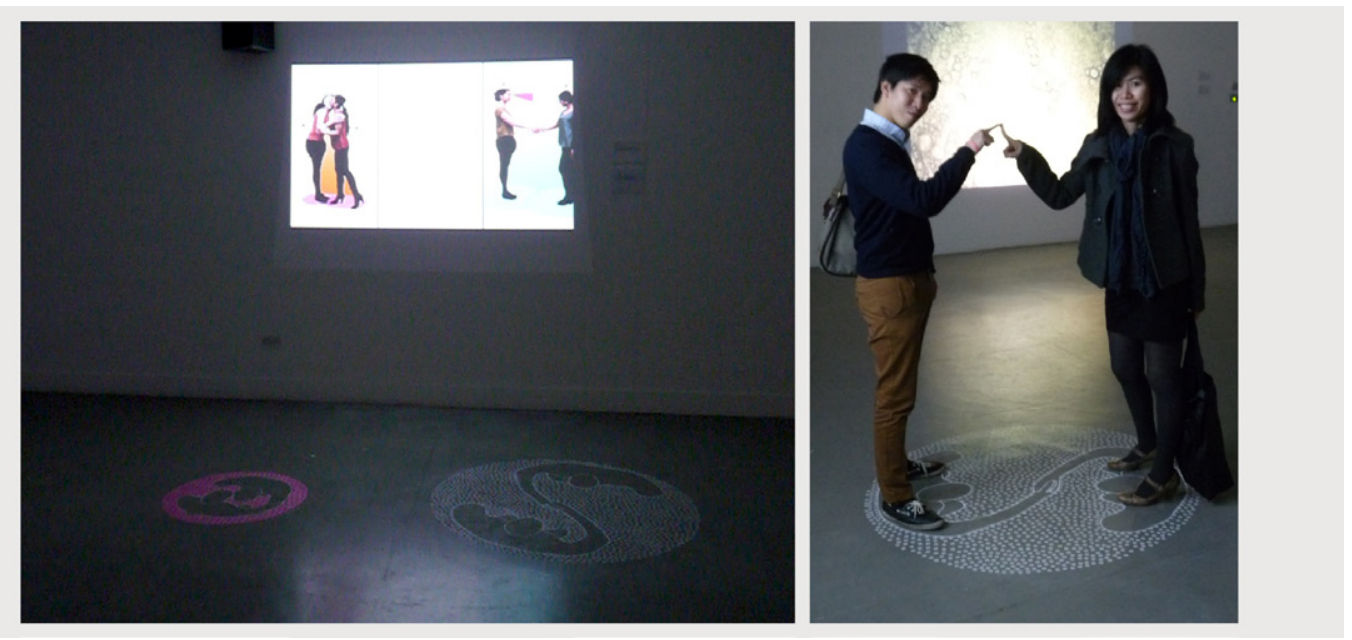

Fig. 199 - Work installed at UCA Student Research Conference 2012: Process. Perception. Phenomena. Visitors interacting with the floor piece

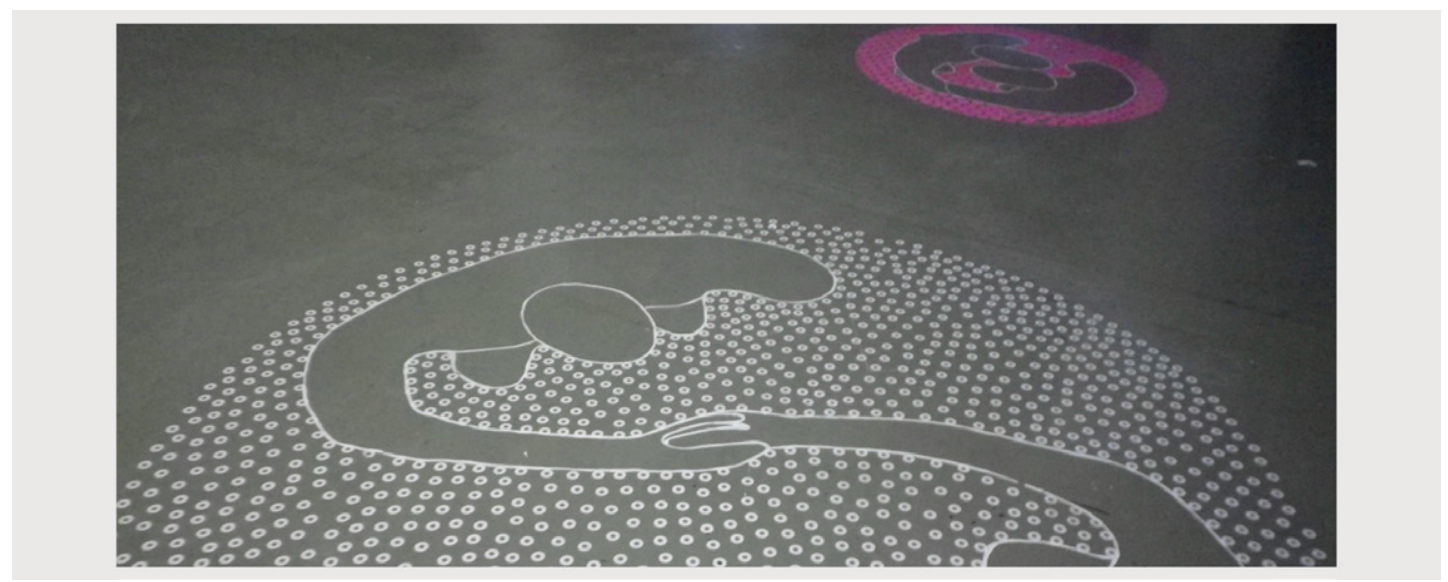

Fig. 200 - Work installed at UCA Student Research Conference 2012: Process. Perception. Phenomena. Floor piece 
Greetings were filmed in a studio with a white background, and edited in Final Cut Pro (7.0.3) into a triptych video (three screens that seem to interact with each other), in order to create a narrative of walking in and out of different social situations. The triptych video was then animated in Adobe After Effects CS5. The piece was an experiment to determine whether After Effects would be better software to use for animating my greeting videos than Adobe Flash.

During the exhibition only a few visitors actually stepped onto the floor graphic after seeing others doing so (see fig. 199). Overall the interactive aspect I had intended for the piece was unsuccessful, but it received a positive response as an artwork. The video seemed to be interpreted as video art rather than a video revealing information about the experiences of the greetings. However, my intention was to make a piece that was visually interesting as well as an informative one, so perhaps it ended up caught between different genres, somewhere between video art and information graphics. Adobe After Effects is excellent software for adding animation to videos; however I still choose to continue using Adobe Flash to create my vector animations, due to my familiarity with it, the graphic style I require, and more importantly because of its capacities of adding interactivity though programming.

Interactive Greetings in a Mediated Performance Space - Prototype My first experiment with using interactive media for performance/installation was a collaborative project with Bahar Sert, in which we explored some common research interests such as interaction between performer, audience and technology, and the representation of personal space inspired by Bauhaus performances and costumes.

In this piece, which could be further developed in the direction of either performance or installation, two performers interact on stage in front of a wall projection. The main performer wears a costume that is made to 
physically represent different personal space zones. The costume makes it difficult for other performers to get too close to the main performer (if for example approaching for a 'kiss on the cheek' greeting). The wall projection reacts to the distance between the two performers on stage. There are four zones, which trigger different colours that reflect the colours used for the four intimate zones in my graphic greeting notation system (see fig. 201). Software and a web camera are programmed to measure the space between the two people, and react to it. For example, if the distance is within the space that has been set for zone four, animation four will be triggered.
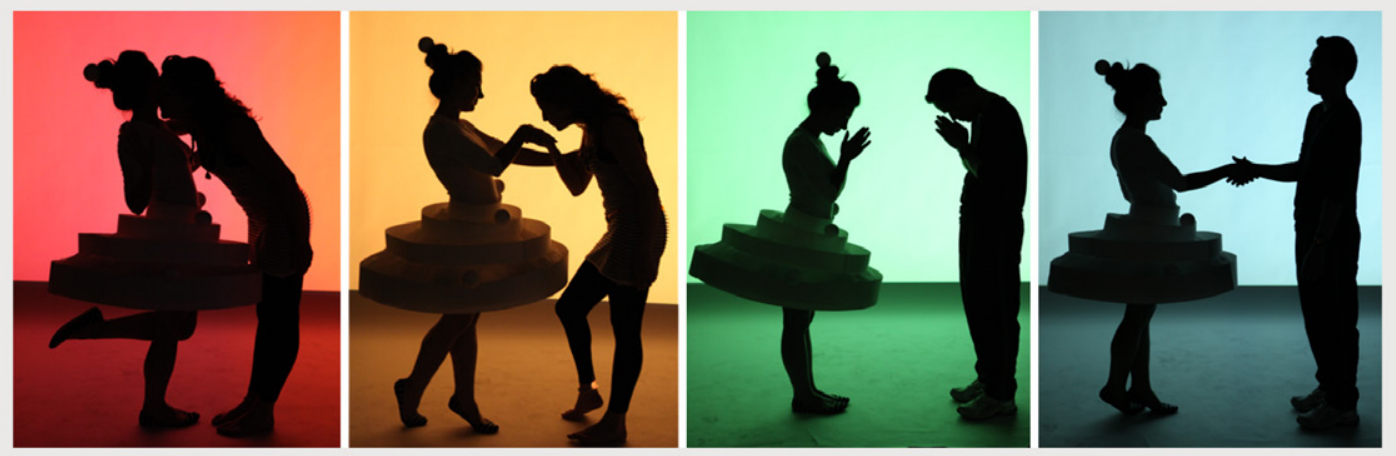

Fig. 201 - Photos from greeting space prototype. Bauhaus inspired costume made by Bahar Sert

Two different types of technical and software solutions were explored in the attempt to achieve this result - Isadora software and Arduino sensors and software. Both Bahar and I were familiar with the Isadora (v.1.2.9) software and decided to try to use it to create the interaction; we were able to do so, to an extent, by connecting Isadora to a webcam that was programmed to track motion (see fig. 202). However, the result was not as accurate as we needed it to be. We would need an infrared webcam and up-skilling in Isadora in order to carry out this project successfully. 


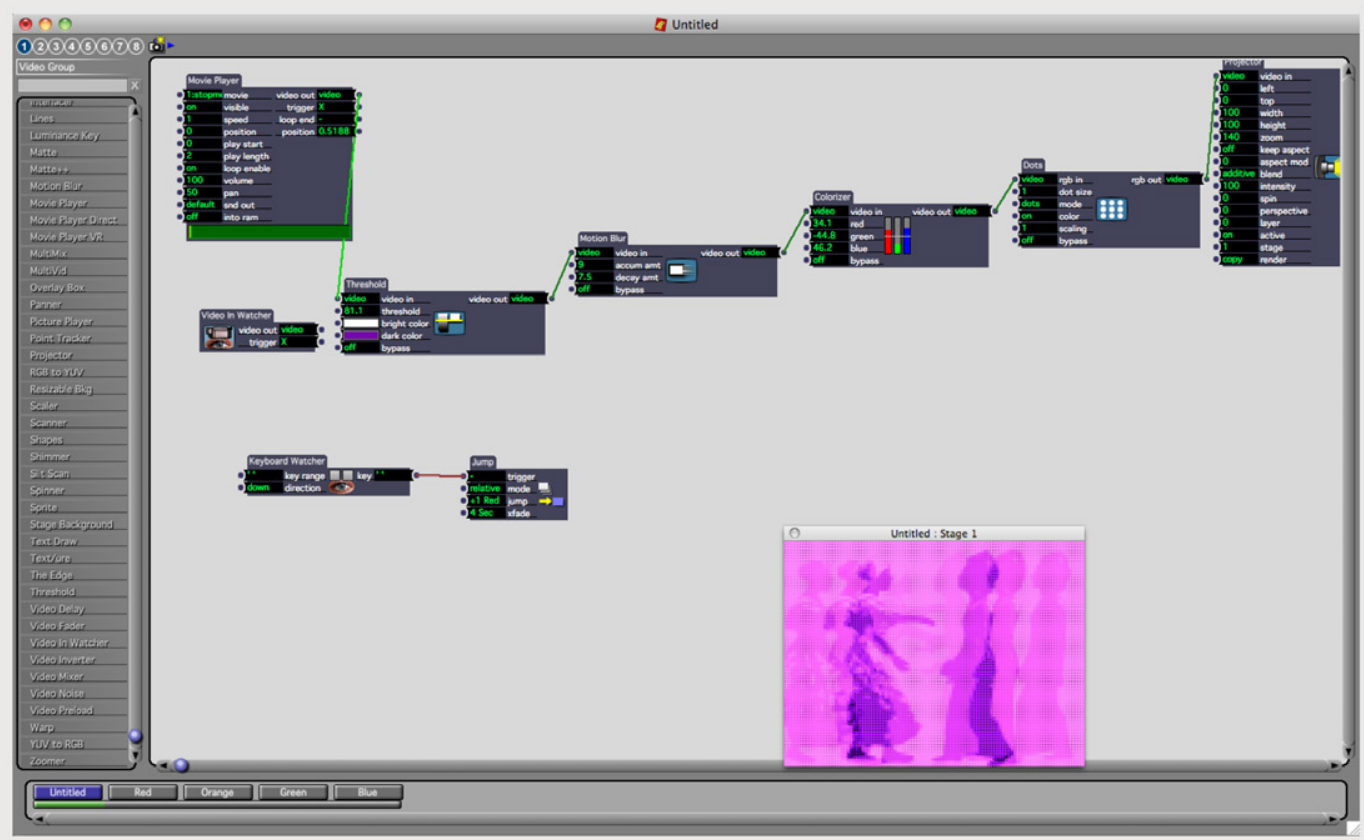

Fig. 202 - The set-up in Isadora software. A webcam is used for motion tracking
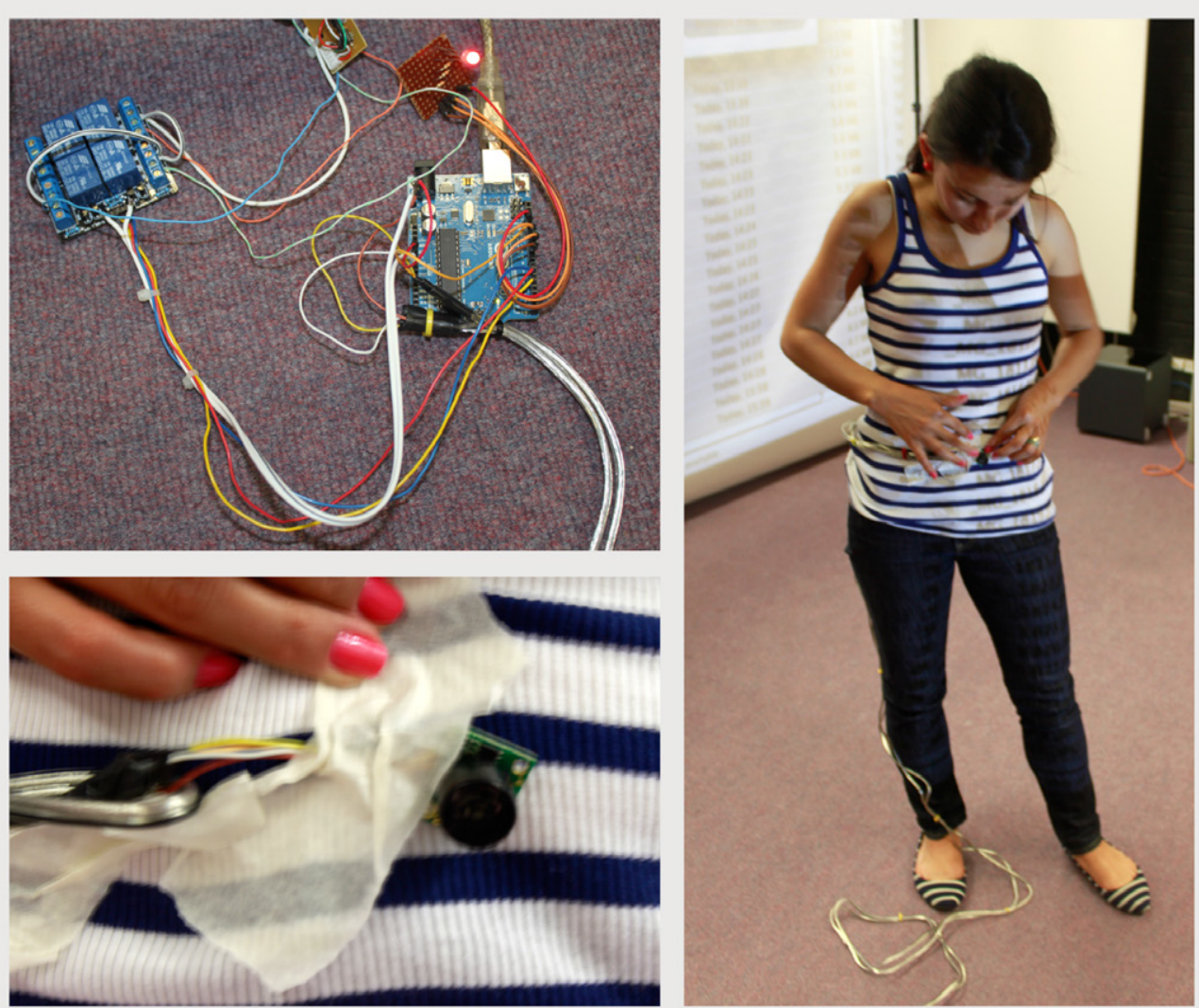

Fig. 203 - Testing the Arduino sensor attached to the performer 
We then discussed using the Arduino platform and open source software with Phil Marsh at UCA Rochester, and he offered to assist us with the programming in Arduino. We used an Arduino Ultrasonic Range Finder Maxbotix LV-EZO and Arduino UNO R3 board that Phil helped to programme to trigger the four different colours, depending on the distance between the two people greeting. We tried using the range finder (sensor) in two different ways. First we tried attaching it to the floor on the middle of the stage, where the two performers meet to greet each other; the problem with that solution was that the performers would have to make sure to always greet in the same spot, and this would not give the user much freedom of movement. Then we tried attaching the sensor to one of the performers, in order to be more flexible with regards to use of the space; however, because the cables attached to the performer were also connected to the laptop, this was not ideal either (see fig. 203). These experiments focused mainly on the technical aspects of the interaction rather than on creating a narrative, and were not completed into finished pieces of work. For a video of this prototype, see Appendix 17b.

\section{Interactive Greeting Space - Installation}

As a continuation of the other experiments, I created an installation titled The Interactive Greeting Space to present at the UCA Student Research Conference Exhibition 2013: Time - Place - Space. It was an interactive greeting space in which the proximity of two people who enter the space changes the mood and overall look of the space. During real-time greetings, different feelings and emotions are triggered by interaction with the other person. This installation tried to capture some of this reality in different ways. The greeting space 'reacts' to two aspects of movement: three different colour zones - green, orange and red (it will ideally include a fourth colour, blue, in future developments), are triggered depending on which zone the two people greeting find themselves in; and the actual movement is tracked and reflected in two circles projected on the floor - one circle surrounding each person greeting - that follows their movements and is meant to represent 
their personal space. So when they move towards each other to greet, the circles follow them. Figs. 204-206 show the initial sketches and simulations of the greeting space.

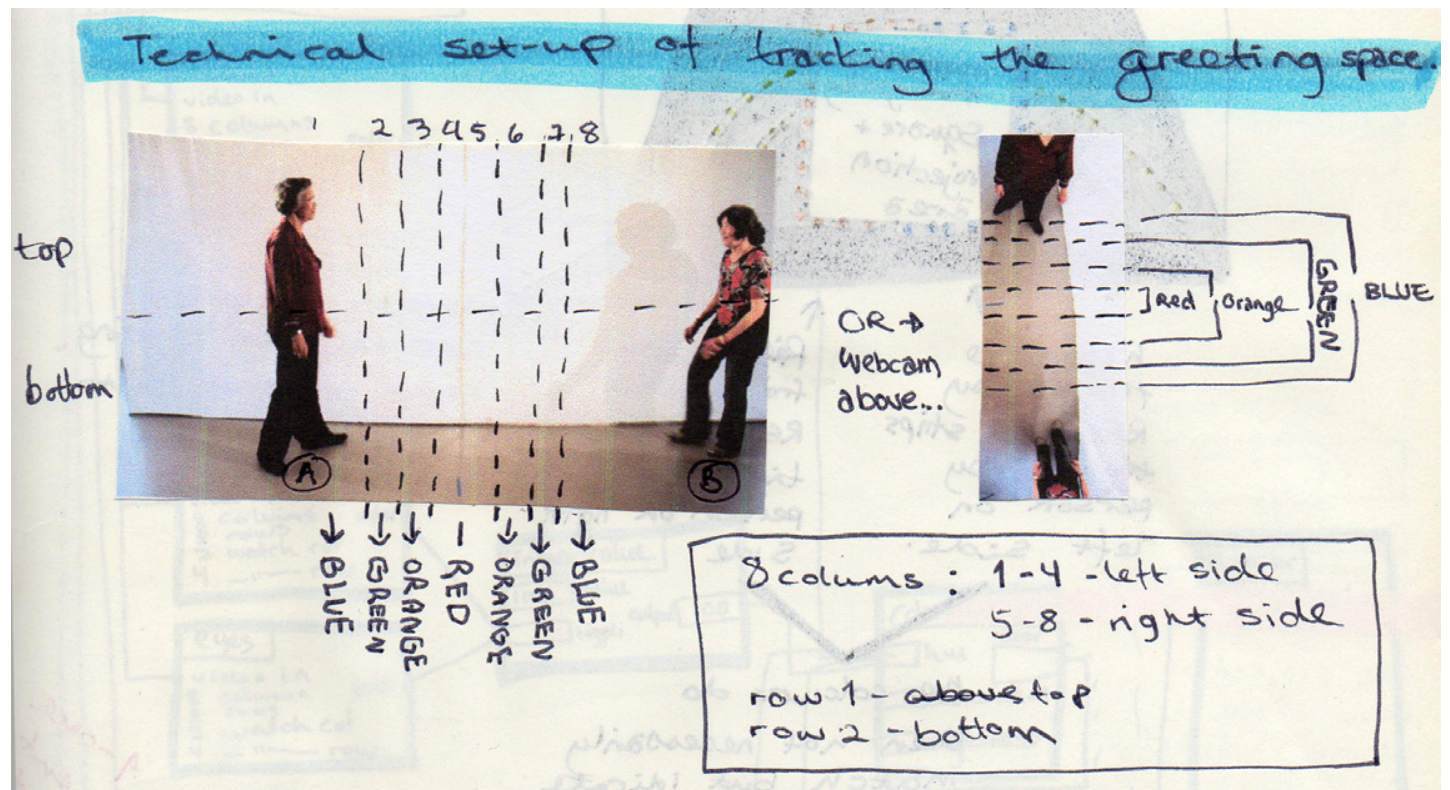

Fig. 204 - Initial sketches of greeting space set-up

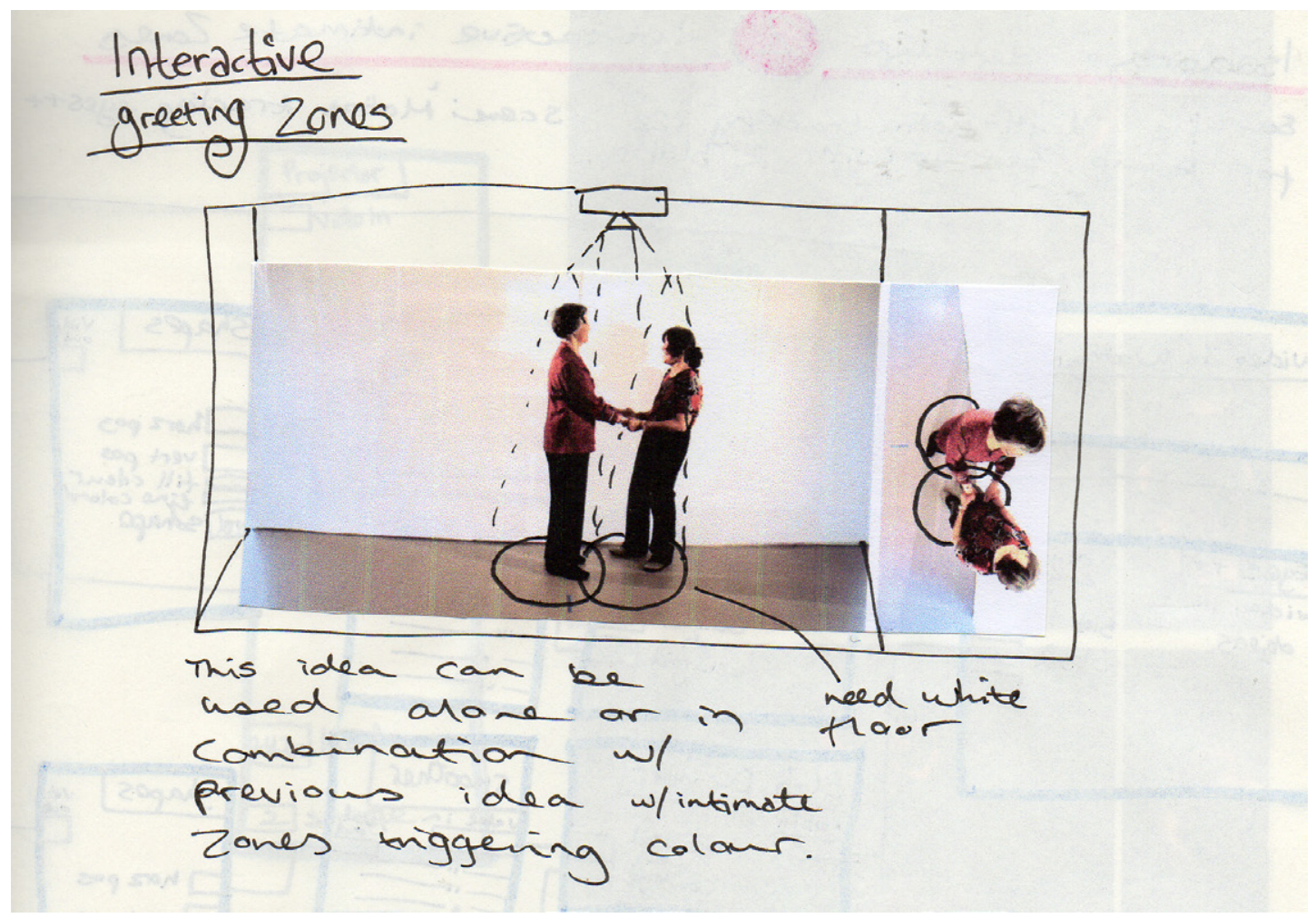

Fig. 205 - Initial sketches of greeting space set-up - placement of projector 

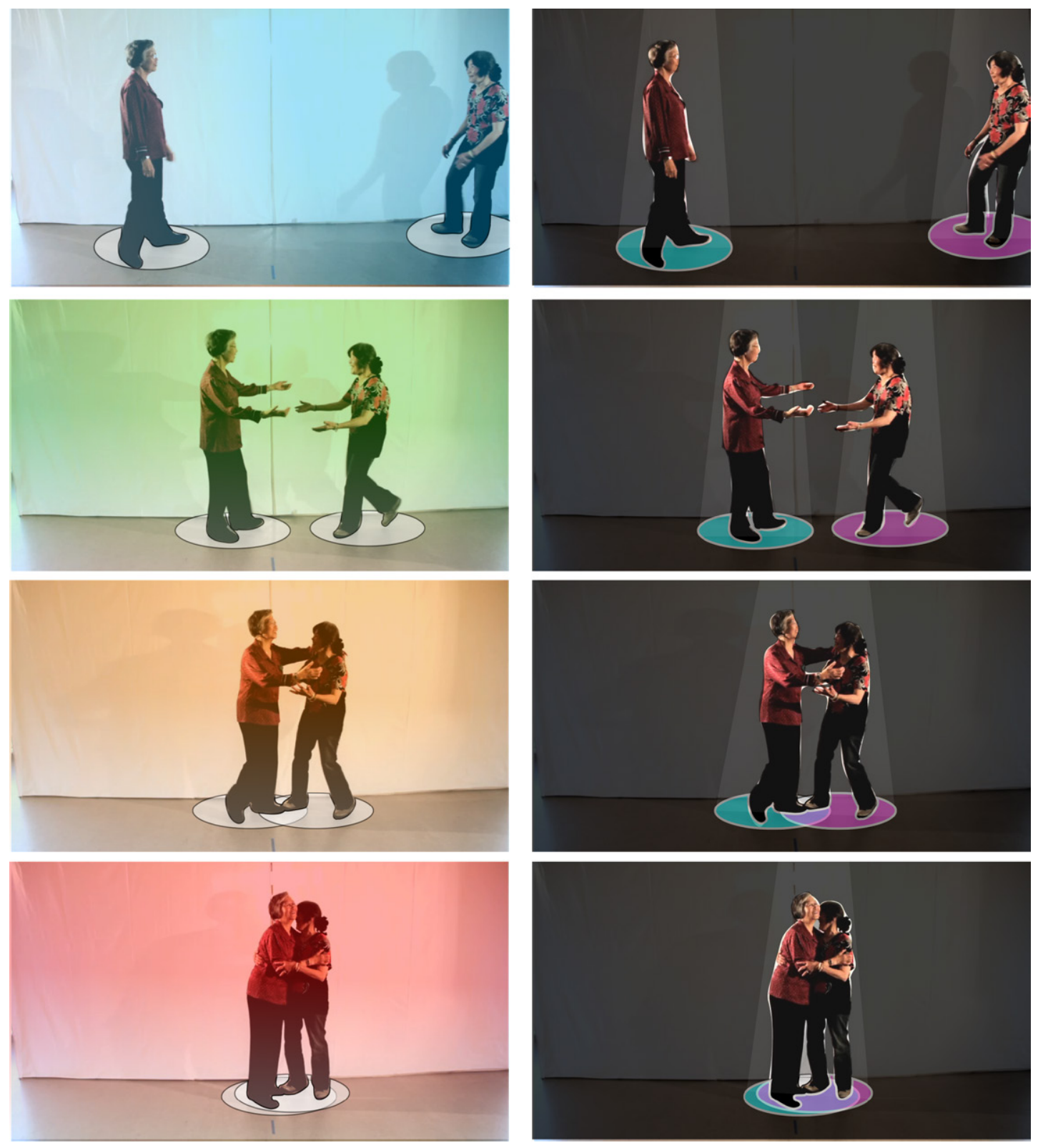

Fig. 206 - Simulation of the greeting space

The installation was built using Isadora (v.1.3.0f24), a software used for live performance, VJ-ing and similar. A Kinect X-Box camera was used for motion tracking; the camera used an infrared laser projector and depth sensor and has the ability to work well in any ambient light conditions. The camera was connected to Isadora on a laptop. In the Isadora program I divided the greeting space into columns, which were programmed to project different colours. If motion was detected in any of these columns, the corresponding colour would be projected (see figs. 207-209). 


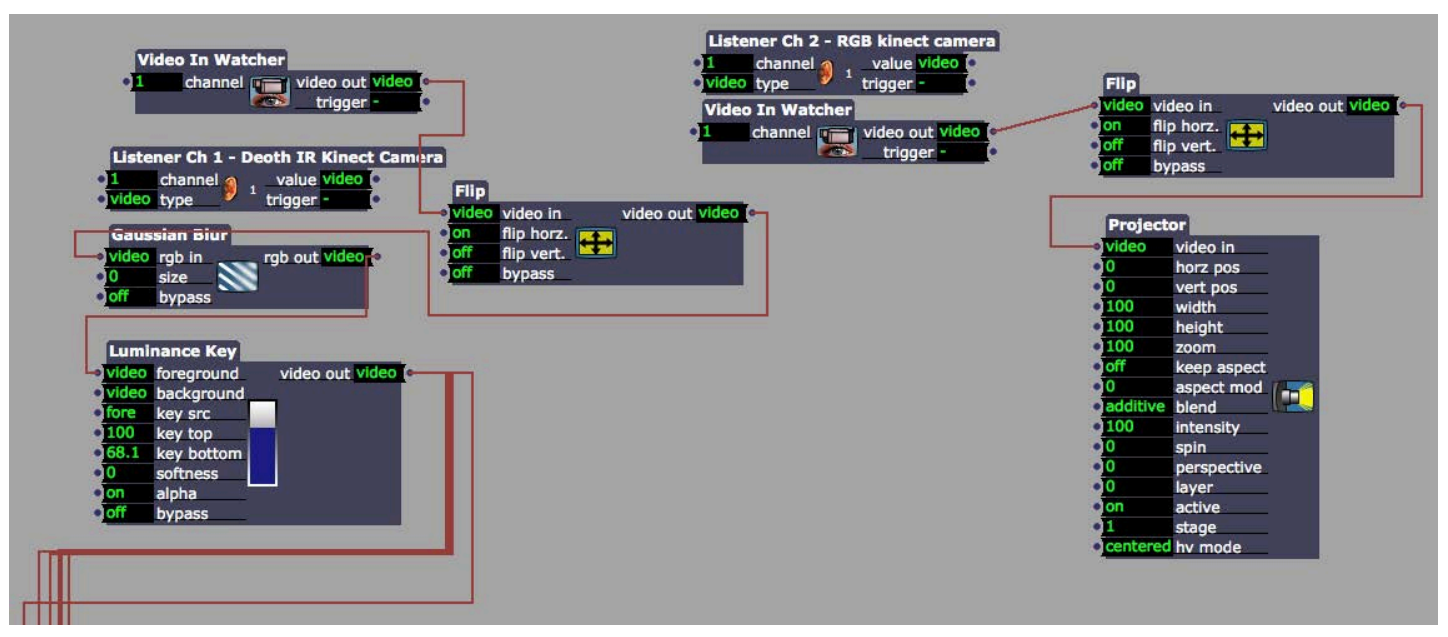

Fig. 207 - Connects and feeds the Kinect camera into Isadora

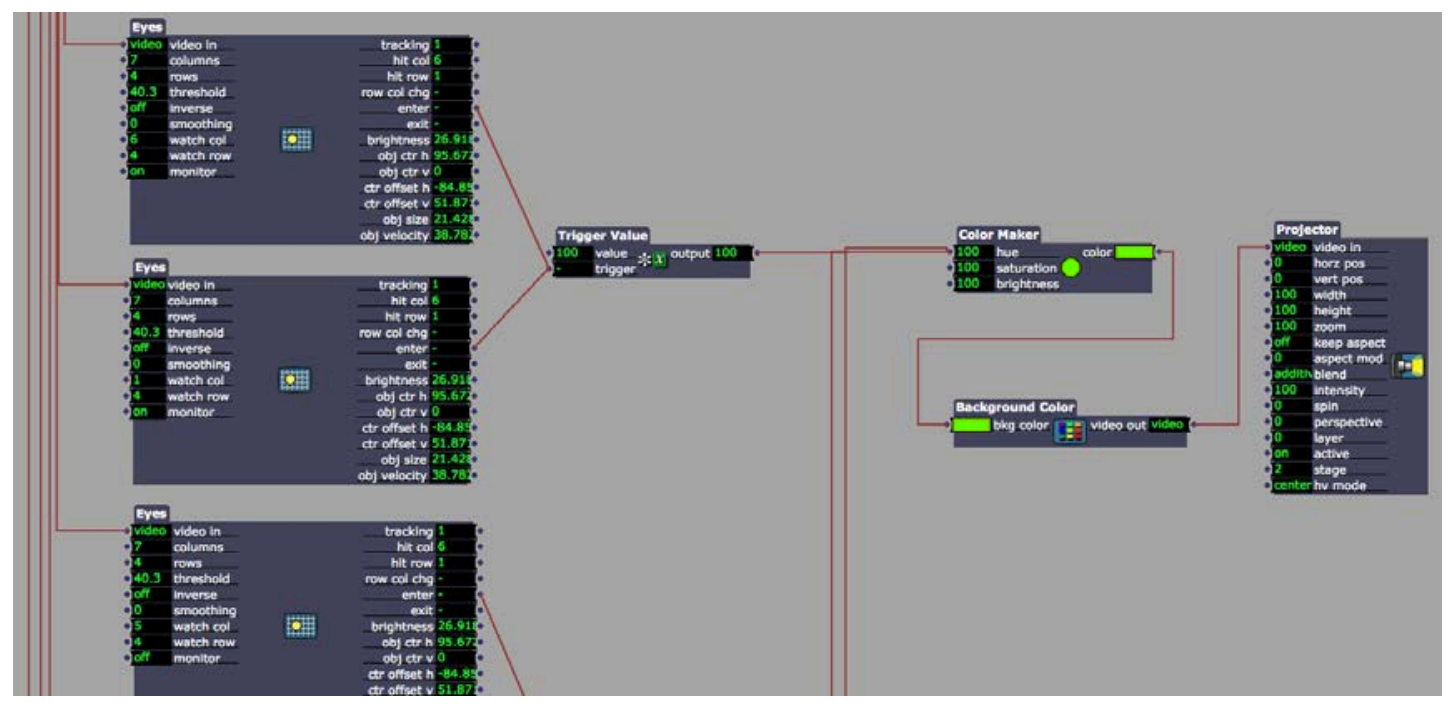

Fig. 208 - Set-up of motion tracking and columns to project green

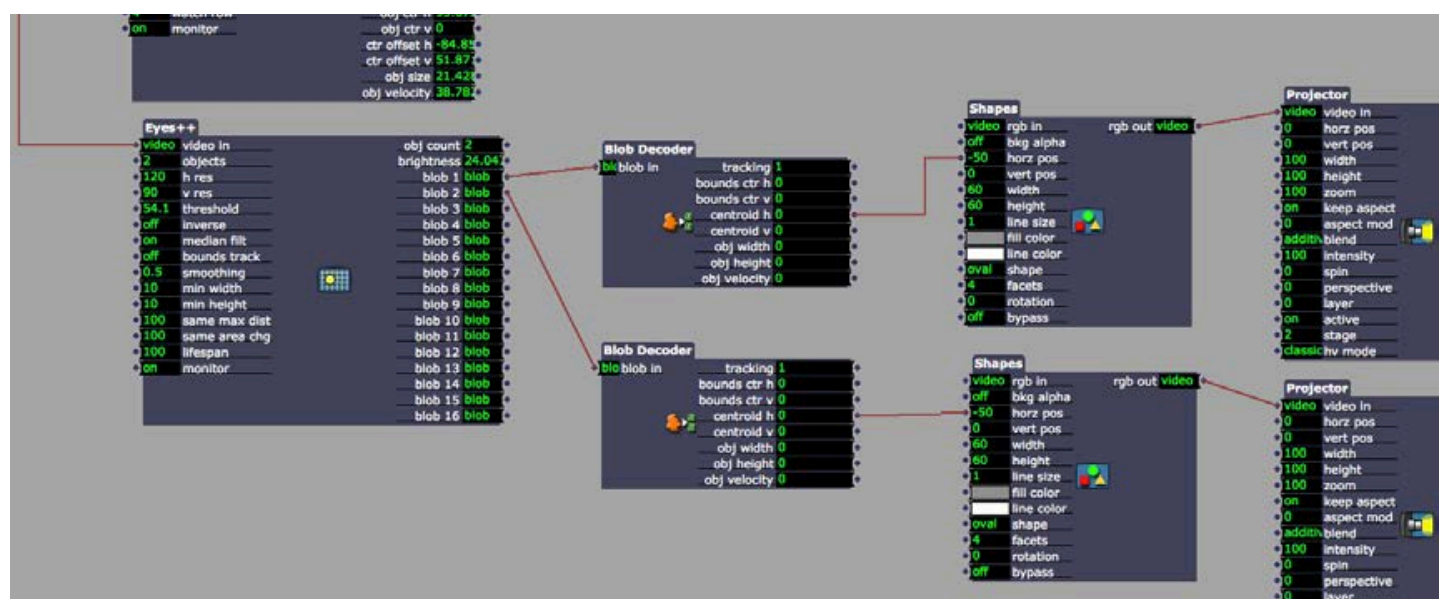

Fig. 209 - Set-up of motion tracking to project shapes (circles) 
If only one person was tracked, only one circle would move within the screen/ greeting area. Fig. 210 shows the set-up of the exhibition space, which included the following equipment: a MacBook Pro, a Kinect camera, and two projectors. Fig. 211 shows the graphics that were mounted on a wall in the space, instructing the visitors on how to use the space. Fig. 212 shows the space in use during the exhibition.

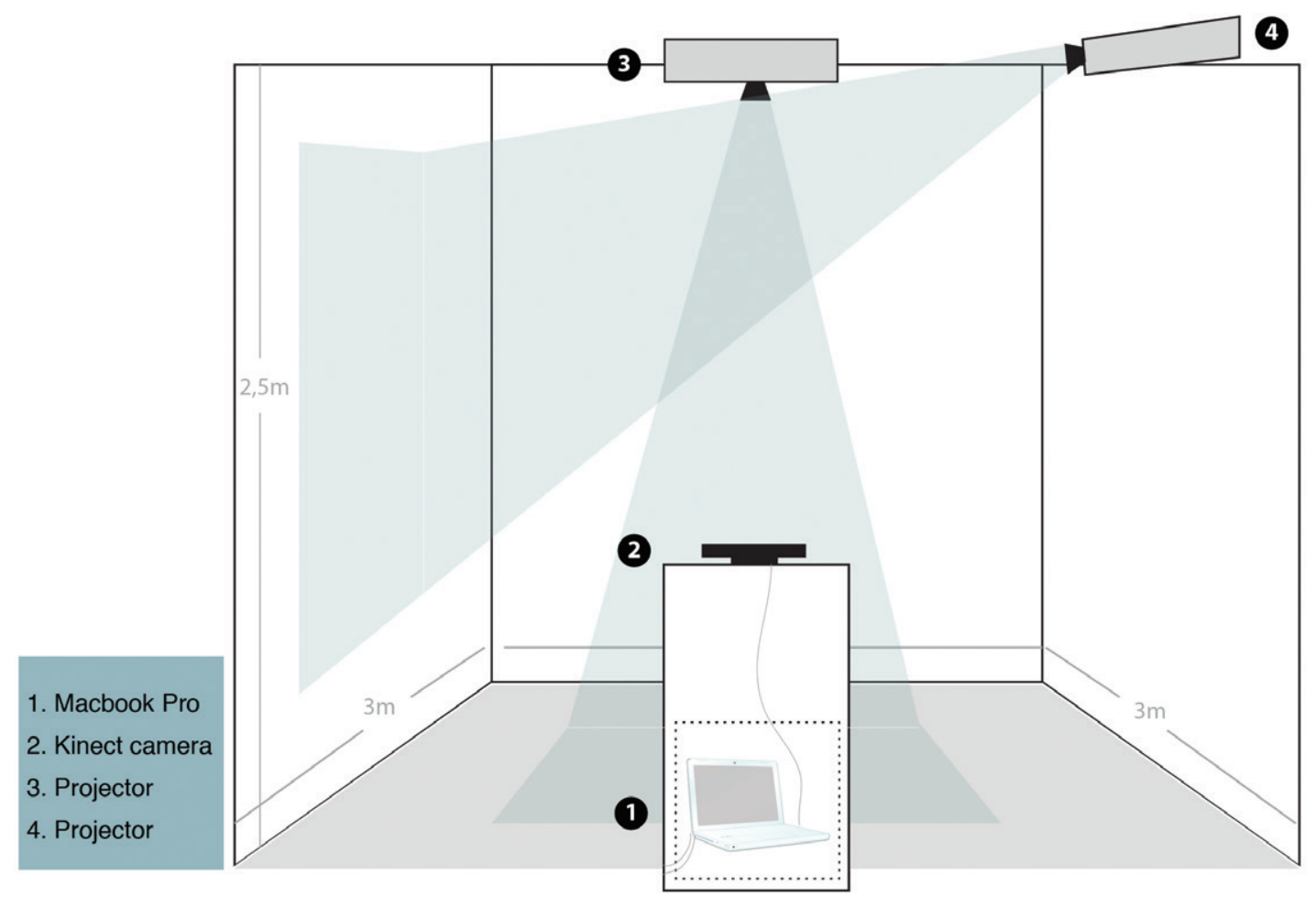

Fig. 210 - Interactive Greeting space: Set-up of equipment/technology
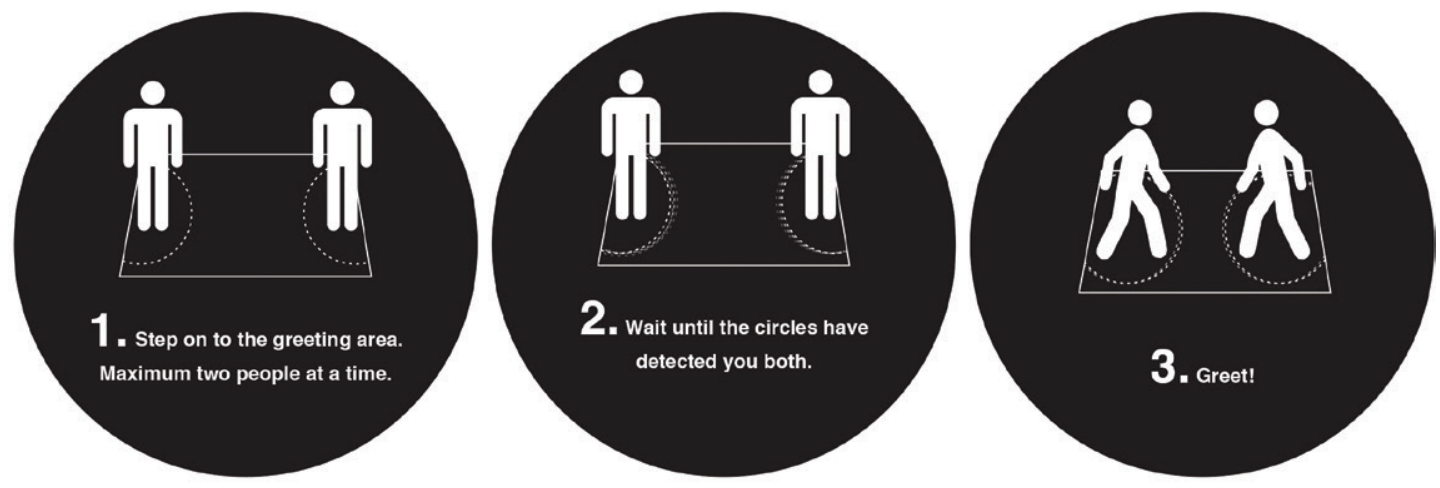

Fig. 211 - Instructions that were printed and attached to the wall of the greeting space 

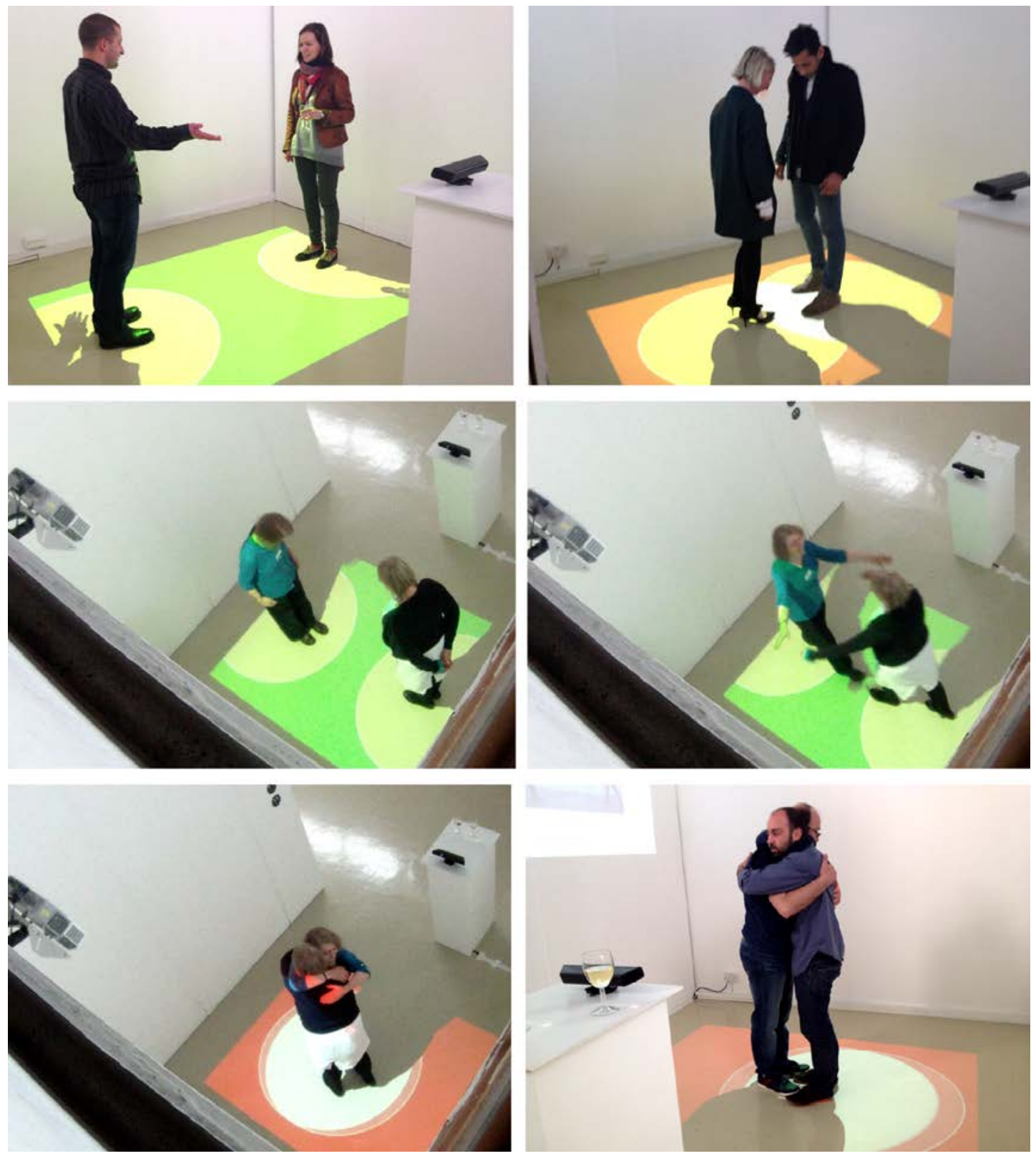

Fig. 212 - The greeting space in use

During the exhibition visitors were seen exploring and playing around in the greeting space: trying out a variety of greetings and attempting to trigger different colours by changing how close they would come to greet each other. Though my ideas of adding more conceptual elements to it such as sound and graphics were dropped owing to time and technical limitations, this simpler experiment seemed to work and attract interest beyond my 
expectations. For a video recording of the space in use during the exhibition, see Appendix 17c.

A number of technical and conceptual developments could be made in order to improve this installation. There was a slight delay from when the visitors stepped onto the greeting area until the motion tracking started: this caused some confusion, and would need to be reduced. The circles that were projected on the floor should grow and reduce in size depending on how close or far apart the two people greeting are, to reflect how our personal space changes depending on proximity.

In this chapter I have given a detailed description of the process of developing my system for the visual notation of greetings. While the system is a work in progress, and can still be developed further, I have achieved many of the improvements and developments of the earlier version that I set out to achieve.

My fieldwork generated completely new greeting documentation, in the form of video recordings of greetings and audio recorded interviews. This material has been a key resource for all my practical work, and the fieldwork has expanded my network in multicultural Stavanger. I am still in contact with some of the participants of my study, and I have been invited to cultural events since.

With regard to the structure and content of my system, I have redefined and expanded the types of greetings documented, so that they reflect more specific greeting situations. I have visualised more types of greetings: social greetings, family greetings and intercultural greetings. By including intercultural greetings in my research, the system has developed in many new and unexpected directions that explore cultural diversity. The current 
interface visualises and represents a range of dimensions of everyday multiculturalism found in Stavanger.

Another crucial development of my system regards movement and interactivity. In Kinesics I had annotated still vector images of greetings, whereas in my current system I present annotated animations that analyse each greeting as a sequence in time. Furthermore, the three greeting diagrams have been developed to allow users of the system to explore comparisons between greetings, and view multiple analyses of individual greetings, in an interactive and dynamic way.

Important developments have also been made in terms of the information represented in the new system. To indicate personal space, I changed the notation to visualise each person's individual space rather than a shared space. The visualisations now show how in some greetings, usually intracultural ones, people have the same relations to personal space, while in others, particularly intercultural greetings, people who greet are accustomed to different personal space patterns. By colour coding each person's use of personal space, such differences are communicated clearly. Another subsequent improvement arising from the separate coding of individual space was that it enabled the combined visualisation of personal space and subjective experiences, thereby also showing how these two dimensions, and different semiotic codes, may influence each other.

The visualisation of subjective experiences was itself a key development of my system. Previously, I had limited my visual annotations to represent signals that I could observe from the videos and animations. However, the cultural meanings of nonverbal signaling, and how meanings are interpreted differently by people, have been of great interest to my study, and in this project I have been able to explore ways to visualise them. 
As well as explaining the visual and technical processes of creating my system, I have explained in this chapter how to use it, and given examples of how it can visualise cultural differences (see fig. 153, and figs. 186-189). I have also shown that my system is not limited to this particular study, but can be applied to other greetings, such as the Obama greetings and those analysed textually by Alessandro Duranti.

While my main focus has been on the development of my system, I have also described, in section 4.6, how I experimented with using other technologies such as motion tracking. Such technologies are promising and could in the future be used to develop this project further, for example to create interactive physical spaces.

Although the present version of the system has a number of limitations, particularly in terms of technology, as a stand-alone piece it is fully functional. In the next and final chapter I will illustrate specific examples of how cultural knowledge can be extracted using the system, reflect on the scope for improvements, and suggest potential uses. 


\section{Findings and Conclusions: A Reflection on the System for the Visual Notation of Greetings}

In previous chapters I have described the contexts of my study as well as my own practice and processes. Chapter 1 sets out the multicultural context and the state of immigration in Scandinavia and in Stavanger. It illustrates the diversity of cultures that make up this multicultural city in which my fieldwork took place. Chapter 2 outlines the anthropological context of my study. It provides a historical overview of greetings studies within anthropology - how greetings have been understood in the past - and it established that greetings are now considered as exchanges of important verbal and nonverbal information. It also discussed notation systems developed within anthropology. I concluded that while these systems and techniques are important developments when it comes to using visual methods for studies of greetings, the systems and techniques have not been followed up and further developed at pace with technological developments and digital media. Chapter 3 sets out the visual and technological context of my study, discussing both past and present-day visualisation techniques that display great potential for creating visual representations of greetings. My system was developed utilising some of these concepts and techniques, and the practical process of developing the system is discussed in detail in chapter 4.

My task as a designer was to devise a system for the visual notation of greetings. As discussed in chapter 4 , the process of devising the system was a responsive one, and the field study in Stavanger influenced some of the structuring of my system and its interface. However, while the fieldwork played a valuable role in the design process, its key purpose was to gather documentation that would make it possible to create a working model of the system. My study in Stavanger is an example, the first example, of using my system to visually document, analyse and present a 'real' study and is a means of showing how the system works. 
In this final chapter I will discuss what kinds of knowledge and understandings my system makes visible and comparable through discussing selected examples from my research in Stavanger. I will also discuss limitations of the system and its scope for future developments, and finally, what kinds of uses and applications it may have.

\subsection{Cultural Knowledge Through Visual Notation}

This particular project contains important knowledge about cultural diversity and everyday multiculturalism in Stavanger. This knowledge has been made visible through the use of my system which has enabled me to communicate layers of information about how people in Stavanger interact during greeting and how their interactions have been patterned by their different cultural backgrounds. Local knowledge about what kinds of interactions occur during greetings, why they occur, and how they are experienced differently by people from different cultural backgrounds, can be found in my current project. Examples of how behaviour relating to gender and generational differences is patterned differently across cultures can be viewed in my animated visualisations of space, proximity, touch and subjective experiences. In the following two sections I look at specific examples from my findings that display such cultural patternings. (Images used in this chapter are all screen captures from the greeting animations.)

\subsubsection{Intracultural Greetings}

Intracultural greeting patterns are, according to many participants in my study, experienced as 'safe'. One Vietnamese female participant told that she considers them comfortable greetings because she knows when and how she should greet other Vietnamese people.

My own culture... When we greet each other it does not make me uncomfortable, because I know when and how I should greet the others. So for me it is not uncomfortable. (For a full transcript of this interview see Appendix 10f.) 
My study found that, as expressed by this Vietnamese participant, people know how they are supposed to greet people from their own culture in most situations. Fig. 213 shows a greeting between two Vietnamese females who are close friends, and highlights the distance they are both comfortable with in this greeting situation. Fig. 214 shows the level of intimacy they are accustomed to - in the orange intimate zone - which represents a small personal space and close proximity, and fig. 215 shows their subjective experiences; that they both experience it as a warm greeting. To summarise,
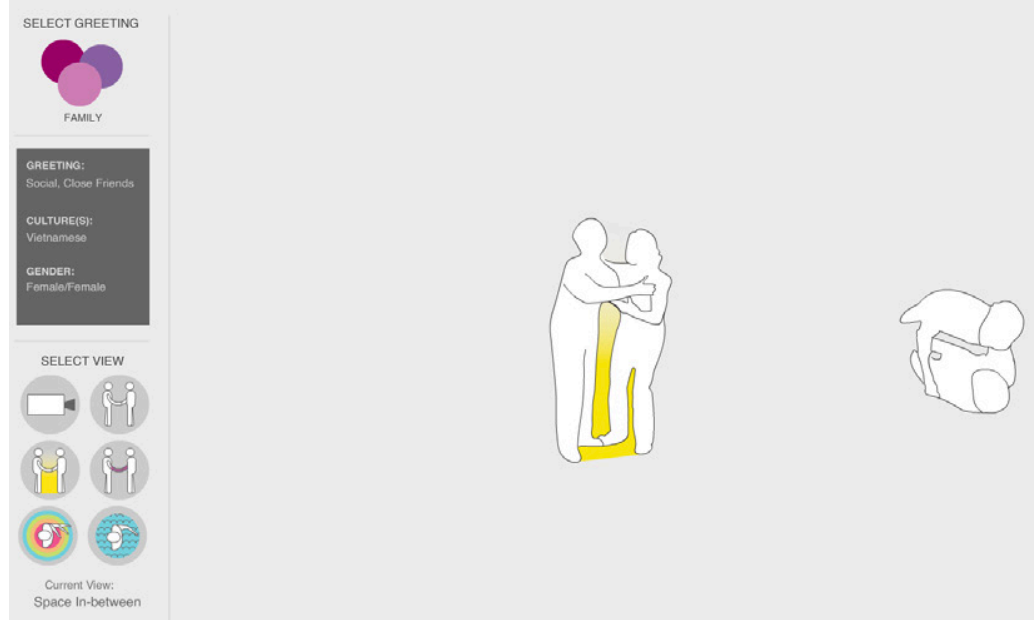

Fig. 213 - Still images from animations of a greeting between two Vietnamese females who are close friends (Vietnamese - close friends $-\mathrm{f} / \mathrm{f}$ ) - highlighting the distance between them

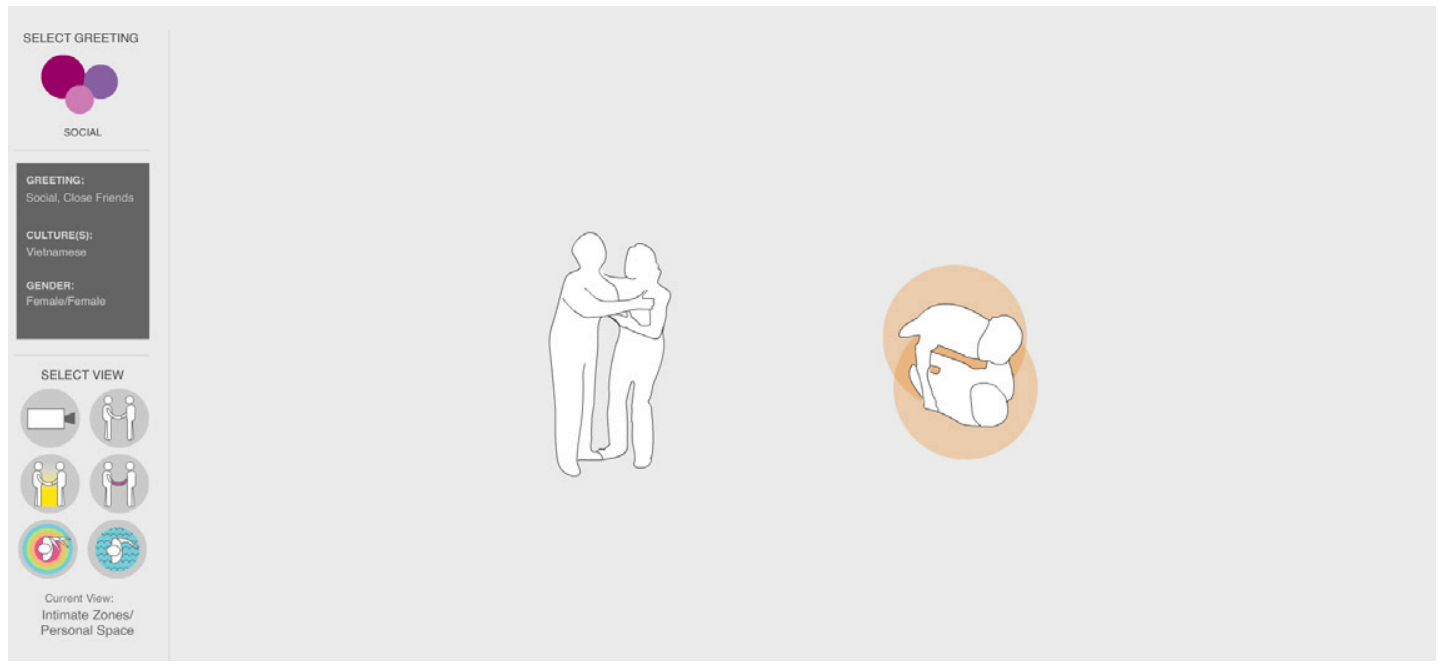

Fig. 214 - Greeting: Vietnamese - close friends $-f / f$. This image shows they are both in the orange intimate zone (1/2 arm's distance to $1 / 4$ arm's distance) at this moment of the greeting 


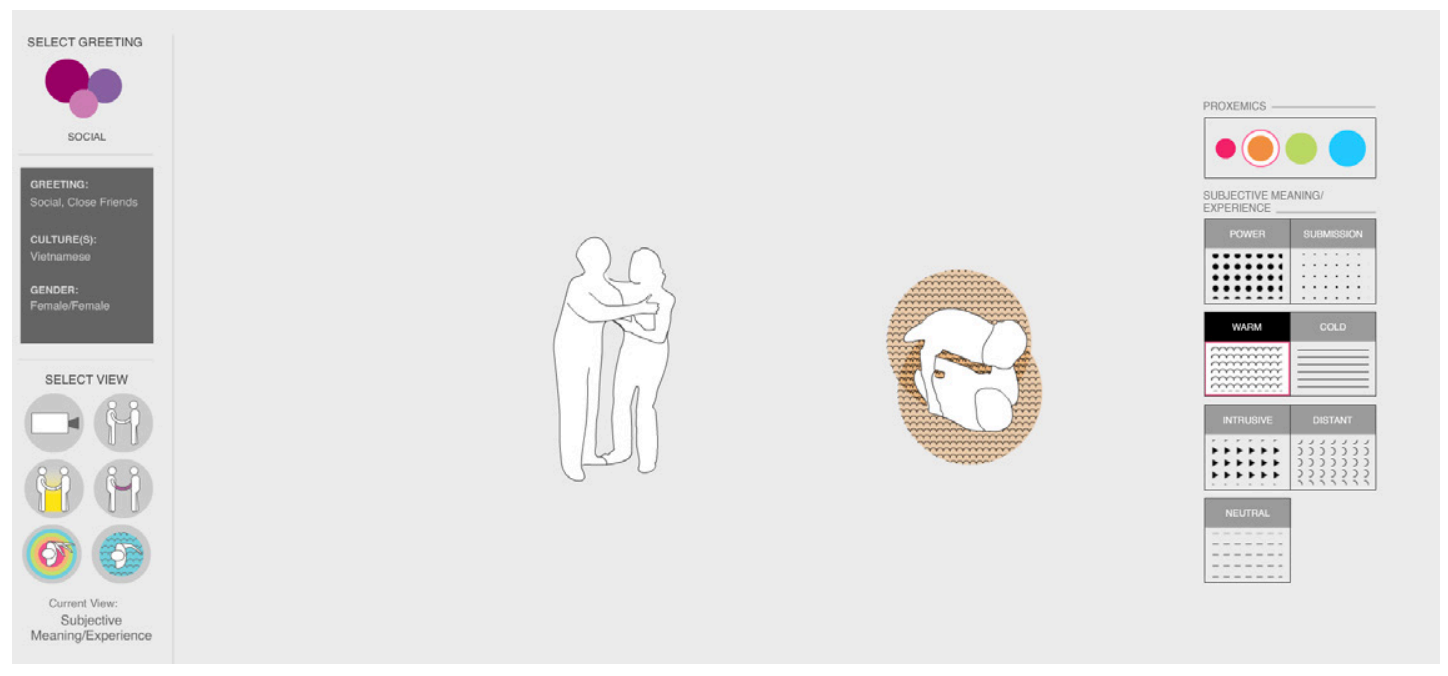

Fig. 215 - Greeting: Vietnamese - close friends - f/f. This image shows the intimate zones, as in fig. 214, and patterns of subjective experiences show that they are both experiencing this as a warm greeting

the images show us that their movement, use of space, and experiences are synchronised and patterned in a similar way so that the interaction is smooth, familiar, and without any notable hesitation or discomfort.

While intracultural greetings often follow a familiar pattern, such as this Vietnamese greeting and most other intracultural greetings I documented, I also found that intracultural greetings could be subject to cultural (and subcultural) differences. An Angolan female participant told of confusion and awkwardness sometimes arising when greeting other Angolans:

... the good thing is that in my culture in Angola we normally give two kisses when we say hello... even if we don't know them very well, we always do that. But sometimes... it is a typical thing, you expect them to give the two kisses, and then they put [out] the hand. We have so many kinds of greetings that sometimes it gets awkward because we don't know which one is going to be the chosen one. ... automatically we have this kind of code that maybe the girl goes to the left - first kisses left and then right. But sometimes people do the other way around, and then we are about to kiss in the mouth. It is really awkward... (For a full transcript of this interview see Appendix 10g.) 
A Norwegian female participant also told of sometimes feeling uncertain of how to greet other Norwegians:

Sometimes people I meet want to hug me and I'm not prepared for it. And other times I think, ok I should be better at hugging people, so I go and hug, and maybe they are not prepared for it, because it can be quite difficult to know how often and when you should hug someone. I don't know how to follow social guidelines for it, and I think people have very different social guidelines. So some people always hug, and some people don't, and some people hug in these situations but not those. And it depends when did you last see the person, and that is very relative, if you feel it is long ago or not long ago. (For a full transcript of this interview see Appendix 10b.)

This uncertainty was apparent during the studio recording sessions when the same participant greeted a male close friend and a male acquaintance. When greeting as close friends, both participants greeted with an embrace. When greeting as acquaintances they were unsure whether to embrace or not. They explained that sometimes, depending on how close they are to the person, they do embrace and at other times they simply greet with a verbal greeting. Figs. 216-217 show these two different greetings. When comparing the images, it is clear that there are obvious differences in all nonverbal dimensions. The visualisations of space in-between show much larger space between acquaintances than between close friends. The visualisations of touch show that there is a lot of touch between close friends (see fig. 216) compared to none between acquaintances (see fig. 217). The visualisations of intimate zones show that they interact in the red zone as close friends and in the green zone as acquaintances.

Finally, the visualisations of subjective experiences show that the greeting between close friends is experienced as warm and the greeting between acquaintances is experienced as neutral. These graphic visualisations do not show the uncertainty or frustration that the participants sometimes feel in greeting situations, as expressed in the interviews. In real life the borders 


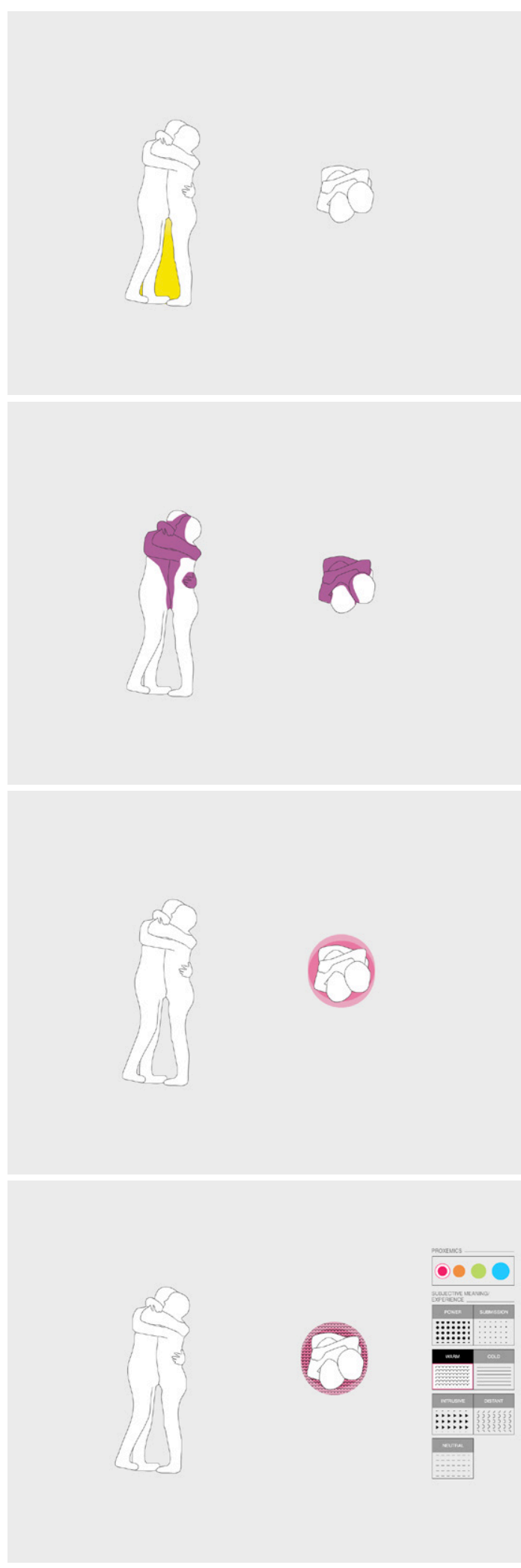

Fig. 216 - Greeting: Norwegian - close friends $-\mathrm{m} / \mathrm{f}$. Images are stills from animations highlighting space in-between (top), touch (second from top), intimate zones (third from top), and subjective experience (bottom)
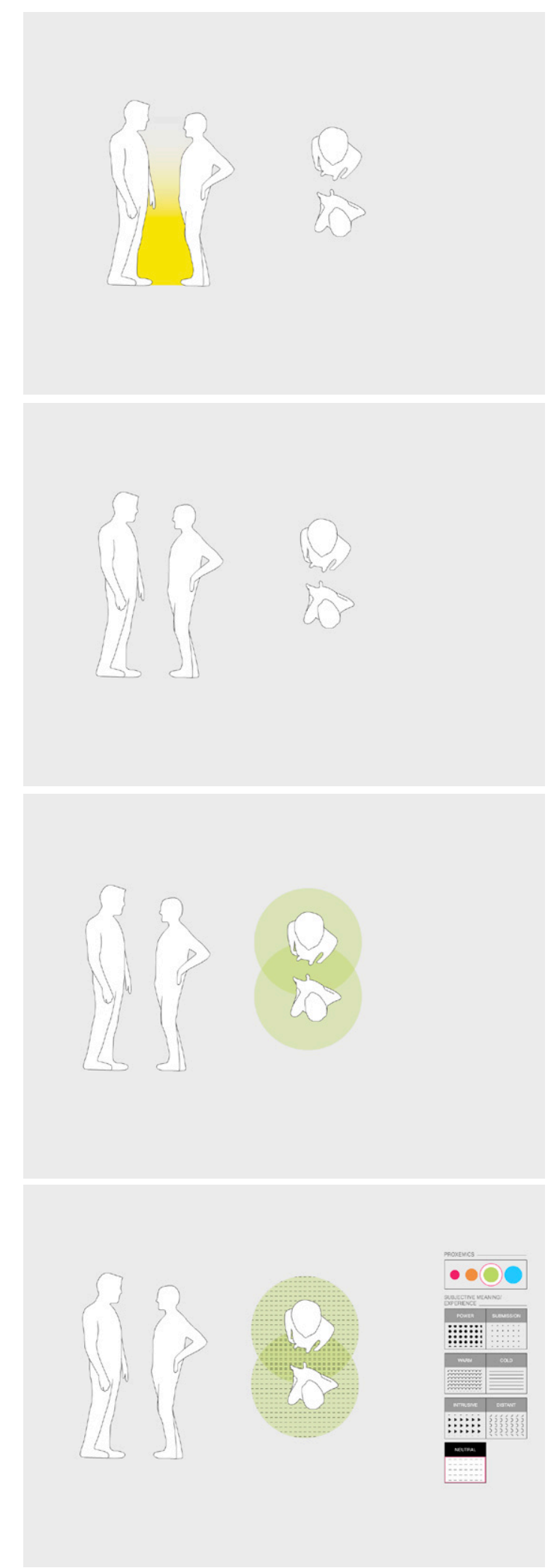

Fig. 217 - Greeting: Norwegian acquaintances $-\mathrm{m} / \mathrm{f}$. Images are stills from animations highlighting space in-between (top), touch (second from top), intimate zones (third from top), and subjective experience (bottom) 
between a close friend, a friend and an acquaintance may be more blurred than the system's categories can express, and how blurred these borders are varies from culture to culture and from one person to the next. However, the visualisations do highlight the fact that not all intracultural greetings follow the same patterns of nonverbal communication, and that each greeting situation can trigger very different subjective experiences.

\section{Gender}

In my research the most noticeable and perhaps severe intracultural difference in greeting behaviour was documented between two Turkish participants. The difference was caused primarily by their different religious backgrounds, which meant that they had opposing expectations of how the sexes should interact with each other. This difference became apparent during the initial, unrecorded, meeting of the pair, and later in the interview audio recordings. Upon arriving at the studio before the actual recording took place, the two Turkish participants approached each other to greet. The male put his hand forward to greet her with a handshake, and the female responded with folding her hands and greeting verbally with a smile. She explained that since this research deals with greetings she wanted to show how she prefers to greet men in a social context - which is with no physical contact. She made clear that she usually would respond to a handshake if the person she greets were unaware of this Muslim custom.

The Turkish male later explained that he was offended by her greeting, and claimed that what she was showing was not representative of a Turkish greeting, or of their culture. In a separate interview, the female explained that it made her very uncomfortable when people, such as the Turkish man, who should be aware of the Muslim custom, do not respect it. She felt embarrassed on his behalf because in her view he should, when he saw she was wearing a headscarf, have respected her customs although he was not Muslim himself. The Turkish male got angry because he felt that she was generalising about Turkish people and Turkish women. He said that if this 
situation had happened to him in a different context he would never want to speak to that person again, and he would find it very disrespectful if someone did not respond to his handshake. He did admit that he was very sensitive to these issues due to the political situation in Turkey at the time ${ }^{1}$. (For full transcripts of the interviews, see Appendices 10d-e.)

In the greetings that were visually recorded, the Turkish female participant also greeted a different Turkish male participant. They greeted with no touch when showing a greeting between a male and female who are close friends. She said in the interview that because her close friends are aware of her beliefs she greets them the way she is most comfortable with. This male participant said he would usually give two kisses on the cheeks to his close female friends, but if they are Muslim and prefer to not touch, he respects it and follows their way of greeting. In the greeting between female and male strangers, showed by the same pair, the female adapted to his way of greeting. She said that although she prefers not to greet with a handshake she would respond to it if she greets someone for the first time and they are not aware of her customs. Figs. 218-219 show that these two greetings are similar in terms of proximity and personal space, and differ only in that their hands are touching. The Turkish participants did share the same greeting customs for other situations that were documented, such as family greetings, but the greeting between the Muslim female and the non-Muslim male illustrates that clear cultural differences can be found within the Turkish culture.

Intracultural differences also arise when people within a culture adhere to cultural traditions and customs to varying degrees, as was explained by four young Muslim Somali female participants. To them, they said, there is

\footnotetext{
1 At the time of conducting this interview, in 2013, there was serious unrest in Turkey. There were clashes between the police and anti-government protesters in Istanbul's Taksim Square. Thousands of protesters gathered to protest against the government and the apparent authoritarian rule of prime minister at the time Recep Tayyip Erdoğan. Turkey was also approaching the 2014 presidential election, which Erdoğan won (BBC News Europe, 2014a, 2014b; Letsch, 2014; Uras, 2014).
} 


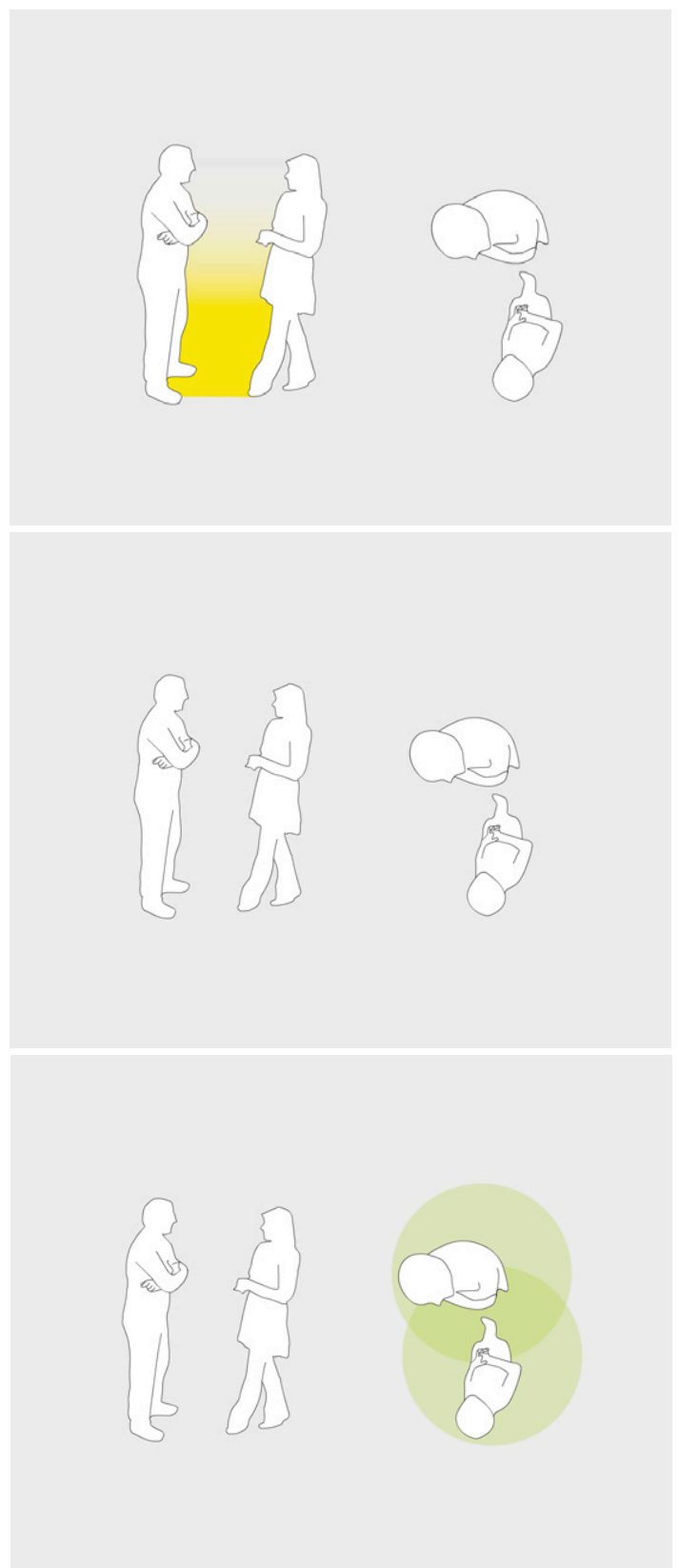

Fig. 218 - Greeting: Turkish - close friends $-\mathrm{m} / \mathrm{f}$. Images are stills from animations highlighting space inbetween (top), touch (middle), and intimate zones (bottom)

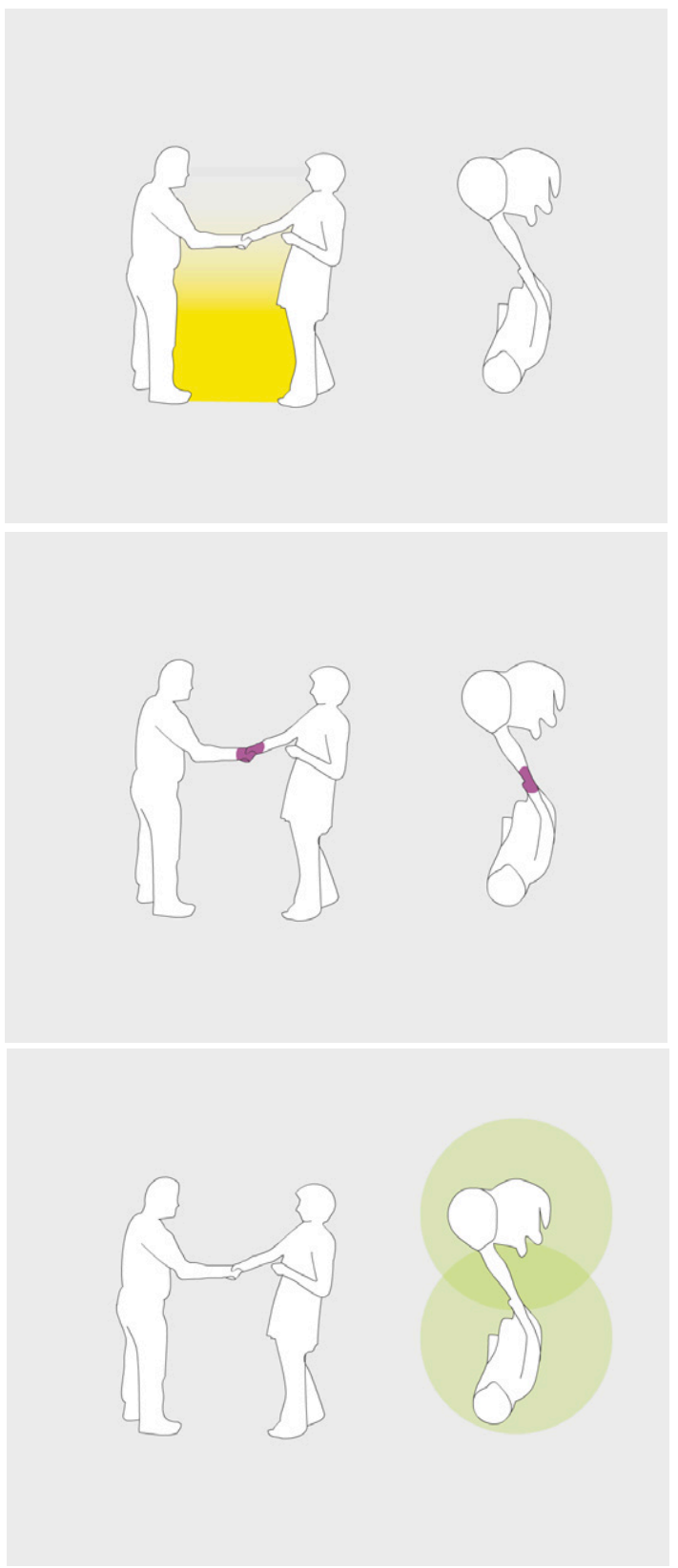

Fig. 219 - Greeting: Turkish - strangers $-\mathrm{m} / \mathrm{f}$. Images are stills from animations highlighting space in-between (top), touch (middle), and intimate zones (bottom) 
a difference between following their religion and following secular Somali culture. For instance, if they follow (Muslim) religion, and depending on how religious they are, they should not touch the opposite sex. This means they should not, as already seen in the Turkish female to male greeting, shake a man's hand during a greeting. However, they explained that if they follow secular Somali culture they can shake the hand of the opposite sex. What greeting a Somali person living in Stavanger performs depends on how religious the person is, within the variations of Somali culture, as well as to what extent he or she has adapted to the Norwegian culture.

Intracultural difference can also be found in greetings between two people of the same gender. The same female Turkish participant, who was born in Norway to Turkish parents (refugees), and who identifies as being Turkish, explained that sometimes it is confusing when she meets Turkish people who are unaware of how her and her friends greet each other in the Turkish community of Stavanger. Between female friends, they do three kisses on the cheeks rather than two. The three kisses, she explained, is a greeting used by close female religious friends who are active in the mosque in Stavanger. However, it is not a common greeting for Turkish people living outside Stavanger: they usually kiss twice, or two kisses followed by two hugs. So the extra kiss on the cheek distinguishes Turkish females born in Stavanger from Turkish females born in Turkey or even elsewhere in Norway (see figs. 220-221). Sometimes if the other person is not aware of this greeting, the third kiss can cause confusion and embarrassment as it may be experienced as invasive.

One Turkish male participant, a labour immigrant who is non-religious and has been living in Norway for the past seven years, said that confusion can arise when he greets other Turkish men because in Turkey men often kiss each other on the cheeks, but some men prefer to give a handshake, so sometimes there is confusion if one person goes for the handshake while the 


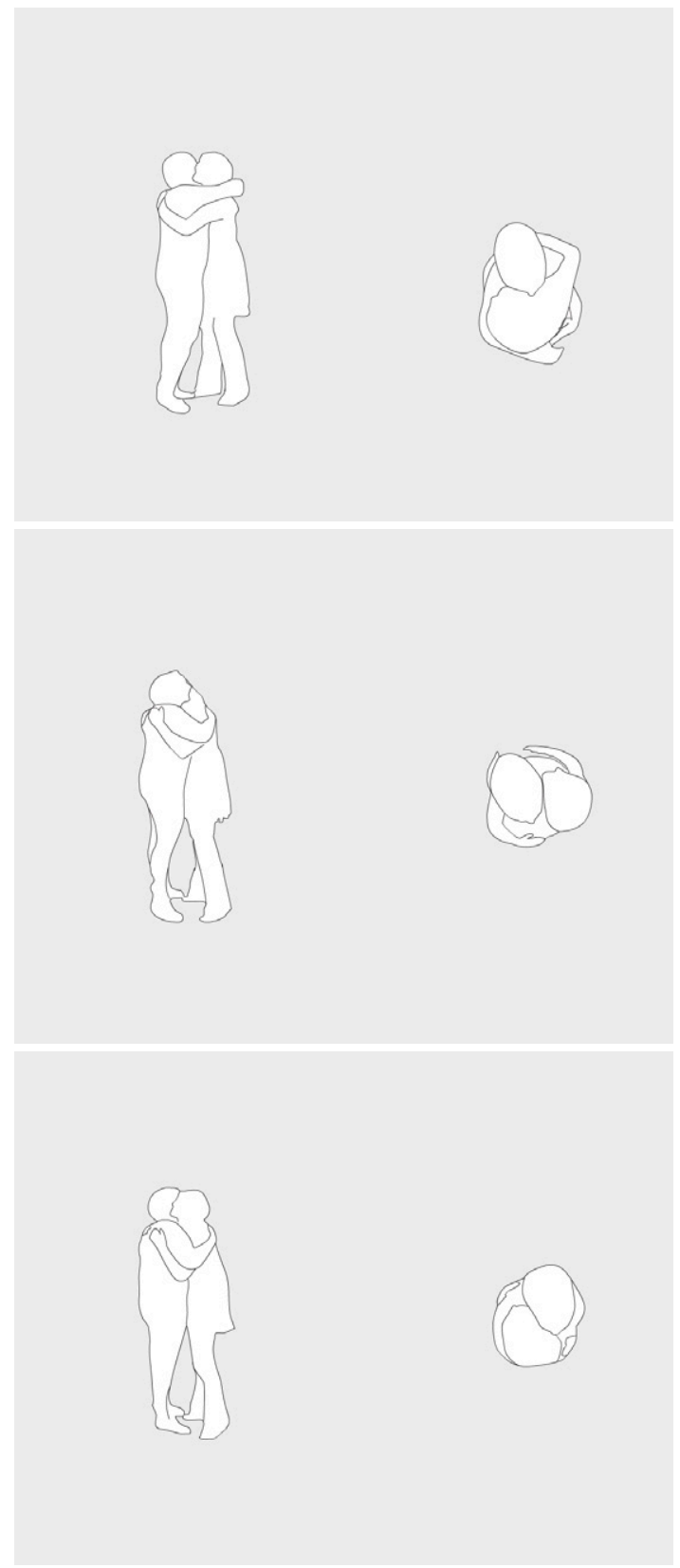

Fig. 220 - Greeting: Turkish - close friends $-f / f$. This greeting, involving three kisses, is used by Turkish female close friends (Muslims) in Stavanger

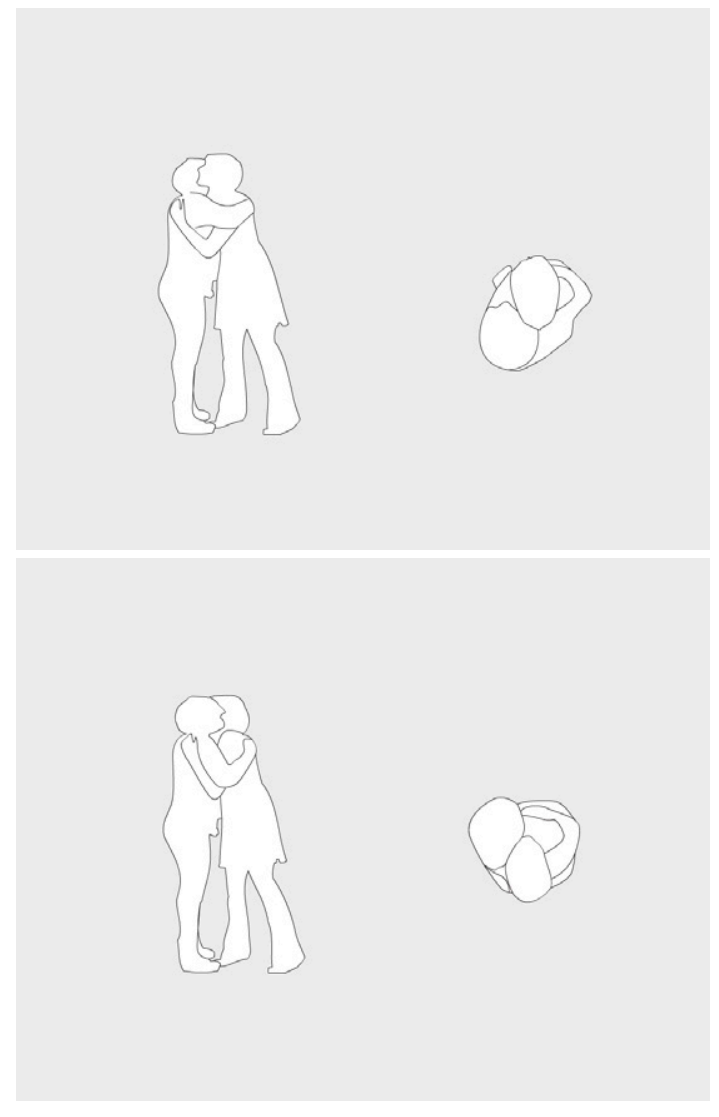

Fig. 221 - Greeting: Turkish acquaintances $-\mathrm{f} / \mathrm{f}$. This greeting, involving two kisses, is generally used among Turkish female close friends (Muslims) outside Stavanger 
other one leans forward to kiss. Because he has been living in Norway for so long he says he is not used to the male-to-male kissing greeting anymore and can sometimes experience this greeting as awkward when he goes back to Turkey.

These differences in behaviour, beliefs and opinions within a national culture show how intracultural greeting customs can have significant variations. The Turkish intracultural differences were the most notable ones observed during my fieldwork, but cultural and sub-cultural variation and difference exist in every country and culture. While these examples show that difference in behaviour and customs can be experienced intraculturally, I found that such differences were even more prominent in intercultural greetings.

\subsubsection{Intercultural Greetings}

My study shows that confusion, uncertainty, unfamiliarity, and discomfort are common experiences and feelings that arise during intercultural greetings. I also found that different factors were responsible for triggering such feelings. Two highly educated (and well-travelled) Norwegian participants, a male and a female, expressed that they find it confusing and difficult to know how to greet people from different cultures, such as not knowing if one kiss, two kisses, three kisses, a hug, or just a handshake is the appropriate greeting. Also not knowing whether to start to the left or the right side when greeting with a kiss, can cause great confusion when greeting people from what the male participant termed 'warmer cultures'. The female had experienced that personal space could also be an issue when greeting people from other cultures who are often comfortable with coming up close when greeting, while she prefers more distance. They both spoke of feeling awkward, uncertain, stupid and uncomfortable during such greeting miscommunications. (For full transcripts of these interviews see Appendices 10b-c.) A Vietnamese participant expressed the same confusion and nervousness, and that she does not know how to greet people from other cultures: whether to do an 
embrace, a handshake or just a 'hi'. (For a full transcript of this interview see Appendix 10f.)

Many participants in the field study mentioned that a firm handshake is seen as a standard and 'safe' greeting in Norway, particularly when meeting someone for the first time. One Turkish male participant's solution to situations where he is unsure of whether to give one, two, or three kisses is to just shake hands. The handshake is a straightforward greeting that seems to work cross-culturally. However, some participants view the handshake as a very Western way of greeting, a Turkish Muslim female participant speaks of the handshake:

If I greet a Norwegian, a non-Muslim, I make sure to give a very firm handshake to show my personality. It might be more of a conscious thing, I am Muslim, I am a woman, I wear a headscarf, but I also have power. If there is something I can't stand, it is those sloppy, weak handshakes, making you look suppressed. I am SO for showing that this [wearing a headscarf] is our choice, that we are independent, have power, and so on. (For a full transcript of this interview see Appendix 10d.)

Here the Turkish female admits to occasionally adapting to the Norwegian style of greeting because she does not want to be seen as a suppressed Muslim woman. While it is important to her to maintain her identity and to practice her cultural and religious customs, such as no physical contact with men other than her husband, she sometimes adapts in order to defy the stereotype - of a suppressed woman - that she knows she is associated with. So, she adapts to the local, or Western, way of greeting in order to be considered an equal.

One might expect that the Norwegian way of greeting, the way of the host, is the accepted form of greeting in Stavanger, and that immigrants are expected to learn and adapt to it. I found this to be true only to some extent. With Stavanger becoming increasingly multicultural, international customs 
and greetings do mix with more traditional Norwegian ones. On the subject of whether greetings have changed or are changing in Stavanger in the past years, many participants agreed that they have. Many believed that part of the reason for this change was immigration from people from all over the world. One female Norwegian participant found it difficult to determine whether greetings had changed or if just her own way of greeting had changed since she moved to Stavanger. Interestingly this change had not affected how she greets her Norwegian friends. For instance, she would never greet her Norwegian friends with kisses on the cheeks, while she would apply this greeting when meeting her French friends in Stavanger. She had learnt to read the French way of greeting, and to adapt to it. Her view was that in Norway there is not a very strong culture for greeting, because she, and other Norwegians, tend to adapt to the immigrants' ways of greeting instead of them adapting to the Norwegian way of greeting.

Figs. 222-224 show a similar scenario. These figures compare a social greeting between two Norwegian males with the same greeting between a Chilean and a Norwegian male, and between a Turkish and Norwegian male. They show that the Norwegian male, who took part in all three greetings, adapts to the greetings of the immigrants. The greeting between him and the Norwegian male is the greeting he said he is most comfortable with. This greeting involves a handshake, and as fig. 222 shows, there is a larger space in-between them, very little touch - only the hands touching, and they interact in the green intimate zones - with half an arm to an arm's distance. In the other two greetings, between him and a Chilean male (see fig. 223) and between him and a Turkish male (see fig. 224), the use of space and levels of touch are quite different. As the images highlight, there is no distance inbetween them, more touch, and they interact within the red intimate zones. So the Norwegian adapts the way he greets these immigrants to suit the ways of greeting that are familiar to them. 


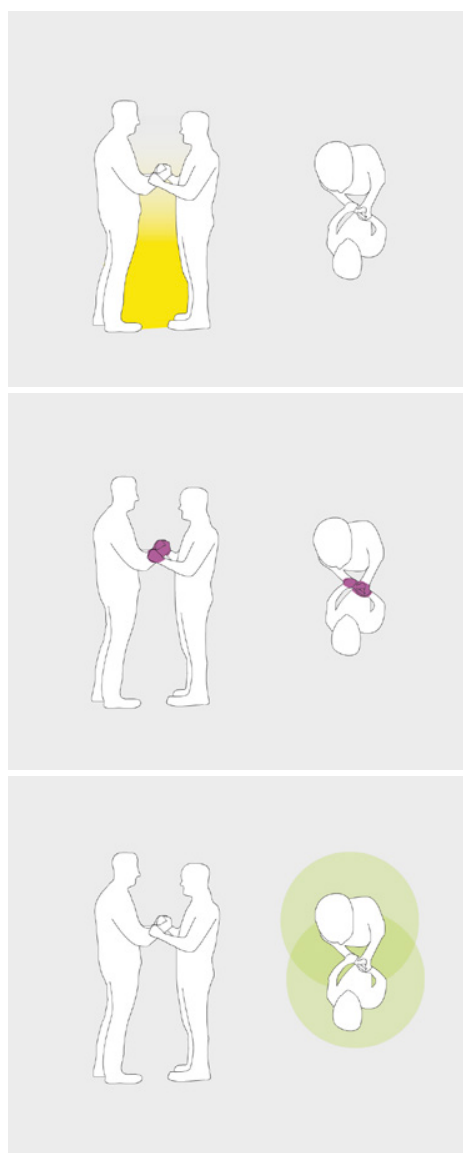

Fig. 222 - Greeting: Norwegian - close friends $-\mathrm{m} / \mathrm{m}$. Space in-between (top), touch (middle), and intimate zones (bottom)

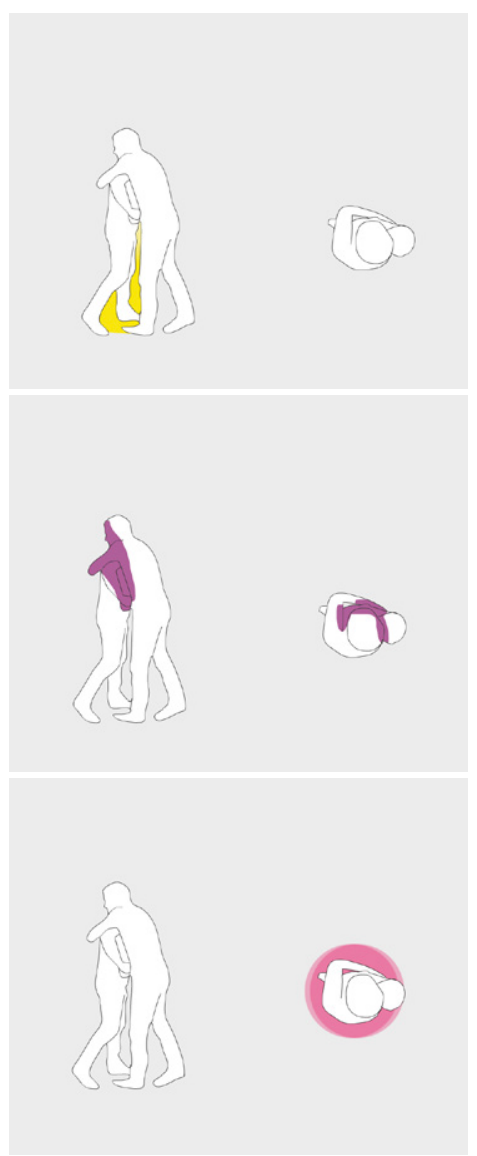

Fig. 223 - Greeting: Chilean/Norwegian - close friends $-m / m$. Space in-between (top), touch (middle), and intimate zones (bottom)

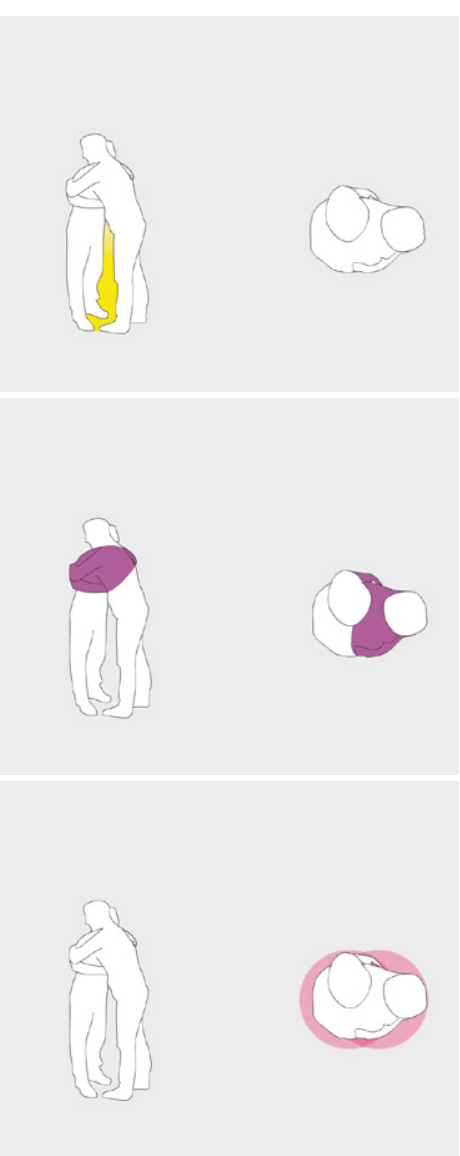

Fig. 224 - Greeting:

Turkish/Norwegian - close friends $-m / m$. Space in-between (top), touch (middle), and intimate zones (bottom)

As these examples show, it is not always the immigrants that adapt to the ways of the host society - some adaptation occurs from the host too. Regarding her fieldwork in the Australian neighbourhood of Ashfield, Amanda Wise (2009) talks of 'mutual care'. Residents there have developed a mutual care for each other, which has resulted in an equal relationship between them. This relationship does not follow a host/guest relationship in which the migrant is constantly expected to be grateful towards the host. It is based on mutual respect, and both sides adapt to each other's ways and customs (Wise, 2009). My study shows that 'mutual care' can be seen in some greeting situations in Stavanger too, such as in the last example: it is quite often Norwegians who adapt to the greetings of immigrants, and not always the other way around. 


\section{Gender}

As the previous example of the greeting between the Muslim and nonreligious Turkish participants illustrated, the handshake is not always a 'safe' option. Having learnt about the preference of the Turkish female when greeting a male during my first studio session, a Norwegian male participant (who attended both studio sessions) spoke of feeling uncertain whether this same greeting 'rule' applied during his first meeting with four Somali female participants, who were all Muslim. He explains:

Yes, if you meet a woman wearing a hijab or a head-scarf or something, you instantly become uncertain about how to greet her, I have just learnt during the filming of the Turkish greetings that not all women will give you a handshake due to their religion, so now when I meet people who are wearing a hijab, I naturally start thinking about it, 'If I reach out my hand now, am I being impolite or rude?' . (For a full transcript of this interview see Appendix 10c.)

Important to note here is the fact that the Somali girls responded to his handshake without hesitation. The Somali participants explained that although the handshake is more typical of Western cultures, and that it feels unnatural for them to do a handshake, especially woman-to-woman, they often adjust to the Norwegian way of greeting. This example, and others, shows that there are distinct cultural differences in how members of the different sexes are expected to act and interact, and that intercultural greetings are seldom pre-defined with a set of definitive behavioural guidelines to follow.

When comparing the Turkish and Norwegian cultures, for instance, a Turkish female participant said she finds the Norwegian greetings between the opposite sexes very strange. During greetings between Norwegians, she explained, women hug women, and women hug men, but you seldom see men hugging men. Her Turkish husband also found this very irrational when he first came to Norway - that he should hug the wife or girlfriend of his male friend, but not his own male friends. In Turkey it is the opposite: 
there he hugs and kisses his close male friends, but not their girlfriends (see figs. 225-226). The Turkish couple found this Norwegian greeting amusing because it was such a contrast to their own Turkish (Muslim) culture where they share intimate greetings with the same sex, but maintain

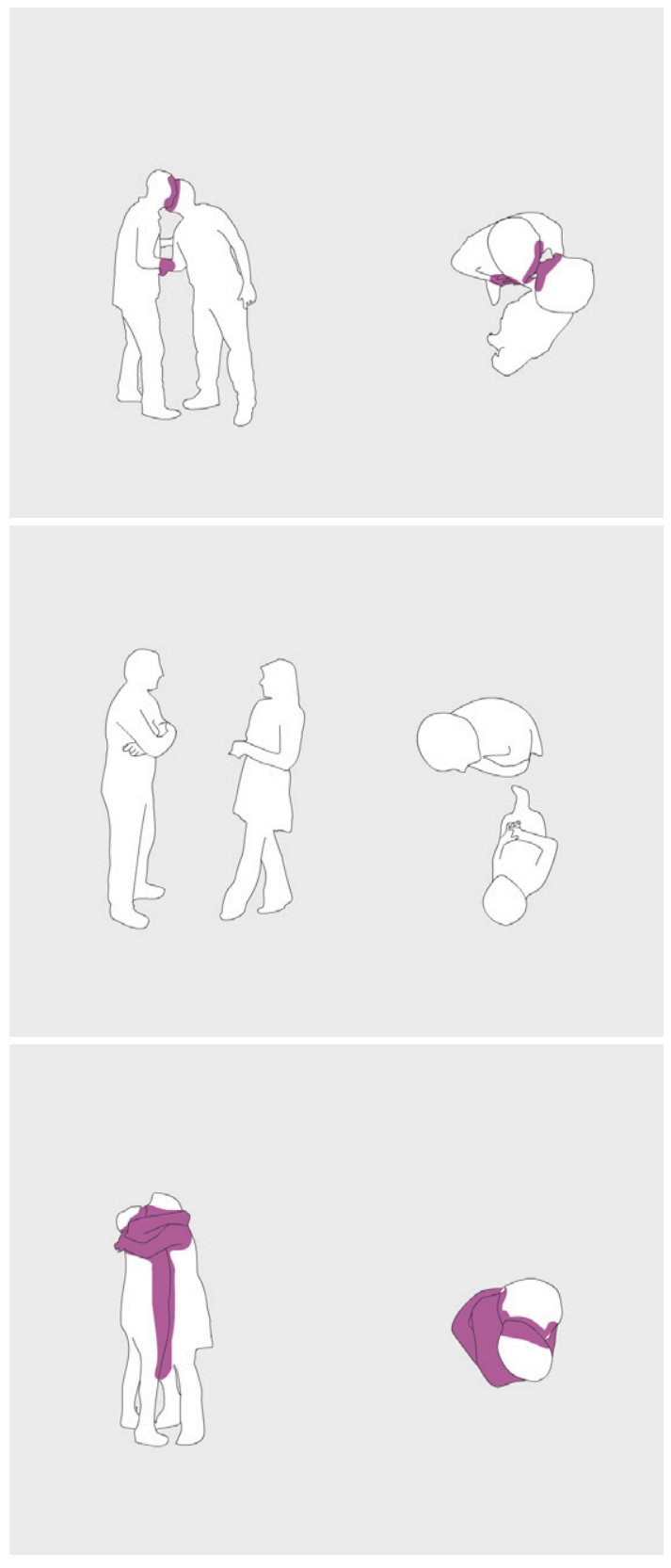

Fig. 225 - Turkish social greetings between close friends $-\mathrm{m} / \mathrm{m}$ (top), $\mathrm{m} / \mathrm{f}$ (middle), $\mathrm{f} / \mathrm{f}$ (bottom). The visualisations show levels of touch during a moment of the greetings

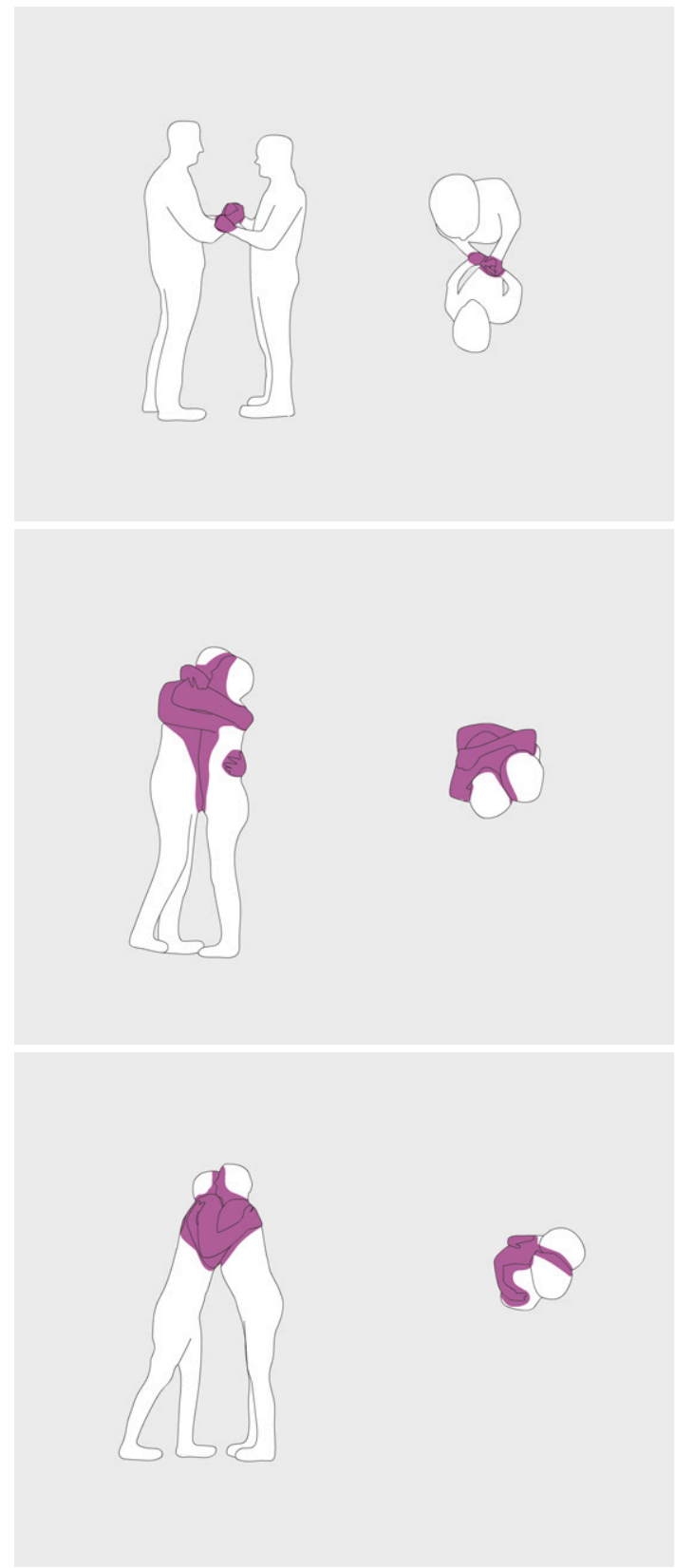

Fig. 226 - Norwegian social greetings between close friends $-\mathrm{m} / \mathrm{m}$ (top), $\mathrm{m} / \mathrm{f}$ (middle), $\mathrm{f} / \mathrm{f}$ (bottom). The visualisations show levels of touch during a moment of the greetings 
more of a distance with the opposite sex. When asked about this same sex and opposite sex greeting, a Norwegian male participant said he would get scared if another male tried to kiss him during a greeting.

Yes, that is too much for me. Then I get scared. Well you feel like you almost get... almost like you get sexually assaulted. To get kissed by a man... that is really strange for me. Their lips have nothing to do on my body to be honest. (For a full transcript of this interview see Appendix 10c.)

In these two greeting examples by the Turkish and Norwegian males we find very different and contrasting views on what is a preferred and culturally accepted greeting between two men, and between a man and a woman. While the Turkish Muslim male finds it awkward to hug and kiss a female friend, he is comfortable with kissing his male friends on the cheeks - a greeting the Norwegian male would find very uncomfortable. To each participant the greeting of the other is strange and unfamiliar.

First-time cross-cultural greetings between strangers seem to follow a slightly more fixed pattern. Although a sense of uncertainty regarding how to greet the other is usually present, most participants said that they usually give a handshake the first time they meet someone. Many defined the handshake as a 'safe' greeting. But sometimes a handshake is not responded to as one had anticipated, as seen in yet another recording of a greeting - between an Angolan female participant and a Norwegian male participant who were strangers greeting for the first time. He approached her preparing for a handshake, while she embraced him and gave him a kiss on each cheek, unaware of his attempted handshake. The Norwegian participant, who said he is most comfortable with a handshake at arms-length, explained that greeting situations like this were very intense for him, and could leave him feeling invaded (see figs. 227-228). Many other participants said they had also experienced unpleasant or awkward moments during intercultural greetings. 


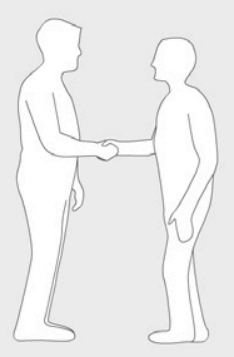

Fig. 227 - Greeting: Norwegian strangers $-\mathrm{m} / \mathrm{f}$. Greeting in the green intimate zone, showing both participants staying within half an arm to an arm's distance

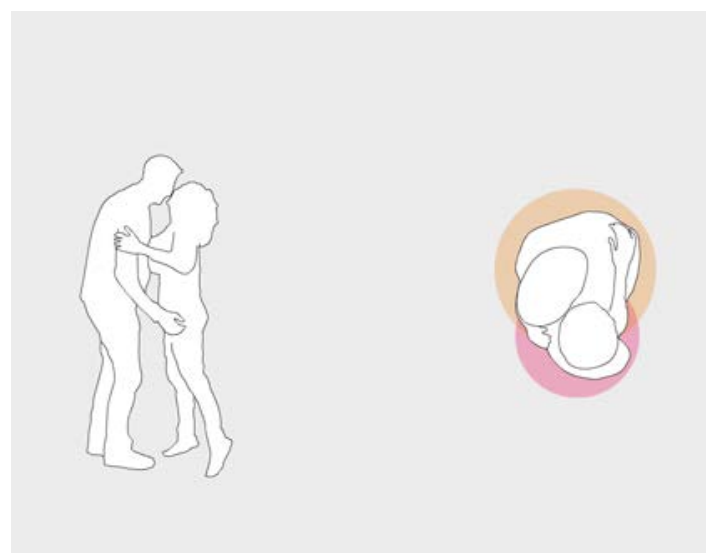

Fig. 228 - Greeting: Norwegian/Angolan - strangers $-m / f$. Greeting in the orange and red intimate zones, showing participants' different relations to the closer proximity of the greeting

As shown in the examples mentioned, greetings in the multicultural city of Stavanger are shaped by, and can reveal, gender-related cultural difference. An attempted handshake greeting by a Norwegian male participant, documented in my study, illustrates this point further. His greetings, as mentioned, triggered very different responses from women depending on their cultural backgrounds, and depending on how strict they were in their cultural practice. The fact that his attempted handshakes were met with: a full embrace followed by two kisses by an Angolan woman; a 'rejection' of the hand due to religion by a Turkish Muslim woman; and a response to his handshake by a Somali Muslim woman, illustrates some of the complexities that can, and do, occur during intercultural greetings in Stavanger and elsewhere. Though a handshake is perhaps the most common way of greeting a stranger from any culture for the first time, many of my examples have shown that even this straightforward gesture is not completely accepted and practiced cross-culturally. The example of the greeting between the Turkish Muslim female and the Norwegian male, makes clear that what is accepted in one culture can be viewed as inappropriate in another. 


\subsubsection{Generation \& Hybrid Identity}

I have so far looked at examples from my study of intracultural and intercultural differences and of gender issues within each of these categories. However, it is not always possible to divide greetings into intracultural or intercultural ones, as they may be both at once. A grandparent from an immigrant culture and a grandchild born in the host country, for example, may have different cultures as well as a shared culture. The greeting patterns of the grandparent and grandchild will vary in many situations, while in other situations will be identical owing to their shared cultures. This section looks at differences in greetings across generations, both in terms of how greetings have changed and continue to change across generations, and of how culture is practiced to different degrees across generations, in particular with regard to people with hybrid identities.

During conversations with participants in my study, it became clear that social greetings in Stavanger have changed a great deal in the past 20-30 years. A Turkish female born in Norway pointed out that Norwegians in Stavanger have taken the positive qualities from different cultures and integrated these into their ways of behaving during greetings. She explained that during her years in secondary school in Stavanger it was only her and other foreign girls who used to hug each other when greeting, then at some point a few years later it became fashionable for the Norwegian teenagers too to hug and kiss each other. Today, she said, Norwegian teenagers hug each other much more intimately than she is used to. (For a full transcript of this interview see Appendix 10d.) A Norwegian male participant, in his late 30s, also spoke of this change:

Well, I think that teenagers now are much more 'huggy' and cosy with each other than we were when we were teenagers. Now it is much more physical, or my impression when I see teenagers now is that it is much more physical - how should I put it - much more continental. It could have to do with the society being much more integrated, that there is much more international influences around. I also think we 
are living in a more and more globalised world. We are much more international now, the distance from Norway to the US is very short, just a fraction of a second on the Internet and you are there...TV, and everything. You get influences from all directions. (For a full transcript of this interview see Appendix 10c.)

An Angolan female participant, who has been living in Stavanger for the past ten years, shared a similar experience, and spoke of the changes she had seen during her time there:

I think that Stavanger became much more multicultural, and people have adapted more to the other cultures, and they are very curious and open, so they also try to copy our kind of customs, and we are all exchanging. We adapt to their culture, so we are all meeting in the middle in some ways. And in a group, if we are a big group, some will hug, some will kiss, some will do two kisses, some will do three kisses, some will shake [the hand], and in the end we hug. So it definitely changed for the better. It is much nicer. (For a full transcript of this interview see Appendix 10g.)

While TV, media and outside sources influence behaviour, so do people's own multicultural backgrounds and connections, as illustrated in my earlier example in chapter 1 of the Dutch person with hybrid identities who also identified with contemporary hip-hop culture. For children of immigrants, cultural diversity is part of their everyday lives and identity. A female participant from Vietnam, for example, explained that there is a difference between people like her who were born in Vietnam, and those born in Stavanger to Vietnamese parents. Her younger cousins who were born in Stavanger, for instance, greet their Norwegian friends with a hug, while they greet other Vietnamese with just a 'Hi', keeping with the Vietnamese customs. (For a full transcript of this interview see Appendix 10f.) The greeting here acts as an identity marker for the combined Vietnamese and Norwegian cultures these young people identify with. They were born in Norway but they still practice their Vietnamese culture when meeting and 
socialising with other Vietnamese. This point cannot be generalised to apply to all second-generation or third-generation immigrants, as some people embrace their parents' culture(s) while others may not have much interest in retaining these cultural traditions. The extent to which a person retains and practices the cultural customs of their parents varies from one community to the next, and from one person to another. Another Vietnamese female participant talked of a similar situation within her own culturally diverse family: Our way of kissing is completely different. The Vietnamese use their nose when kissing their children. My kids do not like it. In Vietnam only couples/lovers kiss on the mouth, but when I kiss my kids it is with my nose.

My partner is from Iraq - there men kiss each other when they greet two men, they do not actually kiss, but they make a kissing sound when they greet each other. (For a full transcript of this interview see Appendix 3.)

Her children adjust to and practice their three cultural identities to different degrees. She kisses her son's cheek with the 'nose kiss' greeting she is used to from South Vietnam. Her partner greets their son in the Iraqi way, with kisses on the cheeks, and when meeting his Norwegian friends, the boy greets them in the Norwegian way, usually just a 'hello' or a hug. There were many similar examples of diversity found within families in my study, in which different generations of immigrants would greet differently depending on who they greeted and depending on the extent to which they identified with their different cultures. As one Somali girl explained, there is a big difference in behaviour between her, a second-generation immigrant, and her mother, a first-generation immigrant. While she will greet Norwegian males with a handshake, her mother will not. These examples show that greetings are changing and mixing according to the multiple identities that are found within families and in the wider culturally diverse society of Stavanger. 
What has become apparent during my study is that the way a person performs a greeting in a given situation tells us something about the person's cultural identity, or indeed identities. It is a physical pattern of behaviour, whose meanings operate through the wider cultural codes that in turn pattern experience and identity. My study illuminates some of the complexities of cultural identity and interaction in a multicultural society, and how these complexities are reflected in greetings. As discussed by Wise and Velayutham, the study of human interaction, in this case intercultural greetings, can reveal everyday strategies that residents use to negotiate cultural difference during interaction (Wise and Velayutham, 2009). When people from mono-, bi- or multicultural backgrounds interact, differences and similarities are manifold, and confusion and embarrassment are among the emotions that commonly occur. The examples I have outlined demonstrate why and how a simple universal ritual like a greeting can be an important source of knowledge through which better understanding of everyday multiculturalism can be reached.

\subsection{Present Limitations of the System}

In the previous section I have outlined some of the specific cultural knowledge produced by my research in Stavanger, which shows at a more general level the kinds of understanding that can be visualised and made accessible by my system. However, reflecting on the design, structure, interface and technology used for the system, there are a number of limitations that could be improved upon in future developments. Some of the key limitations concern the structure of the current interface; others involve technical processes and their effects on accessibility.

\section{Structural Limitations}

1) The interface in which my system is presented is structured to represent cultural diversity according to national cultures, and in its current format it does not cater for all complexities relating to cultural identity, such as people 
having hybrid identities ${ }^{2}$. However, the cross-cultural diagram does, to some extent, show how people adapt to different cultures during greeting. As I have also pointed out, there are generational, gender, and personal differences in how people within a culture greet each other. Generational differences have been represented in family structures/greetings - in how parents and grandparents are greeted - but not in all greeting types. This study did not document generational differences in social greetings, for example how a Vietnamese teenager greets a stranger vs. how a Vietnamese elderly person greets a stranger. However, such specific studies could be carried out in the future.

2) Another limitation of my interface is its inability to show different variations of a greeting. Often there are different ways of greeting within a situation owing to personal preference, region, religion or other reasons. As seen in the examples of the male to female Turkish greeting, there was significant difference in how they preferred to greet owing to their different religious beliefs. I have not included an option to show multiple varieties of each greeting in the current interface.

3) In the cross-cultural diagram there is a limitation, or unclarity, in male to female greetings. I have not recorded two versions of each greeting, so that both genders from both cultures are represented. I have, for example, recorded a greeting between a Norwegian female and a Somali male, but not a greeting between a Norwegian male and a Somali female. In hindsight it has become obvious that a greeting could possibly take a different form if the Norwegian was female and the Somali was male, or vice versa, but such a distinction has not been represented in the system.

2 I have adjusted the diagram for family greetings slightly to adapt to the fact that one family attending was intercultural - Chilean/Norwegian. The label originally named 'Chilean' has been renamed to 'Chilean/Norwegian' in this diagram because the greetings recorded of this family are greetings between a Chilean parent and a Chilean/Norwegian daughter, a Norwegian parent and a Chilean/Norwegian daughter and so on. 


\section{Technical Limitations}

1) The process of creating the greeting animations is, as discussed in chapter 4, still very manual. This means that if other researchers were to analyse their greeting documentation visually, they would need to master the technologies and software I have used to achieve the same visual outcome. This would be a very time-consuming and challenging task.

2) There are limitations with having used Adobe Flash (and Actionscript 3.0) for a variety of reasons. Adobe Flash is not an open platform, it is owned by Adobe and sold by Adobe only, as opposed to other programming languages/ platforms such as HTML 5, CSS, and JavaScript that are all open web standards (Jobs, 2010). So, in order to play the Flash file containing the interactive greetings diagrams interface, the user must have Flash Payer installed on their computer. As most computers do have it installed, and it is free to download and install for those who do not, this is not a major problem. A more significant problem is that Flash files do not work on Apple mobile and tablet devices such as the iPad and iPhone. My interface is made for computer screen playback, for both PC and Apple computers, but has not been optimised for touch screens.

Although there are clear limitations to the system and interface as implemented in my current project, this chapter has argued that this research project is useful in representing many dimensions and complexities of human interaction, and I have demonstrated this in the example of the multicultural city of Stavanger. There are a number of potential developments of the system and its interface that could be explored in post-doctoral work, in terms of content, visual presentation and technical processes. 


\subsection{Potential Developments of the System}

\section{Content/Information}

As mentioned in chapter 4, there were further dimensions of both the data gathering phase and the analysis phase that time did not allow me to explore. As described in that chapter, I documented greetings from four different perspectives with the aim to study the relevance and signification of facial communication such as eye-contact during greeting. This dimension of the greeting analysis has remained relatively unexplored. The significance of eye contact, smiling, and other facial expressions, was mentioned in many interviews with participants, and so these elements have had some influence on the visualisations of subjective experiences. However, they have not yet been explored in terms of creating unique visual notation purely for facial expressions. This area opens up a whole new field of informational content for which notation could be devised in the future.

\section{Visual Presentation}

There is a variety of ways the project can be developed in terms of the visual expression and the way information is presented to the user. While I am equally concerned with the graphic and visible qualities of the system interface as with the technology, at this particular stage of devising the system I have had slightly more focus on the visual. The key concern was to create a graphic notation 'language' and an interactive structure for presenting the animations - in the form of an interface - rather than to explore different forms of technology and programming languages.

I have identified four main visual features that should be developed and refined:

1) The first one concerns the quality of the vector drawings. Some of the vector illustrations of people could benefit from additional treatment because some of the lines are quite rough, and there are slight inconsistencies in how much detail is included in the outlines of the bodies, such as hair and clothing (see fig. 229). Participants of my study wore different types of clothes and 

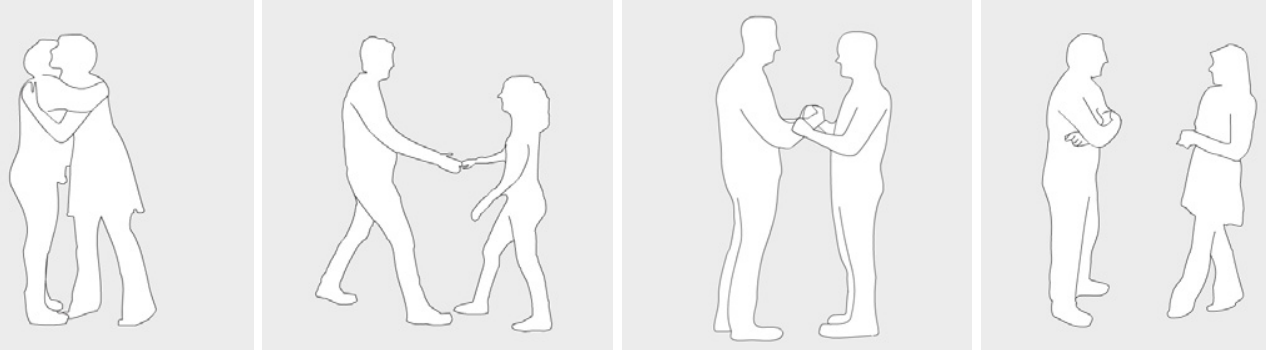

Fig. 229 - Images showing inconsistencies in vector drawings such as clothing and hair - vs. no clothing/hair

headwear, which sometimes made it difficult to know what lines to follow in the line drawings. The sole purpose of such a development would be to improve the visual presentation of the greetings in the system.

2) The second visual development has to do with adding a function to show different variations of a greeting. As it is now, although I had recorded different variations of some greetings, I selected the one I felt was most representative and most used. Therefore it would be desirable, as already discussed, to have the possibility to show multiple versions of a greeting type. This would give a more complete and realistic view of greetings in a multicultural society. This function could also be used to show the different versions of male to female combinations in cross-cultural greetings, and would be an important further development of the current structure of the interface.

3) The third visual development is to add a function that would allow the viewer to switch on and off the different layers of visual notation, so that it would be possible to view the animation with any number of visual dimensions added to it at the same time. At the moment each view is a separate timeline animation, so must be viewed separately. It would make more sense if the user were able to control this view better, to also see that 
all these nonverbal cues are layers of information that happen at the same time. This development would make the viewing of and interaction with the notation more intuitive and free. Fig. 230 is a simulation of what a greeting between a Norwegian and a Somali male, who are strangers, would look like with all the nonverbal visual layers switched on. This view draws attention to all the various information channels that are at work: space in-between, touch, intimate zones and subjective experiences patterns.

4) The final visual development I have identified so far that could give the viewer a clearer perspective on specific differences across cultures would be to create another way of viewing the greetings. An option could be added that would make it possible to compare two greetings, and all their dimensions, side by side to reveal clear differences or similarities between greetings and across cultures. This development would perhaps be most interesting

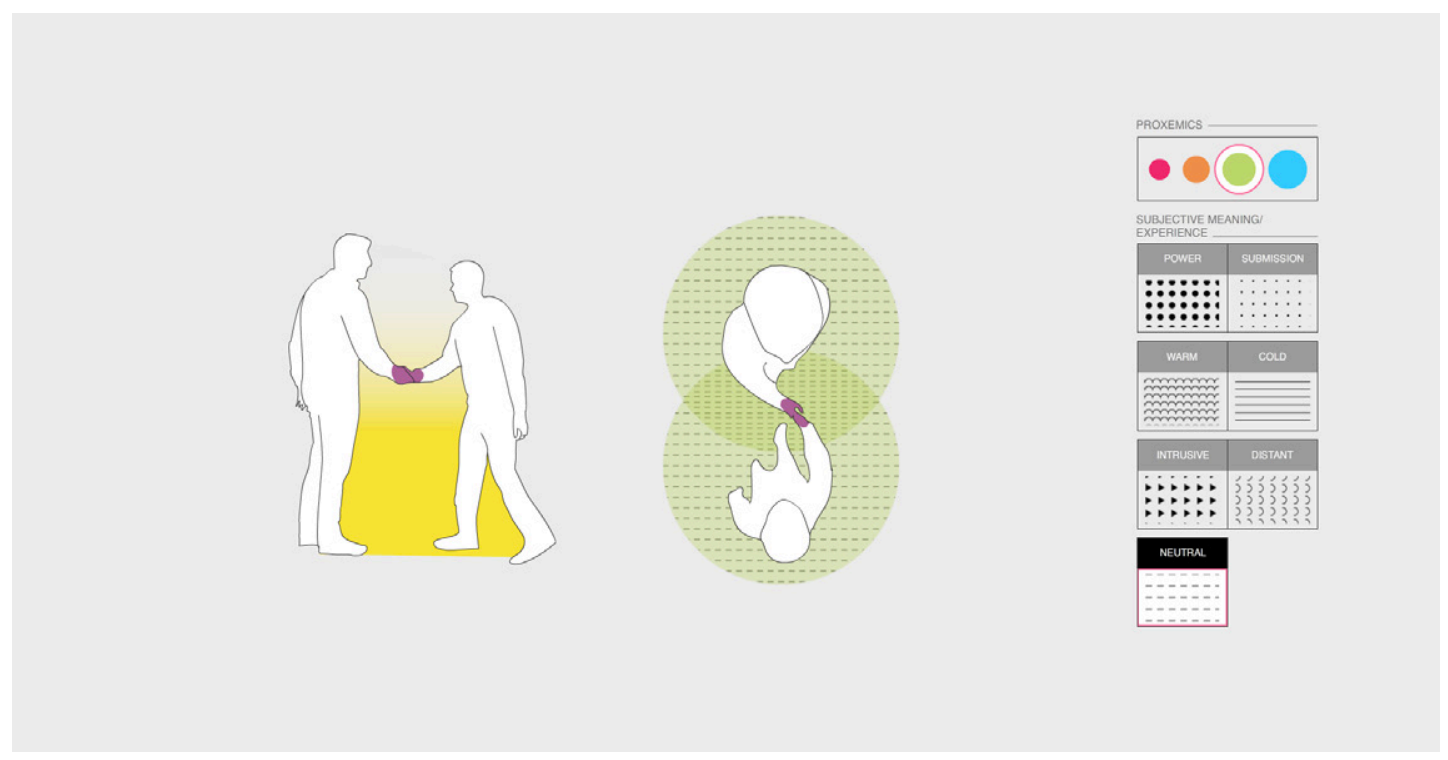

Fig. 230 - Greeting: Somali/Norwegian - strangers - m/m. Image shows all layers of notation applied in the same greeting animation 


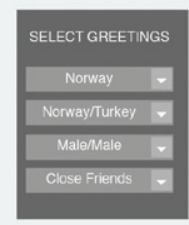

SELECT VIEW

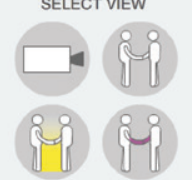

(2)

Current View:
Physical Contact
Towht

Touch
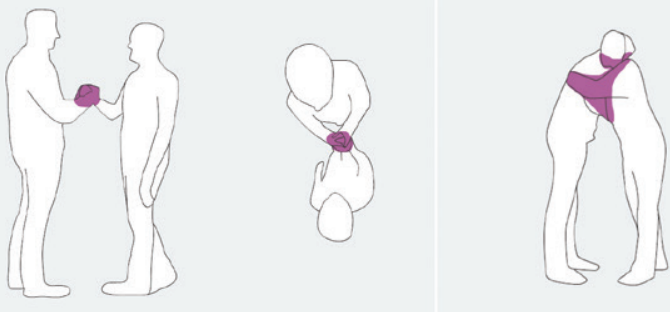

[Norwegian/Turkish]

Fig. 231 - Simulation of interface for comparing two greeting animations side by side

for anthropologists who could use it to analyse differences across cultures, visually side by side. Fig. 231 shows a simulation of what such an interface would look like.

\section{Technical Processes}

Reflecting on the process of creating the system, and the way information is presented, I have identified a number of technical aspects of the system that could be improved and further developed. While I have identified four key potential visual developments, the features that stand out as the most pressing when it comes to further development of the system relate to its technological construction and output of the system. In the current version of the system, the methods used are very manual, as discussed in chapter 4, and complicated for other (non-designer) researchers to use. So it would be essential, if others were to benefit from the system, to make the visualisation process a more automated one. This could be done in at least two ways - by using existing software to help speed up the editing/visualising process, or by creating new software that would be custom-made for the purpose of creating visual representations of greetings. 
1) The first option of using existing software would be the least expensive and least time-consuming. Sophisticated technologies and advanced programming, as used in projects such as Motion Bank and by Random International, could potentially improve this process in the future. Although I would need to do more research into existing software and technologies to find the most suitable solutions before pursuing this option, I have found some available software that could potentially be used for parts of the visualisation process. Piecemaker (PM2GO), which is a video annotation software that enables cue editing and colour categorisation, developed through the Motion Bank projects, could be used to create a timeline to map moments of the greetings in which important motion and exchange happen. This could possibly reduce the number of frames necessary to vectorise per greeting. Another potential technique involves using motion-tracking technologies. Motion tracking software will become more affordable and available in the future, which could help speed up my process of recording the greetings as graphic shapes rather than video, and thereby cutting the lengthy process of editing videos and converting them to graphic animations. I have also been advised by a programmer in the Motion Bank project, that the programming language and development environment Processing could perhaps be used for my visualisations, and to make my process more automated $^{3}$. These steps could help reduce the time it takes to edit the video material in the initial stages of the visualisations. However, using additional software such as these would not be much use for a non-designer, such as an anthropologist, because the process would still involve a lot of manual labour in the stages of creating the graphic animations and diagrams.

3 This advice was given during the Digital Heritage International Congress 2013 where I presented my paper Patterning Culture: Developing a System for the Visual Notation of Greetings (Shortt, 2013), and where participants of Motion Bank/The Forsythe Company presented their paper Capturing of Contemporary Dance for Preservation and Presentation of Choreographies in Online Scores (Kahn, Keil, Müller, Bockholt and Fellner, 2013). 
2) The second and more favourable development would be to develop new software that could make the visualisation process more automated. The aim would be to create a programme that would automatically add the visual annotations to any greeting video that was input into the program, by the click of a few buttons within the software. To undertake such a sizeable development I would need to collaborate with programmers, and receive funding to make such collaboration possible. As mentioned I have used Actionscript 3.0 programming language, which has a number of limitations. If software is to be developed, programming languages HTML5, CSS, and JavaScript that are open web standards should be used instead of ActionScript 3. The system could then also be made responsive and be developed into Apps for mobile technologies, including Apple products, such as games, and travel and business relations guides.

\subsection{Uses \& Applications}

In this section I will discuss a number of potential uses and applications of my system. The two main categories of uses are anthropological and multicultural/social. The most obvious use is for anthropological analysis. Although I am a designer, not an anthropologist, the potential for the anthropological use of my system is evident. As discussed in chapter 2 , visual notation systems developed in the past for use in anthropology were mainly created for documentation and analysis purposes, and not for presentation of research. Like those systems, my system attempts to make sense of nonverbal communication through visual means. However, unlike those systems, my system can be used for the documentation, analysis and the visual presentation of anthropological research. Its aim is to provide researchers and the field of anthropology with a new method of analysis and way of presenting research that could work alongside current methods that are primarily textual (as seen in Duranti's analysis of the Hillary Clinton greetings in chapter 4). This visual method will allow anthropologists to share their research and findings with a wider public than current textual transcripts and analysis seem to do. Further, the system can be used to 
study intracultural and intercultural greetings alike, expanding the field of greetings studies which has so far primarily been studied intraculturally, as also discussed in chapter 2 .

The fieldwork in Stavanger was undertaken in order to devise the initial model/prototype of the system. However, in the future the same process could be used to analyse any greeting situation in any society, and it could be developed to include other types of greeting situations, and to highlight other subjective experiences such as a greeting being experienced as; insulting, friendly vs. rude, polite vs. impolite, authoritative, and so on. It could also be used to study other forms of interaction such as parting rituals, group interactions, or similar (see fig. 232).

The second use of the system is multicultural/social. As discussed in chapter 1 , this study aims to create awareness and understanding of everyday multiculturalism - of how people live and experience cultural difference in multicultural societies (in this instance, Stavanger). By analysing and presenting the findings through a visual system that can communicate across language barriers, the findings can also be accessible to participants of the study no matter what their language or cultural backgrounds. The use of the system for this study is socially valuable as it can be used to learn about the
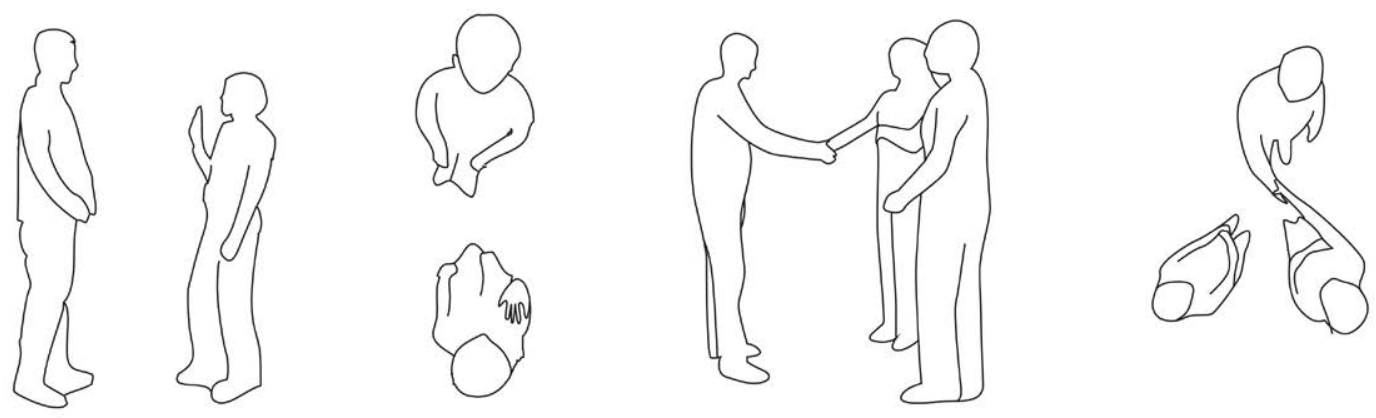

Fig. 232 - Potential development: using my visual methods to represent other forms/rituals of nonverbal communication such as parting rituals (left) or group interactions (right) 


\section{WELCOME TO NORWAY}

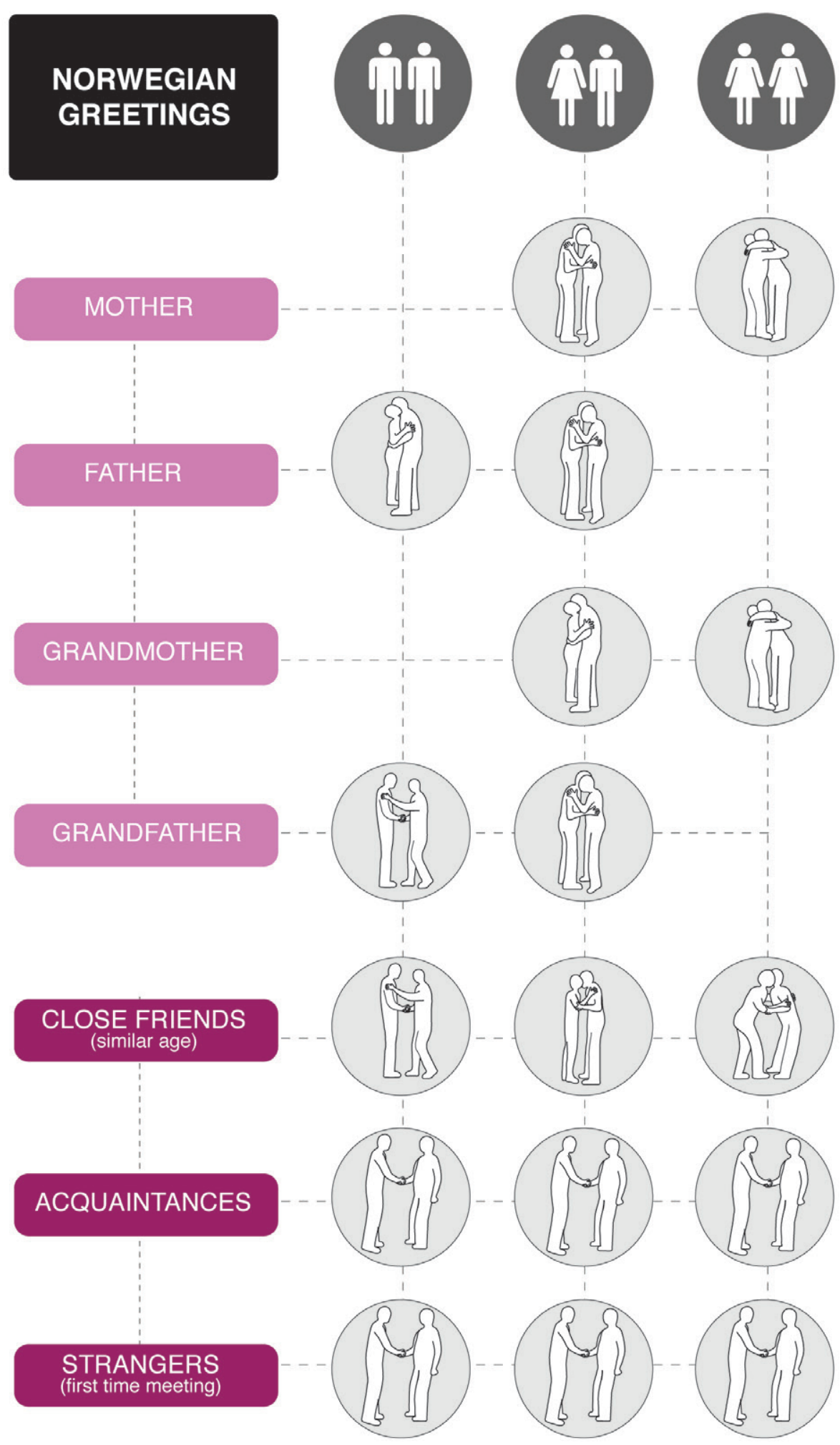

Fig. 233 - Potential development: visual diagrams aimed at teaching immigrants about local greeting customs 
multicultural diversity in Stavanger, and could function in a range of contexts. It could be used at induction courses in Stavanger to teach immigrants about local (and national) greeting behaviours (see fig. 233). It could be used in business settings, such as during or preparing for intercultural business meetings to create cultural understanding. A visual system such as this could become invaluable in assisting teachers, educators, and businesses in training people in cross-cultural understanding and empathy. One participant in my field study who works as a senior consultant for a Norwegian oil services company, said (in an unrecorded discussion) that such a system would be very useful in this company and industry because they do business worldwide and it could help up-skill staff and prepare them for cross-cultural business meetings.

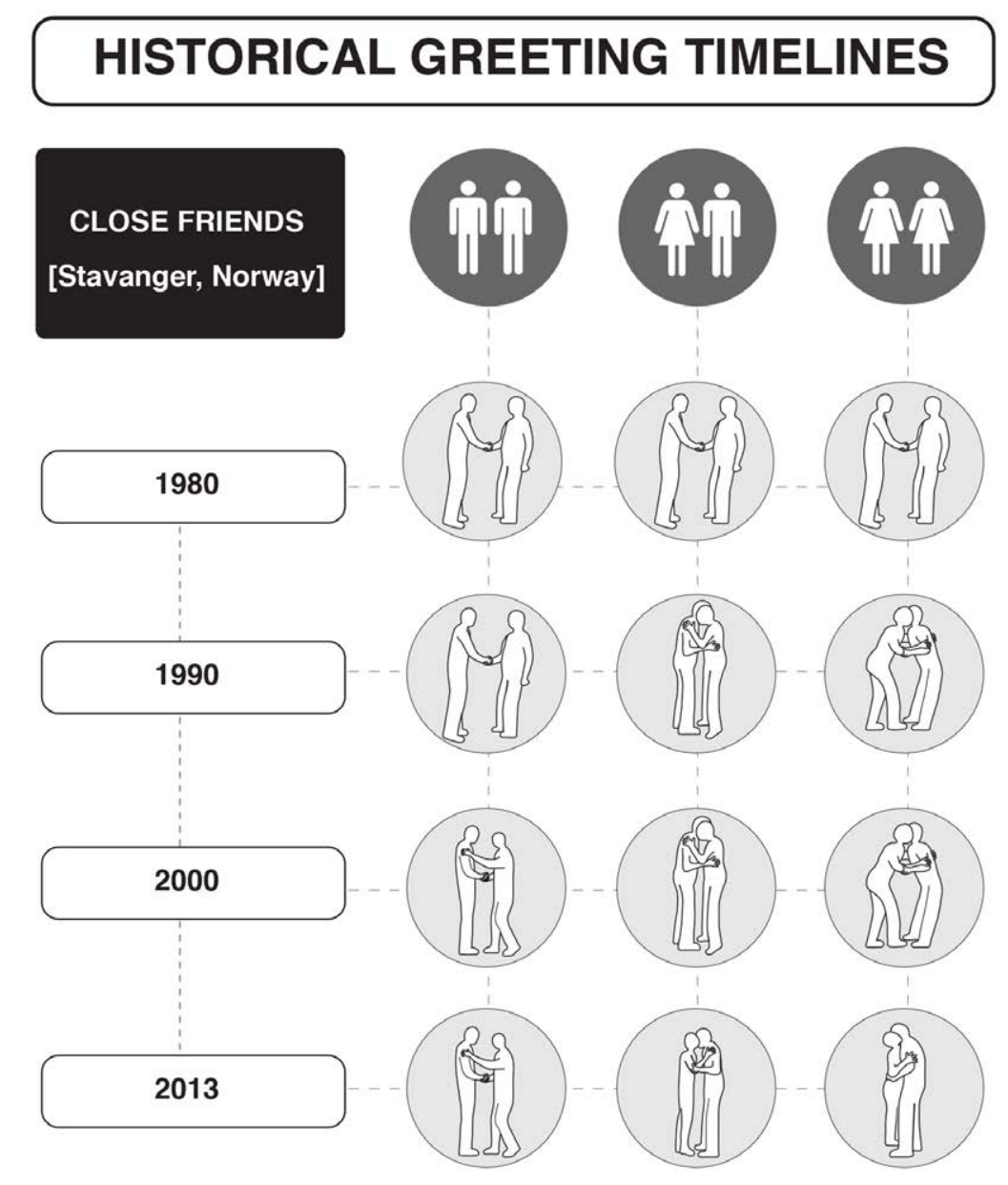

Fig. 234 - Potential development: visual diagrams mapping changes in greeting patterns over a period of time (years) 
Finally, the system, if updated with new content regularly, could also be used for archiving and visual preservation of greeting patterns, to map changes in greeting behaviour over a period of time (see fig. 234). If it were updated (e.g. annually) it could serve as an indicator of how the growing multicultural population influences local greeting behaviours. As these examples show, there are a number of potential expansions, applications and practical uses of the system.

\subsection{Conclusions}

In this study I have developed a system for the visual notation of greetings, using fieldwork in Stavanger as an example to illustrate the use of the system and what kind of knowledge it could produce. I have been able to carry out visual analyses of specific greetings that were recorded, and to present layered information about some of the cultural complexity found in Stavanger, in an understandable visual language. This study illustrates through a simple and basic form of human interaction - the greeting - some of the diversity found in multicultural societies: intracultural and intercultural differences, same sex and opposite sex issues, hybrid cultures, and more. As I now have a working model of the system, the aim, as discussed in this chapter, is to carry on the research and development of this system into one that can, in the future, be used by other researchers across many disciplines such as: anthropology, sociology, psychology, art, international business and teamwork; and for many different purposes: learning about local greeting customs and cultural diversity, preparing for travels or emigration, and preparing for international business meetings and negotiations. This is an ongoing project that could, in time, contribute to the field of anthropology, and beyond it, new methods of gathering, analysing and representing information and data in a visual language, using contemporary technologies. It would make possible extended opportunities of sharing the research visually and digitally through a number of digital platforms. 
Most of all, I hope it will make a real contribution to knowledge and understanding of and between cultures, in our increasingly globalised world where migration and travel make such understandings both urgent and necessary. Everyday multiculturalism is the arena in which individuals from different cultures encounter their own, and each other's, patterns of culture: my research is intended to contribute towards making these interactions a positive, rather than a destructive, element in today's multicultural societies. 


\section{Bibliography}

A

Aalen, Kristin (2014) 'Nye Debattdeltakere i Rasismedebatt' In: Stavanger Aftenblad

[Online] At: http://www.aftenbladet.no/meninger/idagdiskuterervi/Nye-debattdeltakere-irasismedebatt-3383581.html\#.U7K5Idwi7T4 (Accessed on 25.03.14)

Abromovic, Marina (2010) Marina Abramović - Uley Visits The Artist is Present [Video File] At: https://www.youtube.com/watch?v=R4lp4w8INYs (Accessed on 10.02.12)

Ameka, Felix K. (2009) 'Access Rituals in West African Communities: An Ethnopragmatic Perspective' In: Senft and Basso (eds.) Ritual Communication. Oxford: Berg. pp.127-151 Andersen, Ingunn., Wernersen, Camilla., Døvik, Olav and Wergeland, Paal (2012) 'Breivik Gjorde Ikke Hilsen' In: NRK [Online] At: http://www.nrk.no/227/artikler/breivik-gjorde-ikkehilsen-1.8082746 (Accessed on 20.04.12)

Anderson, Benedict (2006) Imagined Communities - Reflections on the Origin and Spread of Nationalism. (3rd ed., first published in 1983) London: Verso.

Arendsen, Jeroen (2008) 'Garrick Mallery (1891). Greeting by Gesture’ [Book Review] In: Gesture, 8 (3) pp.385-390

Argyle, Michael (1988) Bodily Communication. (2nd ed., first published in 1975 by Methuen \& Co Ltd) New York: Routledge.

Askham, Giles (2009) ، "Shotgun Muzzle Blast Photographs” by Andrew Dávidházy’ In: St George (ed.) Sequences - Contemporary Chronophotography and Experimental Digital Art. London: Wallflower Press. pp.120-125

Aston, Adam (2009) 'Tufte's Invisible Yet Ubiquitous Influence' In: Bloomberg Businessweek Innovation \& Design [Online] At: http://www.businessweek.com/stories/2009-06-10/tuftesinvisible-yet-ubiquitous-influencebusinessweek-business-news-stock-market-and-financialadvice (Accessed on 04.07.14) 
Bartebueff, Irmgard and Lewis, Dori (1980) Body Movement - Coping with the Environment. London: Gordon \& Breach.

Barthes, Roland (1964) Elements of Semiology. New York: Hill and Wang.

BBC (no author name) (2012) 'Breivik Trial: Key Moments of Opening Day' In: BBC News Europe [Online] At: http://www.bbc.co.uk/news/world-europe-17733869 (Accessed on 20.04.12)

BBC News Europe (2014a) 'Turkish Mass Protests: “Two Dead” After Teenager's Funeral' In: BBC News Europe [Online] At: http://www.bbc.com/news/world-europe-26555745 (Accessed on 11.08.14)

BBC News Europe (2014b) 'Turkish Police Tear Gas Protesters on Taksim anniversary' In: BBC News Europe [Online] At: http://www.bbc.com/news/world-europe-27649472 (Accessed on 11.08 .14$)$

BBC Newsnight: Information Graphics. YouTube clip of discussion on BBC Newsnight (2010, August 9) At: https://www.youtube.com/watch?v=q2Wnu1SOhKs (Accessed on 04.07.14) Beban, Brida (2008-11) Arte Vivo. At: http://msu.hr/\#/en/19984/ (Accessed on 04.07.14) Beck, Tine (2010) Catch Me Now - Interactive Light Installation. At: http://www.tinebech.com/ Artwork/Interactive/CatchMeNow/ (Accessed on 10.06.13)

Bill Viola - the eye of the heart [DVD] (2007) Directed by: Mark Kidel. Quantum Leap.

Birdwhistell, Ray L. (1970) Kinesics and Context: Essays on Body Motion Communication. Philadelphia: University of Pennsylvania Publications.

Blom, Svein (2013) Holdninger til Innvandrere og Innvandring, 2013 [Online] At: http://ssb. no/befolkning/artikler-og-publikasjoner/holdninger-til-innvandrere-og-innvandring-2013 (Accessed on 29.01.14)

Bolongaro, Kait (2014) 'Is Multiculturalism Good for Europe?' In: Aljazeera [Online] At: http://www.aljazeera.com/indepth/opinion/2013/12/multiculturalism-goodeurope-2013123045413908478.html (Accessed on 29.01.14) 
Bowen, John R. (2011) ‘Europeans Against Multiculturalism' In: Boston Review [Online] At: http://www.bostonreview.net/john-r-bowen-european-multiculturalism-islam (Accessed on 21.05.14)

Braun, Marta (2009) 'Chronophotography: Photographing Movement’ In: St George (ed.) Sequences - Contemporary Chronophotography and Experimental Digital Art. London: Wallflower Press. pp.20-47

Braun, Marta (2010) 'Animal Locomotion' In: Brookman (ed.) Eadweard Muybridge. London: Tate Publishing. pp.271-284

Braunstein, Roger (2010) ActionScript 3.0 Bible. (2nd ed., first published in 2007) Indianapolis: Wiley Publishing, Inc.

Brick Lane (2009) Directed by Sarah G. [Videorecording]. London: Film4 Brochmann, Grete and Djuve, Anne B. (2013) 'Multiculturalism or Assimilation? The Norwegian Welfare State Approach' In: Kivisto and Wahlbeck (eds.) Debating Multiculturalism in the Nordic Welfare States. London: Palgrave Macmillan. pp.219-245 Brookman, Philip (2010) Eadweard Muybridge. London: Tate Publishing. Brown, Ann Kipling and Parker, Monica (1984) Dance Notation for Beginners. Labanotation \& Benesh Movement Notation. London: Dance Books Ltd.

Bullock, Alan and Trombley, Stephen (1999) The New Fontana Dictionary of Modern Thought (3rd ed., first published in 1977) London: HarperCollinsPublishers.

\section{C}

Canguilhmem, Denis (2009) 'The True Retina of the Scientist: Scientific Photography under the Microscope' In: St George (ed.) Sequences - Contemporary Chronophotography and Experimental Digital Art. London: Wallflower Press. pp.69-79

Carlsen, Helge (2012) 'Jeg Kan Ikke Anke Fordi Jeg Ikke Anerkjenner Retten' In: NRK [Online] At: http://www.nrk.no/227/artikler/breivik_-jeg-anker-ikke-1.8294369 (Accessed on 28.08.12) 
Cashmore, Ellis (2008) Encyclopedia of Race and Ethnic Studies (first published in 2004) New York \& London: Routledge.

Caton, Steven C. (1986) 'Salam Tahiyah: Greetings from the highlands of Yemen’ In: American Ethnologist, 13 (2) pp.290-308

Chandler, Daniel (2007) Semiotics: The Basics [Online]. London: Routledge.

Cloninger, Curt (2009) ' "Stop Motion Studies (series 3)” by David Crawford' In: St George (ed.) Sequences - Contemporary Chronophotography and Experimental Digital Art. London: Wallflower Press. pp.112-119

Cookson, Clive (2013) 'The Graphics Guru has Shown Us how to Visualise Data with Simplicity, Clarity and Elegance' In: FT Magazine 26.07.13 [Online] At: http://www.ft.com/ cms/s/2/dda1cb5c-f4c0-11e2-a62e-00144feabdc0.html\#axzz39iD81K4P (Accessed on 04.07.14)

Crow, David (2010) Visible Signs: an Introduction to Semiotics in the Visual Arts. Lausanne: AVA Publishing SA.

D

Dance Notation Bureau (2012) Labanotation Basics. At: http://www.dancenotation.org/ Inbasics/frame0.html (Accessed on 11.10.12)

Darwin, Charles (1999) The Expression of the Emotions in Man and Animals. (3rd ed., first published in 1872) London: HarperCollins.

Davies, Eden (2001) Beyond Dance - Laban's Legacy of Movement Analysis. London: Routledge.

D'Efilippo, Valentina and Ball, James (2013) The Infographic History of the World. London: Collins.

Duranti, Alessandro (1992) 'Language and Bodies in Social Space: Samoan Ceremonial Greetings'In: American Anthropologist, 94 (3) pp.657-691 
Duranti, Alessandro (1996) Alessandro Duranti - Professor of Anthropology:: University of California at Los Angeles. [Hillary Clinton Meets the Capps] At: www.sscnet.ucla.edu/anthro/ faculty/duranti/audvis/greet_capps.htm (Accessed on 10.12.12)

Duranti, Alessandro (1997a) 'Polyphonic Discourse: Overlapping in Samoan Ceremonial Greetings' In: Text, 17 (3) pp.349-81

Duranti, Alessandro (1997b) 'Universal and Culture-Specific Properties of Greetings' In: Journal of Linguistic Anthropology, 7 (1) pp.63-97

Duranti, Alessandro (2001) 'Performance and Encoding of Agency in Historical-Natural Languages' In: Texas Linguistic Forum, 44 (2) pp.266-287

Duranti, Alessandro (2003) 'The Voice of the Audience in Contemporary American Political Discourse' In: Alatis and Tannen (eds.) Georgetown University Round Table on Languages and Linguistics. Linguistics, Language, and the Real World: Discourse and Beyond. Georgetown: Georgetown University Press. pp.114-134

Duranti, Alessandro (2006) 'Narrating the Political Self in a Campaign for U.S. Congress' In: Language in Society, 35 (4) pp.467-97

Dzamarija, Minja Tea., Andreassen and Kristina Kvarv. (2014) Personer med Flyktningbakgrunn, 1. Januar 2013 [Online] http://ssb.no/befolkning/statistikker/flyktninger/ aar/2013-09-04\#content, (Accessed on 29.01.14)

E

Egge-Hoveid, Kristin (2014) Innvandringer, etter region, innvandringsgrunn, tid og statistikkvariabel [Email attachment, spreadsheet, sent from Kristin Egge-Hoveid from Statistics Norway to Marie Therese Shortt, 23rd September 2014].

Ekman, Paul., Friesen, Wallace V. and Hager, Joseph C. (2002) Facial Action Coding System. At: http://face-and-emotion.com/dataface/facs/manual/TitlePage.html (Accessed on 19.08.13) 
Eshkol, Noa (1972) Folk Dances of Israel: The Yemenite Dance: 142 Steps. Tel Aviv: The Movement Notation Society Israel.

Eshkol, Noa and Harries, John (1988) EW Movement Notation: The System of Reference. China/Nanjing: International Congress for Movement Notation.

Examined Life (2010) Directed by A. Taylor [Videorecording] London: ICA.

Eytan, Ariel (2004) 'Globalisation and Biculturalism' In: The British Journal of Psychiatry, 184 (4) pp.362-363

\section{$\mathbf{F}$}

Faist, Thomas (2013) 'Multiculturalism: From Heterogeneities to Social (In)equalities' In: Kivisto and Wahlbeck (eds.) Debating Multiculturalism in the Nordic Welfare States. London: Palgrave Macmillan. pp.22-47

Falle, Rainer (2007) East Meets West: An Infographic Portrait by Yang Liu. At: http://bsix12. com/east-meets-west/ (Accessed on 10.06.13)

Farnell, Brenda (1996) 'Movement Notation Systems' In: Daniels and Bright (eds.) The World's Writing Systems. New York: Oxford University Press. pp.855-879

Firth, Raymond (1954) 'Anuta and Tikopia: Symbiotic Elements in Social Organization' In: The Journal of the Polynesian Society, 63 (2) pp. 87-132

Fox, Kate (2005) Watching the English - the Hidden Rules of English Behaviour. London: Hodder \& Stoughton.

Frafjord, Magne., Selmer-Anderssen, Ina and Topdahl, Rolv Christian (2014) 'Han vil ha borgervern mot asylmottak' In: NRK [Online] At: http://www.nrk.no/rogaland/vil-haborgervern-1.11598677 (Accessed on 25.03.14)

Frijhoff, Willem (1992) 'The Kiss Sacred and Profane: Reflections on a Cross-Cultural Confrontation' In: Bremmer and Roodenburg (eds.) A Cultural History of Gesture. Ithaca: Cornell University Press. pp.210-236 
Gapminder (2014) Gapminder - For a Fact-Based Worldview. At: http://www.gapminder.org (Accessed on 10.06.13)

Georges, Fanny (2009) “ “One Day in Japan, 15 September 2002” by Studiometis' In: St George (ed.) Sequences - Contemporary Chronophotography and Experimental Digital Art. London: Wallflower Press. pp.176-182

Ghetto Britain: 30 Years of Race (2006) [Videorecording] London: Channel 4.

Gonsalves, Tina (2009) The Chameleon Project. At: http://www.tinagonsalves.com (Accessed on 10.06.13)

Goodall, Heather., Wearing, Stephen., Byrne, Denis and Cadzow, Allison (2009) 'Fishing the Georges River: Cultural Diversity and Urban Environments' In: Wise and Velayutham (eds.) Everyday Multiculturalism. London: Palgrave Macmillan. pp.177-198

Gregory, Derek., Johnson, Roy., Pratt, Geraldine., Watts, Michael and Whatmore, Sarah (2000) The Dictionary of Human Geography. (4th ed., first published in 1981) Oxford: Blackwell Publishing.

Griesbeck, Christian (1996) Introduction to Labanotation. At: http://user.uni-frankfurt. de/ griesbec/LABANE.HTML (Accessed on 17.06.14)

H

Hall, Edward T. (1963) 'A System for the Notation of Proxemic Behavior' In: American Anthropologist, 65 (5) pp.1003-1026

Hall, Edward T. (1983) The Dance of Life. New York: Anchor Books.

Hall, Edward T. (1989) 'Proxemics - A Complex Cultural Language' In: This Week's Citation Classic. [Illinois Institute of Technology, Chicago] (5) p.14

Hall, Edward T. (1990) The Silent Language. New York: Anchor Books. 
Hall, Stuart (1997) Representation: Cultural Representations and Signifying Practices. London: Sage Publications Ltd.

Hart, Keith (2004) 'Notes Towards an Anthropology of the Internet' In: Horizontes Antropológicos, 10 (21) pp.15-40

Hérbert, Louis (2005) Elements of Semiotics. At: http://www.signosemio.com/elements-ofsemiotics.asp (Accessed on 01.10.14)

Hervik, Peter (2012) 'Ending Tolerance as a Solution to Incompatibility: The Danish "Crisis of Multiculturalism" ' In: European Journal of Cultural Studies, 15 (2) pp.211-225

Hine, Christine (2000) Virtual Ethnography. London: Sage Publications Ltd.

History.com Staff (2010) The Invention of the Internet. At: http://www.history.com/topics/ inventions/invention-of-the-internet (Accessed on 10.09.13)

Holmes, Nigel (2005) Wordless Diagrams. London: Gerald Duckworth \& Co. Ltd.

Horsti, Karina (2009) 'From Multiculturalism to discourse of cultural diversity: European public service broadcasting and challenge of migration', paper presented at Interethnic Relations: Multidisciplinary Approaches, IMISCOE International Migration, Integration and Social Cohesion Conference. Portugal: Universidade Lisboa.

Hudson, Maria., Phillips, Joan and Ray, Kathryn (2009) ' "Rubbing Along With the Neighbours" - Everyday Interactions in a Diverse Neighbourhood in the North of England' In: Wise and Velayutham (eds.) Everyday Multiculturalism. London: Palgrave Macmillan. pp.199-215

Hæreid, Marit (2014) 'Fikk Nabovarsel om Asylmottak i Posten' In: Stavanger Aftenblad [Online] At: http://www.aftenbladet.no/nyheter/lokalt/sandnes/Fikk-nabovarsel-omasylmottak-i-posten-3373083.html\#.UzaM79xMAZt (Accessed on 25.03.14)

I

Imagine: The World According to Parr: Season 2, Episode 4. (2003) BBC1: December 3 
Jobs, Steve (2010) Thoughts on Flash. At: https://www.apple.com/hotnews/thoughts-onflash/ (Accessed on 12.11.14)

K

Kahn, S., Keil, J., Müller, B., Bockholt, U., and Fellner, D.W. (2013) ‘Capturing of Contemporary Dance for Preservation and Presentation of Choreographies in Online Scores' In: 2013 Digital Heritage International Congress, 1 pp.273-280

Kapuściński, Ryszard (2008) The Other. London: Verso.

Keating, Elizabeth (2003) 'Introduction' In: Journal of Linguistic Anthropology [Special Issue on Visual Communication], 13 (1) pp.3-6

Kelly, Jon (2012) ‘Breivik: What's Behind Clenched-fist Salutes?' In: BBC News Magazine [Online] At: http://www.bbc.co.uk/news/magazine-17739105 (Accessed on 20.04.12)

Kemp, Geoffrey and Harkavy, Roberr (1997) The 1973 Arab-Israeli War and the Oil Crisis of the 1970s. At: http://acc.teachmideast.org/texts.php?module_id=4\&reading_ $\mathrm{id}=120 \&$ sequence $=21($ Accessed on 04.07.14)

Kendon, Adam (2009) Conducting Interaction - Patterns of Behavior in Focused Encounters. (2nd ed., first published in 1990) Cambridge: Cambridge University Press.

Kipfer, Barbara A. and Chapman, Robert L. (2007) 'B-boy’ definition 3 [Online] At: http:// dictionary.reference.com/browse/bboy (Accessed on 08.01.13)

Kivisto, Peter and Wahlbeck, Östen (2013) (eds.) Debating Multiculturalism in the Nordic Welfare States. London: Palgrave Macmillan.

Kress, Gunther R. and Leeuwen, Theo V. (1996) Reading Images - The Grammar of Visual Design. London: Routledge. 
Kunin, V., Goldovsky, L., Darzentas, N., and Ouzounis, C. A. (2005) Microbial Evolution. At: http://www.visualcomplexity.com/vc/project_details.cfm?id=299\&index=30\&domain=Biology (Accessed on 04.07.14)

$\mathbf{L}$

Leeuwen, Theo V. (2006) Introducing Social Semiotics. London: Routledge.

Lefebvre, Thierry (2009) 'Scientific Cinema in the Public Eye' In: St George (ed.) Sequences - Contemporary Chronophotography and Experimental Digital Art. London: Wallflower Press. pp.80-95

Letsch, Constanze (2014) ‘Turkey’s Economic Success Threatened by Political Instability’ At: http://www.theguardian.com/world/2014/jan/09/turkey-instability-threatens-economicsuccess-erdogan (Accessed on 11.08.14)

Lewis, Mark (1998) The Pitch. At: http://marklewisstudio.com/the-pitch/ (Accessed on 10.02.12)

Lima, Manuel (2011a) Visual Complexity - Mapping Patterns of Information. New York: Princeton Architectural Press.

Lima, Manuel (2011b) The Power of Networks: Knowledge in an Age of Infinite Interconnectedness [video of presentation] Pres. RSA At: https://www.youtube.com/ watch?v=_0LVSIwifpl\#t=48 (Accessed on 10.06.13)

Lima, Manuel (2011, March 16) Manuel Lima: Keynote on Visual Complexity [Video File] Pres. Broad Institute At: https://www.youtube.com/watch?v=PWMFgS6w3BY (Accessed on 04.07.14)

Lima, Manuel (2011, April) Manuel Lima - El Poder de las Redes [Video File] At: https://www. youtube.com/watch?v=xwiln7kHpnM (Accessed on 04.07.14)

Lister, Richard (2012) 'Breivik Takes Stand in Norway Massacre Trial' In: BBC News Europe [Online] At: http://www.bbc.co.uk/news/world-europe-17737685 (Accessed on 18.05.12) 
Lithman, Yngve (2013) 'Norwegian Multicultural Debates in a Scandinavian Comparative Perspective' In: Kivisto and Wahlbeck (eds.) Debating Multiculturalism in the Nordic Welfare States. London: Palgrave Macmillan. pp.246-269

Liu, Yang (2007) Ost Trifft West. Germany: Schmidt Hermann Verlag.

Liu, Yang (2014) Man Meets Woman. Berlin: Taschen.

Liyama, Genji (1996) Global Vision: United Colours of Benetton. Tokyo: Robundo Publishing Inc.

Logan, Gene Adams and McKinney, Wayne C. (1970) Kinesiology. United States: Wm. C. Brown Company Publishers.

Lott, Joey., Schall, Darron and Peters, Keith (2006) ActionScript 3.0 Cookbook. US/ Sebastopol: O'Reilly Media, Inc.

M

Macalister, Terry (2011) ‘Background: What caused the 1970s oil price shock?' In: The Guardian [Online] At: http://www.theguardian.com/environment/2011/mar/03/1970s-oil-priceshock (Accessed on 04.07.14)

Making Bradford British (2012) [Videorecording] London: Channel 4.

Manchot, Melanie and Honnef, Klaus (2001) Melanie Manchot: love is a stranger: photographs 1998-2001. Munich and London: Prestel.

Marsh, Peter E. (1988) Eye to Eye: How People Interact. Massachusetts: Salem House. McCandless, David (2009) Information is Beautiful. London: Collins.

McCandless, David and AlwaysWithHonor.com (2009) Colours In Culture. At: http://www. informationisbeautiful.net/visualizations/colours-in-cultures/ (Accessed on 10.06.13)

McCandless, David (2010, July) David McCandless: The Beauty of Data Visualization [Video File] At: http://www.ted.com/talks/david_mccandless_the_beauty_of_data_visualization (Accessed on 04.07.14) 
McCandless, David (2013a) Snake Oil Supplements? - Scientific Evidence for Popular Health Supplements. At: http://www.informationisbeautiful.net/play/snake-oil-supplements/ (Accessed on 10.06.13)

McCandless, David (2013b) VizSweet. At: http://www.informationisbeautiful.net/vizsweet/ (Accessed on 10.06.13)

McCandless, David (2014) Knowledge is Beautiful. London: Collins.

McFarland, Dan and Bender-deMoll, Skye (2004) SoNIA - Streaming Classroom Interactions. At: http://www.visualcomplexity.com/vc/project.cfm?id=165 (Accessed on 04.07.14)

McGraw-Hill Dictionary of Scientific \& Technical Terms, 6E. (2003a) 'Convex' definition 3 [Online] At: http://encyclopedia2.thefreedictionary.com/convex (Accessed on 08.11.14)

McGraw-Hill Dictionary of Scientific \& Technical Terms, 6E. (2003b) ‘Convex Hull' definition 1 [Online] At: http://encyclopedia2.thefreedictionary.com/convex+hull (Accessed on 08.11.14)

MODassic Marketing (2015) 'Vector, Raster, JPG, EPS, PNG - What's the Difference?'At: https://modassicmarketing.com/understanding-image-file-types/ (Accessed on 21.03.2015)

Monaghan, Adam Lloyd (2009) “ “Timepiece” by Tess Glanville’ In: St George (ed.) Sequences - Contemporary Chronophotography and Experimental Digital Art. London: Wallflower Press. pp.126-131

Morris, Desmond (1979) Manwatching - A Field Guide to Human Behaviour. (3rd ed., first published in 1977) London: Jonathan Cape Ltd.

Motion Bank (2013a) Motion Bank. At: http://motionbank.org/en (Accessed on 11.11.2013) Motion Bank (2013b) Impulse. At: http://scores.motionbank.org/two/\#/set/impulse (Accessed on 11.11.13)

Motion Bank (2013c) Using The Sky, Deborah Hay. At: http://motionbank.org/en/content/ deborah-hay (Accessed on 11.11.13) 
Muybridge, Eadweard (1955) The Human Figure in Motion. New York: Dover Publications.

$\mathbf{N}$

New Media Institute (2014) History of the Internet. At: http://www.newmedia.org/history-ofthe-internet.html?page=4 (Accessed on 12.11.14)

Noble, Greg (2009) ‘Everyday Cosmopolitanism and the Labour of Intercultural Community’ In: Wise and Velayutham (eds.) Everyday Multiculturalism. London: Palgrave Macmillan. pp.46-65

Nöth, Winfried (1995) 'Proxemics: The Semiotics of Space' In: Nöth (ed.) Handbook of Semiotics. Bloomington: Indiana University Press. pp.410-414

Notcot (2007) Interview with Yang Liu 11.13.07. At: http://www.notcot.com/archives/2007/11/jean-posted-ab.php (Accessed on 10.06.13)

Nova, Nicolas., Miyake, Katherine., Chiu, Walton and Kwon, Nancy (2012) Curious Rituals Gestural Interaction in the Digital Everyday. Pasadena: Media Design Program at Art Center College of Design.

\section{0}

Olwig, Karen Fog (2011) “ “Integration”: Migrants and Refugees between Scandinavian Welfare Societies and Family Relations' In: Journal of Ethnic and Migration Studies, 37 (2) pp.179-196

O’Neill, Brendan (2012) ‘Breivik: A Monster Made by Multiculturalism’ In: The Telegraph [Online] At: http://blogs.telegraph.co.uk/news/brendanoneill2/100151677/breivik-a-monstermade-by-multiculturalism/ (Accessed on 19.05.12)

Oxford Dictionaries (2014) '-ism' definition 2 [Online] At: http://www.oxforddictionaries.com/ definition/english/-ism (Accessed on 08.01.13) 
Parker, Monica (1996) Benesh Movement Notation. Elementary Solo Syllabus, Ballet Application. London: The Benesh Institute.

Parr, Martin (1995) Small World. Stockport: Dewi Lewis Publishing.

Parr, Martin (2005) Fashion Magazine. New York: Magnum Photos.

Pease, Allan and Pease, Barbra (2006) The Definitive Book of Body Language. London: Orion Books Ltd.

Pettersen, Silje Vatne and Østby, Lars (2013) 'Skandinavisk Komparativ Statistikk om Integrering: Innvandrere i Norge, Sverige og Danmark' In: Samfunnsspeilet, 5 pp.76-82

Pillet-Shore, Danielle (2008) Coming Together: Creating and Maintaining Social Relationships Through the Openings of Face-To-Face Interactions [PhD Dissertation in Sociology] University of California, Los Angeles.

Pillet-Shore, Danielle (2012) 'Greeting: Displaying Stance through Prosodic Recipient Design' In: Research on Language and Social Interaction, 45 (4) pp.375-398

Pink, Sarah (2007) Doing Visual Ethnography. London: Sage Publications Ltd.

Pink, Sarah (2009) Doing Sensory Ethnography. London: Sage Publications Ltd.

Pisano, Guisy (2009) 'The Photographic Instant and the Chosen Instant in Painting and Sculpture' In: St George (ed.) Sequences - Contemporary Chronophotography and Experimental Digital Art. London: Wallflower Press. pp.58-68

Preston-Dunlop, Valerie and Sayers, Lesley-Anne (2010) The Dynamic Body in Space Exploring and Developing Rudolf Laban's Ideas for the 21st Century. London: Dance Books Ltd. 
Radice, Martha (2009) 'Everyday Cosmopolitanism and the Labour of Intercultural Community' In: Wise and Velayutham (eds.) Everyday Multiculturalism. London: Palgrave Macmillan. pp.140-160

Random International (2014) Random International. At: http://random-international.com/ (Accessed on 04.07.14)

Rath, Jan (2011) ‘Debating Multiculturalism' In: Harvard International Review 06.01.11

[Online] At: http://hir.harvard.edu/debating-multiculturalism (Accessed on 21.05.14)

Reker, Judith and Grosse, Julia (2010) Don't Get Me Wrong! The Global Gestures Guide. Munich: Bierke Publishing.

Robertson, Ian (2009) ' "Border" by Darren Almond and "CineSpinner" by Rufus Butler Seder' In: St George (ed.) Sequences - Contemporary Chronophotography and Experimental Digital Art. London: Wallflower Press. pp.168-175

Rogers, Simon (2013) Facts Are Sacred. London: Faber and Faber Limited [Published with Guardian Books].

Rosenberg, Scott (1997) The Data Artist. At: http://www.edwardtufte.com/tufte/salon_0310 (Accessed on 04.07.14)

Rosling, Hans (2006, February) Hans Rosling: The best stats you've ever seen [Video File] At: http://www.ted.com/talks/hans_rosling_shows_the_best_stats_you_ve_ever_seen (Accessed on 10.06.13)

Royal Academy of Dance (2014) The Benesh Institute \& Benesh Movement Notation. At: https://www.rad.org.uk/study/Benesh/how-benesh-movement-notation-works (Accessed on 11.08.14)

Rutledge, Dennis M. (2008) Biculturalism, Self Identity and Societal Transformation. Bingely: Emerald Group Publishing Limited. 
Samovar, Larry A., Porter, Richard E. and McDaniel, Edwin R. (2007) Communication Between Cultures. (6th ed., first published in 1991) United States of America: Thomson Wadworth.

Sandvik, Siv., Helljesen, Vilde., Brekke, Anders., Nilsen, Amund Aune and Skille, Øyvind Bye (2012) ‘Breivik: - Ser på Dem Som Dyr’ In: NRK [Online] http://www.nrk.no/227/artikler/ breivik_-_-ser-pa-dem-som-dyr-1.8177026 (Accessed on 06.06.12)

Schleicher, Antonia F. (1997) 'Using Greetings To Teach Cultural Understanding' In: Modern Language Journal, 81 (3) pp.334-343

Schwartz, Seth J. and Unger, Jennifer B. (2010) 'Biculturalism and Context: What is Biculturalism, and When is it Adaptive?' In: Human Development, 53 (1) pp.26-32

Semi, Giovanni., Colombo, Enzo., Camozzi, llenya and Frisina, Annalisa (2009) 'Practices of Difference: Analysing Multiculturalism in Everyday Life' In: Wise and Velayutham (eds.) Everyday Multiculturalism. London: Palgrave Macmillan. pp.66-86

Shermer, Michael (2005) The Feynman-Tufte Principe. At: http://www.edwardtufte.com/tufte/ si_05 (Accessed on 04.07.14)

Shortt, Marie T. (2013) 'Patterning Culture: Developing a System for the Visual Notation of Greetings' In: Digital Heritage International Congress (DigitalHeritage), 2013, 1 pp.403-406

Solnit, Rebecca (2004) Motion Studies: Time, Space and Eadweard Muybridge. London: Bloomsbury.

Song, Sarah (2010) 'Multiculturalism' [Online] At: http://plato.stanford.edu/archives/win2010/ entries/multiculturalism/ (Accessed on 29.01.14)

Statistics Norway (2014a) Lønn, Alle Ansatte, 2013 [Online] At: http://ssb.no/arbeid-og-lonn/ statistikker/lonnansatt (Accessed on 25.03.14) 
Statistics Norway (2014b) Holdninger til Innvandrere og Innvandring, 2014 [Online] At: http:// ssb.no/befolkning/statistikker/innvhold/aar and https://www.ssb.no/befolkning/artikler-ogpublikasjoner/_attachment/208570?_ts=14a04ea4e40 (Accessed on 22.12.14)

Statistics Norway (2014c) Flyttinger, 2013 [Online] At: http://www.ssb.no/befolkning/ statistikker/flytting/aar/2014-04-28?fane=tabell\&sort=nummer\&tabell=174041 (Accessed on 22.12.14)

Statistics Norway (2014d) Innvandrere Etter Innvandringsgrunn, 1. Januar 2014 [Online] At: http://www.ssb.no/befolkning/statistikker/innvgrunn (Accessed on 22.12.14)

Stavanger-statistikken (2014) Innvandrere og norskfødte med innvandrerforeldre [Online] At: http://statistikk.stavanger.kommune.no/befolkning_15s.html (Accessed on 29.01.14)

Stefaner, Moritz (2010) Map Your Moves. At: http://www.visualcomplexity.com/vc/project. cfm?id=738 (Accessed on 04.07.14)

St George, Paul (2009) (ed.) Sequences - Contemporary Chronophotography and Experimental Digital Art. London: Wallflower Press.

Stokke, Hugo (2013) 'Nordic Multiculturalism: Commonalities and Differences' In: Kivisto and Wahlbeck (eds.) Debating Multiculturalism in the Nordic Welfare States. London: Palgrave Macmillan. pp.74-109

Sølvberget (2014) Sølvberget Stavanger Kulturhus - Internasjonal Kultur At: http://www. stavanger-kulturhus.no/INTERNASJONAL-KULTUR (Accessed on 25.03.14)

$\mathbf{T}$

Tiller, Alise Lea (2014) ‘Demonstrerer mot rasisme' In: Stavanger Aftenblad [Online] At: http:// www.aftenbladet.no/nyheter/lokalt/stavanger/Demonstrerer-mot-rasisme-3384462.html\#. UzaMIdxMAZt (Accessed on 25.03.14)

Toscani, Oliviero and Broomberg, Adam (1998) Touch [special edition of] COLORS magazine (28). 
Tufte, Edward R. (1990) Envisioning Information. Cheshire, Connecticut: Graphics Press LLC.

Tufte, Edward R. (2006) Beautiful Evidence. Cheshire, Connecticut: Graphics Press LLC.

Tulane University (2000) 'Agency’ [Online] At: http://www.tulane.edu/ rouxbee/kids00/ mexico1.html (Accessed on 10.08.14)

Turner, Terence (1993) 'Anthropology and Multiculturalism: What is Anthropology that Multiculturalists Should be Mindful of it?' In: Cultural Anthropology, 8 (4) pp.411-429

U

Uras, Umut (2014) ‘Erdogan wins Turkey’s presidential Election’ In: Aljazeera [Online] At: http://www.aljazeera.com/news/middleeast/2014/08/erdogan-wins-turkey-presidentialelection-2014810172347586150.html (Accessed on 11.08.14)

Urban Dictionary (2006) ‘B-boy’ definition 2 [Online] At: http://www.urbandictionary.com/ define.php?term=b+boy (Accessed on 08.01.13)

V

Valenta, Marko and Bunar, Nihad (2010) ‘State Assisted Integration: Refugee Integration Policies in Scandinavian Welfare States: the Swedish and Norwegian Experience' In: Journal of Refugee Studies, 23 (4) pp.464-483

Viola, Bill (1995) Bill Viola - The Greeting [Video File] At: https://www.youtube.com/ watch?v=DgolyGUVXaQ (Accessed on 10.02.12)

Visual Complexity (2014d) Visual Complexity. At: http://www.visualcomplexity.com (Accessed on 04.07 .14$)$ 
Wainwright, Jean (2004) 'Telling Times: Revisiting the Greeting' In: Townsend (ed.) The art of Bill Viola. London: Thames and Hudson. pp.110-123

Wallinger, Mark (2000) Threshold of the Kingdom [Video File] At: http://vimeo.com/18551262 (Accessed on 10.02.12)

Walter, John (2009) 'Gesturing at Authority: Deciphering the Gestural Code of Early Modern England' In: Past and Present, 203 (supplement 4) pp.96-127

Watson, Sophie (2009) 'Brief Encounters of an Unpredictable Kind: Everyday

Multiculturalism in Two London Street Markets' In: Wise and Velayutham (eds.) Everyday Multiculturalism. London: Palgrave Macmillan. pp.125-139

Wernersen, Camilla and Sandvik, Siv (2012) 'Advarer mot Breiviks Smitteeffekt' In: NRK [Online] At: http://www.nrk.no/227/artikler/advarer-mot-breiviks-smitteeffekt-1.8081331 (Accessed on 20.04.12)

Wex, Marianne (1979) Let's Take Back Our Space: 'Female' and 'Male' Body Language as a Result of Patriarchal Structures. Germany: Frauenliteratur Verlag.

Wikan, Unni (1999) 'Culture: A New Concept of Race’ In: Social Anthropology, 7 (1) pp.57-64 Wilkins, Adrien (2012) Demonstrasjonsrapport: Folkefest mot rasisme 23. Juni [Online] At: http://www.motmakt.no/aktuelt/demonstrasjonsrapport-folkefest-mot-rasisme-23-juni (Accessed on 25.03.14)

Williams, Raymond and Rossi-Landi, Ferruccio (1981) Contact: Human Communication and its History. London: Thames and Hudson.

Williams, Val (2004) Martin Parr. (2nd ed., first published in 2002) London: Phaidon.

Wise, Amanda (2005) ‘Hope and Belonging in a Multicultural Suburb’ In: Journal of Intercultural Studies, 26 (1-2) pp.171-186

Wise, Amanda (2009) ‘Everyday Multiculturalism: Transversal Crossings and Working Class Cosmopolitans' In: Wise and Velayutham (eds.) Everyday Multiculturalism. London: Palgrave Macmillan. pp.21-45 
Wise, Amanda and Velayutham, Selvaraj (2009) (eds.) Everyday Multiculturalism. London: Palgrave Macmillan.

Women in Black (2008) Directed by Yemisi Brookes. [Videorecording] London: BBC2.

Y

Youssouf, Ibrahim A.G., Grimshaw, Allen D. and Bird, Charles S. (1976) 'Greetings in the Desert' In: American Ethnologist, 3 (4) pp.797-824 


\section{Appendices}

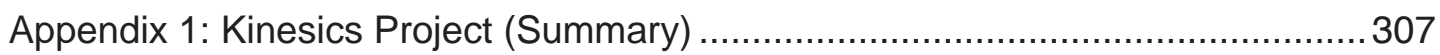

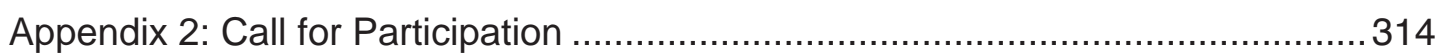

Appendix 3: Conversations During Breakfast with Vietnamese Participants...........316

Appendix 4: Programme for Greeting Documentation.......................................... 320

Appendix 5: Research Information Sheet.......................................................... 322

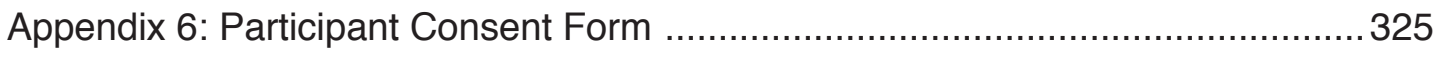

Appendix 7: Research Ethics Approval Application Outcome ...............................328

Appendix 8: Conversations During Video Recording of Greetings........................ 330

Appendix 9: Report on Greetings Documented During Fieldwork.......................... 336

Appendix 10: Interview questionnaire and transcripts ........................................... 356

a: Interview Questionnaire ...................................................... 356

b: Interview with Norwegian female participant..............................357

c: Interview with Norwegian male participant.................................365

d: Interview with Turkish female participant ....................................382

e: Interview with Turkish male participant .....................................395

f: Interview with Vietnamese female participant ..............................405

g: Interview with Angolan participant.........................................416

Appendix 11: Actionscript 3.0 code used to programme greeting diagrams........... 425

Appendices 12-20 are found on the enclosed DVD.

Appendices 21-22 are enclosed as a separate notebook. 


\section{Appendix 1: Kinesics Project (Summary)}

This provides a complete outline and overview of my earlier project referred to in chapter 4.

In my MA project, Kinesics - Creative Intercultural Learning, I developed a version of my system for graphically visualising greeting rituals. The study was conducted through fieldwork in Eindhoven in the Netherlands, and included participants from nine different cultural backgrounds. The project was completed in 2007. The main purpose of the system was to enable the comparison of greetings across different gender combinations - within, and between those cultures. I documented three broad greeting situations: informal greetings, formal greetings and greeting the elderly. Greetings were filmed in a studio and I used two video cameras to document the greetings. The cameras were placed at angles that captured posture - from a front view - and use of space - from an overhead view. The video material was developed into illustrations and vector graphics (see figs. 235-236).
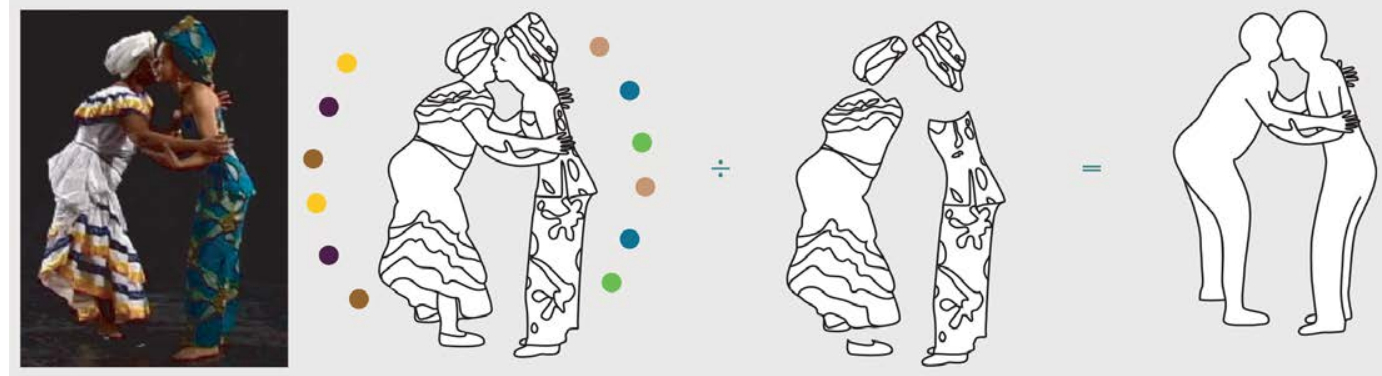

Fig. 235 - Kinesics visuals: From video still to vector graphic
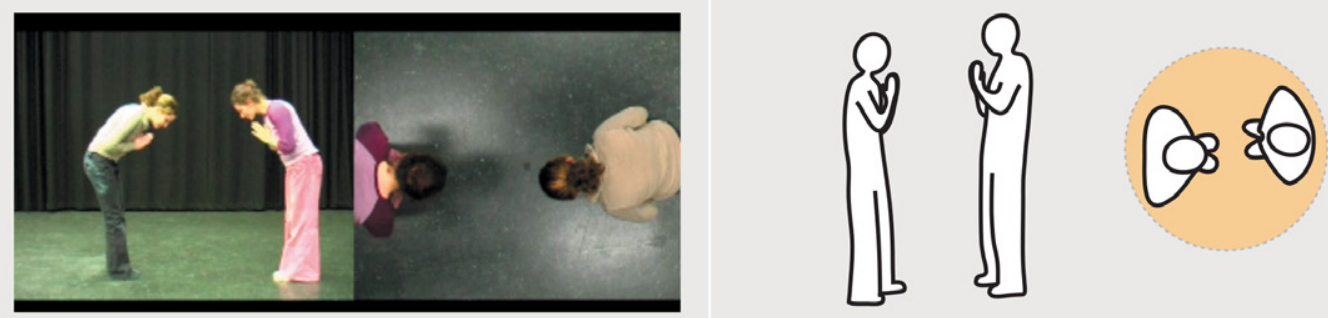

Fig. 236 - Kinesics visuals: video angles used, and a graphic visualisation of the video stills 
I also created some vectorised animations from the video recordings. However, these were not used for annotation (as the still images were - see below) and their timings did not correspond proportionately to those of the actual greetings (see p.313).

The key focus of the visualisations was to highlight the use of personal space. Personal space was visualised graphically as a shared space of the two people greeting. I created four different 'intimate' zones, that were scaled and colour coded, to visualise personal space, and I annotated each greeting visualisation using this system (see fig. 237). I created graphic diagrams, both interactive and non-interactive, of the greeting illustrations, and these diagrams enabled visual comparisons of the greetings and use of personal space, across cultures and gender (see fig. 238).

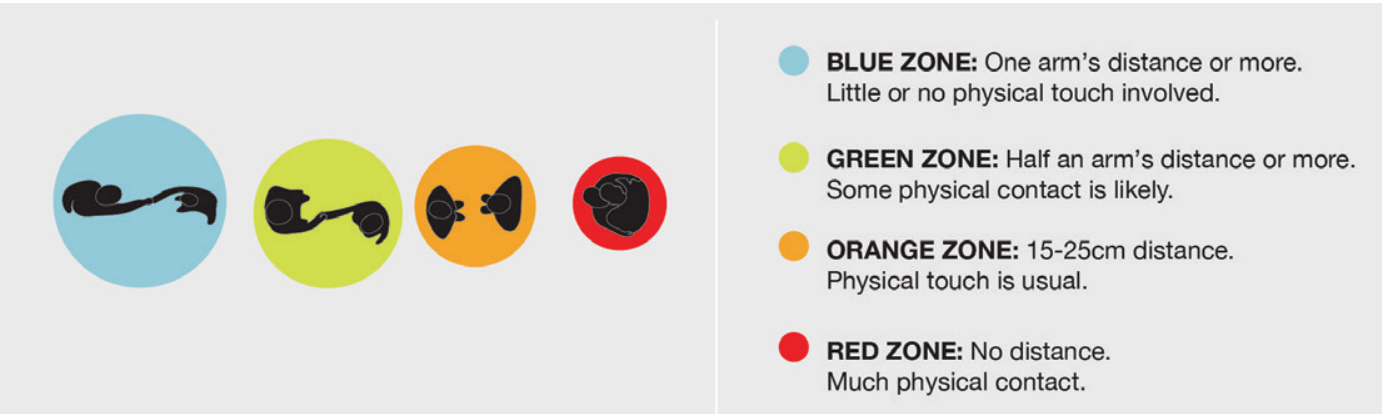

Fig. 237 - Kinesics visuals: colour coded intimate zones and their measurements

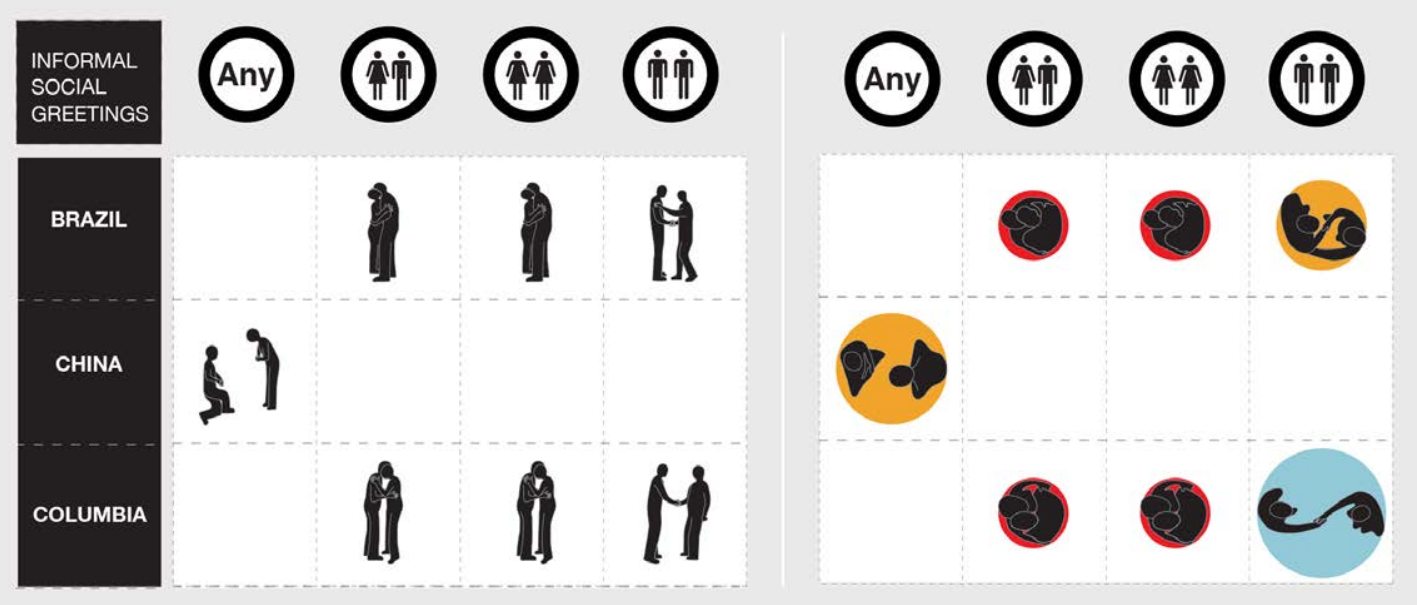

Fig. 238 - Kinesics visuals: graphic greetings diagrams for comparisons across genders/cultures 
The final presentation of the Kinesics project consisted of nine printed greetings diagrams (see fig. 239), screen based interactive diagrams (see fig. 240), a screen-based interactive world map of greetings (see fig. 241), and a number of stand-alone greeting animations (see fig. 242).
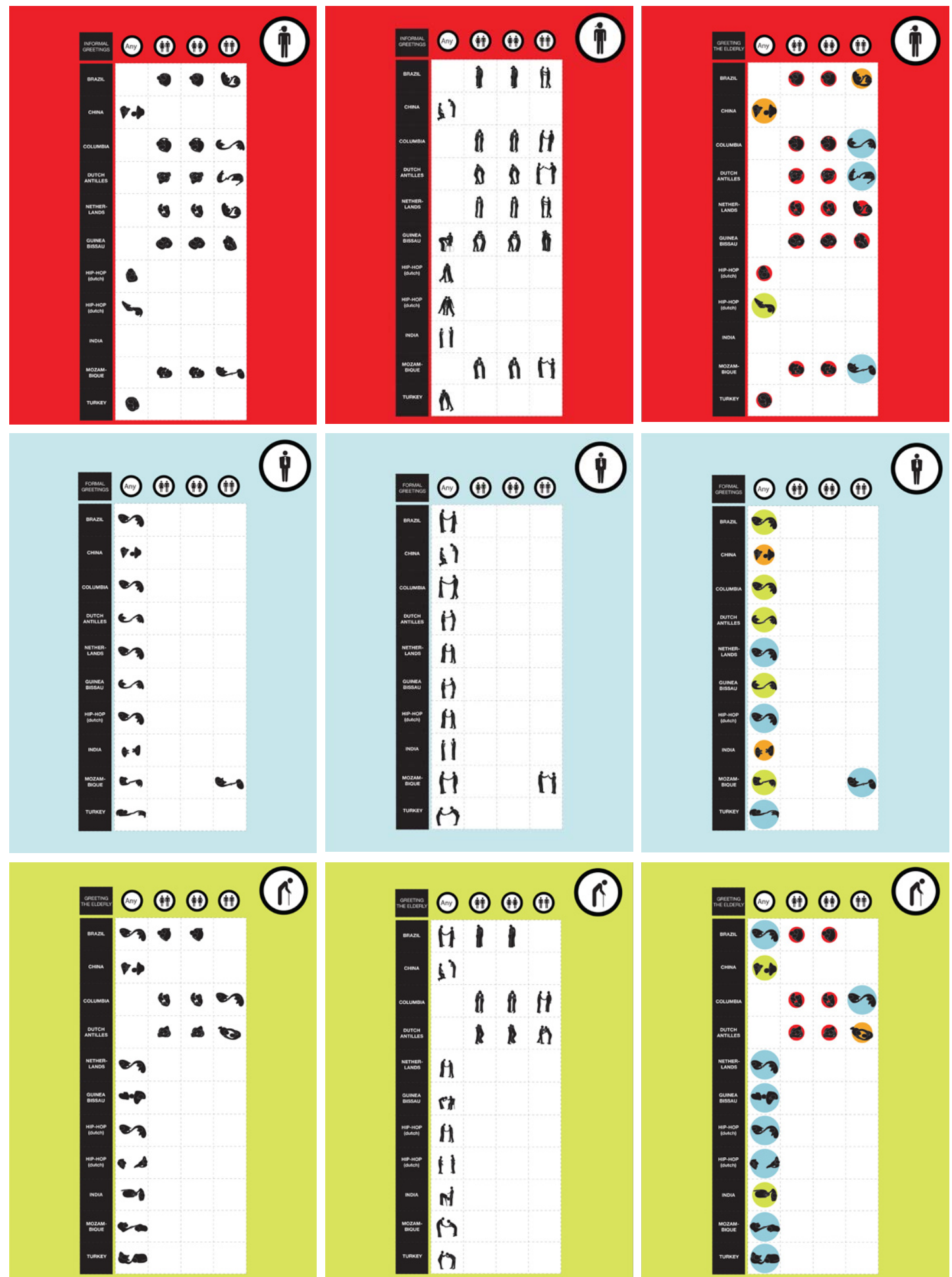

Fig. 239 - Kinesics visuals: printed greetings diagrams. Informal greetings (top), greeting the elderly (middle), and formal greetings (bottom) 
The static diagrams of each greeting situation - informal greetings, formal greetings, and greeting the elderly - were printed in three versions: greetings in front view, overhead view, and overhead view with added intimate zones (see fig. 239). The interactive diagrams were interactive in so far as it was possible to select which greeting situation to view, and whether to view the front view, or the overhead view annotated with intimate zones. It was also possible to compare two greetings at a time. However, the diagrams did not show the animated versions of each greeting, only the static graphic greeting images as shown in fig. 240. It was also not possible to view each greeting separately. The interactive maps were similar to the diagrams in that they displayed static versions of the greetings, but with the added function
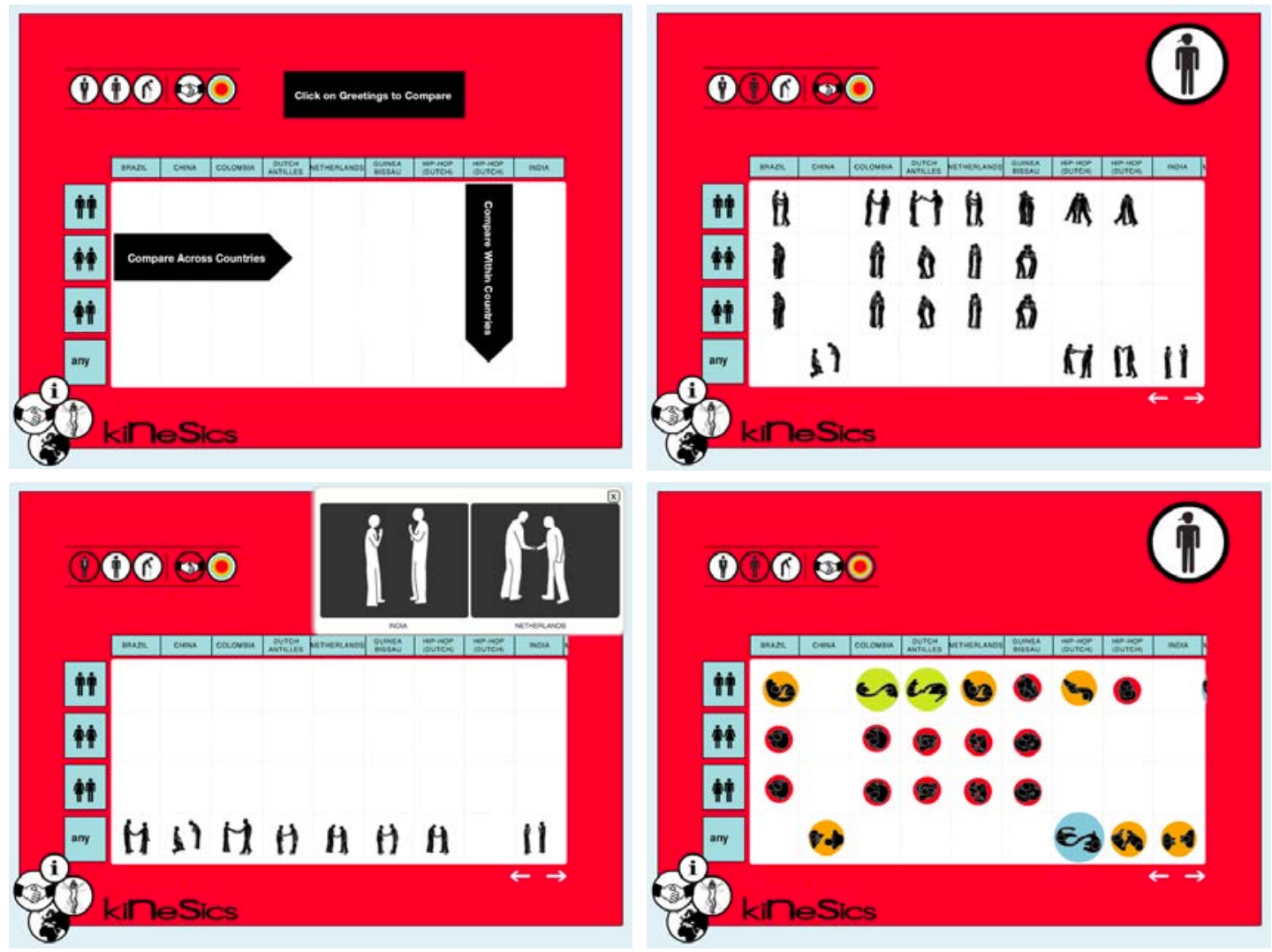

Fig. 240 - Kinesics visuals: screenshots of interactive greetings diagrams interface 

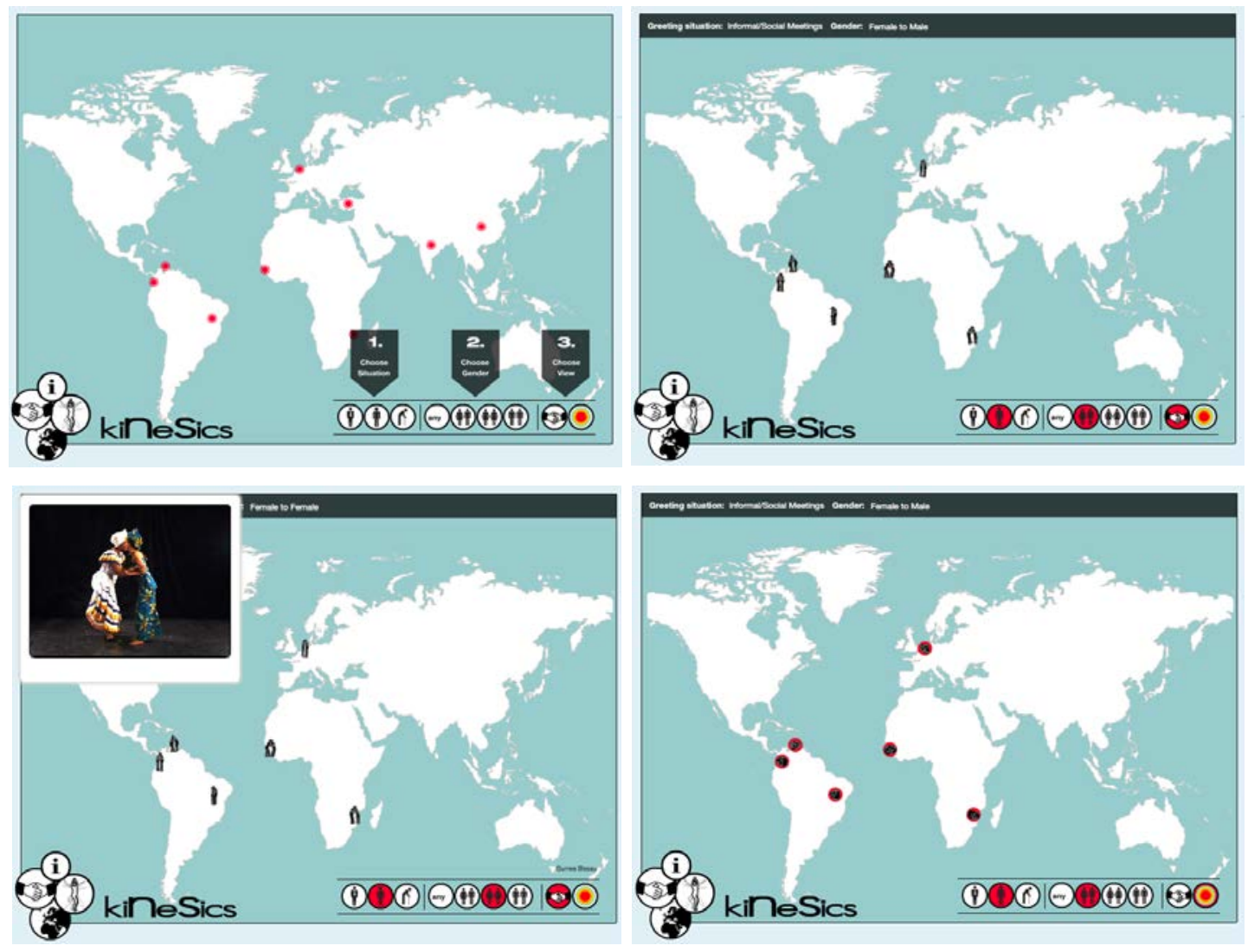

Fig. 241 - Kinesics visuals: screenshots of interactive greetings maps interface

of being able to view the greeting footage by scrolling over the greeting graphics. The purpose of the maps was to give a geographical view of the origin of the greetings (see fig. 241). The greeting animations showed multiple animated vectorised greeting sequences that played continously in a loop, and included the front and overhead views (see fig. 242). 


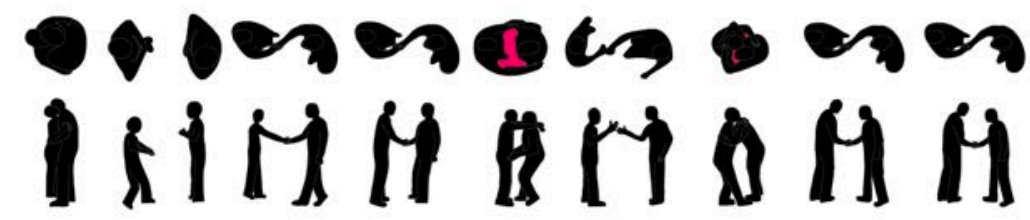

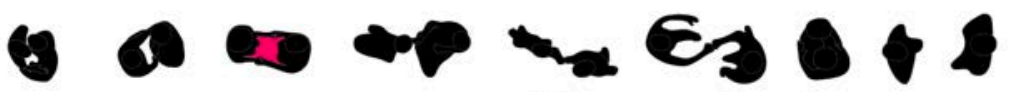

II Hith HAll

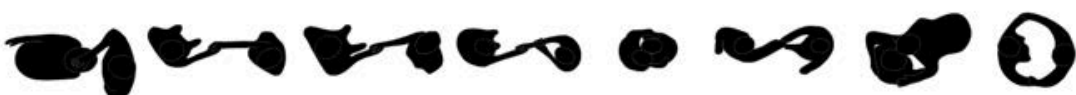

TH HMHAMA

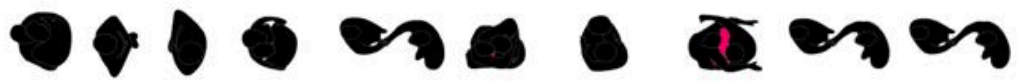

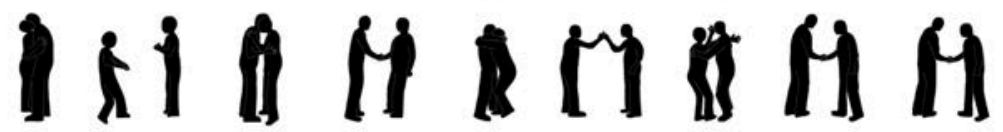

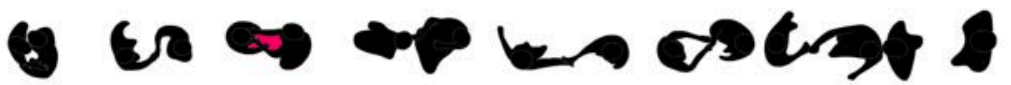

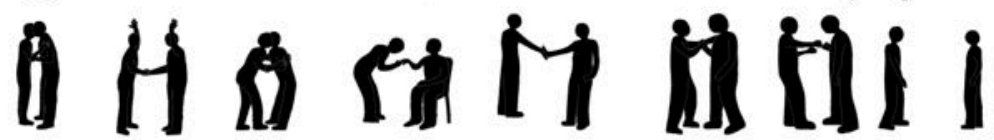

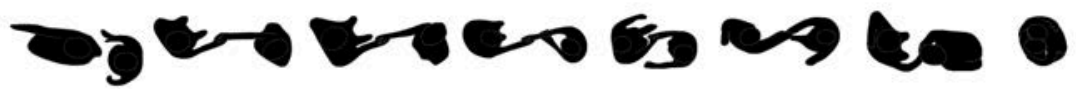

(1) HHHHA A

Fig. 242 - Kinesics visuals: two still images from a greeting video in which all greetings were animated 
The technical process of how the greeting visualisations were developed is outlined below.

Kinesics Project Summary of Technical Process of Visualisation:

1. FILMING - Greetings were filmed from the front and from overhead.

2. SELECTION OF KEY MOMENTS - Still images of key moments of each greeting were extracted from the video footage.

3. VECTORISATION - The images were then vectorised. This was done by drawing over the still images, onto tracing paper. The drawings were then scanned and drawn/vectorised in Adobe Illustrator. Colour coded intimate zones were added to the overhead view vector graphics.

4. ANIMATION - Some of the vector graphics were animated in Adobe Flash (CS5) using frame-to-frame animation. The greeting animations, at $12 \mathrm{fps}$ (frames per second), show key moments of each greeting, but are not true to the real duration/ timings of the greeting.

5. GREETINGS DIAGRAMS - the greetings are organised into static diagrams for print, using Adobe Illustrator (CS5), and interactive diagrams for screen-based viewing, using Adobe Flash (CS5) and ActionScript 2.0. 


\section{Appendix 2: Call for Participation}

\section{Participants needed for research project about cultural}

\section{GREETIIGS In STAVANGER}

As part of my PhD project Patterning Culture, at the University for the Creative Arts in England, I need participants for a case study in which I will film greeting rituals from different cultures.

\section{WOULD YOU LIKE TO PARTICIPATE?}

Are you or your parents from VIETNAM, SOMALIA, TURKEY or CHILE, and would like to participate with showing greetings from your culture?

I need participants from: Vietnam, Somalia, Turkey, Chile and Norway

Age: $20+$

Permanent residence: Stavanger

Participants per culture: 4 people - 2 men and 2 women.

Place: Stavanger Kulturhus, 2. etasje.

Date: 15th September

Time: Kl.10.30 - 15.00 (each group will be given a specifc time to meet up)

Participants will be asked to show different greetings from their culture, and to show how they greet across cultures. The greetings will be filmed and the video documentation will be used for further visualisations of nonverbal differences of the multicultural society of Stavanger. The video documentation will take 1-2 hours to complete per group. Please contact me if you would like to participate.

\section{Contact me on:}

Marie Therese Shortt

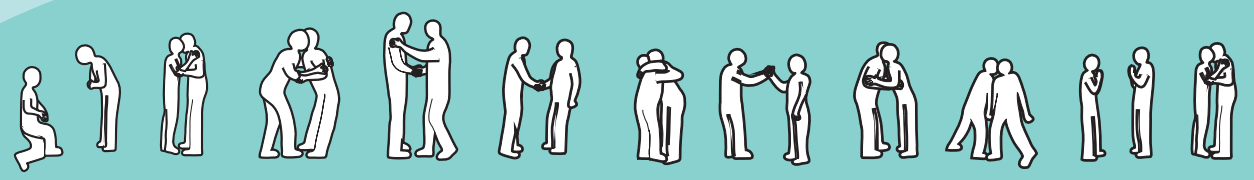




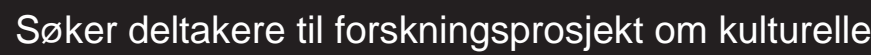 HILSENER I STAVANGER}

Som del av mitt PhD prosjekt Patterning Culture ved University for the Creative Arts i England, trenger jeg deltakere til en case study der jeg skal filme hilsener fra forskjellige kulturer.

\section{VIL DU VAERE MED?}

Er du/ dine foreldre fra VIETNAM, SOMALIA, TYRKIA eller CHILE, og ønsker du å delta med å vise hilsener fra din kultur?

Jeg trenger deltakere fra: Vietnam, Somalia, Tyrkia, Chile og Norge

Alder: 20+

Bosted: Stavanger

Deltakere per kultur: 4 personer - 2 menn og 2 kvinner.

Sted: Stavanger Kulturhus, 2. etasje.

Dato: 15. september

Tid: Kl.10.30 - 15.00 (hver gruppe vil bli gitt et tidspunkt for oppmøte)

Deltakere vil bli bedt om å vise forskjellige hilsener fra deres kultur, og også vise hvordan de hilser på tvers av kulturer. Hilsenene vil bli filmet og brukt til videre visualisering av nonverbale aspekter ved det kulturelle mangfoldet i Stavanger. Filmingen vil ta ca 1-2 timer per gruppe. Vennligst ta kontakt dersom du er interessert i å delta, eller har spørsmål om dette forskningsprosjektet.

\section{Ta kontakt:}

Marie Therese Shortt mt@shorttdesign.com Mob: 99452765

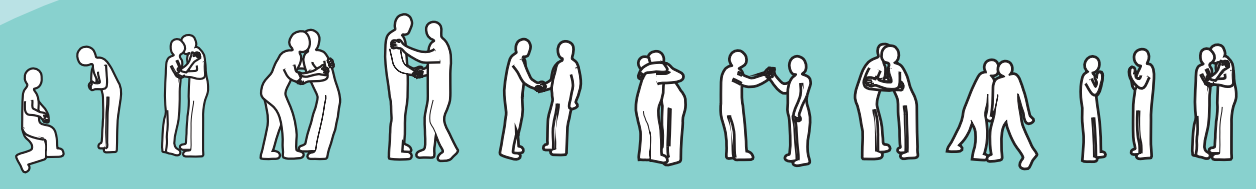

[Call for participants in Norwegian] 


\section{Appendix 3: Conversations During Breakfast with Vietnamese Participants}

\section{9th September 2012}

The following transcript are quotes taken from audio recordings of conversations held during breakfast with Vietnamese participants Stavanger 9th September 2012. (The recorded conversations were conducted in Norwegian. The transcript below is translated into English.)

- 46 year old female $(\mathrm{J})$ -

Young people are not keeping traditions anymore. Young people in Norway with Vietnamese parents, the teenagers, well they do not necessarily keep the traditional greetings anymore, like for instance greetings that show respect to the elderly.

$-\mathrm{J}-$

An older person can touch the head of a young person or a child, it is a form of respect, but a young person cannot do the same to an older person, as it would not be respectful.

- 82 year old male $(\mathrm{C})$ translated by $\mathrm{J}-$

In Vietnamese culture if there is a party or social/family gathering, when you enter the house or room, you should always greet the oldest person first, then second oldest and so on. But kids that are growing up in Norway do not necessarily practice such traditions anymore.

Most young people growing up in Norway become more Norwegian, they might mix Norwegian and Vietnamese cultures. Their parents will teach them Vietnamese culture and traditions, but they do not always listen or are not interested as they do not see the point in why they should practice these traditions in Norway. 
$-\mathrm{C}-$

You are giving me an idea, to gather everyone in our Vietnamese community to talk about greetings, because it is so important to keep our identity and our greetings, they are starting to fade now with the younger generations.

$-J-$

We do not use much physical contact when we greet, but that does not mean that we do not care about each other.

- 65 year old female $(\mathrm{T})$ -

To show respect we do not use a lot of bodily contact, but smiling is very important, not hugging, but eye contact and smiling shows a lot of respect.

$-\mathrm{J}-$

My partner is from Iraq - there men kiss each other when they greet - two men, they do not actually kiss, but they make a kissing sound when they greet each other.

$-\mathrm{T}-$

We do not use a lot of body language, but they eyes tell a lot. Being polite and smiling is very important. Not hugging, not kissing.

$-\mathrm{J}-$

Our way of kissing is completely different. The Vietnamese use their nose when kissing their children. My kids do not like it. In Vietnam only couples/ lovers kiss on the mouth, but when I kiss my kids it is with my nose. When other's see it, it looks like I am smelling my child, but it is our way of kissing. Kissing on the mouth is only for lovers.

$-\mathrm{T}-$

In North of Vietnam we hug normally, with the cheeks, but in the South where she is from, the kissing with the nose is more like they 'smell' their children. 
- J translating for $\mathrm{C}$ -

He wants to thank you for doing this project. He thinks it is very diverse and would like to help with any information he can if you would like to go deeper into this topic.

- 60 year old male (C's nephew) -

They will try their best, they will prepare their costumes, because they want to represent the culture, and they want to do their best. We are going to help your project to become the best.

- MT (myself) -

Is there a difference between how you greet in Vietnam depending on religion?

$-\mathrm{T}-$

I am Buddhist, if I greet a monk, I have to be very, very polite, and I bow. You always have to greet the monk first if it is a gathering with a lot of people.

There has to be 2 metres distance between us and the monks and the nuns, and no physical contact, we cannot touch them.

$-\mathrm{C}-$

If it is a priest, I give him a handshake and put my other hand on top of the handshake to show respect to the priest. This is a form of tradition - before we would kiss the priests' hand, or even kneel for him, but this has changed and become simplified. If you are a man and you greet a nun you should not have any physical contact with her. If you are a Catholic meeting a Buddhist monk, you have to be polite and greet him like the Buddhists do.

$-\mathrm{T}-$

Family members that are dead, it is important to mark the day they died, their birthday is not important. You should greet them every day - greet pictures of them that are hanging on the wall, and you should pray to them. 
(T explains how she prays/greets photos of her husband and parents.)

$-\mathrm{C}-$

After communism came, some of the manners and polite ways disappeared, the focus turned too much towards money and things. Politeness should be the first thing one learns in school.

$-\mathrm{C}-$

I know a Norwegian man who was a teacher 25 years ago, and he says that back then Norwegians were very polite towards each other, today, however, pupils sit there with their feet on the desk and so on, so in Norway too, manners have changed.

$-\mathrm{T}-$

I came to Norway as a refugee. We lost our country when communism came 37 years ago in 1975, communism came from the North to the South, so we fled, we could not live together with the communists. We are very grateful towards Norway, it was a Norwegian boat/ship that eventually saved us when we had fled to Japan. We were 52 people that got saved by the ship. 


\section{Appendix 4: Programme for Greeting Documentation}

15th September 2012, Kiellandrommet, Stavanger Kulturhus

Time:

$\mathrm{KI}: 10.30$ - $11.30 \quad$ Vietnam

K: $11.30-12.30$ Somalia

$\mathrm{KI}: 12.30-13.30 \quad$ Norway

$\mathrm{Kl}: 13.30-14.30 \quad$ Chile

KI: $14.30-15.30 \quad$ Turkey

Please show up 10 minutes before your given time. If some people per group have the possibility to stay for longer than 1 hour, that would be great as I would like to film greetings across cultures too.

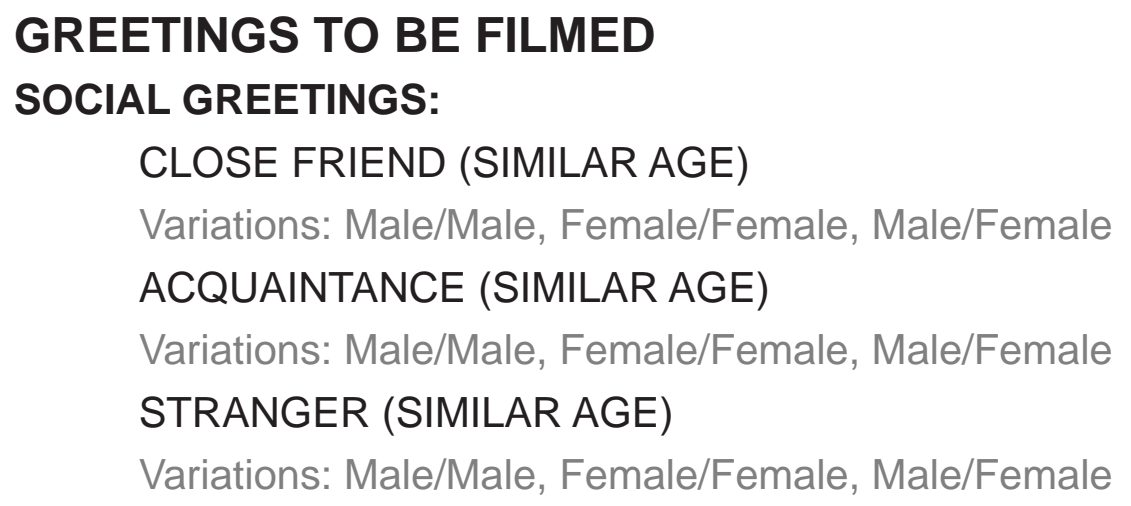

FAMILY GREETINGS:

MOTHER

Variations: Son/Mother, Daughter/Mother

FATHER

Variations: Son/Father, Daughter/Father

GRANDMOTHER

Variations: Male/Grandmother, Female/Grandmother

GRANDFATHER

Variations: Male/Grandfather, Female/Grandfather

GREETINGS ACROSS CULTURES:

SOCIAL GREETING - GREETING A STRANGER (First time meeting) (SIMILAR AGE)

Variations: Male/Male, Female/Female, Male/Female 


\section{Programme for Greeting Documentation [Somali Greetings]}

27th September 2012, Kiellandrommet, Stavanger Kulturhus

Time:

$\mathrm{KI}: 14-16.00 \quad$ Somalia

\section{GREETINGS TO BE FILMED}

SOCIAL GREETINGS:

CLOSE FRIEND (SIMILAR AGE)

Variations: Male/Male, Female/Female, Male/Female

ACQUAINTANCE (SIMILAR AGE)

Variations: Male/Male, Female/Female, Male/Female

STRANGER (SIMILAR AGE)

Variations: Male/Male, Female/Female, Male/Female

\section{FAMILY GREETINGS:}

MOTHER

Variations: Son/Mother, Daughter/Mother

FATHER

Variations: Son/Father, Daughter/Father

GRANDMOTHER

Variations: Male/Grandmother, Female/Grandmother

GRANDFATHER

Variations: Male/Grandfather, Female/Grandfather

\section{GREETINGS ACROSS CULTURES:}

SOCIAL GREETING - GREETING A STRANGER (First time meeting) (SIMILAR AGE)

Variations: Male/Male, Female/Female, Male/Female 


\section{Appendix 5: Research Information Sheet}

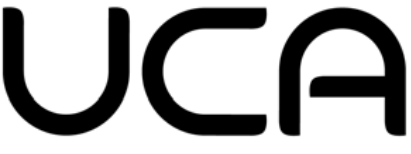

university for the creative arts

Ethics Committee

\section{Research Information Sheet}

Project Title: Patterning Culture: Developing a System for the Visual Notation of Greeting Rituals

\section{Data Controller:}

Student Name: Marie Therese Shortt

Study: MPhil/PhD Researcher, University for the Creative Arts

Supervisors: Jean Wainwright, Judith Williamson \& Ricardo Leizaola

\section{Nature/purpose/use of data:}

I am documenting greeting rituals within and across 5 different cultures in Stavanger, Norway, using video, still photography, and audio documentation. Voluntary participants, a minimum of four - two men and two women - per culture, are filmed and photographed from different angles while performing a number of different greeting rituals. Participants will also be interviewed before or after the greeting documentation, interviews will be audio recorded.

The purpose of this data collection is to use it to visualise how culture patterns behaviour, and to use this raw material to further visualise the cultural diversity and varied behavioural patterns that coexist in Stavanger city. 
The video and audio material will be used to create a graphic mapping of cultural greetings across these 5 cultures living in Stavanger, and to visualise different layers of communication when it comes to the diverse meanings different cultures put into their and others' behaviour.

\section{The use or potential benefits of the study:}

My investigation will contribute to making visible some of the diversity of Stavanger today, its cultures, and their culturally patterned behaviour. This will cater for increased intercultural understanding in a city and region that is becoming increasingly multicultural.

\section{Likely duration of the project and location:}

The video documentation takes place on 15th/27th September 2012, at Stavanger Kulturhus, Norway, and audio documentation/ interviews will take place during the month of September 2012 and September 2013. However, participants may be asked to participate in additional studies during 2012-2014, if further documentation or re-takes are required.

\section{Obligations and commitments of the participant during the study:}

All participants will be expected to perform a number of different greetings, with other participants within their culture, and to greet participants from different cultures, male and female. These greetings will all be filmed and photographed from different angles, and the main purpose of this material is to turn it into graphic symbols and animations, in a greeting mapping of Stavanger. This final outcome will be presented as my final PhD project, and photographic and video material may be included in international exhibitions and publications of my research findings.

\section{The rights of the participants:}

Participants are free to withdraw from the study at any time without needing to justify their decision and without prejudice. In case of withdrawal of consent no further data regarding their participation should be added to the project.

All personal data relating to volunteers is held and processed in the strictest confidence, and in accordance with the Data Protection Act (1998) and Norwegian Datatilsynet. 
In consideration for the research nature of the study participants shall not receive any reimbursement, payment or rewards.

Information for further concerns or complaints:

Any concerns or complaint about any aspects of the way you have been dealt with during the course of the research will be addressed; please contact:

Marie Therese Shortt

MPhil/PhD Researcher

University for the Creative Arts - Farnham (UCA)

Tel: (+47) 99452765 / (+44) 07414646341

Email: mt@shorttdesign.com / MShortt@students.ucreative.ac.uk 
Appendix 6: Participant Consent Form

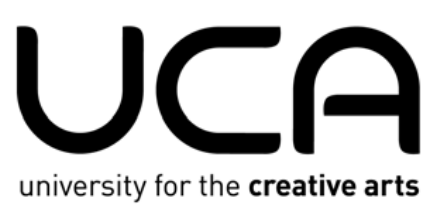

Ethics Committee

\section{Consent Form}

Project title: Patterning Culture: Developing a System for the Visual Notation of Greeting Rituals

\section{Data Controller:}

Student Name: Marie Therese Shortt

Study: MPhil/PhD Researcher, University for the Creative Arts

Supervisors: Jean Wainwright, Judith Williamson \& Ricardo Leizaola

\section{Participant Name:}

Participant Location: Stavanger, Norway.

$\square$ I the undersigned voluntarily agree to take part in the study on Patterning Culture; Developing a System for the Visual Notation of Greeting Rituals, a practice-based research project. 
I have read and understood the Information Sheet provided. I have been given a full explanation by the investigator of the nature, purpose, location and likely duration of the study, and of what I will be expected to do. I have been advised about any discomfort and possible ill-effects on my health and well-being which may result. I have been given the opportunity to ask questions on all aspects of the study and have understood the advice and information given as a result.

I agree to comply with any instruction given to me during the study and to co-operate fully with the investigator. I shall inform them immediately if I suffer any deterioration of any kind in my health or well-being, or experience any unexpected or unusual symptoms.

I I understand that all personal data relating to volunteers is held and processed in the strictest confidence, and in accordance with the Data Protection Act (1998). I agree that I will not seek to restrict the use of the results of the study on the understanding that my anonymity is preserved.

$\square$ I understand that I am free to withdraw from the study at any time without needing to justify my decision and without prejudice.

— I acknowledge that in consideration for completing the study I shall not receive any reimbursement, payment or rewards.

$\square$ I confirm that I have read and understood the above and freely consent to participating in this study. I have been given adequate time to consider my participation and agree to comply with the instructions and restrictions of the study. 
Name of volunteer

(BLOCK CAPITALS)

Signed

Date

Name of witness

(BLOCK CAPITALS)

Signed

Date

Name of researcher/person taking consent

(BLOCK CAPITALS)

Signed

Date 


\section{Appendix 7: Research Ethics Approval Application Outcome}

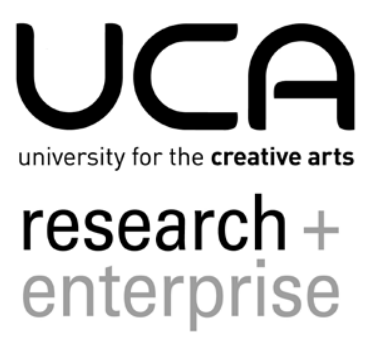

6 September 2012

Direct telephone 01252892922

Email: Mohagan@ucreative.ac.uk

Marie Therese Shortt

Lauvveien 8

4330 Algard

Norway

Dear Marie Therese

Research Ethics Approval application outcome

I can now confirm that your application for research ethics approval has been considered and approved.

The Chair of the Research Ethics Committee, Professor Kerstin Mey, has considered your ethical self-evaluation for your proposed project and confirmed that the rationale and informed consent of participants is well detailed and meets the criteria for approval. 
Professor Mey has recommended that the ethics consent form is revised to include publications of the research findings.

Overall attention to the Code of Practice has been carried out with due care and attention and does not, in itself, raise any substantive concerns.

However, the Chair advises that as your project involves the handling of personal data it is essential that you familiarise yourself with the 8 Principles of the Data Protection Act and as some of your research will stretch to Norway, particular attention should be paid to Principle 8.

Please study the information at the following link which sets out the key definitions in the Act, and explains what they mean, and shows how they often relate to each other. http://www.ico. gov.uk/for_organisations/data_protection/the_guide/principle_1.aspx

You should discuss with your supervisor the logistics of securely generating, storing and processing data both electronically and in hardcopy to ensure there is no breach of the Data Protection Act.

You should also consider if there are similar laws in Norway, which you will need to adhere to.

If you require any further information please don't hesitate to contact me.

Yours sincerely

Mary O'Hagan

Research + Enterprise Manager (Research Degrees) 


\section{Appendix 8: Conversations During Video Recording of Greetings}

\section{5th \& 27th September 2012}

The following comments and conversations were recorded during the 2 days of recording greetings in Stavanger, on the 15th and 27th of September 2012. Participants (and visitors) across different cultures discussed issues surrounding greetings. Some of the conversations were in Norwegian and have been translated into English. There is no single audio file for these conversations as the comments are taken from different video/audio footage gathered during the two days.

\section{5th September 2012}

- Male \& female from Estonia (visitors) -

[Discussing greetings in general:]

In Estonia, if I am sitting and the person is coming, I stand up to greet.

One thing that is special in Estonian greetings, is that you never greet on the doorstep - this is considered as bad luck. You go outside to greet them, or inside.

- Norwegian female -

I have one grandmother that never hugs me and one that squeezes me until I almost die.

I have never hugged my grandfather.

- Another Norwegian female -

I do not usually hug my grandfather either. My grandfather always takes my hand but he never hugs me. 
- Norwegian male -

[About the greeting he did with the lady from Chile:]

When she kisses my cheek, it weird for me every time, it always makes me wonder - is it one time, two times, three or four? I have never learnt it. I have a female friend from Brazil and it is awkward every time I greet her, sometimes she gives two kisses and sometimes three.

- Turkish female -

[When she entered the room and the other participants stood up to greet her, many reaching forward for a handshake:]

I usually do not greet men with a handshake, but I can do it here.

If you are religious and the person with whom you are interacting is aware of it, like a Turkish man, I do not greet with a handshake. If the person with whom I am meeting is Norwegian and reaches out his hand, then I will respond by doing a handshake.

On holy days and religious holidays children will kiss the hand of their mother.

- Interviewer (MT) -

So you greet grandparents with 3 kisses?

- Turkish female -

Well - that was a religious greeting. A normal greeting is 2 kisses. 3 kisses is a usual daily greeting if you are from a religious family. However, when I am in Turkey I use 2 or 4 kisses, as they are not familiar with the 3 kisses greeting there. There 2 is the daily greeting, and if you have not seen each other in a long time it is 4 . However, here in Stavanger they use 3 kisses - it is sort of a Stavanger Turkish greeting. 
[During a conversation between participants, the Turkish female explains the rules for headscarves, and when asked why her daughter is not wearing a headscarf, she explains:]

All rules start to count from puberty onwards - when girls get their period and when the boys' voices start changing. This is when the girls start wearing headscarves, and the greeting rules also comes into count.

- Angolan/Portuguese female -

[After her greeting with the Norwegian male participant who got surprised when she kissed/hugged him when they did a Social strangers greeting:] Oh, I showed how I would greet in Angola... but in Angola people would treat him like that, he would get a shock!

\section{7th September 2012}

- Somali females -

[The ladies explain the social greeting between a male and a female who are close friends:]

Either you follow culture or you follow religion - this is a dilemma. If you follow religion, depending on how religious you are, you should not touch each other, no handshake or anything. If you follow culture, you can, it really depends on the person.

It is the same if you are acquaintances of close friends. Some people hug their good friends, but personally I do not. If I do not know the person very well, especially if it is the opposite sex, I usually fold or hold my own hands and just do a verbal greeting without touching the other person.

[When asked if they hug once or twice, because they showed both during the documentation. One Somali female answers:]

It depends, I usually hug two times when greeting close female friends. 
[Another Somali female answers:]

You are really supposed to hug one time, but it is a matter of taste.

$-\mathrm{MT}-$

What is a common greeting if you are introducing two of your friends to each other?

- Somali females -

If I introduce two friends, one male and one female, and if the female is going to pray afterwards, then she will not take his hand, then she just says 'hi my name is Farhia' or similar. But if we do the usual Norwegian way, like if the female is not religious or if she is not going to pray afterwards, she can do a handshake, it depends really.

$-\mathrm{MT}-$

If I were from Somalia, would you have greeted me differently this morning when you arrived?

- Somali females -

No we think we would have greeted in the same way. I never think over how I greet people. I greet if I am bothered to greet. If I meet someone I kind of respond to how they are, if they lean in to greet, then I do too.

[After two girls were asked to show how two women that are strangers would greet, and they do a handshake greeting, they stop and laugh because they realise that this is an unnatural greeting to them, they discuss:]

Somali women do not greet with a handshake. Handshakes are typical for Western cultures. If both are women, then it is unnatural that we do that either we kiss or hug each other.

$-\mathrm{MT}-$

[When filming the greeting between grandmother and granddaughter:] 
Who is acting like the older person now in this greeting?

- Somali female -

I cannot play the older person, because I am wearing trousers today, it is a cultural thing.

[When filming the greeting between mother and daughter:]

It is in the (verbal) language if you are greeting your mother or your aunt, what you say might be different, but the greeting is the same.

[When filming the grandmother/granddaughter greeting:]

When greeting an older person like a grandparent, if the younger person is sitting, he or she has to stand up when greeting the older person or grandparent. We hug our grandparents, both grandmother and grandfather.

[Regarding generational differences:]

If my mum and I go to a doctors appointment with a male (Norwegian) doctor, then my mum will greet him without taking his hand, while I do reach out for his hand to do a handshake, then he gets very surprised as he is not expecting me to do a handshake when my mum does not.

- Norwegian male in conversation with Somali females Last time we were here there was a Turkish woman here who was also Muslim, and she did not want to greet me with a handshake. So when I came here today and saw your head scarves, I became very unsure/insecure, I was prepared that my hand could get stuck hanging in the air... But you did girls did respond to my handshake.

$-\mathrm{MT}-$

Have any of you got any 'street greetings' you use? 
- Somali female -

No we are not really in to that, it is more the guys that do greetings like that.

- Somali male -

I do these kinds of greetings with my friends.

[He shows a street greeting - a high-five style greeting followed by some different hand gestures.] 


\section{Appendix 9: Report on Greetings Documented During Fieldwork}

The images in this section are all stills from greeting videos recorded in Stavanger on 15th September 2012, and for Somali greetings on 27th September 2012.

The observations are based on my notes made at time of filming.

\section{GREETINGS - SOCIAL (ON MUTUAL GROUND)}
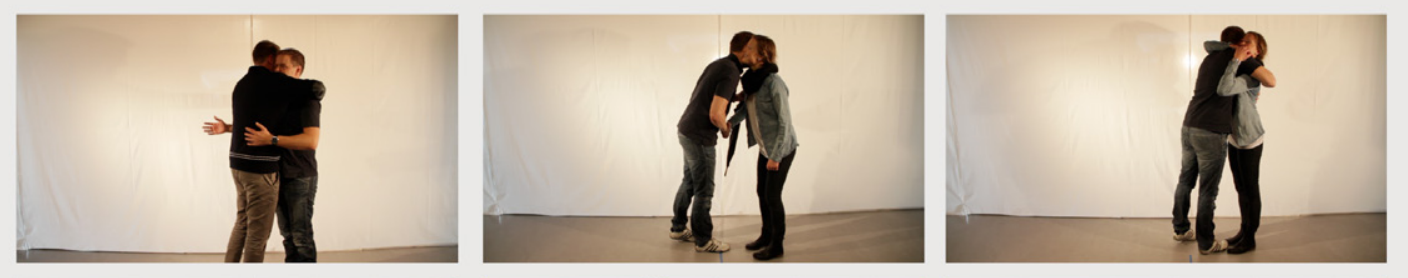

Fig. 243 - Chilean social greetings

\section{Chile}

Close friends: The same greeting, one kiss on the right cheek, is used for close friends, acquaintances and strangers, regardless of the gender combination. The close friend greeting involved a longer hug leading in to the kiss, and did not involve the handshake.

Both these greetings ( $f / f$ and $m / f)$ involved a handshake/ holding hand that led into the kiss. There was slight difference in the amount of touch during the kiss and after, depending on how close friends they were. The main difference was in the verbal greeting and conversations.

Did not film: $m / m$ and $f / f$ versions of greetings between close friends, acquaintances and stranger due to lack of participants. 


\section{Norway}
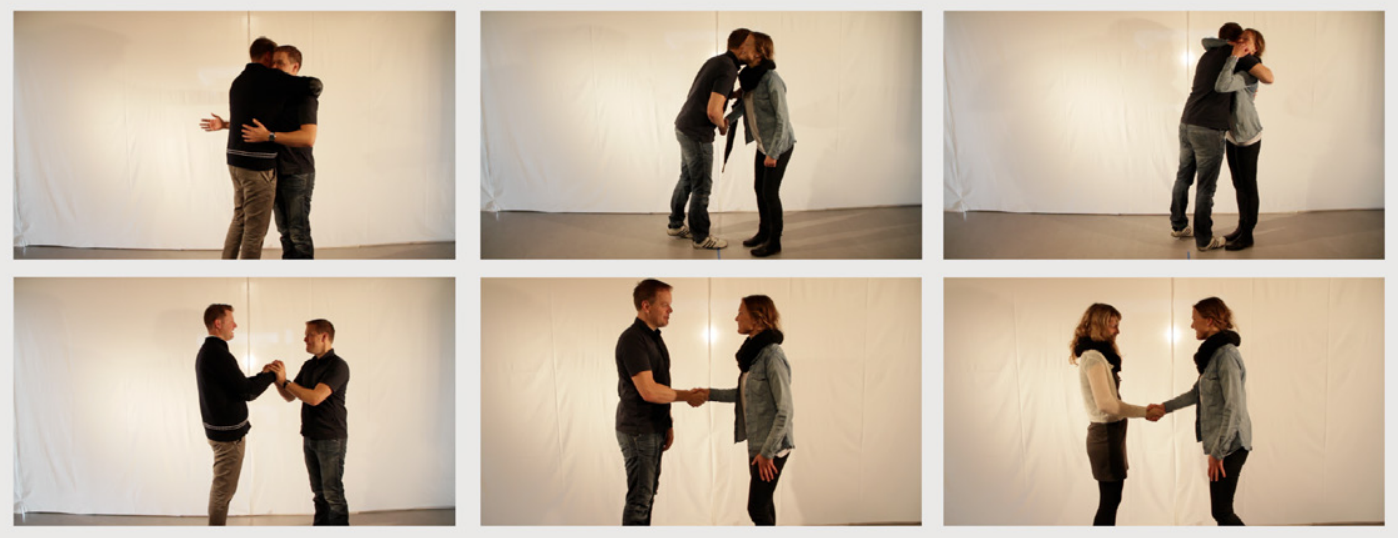

Fig. 244 - Norwegian social greetings

Close friends: Female to female close friends greetings usually consists of a hug, however if they have seen each other recently a simple 'hello' is ok, whereas the male to male close friends greeting varies from a 'hello' without any real gesturing, to a high-five type greeting, to a handshake or a handshake with a hug and pat on the back.

Two female acquaintances hug or just say 'hello', depending on the situation and amount of time since their last meeting. Two males either do a handshake with a pat on the back hug, or just nod. A male and a female either hug or just nod with a 'hello', also depending on the situation and time since last meeting.

Strangers: $\quad$ Two strangers, whether $\mathrm{m} / \mathrm{f}, \mathrm{m} / \mathrm{m}$ or $\mathrm{f} / \mathrm{f}$, meeting for the first time usually shake hands, and a firm handshake is recommended/ the norm. 


\section{Somalia}
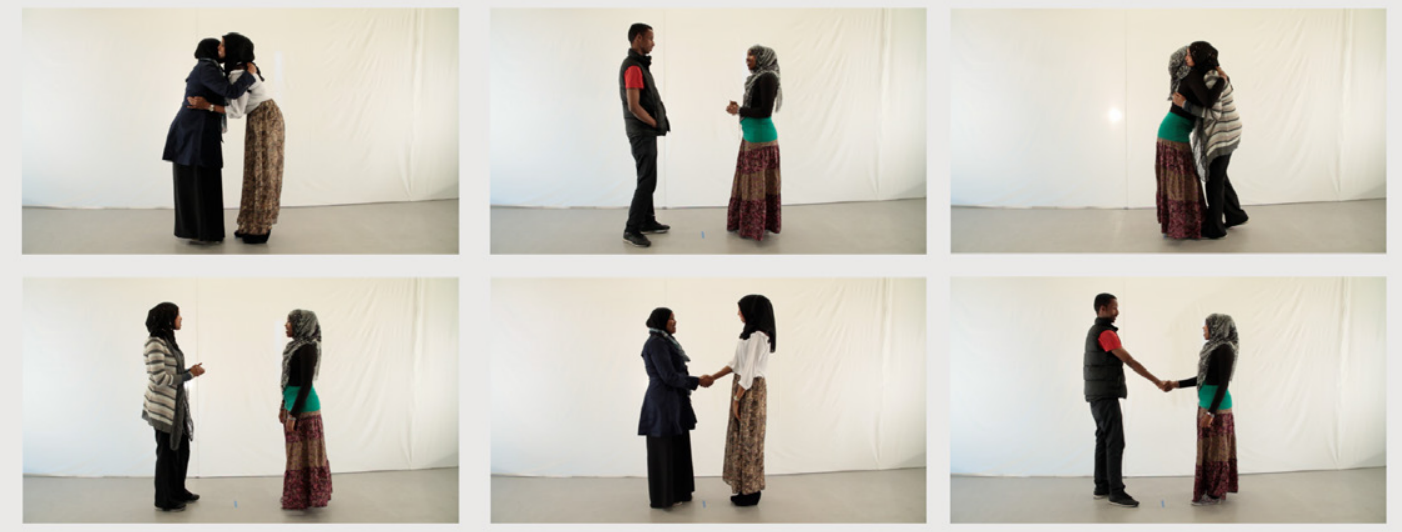

Fig. 245 - Somali social greetings

Close friends: For the female to female close friends greeting they have 2 variations - some people give 1 hug some give 2 hugs, the female to male greeting depends on how close friends they are - some hug the opposite sex, and some don't because of religious beliefs. If you are very religious you will not touch the opposite sex.

Greetings between acquaintances, female to male is the same greeting as the greeting between close friends - some people hug, some do a handshake, and some don't touch.

Strangers: $\quad$ Two female strangers meeting for the first time greet with 3 kisses (it is unnatural for two Somali females to shakehands). In a male-to-female greeting, some shake hands and some do not touch. The participants said that when greeting you either follow culture or you follow religion, which will result in very different greetings especially for $\mathrm{m} / \mathrm{f}$ greetings.

Did not film: $\mathrm{m} / \mathrm{m}$ lose friends, acquaintances and strangers due to lacking one male participant. 


\section{Turkey}

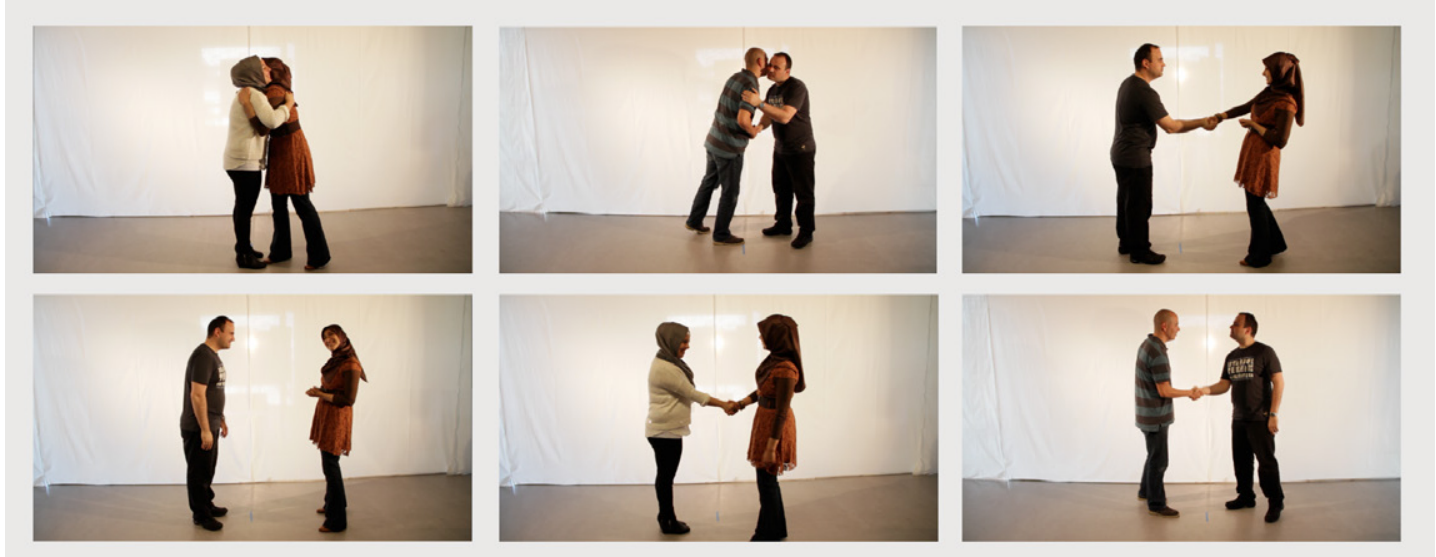

Fig. 246 - Turkish social greetings

Close friends: Two male close friends give a handshake and 2 kisses (right then left). Close friends, $\mathrm{m} / \mathrm{f}$, if female is not too religious they use the same greeting as $\mathrm{m} / \mathrm{m}$ greeting (kiss), if she is religious, they do not touch, but show more body language compared to when greeting an acquaintance. Two females that are close friends will give 3 hugs with a lot of physical contact/touch.

Two female acquaintances greet with 2 hugs, male to female acquaintances greet without touching, just a nod and eye-contact if the female is religious (Muslim), however depending on how religious you are, some females will do a handshake with a male. Male to male acquaintances give a handshake.

Strangers: $\quad F / f$ and $m / m$ strangers give a handshake, as does female to male, or if she is very religious, they do not touch, they just do a verbal greeting and maintain eye contact. 


\section{Vietnam}

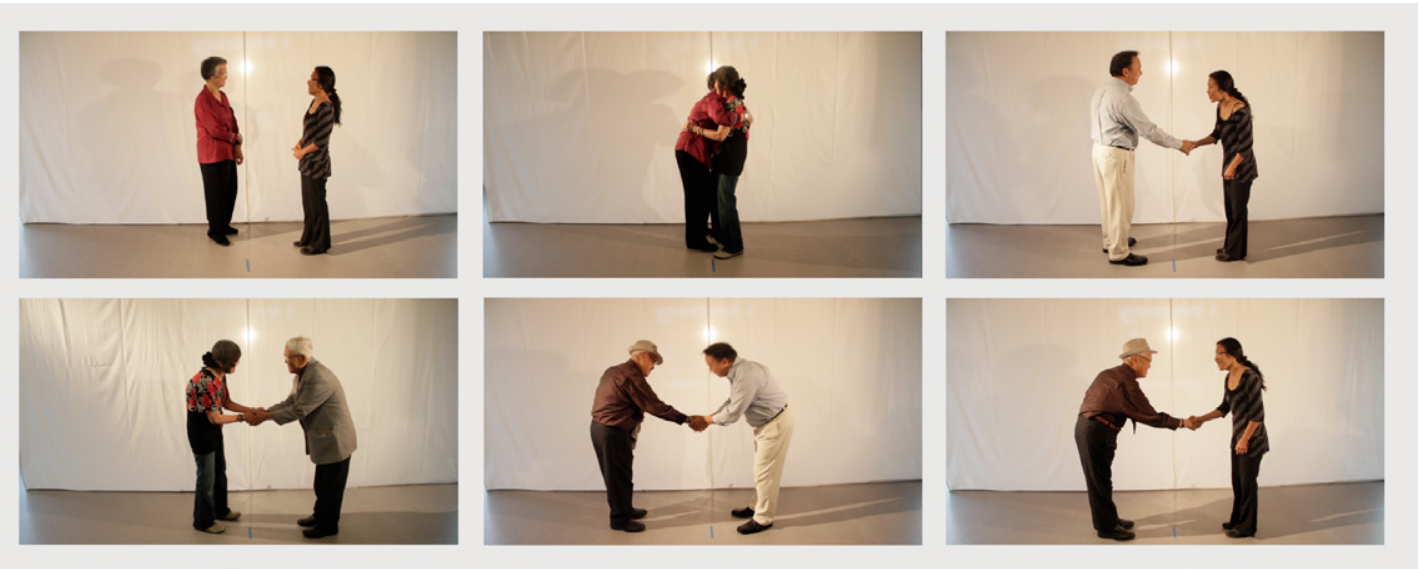

Fig. 247 - Somali social greetings

Close friends: Two female close friends greet with a hug/embrace, then they hold on to each others hands as the conversation starts, $\mathrm{m} / \mathrm{f}$ is similar - first a slight bow, then they hold hands for some seconds, then bow again after short conversation. Male to male close friends greet with a handshake then bow (only the youngest person bows).

Two female acquaintances greet with a slight bow/nod, there may be some physical contact, like a hand on the other's shoulder, but most important is the gaze and the smile. F/m and $\mathrm{m} / \mathrm{m}$ use a slight bow then a gentle handshake followed by smiles and conversation.

Strangers: A greeting between two female strangers involves no touch, just gaze and smiles, the same goes for male to female, and the $\mathrm{m} / \mathrm{m}$ greeting is a bow (by youngest person) and a handshake. However, they said it is personal so it can vary, some touch while others do not. 


\section{GREETINGS - FAMILY}

\section{Chile}
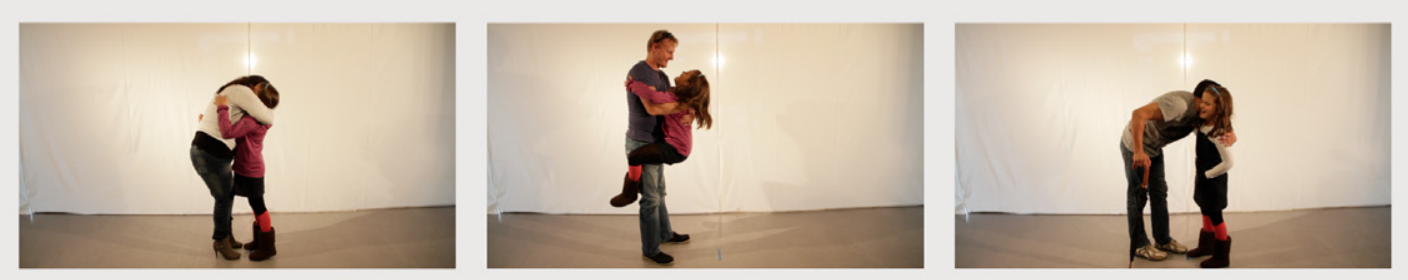

Fig. 248 - Chilean family greetings

Mother: $\quad$ Chilean Mother and Norwegian/Chilean daughter (child) greeting is a hug/kiss on cheek with an embrace and a lot of physical contact.

Father: $\quad$ Norwegian Father and Norwegian/Chilean daughter (child) greeting is an embrace/hug.

Grandmother: Norwegian grandmother and Norwegian/Chilean granddaughter (child) is a hug.

Grandfather: Chilean grandfather and Norwegian/Chilean granddaughter (child) is a hug/kiss on left cheek, then a pat on the back/ embrace.

Did not film: mother/son, father/son, grandmother/grandson and grandfather/ grandson. 


\section{Norway}
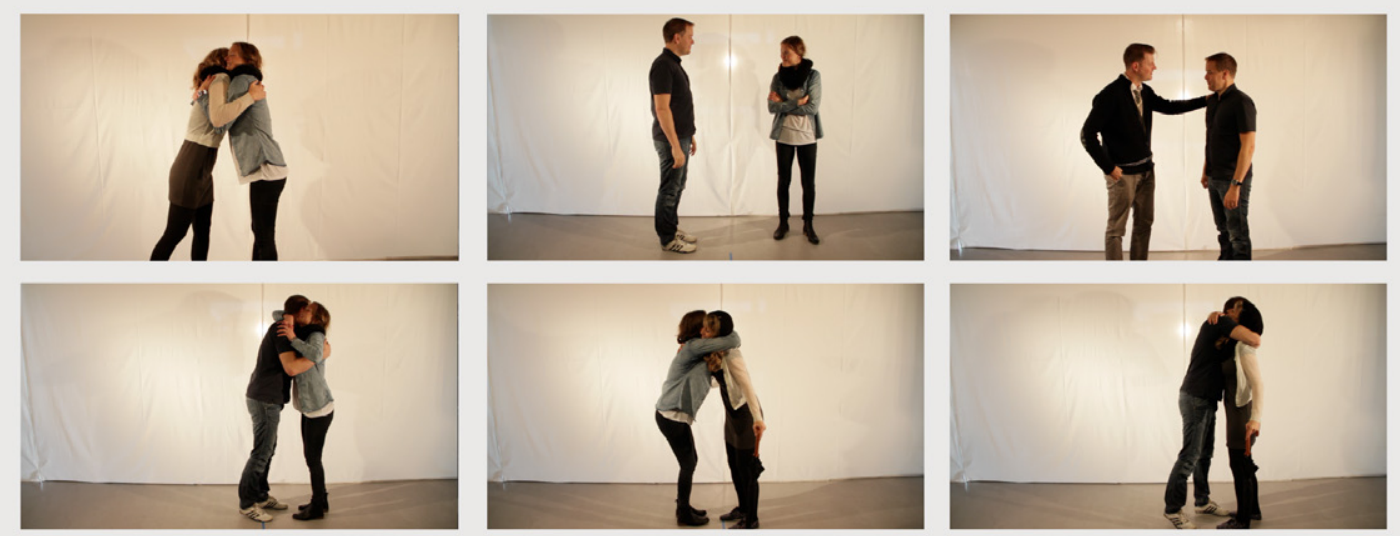

Fig. 249 - Norwegian family greetings

Mother: $\quad$ A mother/daughter and mother/son greeting changes whether they have recently seen each other or if they haven't seen each other in a long time. If they have seen each other recently a simple 'Hei' ('hi') or similar short verbal greeting while smiling and giving eye contact is used, and if it has been a long time since the last meeting, a hug is usual.

Father: $\quad$ Same as above, father/daughter and father/son greeting is either a hug or just a 'hi'.

Grandmother: Depending on whether they have recently seen each other or if it has been a long time. Some hug their grandmother, others just say 'hi' or similar without any physical contact.

Grandfather: Two variations - depending on whether they have recently seen each other or if it has been a long time. Both female participants said they never hug their grandfathers, but that their grandfather will hold their hand and squeeze it a little bit. Some males hug their grandfather, others just have eye contact and/or nod. 


\section{Somalia}
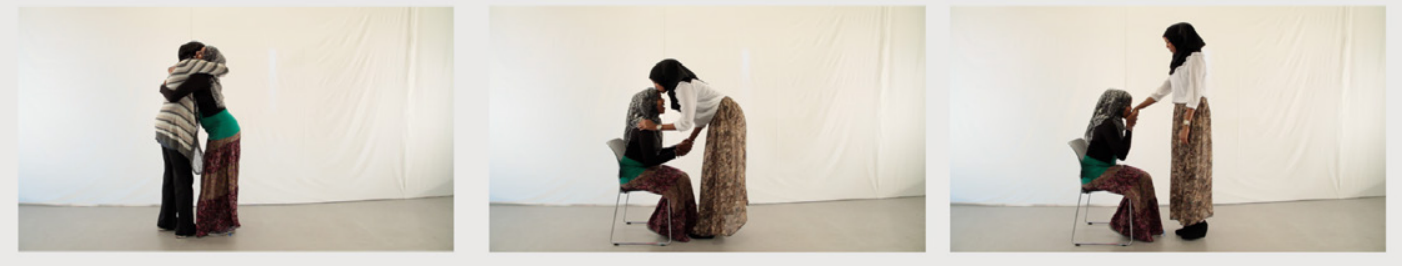

Fig. 250 - Somali family greetings

Mother: $\quad$ Mother and daughter kiss with a hug 4 times, starting left right - left - right. The same greeting is used for aunts and older female family members, but greeting grandmothers is different.

Father: $\quad$ A greeting between father and son is usually a handshake, or sometimes a handshake followed by a hug.

Grandmother: Granddaughter \& granddaughter - If the grandmother is sitting, grandchild has to bow down and greet her, if the grandchild is sitting, he/she has to stand up to greet the standing grandmother. If the grandmother is sitting the grandchild approaches, takes her hand, kisses and gives hugs left then right then on head of the grandmother. The grandmother then kisses the grandchild's hand.

Grandfather: The same as greeting a grandmother.

Did not film: mother/son, father/son, father/daughter, grandmother/grandson and grandfather/granddaughter. 


\section{Turkey}

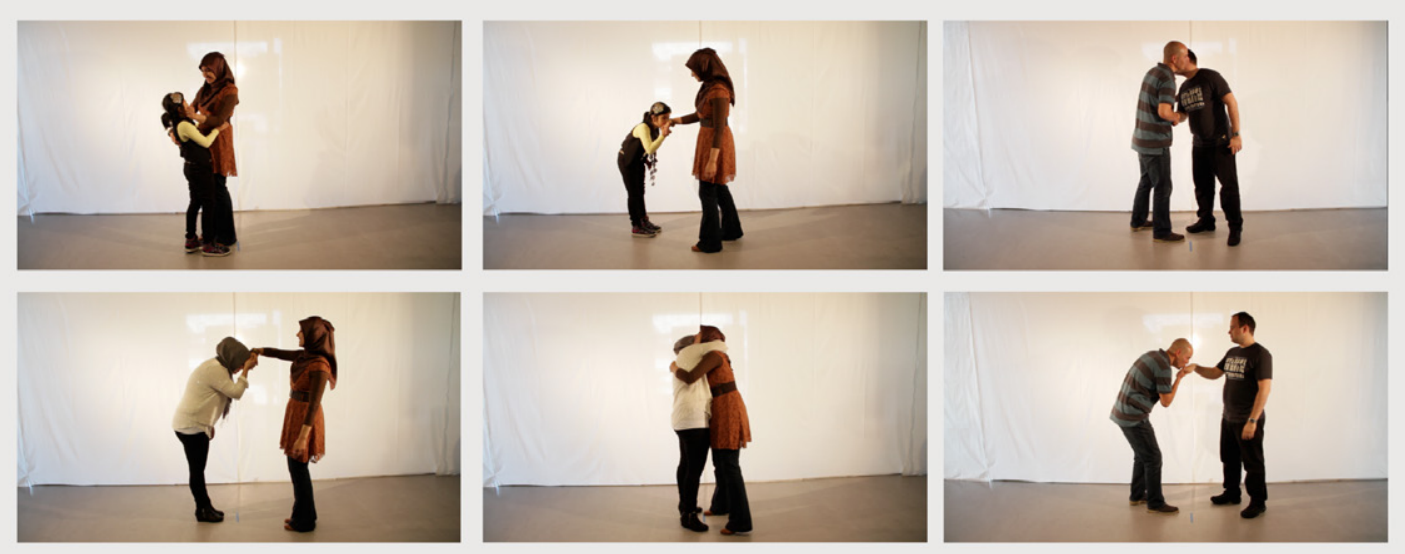

Fig. 251 - Turkish family greetings

Mother: $\quad$ The daily greeting between mother and daughter (child) is an embrace/hug, while the greeting on a religious day like Eid is daughter taking mother's hand, kissing it, putting it to her forehead, then kiss/hug twice - right then left - they hold each other's hands throughout the greeting.

Father: $\quad$ Son and father is the same as a $\mathrm{m} / \mathrm{m}$ social greeting - a handshake and two kisses (right then left).

Grandmother: There are different versions of the grandmother/ granddaughter greeting: daily religious; non-religious; and religious. The daily greeting between a religious (Muslim) grandmother and granddaughter is three kisses on the cheeks - left-right-left. The greeting for non-religious is two kisses left then right, or handshake leading into two kisses left then right, followed by two hugs right to left, holding each others' hand throughout. The grandmother/granddaughter greeting when it is a religious holiday/event like Eid is as follows: the granddaughter approaches, takes the hand of her grandmother, kisses the hand, puts the hand to her 
(granddaughter's) forehead, then kisses three times right left - right, holding the handshake throughout the greeting.

Grandfather: Grandfather/grandson - same as father/son greeting sometimes they greet with kisses like $\mathrm{m} / \mathrm{m}$ social greetings and sometimes with a more elaborate greeting: the grandson approaches, takes the hand of the grandfather, kisses the hand, puts the hand to his (grandson's) forehead, then kisses twice - right then left side - holding the handshake throughout the greeting.

Did not film: mother/son, father/daughter, grandmother/grandson and grandfather/grandson.

\section{Vietnam}
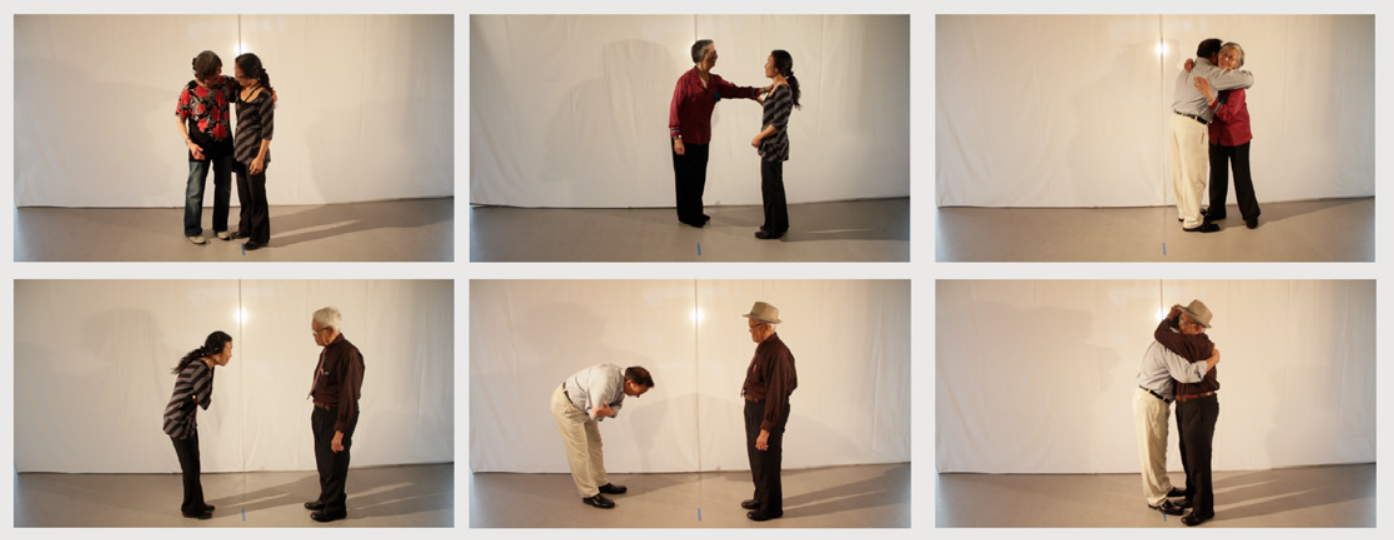

Fig. 252 - Vietnamese family greetings

Mother:

I recorded a mother greeting her adult daughter and adult son. The greeting changes if the daughter or son is a child. Daughter folds hands gently as she is approaching her mother, bowing gently too, mother puts her arm around her daughter's back, in a one-sided open hug, touching the 
daughter's head, while daughter places her hand on her mother's back. They continue into conversation holding on to each other's arms/hands. The mother and son greeting is a hug, and the mother pats his back and head. They also hold on to each other's arms when entering into conversation.

Father: In the father/daughter greeting, the daughter bows (gently) to her father a couple of times, both are smiling and maintaining eye contact. In this recorded greeting they have a short conversation, father gives a present to daughter, and daughter bows again. The space between them remains quite large throughout the greeting. In the father and son greeting, the son bows first keeping his arms at his sides, he bows quite low, then father bows a little, not as low as son, while son is bowing. After bowing they have a short conversation with eye contact before they shake hands leading in to an embrace with father patting son's back.

Grandmother: Same as mother/daughter greeting.

Grandfather: In the grandfather/grandson greeting, the grandson folds his arms, holding the hands on the opposite bicep, keeping a good distance then bows almost 90 degrees, grandfather does not bow. Then they embrace in a hug with grandfather patting the grandsons' head. The grandfather/ granddaughter greeting is similar without the embrace at the end. Granddaughter folds arms, then bows gently (not 90 degrees), grandfather responds with a slight bow, they keep that distance when they go into conversation. The second version of this greeting included the grandfather giving some money to his granddaughter and then embracing her in a hug gently holding on to her head. 


\section{GREETINGS ACROSS CULTURES}

\section{Norway/ Chile}
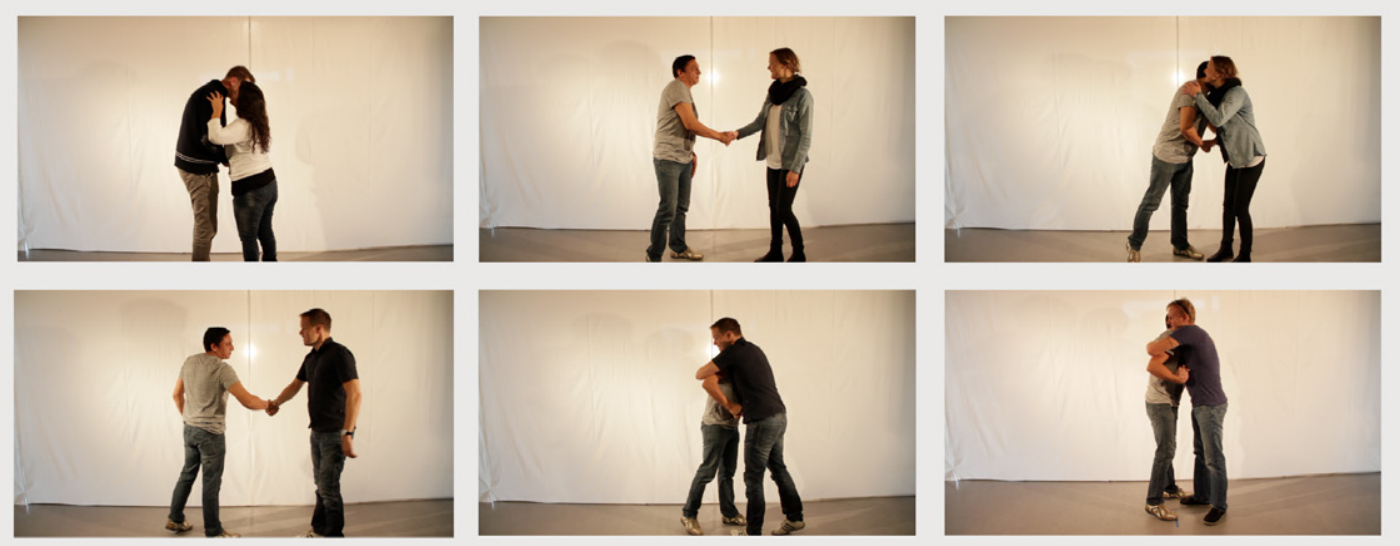

Fig. 253 - Norwegian/Chilean cross-cultural greetings

Strangers: In the greeting between the Norwegian male and the Chilean female, they approached each other with a handshake initiated by the Norwegian male, he seems to think the greeting is finished there and hesitates a slight moment as she touches his shoulder and reaches up to give him a kiss on the cheek, he responds to her kiss on the cheek. The Norwegian male said he gets confused and never knows how many hugs and kisses he should give, so it tends to become awkward. In the Chilean male and Norwegian female greeting, they do a handshake keeping good distance, after the handshake the Norwegian female steps back. The Chilean male and Norwegian male greeting was also a handshake, a firmer handshake, accompanied with a nod of their heads and both leaning in slightly.

Close friends: The greeting between the Chilean male and Norwegian female was a handshake leading into a kiss on the cheek - 
a typical Chilean greeting. The female hesitated before the handshake, unsure whether the greeting was going straight into a hug or not. The Chilean male initiated every move. The Chilean male and Norwegian male greeting was a hand-slap/ handshake leading into a greeting - the Chilean male going to the left for a hug as is usual in Chilean greetings, while the Norwegian male was leaning to the right for a hug, but changed direction when he realised the Chilean male was going left, they hugged while slapping each others' backs. In the greeting between the Chilean male and a Norwegian male who had lived in Chile for 3 years and is married to the Chilean female participant, the greeting went more smoothly, both males knowing to go for the hug/kiss to the left after the handshake.

\section{Norway/ Somalia}

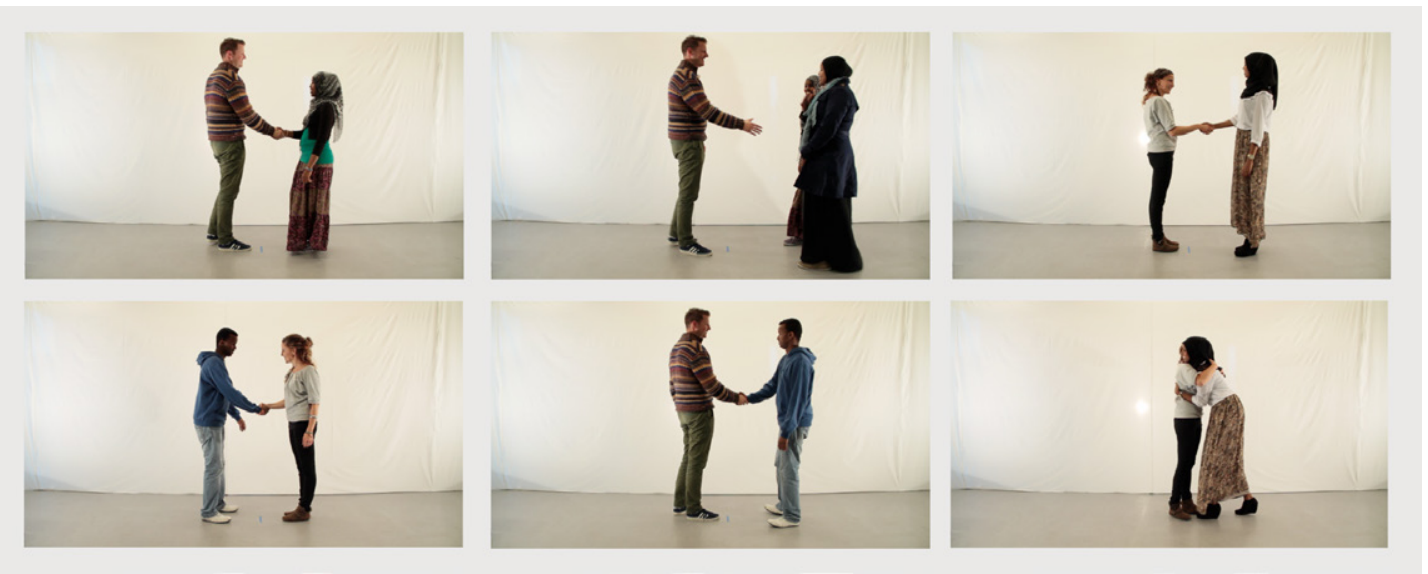

Fig. 254 - Norwegian/Somali cross-cultural greetings

Strangers: The greeting between the Norwegian male and Somali female was a handshake. Somali females sometimes do not want to touch the male, it depends on their personal beliefs and how religious they are - if they follow religion or culture. 
The Norwegian female and Somali female greeting was also a handshake, with the Somali female bowing very slightly while holding her skirt with her left hand. The Somali male and the Norwegian female (me) greeting was a handshake that obviously lasted a lot longer than what the Norwegian female expected and was used to. The Somali females also said that it is changing with the different generations, that they would shake the hand of a Norwegian male but their mothers would not. We recorded such a greeting, where a Norwegian male tried to shake hands with the 'mother' who rejected the handshake responding by putting her right hand on her heart, while the daughter responded by shaking his hand. This had happened to the girl in real life, and she said the Norwegian male was very surprised when she shook his hand after her mother rejecting it. The Norwegian male and Somali male greeting was a handshake that lasted a bit longer than a usual Norwegian handshake.

Close friends: The Norwegian female and Somali female greeting was a hug with smiles and eye contact. One Norwegian male and Somali male street greeting was also recorded but the Norwegian male did not know it very well so there was some confusion and unsynchronised hand-slaps involved. The Somali male uses this greeting with his close friends (Somali and friends from other cultures). The Somali girls said they sometimes hug Norwegian male friends if they have not seen them in a while. 


\section{Norway/Turkey}

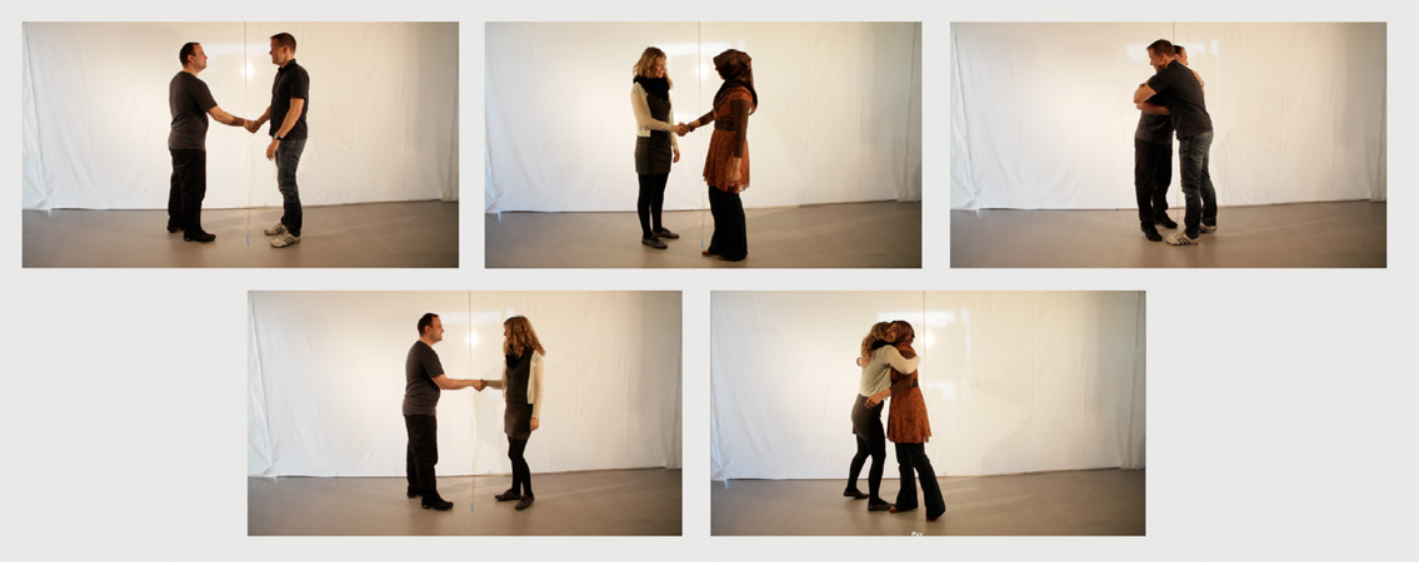

Fig. 255 - Norwegian/Turkish cross-cultural greetings

Strangers: The Norwegian and Turkish female greeting was a handshake, as was the Turkish male and Norwegian female and the Turkish and Norwegian male greetings.

Close friends: The Norwegian female and Turkish female greeting was a hug with smiles and eye contact and patting on the back. The Turkish male and Norwegian male greeting was a hand-slap/ handshake leading in to a hug/pat/ slap on the back.

\section{Norway/Vietnam}
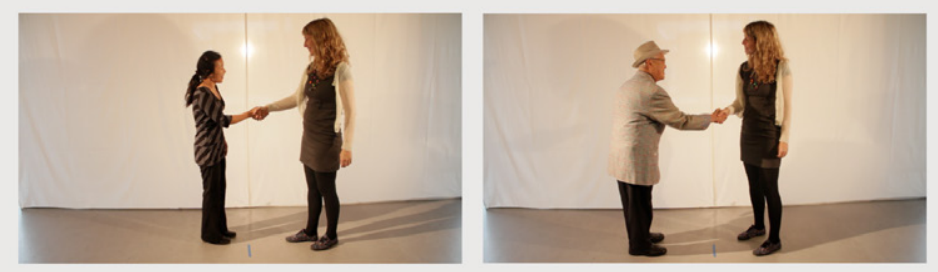

Fig. 256 - Norwegian/Vietnamese cross-cultural greetings

Strangers: The $\mathrm{f} / \mathrm{f}$ Norwegian and Vietnamese greeting was a soft handshake, the Vietnamese female bowing/nodding slightly 
as she greeted. The Vietnamese male and Norwegian female greeting was also a handshake followed by conversation.

Close friends: Not recorded.

\section{Norway/Angola}
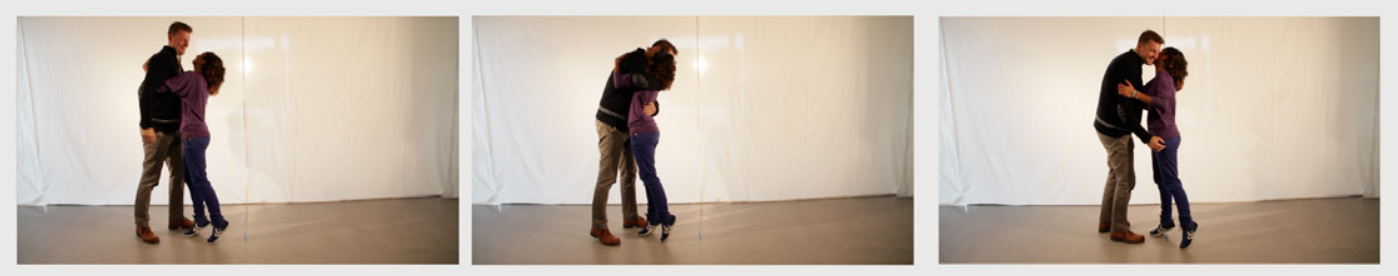

Fig. 257 - Norwegian/Angola cross-cultural greetings

Strangers: The Angolan female misunderstood what she was supposed to show, and showed how she would greet a stranger she is meeting for the first time in Angola - a handshake followed by two kisses on the cheeks and hugs. The Norwegian male was taken by surprise as he was expecting just a handshake (how he usually greets strangers), laughing and blushing he said 'That's exactly how it feels like' explaining that this awkward feeling is what he usually feels if he meets a female from a country where they hug/kiss. He never knows if he is supposed to kiss one, two or three times.

Close friends: This time, the Norwegian male is more prepared, embracing the Angolan female in a hug, thinking they will greet with one hug, then she releases and goes for another kiss/hug on the other side, and again back to the left for a final kiss/hug, the Norwegian male is again taken by surprise trying to follow the greeting awkwardly, they both burst out in laughter at this clumsy greeting. 


\section{Turkey/Angola}

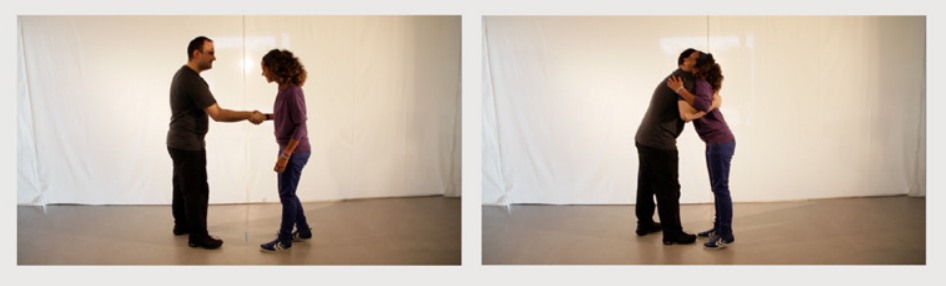

Fig. 258 - Turkish/Angolan cross-cultural greetings

Strangers: The Turkish male and Angolan female greeted with a handshake, the Angolan female nodded her head and leaned in slightly. (The Norwegian male from the Angolan/Norwegian greeting objected, laughing, from the back of the room saying 'Hey hey where is the kiss?', everyone laughed, and the Angolan female explained that in the greeting with him she showed how they do in Angola while for this one she showed how she would usually greet in Stavanger.)

Close friends: The Turkish male and Angolan female approached each other, both reaching both arms out, the Turkish male prepared his right hand for a handshake (then hug), while the Angolan female went straight in for the hug - the Turkish male then adjusted his arm into a hug instead, they hug two times, first left then right, patting/rubbing each others backs.

On the following three pages I have included the excel diagrams I used during the greeting recordings to keep track of what greetings were recorded and notes about particular greetings that had multiple variations, of greetings that were the same as other greetings that were recorded, or of any other relevant information. 


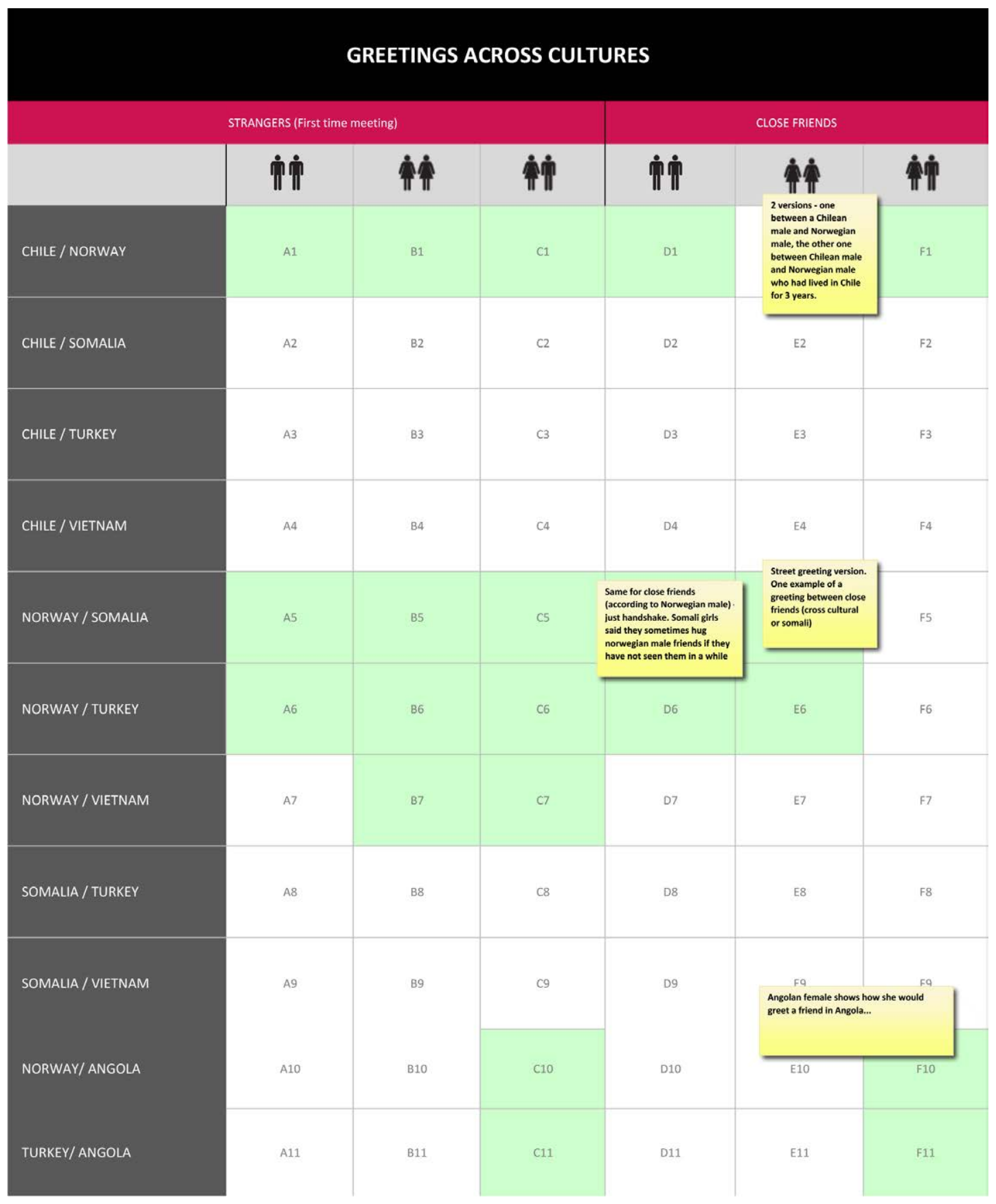




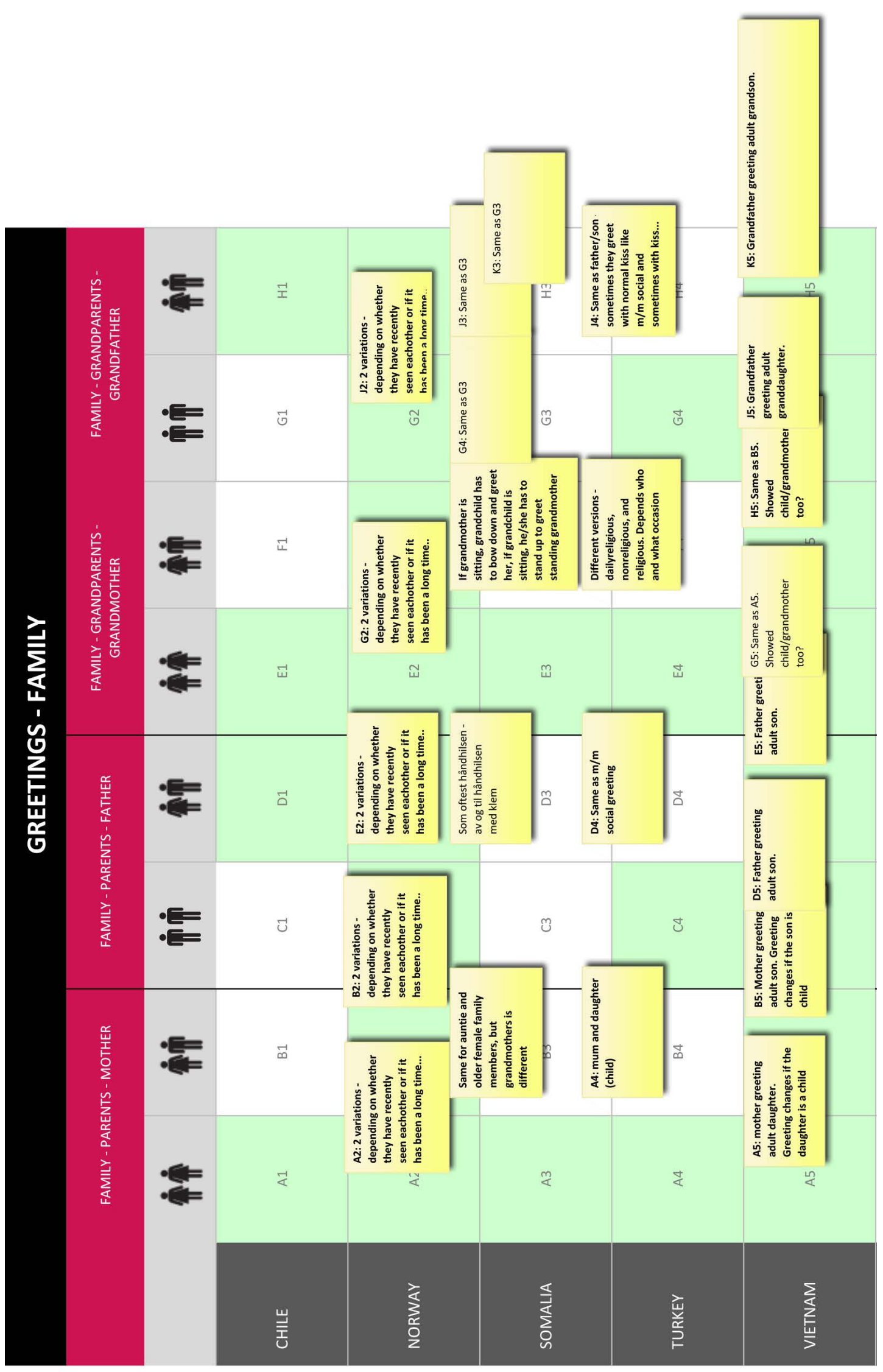




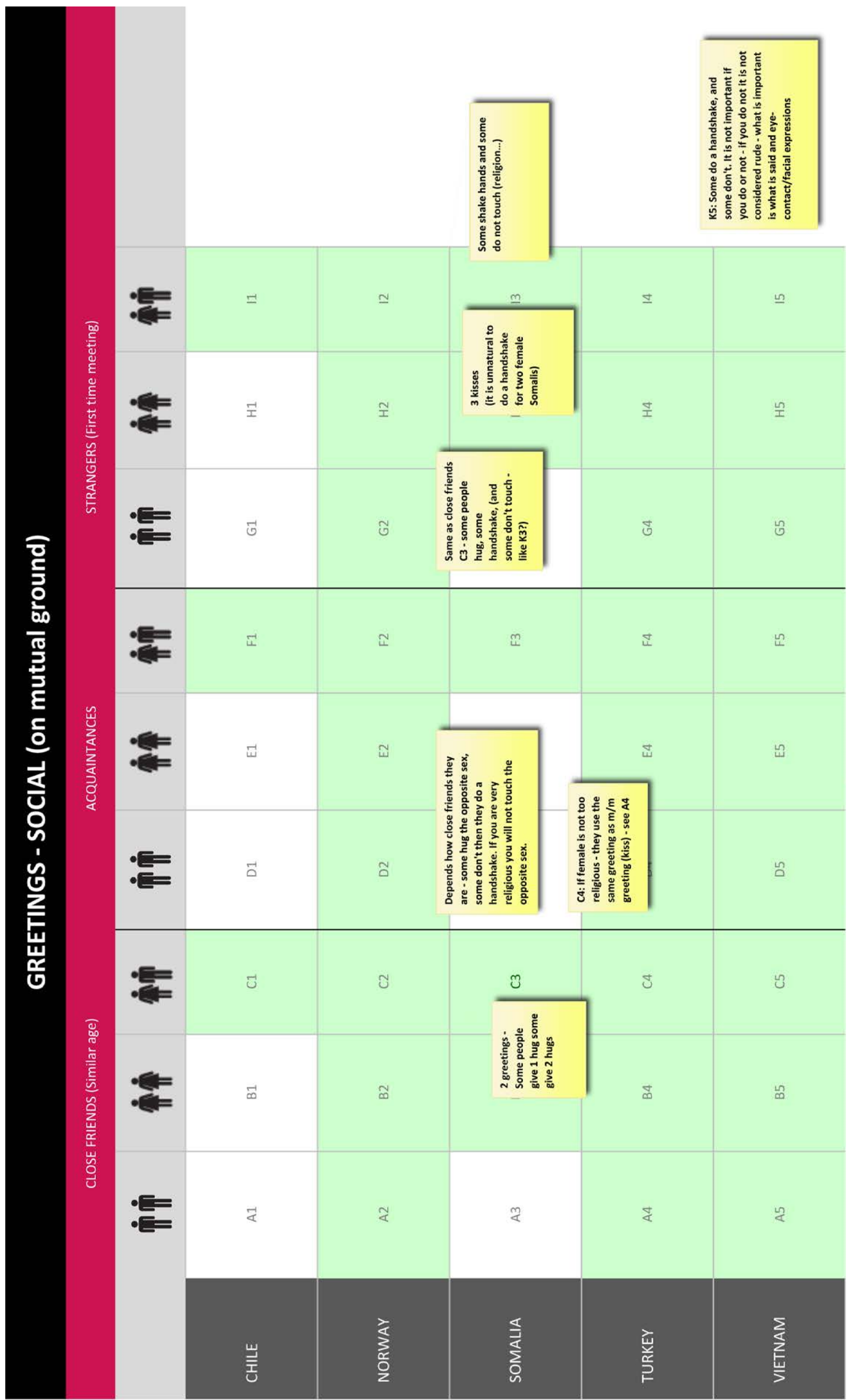




\section{Appendix 10: Interview questionnaire and transcripts}

a: Interview Questionnaire

\section{QUESTIONS FOR INTERVIEWS}

1. Have you ever experienced any awkward, funny or uncomfortable situations when greeting people from your own culture?

2. What in your view caused this uncertainty?

3. Have you ever experienced any awkward, funny or uncomfortable situations when greeting people (in Stavanger) from a different culture?

4. What in your view caused this uncertainty?

5. In Stavanger - how do you feel when greeting someone from a different culture for the first time? Is there a general greeting?

6. Do you greet people differently depending on their cultural background?

7. How do you think your relation to/comfort with touch and personal space varies from that of people from other cultures?

8. Have you ever been or felt judged or stereotyped because of your cultural background and behaviour? (i.e. cold Norwegians etc.) and if so, why do you think that is?

9. What in your view and in your culture is considered a:

- Respectful greeting

- A submissive greeting

- A warm greeting

- A cold greeting

- A masculine greeting

- A feminine greeting

- Powerful greeting (display status)

10. Of all the greetings we filmed, what would you say is your most comfortable greeting? And your most uncomfortable one?

11. Do you feel like greetings are changing or have changed in Stavanger?

12. What to you is a typical Norwegian greeting?

13. What to you is a un-typical Norwegian greeting? 
b: Interview with Norwegian female participant

\section{0th July 2013}

- Interviewer (MT) -

Have you ever experienced any awkward, funny, or uncomfortable situations when greeting people from your own culture?

$-\mathrm{T}-$

Yes! Ok, for example, sometimes people I meet want to hug me and I'm not prepared for it. And other times I think, ok I should be better at hugging people, so I go and hug, and maybe they are not prepared for it, because it can be quite difficult to know how often and when you should hug someone. So then it is either... either you just stand at a distance and say hello, or you go very close to a person and do a hug greeting, it is not often anything in between, unless it is older people that you show a bit of respect by handshaking, for example my uncles or my colleagues or...something like that.

$$
\text { - MT - }
$$

And what, in your view, caused this uncertainty?

$$
-\mathrm{T}-
$$

What caused it? I think it is because there is never a set rule for it. There is no, for me, I don't know how to follow social guidelines for it, and I think people have very different social guidelines. So some people always hug, and some people don't, and some people hug in these situations but not those... And it depends when did you last see the person, and that is very relative, if you feel it is long ago or not long ago. 


\section{$-\mathrm{MT}-$}

Have you ever experienced any awkward, funny or uncomfortable situations when greeting people, we can say in Stavanger, from a different culture?

$-\mathrm{T}-$

Yes, it is generally the same. I know I can come across a bit cold, because maybe people from other cultures have more... what do you call it, that they might not feel awkward of coming up close to you when they are greeting. Whereas Norwegians can maybe say a greeting from a longer distance. So I think that could have been one of the reasons why it has been a bit difficult sometimes.

\section{$-\mathrm{MT}-$}

In Stavanger, how do you feel when you greet someone from a different culture for the first time? Is there a general greeting?

$$
-\mathrm{T}-
$$

I generally put out my hand, and then I'm kind of paying attention to the person to see what direction he or she wants to go, and what they are trying to do.

$$
-\mathrm{MT}-
$$

And do you greet people differently depending on their cultural background?

$$
-\mathrm{T}-
$$

Mmm, yes, I would say yes, because for example, I could meet someone from the UK vs. someone from Ghana I would maybe, subconsciously I would perceive them a bit different, but I still think I try to pay attention to how they are used to be greeted. Then I try to adapt to it. So I don't try to make them do the Norwegian thing, I try to adapt to how they greet. 


\section{$-\mathrm{MT}-$}

How do you think your relation to and comfort with touch or personal space, varies from people from different cultures? Well you already kind of answered that, you said about the space being larger here.

$-\mathrm{T}-$

Yes.

$-\mathrm{MT}-$

Have you ever been or felt judged or stereotyped because of your cultural background and behaviour, like 'cold Norwegians' for example, and if so, why do you think that is?

$-\mathrm{T}-$

Yes because I think that, for example I come from a family where hugs are very awkward, but it doesn't mean that we don't love each other, and we do have a strong connection somehow, but, I feel that it can be judged, and that other people don't understand that I still can have a lot of love for someone or respect for someone without going very close up in their personal space for hugging every time I see them, or these things... mmm...and it is a... if I feel judged, yes I would say so.

$$
-\mathrm{MT}-
$$

So you feel like people associate touch and personal space with being warm?

$$
-\mathrm{T}-
$$

Yes, I would say so.

$$
\text { - MT - }
$$

Ok so that relates to the next question. What in your view and in your culture is considered a respectful greeting? 


\section{$-\mathrm{T}-$}

A respectful greeting is a handshake... yes, a proper handshake, or maybe a handshake with a tap on the shoulder or the elbow.

$-\mathrm{MT}-$

And a powerful greeting, or if you want to show status?

$-\mathrm{T}-$

Then it's a firm handshake, I would say.

- MT -

And submissive, like giving power to the other person? I don't know if you have experienced that at work or anything?

$-\mathrm{T}-$

If I greet my boss for example?

- T's partner $(\mathrm{N})$ -

Then it is a bow.

$-\mathrm{T}-$

Yes but a little kind of nod. It is how you kind of, if you have been chatting to the boss, but you don't quite know him, but you want him to see that you greet him, then you would, like if you are walking past him, then you would try to get eye contact and give a nod. Then it is kind of 'I see you and you see me and I know who you are', kind of submissive greeting I would say.

$-\mathrm{N}-$

You would also open your body to them wouldn't you? If you are talking to your boss, you never turn your side or your back, you would always open your body for respect wouldn't you, to show that you are open to communicate. Like with a friend you could chat away and be like..., but if it is 
kind of formal you open your body maybe. Then you are showing attention to them. To give full attention.

$-\mathrm{MT}-$

And in your culture, what is considered a warm greeting?

$-\mathrm{T}-$

A warm greeting is a hug, a good hug, a strong hug. And sometimes if you can take ... use both your hands, and hold around the elbows or the shoulders... if it is, well for example at a funeral, it is kind of respectful and warm at the same time.

$$
-\mathrm{MT}-
$$

And does it have to be a hug, is that the only warm greeting?

$$
-\mathrm{T}-
$$

Ehm.... no, not really, a warm greeting is also a happy face. So for example, if I come in to my grandmother's house, we don't always hug when we meet each other, or if I come in to the house, I wouldn't maybe go over and give a hug straight away, but she would have a big shining happy face, and maybe clap her hands together, it is a warm kind of welcome. And clap her hands, and I would shake them a bit 'oh it is so lovely to see you', and that is a warm greeting as well. And then maybe we would give a hug as I was leaving the house again. And also... That is one grandmother, but another grandmother I have, she would hit me as a warm greeting, she would box, give me a little box, she would fold her hand and rub a bit on my shoulder and give a little hit or rub a bit on the cheeks and a little kind of punch or a hit. That is kind of her way. But I know it is coming.

$$
-\mathrm{MT}-
$$

Ok, and a cold greeting then? 
A cold greeting is if someone doesn't look happy when they see you.

Sometimes I think it can be mistaken by being tired as well, that you can feel it is cold, but it is just how you interpret body language, if you are not bringing your face up to a natural big smile, and a cold greeting would be on a distance maybe just, inside a house, if you just lift your hand, could be seen as cold in some situations, or if you do not get up off the seat, it can be a bit cold.

$-\mathrm{T}-$

And also, it depends very much on the situation, but sometimes, if it is a person you know, or you have been chatting to a lot, they can come with a big happy smile, and 'hello' and all of that, but if they leave too quick, they don't stop to have a little chat, it can still be seen as cold, even though they are smily and bubbly when they see you, because why didn't they give me time, for example? Yes that is a bit more complex, that is a hidden cold greeting. Yes so that adds to what is a warm greeting - to pay attention, give time, and have ... yes, give a bit of space and be open for whatever interaction is coming. Because that is the whole intention of a greeting.

$-\mathrm{MT}-$

Ok, and a masculine greeting?

$-\mathrm{T}-$

Yes, they would maybe shake each others hands really hard, and they would pull each other towards, almost as if they are giving a hug, but they are not touching, and tap the shoulder and then push, they give a smack on the back or something but the ears never touch! Or the cheeks never touch. Shoulder to shoulder as well yes, a smack on the shoulder. Or a hi-five could be a bit masculine.

- T's partner -

Back in 1987!! 
[laughter]

$-\mathrm{T}-$

A small nod, yes.

$-\mathrm{MT}-$

And a feminine greeting?

$-\mathrm{T}-$

It can be seen as quite feminine to give a really good hug, that is kind of a hearty kind of mother's hug or something. A kiss on the cheek. I am not very used to that in my culture, so when I went abroad and people started kissing me I felt awkward, but then I got used to it. That is quite a feminine greeting!

$-\mathrm{MT}-$

Of what we filmed, what would you say is your most comfortable, and your most uncomfortable greeting?

$-\mathrm{T}-$

Well I think, in general, I would be most comfortable with shaking the hand of a stranger, to be honest, because then I know what to do, I always know what to do when I meet someone I haven't met before because that is just right out of the book, put your hand forward and shake it and say your name and listen to that other person's name. So then it is kind of safe and a given guideline, but even with close friends, sometimes it is a bit unsure what is happening.

$-\mathrm{MT}-$

Do you feel like greetings are changing or have changed in Stavanger? 


\section{$-\mathrm{T}-$}

If they have changed since I was a kid? Coming from a village where you do not give out hugs so much, then it was kind of a new thing for me to start hugging people when i grew up and went out travelling on my own and met new people and new cultures. But in Stavanger, if it has changed, it is difficult to say. But I think, well in for myself, we have a lot of international friends and some people I kiss on the cheek, and some people I hug, and some people I shake their hand, and... so in that sense, I have changed my greetings a lot since I came here. And I suppose if I have, then I'm sure a lot of other Norwegians have changed as well. But I don't know how much would it effect greetings between Norwegians, but with my friends it is mostly handshakes or a hug, so I haven't been adapting the kiss on the cheek or these things, and I have only gotten three kisses on the cheeks from French people, for example. It is funny like that, because it shows that maybe Norwegians maybe don't have a very strong culture for it, because we always adapt to the people that come here, instead of them adapting to our way of greeting. So I think that we are kind of just going along with whatever other people bring.

$$
\text { - MT - }
$$

So what would you say is a typical Norwegian greeting?

$$
-\mathrm{T}-
$$

A typical Norwegian greeting is 'good morning', you would just look at someone and say 'good morning'. Or, 'see ya later'... and the handshake.

$$
-\mathrm{MT}-
$$

And last one, what is an untypical Norwegian greeting?

$$
-\mathrm{T}-
$$

It is very untypical to kiss. I think that is the furthest away from our greeting. 
c: Interview with Norwegian male participant

\section{7th September 2012}

(The recorded interview was conducted in Norwegian. The interview transcript below is translated into English.)

- Interviewer (MT) -

Have you ever experienced any awkward, funny or uncomfortable situations when greeting people from your own culture?

$-\mathrm{R}-$

Yes I have, but it is when you... greet in the wrong way, when you meet people that belong to a different social environment than you do, then it is easy to get burnt because you are not familiar with... how should I put it... how people greet in different sub-cultures. There is a very big difference between where I come from (Trondheim) and here (Stavanger).

- MT -

Ok, what kind of difference?

$-\mathrm{R}-$

Well, like when you say 'Javel' ('Okay') - for me that makes me think 'Oh, now l've done something wrong... while for you it is a natural thing to say when you meet someone 'javel, hva skjer' ('Okay, what's happening?'), while for me this feels like an accusation like: 'Javel hva har du gjort nå?' ('Okay, what have you done now?')

- MT -

Have you ever experienced any awkward, funny or uncomfortable situations when greeting people (in Stavanger) from a different culture? 
Yes, absolutely, when you meet someone who is used to kisses and hugs. I am not used to kisses and hugs, I am not at all used to kisses and hugs. Arms-length always, right, 'Hi, hi', very stiff and cold. So when you then meet... people from a warmer culture, especially if you meet people from South-America and more southern latitudes, where they are used to kisses on the cheeks and hugs and stuff like that, then.... Well for me it feels quite weird, I get very surprised, always surprised... and then you often feel uncertain when you do meet someone, should I hug her, kiss on the cheek? How many kisses on the cheek? Right or left? Where do we start? How many? And so on...

$-\mathrm{MT}-$

How do you feel in those situations?

$-\mathrm{R}-$

Well you just feel stupid. You feel like an idiot. When you are greeting someone, you are trying to give a good first impression, right? Eh....and... it is obvious then when you get all insecure about how to give that person a good impression of yourself, eh... and you kind of stumble through a greeting, then you just look stupid, and you just feel like an idiot.

$-\mathrm{MT}-$

Yes I know that feeling. But this thing about how many kisses to give, this can be tricky, because you just got past this other barrier, and think ok I don't mind to give a...

$-\mathrm{R}-$

Yes! ... I'll give you a hug, but how many times should we kiss?

$-\mathrm{MT}-$

Yes, and sometimes it ends in a nose-crash. I have had a few of those! 
$-\mathrm{R}-$

Yes and and you've seen that it just goes wrong, right? Then you get uncertain if they will go to the left of to the right, and if you go to the same side it can almost seem like you are trying to kiss them on their mouth. You don't want to kiss them on their mouth, that's just wrong, or at least it would be very uncomfortable, especially if the other person is a bit shy... then you'll have entertainment all night.

$-\mathrm{MT}-$

In Stavanger - how do you feel when greeting someone from a different culture for the first time? Is there a general greeting?

$-\mathrm{R}-$

Just 'Hei god dag, hyggelig å hilse på deg' ('Hi, good day, nice to meet you'). Safe, safe, safe, always safe, always very safe! Then you have people that just attack you, people that you almost feel like you have had sex with the person when the greeting is finished, right, because these people do exist. It is almost as if you are standing there and start checking if you still have your wallet, and go through your pockets after to check if you have been robbed, just because the greeting is so intense. So, usually I use safe armslength distance, but Stavanger is kind of a unique city in Norway. It is a very international city, so you are almost guaranteed to meet people from different cultures, unless you lock yourself in at home. So I guess here people are a little bit more used to it. But the first time I came to Stavanger, 10 years ago, then... there were lots of strange things. Now I am starting to get used to it, but still I fall in the trap sometimes when it comes to...

$-\mathrm{MT}-$

What did you do, what happened then? 
$-\mathrm{R}-$

I am from Trondheim, there we hardly say hi to each other, right. It is kind of a 'Hei' ('Hi') and you look down, do not look people in the eyes, because that is almost seen as aggressive, right. So you just walk along and should not say too much. And it is very quiet, it should be soberly and reserved. You should be naturally sceptical to everything that is new. Here in Stavanger is it the complete opposite. You should not be sceptical to new things, rather the opposite. You should just embrace what is new. So for me, when I moved here, it was a big cultural change. I could just as well have moved to a different country. I told people at home that I had moved to 'Syden' when I moved here. And that is not because of the weather. ['Syden' is a word used for holiday places like Spain, Italy, Canary Islands etc.]

$-\mathrm{MT}-$

Oh, I actually would have thought that they were warmer or more approachable in...

$-\mathrm{R}-$

In Trøndelag? No, then you need to go further north. No, we are sceptical, very sceptical, that is one of the differences you notice on the people and their behaviour.

$-\mathrm{R}-$

Like when I moved from Trondheim, there had just been two huge concerts that year. Elton John played on the market place, and so did Bob Dylan. Two artists that usually fill up an arena. In Trondheim, people hardly bothered leaving their houses although Elton John and Bob Dylan played in the market place. In Stavanger, when Elton John played, it was sold out in five minutes.

$-\mathrm{MT}-$

Do you greet people differently depending on their cultural background? 
$-\mathrm{R}-$

Yes, if you meet a woman with a hijab or a head-scarf or something, you instantly become uncertain about how to greet her, I have just learnt that not all women will give you a handshake due to their religion, so now when I meet people who are wearing a Hijab, I naturally start thinking about it, 'If I reach out my hand now, am I impolite or rude?'. So yes, I have started thinking about that lately, but apart from that I usually greet with a handshake, say 'good day', am polite and say my name, blablabla.

$-\mathrm{MT}-$

Yes, and I guess you do not want to treat people differently either, if there are many people in the room...

$-\mathrm{R}-$

Exactly, because for us there is no difference. Like for me, there is no difference if you are a woman or a man, if you are Spanish or French or Turkish or Iranian, a woman is a woman and a man is a man, full stop.

$-\mathrm{MT}-$

In South-America, men greet each other with two kisses and hugs. What do you think about that?

$-\mathrm{R}-$

Yes, that is too much for me. Then I get scared.

$-\mathrm{MT}-$

And how does it make you feel?

$-\mathrm{R}-$

Well you feel like you almost get... almost like you get sexually assaulted. To get kissed by a man... that is really strange for me. Their lips have nothing to do on my body to be honest. 
$-\mathrm{R}-$

Yes, well maybe before I turned 4 I used to hold my dad's hand, but not after that. We just don't do that.

$-\mathrm{MT}-$

How do you think your relation to/comfort with touch and personal space varies from that of people from other cultures?

$-\mathrm{R}-$

I think we have more, in my opinion we have more distance, preferably as long an arm as possible. It is seldom that you pull people in towards you, and when people pull you in towards them it can often be experienced as hey hey hey too close now, back off. The closest we get is to do a handshake and perhaps put the hands on the shoulders or the arms on top of the other person's arms, but to pull in and give a hug, then you should... then it should be someone close, then you should know the other person quite well.

$-\mathrm{R}-$

Or actually, it depends, because if you are out on 'the pull' for example, yes I just thought of that now... then it is different.

$-\mathrm{MT}-$

Yes, but with other men too?

$-\mathrm{R}-$

No, no, not with other men.

$-\mathrm{R}-$

After the first hug, then it becomes easier later on, but the first one, to know when it is supposed to come, that is just impossible to know, unless the other person initiates it. You are scared that people will feel like you are assaulting them, you do not want to be that person that is intrusive either. 
$-\mathrm{MT}-$

Have you ever been or felt judged or stereotyped because of your cultural background and behaviour, i.e. cold Norwegians, and if so, why do you think that is?

$-\mathrm{R}-$

Oh yes indeed. You do not need to go any further than to Sweden to be called a cold Norwegian. I think the only place we do not get called cold Norwegians is in England, there it is worse, the English are even stiffer than us. It is not like they walk around with a 'stick up their bum', but it is a bit like that, right? But yes, I have heard that Norwegians are considered a cold people, but they do say it sounds like we are singing when we talk.

$-\mathrm{MT}-$

Why do you think they say we are cold? Do you think we are cold?

$-\mathrm{R}-$

I think we are... well... we are polite to start with. We are easy to say hi to, but not easy to get to know. To get to know someone from Norway, well that is a challenge, and I actually think that Stavanger is in a league of its own, oddly enough, when you consider that it is such an international city. To get to know someone from Stavanger is difficult, but to get to know someone who is not from Stavanger but living in Stavanger, is very easy. 'Siddiser' and 'Rogalendinger' are really difficult to get to know. I think this is caused by the fact that people from Rogaland are so used to constant exchange of 'the others', people that are not originally from there, they come for a few years, then they leave again, so they just think 'oh well, we won't waste time and energy getting to know them because they are leaving soon anyway', so we are polite and friendly with them as far as our front door, but then the door is shut. I am pretty sure that this is quite an accurate view on 'Rogalendinger'. Do you identify with this? 
$-\mathrm{MT}-$

Well yes, a little bit... I do realise it is very difficult to get to know people here.

$-\mathrm{R}-$

Yes it is, it is shit hard!

$-\mathrm{R}-$

Everyone is just sitting at home complaining that it is difficult to get to know people, that no one wants to go out and socialise, and to organise something becomes quite difficult and heavy.

$-\mathrm{MT}-$

Yes, but then I do not do anything about it myself, I do not call these people, I just get annoyed that it is like this, but I do not do anything about it.

$-\mathrm{R}-$

Yes exactly, but I think this, the fact that we do not call and invite people etc., is because we do not want to be intrusive and annoying. No you do not want to be intrusive. No one wants to be that friendless person that keeps calling and keeps hassling people. No, then it is better to follow them on Facebook in a way. Let's all just sit here and pretend that our lives are so fantastic, right. Because people do that, you never see anyone on Facebook saying 'The fucking kids have spilt coffee all over my new white sofa', it is more like 'ooooooh we have such fantastic lives', so everyone is just living in their own little bubble. That's what I think.

$-\mathrm{MT}-$

I have many friends from Spain and Italy, and they often say things like 'aah you cold Norwegians', but then I talked to someone from Vietnam participating in my project, and I asked them what is considered warm behaviour in their culture, because my Latin friends that say that we are so cold, it is because we do not touch each other much, and we are quiet, so I 
feel like it is always physical. But the Vietnamese said that to them a warm greeting is if you are smiling and have eye-contact, they do not necessarily need to touch each other, because the warmth is more in the smile and eye contact. It is very different in different cultures what is considered warm and cold behaviour, what is respectful and what is not. Do you think that the Norwegians feel that they are cold, it is not just another way of being?

$-\mathrm{R}-$

I think it is connected to the fact that we do not have a lot of facial expression or use much body language. There are many ways to be warm and many ways to be cold, but I can imagine that many people and cultures experience us as cold because we lack this use of facial expressions and bodily gestures. We do not really speak a lot with our bodies, we use words.

$-\mathrm{MT}-$

Yes, and it is quiet and always controlled.

$-\mathrm{R}-$

It is not always controlled. Talk about politics with a Norwegian, then it is up to a hundred and back down to zero five seconds later. Look at French farmers, for example, when they are unhappy with something, they block the street, dump tomatoes on the highway, there is an anger there that you do not find anywhere else, especially not in Norway, when we are unhappy with something we just say 'ah, someone should really do something about this', then we are moody inside, irritated, say awful things, and that is where it stops. You will never see the same kind of rebellion and uprisings that took place in Libya and Egypt, in Norway, never, at least not by Norwegians, we just do not do that, it is not how we were raised.

$-\mathrm{MT}-$

What in your view and in your culture is considered a respectful greeting? 
$-\mathrm{R}-$

A respectful greeting, to be polite: eye contact, firm handshake, short and precise.

$-\mathrm{MT}-$

If you want to show power in a greeting, how would you do that?

$-\mathrm{R}-$

Then it is a bit friendlier. Still a firm handshake, you keep this friendly tone, like at work I have a contract position above a number of workers, and when I greet them I often lift myself up by talking to them as if they were a couple of years younger than me. Make them feel like they are a bit younger than me, smaller than me. So I talk to them in a very friendly way 'Hey, how are you doing?', firm handshake... you become kind of the 'jock' on the handball or football team. You become a bit of a cool guy, add some cheeky comment to the greeting, kind of rude but not.

$-\mathrm{MT}-$

Back to respect, what is a respectful greeting?

$-\mathrm{R}-$

Well, it depends on how much older the person is, if the person is in their 70-80s, or older, then you are very pronounced when you speak. This is the important thing that the communication is clear, that you are strong and clear when you speak, and spend time to be understood.

$-\mathrm{MT}-$

If it is a person above you, authority so to speak, how does that influence the greeting? 
$-\mathrm{R}-$

I am not very good at taking that, I prefer and am used to being at the other end. When I think back to when the company's CEO was here, well then I behaved in a very business-like, professional manner, you should not make any mistakes, be correct and educated. You should show that 'here is a real worker, this guy really does his job'.

$-\mathrm{MT}-$

So you adjust your behaviour?

$-\mathrm{R}-$

Oh yes! But, in Norway, unlike most other countries, the respect for authority is quite broken. If you go to Sweden, for instance, there is a much clearer hierarchy, in Norway the hierarchy is not very clear. Everyone here is kind of on this 'greet the prime minister' in a way, everyone is on a greeting-basis with the boss, and in Norway you do not work for the boss, but the boss works for you, what can the boss do for me... You do not get that many other places. We have a lot of foreigners working at my company, we have a lot from Poland and the Baltic states working on the construction site, and none of them would be rude when talking to the boss. They are polite, always say 'good day' and greet very politely every morning. While the Norwegian guys, similar age, have more of an attitude and do not really give a shit. So I do not think that we are very good at this submissive tone to be honest.

$-\mathrm{MT}-$

Well, you can even see that in schools and when you are studying. You have no respect for teachers, there is no 'Hi Miss' or anything like that.

$-\mathrm{R}-$

It is easier to tell the teacher to go to hell than or to shut up than to give them respect. Things have no consequence in this country, you can do whatever you want, and it has no consequence. We do not have a lot of discipline, we 
are great workers, we are very productive, but we lack discipline, it is not like in England, not at all.

$-\mathrm{R}-$

It is noticeable. We are not very good at holding the doors open for women for instance.

$-\mathrm{MT}-$

What in your view and in your culture is considered a warm greeting?

$-\mathrm{R}-$

To me - like we greeted today, that is a warm greeting. It is friendly, you feel welcome.

$-\mathrm{MT}-$

Does it have to involve a lot of body contact?

$-\mathrm{R}-$

No, but you feel welcome, it is not just a 'hi, nice of you to come', but it is a 'heeeeey, so nice of you to come, great, it is lovely to have you here!'. You get the impression that your being there contributes and is positive for everyone.

$-\mathrm{MT}-$

And what about a cold greeting?

$-\mathrm{R}-$

Well, that is the opposite, when you feel like 'do I really need to be here'.

- MT -

A masculine greeting? 
$-\mathrm{R}-$

A little bit rough. Kind of when the boys are out on town 'eeeeeeeyyy' shouting half way down the street from each other, and the greeting starts when they are far away from each other, then you meet 'eeeey', and the greeting is kind of messy, hard.

$-\mathrm{MT}-$

And a feminine greeting?

$-\mathrm{R}-$

It could be the same, but with a higher pitch, kind of teenager style screaming, and lots of hugging and 'oh my God it is soo good to see you again! Lalala...' 'God, your hair looks amazing, have you been to the hairdressers?' you know very feminine, we would never greet like that.

$-\mathrm{MT}-$

Does the feminine greeting have more body contact and compliments than the masculine greeting?

$-\mathrm{R}-$

Yes, and very much 'now I have to make you feel good, and after that you have to make me feel good', while us boys, we are more like 'hey you asshole, what are you up to?' and 'Shit you look ugly today, did you dress yourself?'.

$-\mathrm{MT}-$

But they way you say it, you know that it is just messing about, right?

$-\mathrm{R}-$

Yes yes yes, we are just joking, pulling each other's leg. 
$-\mathrm{MT}-$

Of all the greetings we filmed, which one were you most comfortable with?

$-\mathrm{R}-$

That would be the normal 'hi', handshake and 'nice to meet you'. But the one that really made an impression on me, resulting in me talking about it for days afterwards, that was the Angolan greeting with your sister in-law. That was like 'wooooowww, hehehe', I felt like I had made her unfaithful. It was just like 'heeeey heeeeey hey hey heeeey!'

$-\mathrm{MT}-$

But when I looked at the footage, I realised that she thought she was supposed to show how she greets strangers in Angola, so the whole thing became really funny.

$-\mathrm{R}-$

Yes, I was just really baffled, it worked so well when you think of it as a cultural clash! I was just like 'wooow woo000000w wooow wow'...

$-\mathrm{MT}-$

I think I have to look at it again in slow motion.

$-\mathrm{R}-$

Yes, she was everywhere, she was just everywhere!

$-\mathrm{MT}-$

But to me it does not look as bad as you probably felt it...

$-\mathrm{R}-$

No, but I just felt like a complete idiot. 
$-\mathrm{MT}-$

Yes, I know that feeling.

$-\mathrm{R}-$

Yes, you just feel like a total idiot, thinking 'Oh no, what just happened? Oh no, I just did a Jens Stoltenberg and Trond Giske kind of hug'.

$-\mathrm{R}-$

It is like...ah it is just, it makes you cringe thinking about it, it was just so strange, but it was funny at the same time, in this context it was funny, because that was kind of the point, to get this kind of crash.

$-\mathrm{MT}-$

But then after you did the same to me, because I was not expecting it.

$-\mathrm{MT}-$

Do you feel like greetings are changing or have changed in Stavanger?

$-\mathrm{R}-$

Well, I think that teenagers now are much more huggy and cosy with each other than we were when we were teenagers. But I think that is typical, we always move away from what the generation before us were doing. Now, it is much more physical, or my impression when I see teenagers now is that it is much more physical, how should I put it, much more continental, it could have to do with the society being much more integrated, that there is much more international influences around. But it could also be a sort of a 'rebel'culture towards what was before, like, there is a reason why people during the 60-70s stopped wearing hats, our parent's generation stopped wearing hats, while now we are wearing hats again.

$-\mathrm{MT}-$

Do you think it has anything to do with Stavanger being so international? 
$-\mathrm{R}-$

Yes I think so, but I also think we are living in a more and more globalised world. We are much more international now, the distance from Norway to the US is very short, just a mili-second on the Internet and you are there...TV, and everything. You get influences from all directions. So, I think the world is starting to become a sort of a McDonald's culture.

$-\mathrm{MT}-$

What to you is a typical Norwegian greeting?

$-\mathrm{R}-$

A typical Norwegian greeting... In Stavanger: 'Javel' ('Okay'). Not really a physical greeting, it is a bit more formal to shake people's hand. When you meet your male friends you do not shake their hand. Either you say 'hi how are you doing', and you might give them a hug, but you do not just throw yourself around their necks and say 'heeeeeeey...' maybe you did when you were $20 \ldots$

$-\mathrm{MT}-$

But I do notice a difference here and Ålgård, because I hug a lot, which is not something I grew up with in Ålgård, so when I go back there and hug my friends, they go 'Oh yes, you always hug us'... because I have travelled a lot and lived away, I notice a change in myself, which I only really notice in these situations because they do not greet like I do. In Stavanger, however, it is quite normal.

$-\mathrm{R}-$

Yes, but Ålgård, although it is very close to Stavanger, it is still very much on the countryside really.

- MT -

What to you is an untypical Norwegian greeting? 
$-\mathrm{R}-$

Untypical Norwegian? Like they do in Angola! That is very un-Norwegian.

'Rape me' in a way.... To put it like this - if your have no friends and are missing physical contact - then go to Angola for God's sake. At least you'll get physical contact with people. 


\section{d: Interview with Turkish female participant}

\section{1th July 2013}

(The recorded interview was conducted in Norwegian. The interview transcript below is translated into English.)

$-\mathrm{F}-$

Older people you kiss their hand, like when it is Eid or other celebrations, then that is a very common greeting, but also out of tradition and respect you kiss the hand of the older person. But you do not kiss like a normal kiss, you kiss their hand and then you put it on your forehead. It depends where in Turkey you are from and how religious it is there, but if it is religious you do not have intimate eye contact with the opposite sex. The number three is a very symbolic number in Islam...

- Interviewer (MT) -

Oh, is that why you do three kisses?

\section{$-\mathrm{F}-$}

Yes, because in Turkey it is the people that are more religious, or the ones that are in.... what should I say... groups... so not everyone is aware that we do three kisses, so often it gets a bit....you end up standing there on the third one. I think that is very embarrassing.... because we also hug people we meet for the first or second times, and when you are greeting, it is like....oh, ok... and you feel like you are being intrusive and the other person feels that too, because not everyone are aware of it, not everyone practices it, but us religious in Stavanger that are still somewhat active in the Mosque and keep traditions, do practice it. So the greeting you showed me, was a greeting between two religious female friends, so they kissed three times. What is normal really depends, you know. One kiss is definitely not normal, like the Norwegians do, that is very typical Norwegian. We use the little bit superficial 
one if you know the person, mwah mwah, you kiss the person twice, and if you know the person well you kiss twice followed by two hugs, and then you have the one with three kisses, which is used by more religious Turkish. It became a kind of trend a few years ago to give three... it kind of came from religious groups/organisations. But it is not from the Turkish culture, a lot of Turkish people that do not live here ask 'why do you give three kisses?', so it is kind of symbolic. I do not really know the history of it, but it could be something the Muslims here made up to stand out. What I think is quite special when you compare the Norwegian culture and the Turkish one, and my husband and most Turkish who come here and are not familiar with the Norwegian culture think the same, is that in Norway it is usual for the opposite sexes to give each other a hug. Like 'heeeey Ola' and 'heeey Marit'.... They react to that, why should he kiss the wife or girlfriend of his friend? It is like 'what??'... I have noticed that here in Norway that hugging is more a girly thing. Men hug women, but you do not often see men hugging other men, but they do hug the opposite sex. The Turkish think that is just.... is it possible? They ask 'but why don't you hug your mates?', that is just totally different!

\section{$-\mathrm{MT}-$}

Have you ever experienced uncomfortable or strange situations when you have greeted people from your own culture? I do not know if you consider 'your' culture the Turkish or Norwegian one?

$$
-\mathrm{F}-
$$

It is Turkish, yes. Emm...uncomfortable...well how uncomfortable can a greeting be? But well, when people know.... Like that situation that happened at the library. I experienced that as uncomfortable. It was embarrassing on his behalf, he said people from MY culture do not practice that, but like, there are a lot of cultural differences within cultures too... You can not compare a 'Trønder' with a 'Nordlenning', so I find that uncomfortable, when people from my own culture... Like, I do not find it wrong at all if a Norwegian man sticks 
out his hand to greet me, but if they are muslim, I think... 'can you not see that I am wearing a scarf'... well think a little... Then I just say 'hi', I think he is the one who should be embarrassed. I find it a bit uncomfortable, or what do I have to loose here?, but I do think that he should think it through because he should know this. I feel like he is the one creating this situation, you know the awkwardness that leads to his hand just hanging there in the air. But I do not like to hurt people, so I think oh well... I have experienced this many times. Once at a restaurant here at Rica Forum Hotel, the chef was trying it on. I thought it was so strange, because there were so many lightly dressed girls there, a lot, and he sent the waitress over to me and she said 'could you come over here for a bit, the chef wants to talk to you', so I thought he wanted to apologise because my food, my fish, was delayed, but then he just said 'hi' and stuck out his hand, so I said 'hi', then he tried to greet me with a handshake, and I just said 'I am muslim, so are you, I will not give you my hand', and then he just says 'oh yes', then I said 'can you not see my ring' and 'I have two kids'... and just...yeah... It is uncomfortable when people who should be aware of it do not behave.

There is a big difference between strong practicing and not as strong practicing ones. I have experienced this with my husband's mates in Turkey, they are from different cities, some he has studied with, and some he has lived with, and we went to meet them. They had completely different expectations and certain preconceptions about me, thinking she is born and grew up in Norway, she must be quite modern... Then I arrive, completely covered up. I always say that whoever I am meeting, if it is the most holy person on earth, I will never give away my identity, if I am to be liked it is because I am myself. They looked like question marks, they were so fascinated, 'you are so religious but at the same time you talk about taboos like sex, lesbians, homosexuality....'. Then the next summer holiday when we went back to visit them, one of the guys stuck out his hand to greet me, so I thought - now I have to teach them... because we were really good friends, so I said 'Do you know what Ismail, you know me now, so please do not get 
offended if I do not reach out my arm', then we had a laugh about it, and he turned red, and I said that was a lesson learnt for him. But I am the same way here in Norway too, ok, if it is a person I meet for the second or third time and I have had the possibility to tell them about myself and my religion. You shouldn't have body contact with the opposite sex.

$$
-\mathrm{MT}-
$$

But should this work both ways?

$$
-\mathrm{F}-
$$

Well we do greet the other parents and stuff like that, within the Norwegian society. But we have had many discussions.... 'Why did you reach out your hand to say welcome?' - my opinion is that this is not part of our culture, it came from Europe in more recent times. I tell my husband that if I have said welcome and hello to the men only using head nods and body language , then he can do the same with the women. Then my husband says: 'Ok, how do we do that again?' - well it really depends what group and who are visiting, but you can stand at a distance, and if it is my friends, you can stand at a distance and say 'welcome' without touching them...

$$
-\mathrm{MT}-
$$

But what if they arrive and say 'hiii'?

$$
-\mathrm{F}-
$$

Yes I think it has to do with personality, when they come like that he says 'I could not just let them stand there with the hand in the air'. It is funny, we hug and kiss strangers too, so we are very intimate with the same sex, but then we have this distance with the opposite sex.

$$
-\mathrm{MT}-
$$

So, the same question, but have you experienced uncomfortable or strange situations when you have greeted someone from a different culture? 


\section{$-\mathrm{F}-$}

Well, some Norwegian teachers that I had a really good relationship with, well, it is not a greeting, more a farewell when I quit my job. He knew, because I always spoke to him joking 'don't touch me', we had a great dialogue. So one of them called Oddgeir, on my last day, because he knew I was getting married, and he just wanted to hug me, so he came over, and I didn't have to take exams so I was just completely happy... so he said 'then I will take advantage of this situation that you do not have to sit exams, it is ok for me to hug you, right?' and I just laughed and said 'don't touch me'. But it does make me very uncomfortable, if I have been hugged by the opposite sex, my thoughts start racing, what could I have done to avoid this situation?

$$
-\mathrm{MT}-
$$

Do you greet people from different cultures differently?

$$
-\mathrm{F}-
$$

Yes, like the pressure during a handshake. If I greet a Norwegian, nonmuslim, I make sure to be very 'click' to show my personality. It might be more of a conscious thing, I am muslim, I am a woman, I wear a head scarf, but I also have power. It is a bit of...my personality, because I am like that, with a straight back and yes... If there is something I can't stand, it is those...suppressed. I am SO for showing that this is our choice, that we are independent, have power, and so on. It is unconscious, I do not think about it every time I greet, it has just become a part of me. But I definitely do not do that with my few Turkish or muslim friends I greet...then they would definitely misunderstand my intentions.

$$
-\mathrm{MT}-
$$

What is your relation to and tolerance to personal space, and how do you think that differs from other cultures? 


\section{$-\mathrm{F}-$}

I think I am quite concerned with it, with my zone...

I do not like it if people are too intrusive, that creates misunderstanding and sometimes suspicion, so personally I do not like it. But, I'm just thinking, in my culture, all the hugging, but it is not the kind of intimate hugging, no you keep a distance, some call it the 'fake mwah mwah', but for us this is a way to maintain a distance, we do not do that kind of 'mmmm'.

\section{$-\mathrm{MT}-$}

Have you ever been judged or stereotyped because of your cultural background?

\section{$-\mathrm{F}-$}

Judged - oh yes, all the time! I am just thinking where to start...

Well now, it is not because I am a foreigner, but because I am Muslim, and I show it with my whole appearance that I am a Muslim, because I use a headscarf. But we are more used to being judged, or of people being sceptical because people have preconceptions, so I react more if I am treated normally. Especially when we didn't have the car we used to get the bus, and some days I would ask my husband 'why are people not turning around to look at me?' because I was so used to that happening, and he was like 'why do you ask' and I would say 'do I look too normal today, or what is wrong?' and he just thought that I was so used to people turning to look at me, you know that extra look, so when people don't do it, it is like 'what? am I missing something today?' - but yes like I said I am so used to it now that I react more if people don't do it.

I get so happy when people speak in real Stavanger dialect to me, and when they don't try to speak extra clear and pronounced to me. I say, try Norwegian first, then English, it really makes me angry when they 
don't because I am not even comfortable speaking English! Or if people start speaking to me in a very clear and over-pronounced way 'Do you understand?' and I'm just like 'Yes I know this' - that particular setting was one in which I got a lot of attention of people around me anyway, so then I just had to speak extra loud with a strong Stavanger accent to show that yes I do speak Norwegian and I have voted before, ok.

$-\mathrm{MT}-$

Does it feel heavy?

$-\mathrm{F}-$

Not really, but I do notice that the older I get, and if I have the kids with me, like my daughter, she has completely different... to her I am the answer to everything, I am the strong mother who is a representative in her school, a leader for something else...so she looks up to me. Maybe that is why I got so offended because she is not used to seeing me helpless. Like today at work, there was me and another assistant at work, then a mother arrives and asks for some forms and general things, then I said 'I can help you with that', then I see that she approaches the Norwegian assistant instead, because she thinks she knows more about it, but I am the boss there... I often experienced that when I was doing my work experience too. But when it is just against me, when I am alone, I deal with it alright, but if I am in a group where I want status, then it is different. I guess us, the second generation have a bit more pressure, and the third generation maybe even more, that I feel like I have to be a sort of octopus that is everywhere, we have to show that we are useful and that we can actually contribute with different things.

$$
-\mathrm{MT}-
$$

What is a respectful greeting? 


\section{$-F-$}

It depends. If it is in my culture then we have to maintain those limits and rules...yes... I think a greeting is really important. It has to do with the message one receives, kind of telepathy, but you do not have to take my hand, but show it with your body, the body language, the eyes, all of it....you say it with your body.

$-\mathrm{MT}-$

Ok, what about a greeting that shows power or status?

$-\mathrm{F}-$

That was that pressure I talked about... And body language, keep a straight spine, to show that you have confidence and believe in yourself.

$-\mathrm{MT}-$

A submissive greeting?

$$
-F-
$$

With older people... when you kiss them you bow for the person. We have a lot of respect for the elderly. Bodylanguage and how you carry yourself is very important.

$$
-\mathrm{MT}-
$$

And a warm and a cold greeting?

$$
-F-
$$

In a warm greeting you are more physical I think, and you use more body language. In a cold greeting you use less facial expressions. Like when you go for an interview, then you are more neutral. 
$-\mathrm{MT}-$

And we already talked a bit about this, masculine and feminine greetings? Like in Norway it is not considered very masculine for two men to hug each other.

$-\mathrm{F}-$

I find it just lovely to watch my husband and his brothers hug each other, it is just lovely, it is beautiful in a masculine sort of way. It touches me to watch them, but that is because we see so little of that here, so it really fascinates me. But you see the body language too, it is a bit more masculine... They also do this head to head bumping... it is very masculine. when hugging, men are a bit more, while us women are more like this...

$$
-\mathrm{MT}-
$$

Of all the greetings we filmed, which ones are you most and least comfortable with?

$$
-\mathrm{F}-
$$

I am most comfortable with greeting people close to me, because then I know, I can't really make any mistakes. You do not think it over, it is spontaneous and just happens. Like with my daughter or my best friend, right...But when greeting a stranger, that is more... even if you do not plan how to greet, it is more, you think it through more, you are not as free in your movements, and what you do is important. And for me it is with the opposite sex that becomes more, well how should I greet.... I can think about how religious is he, out of nowhere you could be approached with a handshake. Then I have to think, should I refuse the hand shake?

A lot of people ask me 'what do you do if the doorbell rings?'. Then I tell them that I always have a head scarf available close by. The thing is, I am not only Feiza, I am judged as a Muslim, and as a Turk, so I always represent more people, so I have to always consider things twice. It is a kind of pressure. It 
is not always easy being the minority... I do notice that, it does take a lot, but it depends how you deal with it. I am really against this attitude that people abuse 'he is a rasist' - like a friend of mine, she failed her work practice, and then she said the teacher was a rasist. That excuse is too easy... And it is lack of knowledge. You have to be judged the same way as others, so in my opinion, if you are not any good at Norwegian and you want to become a teacher, then it is no wonder you fail...

$-\mathrm{MT}-$

Do you feel that greetings in Stavanger are changing or have changed in the recent years?

$$
-\mathrm{F}-
$$

Yes, a lot! Compared to my childhood and now, now people are used to hug when greeting. Before... I remember when I was in secondary school, it was only us foreign girls that hugged each other, and then it became popular... you know that fake 'mwah mwah' greeting. But before that in secondary school, the others thought it was strange, a bit disgusting to hug, they were like 'why should you?'... but then it became a fashionable thing to do the mwah mwah. Now I feel like it has become more normal with body contact and more close contact actually in Stavanger. They say that, or at least I think so, that is has to do with immigration. Integration from the Norwegian people, that they have taken the positive sides from different cultures. I absolutely think so. Today a lot of young people hug, almost a bit too intimate, it wasn't like that in our time, and it was us who brought it here. I have seen a really clear change, even amongst colleagues and adults, it wasn't like that before.

[Feiza's husband comes home. Before he enters the living room we discuss, laughing, how I should greet him...]

$$
-\mathrm{MT}-
$$

What is a typical Norwegian greeting? 


$$
-\mathrm{F}-
$$

I think it really depends. But what you really notice if you are looking at a distance, then, like I mentioned, men hug the other man's woman but not the man. That is typical Norwegian.

Alright you two, let's see how you greet now!

$$
\text { - F's husband - }
$$

Welcome!

$$
-\mathrm{F}-
$$

Oh I have taught you well!

I have just been telling MT that we do not greet the other sex with a handshake...

$$
-\mathrm{MT}-
$$

I was a bit unsure how to greet you.

$$
\text { - F's husband - }
$$

Yes, well if people know about it it is fine, but if not then of course we respond!

$$
-\mathrm{F}-
$$

[Jokinly:]

I told her that if you do that, I will tear up all the papers!

$$
-\mathrm{MT}-
$$

I said, if I do this... will you throw me out of your house then?

$$
\text { - F's husband - }
$$

oh yes? Is that what you were talking about? Ok, I am S....! 
- MT -

I am Marie Therese

$-\mathrm{MT}-$

Yes and the last question is, what is an untypical Norwegian greeting?

$-\mathrm{F}-$

Untypical Norwegian? It is that one, well if I compare to our culture, it is the 'mwah mwah' greeting. Close contact with strangers, body contact with strangers, that is untypical.

\section{- F's husband -}

In Turkey we hug more the same sex, in big cities they might hug the opposite sex, and maybe at University. When I saw it here, I asked if they share the girl.

$$
-\mathrm{F}-
$$

Yes and it is very untypical for men to hug each other.

[F asks her husband:]

Have you hugged a Norwegian man? Like when you worked with your friends at the school? When you greeted each other, it was more just a 'hi', not like you would hug each other in Turkey?

\section{- F's husband -}

No not the complete Norwegians. But one Norwegian, he is a Muslim, he lived in Egypt, I hugged him some times, because he had lived in Egypt he was used to it. 


\section{$-\mathrm{F}-$}

Yes, he was used to it, but didn't the other guys react when they saw you do that?

- F's husband -

No because he did the same to our Norwegian friends. He hugged. He is Norwegian but he has converted and has lived in Egypt.

$$
-\mathrm{F}-
$$

I often use the word 'cold', but that is.... it is very reserved. They hide their feelings. It is quite reserved here. So then I think that they are cold. It has to do with openness. Just the fact that you hugged me, it made me so happy. You get a different relationship with the person when you hug at the entrance.

I have always struggled with eye contact, I do not have that intimate thing. I forgot to say about one of our greetings, it is a very religious greeting between muslims, then you take each other's hand, and look straight into each others eyes. I struggle with that one, especially if there are holy participants that are celebrating, then they walk around and say 'look into my eyes Feiza' and I'm just like 'no, you know I can't manage it'. It is very important, and you say a short verse, I just really struggle with it, and the more intimate it is, the more I struggle with it. I have always struggled with it. 
e: Interview with Turkish male participant

\section{7th July 2013}

- Interviewer (MT) -

Have you ever experienced any awkward or funny or uncomfortable situations when you greet people from your own culture?

\section{$-\mathrm{T}-$}

Like sometimes, it is, you don't know what to do... some people are very open, and because in Turkey we kiss men as well not like a juicy kiss, but you kind of touch the cheeks on both sides, and sometimes it is awkward because some people just want to shake hands and then some people will just bend already, and you don't want to bend, you bend, he bends, you don't bend, you bend he doesn't bend, and stuff like that. And especially after living so long here, now when I go and meet my friends, and they kind of bend over to kiss me and I'm like 'What the fuck are you doing, just hold it ok?' because I am not used to it anymore.

$$
-\mathrm{MT}-
$$

So, the same question, only when you greet people from a different culture in Stavanger?

\section{$-\mathrm{T}-$}

Well yes in Stavanger, here you pretty much just shake hands basically. And sometimes I don't know what to do with women, because usually what I do is I just kiss, but maybe some women are not very comfortable with it,

$-\mathrm{MT}-$

But like two kisses or? 


\section{$-\mathrm{T}-$}

Yes... But I know that for example Dutch people they kiss three times, some French people they kiss three times... So with my friends I know what to do, but if it is somebody that I don't know, then sometimes I just take the most safe way and just shake hands if it is somebody that I don't know, and if it is a friend of my friend that I know very well, I just kiss. And sometimes of course you go into this... if it is men here, it is just shake hands, right? And then sometimes you go into this cycle with women here that like bend? kiss? no kiss? you kiss? she doesn't want to maybe? You know...

\section{$-\mathrm{MT}-$}

So, in Stavanger, how do you feel when you greet someone from a different culture for the first time? Is there a general kind of greeting? I guess the safe one is the handshake?

\section{$-\mathrm{T}-$}

Not that I am racist or something, but we all have our kind of ideas about different cultures right, and then if I think that this person might be offended or may not want to do certain things, for me I just shake hands, really simple. Because it is not about what you want to do, it is about what the other person wants to do as well, and then the most common thing is to shake hands.

$$
-\mathrm{MT}-
$$

Are there some cultures you know better so you know how to greet the first time?

$$
-\mathrm{T}-
$$

Well if that hand is pulling your hand towards himself and wants to kiss you then... 


\section{$-\mathrm{MT}-$}

Do you greet people differently depending on what culture they are from/ their cultural background?

\section{$-\mathrm{T}-$}

All the same, but like, say we went to a gathering, and you know how it is here, right you can have a German, Italian, French, Turkish, Portuguese, Angola, you name it... right, so, just like a basic shake hands with people that I do not know, and then if they are my friends, then of course more ... more warm and welcoming.

$$
-\mathrm{MT}-
$$

How do you think your relation to or your comfort with touch and personal space, how close you get, is that different from people from other cultures?

$$
-\mathrm{T}-
$$

Yes I think I'm more that 'warm country profile', I would fit into it. Like I don't mind being close, if someone gives a hug I'm like 'ohh', I'm not like 'oh what the hell is happening?', I'm like 'oh yes we are hugging, that's ok'.

$$
-\mathrm{MT}-
$$

Have you ever felt or been judged or stereotyped because of your cultural background or behaviour? Like for example hear 'ah you're cold'...

$$
-\mathrm{T}-
$$

I'm not so much exposed to that stereotype because of the circles that I am in. Because you know like here it is oil business and it is like, well, the people that we see, the people that we work with, the people that we hang around with, are usually open people because he has lived in Angola, he has lived in Siberia, he has lived in Spain... you know. They are very open, so I haven't been exposed to it that much. But of course when we gather around and 
there are some people, it is not necessarily Norwegians, but people coming from North Germany, and you know...

$-\mathrm{MT}-$

Have you ever been stereotyped because of your culture?

$-\mathrm{T}-$

No, not really. Not at all actually. I have never had a negative thing, that people would give me 'the look' because I am Turkish, I never had that. The only thing that I had was 'Ah? But, you don't look Turkish'. That I had. So people have some kind of understanding of how Turkish people should be, looking at the Turkish people that live here maybe, and that is very general comment in Europe, and especially in Germany, right, 3 million Turkish people live there, it is the conception of the locals of the Turkish people that live there, and especially, they have been living there for 30 years, and they are kind of living and get stuck in the Turkey of 30-40 years ago, and if you look at the education level, or ... you know these people were not the highereducated people in Turkey when they came to Europe at that time, so you can understand why, so that is why I don't take it personally, and then I say 'Ah, where do you think I am from?' just to change the negative, because people feel bad as well you know when they say it, because they do not say it with a bad intention, so then I just say 'hey where do you think I am from then?'. A lot of, $99 \%$ of my friends are like me. Because I lived my entire life in Turkey, until I moved to Stavanger, I am 35 now, and then I have been here $71 / 2$ years.

$-\mathrm{MT}-$

So this is your first country abroad?

$-\mathrm{T}-$

Yes, well I have been abroad before, but it is the first country that I lived abroad. 
$-\mathrm{MT}-$

So in Turkey, or in your background, what would consider as a respectful greeting?

$-\mathrm{T}-$

If it is like a, well let's separate these two things. For me, somebody that I respect would be an older person, and that you show it the way that we did, you kiss the hand and you put it to your forehead, and then you kiss normally. That is the normal way, and I would only do it to older people. But some people, they also do this to the same aged people, because you have different levels of class... The difference between the lower class and upper class is huge in Turkey. So some people, if they are in a lower class, and if they see somebody they would have the tendency to do the same no matter what the age. But this also buys in with the education level as well. Because I am not upper class at all, when I say upper class, it is better economic situation...blablabla... but then I am educated, so I know I should not, there is no point. So it is education and the level of economic income as well. If you are not educated enough, and if your income is low and you are living a hard life, and if you see somebody really rich, and even if that person is educated or not...

$-\mathrm{MT}-$

Ok, and what would you consider a powerful greeting that you show status or power?

\section{$-\mathrm{T}-$}

Maybe the look of the person, how it looks. Some people try to look down to you, and you know... But if it is somebody that I respect because of what he wrote or what he did or something then I would probably want to shake his hand with two of my hands, and say 'hey, I am very honoured to meet you', I would say that. 
- MT -

Yes, that relates to the next question which was a submissive greeting, because you are showing him your respect.

$-\mathrm{T}-$

Yes

- MT -

What do you consider a warm greeting?

$-\mathrm{T}-$

It is a hug. A big hug and long hug, and followed by a kiss.

$-\mathrm{MT}-$

And what about when you just do the kisses, is that considered a warm greeting?

$-\mathrm{T}-$

Well, if you shake hands and just kiss, it is not necessarily warm. But if you hug first and then kiss, 'aww I missed you so much'...then it is warm.

- MT -

And cold, what is a cold greeting?

$-\mathrm{T}-$

A very weak handshake... 'this mother fucker, yes I don't want to shake his hand anyway'. Or if he doesn't put his hand, to avoid to shake hands, and then when he puts his hand I think 'fuck he wants me to shake his hand'.

$$
-\mathrm{MT}-
$$

What is a masculine greeting? I know it is a very big difference in Turkey and here because in Turkey men do kiss and hug... 
No I can't think of anything to be honest.

$-\mathrm{MT}-$

And feminine, is there any more typical feminine greeting?

$-\mathrm{T}-$

I think women would be more open and warm, more hugs. Whereas men would be a bit more conservative. But not as conservative as up North of Europe.

$-\mathrm{MT}-$

Out of all the greetings we filmed, which would you say you are more comfortable doing? And is there a greeting that you are not so comfortable with?

$-\mathrm{T}-$

I don't think it has something to do with the way of greeting someone, whether it is more comforting or not comforting, it is more if you know the person or not. I think. Because if it is someone I know, that I would not have problems kissing their hand and putting it on the forehead and getting close, you know, but if it is somebody I don't know and everyone is kissing his hand I would just be like 'fuck I'm not going to kiss his hand'. And it is the same thing for women as well, right, if it is somebody that I know I would just hug and kiss, but if it is somebody I don't know, I would probably just shake hands.

$$
-\mathrm{MT}-
$$

Like there was one, I know it was the other guy, Ufuk, he greeted another Turkish girl, who is muslim, and when greeting men, because I know you had a reaction to this, if that was you greeting her, would that make you uncomfortable if you could not shake her hand? 


\section{$-\mathrm{T}-$}

I would not talk to that girl ever in my life to be honest. If I put my hand out and she would not want to shake it, I would say 'who the hell are you?'. Because, to be honest what made me angry there, is that she generalised Turkish people but especially Turkish women, that they do not shake hands because of their beliefs, and that is just bullshit to be honest. Maybe she did not mean to generalise to Turkey, but it was something she said which I heard, so then I just went ballistic basically, and that is why I had to excuse (and leave) because of how the situation is now in Turkey, I am very sensitive.

$-\mathrm{MT}-$

I did talk to her after, and she said that with herself she is very strict in her belief, and that is why since we were dealing with greetings as well, that she kind of wanted to make a point of it.

\section{$-\mathrm{T}-$}

Also, in general, I don't like that way of life, it doesn't matter if it is Turkish or any place. I think that women are being put in a situation where they believe all those things, but they always suffer from it but they just don't have the courage to stand up, and they just live with it because whatever she is doing, she thinks it is her belief, right, but actually it is limiting her life. It is a big step that she has to go over, and it is stopping her from living as a free human. And I think women should be exactly the equal as men. And this belief thing is kind of, that way of by men are putting barriers on women basically. And unfortunately there is a direct relationship between education level and ending up in this situation. If all women are educated the same way, maybe $0.00001 \%$ of the women will be like that, but that is a big discussion so...

$$
-\mathrm{MT}-
$$

No but I wonder, is that a situation you would come across in Turkey? 


\section{$-\mathrm{T}-$}

Not around the circles that I am in, no. And I have been in a lot of places in Turkey, in the South East, up North, East, in the most South East, you know where all this kind of places with leaders I mentioned, even in those regions you expect them to be very conservative, but there people are not conservative because of the religion they are more conservative because they are not used to seeing other people, foreigners, they live in a village with 200 people and I am the 255th person she saw, but even she, when I put my hand, she doesn't hesitate to shake it, maybe you don't shake hands, but you touch the hand and hold it, you know. And after half an hour when you leave she is in love with you, this old lady, and she gives you the biggest hug you have ever got in your life. So that is why I am kind of, it is not our culture.

\section{$-\mathrm{MT}-$}

It is not such a long time, but since you have been living in Stavanger, do you feel like the greetings here are changing? Like when I grew up, the only way we would greet would be a handshake or 'hello', we wouldn't hug very much or anything. Have you seen a change since you have been here?

\section{$-\mathrm{T}-$}

Umm no.

$$
-\mathrm{MT}-
$$

What to you is a typical Norwegian greeting?

$$
-\mathrm{T}-
$$

I think men just shake hands. I think I only have one very very very, oh no, I have a couple of very close guy friends here. We just shake hands, and with the very very close one, we give a big hug like 'agh', but with the other one just the half one. But these people are really close to me. With the less close ones just shake hands, and women I think it is a half hug and a kiss always. If it is people I know and we see each other on a regular basis, once every 
other day or something, it is like a kiss with a half hug. That is worst case scenario, best case scenario - a big hug.

$-\mathrm{MT}-$

What is a very non-typical Norwegian greeting?

$-\mathrm{T}-$

If a guy would kiss me, I would say that would be very non-typical Norwegian.

$-\mathrm{MT}-$

Has it happened yet?

$-\mathrm{T}-$

No... I think I kissed Tor once, and I think we both felt horrible. 


\section{f: Interview with Vietnamese female participant}

\section{8th July 2013}

(The recorded interview was conducted in Norwegian. The interview transcript below is translated into English.)

- Interviewer (MT) -

Have you ever experienced uncomfortable or strange situations when greeting people from your own culture - perhaps that they greet differently than you do?

\section{$-\mathrm{K}-$}

mmmm.... no, not really... my own culture... When we greet each other it does not make me uncomfortable, because I know when and how I should greet the others. So for me it is not uncomfortable.

$$
-\mathrm{MT}-
$$

Ok, so the same question as before, but when you greet someone from a different culture?

$$
-\mathrm{K}-
$$

Yes a lot! Because... another culture... I do not know how I should greet them, for instance...dmm... When we meet I do not know if we should embrace or if we should shake hands, or if we should only say 'hi', I get a bit nervous about it. I remember my first day at work... for my boss, and the first time I met her, she came over to me, and I was thinking to just say hello, but then she came straight over to me and hugged me... I don't know... I just smiled, but the second time she did it again, it still made me a bit nervous, but the third time I was becoming familiar with her culture. But...yes, perhaps the first or second time it makes you nervous, but after that you are ok with it. 
$-\mathrm{MT}-$

And where was she from, your boss? Was she Norwegian?

$-\mathrm{K}-$

She was from Mexico, but she has been living in Norway for six-seven years.

$-\mathrm{MT}-$

Have you met people from other cultures that you did not know how to greet? Is the difference more if they are more...?

$-\mathrm{K}-$

Yes, when a man wants to hug, yes to hug... and when he kisses my cheek I get nervous, because in Vietnam, girls and boys, or men and women, do not hug each other like that. We do not kiss when we meet each other, so for me, it makes me a bit nervous... hehe. Yes it is very hard or strict in Asia, especially in Vietnam. It is not allowed to hold hands, or at least it wan't in my time, $15-20$ years ago. It is more common now, it is not as strict as before.

$$
- \text { MT - }
$$

Ok, but what if you are seeing someone? Is it not normal to hold hands then if you are out in public?

$$
-\mathrm{K}-
$$

No it is not common to hold hands in public. If you and your boyfriend walk together, to hold hands or kiss in public, well that would make me a bit nervous. Maybe Asia is stuck deep in my body.

$-\mathrm{MT}-$

Why do you think these situations make you feel uncomfortable? 


\section{$-\mathrm{K}-$}

Maybe because, growing up I lived with my Vietnamese family and we had some rules. Then in school they taught us this way, and home the same, so it has become our rules, I think that is why we feel uncomfortable when we greet others, or other cultures.

\section{$-\mathrm{MT}-$}

In Stavanger, what do you feel when you greet someone from a different culture for the first time? Do you use a standard greeting, or is it like you mentioned earlier, that you are not sure how to greet? For instance, if a Mexican, a Norwegian and an African person, or wherever they are from...

$$
-\mathrm{K}-
$$

Well, for me, the first time you always have to think how to do or how to greet. I think that the first time for me is always a bit... I'm always a bit unsure of what to do, or how to greet them. Because you know there are so many different cultures in the world. Some hold hands or shake hands, others embrace or kiss, while in another culture they do it differently, they might not like to embrace or hold hands, that is why you have to keep some distance, or be aware.

$$
-\mathrm{MT}-
$$

But like when we greeted today, I was a bit...eeehhh... too, I didn't know if I should shake your hand or give you a hug. It is always like that, some people kiss twise, some three times while others once, it is always a bit uncertain. Do you greet people from different cultures differently? Say you meet a Spanish person or a Turkish person, do you greet them the same way? Or say you meet three people that are together, one is Mexican, one is Norwegian and the third is Vietnamese? 


\section{$-\mathrm{K}-$}

If I know their culture and how they greet, then I can greet them their way. But if it is the first time meeting them, then I just greet all of them the same, say 'hi' or something. If I know their culture, how they greet in Mexico or Norway, then I greet them like that, then I will respect their culture. But for the first time, if you do not know the culture, then I just greet normally, a handshake or a 'hi'.

$-\mathrm{MT}-$

In your opinion, what is a respectful greeting?

$-\mathrm{K}-$

Like I said, my background is Asian, Vietnamese. We have a very strict culture, and greetings are very important to us. We divide differently how we greet friends, mother, father, grandmother and grandfather. I have been living in Norway for five years, so I have seen how they greet each other here. Personally I like our culture, we divide differently how we greet people around us, and particularly how we greet the elderly.

$$
-\mathrm{MT}-
$$

And how do you show respect? Is it different how you show respect to an older person and to a person your own age? In Japan, for instance, it is like that, it depends on how far you bow down to show respect to the elderly.

$-\mathrm{K}-$

For us, we hold two hands together like this and bow, then you show respect.

$$
-\mathrm{MT}-
$$

Is there a greeting that shows status, or power? Are there people in society that you have to show respect, like a priest or a monk? 
$-\mathrm{K}-$

Yes, to a priest you have to show respect, and to your mother and father, but not to your friends. Respect is always shown to those who are older than us. And especially to teachers. It is quite strict at school, between teachers and pupils. We cannot shake hands or anything, you always have to show respect, even if you are 5 or 6 years old, and you do the same to your mother and father.

$-\mathrm{MT}-$

In Vietnam, what is.... Like in Mexico they consider a warm greeting to be an embrace or kiss, but the other Vietnamese participants I spoke to, said that in Vietnamese culture it is more important to smile and have eye-contact.

$$
-\mathrm{K}-
$$

Yes, to have eye-contact when you greet! You have to look at the other person.

$$
-\mathrm{MT}-
$$

So that is considered more friendly than necessarily having to...

$$
-\mathrm{K}-
$$

hmmm, I think that in our culture, to show respect when we greet we mostly use the eyes and smile, we do not embrace or have very near physical contact like they do in other cultures.

$$
-\mathrm{MT}-
$$

Ok, is there anything you consider a cold or unfriendly greeting?

$$
-\mathrm{K}-
$$

When you greet you always show... even if you do not feel like it, or if you are tired, but when you greet you have to show respect. You can not be distracted or grumpy or anything, no, when you greet you must be respectful. 
I do not mean respect in the same sense as when you greet someone older, but even with friends when we greet we respect each other. We respect the others.

$-\mathrm{MT}-$

In Norway, if two men greet each other, then they do not hug, they do more... Is there anything in Vietnamese culture you would consider a more masculine greeting? Or is there any difference?

$$
-\mathrm{K}-
$$

Yes there is a difference, when I greet another girl or woman then we can hug, but when I greet a man then I just want to shake hands, not to hug or embrace.

$$
-\mathrm{MT}-
$$

Is this so when you greet your father or grandfather too?

$$
-\mathrm{K}-
$$

Yes it is the same, I think it is the same for me.

$$
-\mathrm{MT}-
$$

What about when two men greet?

$$
-\mathrm{K}-
$$

When two men greet each other, they shake hands, and if they are very close, like best friends, then they can hug. But usually they just shake hands.

$$
-\mathrm{MT}-
$$

And what about a feminine greeting? 
$-\mathrm{K}-$

Well then there is more emotion shown, then you can hug and embrace each other.

- MT -

Out of the greetings that we filmed, which one are you most comfortable with, and least comfortable with? Or does it depend on the situation? Would you greet your female friends with a hug?

$$
-\mathrm{K}-
$$

Yes I would hug, if they also hug.

$$
-\mathrm{MT}-
$$

But, for instance, the greeting I showed you between Norway and Angola, if someone greeted you like that the first time you met them, would that be strange?

$$
-\mathrm{K}-
$$

Yes if it is the first time I met them, but if it is the second, third or fourth time meeting them I think it is ok.

$$
\text { - MT - }
$$

You have been in Stavanger for 5 years, so I do not know if you have noticed any big changes, but do you feel like greetings have changed in Stavanger at all?

$$
-\mathrm{K}-
$$

When I greet Norwegian friends I have started to hug or kiss, we are quite close, but with my Vietnamese friends I still keep my own culture, I just say 'hi' or something, we do not hug or kiss. 
$-\mathrm{MT}-$

And what about with young Vietnamese?

$-\mathrm{K}-$

Mmm...young Vietnamese, do you mean the ones living in Stavanger?

$-\mathrm{MT}-$

Yes if they are born or live in Stavanger.

$-\mathrm{K}-$

If they are born in Stavanger they are different to us when they greet their friends. I have seen my younger cousins here when they greet their friends they hug, but when they greet other Vietnamese, then it is the same as us, they just say 'hi' or hug or something. I was a bit surprised to see this at first. I think that they are born in Norway but they understand the Vietnamese culture.

$-\mathrm{MT}-$

Ok, and what do you consider a typical Norwegian greeting?

$$
-\mathrm{K}-
$$

A typical Norwegian greeting.... To hug and kiss. I think so, because I have some Norwegian friends, and when I meet them they hug and kiss on the cheek, yes, I think that is typical Norwegian.

$$
-\mathrm{MT}-
$$

Well, I grew up in Ålgård, and it is not as international as Stavanger, so I grew up with either greeting with a handshake or a hug. If I haven't seen my friends in a while we give a hug, but if I see them often we just say 'hi'. I think it is different in Stavanger because it has become more international. 


\section{$-\mathrm{K}-$}

Yes, when it is friends or someone you have not seen in a long time you hug or kiss, buy if you meet every day, I think we just say 'hi'.

$-\mathrm{MT}-$

And the last question is, what is an un-typical greeting? Some Norwegians say we do not hug, but I think it might be different in the city and outside the city?

$$
-\mathrm{K}-
$$

I think we hug more in the city. At art school, when we met each other we gave a hug, but after we just say 'hi' or 'good morning' when we meet, we do not hug all the time.

$$
-\mathrm{MT}-
$$

What about at home? Do you live with family here in Stavanger?

$$
-\mathrm{K}-
$$

Yes, I live at my aunt's house.

$$
-\mathrm{MT}-
$$

And is she Vietnamese?

$$
-\mathrm{K}-
$$

Yes she is Vietnamese, she has been living here for $15-16$ years.

$$
-\mathrm{MT}-
$$

And does she keep on to her Vietnamese culture?

$$
-\mathrm{K}-
$$

Yes, especially the older Vietnamese people, they like to hold on to the Vietnamese culture. 
$-\mathrm{MT}-$

And what about the young ones?

$-\mathrm{K}-$

The young change a lot. At home they speak some Vietnamese but not too much. The Vietnamese that are born in Noway, they speak mainly Norwegian and not Vietnamese when they meet each other.

$-\mathrm{MT}-$

And with you, do they speak Norwegian to you too?

$-\mathrm{K}-$

Yes, because I want to learn Norwegian, but at home we only speak

Vietnamese. I think almost $99 \%$ of Vietnamese here live with Vietamese and speak mostly Vietnamese at home. It is easy for us, easy to understand, it is natural.

$-\mathrm{MT}-$

The greetings I mentioned, do they still greet like that in Vietnam, or has it changed at all?

$-\mathrm{K}-$

I have not been to Vietnam in 5 years, but...

$-\mathrm{MT}-$

Because when I contacted her, she contacted others to join the project and get filmed, and she chose to invite the older people because she felt they were better at keeping culture and traditions, that they know more about the Vietnamese culture than the ones that are born here. 


\section{$-\mathrm{K}-$}

Yes of course. But you know, parents.... Culture is for older people now. I know that in Vietnam, they young people do not greet as respectfully anymore, not like we do. They learn a lot of new stuff, they watch new movies... so it is changing.

\section{$-\mathrm{MT}-$}

Here in Norway, we don't really have any.... well we greet young and old the same way, is this strange for you?

$$
-\mathrm{K}-
$$

At first I was nervous, because I am living in a different country, it is not our culture. At first I got very surprised when I saw Norwegians greet or say 'hi'. It is the same, they do not distinguish between older people, mother, father or friends... I noticed that the greeting was exactly the same, it made me feel a bit uncomfortable. But now I understand, because that is their culture, not ours. But for someone from Asia or Vietnam seeing this for the first time... when my mother and grandmother came to visit us from Vietnam.... when they saw that here they greet mother, father and friends the same way, they were like 'what??'.

In Vietnam, when you sit at a table and eat together, you have to let the oldest person eat first, when the oldest person has started to eat, the rest can start too. We can not eat first, we have to wait. Or greetings, you always have to greet the oldest person first. At school you have to greet the teacher first. If you are with your friends, mother, father, grandmother and grandfather, you are not allowed to greet your friends first, that is not respectful in Vietnamese culture. 
g: Interview with Angolan participant

\section{3th December 2014}

- Interviewer (MT) -

Have you ever experienced any awkward, funny, or uncomfortable situations when greeting someone from your own culture?

\section{$-\mathrm{J}-$}

Yes eh... uncomfortable maybe because....eh...it is a mix of feelings, the good thing is that in my culture in Angola we normally give two kisses when we say hello, and generally eh... even if we don't know them very well, we always do that. But sometimes... yes, sometimes some people... it is a typical thing, you expect them to give the two kisses, and then they put the hand. We have so many kinds of greetings that sometimes it gets awkward because we don't know which one is going to be the chosen one. I prefer... for me I'm very flexible, I would give two kisses, but if it is an older person that is a stranger, that is just a worker in some company... then you have to probably shake the hand, but if it is close enough to your family then you don't know where you stand. So then it can become a bit, kind of awkward. Ehh... yes when it is funny, it is just things that happen with everybody, because with this thing of two kisses sometimes people turn the face in the same side as you. So normally, I don't know...automatically we have this kind of code that maybe the girl goes to the left - first kisses left and then right. But sometimes people do the other way around, and then we are about to kiss in the mouth. It is really awkward... or it is in the corner of the lip, because people are different. Or sometimes the person is a bit more wet... it is disgusting, but normally it should be not a proper kiss, it is just the movement. That's really what it is, but sometimes it can be confusing, or... depends on the situation. But it is ok! 
- MT -

The next one - but you kind of answered it - what in your view what caused this uncertainty? It is just because you have so many options in a way, isn't it?

$-\mathrm{J}-$

Yeah!

- MT -

Have you experienced any awkward, funny, or uncomfortable situations when greeting people (in Stavanger specifically) from a different culture?

$-\mathrm{J}-$

Yes, then it is even more awkward because...well, generally here, I have learned being in Europe that you just shake hands. It is the safest approach. But yes, sometimes, because here it is so many international people, there are some people that just give one kiss, there are people that give two, people shake hands, people hug... I think here maybe it is in between shaking the hand and a very like...eh... diplomatic hug. It is not a real hug, that is just with general people. Ehm... then with friends it is nice, because it is warm hugs and warm kisses, like proper. Closer, with more kind of body contact. But also here in Stavanger, there is also the thing of not even... the greeting is just to say 'hello', people don't touch each other. So there is actually a lot of mix here.

Depends where you meet them. If you meet them at night in a bar, it is one way of greeting, if you meet them at work they might ignore you... they don't even say hi because they are awkward. And if you meet in the street you just say hi.

So it is actually... And here what I would say is the most awkward is that, I tend to say hello all the time and people pretend they don't see you. And 
they pretend that you don't exist. That is the most common annoying cultural shock that I have with being in Stavanger and being from Africa that I struggle until today. With some of my colleagues at work, it took me a year for them to actually say hello. I would say hi and they would just walk past. So it is really... Norwegians can be...even foreigners do that as well in Norway, not just Norwegians actually, it is like contagious, it is something that everybody ends up doing...

$-\mathrm{MT}-$

And what...well I guess you answered, what caused this uncertainty? The uncertainty between you and them - because it is so different, or?

$-\mathrm{J}-$

I don't know if it is the weather because in the summer everybody is much more open. People here are a bit introverted, and I don't know... I have heard here that also culturally, the families don't really say 'I love you', generally there is not so much show of affection, and show of... people don't touch themselves so much, so ehm... it is still warmer than Japan or other countries, but... so maybe it is a cultural thing from birth. But it is changing, people are getting more international, at least in Stavanger.

$-\mathrm{MT}-$

Ok so in Stavanger then, how do you feel when you greet someone from a different culture for the first time? Is there a general kind of greeting, or? So it can be Norwegian or other cultures...

\section{$-\mathrm{J}-$}

Yes it depends...there is so many kinds of greetings. That's very interesting. If I meet someone from my country we give two kisses. If I meet a Norwegian person randomly on the street, I might just say hi. Of course also it depends on the place and time where we are. If it is someone from Africa, maybe I would bow a bit, we bow... and we might do some more movements with 
our hands differently. Out of respect we...I tend to bow a little bit anyways. I try to impose my...even if sometimes I'm uncomfortable, I try to pretend I am comfortable so that I don't make the other person uncomfortable. So I try to break the ice because I am outgoing, so...but without stepping the boundaries. I think the ideal is to just say hi, without touching, and lifting your hand and make a bow and that's it, I think that's the safest. Then there is no misunderstandings.

$-\mathrm{MT}-$

So you already answered if you greet people differently depending on their cultural background. So, how do you think your relation to or comfort with touch and personal space varies from that of people from other cultures?

$-\mathrm{J}-$

Culturally we are much more open in terms of personal space. But at the same time, I feel like, because I have been in Europe so much, I have adapted enough to... I only feel like I open my personal space with very close friends or people from my country now. But everyone else I always keep a bit of distance because I don't want to have any awkwardness because I know that people don't feel comfortable. Even with my students...I do touch them sometimes, and they get uncomfortable, but I do it on purpose because I feel like they appreciate it in a way. I think people want it, not everybody, some people just feel awkward with that so we have to respect it. But generally people like it I think, it's just they don't know how to approach it. I think Norwegians are very warm. So in Stavanger I think it is ok, it is not an issue, but of course... It depends who you are also relating with.

It is very... it really varies I think this, it is very hard to generalise too much I think. 
Have you ever been or felt judged or stereotyped because of your cultural background and behaviour, like for example Norwegians often hear that they are cold - it is kind of a stereotype, and if so why do you think that is?

$-\mathrm{J}-$

Yes, I'm definitely stereotyped. When people look at me I think they assume I'm Brazilian. So they have this stereotype that I am Brazilian. Then they find out that I'm from Angola and they get a bit confused, they understand our cultures are quite similar, so I think they expect me to be quite outgoing anyways, outspoken and...eh... Here in Norway I don't have so much of a negative stereotype, generally. Sometimes people make comments. I think when I was in Portugal I had more of a negative stereotype. Because there they talk of Africans to be like this or like that, but here I think that times are different because there is a lot of political and economical situations going on there, so now everybody knows about Angola, but if you asked me this about ten years ago then they don't know. But when I say Africa I still feel that it's AFRICA - you know, it's...I don't feel...I feel like I am exotic. They don't know what it's about. They don't know anything about it, they don't understand it but they are curious, so that's a good thing. But yes...because I am a little bit light skinned, I think for me it is easier, but if it was someone darker I think they get more stereotyped probably, more negatives. For me they don't know if I'm from South America...so it is a bit more...they don't know where to place me so much. But I have had a good experience in Stavanger. I have been in Stavanger city all the time. I don't know... If it is in other small towns, it is different, then it is more negative stereotypes.

$-\mathrm{MT}-$

What in your view and in your culture is considered a respectful greeting? 
I think the best is to nod or bow a little bit, and smile and be humble, show your humble nature, and look in the eyes and smile. I think eye contact is very important, with smile and genuine. And the shaking of the hand I think is very respectful. Bow, eye contact, smile and shake the hand, to any age, group or... I think that is very beautiful.

$-\mathrm{MT}-$

And a powerful greeting, or when someone is kind of showing status?

$-\mathrm{J}-$

I have heard that there is ways of shaking the hands. There are people that shake the hands with their hand on top. And if they do that they are in power, they are on top. I have heard of these kinds of handshakes, and also the pressure. That you have to make pressure to show that you are confident and... actually I don't like to shake a hand that is loose and soft myself. I don't like that kind of handshake. But, I think it has to be a balance, not too strong, not putting your hand... and not too physical, like... and also with enough distance to each other, because if it is too far apart I think it is also showing that you are not interested to get to know the other person. So it should be like half ways, both half ways, and same balance so we are together in the same level... and of course I think a smile does a lot, so if you are not smiling...the eye contact, the facial expressions are definitely also a lot.

$-\mathrm{MT}-$

And what is a submissive greeting, so if you are...say if you are greeting someone who is more in authority for example, how do you show submission?

$-\mathrm{J}-$

I think it goes back to the bow. It is the same one as the respectful one. And another way of...going back to the people that want to show power, it 
might be also to reject someone's hand or offering, that is very rude. But I think culturally it is good to be humble, so the first and third question I would answer the same way.

$-\mathrm{MT}-$

And a warm greeting?

$-\mathrm{J}-$

A warm greeting is the best. It's eh... I can imagine it is a person laughing actually loud, making noise, and hugging you and really really warm and tight and with laughs. Patting on the back and...the Angolans, we are loud, so we would go like 'hahahaha' and really express love and we express it, so that is the best.

$-\mathrm{MT}-$

And the opposite then, a cold greeting?

$-\mathrm{J}-$

A cold greeting is these hugs that I was talking about like... If you want to hug it depends. If it's a hug it's that hug that is like, I don't know how to say... robotic but nobody touches and it is just like awkward, that's one. And then that one of the shaking hands that is soft that is weird. Or if someone that is stiff, and the other comes to give two kisses, but the other has to reach out of all their space, all the way to the other person's space, and the other is just a stone... and that is not very nice.

$-\mathrm{MT}-$

And masculine, what is the typical masculine greeting in your culture?

$-\mathrm{J}-$

Masculine... Yes, this hug that I was talking about that the guy goes and takes control, that is one masculine hug, I think it is more...the men do that. 
The shaking the hand also, shaking a lot and strong and holding the whole hands, that's very masculine. Yes I think those two are probably the most masculine.

$-\mathrm{MT}-$

And feminine?

$-\mathrm{J}-$

The feminine...we are much more gentle. If we hug we don't hug so strong like that, but we probably rub more the back, in a soft way. When we shake... we let people have control. And the kissing...the girls get kisses, the guys shake each other's... sometimes if it is a father and a son, family, they give two kisses, like my father and my brothers they kiss. But a girl always gets a kiss...two kisses, it is very sweet.

$-\mathrm{MT}-$

Of all the greetings we filmed, what would you say is your most comfortable greeting and what is your most uncomfortable one?

$-\mathrm{J}-$

The comfortable one is when it comes natural, when two people come to each other naturally with the same kind of...eh how do you say...feelings, they come together, they have acknowledged each other at the same time, they come together, they smile, they shake their hands and they smile. That is the perfect greeting. The uncomfortable is when you are going to hug and he puts your hand and... but it can be solved with a laugh. That one is not so uncomfortable, it is just misunderstanding. Uncomfortable is if there is no laugh at the end. If there is no laugh, then it is uncomfortable. If there is a laugh then it is ok, then it is the start of the conversation. 
$-\mathrm{MT}-$

And do you feel like greetings are changing or have changed in Stavanger since you have been there?

$-\mathrm{J}-$

Yes, I think so. I think that Stavanger became much more multicultural, and people have adapted more to the other cultures, and they are very curious and open, so they also try to copy our kind of customs, and we are all exchanging. We adapt to their culture, so we are all meeting in the middle in some ways. And in a group, if we are a big group, some will hug, some will kiss, some will do two kisses, some will do three kisses, some will shake, and in the end we hug. So it definitely changed for the better. It is much nicer.

$-\mathrm{MT}-$

What to you is the typical and untypical Norwegian greeting?

$-\mathrm{J}-$

The typical Norwegian greeting is 'Hi', it is just a sound and that's it. People are smiling and they look at each other and they say 'Hi' and that's it. Untypical is when they come and give you the kisses, and some of them even give three kisses, so that is untypical, but that's very nice, it is very warm, it is very sweet. But when you have close friends, closer, it changes as well. They hug you, it is nice. They are actually quite warm once you get to know them, they are just a bit shy in the beginning, because they want to understand where you are from, where you come from, but once they understand, they are actually quite warm. 


\section{Appendix 11: Actionscript 3.0 code used to programme greeting}

\section{diagrams}

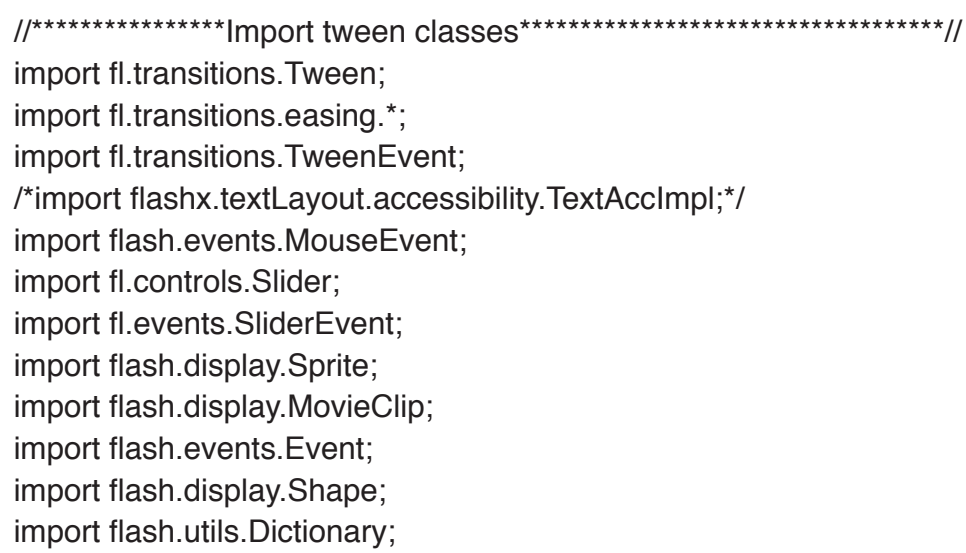

// creating variables for each greeting \& all its views// //BUTTONS//

var fama1_btn = new fam_a1_btn(); var fama2_btn = new fam_a2_btn(); var fama3_btn = new fam_a3_btn(); var fama4_btn = new fam_a4_btn(); var fama5_btn = new fam_a5_btn(); var famb2_btn = new fam_b2_btn(); var famb5_btn = new fam_b5_btn(); var famc2_bth = new fam_c2_btn(); var famc4_btn = new fam_c4_btn(); var famc5_bth = new fam_c5_btn(); var famd1_bth $=$ new fam_d1_btn(); var famd2_btn = new fam_d2_btn(); var famd5_btn = new fam_d5_btn(); var fame1_btn = new fam_e1_btn(); var fame2_btn = new fam_e2_btn(); var fame3_btn = new fam_e3_btn(); var fame4_btn = new fam_e4_btn(); var fame5_btn = new fam_e5_btn(); var famf2_btn = new fam_f2_btn(); var famf5_bth $=$ new fam_f5_bth(); var famg2_bth = new fam_g2_btn(); var famg4_bth $=$ new fam_g4_btn(); var famg5_btn = new fam_g5_btn(); var famh1_btn = new fam_h1_btn(); 
var famh2_btn = new fam_h2_btn(); var famh5_btn = new fam_h5_btn();

var crossa1_btn = new cross_a1_btn(); var crossa2_btn = new cross_a2_btn(); var crossa3_btn = new cross_a3_btn(); var crossb2_bth = new cross_b2_btn(); var crossb3_bth = new cross_b3_btn(); var crossb4_bth $=$ new cross_b4_btn(); var crossc1_btn = new cross_c1_btn(); var crossc2_bth = new cross_c2_btn(); var crossc3_btn = new cross_c3_btn(); var crossc4_bth = new cross_c4_btn(); var crossc5_btn = new cross_c5_btn(); var crossc6_bth = new cross_c6_btn(); var crossd1_bth = new cross_d1_btn(); var crossd2_btn = new cross_d2_btn(); var crossd3_btn = new cross_d3_btn(); var crosse2_bth = new cross_e2_btn(); var crosse3_btn = new cross_e3_btn(); var crossf1_bth = new cross_f1_btn(); var crossf5_bth = new cross_f5_btn(); var crossf6_bth = new cross_f6_btn();

var soca2_bth = new soc_a2_btn(); var soca4_bth = new soc_a4_btn(); var soca5_btn = new soc_a5_btn(); var socb2_btn = new soc_b2_btn(); var socb3_bth = new soc_b3_btn(); var socb4_btn = new soc_b4_btn(); var socb5_btn = new soc_b5_btn(); var socc1_btn = new soc_c1_btn(); var socc2_btn = new soc_c2_btn(); var socc3_btn = new soc_c3_btn(); var socc4_btn = new soc_c4_btn(); var socc5_bth $=$ new soc_c5_bth(); var socd2_bth = new soc_d2_btn(); var socd4_btn = new soc_d4_btn(); var socd5_bth = new soc_d5_bth(); var soce2_btn = new soc_e2_btn(); var soce4_bth = new soc_e4_btn(); var soce5_btn = new soc_e5_btn(); var socf1_btn = new soc_f1_btn(); var socf2_bth = new soc_f2_btn(); var socf3_btn = new soc_f3_bth(); var socf4_bth = new soc_f4_bth(); var socf5_bth $=$ new soc_f5_bth(); var socg2_btn = new soc_g2_btn(); var socg4_btn = new soc_g4_btn(); var socg5_bth = new soc_g5_btn(); var soch2_btn = new soc_h2_btn(); var soch3_bth = new soc_h3_bth(); var soch4_bth = new soc_h4_bth(); var soch5_btn = new soc_h5_bth(); var soci1_bth = new soc_i1_btn(); var soci2_bth = new soc_i2_bth(); var soci3_bth = new soc_i3_bth(); var soci4_bth $=$ new soc_i4_bth(); 
var soci5_bth = new soc_i5_bth();

\section{//MOVIE CLIPS// \\ //CROSS-CULTURAL// \\ ////cross_a1}

var cross_a1: Greeting_cross_a1 = new Greeting_cross_a1();

var cross_a1_zon: Greeting_cross_a1_zon = new Greeting_cross_a1_zon(); var cross_a1_spa: Greeting_cross_a1_spa = new Greeting_cross_a1_spa(); var cross_a1_tou: Greeting_cross_a1_tou = new Greeting_cross_a1_tou(); var cross_a1_mov: Greeting_cross_a1_mov = new Greeting_cross_a1_mov(); var cross_a1_sem:Greeting_cross_a1_sem = new Greeting_cross_a1_sem();

\section{II//cross_a2}

var cross_a2: Greeting_cross_a2 = new Greeting_cross_a2();

var cross_a2_zon: Greeting_cross_a2_zon = new Greeting_cross_a2_zon(); var cross_a2_spa: Greeting_cross_a2_spa = new Greeting_cross_a2_spa(); var cross_a2_tou: Greeting_cross_a2_tou = new Greeting_cross_a2_tou(); var cross_a2_mov: Greeting_cross_a2_mov = new Greeting_cross_a2_mov(); var cross_a2_sem:Greeting_cross_a2_sem = new Greeting_cross_a2_sem(); //

//cross_a3

var cross_a3: Greeting_cross_a3 = new Greeting_cross_a3();

var cross_a3_zon: Greeting_cross_a3_zon = new Greeting_cross_a3_zon(); var cross_a3_spa: Greeting_cross_a3_spa = new Greeting_cross_a3_spa(); var cross_a3_tou: Greeting_cross_a3_tou = new Greeting_cross_a3_tou(); var cross_a3_mov: Greeting_cross_a3_mov = new Greeting_cross_a3_mov(); var cross_a3_sem:Greeting_cross_a3_sem = new Greeting_cross_a3_sem();

\section{II//cross b2}

var cross_b2: Greeting_cross_b2 = new Greeting_cross_b2();

var cross_b2_zon: Greeting_cross_b2_zon = new Greeting_cross_b2_zon(); var cross_b2_spa: Greeting_cross_b2_spa = new Greeting_cross_b2_spa(); var cross_b2_tou: Greeting_cross_b2_tou = new Greeting_cross_b2_tou(); var cross_b2_mov: Greeting_cross_b2_mov = new Greeting_cross_b2_mov(); var cross_b2_sem:Greeting_cross_b2_sem = new Greeting_cross_b2_sem();

\section{//cross_b3}

var cross_b3: Greeting_cross_b3 = new Greeting_cross_b3();

var cross_b3_zon: Greeting_cross_b3_zon = new Greeting_cross_b3_zon(); var cross_b3_spa: Greeting_cross_b3_spa = new Greeting_cross_b3_spa(); var cross_b3_tou: Greeting_cross_b3_tou = new Greeting_cross_b3_tou(); var cross_b3_mov: Greeting_cross_b3_mov = new Greeting_cross_b3_mov(); var cross_b3_sem:Greeting_cross_b3_sem = new Greeting_cross_b3_sem();

\section{//cross_b4}

var cross_b4: Greeting_cross_b4 = new Greeting_cross_b4(); var cross_b4_zon: Greeting_cross_b4_zon = new Greeting_cross_b4_zon(); var cross_b4_spa: Greeting_cross_b4_spa = new Greeting_cross_b4_spa(); var cross_b4_tou: Greeting_cross_b4_tou = new Greeting_cross_b4_tou(); var cross_b4_mov: Greeting_cross_b4_mov = new Greeting_cross_b4_mov(); var cross_b4_sem:Greeting_cross_b4_sem = new Greeting_cross_b4_sem();

\section{//I/cross_c1}

var cross_c1: Greeting_cross_c1 = new Greeting_cross_c1();

var cross_c1_zon: Greeting_cross_c1_zon = new Greeting_cross_c1_zon(); var cross_c1_spa: Greeting_cross_c1_spa = new Greeting_cross_c1_spa(); var cross_c1_tou: Greeting_cross_c1_tou = new Greeting_cross_c1_tou(); 
var cross_c1_mov: Greeting_cross_c1_mov = new Greeting_cross_c1_mov(); var cross_c1_sem:Greeting_cross_c1_sem = new Greeting_cross_c1_sem(); /I

I///cross_c2

var cross_c2: Greeting_cross_c2 = new Greeting_cross_c2();

var cross_c2_zon: Greeting_cross_c2_zon = new Greeting_cross_c2_zon(); var cross_c2_spa: Greeting_cross_c2_spa = new Greeting_cross_c2_spa(); var cross_c2_tou: Greeting_cross_c2_tou = new Greeting_cross_c2_tou(); var cross_c2_mov: Greeting_cross_c2_mov = new Greeting_cross_c2_mov(); var cross_c2_sem:Greeting_cross_c2_sem = new Greeting_cross_c2_sem(); //

//cross_c3

var cross_c3: Greeting_cross_c3 = new Greeting_cross_c3();

var cross_c3_zon: Greeting_cross_c3_zon = new Greeting_cross_c3_zon(); var cross_c3_spa: Greeting_cross_c3_spa = new Greeting_cross_c3_spa(); var cross_c3_tou: Greeting_cross_c3_tou = new Greeting_cross_c3_tou(); var cross_c3_mov: Greeting_cross_c3_mov = new Greeting_cross_c3_mov(); var cross_c3_sem:Greeting_cross_c3_sem = new Greeting_cross_c3_sem();

//cross_c4

var cross_c4: Greeting_cross_c4 = new Greeting_cross_c4();

var cross_c4_zon: Greeting_cross_c4_zon = new Greeting_cross_c4_zon(); var cross_c4_spa: Greeting_cross_c4_spa = new Greeting_cross_c4_spa(); var cross_c4_tou: Greeting_cross_c4_tou = new Greeting_cross_c4_tou(); var cross_c4_mov: Greeting_cross_c4_mov = new Greeting_cross_c4_mov(); var cross_c4_sem:Greeting_cross_c4_sem = new Greeting_cross_c4_sem();

\section{//cross_c5}

var cross_c5: Greeting_cross_c5 = new Greeting_cross_c5();

var cross_c5_zon: Greeting_cross_c5_zon = new Greeting_cross_c5_zon(); var cross_c5_spa: Greeting_cross_c5_spa = new Greeting_cross_c5_spa(); var cross_c5_tou: Greeting_cross_c5_tou = new Greeting_cross_c5_tou(); var cross_c5_mov: Greeting_cross_c5_mov = new Greeting_cross_c5_mov(); var cross_c5_sem: Greeting_cross_c5_sem = new Greeting_cross_c5_sem();

//cross_c6

var cross_c6: Greeting_cross_c6 = new Greeting_cross_c6();

var cross_c6_zon: Greeting_cross_c6_zon = new Greeting_cross_c6_zon(); var cross_c6_spa: Greeting_cross_c6_spa = new Greeting_cross_c6_spa(); var cross_c6_tou: Greeting_cross_c6_tou = new Greeting_cross_c6_tou(); var cross_c6_mov: Greeting_cross_c6_mov = new Greeting_cross_c6_mov(); var cross_c6_sem:Greeting_cross_c6_sem = new Greeting_cross_c6_sem();

\section{////cross_d1}

var cross_d1: Greeting_cross_d1 = new Greeting_cross_d1();

var cross_d1_zon: Greeting_cross_d1_zon = new Greeting_cross_d1_zon(); var cross_d1_spa: Greeting_cross_d1_spa = new Greeting_cross_d1_spa(); var cross_d1_tou: Greeting_cross_d1_tou = new Greeting_cross_d1_tou(); var cross_d1_mov: Greeting_cross_d1_mov = new Greeting_cross_d1_mov(); var cross_d1_sem:Greeting_cross_d1_sem = new Greeting_cross_d1_sem();

//cross_d2

var cross_d2: Greeting_cross_d2 = new Greeting_cross_d2();

var cross_d2_zon: Greeting_cross_d2_zon = new Greeting_cross_d2_zon(); var cross_d2_spa: Greeting_cross_d2_spa = new Greeting_cross_d2_spa(); var cross_d2_tou: Greeting_cross_d2_tou = new Greeting_cross_d2_tou(); var cross_d2_mov: Greeting_cross_d2_mov = new Greeting_cross_d2_mov(); 
var cross_d2_sem:Greeting_cross_d2_sem = new Greeting_cross_d2_sem();

//cross_d3

var cross_d3: Greeting_cross_d3 = new Greeting_cross_d3();

var cross_d3_zon: Greeting_cross_d3_zon = new Greeting_cross_d3_zon();

var cross_d3_spa: Greeting_cross_d3_spa = new Greeting_cross_d3_spa();

var cross_d3_tou: Greeting_cross_d3_tou = new Greeting_cross_d3_tou();

var cross_d3_mov: Greeting_cross_d3_mov = new Greeting_cross_d3_mov(); var cross_d3_sem:Greeting_cross_d3_sem = new Greeting_cross_d3_sem();

\section{/I//cross_e2}

var cross_e2: Greeting_cross_e2 = new Greeting_cross_e2();

var cross_e2_zon: Greeting_cross_e2_zon = new Greeting_cross_e2_zon(); var cross_e2_spa: Greeting_cross_e2_spa = new Greeting_cross_e2_spa(); var cross_e2_tou: Greeting_cross_e2_tou = new Greeting_cross_e2_tou(); var cross_e2_mov: Greeting_cross_e2_mov = new Greeting_cross_e2_mov(); var cross_e2_sem:Greeting_cross_e2_sem = new Greeting_cross_e2_sem();

//cross_e3

var cross_e3: Greeting_cross_e3 = new Greeting_cross_e3();

var cross_e3_zon: Greeting_cross_e3_zon = new Greeting_cross_e3_zon(); var cross_e3_spa: Greeting_cross_e3_spa = new Greeting_cross_e3_spa(); var cross_e3_tou: Greeting_cross_e3_tou = new Greeting_cross_e3_tou(); var cross_e3_mov: Greeting_cross_e3_mov = new Greeting_cross_e3_mov(); var cross_e3_sem:Greeting_cross_e3_sem = new Greeting_cross_e3_sem();

\section{III/cross_f1}

var cross_f1: Greeting_cross_f1 = new Greeting_cross_f1();

var cross_f1_zon: Greeting_cross_f1_zon = new Greeting_cross_f1_zon(); var cross_f1_spa: Greeting_cross_f1_spa = new Greeting_cross_f1_spa(); var cross_f1_tou: Greeting_cross_f1_tou = new Greeting_cross_f1_tou(); var cross_f1_mov: Greeting_cross_f1_mov = new Greeting_cross_f1_mov(); var cross_f1_sem:Greeting_cross_f1_sem = new Greeting_cross_f1_sem();

//cross_f5

var cross_f5: Greeting_cross_f5 = new Greeting_cross_f5();

var cross_f5_zon: Greeting_cross_f5_zon = new Greeting_cross_f5_zon(); var cross_f5_spa: Greeting_cross_f5_spa = new Greeting_cross_f5_spa(); var cross_f5_tou: Greeting_cross_f5_tou = new Greeting_cross_f5_tou(); var cross_f5_mov: Greeting_cross_f5_mov = new Greeting_cross_f5_mov(); var cross_f5_sem:Greeting_cross_f5_sem = new Greeting_cross_f5_sem();

//cross_f6

var cross_f6: Greeting_cross_f6 = new Greeting_cross_f6();

var cross_f6_zon: Greeting_cross_f6_zon = new Greeting_cross_f6_zon(); var cross_f6_spa: Greeting_cross_f6_spa = new Greeting_cross_f6_spa(); var cross_f6_tou: Greeting_cross_f6_tou = new Greeting_cross_f6_tou(); var cross_f6_mov: Greeting_cross_f6_mov = new Greeting_cross_f6_mov(); var cross_f6_sem:Greeting_cross_f6_sem = new Greeting_cross_f6_sem();

\section{//FAMILY//}

//fam_a1

var fam_a1: Greeting_fam_a1 = new Greeting_fam_a1(); var fam_a1_zon: Greeting_fam_a1_zon = new Greeting_fam_a1_zon(); var fam_a1_spa: Greeting_fam_a1_spa = new Greeting_fam_a1_spa(); varfam_a1_tou: Greeting_fam_a1_tou = new Greeting_fam_a1_tou(); var fam_a1_mov: Greeting_fam_a1_mov = new Greeting_fam_a1_mov(); varfam_a1_sem:Greeting_fam_a1_sem = new Greeting_fam_a1_sem(); 
//I/fam_a2 ----> same as soc_e2 !!!

//fam_a3

var fam_a3: Greeting_fam_a3 = new Greeting_fam_a3();

varfam_a3_zon: Greeting_fam_a3_zon = new Greeting_fam_a3_zon(); var fam_a3_spa: Greeting_fam_a3_spa = new Greeting_fam_a3_spa(); varfam_a3_tou: Greeting_fam_a3_tou = new Greeting_fam_a3_tou(); var fam_a3_mov: Greeting_fam_a3_mov = new Greeting_fam_a3_mov(); var fam_a3_sem:Greeting_fam_a3_sem = new Greeting_fam_a3_sem();

//fam_a4

var fam_a4: Greeting_fam_a4 = new Greeting_fam_a4(); var fam_a4_zon: Greeting_fam_a4_zon = new Greeting_fam_a4_zon(); var fam_a4_spa: Greeting_fam_a4_spa = new Greeting_fam_a4_spa(); var fam_a4_tou: Greeting_fam_a4_tou = new Greeting_fam_a4_tou(); var fam_a4_mov: Greeting_fam_a4_mov = new Greeting_fam_a4_mov(); var fam_a4_sem:Greeting_fam_a4_sem = new Greeting_fam_a4_sem();

//fam_a5

var fam_a5: Greeting_fam_a5 = new Greeting_fam_a5();

var fam_a5_zon: Greeting_fam_a5_zon = new Greeting_fam_a5_zon(); var fam_a5_spa: Greeting_fam_a5_spa = new Greeting_fam_a5_spa(); varfam_a5_tou: Greeting_fam_a5_tou = new Greeting_fam_a5_tou(); var fam_a5_mov: Greeting_fam_a5_mov = new Greeting_fam_a5_mov(); var fam_a5_sem:Greeting_fam_a5_sem = new Greeting_fam_a5_sem();

\section{//fam_b2}

var fam_b2: Greeting_fam_b2 = new Greeting_fam_b2();

var fam_b2_zon: Greeting_fam_b2_zon = new Greeting_fam_b2_zon(); var fam_b2_spa: Greeting_fam_b2_spa = new Greeting_fam_b2_spa(); var fam_b2_tou: Greeting_fam_b2_tou = new Greeting_fam_b2_tou(); var fam_b2_mov: Greeting_fam_b2_mov = new Greeting_fam_b2_mov(); var fam_b2_sem:Greeting_fam_b2_sem = new Greeting_fam_b2_sem();

\section{//fam_b5}

var fam_b5: Greeting_fam_b5 = new Greeting_fam_b5();

var fam_b5_zon: Greeting_fam_b5_zon = new Greeting_fam_b5_zon(); var fam_b5_spa: Greeting_fam_b5_spa = new Greeting_fam_b5_spa(); var fam_b5_tou: Greeting_fam_b5_tou = new Greeting_fam_b5_tou(); var fam_b5_mov: Greeting_fam_b5_mov = new Greeting_fam_b5_mov(); var fam_b5_sem:Greeting_fam_b5_sem = new Greeting_fam_b5_sem();

//fam_c2

var fam_c2: Greeting_fam_c2 = new Greeting_fam_c2();

var fam_c2_zon: Greeting_fam_c2_zon = new Greeting_fam_c2_zon(); var fam_c2_spa: Greeting_fam_c2_spa = new Greeting_fam_c2_spa(); var fam_c2_tou: Greeting_fam_c2_tou = new Greeting_fam_c2_tou(); var fam_c2_mov: Greeting_fam_c2_mov = new Greeting_fam_c2_mov(); var fam_c2_sem:Greeting_fam_c2_sem = new Greeting_fam_c2_sem();

/II/fam_c4 ----> same as soc_a4 !!!

//fam_c5

var fam_c5: Greeting_fam_c5 = new Greeting_fam_c5();

var fam_c5_all: Greeting_fam_c5_all = new Greeting_fam_c5_all();

var fam_c5_zon: Greeting_fam_c5_zon = new Greeting_fam_c5_zon(); 
var fam_c5_spa: Greeting_fam_c5_spa = new Greeting_fam_c5_spa(); var fam_c5_tou: Greeting_fam_c5_tou $=$ new Greeting_fam_c5_tou(); var fam_c5_mov: Greeting_fam_c5_mov = new Greeting_fam_c5_mov(); var fam_c5_sem: Greeting_fam_c5_sem = new Greeting_fam_c5_sem();

\section{/I//fam_d1}

var fam_d1: Greeting_fam_d1 = new Greeting_fam_d1(); var fam_d1_zon: Greeting_fam_d1_zon = new Greeting_fam_d1_zon(); var fam_d1_spa: Greeting_fam_d1_spa = new Greeting_fam_d1_spa(); var fam_d1_tou: Greeting_fam_d1_tou = new Greeting_fam_d1_tou(); var fam_d1_mov: Greeting_fam_d1_mov = new Greeting_fam_d1_mov(); var fam_d1_sem:Greeting_fam_d1_sem = new Greeting_fam_d1_sem();

//fam_d2

var fam_d2: Greeting_fam_d2 = new Greeting_fam_d2();

var fam_d2_zon: Greeting_fam_d2_zon = new Greeting_fam_d2_zon(); var fam_d2_spa: Greeting_fam_d2_spa = new Greeting_fam_d2_spa(); var fam_d2_tou: Greeting_fam_d2_tou = new Greeting_fam_d2_tou(); var fam_d2_mov: Greeting_fam_d2_mov = new Greeting_fam_d2_mov(); var fam_d2_sem:Greeting_fam_d2_sem = new Greeting_fam_d2_sem();

\section{//fam_d5}

var fam_d5: Greeting_fam_d5 = new Greeting_fam_d5();

var fam_d5_zon: Greeting_fam_d5_zon = new Greeting_fam_d5_zon(); var fam_d5_spa: Greeting_fam_d5_spa = new Greeting_fam_d5_spa(); varfam_d5_tou: Greeting_fam_d5_tou = new Greeting_fam_d5_tou(); var fam_d5_mov: Greeting_fam_d5_mov = new Greeting_fam_d5_mov(); var fam_d5_sem: Greeting_fam_d5_sem = new Greeting_fam_d5_sem();

\section{////fam_e1}

var fam_e1: Greeting_fam_e1 = new Greeting_fam_e1();

var fam_e1_zon: Greeting_fam_e1_zon = new Greeting_fam_e1_zon(); var fam_e1_spa: Greeting_fam_e1_spa = new Greeting_fam_e1_spa(); varfam_e1_tou: Greeting_fam_e1_tou = new Greeting_fam_e1_tou(); var fam_e1_mov: Greeting_fam_e1_mov = new Greeting_fam_e1_mov(); var fam_e1_sem:Greeting_fam_e1_sem = new Greeting_fam_e1_sem();

\section{//fam_e2}

var fam_e2: Greeting_fam_e2 = new Greeting_fam_e2();

var fam_e2_zon: Greeting_fam_e2_zon = new Greeting_fam_e2_zon(); var fam_e2_spa: Greeting_fam_e2_spa = new Greeting_fam_e2_spa(); varfam_e2_tou: Greeting_fam_e2_tou = new Greeting_fam_e2_tou(); var fam_e2_mov: Greeting_fam_e2_mov = new Greeting_fam_e2_mov(); var fam_e2_sem:Greeting_fam_e2_sem = new Greeting_fam_e2_sem();

\section{I/I/fam_e3}

var fam_e3: Greeting_fam_e3 = new Greeting_fam_e3();

var fam_e3_zon: Greeting_fam_e3_zon = new Greeting_fam_e3_zon(); var fam_e3_spa: Greeting_fam_e3_spa = new Greeting_fam_e3_spa(); var fam_e3_tou: Greeting_fam_e3_tou = new Greeting_fam_e3_tou(); var fam_e3_mov: Greeting_fam_e3_mov = new Greeting_fam_e3_mov(); var fam_e3_sem:Greeting_fam_e3_sem = new Greeting_fam_e3_sem();

//fam_e4

var fam_e4: Greeting_fam_e4 = new Greeting_fam_e4();

var fam_e4_zon: Greeting_fam_e4_zon = new Greeting_fam_e4_zon(); var fam_e4_spa: Greeting_fam_e4_spa = new Greeting_fam_e4_spa(); 
var fam_e4_tou: Greeting_fam_e4_tou = new Greeting_fam_e4_tou(); var fam_e4_mov: Greeting_fam_e4_mov = new Greeting_fam_e4_mov(); var fam_e4_sem:Greeting_fam_e4_sem = new Greeting_fam_e4_sem();

//fam_e5 = fam_a5

//fam_f2

var fam_f2: Greeting_fam_f2 = new Greeting_fam_f2(); var fam_f2_zon: Greeting_fam_f2_zon = new Greeting_fam_f2_zon(); var fam_f2_spa: Greeting_fam_f2_spa = new Greeting_fam_f2_spa(); var fam_f2_tou: Greeting_fam_f2_tou = new Greeting_fam_f2_tou(); var fam_f2_mov: Greeting_fam_f2_mov = new Greeting_fam_f2_mov(); var fam_f2_sem:Greeting_fam_f2_sem = new Greeting_fam_f2_sem();

// fam_f5 = fam_b5

//fam_g2

var fam_g2: Greeting_fam_g2 = new Greeting_fam_g2();

var fam_g2_zon: Greeting_fam_g2_zon = new Greeting_fam_g2_zon(); var fam_g2_spa: Greeting_fam_g2_spa = new Greeting_fam_g2_spa(); var fam_g2_tou: Greeting_fam_g2_tou = new Greeting_fam_g2_tou(); var fam_g2_mov: Greeting_fam_g2_mov = new Greeting_fam_g2_mov(); var fam_g2_sem:Greeting_fam_g2_sem = new Greeting_fam_g2_sem();

/II/fam_g4

var fam_g4: Greeting_fam_g4 = new Greeting_fam_g4();

var fam_g4_zon: Greeting_fam_g4_zon = new Greeting_fam_g4_zon(); var fam_g4_spa: Greeting_fam_g4_spa = new Greeting_fam_g4_spa(); var fam_g4_tou: Greeting_fam_g4_tou = new Greeting_fam_g4_tou(); var fam_g4_mov: Greeting_fam_g4_mov = new Greeting_fam_g4_mov(); var fam_g4_sem:Greeting_fam_g4_sem = new Greeting_fam_g4_sem();

//I/fam_g5

var fam_g5: Greeting_fam_g5 = new Greeting_fam_g5();

var fam_g5_zon: Greeting_fam_g5_zon = new Greeting_fam_g5_zon(); var fam_g5_spa: Greeting_fam_g5_spa = new Greeting_fam_g5_spa(); varfam_g5_tou: Greeting_fam_g5_tou = new Greeting_fam_g5_tou(); var fam_g5_mov: Greeting_fam_g5_mov = new Greeting_fam_g5_mov(); var fam_g5_sem:Greeting_fam_g5_sem = new Greeting_fam_g5_sem();

//I//fam_h1

var fam_h1: Greeting_fam_h1 = new Greeting_fam_h1();

var fam_h1_zon: Greeting_fam_h1_zon = new Greeting_fam_h1_zon(); var fam_h1_spa: Greeting_fam_h1_spa = new Greeting_fam_h1_spa(); var fam_h1_tou: Greeting_fam_h1_tou = new Greeting_fam_h1_tou(); var fam_h1_mov: Greeting_fam_h1_mov = new Greeting_fam_h1_mov(); var fam_h1_sem:Greeting_fam_h1_sem = new Greeting_fam_h1_sem();

//fam_h2

var fam_h2: Greeting_fam_h2 = new Greeting_fam_h2();

var fam_h2_zon: Greeting_fam_h2_zon = new Greeting_fam_h2_zon(); var fam_h2_spa: Greeting_fam_h2_spa = new Greeting_fam_h2_spa(); var fam_h2_tou: Greeting_fam_h2_tou = new Greeting_fam_h2_tou(); var fam_h2_mov: Greeting_fam_h2_mov = new Greeting_fam_h2_mov(); var fam_h2_sem:Greeting_fam_h2_sem = new Greeting_fam_h2_sem();

//fam_h5

var fam_h5: Greeting_fam_h5 = new Greeting_fam_h5(); 
var fam_h5_zon: Greeting_fam_h5_zon = new Greeting_fam_h5_zon(); var fam_h5_spa: Greeting_fam_h5_spa = new Greeting_fam_h5_spa(); varfam_h5_tou: Greeting_fam_h5_tou = new Greeting_fam_h5_tou(); var fam_h5_mov: Greeting_fam_h5_mov = new Greeting_fam_h5_mov(); var fam_h5_sem:Greeting_fam_h5_sem = new Greeting_fam_h5_sem();

\section{/II/SOCIAL//}

//soc_a2

var soc_a2: Greeting_soc_a2 = new Greeting_soc_a2();

var soc_a2_zon: Greeting_soc_a2_zon = new Greeting_soc_a2_zon(); var soc_a2_spa: Greeting_soc_a2_spa = new Greeting_soc_a2_spa(); var soc_a2_tou: Greeting_soc_a2_tou = new Greeting_soc_a2_tou(); var soc_a2_mov: Greeting_soc_a2_mov = new Greeting_soc_a2_mov(); var soc_a2_sem: Greeting_soc_a2_sem = new Greeting_soc_a2_sem();

\section{//soc_a4}

var soc_a4: Greeting_soc_a4 = new Greeting_soc_a4();

var soc_a4_zon: Greeting_soc_a4_zon = new Greeting_soc_a4_zon(); var soc_a4_spa: Greeting_soc_a4_spa = new Greeting_soc_a4_spa(); var soc_a4_tou: Greeting_soc_a4_tou = new Greeting_soc_a4_tou(); var soc_a4_mov: Greeting_soc_a4_mov = new Greeting_soc_a4_mov(); var soc_a4_sem: Greeting_soc_a4_sem = new Greeting_soc_a4_sem();

\section{//soc a5}

var soc_a5: Greeting_soc_a5 = new Greeting_soc_a5();

var soc_a5_zon: Greeting_soc_a5_zon = new Greeting_soc_a5_zon(); var soc_a5_spa: Greeting_soc_a5_spa = new Greeting_soc_a5_spa(); var soc_a5_tou: Greeting_soc_a5_tou $=$ new Greeting_soc_a5_tou(); var soc_a5_mov: Greeting_soc_a5_mov = new Greeting_soc_a5_mov(); var soc_a5_sem: Greeting_soc_a5_sem = new Greeting_soc_a5_sem();

\section{//soc_b2}

var soc_b2: Greeting_soc_b2 = new Greeting_soc_b2();

var soc_b2_zon: Greeting_soc_b2_zon = new Greeting_soc_b2_zon(); var soc_b2_spa: Greeting_soc_b2_spa = new Greeting_soc_b2_spa(); var soc_b2_tou: Greeting_soc_b2_tou = new Greeting_soc_b2_tou(); var soc_b2_mov: Greeting_soc_b2_mov = new Greeting_soc_b2_mov(); var soc_b2_sem: Greeting_soc_b2_sem = new Greeting_soc_b2_sem()

\section{/I//soc b3}

var soc_b3: Greeting_soc_b3 = new Greeting_soc_b3(); var soc_b3_zon: Greeting_soc_b3_zon = new Greeting_soc_b3_zon(); var soc_b3_spa: Greeting_soc_b3_spa = new Greeting_soc_b3_spa(); var soc_b3_tou: Greeting_soc_b3_tou = new Greeting_soc_b3_tou(); var soc_b3_mov: Greeting_soc_b3_mov = new Greeting_soc_b3_mov(); var soc_b3_sem: Greeting_soc_b3_sem = new Greeting_soc_b3_sem();

\section{//soc_b4}

var soc_b4: Greeting_soc_b4 = new Greeting_soc_b4();

var soc_b4_zon: Greeting_soc_b4_zon = new Greeting_soc_b4_zon(); var soc_b4_spa: Greeting_soc_b4_spa = new Greeting_soc_b4_spa(); var soc_b4_tou: Greeting_soc_b4_tou = new Greeting_soc_b4_tou(); var soc_b4_mov: Greeting_soc_b4_mov = new Greeting_soc_b4_mov(); var soc_b4_sem: Greeting_soc_b4_sem = new Greeting_soc_b4_sem();

//soc_b5

var soc_b5: Greeting_soc_b5 = new Greeting_soc_b5(); 
var soc_b5_zon: Greeting_soc_b5_zon = new Greeting_soc_b5_zon(); var soc_b5_spa: Greeting_soc_b5_spa = new Greeting_soc_b5_spa(); var soc_b5_tou: Greeting_soc_b5_tou = new Greeting_soc_b5_tou(); var soc_b5_mov: Greeting_soc_b5_mov = new Greeting_soc_b5_mov(); var soc_b5_sem: Greeting_soc_b5_sem = new Greeting_soc_b5_sem();

/II/soc_c1

var soc_c1: Greeting_soc_c1 = new Greeting_soc_c1(); var soc_c1_zon: Greeting_soc_c1_zon = new Greeting_soc_c1_zon(); var soc_c1_spa: Greeting_soc_c1_spa = new Greeting_soc_c1_spa(); var soc_c1_tou: Greeting_soc_c1_tou = new Greeting_soc_c1_tou(); var soc_c1_mov: Greeting_soc_c1_mov = new Greeting_soc_c1_mov(); var soc_c1_sem: Greeting_soc_c1_sem = new Greeting_soc_c1_sem();

//soc_c2

var soc_c2: Greeting_soc_c2 = new Greeting_soc_c2();

var soc_c2_zon: Greeting_soc_c2_zon = new Greeting_soc_c2_zon(); var soc_c2_spa: Greeting_soc_c2_spa = new Greeting_soc_c2_spa(); var soc_c2_tou: Greeting_soc_c2_tou = new Greeting_soc_c2_tou(); var soc_c2_mov: Greeting_soc_c2_mov = new Greeting_soc_c2_mov(); var soc_c2_sem: Greeting_soc_c2_sem = new Greeting_soc_c2_sem();

//soc_c3

var soc_c3: Greeting_soc_c3 = new Greeting_soc_c3();

var soc_c3_zon: Greeting_soc_c3_zon = new Greeting_soc_c3_zon(); var soc_c3_spa: Greeting_soc_c3_spa = new Greeting_soc_c3_spa(); var soc_c3_tou: Greeting_soc_c3_tou = new Greeting_soc_c3_tou(); var soc_c3_mov: Greeting_soc_c3_mov = new Greeting_soc_c3_mov(); var soc_c3_sem: Greeting_soc_c3_sem = new Greeting_soc_c3_sem();

//soc_c4

var soc_c4: Greeting_soc_c4 = new Greeting_soc_c4(); var soc_c4_zon: Greeting_soc_c4_zon = new Greeting_soc_c4_zon(); var soc_c4_spa: Greeting_soc_c4_spa = new Greeting_soc_c4_spa(); var soc_c4_tou: Greeting_soc_c4_tou = new Greeting_soc_c4_tou(); var soc_c4_mov: Greeting_soc_c4_mov = new Greeting_soc_c4_mov(); var soc_c4_sem: Greeting_soc_c4_sem = new Greeting_soc_c4_sem();

//soc_c5

var soc_c5: Greeting_soc_c5 = new Greeting_soc_c5(); var soc_c5_zon: Greeting_soc_c5_zon = new Greeting_soc_c5_zon(); var soc_c5_spa: Greeting_soc_c5_spa = new Greeting_soc_c5_spa(); var soc_c5_tou: Greeting_soc_c5_tou = new Greeting_soc_c5_tou(); var soc_c5_mov: Greeting_soc_c5_mov = new Greeting_soc_c5_mov(); var soc_c5_sem: Greeting_soc_c5_sem = new Greeting_soc_c5_sem();

III/soc_d2

var soc_d2: Greeting_soc_d2 = new Greeting_soc_d2();

var soc_d2_zon: Greeting_soc_d2_zon = new Greeting_soc_d2_zon(); var soc_d2_spa: Greeting_soc_d2_spa = new Greeting_soc_d2_spa(); var soc_d2_tou: Greeting_soc_d2_tou = new Greeting_soc_d2_tou(); var soc_d2_mov: Greeting_soc_d2_mov = new Greeting_soc_d2_mov(); var soc_d2_sem: Greeting_soc_d2_sem = new Greeting_soc_d2_sem()

//soc_d4 
var soc_d4: Greeting_soc_d4 = new Greeting_soc_d4();

var soc_d4_zon: Greeting_soc_d4_zon = new Greeting_soc_d4_zon(); var soc_d4_spa: Greeting_soc_d4_spa = new Greeting_soc_d4_spa(); var soc_d4_tou: Greeting_soc_d4_tou = new Greeting_soc_d4_tou(); var soc_d4_mov: Greeting_soc_d4_mov = new Greeting_soc_d4_mov(); var soc_d4_sem: Greeting_soc_d4_sem = new Greeting_soc_d4_sem()

//soc_d5

var soc_d5: Greeting_soc_d5 = new Greeting_soc_d5();

var soc_d5_zon: Greeting_soc_d5_zon = new Greeting_soc_d5_zon(); var soc_d5_spa: Greeting_soc_d5_spa = new Greeting_soc_d5_spa(); var soc_d5_tou: Greeting_soc_d5_tou $=$ new Greeting_soc_d5_tou(); var soc_d5_mov: Greeting_soc_d5_mov = new Greeting_soc_d5_mov(); var soc_d5_sem: Greeting_soc_d5_sem = new Greeting_soc_d5_sem();

III/soc_e2

var soc_e2: Greeting_soc_e2 = new Greeting_soc_e2();

var soc_e2_zon: Greeting_soc_e2_zon = new Greeting_soc_e2_zon(); var soc_e2_spa: Greeting_soc_e2_spa = new Greeting_soc_e2_spa(); var soc_e2_tou: Greeting_soc_e2_tou = new Greeting_soc_e2_tou(); var soc_e2_mov: Greeting_soc_e2_mov = new Greeting_soc_e2_mov(); var soc_e2_sem: Greeting_soc_e2_sem = new Greeting_soc_e2_sem();

//soc_e4

var soc_e4: Greeting_soc_e4 = new Greeting_soc_e4();

var soc_e4_zon: Greeting_soc_e4_zon = new Greeting_soc_e4_zon(); var soc_e4_spa: Greeting_soc_e4_spa = new Greeting_soc_e4_spa(); var soc_e4_tou: Greeting_soc_e4_tou = new Greeting_soc_e4_tou(); var soc_e4_mov: Greeting_soc_e4_mov = new Greeting_soc_e4_mov(); var soc_e4_sem: Greeting_soc_e4_sem = new Greeting_soc_e4_sem()

//soc_e5

var soc_e5: Greeting_soc_e5 = new Greeting_soc_e5();

var soc_e5_zon: Greeting_soc_e5_zon = new Greeting_soc_e5_zon(); var soc_e5_spa: Greeting_soc_e5_spa = new Greeting_soc_e5_spa(); var soc_e5_tou: Greeting_soc_e5_tou = new Greeting_soc_e5_tou(); var soc_e5_mov: Greeting_soc_e5_mov = new Greeting_soc_e5_mov(); var soc_e5_sem: Greeting_soc_e5_sem = new Greeting_soc_e5_sem();

III/soc_f1

var soc_f1: Greeting_soc_f1 = new Greeting_soc_f1(); var soc_f1_zon: Greeting_soc_f1_zon = new Greeting_soc_f1_zon(); var soc_f1_spa: Greeting_soc_f1_spa = new Greeting_soc_f1_spa(); var soc_f1_tou: Greeting_soc_f1_tou = new Greeting_soc_f1_tou(); var soc_f1_mov: Greeting_soc_f1_mov = new Greeting_soc_f1_mov(); var soc_f1_sem: Greeting_soc_f1_sem = new Greeting_soc_f1_sem();

I///soc_f2

var soc_f2: Greeting_soc_f2 = new Greeting_soc_f2(); var soc_f2_zon: Greeting_soc_f2_zon = new Greeting_soc_f2_zon(); var soc_f2_spa: Greeting_soc_f2_spa = new Greeting_soc_f2_spa(); var soc_f2_tou: Greeting_soc_f2_tou = new Greeting_soc_f2_tou(); var soc_f2_mov: Greeting_soc_f2_mov = new Greeting_soc_f2_mov(); var soc_f2_sem: Greeting_soc_f2_sem = new Greeting_soc_f2_sem(); 
//soc_f4

var soc_f4: Greeting_soc_f4 = new Greeting_soc_f4();

var soc_f4_zon: Greeting_soc_f4_zon = new Greeting_soc_f4_zon();

var soc_f4_spa: Greeting_soc_f4_spa = new Greeting_soc_f4_spa();

var soc_f4_tou: Greeting_soc_f4_tou = new Greeting_soc_f4_tou();

var soc_f4_mov: Greeting_soc_f4_mov = new Greeting_soc_f4_mov(); var soc_f4_sem: Greeting_soc_f4_sem = new Greeting_soc_f4_sem();

//soc_f5

var soc_f5: Greeting_soc_f5 = new Greeting_soc_f5();

var soc_f5_zon: Greeting_soc_f5_zon = new Greeting_soc_f5_zon(); var soc_f5_spa: Greeting_soc_f5_spa $=$ new Greeting_soc_f5_spa(); var soc_f5_tou: Greeting_soc_f5_tou = new Greeting_soc_f5_tou(); var soc_f5_mov: Greeting_soc_f5_mov = new Greeting_soc_f5_mov(); var soc_f5_sem: Greeting_soc_f5_sem = new Greeting_soc_f5_sem();

/II/soc_g2

var soc_g2: Greeting_soc_g2 = new Greeting_soc_g2();

var soc_g2_zon: Greeting_soc_g2_zon = new Greeting_soc_g2_zon(); var soc_g2_spa: Greeting_soc_g2_spa = new Greeting_soc_g2_spa(); var soc_g2_tou: Greeting_soc_g2_tou = new Greeting_soc_g2_tou(); var soc_g2_mov: Greeting_soc_g2_mov = new Greeting_soc_g2_mov(); var soc_g2_sem: Greeting_soc_g2_sem = new Greeting_soc_g2_sem();

\section{//soc_g4}

var soc_g4: Greeting_soc_g4 = new Greeting_soc_g4();

var soc_g4_zon: Greeting_soc_g4_zon = new Greeting_soc_g4_zon(); var soc_g4_spa: Greeting_soc_g4_spa = new Greeting_soc_g4_spa(); var soc_g4_tou: Greeting_soc_g4_tou = new Greeting_soc_g4_tou(); var soc_g4_mov: Greeting_soc_g4_mov = new Greeting_soc_g4_mov(); var soc_g4_sem: Greeting_soc_g4_sem = new Greeting_soc_g4_sem();

\section{//soc_g5}

var soc_g5: Greeting_soc_g5 = new Greeting_soc_g5();

var soc_g5_zon: Greeting_soc_g5_zon = new Greeting_soc_g5_zon(); var soc_g5_spa: Greeting_soc_g5_spa = new Greeting_soc_g5_spa(); var soc_g5_tou: Greeting_soc_g5_tou = new Greeting_soc_g5_tou(); var soc_g5_mov: Greeting_soc_g5_mov = new Greeting_soc_g5_mov(); var soc_g5_sem: Greeting_soc_g5_sem = new Greeting_soc_g5_sem();

\section{II//soc_h2}

var soc_h2: Greeting_soc_h2 = new Greeting_soc_h2();

var soc_h2_zon: Greeting_soc_h2_zon = new Greeting_soc_h2_zon(); var soc_h2_spa: Greeting_soc_h2_spa = new Greeting_soc_h2_spa(); var soc_h2_tou: Greeting_soc_h2_tou = new Greeting_soc_h2_tou(); var soc_h2_mov: Greeting_soc_h2_mov = new Greeting_soc_h2_mov(); var soc_h2_sem: Greeting_soc_h2_sem = new Greeting_soc_h2_sem();

\section{I/I/soc_h3}

var soc_h3: Greeting_soc_h3 = new Greeting_soc_h3();

var soc_h3_zon: Greeting_soc_h3_zon = new Greeting_soc_h3_zon(); var soc_h3_spa: Greeting_soc_h3_spa = new Greeting_soc_h3_spa(); var soc_h3_tou: Greeting_soc_h3_tou = new Greeting_soc_h3_tou(); 
var soc_h3_mov: Greeting_soc_h3_mov = new Greeting_soc_h3_mov(); var soc_h3_sem: Greeting_soc_h3_sem = new Greeting_soc_h3_sem();

//soc_h4

var soc_h4: Greeting_soc_h4 = new Greeting_soc_h4();

var soc_h4_zon: Greeting_soc_h4_zon = new Greeting_soc_h4_zon(); var soc_h4_spa: Greeting_soc_h4_spa = new Greeting_soc_h4_spa(); var soc_h4_tou: Greeting_soc_h4_tou = new Greeting_soc_h4_tou(); var soc_h4_mov: Greeting_soc_h4_mov = new Greeting_soc_h4_mov(); var soc_h4_sem: Greeting_soc_h4_sem = new Greeting_soc_h4_sem();

//soc_h5

var soc_h5: Greeting_soc_h5 = new Greeting_soc_h5();

var soc_h5_zon: Greeting_soc_h5_zon = new Greeting_soc_h5_zon(); var soc_h5_spa: Greeting_soc_h5_spa = new Greeting_soc_h5_spa(); var soc_h5_tou: Greeting_soc_h5_tou = new Greeting_soc_h5_tou(); var soc_h5_mov: Greeting_soc_h5_mov = new Greeting_soc_h5_mov(); var soc_h5_sem: Greeting_soc_h5_sem = new Greeting_soc_h5_sem();

//soc_i1

var soc_i1: Greeting_soc_i1 = new Greeting_soc_i1();

var soc_i1_zon: Greeting_soc_i1_zon = new Greeting_soc_i1_zon(); var soc_i1_spa: Greeting_soc_i1_spa = new Greeting_soc_i1_spa(); var soc_i1_tou: Greeting_soc_i1_tou = new Greeting_soc_i1_tou(); var soc_i1_mov: Greeting_soc_i1_mov = new Greeting_soc_i1_mov(); var soc_i1_sem: Greeting_soc_i1_sem = new Greeting_soc_i1_sem();

I/I/soc_i2

var soc_i2: Greeting_soc_i2 = new Greeting_soc_i2();

var soc_i2_zon: Greeting_soc_i2_zon = new Greeting_soc_i2_zon(); var soc_i2_spa: Greeting_soc_i2_spa = new Greeting_soc_i2_spa(); var soc_i2_tou: Greeting_soc_i2_tou = new Greeting_soc_i2_tou(); var soc_i2_mov: Greeting_soc_i2_mov = new Greeting_soc_i2_mov(); var soc_i2_sem: Greeting_soc_i2_sem = new Greeting_soc_i2_sem();

//soc_i3

var soc_i3: Greeting_soc_i3 = new Greeting_soc_i3();

var soc_i3_zon: Greeting_soc_i3_zon = new Greeting_soc_i3_zon(); var soc_i3_spa: Greeting_soc_i3_spa = new Greeting_soc_i3_spa(); var soc_i3_tou: Greeting_soc_i3_tou $=$ new Greeting_soc_i3_tou(); var soc_i3_mov: Greeting_soc_i3_mov = new Greeting_soc_i3_mov(); var soc_i3_sem: Greeting_soc_i3_sem = new Greeting_soc_i3_sem();

\section{//soc_i4}

var Soc_i4: Greeting_soc_i4 = new Greeting_soc_i4();

var soc_i4_zon: Greeting_soc_i4_zon = new Greeting_soc_i4_zon(); var soc_i4_spa: Greeting_soc_i4_spa = new Greeting_soc_i4_spa(); var soc_i4_tou: Greeting_soc_i4_tou = new Greeting_soc_i4_tou(); var soc_i4_mov: Greeting_soc_i4_mov = new Greeting_soc_i4_mov(); var soc_i4_sem: Greeting_soc_i4_sem = new Greeting_soc_i4_sem();

//soc_i5

var soc_i5: Greeting_soc_i5 = new Greeting_soc_i5();

var soc_i5_zon: Greeting_soc_i5_zon = new Greeting_soc_i5_zon(); var soc_i5_spa: Greeting_soc_i5_spa = new Greeting_soc_i5_spa(); var soc_i5_tou: Greeting_soc_i5_tou = new Greeting_soc_i5_tou(); var soc_i5_mov: Greeting_soc_i5_mov=new Greeting_soc_i5_mov(); 
var soc_i5_sem: Greeting_soc_i5_sem = new Greeting_soc_i5_sem();

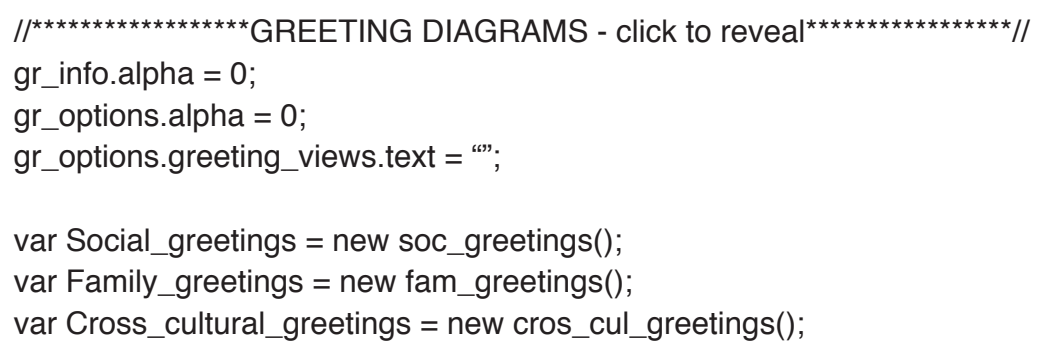




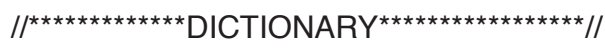

var currentSet: String;

var dict: Dictionary = new Dictionary();

//Cross-Cultural//

$\operatorname{dict}[$ "btncross11"] = crossa1_btn;

$\operatorname{dict}[$ "crossa1"] = cross_a1;

$\operatorname{dict}[$ "crossa11"] = cross_a1_mov;

dict["crossa12"] = cross_a1_spa;

dict["crossa13"] = cross_a1_tou;

$\operatorname{dict}[$ "crossa14"] = cross_a1_zon;

dict["crossa15"] = cross_a1_sem;

$\operatorname{dict}[$ "btncross12"] = crossa2_btn;

$\operatorname{dict}[$ "crossa2"] = cross_a2;

dict["crossa21"] = cross_a2_mov;

dict["crossa22"] = cross_a2_spa;

dict["crossa23"] = cross_a2_tou;

dict["crossa24"] = cross_a2_zon;

dict["crossa25"] = cross_a2_sem;

$\operatorname{dict}[$ "btncross13"] = crossa3_btn; dict["crossa3"] = cross_a3;

$\operatorname{dict}[$ "crossa31"] = cross_a3_mov;

dict["crossa32"] = cross_a3_spa;

dict["crossa33"] = cross_a3_tou;

dict["crossa34"] = cross_a3_zon;

dict["crossa35"] = cross_a3_sem;

$\operatorname{dict}[$ "bthcross22"] = crossb2_btn;

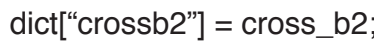

$\operatorname{dict}[$ "crossb21"] = cross_b2_mov;

$\operatorname{dict}[$ "crossb22"] = cross_b2_spa;

dict["crossb23"] = cross_b2_tou;

dict["crossb24"] = cross_b2_zon;

dict["crossb25"] = cross_b2_sem;

$\operatorname{dict}[$ "btncross23"] = crossb3_btn; $\operatorname{dict}[$ "crossb3"] = cross_b3;

$\operatorname{dict}[$ "crossb31"] = cross_b3_mov; $\operatorname{dict}[$ "crossb32"] = cross_b3_spa;

dict["crossb33"] = cross_b3_tou;

$\operatorname{dict}[$ "crossb34"] = cross_b3_zon;

dict["crossb35"] = cross_b3_sem;

$\operatorname{dict}[$ "btncross24"] = crossb4_btn; $\operatorname{dict}[$ "crossb4"] = cross_b4;

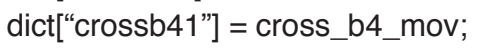
$\operatorname{dict}[$ "crossb42"] = cross_b4_spa; $\operatorname{dict}[$ "crossb43"] = cross_b4_tou; $\operatorname{dict}[$ "crossb44"] = cross_b4_zon; dict["crossb45"] = cross_b4_sem;

$\operatorname{dict}[$ "bthcross31"] = crossc1_btn; $\operatorname{dict}[$ [crossc1"] = cross_c1; 


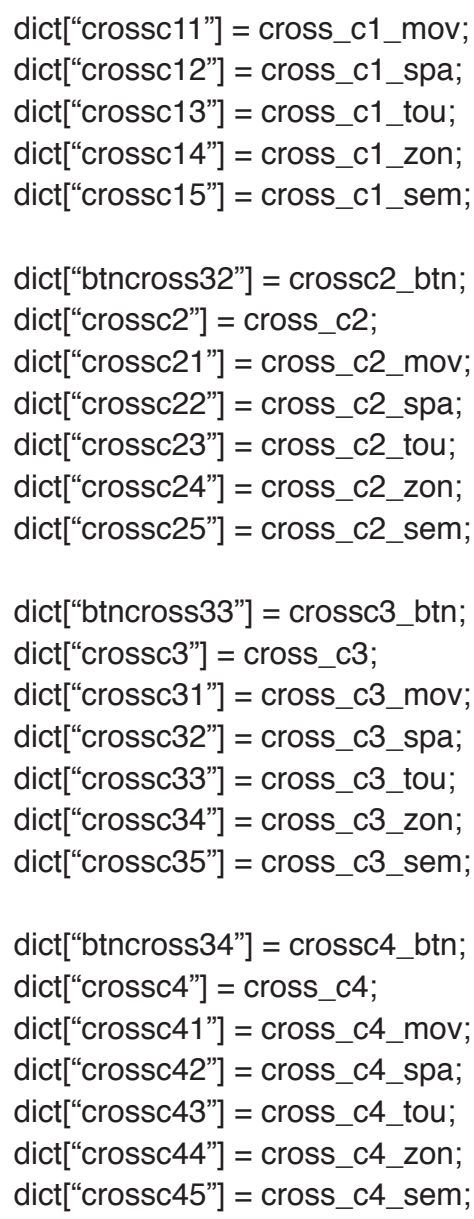

$\operatorname{dict}[$ "btncross35"] = crossc5_btn; $\operatorname{dict}[$ "crossc5"] = cross_c5;

$\operatorname{dict}[$ "crossc51"] = cross_c5_mov; $\operatorname{dict}[$ "crossc52"] = cross_c5_spa; dict["crossc53"] = cross_c5_tou; dict["crossc54"] = cross_c5_zon; $\operatorname{dict}[$ "crossc55"] = cross_c5_sem;

$\operatorname{dict}[$ "btncross36"] = crossc6_btn; $\operatorname{dict}[$ "crossc6"] = cross_c6;

$\operatorname{dict}[$ "crossc61"] = cross_c6_mov; dict["crossc62"] = cross_c6_spa; dict["crossc63"] = cross_c6_tou; $\operatorname{dict}[$ "crossc64"] = cross_c6_zon; $\operatorname{dict}[$ "crossc65"] = cross_c6_sem;

$\operatorname{dict}[$ "btncross41"] = crossd1_btn; $\operatorname{dict}[$ [crossd1"] = cross_d1 ;

$\operatorname{dict}[$ "crossd11"] = cross_d1_mov; $\operatorname{dict}[$ "crossd12"] = cross_d1_spa; dict["crossd13"] = cross_d1_tou; $\operatorname{dict}[$ "crossd14"] = cross_d1_zon; $\operatorname{dict}[$ "crossd15"] = cross_d1_sem;

$\operatorname{dict}[$ "btncross42"] = crossd2_btn; $\operatorname{dict}[$ "crossd2"] = cross_d2;

$\operatorname{dict}[$ "crossd21"] = cross_d2_mov; $\operatorname{dict}[$ "crossd22"] = cross_d2_spa; 
$\operatorname{dict}[$ "crossd23"] = cross_d2_tou; $\operatorname{dict}[$ "crossd24"] = cross_d2_zon; dict["crossd25"] = cross_d2_sem;

$\operatorname{dict}[$ "btncross43"] = crossd3_btn; $\operatorname{dict}[$ [crossd3"] = cross_d3;

$\operatorname{dict}[$ "crossd31"] = cross_d3_mov; $\operatorname{dict}[$ "crossd32"] = cross_d3_spa; dict["crossd33"] = cross_d3_tou; $\operatorname{dict}[$ "crossd34"] = cross_d3_zon; $\operatorname{dict}[$ "crossd35"] = cross_d3_sem;

$\operatorname{dict}[$ "btncross52"] = crosse2_btn; $\operatorname{dict}[$ "crosse2"] = cross_e2;

dict["crosse21"] = cross_e2_mov; $\operatorname{dict}[$ "crosse22"] = cross_e2_spa; dict["crosse23"] = cross_e2_tou; $\operatorname{dict}[$ "crosse24"] = cross_e2_zon; dict["crosse25"] = cross_e2_sem;

$\operatorname{dict}[$ "btncross53"] = crosse3_btn; $\operatorname{dict}[$ "crosse3"] = cross_e3;

$\operatorname{dict}[$ "crosse31"] = cross_e3_mov; $\operatorname{dict}[$ "crosse32"] = cross_e3_spa; $\operatorname{dict}[$ "crosse33"] = cross_e3_tou; $\operatorname{dict}[$ "crosse34"] = cross_e3_zon; dict["crosse35"] = cross_e3_sem;

$\operatorname{dict}[$ "btncross61"] = crossf1_btn; $\operatorname{dict}[$ [crossf1"] = cross_f1; $\operatorname{dict}[$ "crossf11"] = cross_f1_mov; $\operatorname{dict}[$ "crossf12"] = cross_f1_spa; $\operatorname{dict}[$ "crossf13"] = cross_f1_tou; $\operatorname{dict}[$ "crossf14"] = cross_f1_zon; $\operatorname{dict}[$ "crossf15"] = cross_f1_sem;

$\operatorname{dict}[$ "btncross65"] = crossf5_btn; $\operatorname{dict}[$ "crossf5"] = cross_f5;

$\operatorname{dict}[$ "crossf51"] = cross_f5_mov; $\operatorname{dict}[$ "crossf52"] = cross_f5_spa; $\operatorname{dict}[$ "crossf53"] = cross_f5_tou; $\operatorname{dict}[$ "crossf54"] = cross_f5_zon; $\operatorname{dict}[$ “crossf55”] = cross_f5_sem;

$\operatorname{dict}[$ "btncross66"] = crossf6_btn; $\operatorname{dict[}$ [crossf6"] = cross_f6;

$\operatorname{dict}[$ "crossf61"] = cross_f6_mov; $\operatorname{dict}[$ "crossf62"] = cross_f6_spa; $\operatorname{dict}[$ "crossf63"] = cross_f6_tou; dict["crossf64"] = cross_f6_zon; $\operatorname{dict}[$ "crossf65"] = cross_f6_sem;

//Family//

$\operatorname{dict}[$ "btnfam11"] = fama1_btn; $\operatorname{dict["fama1"]~=~fam\_ a1;~}$ $\operatorname{dict}[$ "fama11"] = fam_a1_mov; $\operatorname{dict}[$ "fama12"] = fam_a1_spa; 


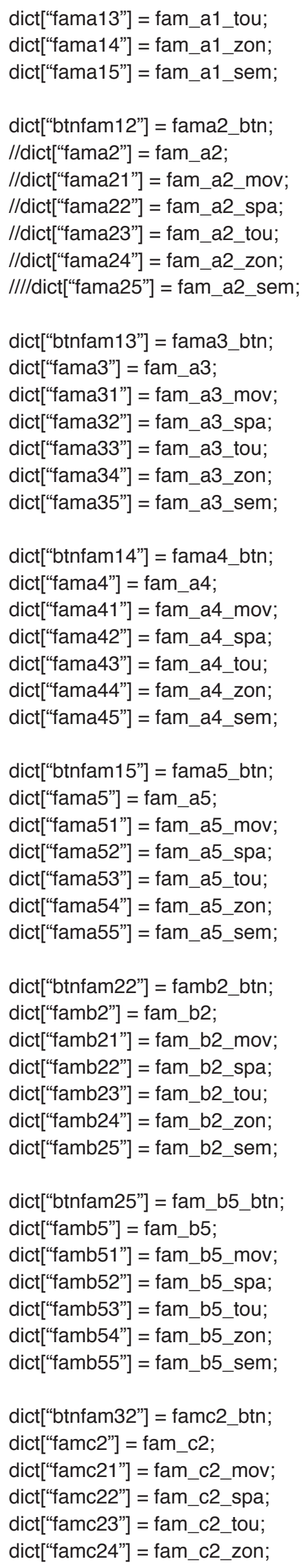




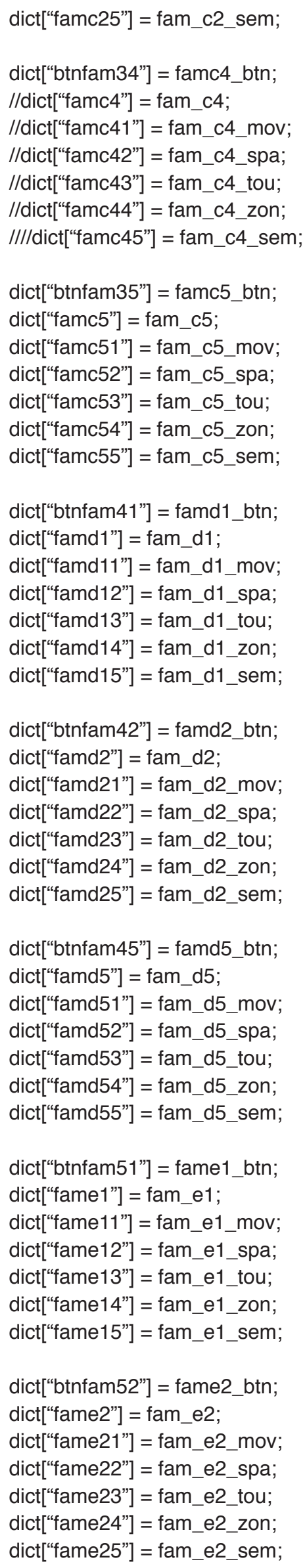




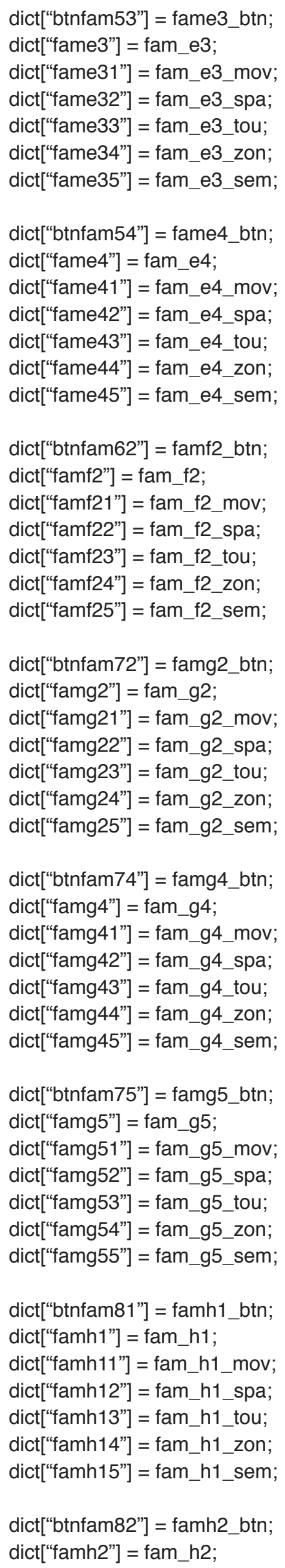




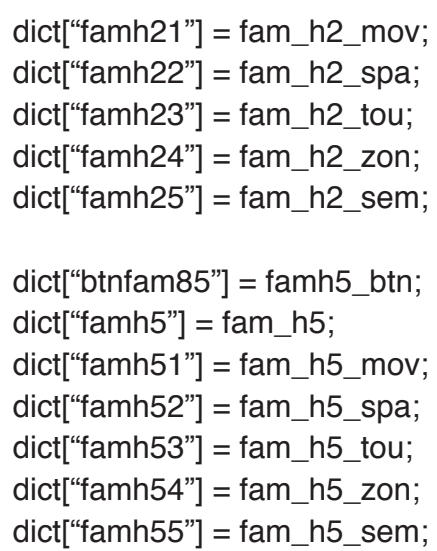

//Social//

$\operatorname{dict}[$ "btnsoc12"] = soca2_btn; $\operatorname{dict}[$ "soca2"] = soc_a2;

dict["soca21"] = soc_a2_mov; $\operatorname{dict}[$ "soca22"] = soc_a2_spa; $\operatorname{dict}[$ "soca23"] = soc_a2_tou; dict["soca24"] = soc_a2_zon; dict["soca25"] = soc_a2_sem;

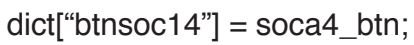
dict["soca4"] = soc_a4;

$\operatorname{dict}[$ "soca41"] = soc_a4_mov; $\operatorname{dict}[$ "soca42"] = soc_a4_spa; dict["soca43"] = soc_a4_tou;

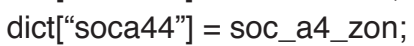
$\operatorname{dict}[$ "soca45"] = soc_a4_sem;

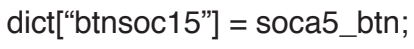
$\operatorname{dict}[$ "soca5"] = soc_a5;

$\operatorname{dict}[$ "soca51"] = soc_a5_mov; dict["soca52"] = soc_a5_spa; dict["soca53"] = soc_a5_tou; dict["soca54"] = soc_a5_zon; dict["soca55"] = soc_a5_sem;

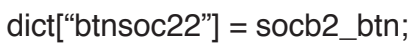
$\operatorname{dict}[$ "socb2"] = soc_b2;

$\operatorname{dict}[$ "socb21"] = soc_b2_mov; dict["socb22"] = soc_b2_spa; $\operatorname{dict}[$ "socb23"] = soc_b2_tou;

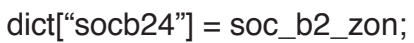
$\operatorname{dict}[$ "socb25"] = soc_b2_sem;

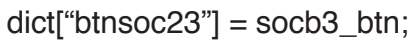
$\operatorname{dict}[$ "socb3"] = soc_b3;

$\operatorname{dict}[$ "socb31"] = soc_b3_mov;

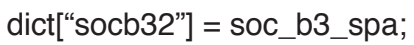
$\operatorname{dict}[$ "socb33"] = soc_b3_tou; dict["socb34"] = soc_b3_zon; dict["socb35"] = soc_b3_sem;

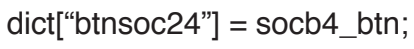
$\operatorname{dict}[$ "socb4"] = soc_b4; 


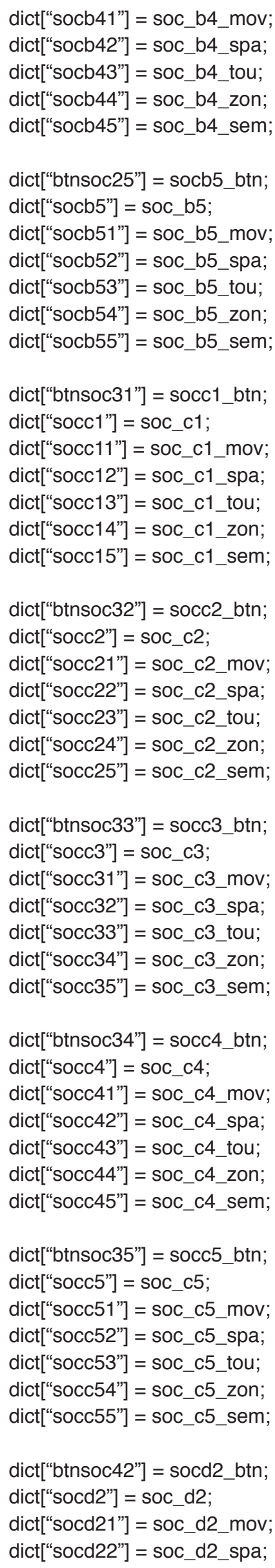




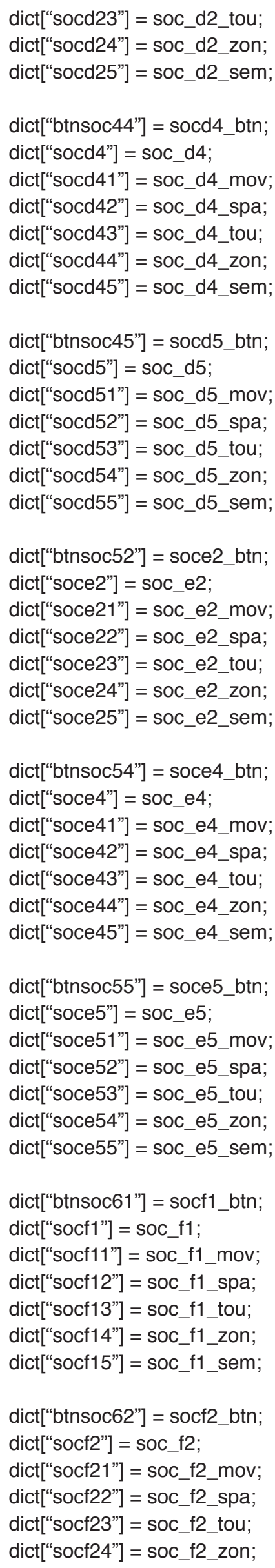




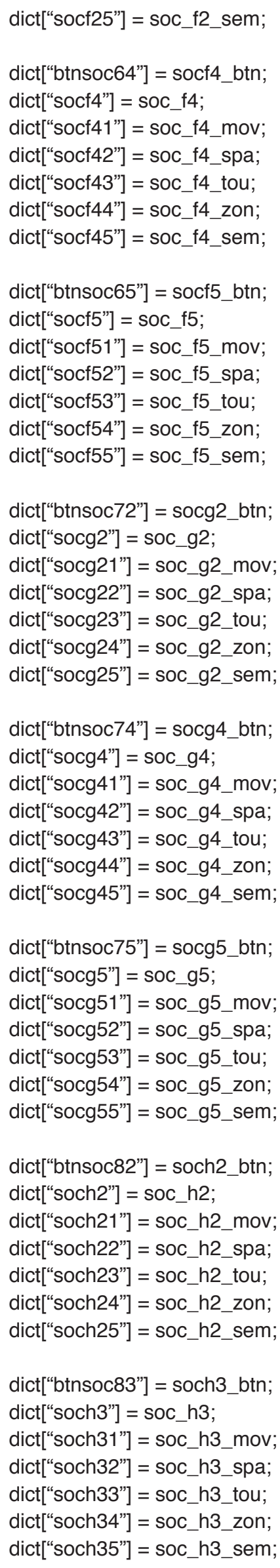




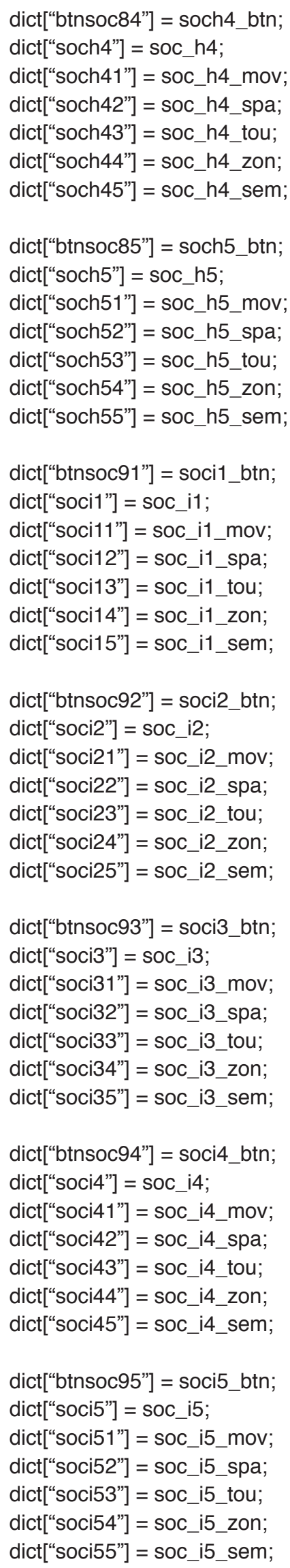


var i: Number;

gr_options.nor_btn.addEventListener(MouseEvent.MOUSE_DOWN, view_greeting_normal); function view_greeting_normal(event: MouseEvent): void \{

trace(dict[currentSet]);

(dict[currentSet]).visible = true;

(dict[currentSet]).gotoAndPlay(1);

$($ dict[currentSet +1$]) \cdot$ visible $=$ false;

$($ dict [currentSet +2$])$. visible $=$ false;

$($ dict $[$ currentSet +3$]) \cdot$ visible $=$ false;

$($ dict [currentSet +4$])$. visible $=$ false;

$($ dict[currentSet +5$]) \cdot$ visible $=$ false;

\}

gr_options.greeting_views.text = "Vector Animation";

gr_options.mov_btn.addEventListener(MouseEvent.MOUSE_DOWN, view_greeting_movie); function view_greeting_movie(event: MouseEvent): void \{

gr_options.greeting_views.text = "Greeting Video";

$\mathrm{i}=1$;

trace(dict[currentSet]);

$($ dict $[$ currentSet $+\mathrm{i}])$.visible $=$ true;

(dict[currentSet + i]).gotoAndPlay(1);

(dict[currentSet]) .visible $=$ false;

$($ dict[currentSet +2$])$. visible $=$ false

$($ dict [currentSet +3$]) \cdot$ visible $=$ false;

$($ dict $[$ currentSet +4$])$. visible $=$ false;

(dict[currentSet +5$]) \cdot$ visible = false;

\}

gr_options.spa_btn.addEventListener(MouseEvent.MOUSE_DOWN, view_greeting_space); function view_greeting_space(event: MouseEvent): void \{

gr_options.greeting_views.text = "Space In-between";

$\mathrm{i}=2$;

trace(dict[currentSet $+i]$ );

$($ dict $[$ currentSet $+i]) \cdot$ visible $=$ true;

(dict[currentSet $+\mathrm{i}]$ ).gotoAndPlay $(1)$;

(dict[currentSet]). visible = false;

(dict[currentSet +1$])$. visible $=$ false;

$($ dict[currentSet +3$]) \cdot$ visible $=$ false;

$($ dict [currentSet +4$])$. visible $=$ false;

$($ dict[currentSet +5$]) \cdot$ visible $=$ false;

\}

gr_options.tou_btn.addEventListener(MouseEvent.MOUSE_DOWN, view_greeting_touch); function view_greeting_touch(event: MouseEvent): void \{

gr_options.greeting_views.text = "Physical Contact/ Touch";

$\mathrm{i}=3$;

trace (dict[currentSet $+\mathrm{i}]$ );

(dict[currentSet $+\mathrm{i}]$ ). visible $=$ true;

(dict[currentSet $+\mathrm{i}]$ ).gotoAndPlay(1);

(dict[currentSet]). visible $=$ false;

$($ dict[currentSet +1$]) \cdot$ visible $=$ false;

$($ dict[currentSet +2$]) \cdot$ visible $=$ false;

$($ dict [currentSet +4$]) \cdot$ visible $=$ false;

$($ dict[currentSet +5$]) \cdot$ visible $=$ false;

\}

gr_options.zon_btn.addEventListener(MouseEvent.MOUSE_DOWN, view_greeting_zone); 


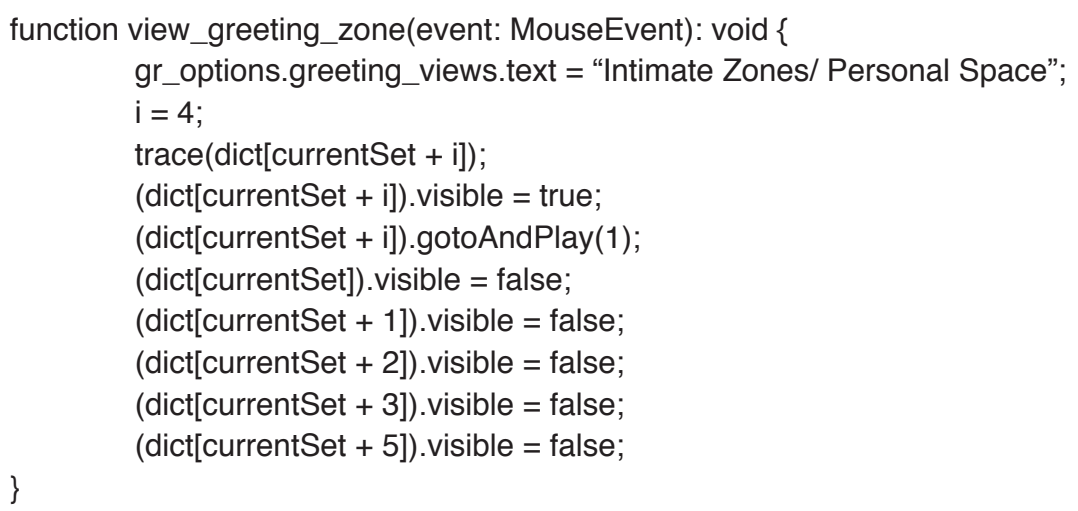

gr_options.sem_btn.addEventListener(MouseEvent.MOUSE_DOWN, view_greeting_semiotics); function view_greeting_semiotics(event: MouseEvent): void \{ gr_options.greeting_views.text = "Subjective Meaning/Experience";

\section{//cross_a1 greeting}

Cross_cultural_greetings.cross_a1.addEventListener(MouseEvent.CLICK, gotoMovieCrossA1); function gotoMovieCrossA1 (myEvent: MouseEvent): void \{ while (container.numChildren) \{ container.removeChildAt(0);

true);

gr_options.alpha = 100;

gr_info.alpha $=100$

gr_info.txt_country.text = "Chilean/ Norwegian";

gr_info.txt_greeting.text = "Cross-Cultural, Strangers";

\}

gr_info.txt_gender.text = "Male/Male";

\}

//cross_a2 greeting 
Cross_cultural_greetings.cross_a2.addEventListener(MouseEvent.CLICK, gotoMovieCrossA2); function gotoMovieCrossA2(myEvent: MouseEvent): void \{ while (container.numChildren) \{ container.removeChildAt(0);

\} for(var i:int $=1 ; i<6 ; i++)$

\{

container.addChild(dict["crossa2" + i]); container.addChild(dict["crossa2"]); (dict["crossa2" + i]) $)$ visible = false; (dict["crossa2"]).visible = true; currentSet = "crossa2";

true); var fadeln_mov: Tween = new Tween(container, 'alpha', Regular.easeln, 0, 1, 1,

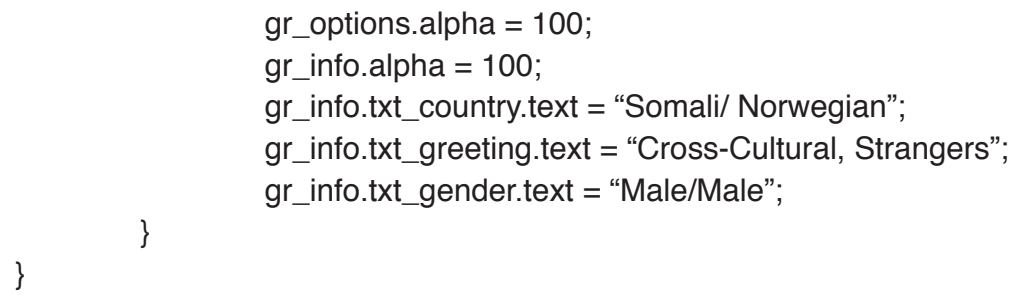




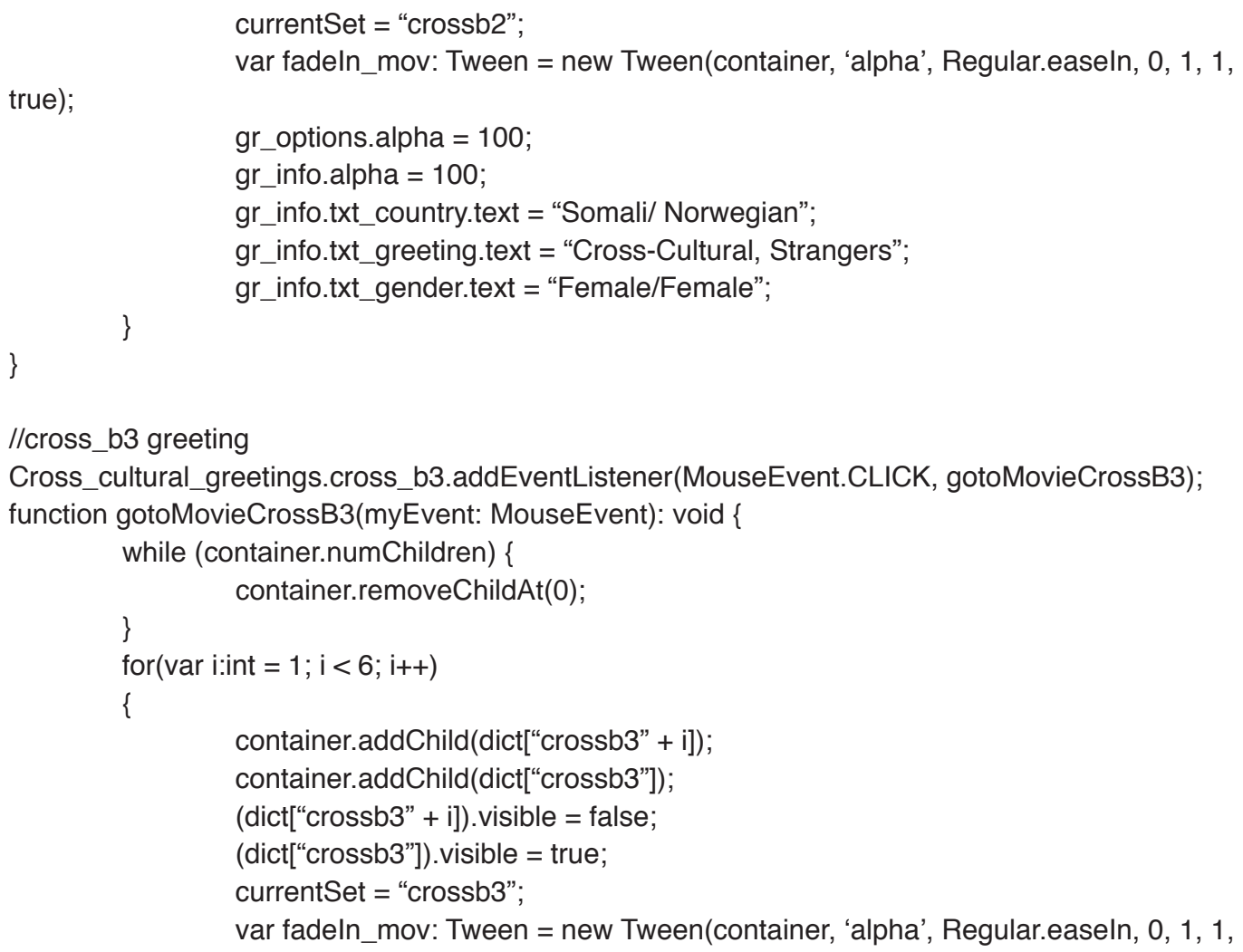


Cross_cultural_greetings.cross_c1.addEventListener(MouseEvent.CLICK, gotoMovieCrossC1); function gotoMovieCrossC1(myEvent: MouseEvent): void \{ while (container.numChildren) \{ container.removeChildAt(0);

\} for(var i:int $=1 ; i<6 ; i++)$

\{

container.addChild(dict["crossc1" + i]); container.addChild(dict["crossc1"]); (dict["crossc1" + i]) $)$ visible = false; (dict["crossc1"]).visible = true; currentSet = "crossc1";

true); var fadeln_mov: Tween = new Tween(container, 'alpha', Regular.easeln, 0, 1, 1,

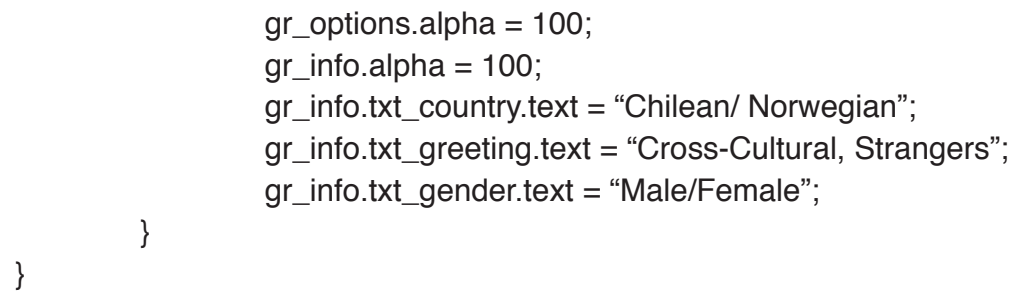




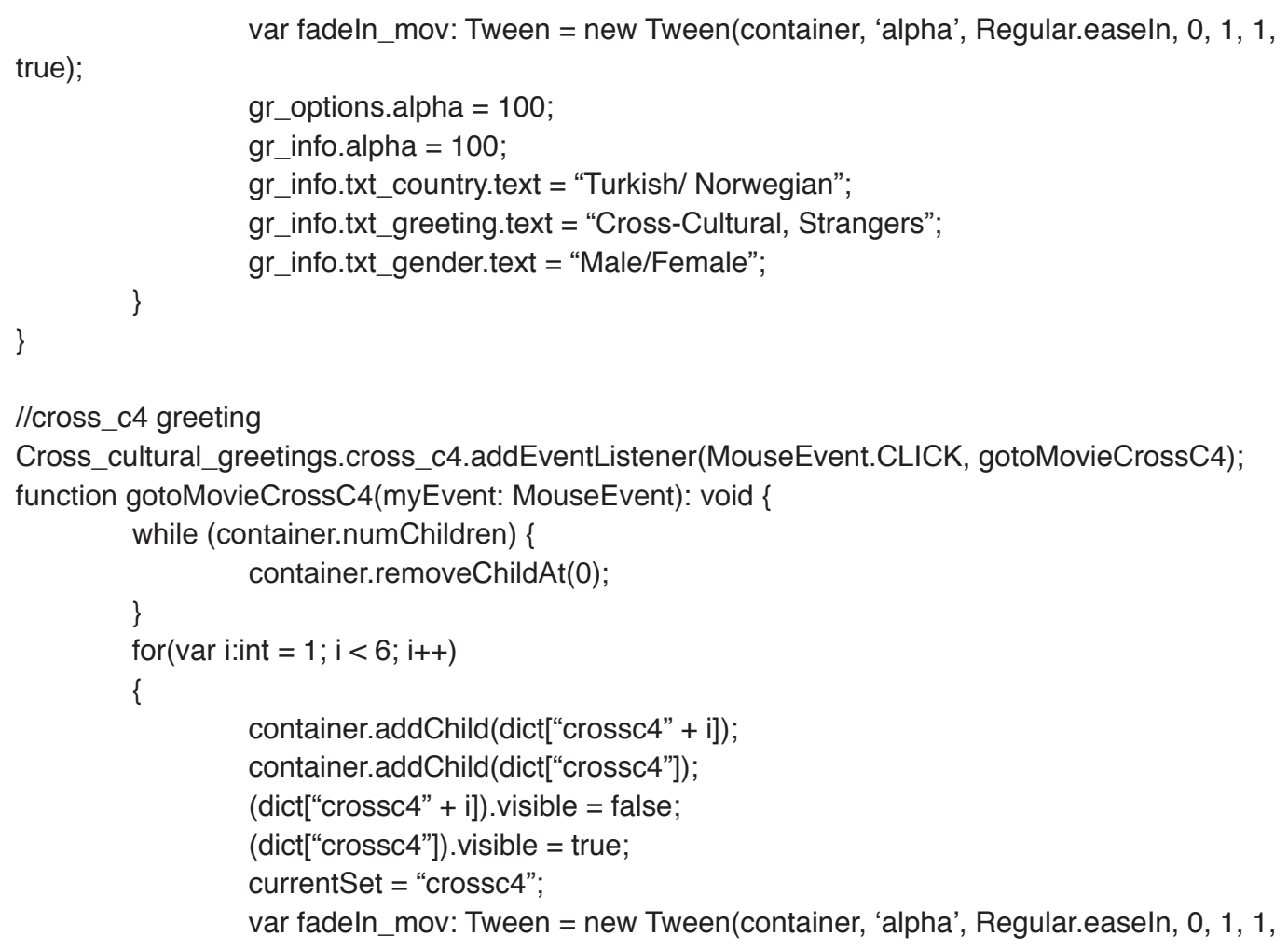

true);

gr_options.alpha = 100;

gr_info.alpha = 100;

gr_info.txt_country.text = "Norwegian/ Vietnamese";

gr_info.txt_greeting.text = "Cross-Cultural, Strangers";

gr_info.txt_gender.text = "Male/Female";

\}

//cross_c5 greeting

Cross_cultural_greetings.cross_c5.addEventListener(MouseEvent.CLICK, gotoMovieCrossC5);

function gotoMovieCrossC5(myEvent: MouseEvent): void \{

while (container.numChildren) \{

container.removeChildAt(0);

\}

for(var i:int $=1 ; i<6 ; i++)$

\{

container.addChild(dict["crossc5" + i]);

container.addChild(dict["crossc5"]);

(dict["crossc5" + i]) $)$ visible = false;

(dict["crossc5"]).visible = true;

currentSet = "crossc5";

true);

var fadeln_mov: Tween = new Tween(container, 'alpha', Regular.easeln, 0, 1, 1,

gr_options.alpha = 100;

gr_info.alpha $=100$;

gr_info.txt_country.text = "Norwegian/ Angolan";

gr_info.txt_greeting.text = "Cross-Cultural, Strangers";

\}

gr_info.txt_gender.text = "Male/Female";

\}

//cross_c6 greeting

Cross_cultural_greetings.cross_c6.addEventListener(MouseEvent.CLICK, gotoMovieCrossC6); 


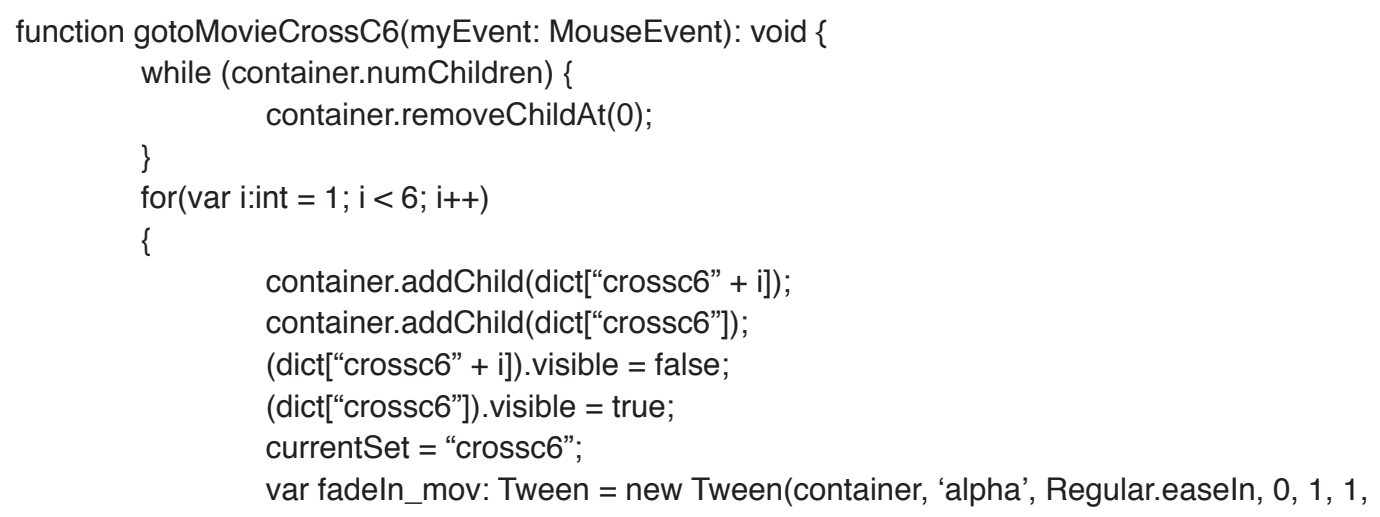

true);

gr_options.alpha = 100;

gr_info.alpha = 100;

gr_info.txt_country.text = "Turkish/ Angolan";

gr_info.txt_greeting.text = "Cross-Cultural, Strangers";

gr_info.txt_gender.text = "Male/Female";

//cross_d1 greeting

Cross_cultural_greetings.cross_d1.addEventListener(MouseEvent.CLICK, gotoMovieCrossD1);

function gotoMovieCrossD1 (myEvent: MouseEvent): void \{

while (container.numChildren) \{

container.removeChildAt $(0)$;

\}

for(var i:int $=1 ; i<6 ; i++)$

\{

container.addChild(dict["crossd1" + i]);

container.addChild(dict["crossd1"]);

(dict["crossd1" + i]).visible = false;

(dict["crossd1"]).visible = true;

currentSet = "crossd1";

true);

var fadeln_mov: Tween = new Tween(container, 'alpha', Regular.easeln, 0, 1, 1 ,

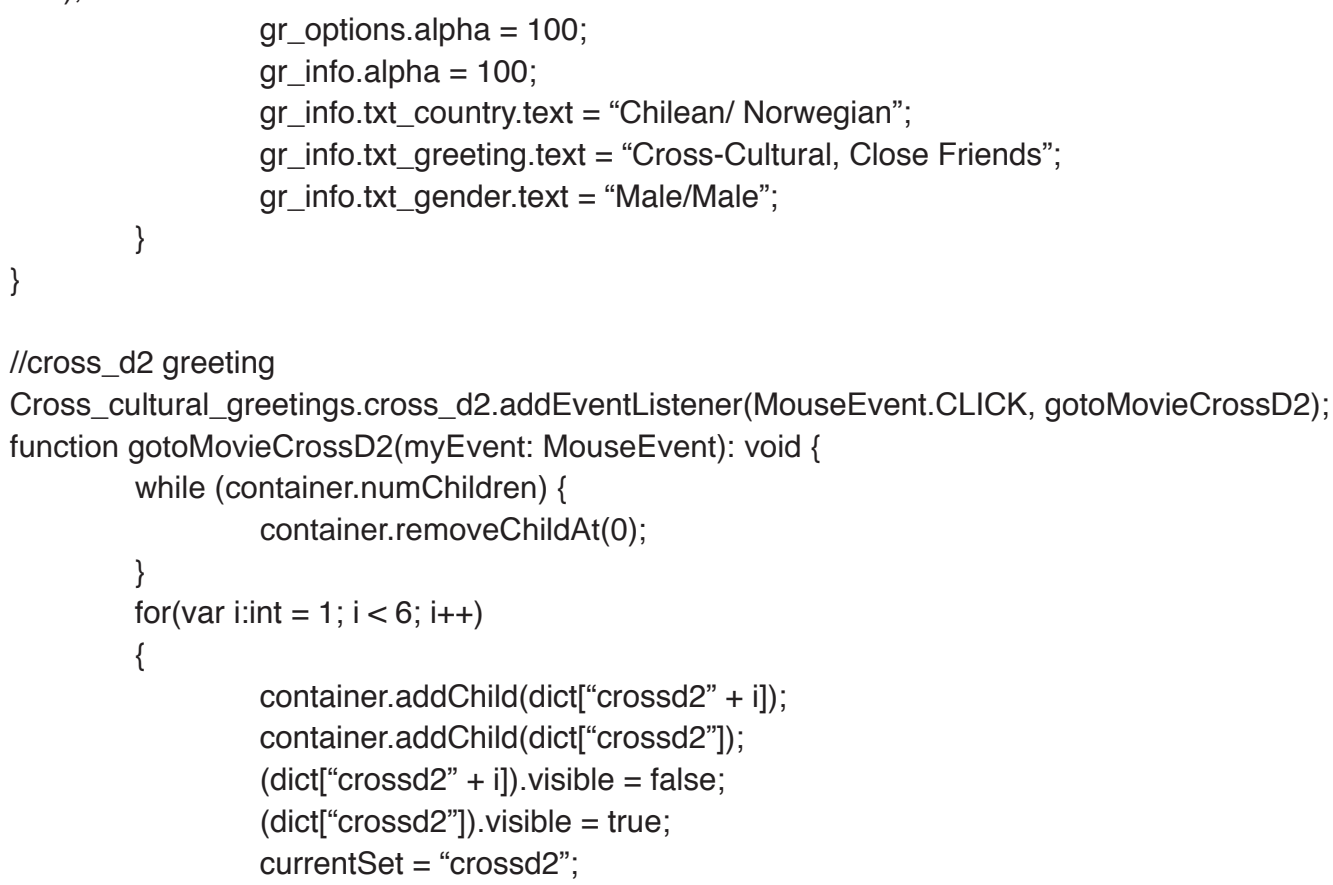




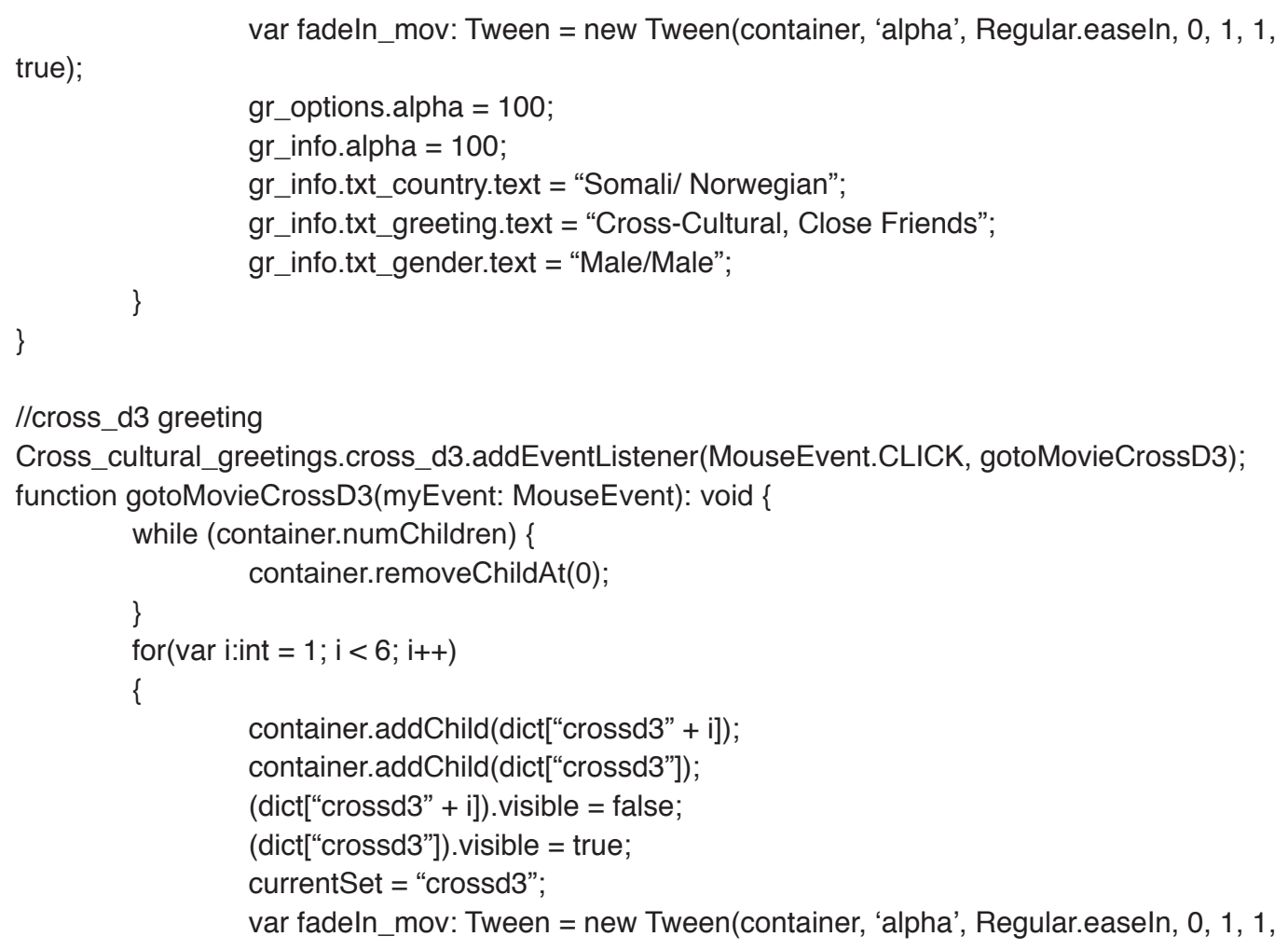

true);

gr_options.alpha = 100;

gr_info.alpha = 100;

gr_info.txt_country.text = "Turkish/ Norwegian";

gr_info.txt_greeting.text = "Cross-Cultural, Close Friends";

gr_info.txt_gender.text = "Male/Male";

\}

//cross_e2 greeting

Cross_cultural_greetings.cross_e2.addEventListener(MouseEvent.CLICK, gotoMovieCrossE2);

function gotoMovieCrossE2(myEvent: MouseEvent): void \{

while (container.numChildren) \{

container.removeChildAt(0);

\}

for(var i:int $=1 ; i<6 ; i++)$

\{

container.addChild(dict["crosse2" + i]);

container.addChild(dict["crosse2"]);

(dict["crosse2" + i]) $)$ visible = false;

(dict["crosse2"]).visible = true;

currentSet = "crosse2";

true);

var fadeln_mov: Tween = new Tween(container, 'alpha', Regular.easeln, 0, 1, 1,

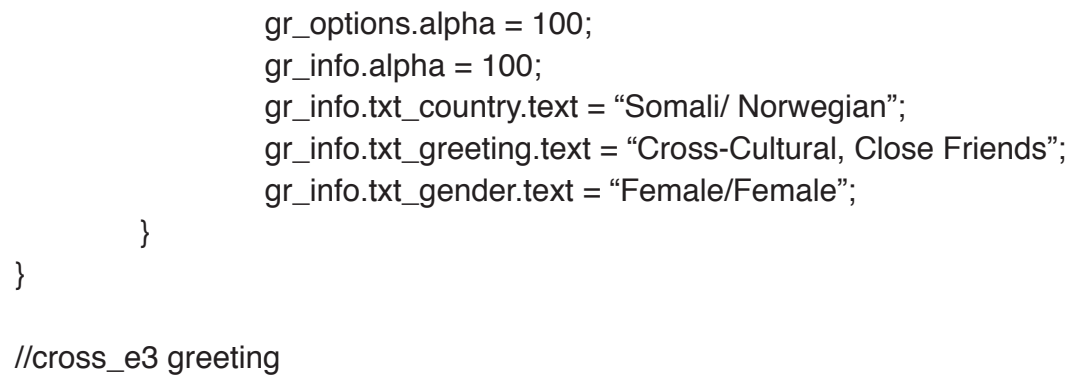

Cross_cultural_greetings.cross_e3.addEventListener(MouseEvent.CLICK, gotoMovieCrossE3); 


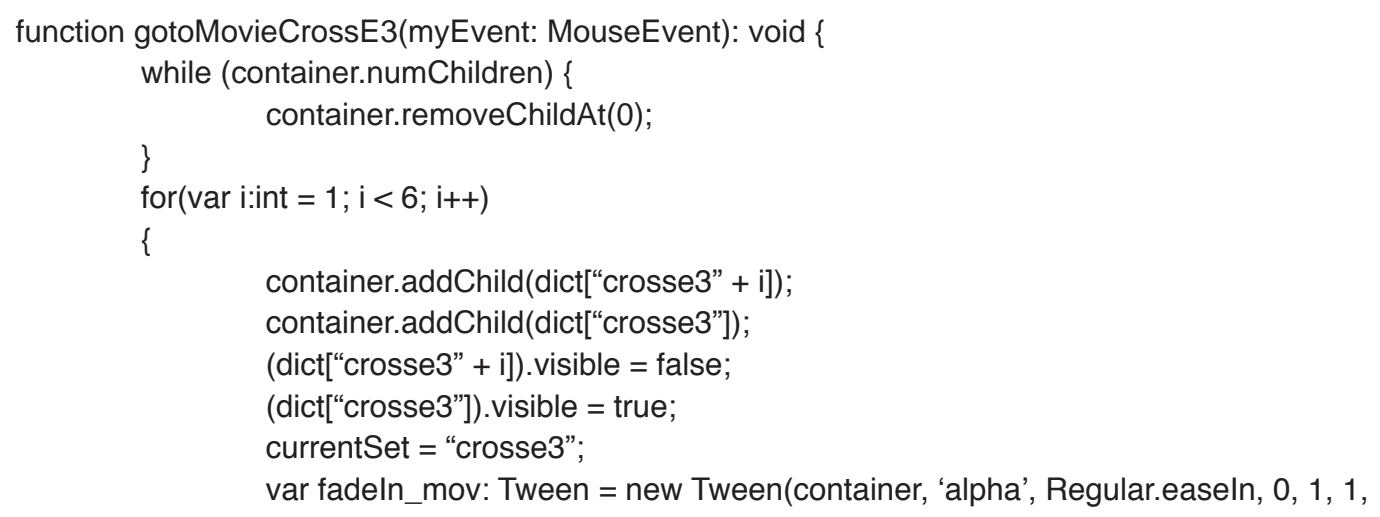

true);

gr_options.alpha = 100;

gr_info.alpha $=100$;

gr_info.txt_country.text = "Turkish/ Norwegian";

gr_info.txt_greeting.text = "Cross-Cultural, Close Friends";

gr_info.txt_gender.text = "Female/Female";

\}

\}

//cross_f1 greeting

Cross_cultural_greetings.cross_f1.addEventListener(MouseEvent.CLICK, gotoMovieCrossF1);

function gotoMovieCrossF1(myEvent: MouseEvent): void \{

while (container.numChildren) \{

container.removeChildAt(0);

\}

for(var i:int $=1 ; i<6 ; i++)$

\{

container.addChild(dict["crossf1" + i]);

container.addChild(dict[“crossf1"]);

(dict["crossf1" + i]).visible = false;

(dict["crossf1"]).visible = true;

currentSet = "crossf1";

true);

var fadeln_mov: Tween = new Tween(container, 'alpha', Regular.easeln, 0, 1, 1 ,

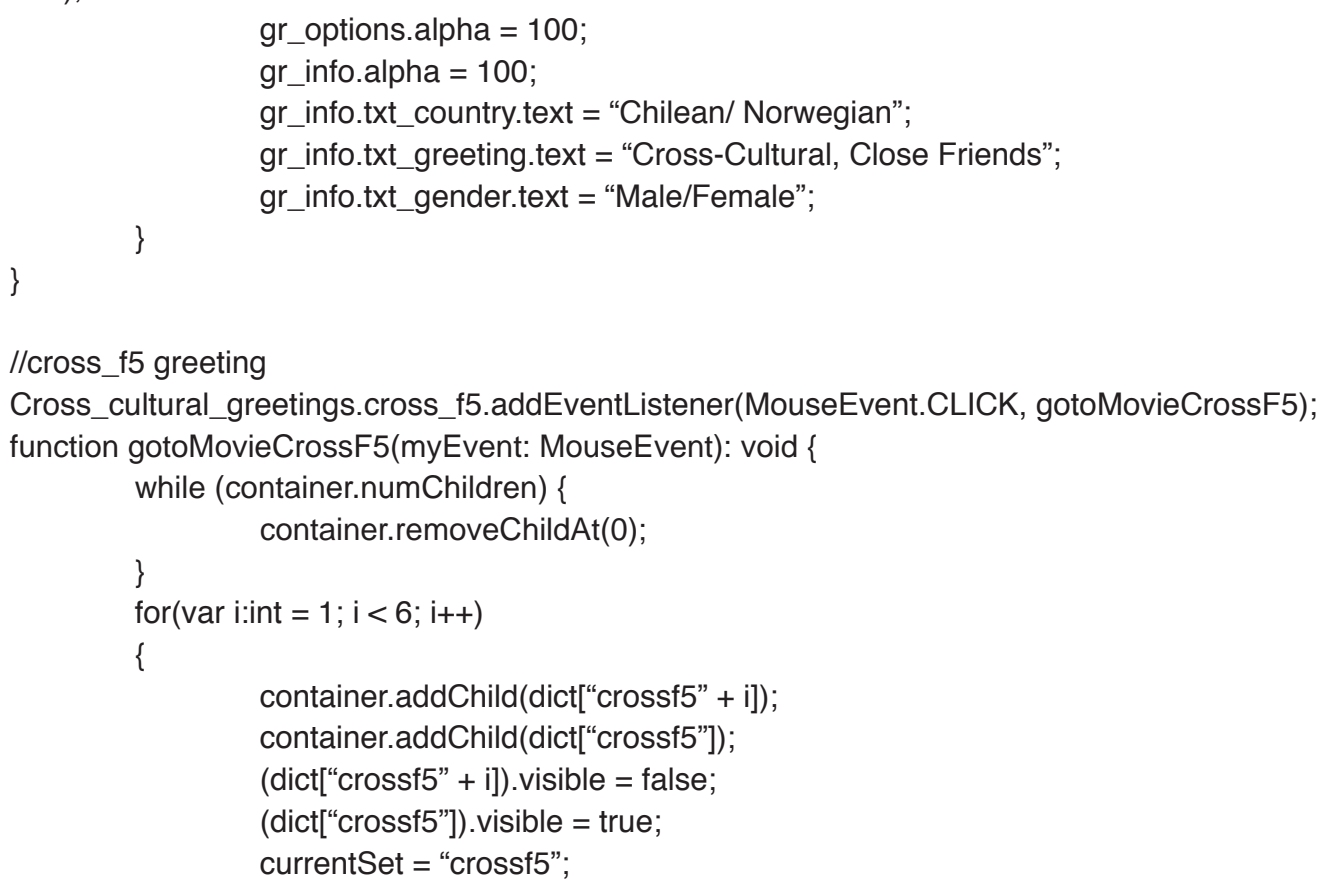




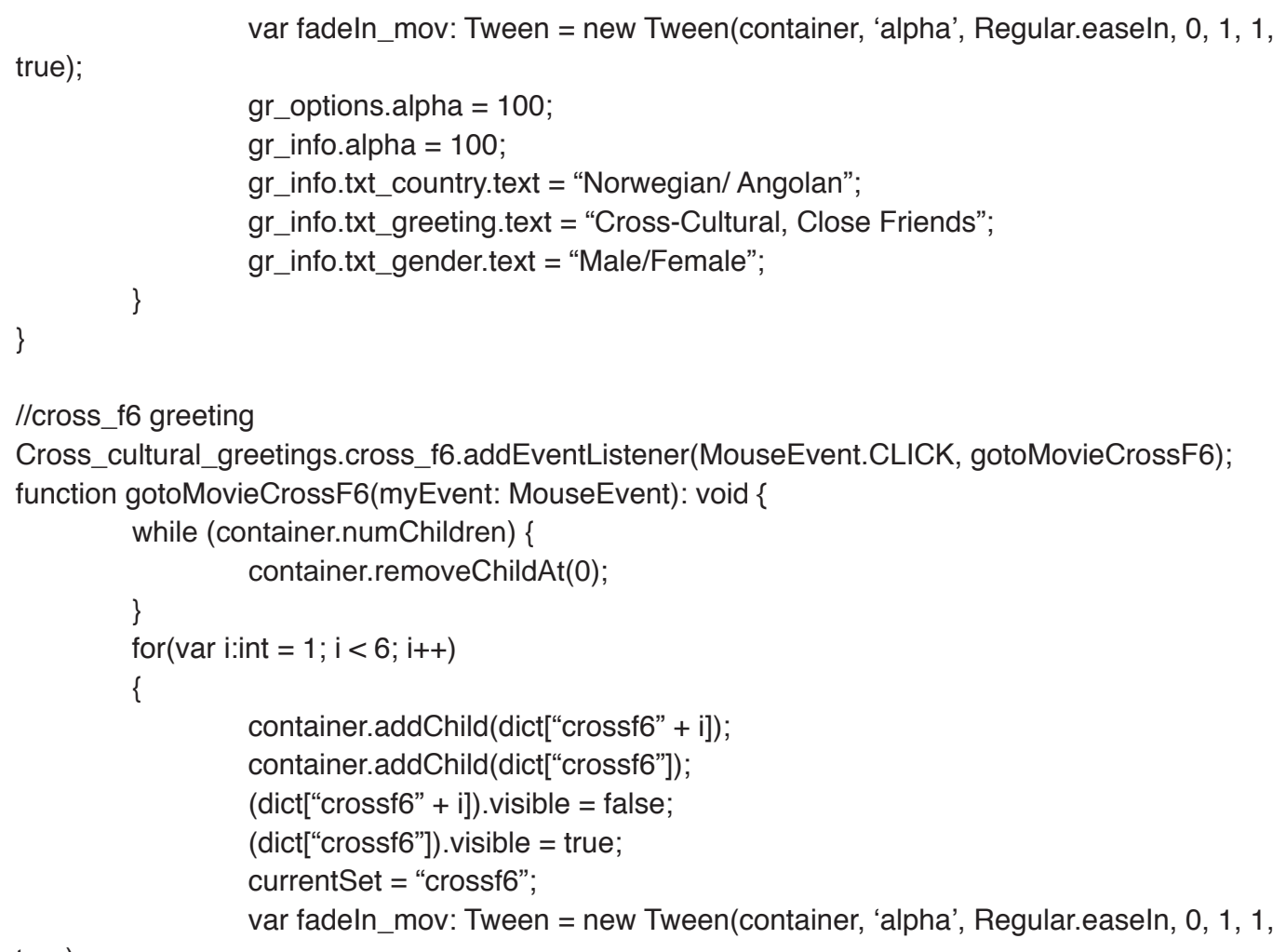

true);

gr_options.alpha = 100;

gr_info.alpha = 100;

gr_info.txt_country.text = "Turkish/ Angolan";

gr_info.txt_greeting.text = "Cross-Cultural, Close Friends";

gr_info.txt_gender.text = "Male/Female";

\}

//fam_a1 greeting

Family_greetings.fam_a1.addEventListener(MouseEvent.CLICK, gotoMovieFamA1);

function gotoMovieFamA1(myEvent: MouseEvent): void \{

while (container.numChildren) \{

container.removeChildAt(0);

\}

for(var i:int $=1 ; i<6 ; i++)$

\{

container.addChild(dict["fama1" + i]);

container.addChild(dict["fama1"]);

(dict["fama1" + i]) $\cdot$ visible = false;

(dict["fama1"]).visible = true;

currentSet = "fama1";

true);

var fadeln_mov: Tween = new Tween(container, 'alpha', Regular.easeln, 0, 1, 1,

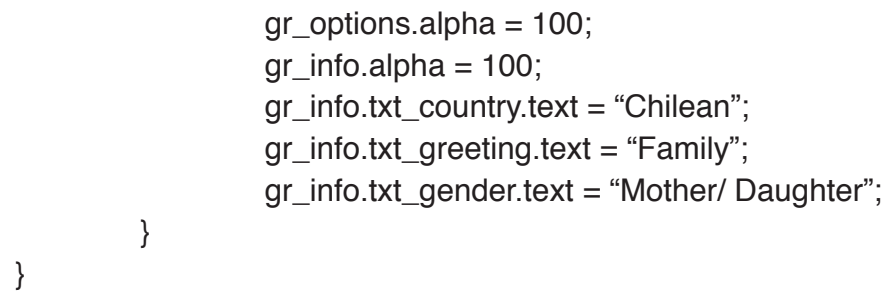

//fam_a2 greeting — same as soc_e2

Family_greetings.fam_a2.addEventListener(MouseEvent.CLICK, gotoMovieFamA2); 


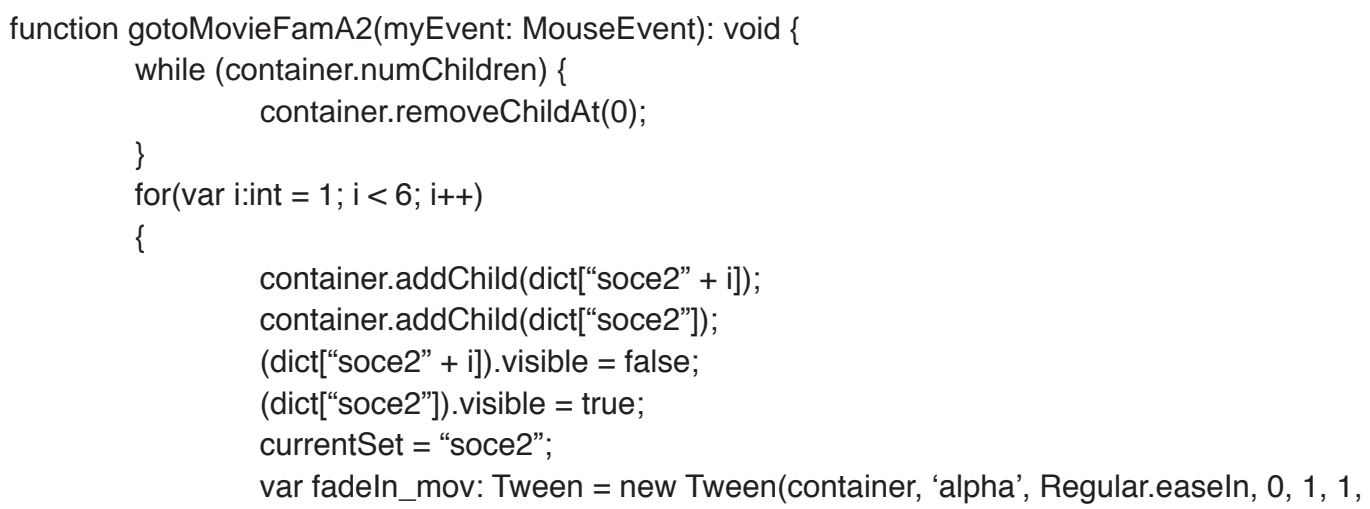

//fam_a4 greeting

Family_greetings.fam_a4.addEventListener(MouseEvent.CLICK, gotoMovieFamA4); function gotoMovieFamA4(myEvent: MouseEvent): void \{

while (container.numChildren) \{

container.removeChildAt(0);

\}

for(var i:int $=1 ; i<6 ; i++)$

\{

container.addChild(dict["fama4" + i]); container.addChild(dict["fama4"]);

(dict["fama4" + i]). visible = false;

(dict["fama4"]).visible = true;

currentSet = "fama4"; 


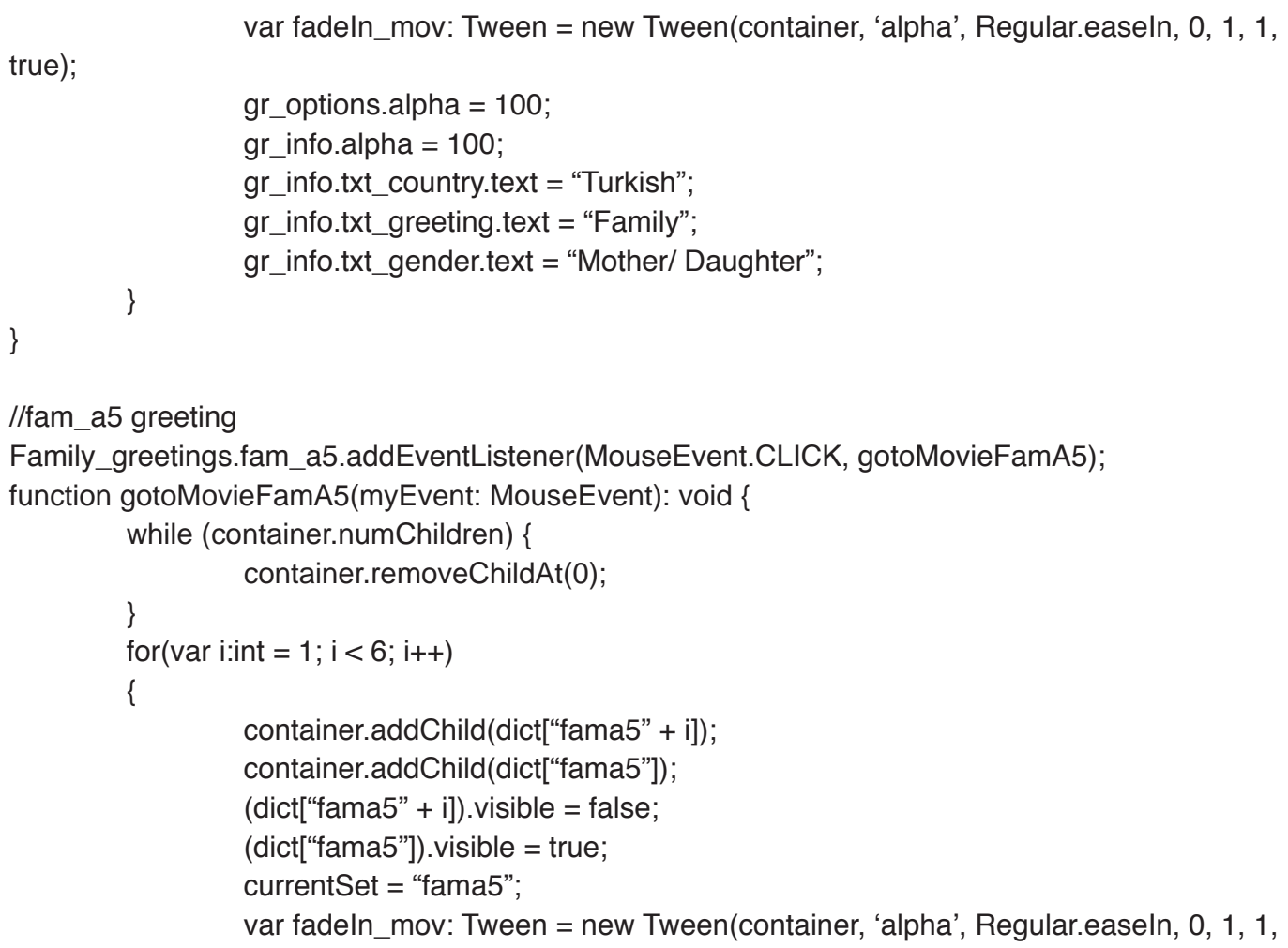




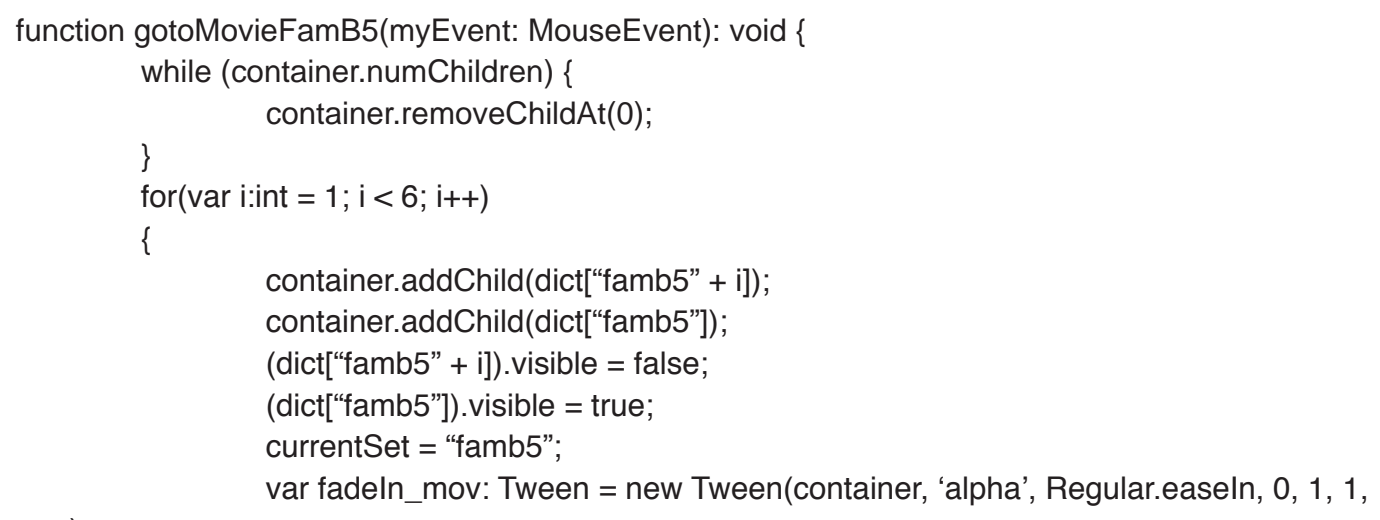

true);

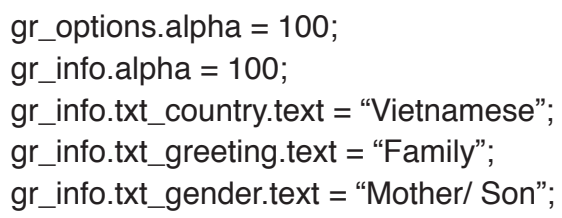

//fam_c4 --- same as soc_a4 greeting

Family_greetings.fam_c4.addEventListener(MouseEvent.CLICK, gotoMovieFamC4); function gotoMovieFamC4(myEvent: MouseEvent): void \{

while (container.numChildren) \{

container.removeChildAt(0);

\}

for(var i:int $=1 ; i<6 ; i++)$

\{

container.addChild(dict["soca4" + i]); container.addChild(dict["soca4"]);

(dict["soca4" + i]).visible = false;

(dict["soca4"]).visible = true;

currentSet = "soca4"; 


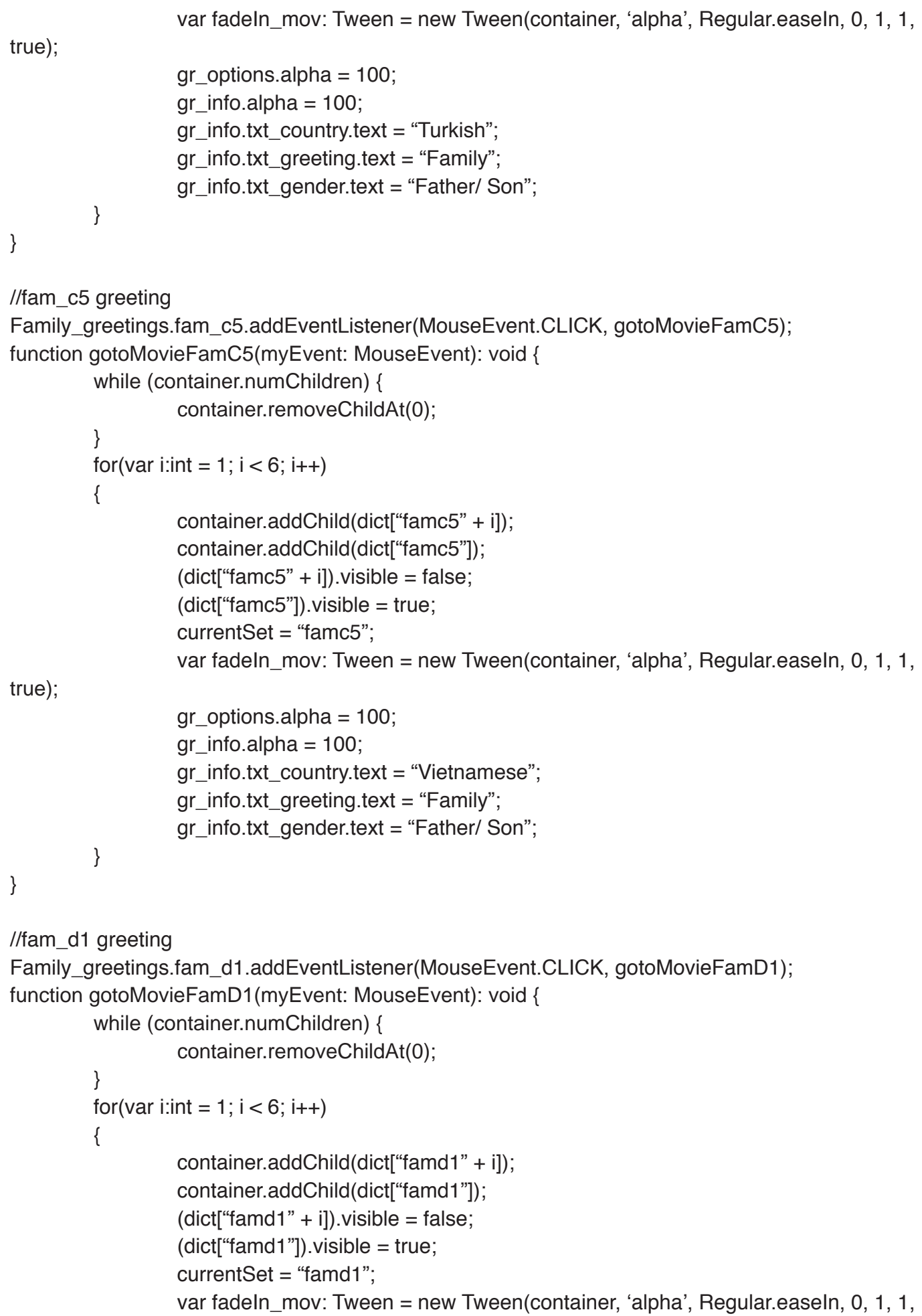




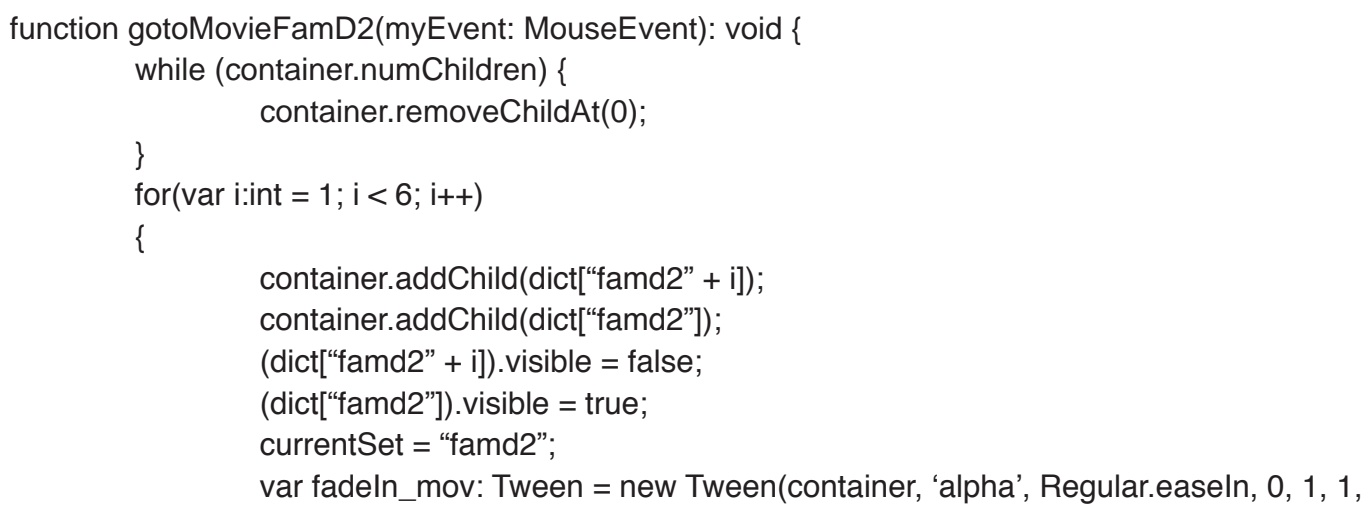

//fam_e1 greeting

Family_greetings.fam_e1.addEventListener(MouseEvent.CLICK, gotoMovieFamE1); function gotoMovieFamE1(myEvent: MouseEvent): void \{

while (container.numChildren) \{ container.removeChildAt(0); 


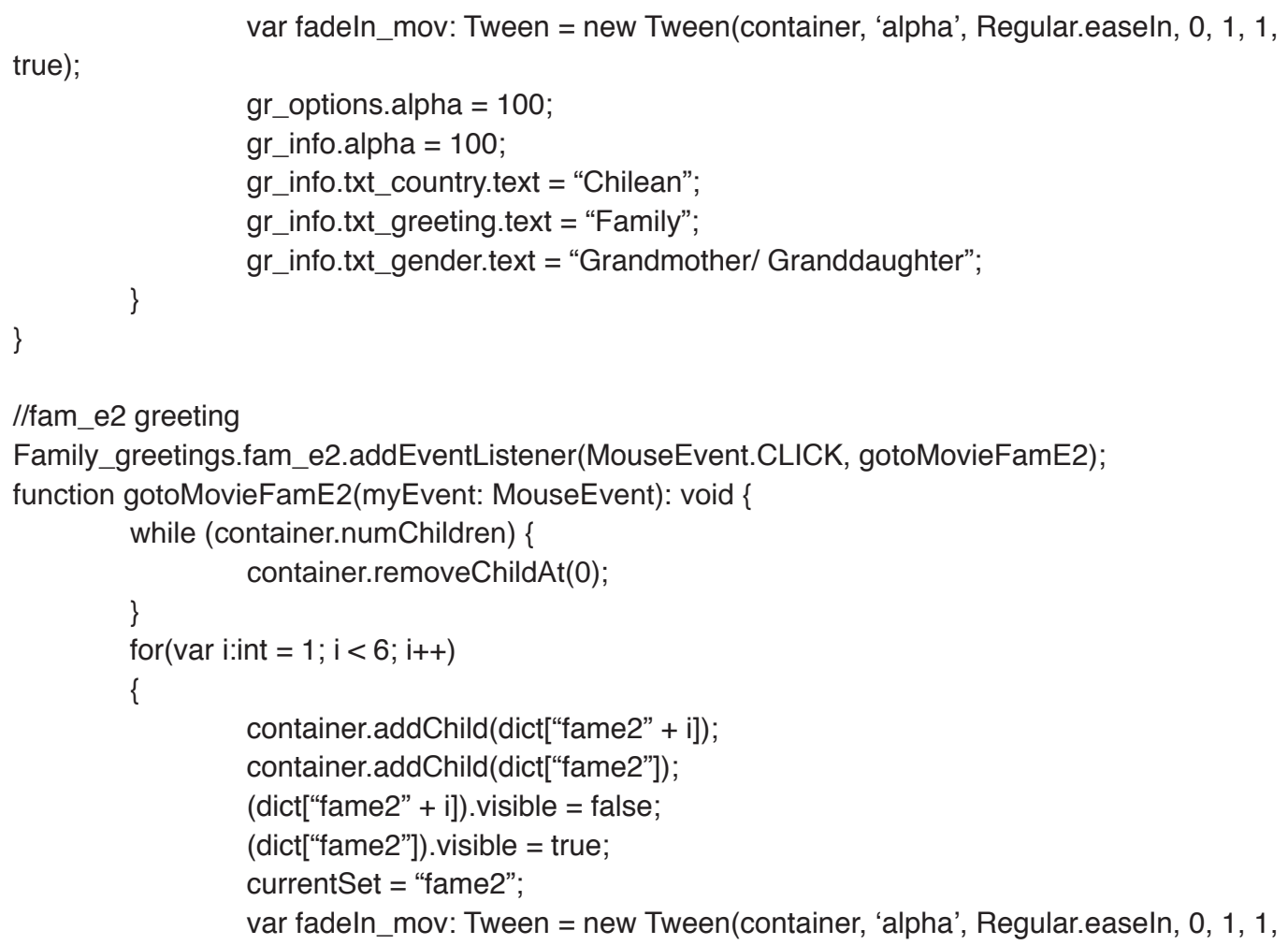




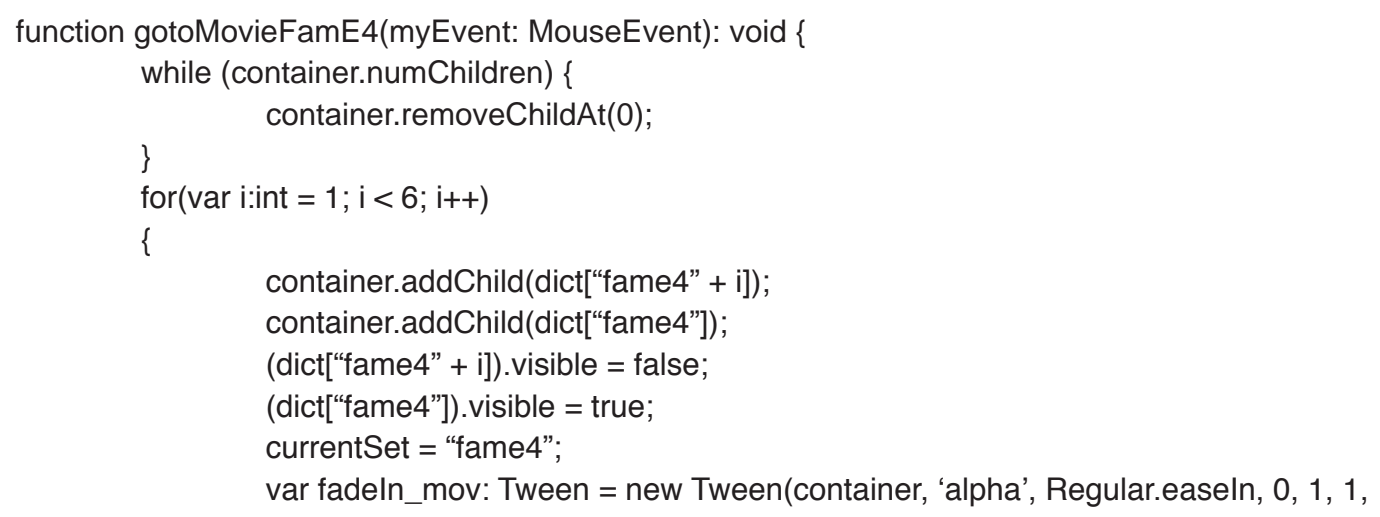

true);

gr_options.alpha = 100;

gr_info.alpha $=100$;

gr_info.txt_country.text = "Turkish";

gr_info.txt_greeting.text = "Family";

gr_info.txt_gender.text = "Grandmother/Granddaughter Religious/Islam";

\}

\}

//fam_e5 greeting

Family_greetings.fam_e5.addEventListener(MouseEvent.CLICK, gotoMovieFamE5);

function gotoMovieFamE5(myEvent: MouseEvent): void \{

while (container.numChildren) \{

container.removeChildAt $(0)$

\}

for(var i:int $=1 ; i<6 ; i++)$

\{

container.addChild(dict["fama5" + i]);

container.addChild(dict["fama5"])

(dict["fama5" + i]) .visible = false;

(dict["fama5"]).visible = true;

currentSet = "fama5";

var fadeln_mov: Tween = new Tween(container, 'alpha', Regular.easeln, 0, 1, 1,

true);

gr_options.alpha = 100;

gr_info.alpha = 100;

gr_info.txt_country.text = "Vietnamese";

gr_info.txt_greeting.text = "Family";

gr_info.txt_gender.text = "Grandmother/ Granddaughter";

\}

\}

//fam_f2 greeting

Family_greetings.fam_f2.addEventListener(MouseEvent.CLICK, gotoMovieFamF2);

function gotoMovieFamF2(myEvent: MouseEvent): void \{

while (container.numChildren) \{

container.removeChildAt(0);

\}

for(var i:int $=1 ; i<6 ; i++)$

\{

container.addChild(dict["famf" + i]);

container.addChild(dict["famf2"]);

(dict["famf2" + i]) $)$ visible = false;

(dict["famf2"]).visible = true;

currentSet = "famf2"; 


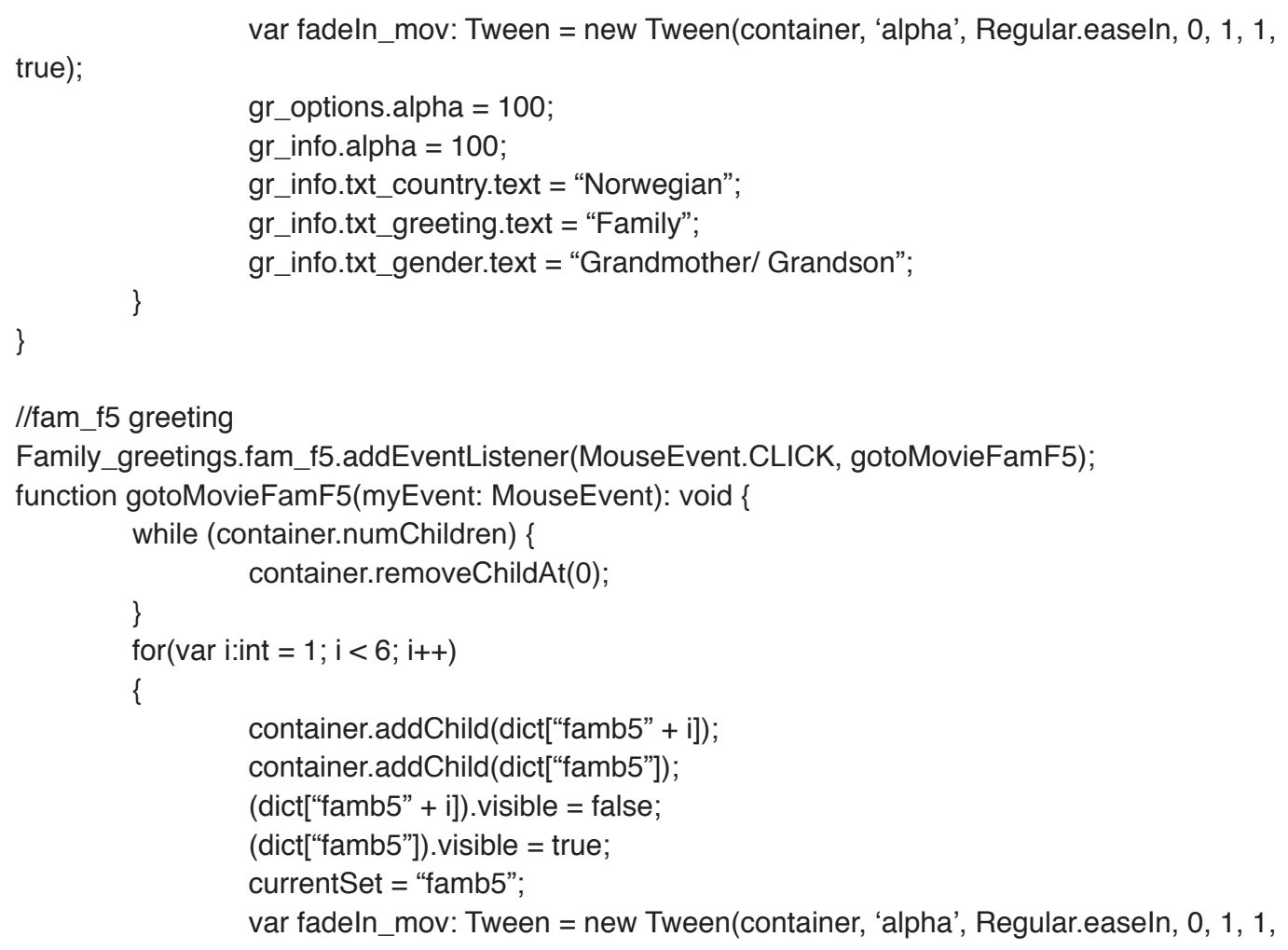

true);

gr_options.alpha = 100;

gr_info.alpha $=100$;

gr_info.txt_country.text = "Vietnamese";

gr_info.txt_greeting.text = "Family";

gr_info.txt_gender.text = "Grandmother/ Grandson";

\}

\}

//fam_g2 greeting

Family_greetings.fam_g2.addEventListener(MouseEvent.CLICK, gotoMovieFamG2);

function gotoMovieFamG2(myEvent: MouseEvent): void \{

while (container.numChildren) \{

container.removeChildAt(0);

\}

for(var i:int $=1 ; i<6 ; i++)$

\{

container.addChild(dict["famg2" + i]);

container.addChild(dict["famg2"]);

(dict["famg2" + i]). visible = false;

(dict["famg2"]).visible = true;

currentSet = "famg2";

true);

var fadeln_mov: Tween = new Tween(container, 'alpha', Regular.easeln, 0, 1, 1,

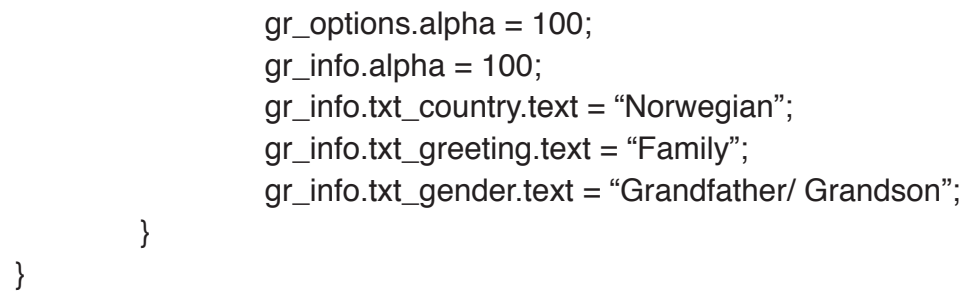

Family_greetings.fam_g4.addEventListener(MouseEvent.CLICK, gotoMovieFamG4); 


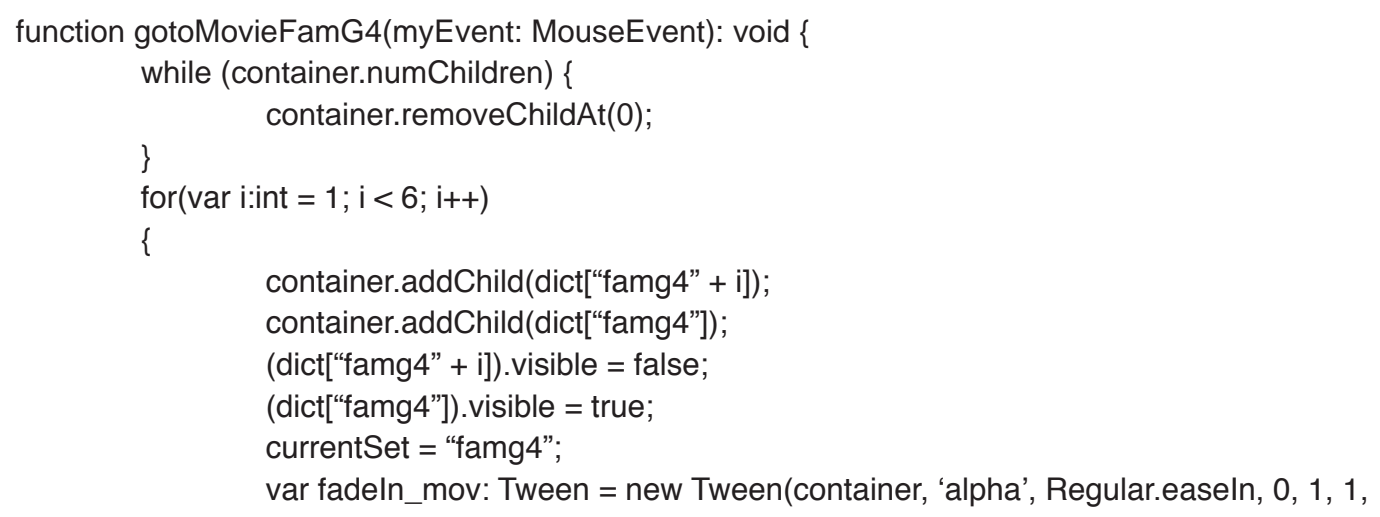

true);

gr_options.alpha = 100;

gr_info.alpha $=100$;

gr_info.txt_country.text = "Turkish";

gr_info.txt_greeting.text = "Family";

gr_info.txt_gender.text = "Grandfather/ Grandson";

\}

\}

//fam_g5 greeting

Family_greetings.fam_g5.addEventListener(MouseEvent.CLICK, gotoMovieFamG5);

function gotoMovieFamG5(myEvent: MouseEvent): void \{

while (container.numChildren) \{

container.removeChildAt $(0)$;

\}

for(var i:int $=1 ; i<6 ; i++)$

\{

container.addChild(dict["famg5" + i]);

container.addChild(dict["famg5"])

(dict["famg5" + i]) $\cdot$ visible = false;

(dict["famg5"]).visible = true;

currentSet = "famg5";

var fadeln_mov: Tween = new Tween(container, 'alpha', Regular.easeln, 0, 1, 1,

true);

gr_options.alpha = 100;

gr_info.alpha $=100$;

gr_info.txt_country.text = "Vietnamese";

gr_info.txt_greeting.text = "Family";

gr_info.txt_gender.text = "Grandfather/ Grandson";

\}

\}

//fam_h1 greeting

Family_greetings.fam_h1.addEventListener(MouseEvent.CLICK, gotoMovieFamH1);

function gotoMovieFamH1 (myEvent: MouseEvent): void \{

while (container.numChildren) \{

container.removeChildAt(0);

\}

for(var i:int $=1 ; i<6 ; i++)$

\{

container.addChild(dict["famh1" + i]);

container.addChild(dict["famh1"]);

$($ dict["famh1" + i]) $)$ visible = false;

(dict["famh1"]).visible = true;

currentSet = "famh1"; 


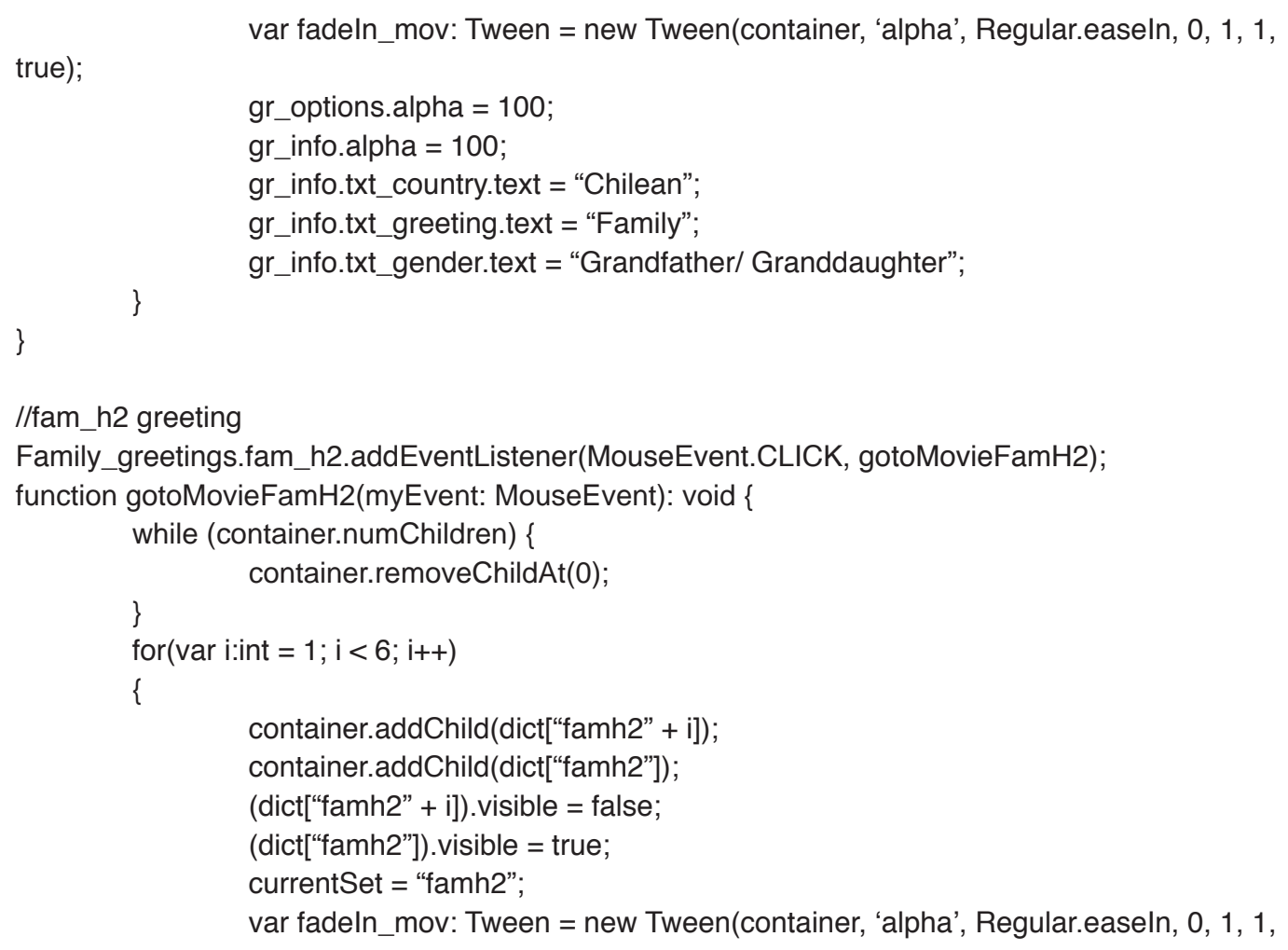

true);

gr_options.alpha = 100;

gr_info.alpha $=100$;

gr_info.txt_country.text = "Norwegian";

gr_info.txt_greeting.text = "Family";

gr_info.txt_gender.text = "Grandfather/ Granddaughter";

\}

\}

//fam_h5 greeting

Family_greetings.fam_h5.addEventListener(MouseEvent.CLICK, gotoMovieFamH5);

function gotoMovieFamH5(myEvent: MouseEvent): void \{

while (container.numChildren) \{

container.removeChildAt(0);

\}

for(var i:int $=1 ; i<6 ; i++)$

\{

container.addChild(dict["famh5" + i]);

container.addChild(dict["famh5"]);

(dict["famh5" + i]) $\cdot$ visible = false;

(dict["famh5"]).visible = true;

currentSet = "famh5";

true);

var fadeln_mov: Tween = new Tween(container, 'alpha', Regular.easeln, 0, 1, 1,

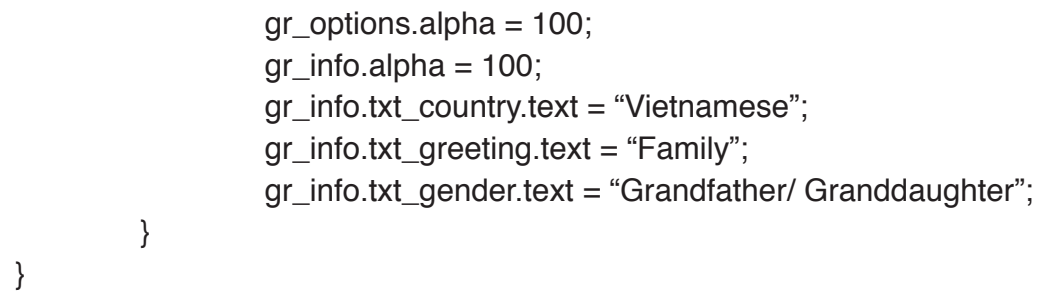




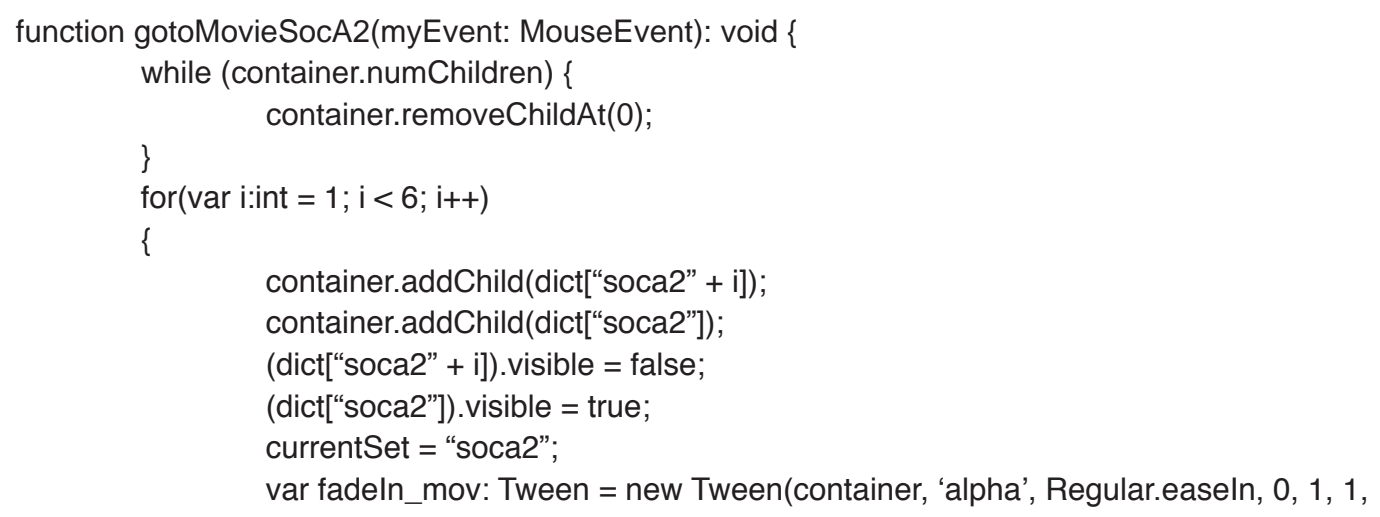

true);

gr_options.alpha = 100;

gr_info.alpha $=100$;

gr_info.txt_country.text = "Norwegian";

gr_info.txt_greeting.text = "Social, Close Friends";

gr_info.txt_gender.text = "Male/ Male";

\}

//soc_a4 greeting

Social_greetings.soc_a4.addEventListener(MouseEvent.CLICK, gotoMovieSocA4);

function gotoMovieSocA4(myEvent: MouseEvent): void \{

while (container.numChildren) \{ container.removeChildAt(0);

\}

for(var i:int $=1 ; i<6 ; i++)$

\{

container.addChild(dict["soca4" + i]);

container.addChild(dict["soca4"]);

(dict["soca4" + i]).visible = false;

(dict["soca4"]). visible = true;

currentSet = "soca4";

var fadeln_mov: Tween = new Tween(container, 'alpha', Regular.easeln, 0, 1, 1,

true);

gr_options.alpha $=100$;

gr_info.alpha = 100;

gr_info.txt_country.text = "Turkish";

gr_info.txt_greeting.text = "Social, Close Friends";

gr_info.txt_gender.text = "Male/ Male";

\}

\}

//soc_a5 greeting

Social_greetings.soc_a5.addEventListener(MouseEvent.CLICK, gotoMovieSocA5);

function gotoMovieSocA5(myEvent: MouseEvent): void \{

while (container.numChildren) \{

container.removeChildAt(0);

\}

for(var i:int $=1 ; i<6 ; i++)$

\{

container.addChild(dict["soca5" + i]);

container.addChild(dict["soca5"]);

(dict["soca5" + i]).visible = false;

(dict["soca5"]). visible = true;

currentSet = "soca5";

var fadeln_mov: Tween = new Tween(container, 'alpha', Regular.easeln, 0, 1, 1,

true); 


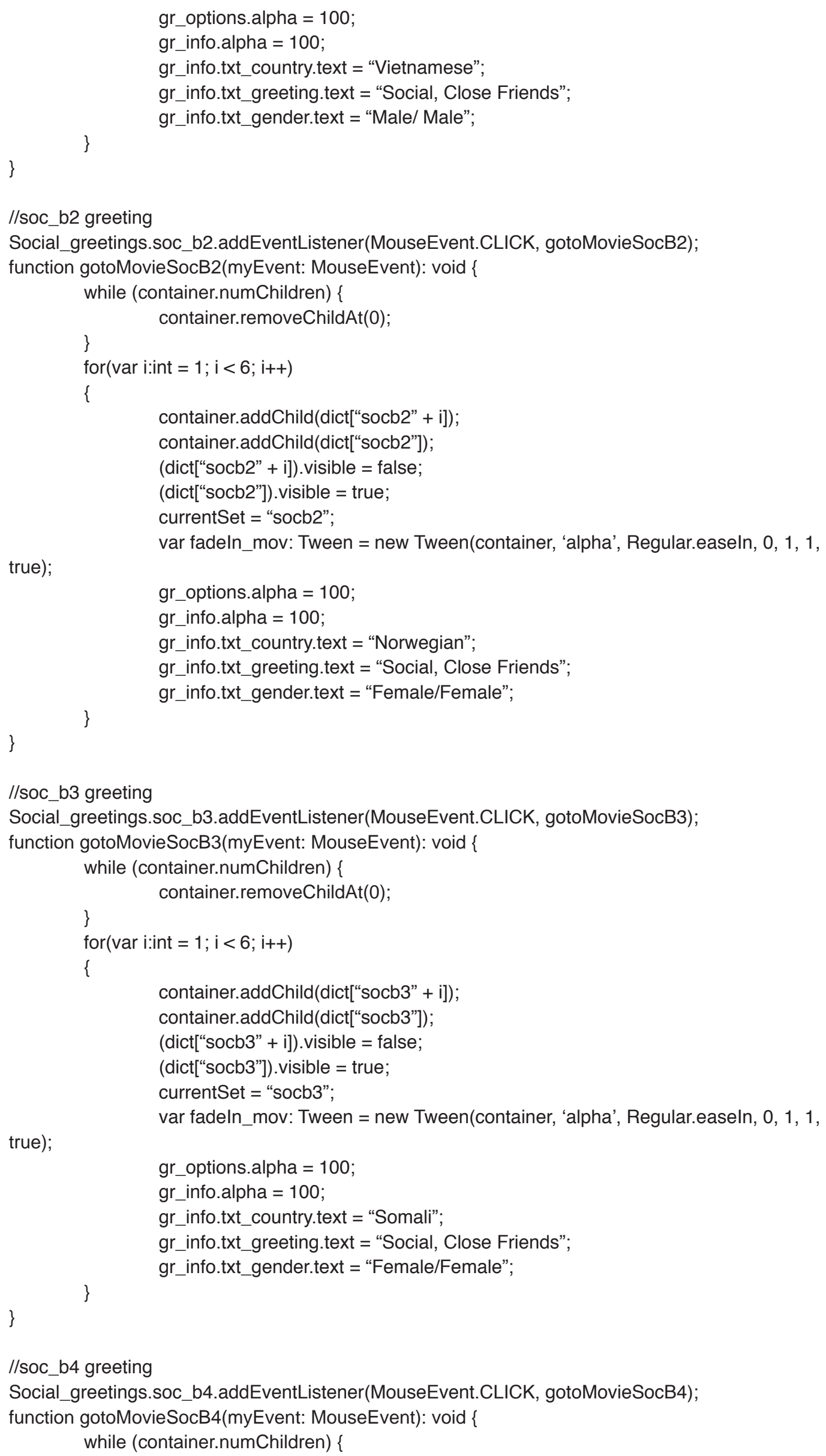




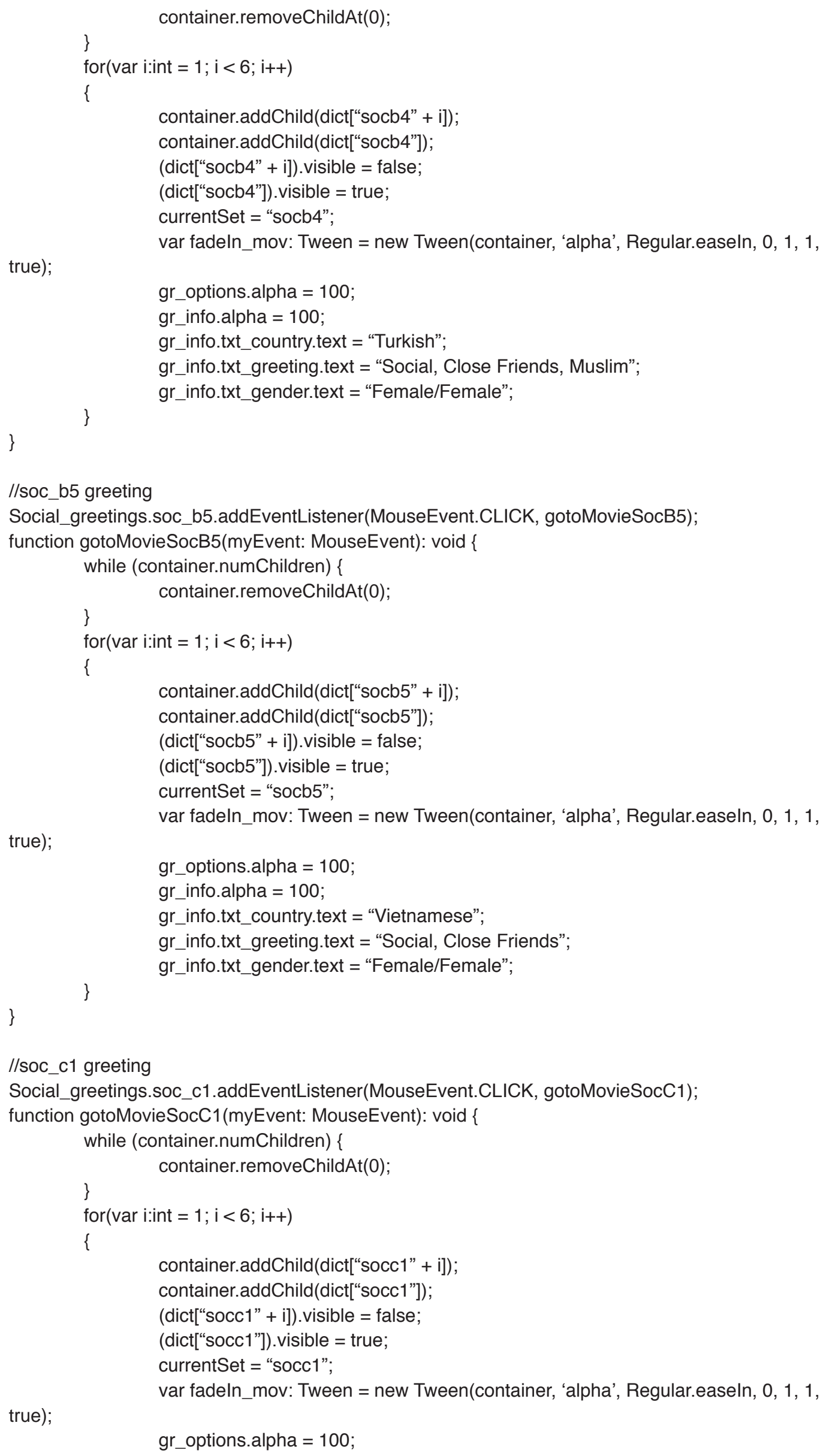




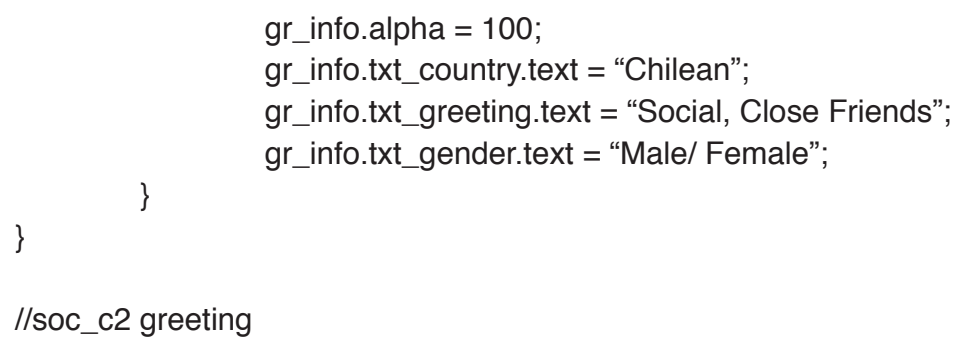


\}

for(var i:int $=1 ; i<6 ; i++)$

\{

container.addChild(dict["socc4" + i]);

container.addChild(dict["socc4"]);

(dict["socc4" + i]). visible = false;

(dict["socc4"]).visible = true;

currentSet = "socc4";

var fadeln_mov: Tween = new Tween(container, 'alpha', Regular.easeln, 0, 1, 1,

true);

gr_options.alpha = 100;

gr_info.alpha = 100;

gr_info.txt_country.text = "Turkish";

gr_info.txt_greeting.text = "Social, Close Friends (Muslim)";

gr_info.txt_gender.text = "Male/ Female";

\}

//soc_c5 greeting

Social_greetings.soc_c5.addEventListener(MouseEvent.CLICK, gotoMovieSocC5);

function gotoMovieSocC5(myEvent: MouseEvent): void \{

while (container.numChildren) \{

container.removeChildAt(0);

\}

for(var i:int $=1 ; i<6 ; i++)$

\{

container.addChild(dict[“socc5" + i]);

container.addChild(dict["socc5"]);

(dict["socc5" + i]).visible = false;

(dict["socc5"]).visible = true;

currentSet = "socc5";

var fadeln_mov: Tween = new Tween(container, 'alpha', Regular.easeln, 0, 1, 1,

true);

gr_options.alpha = 100;

gr_info.alpha = 100;

gr_info.txt_country.text = "Vietnamese";

gr_info.txt_greeting.text = "Social, Close Friends";

gr_info.txt_gender.text = "Male/ Female";

\}

//soc_d2 greeting

Social_greetings.soc_d2.addEventListener(MouseEvent.CLICK, gotoMovieSocD2);

function gotoMovieSocD2(myEvent: MouseEvent): void \{

while (container.numChildren) \{

container.removeChildAt(0);

\}

for(var i:int $=1 ; i<6 ; i++)$

\{

container.addChild(dict["socd2" + i]);

container.addChild(dict["socd2"]);

(dict["socd2" + i]).visible = false;

(dict["socd2"]). visible = true;

currentSet = "socd2";

var fadeln_mov: Tween = new Tween(container, 'alpha', Regular.easeln, 0, 1, 1,

true);

gr_options.alpha = 100;

gr_info.alpha = 100; 


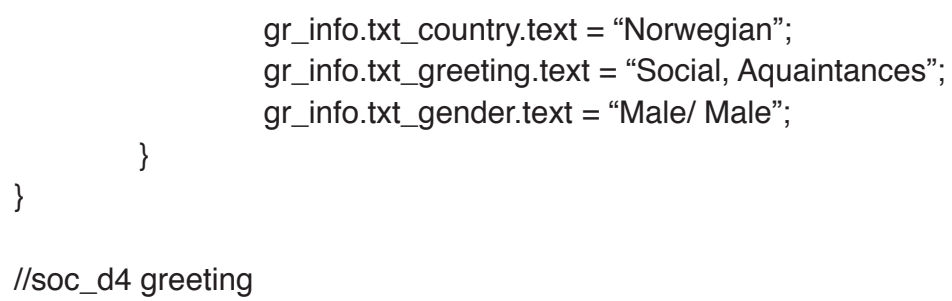




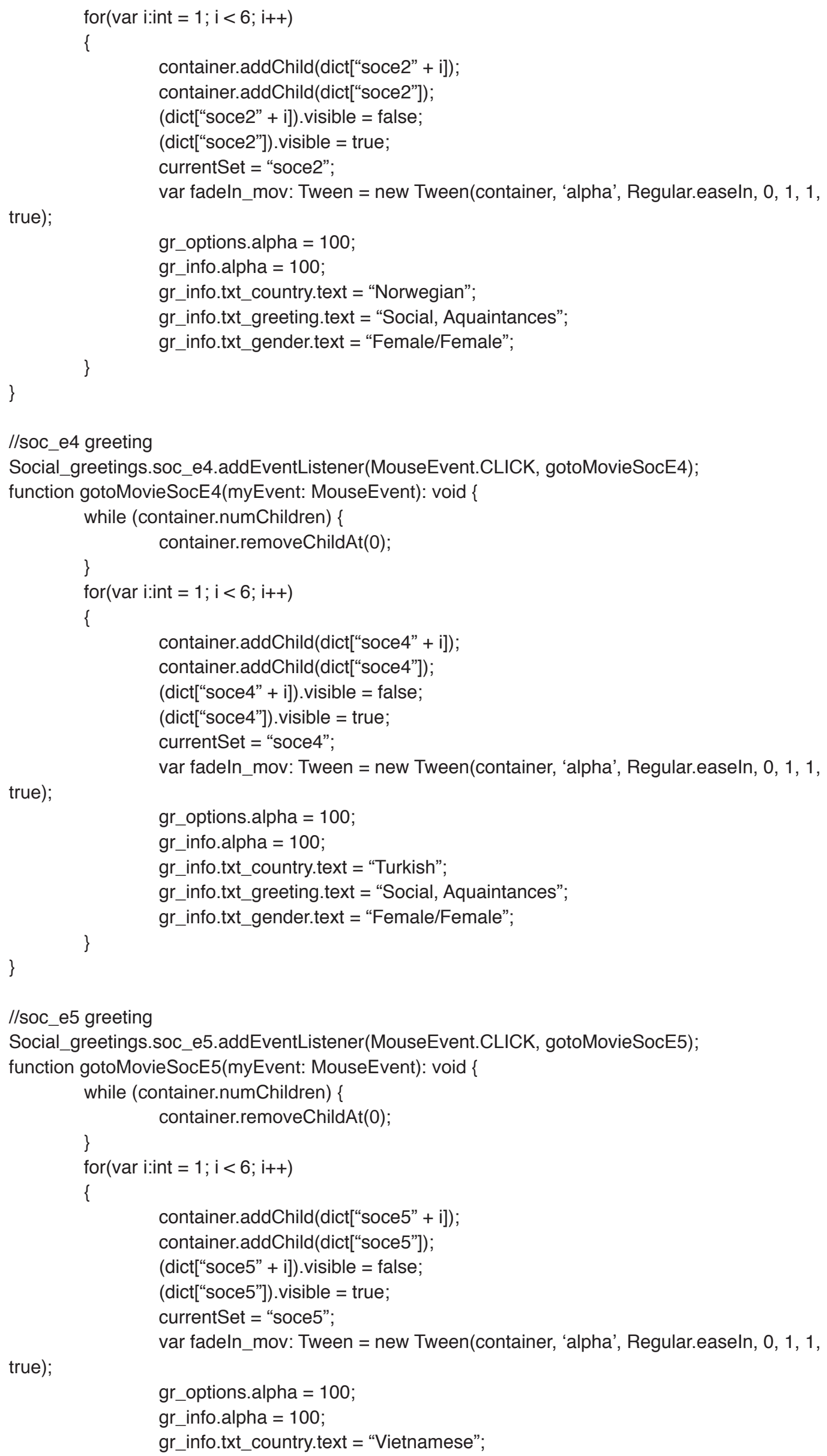




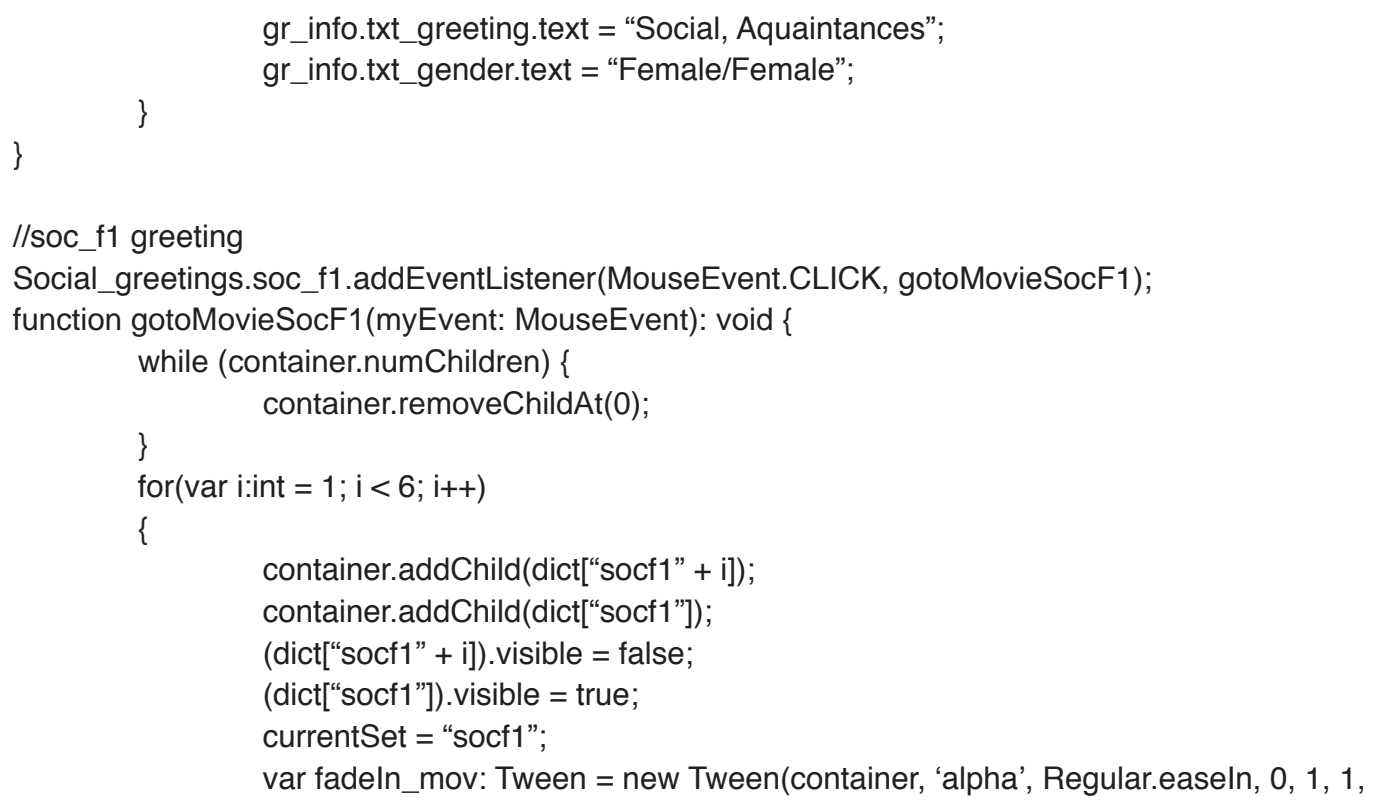




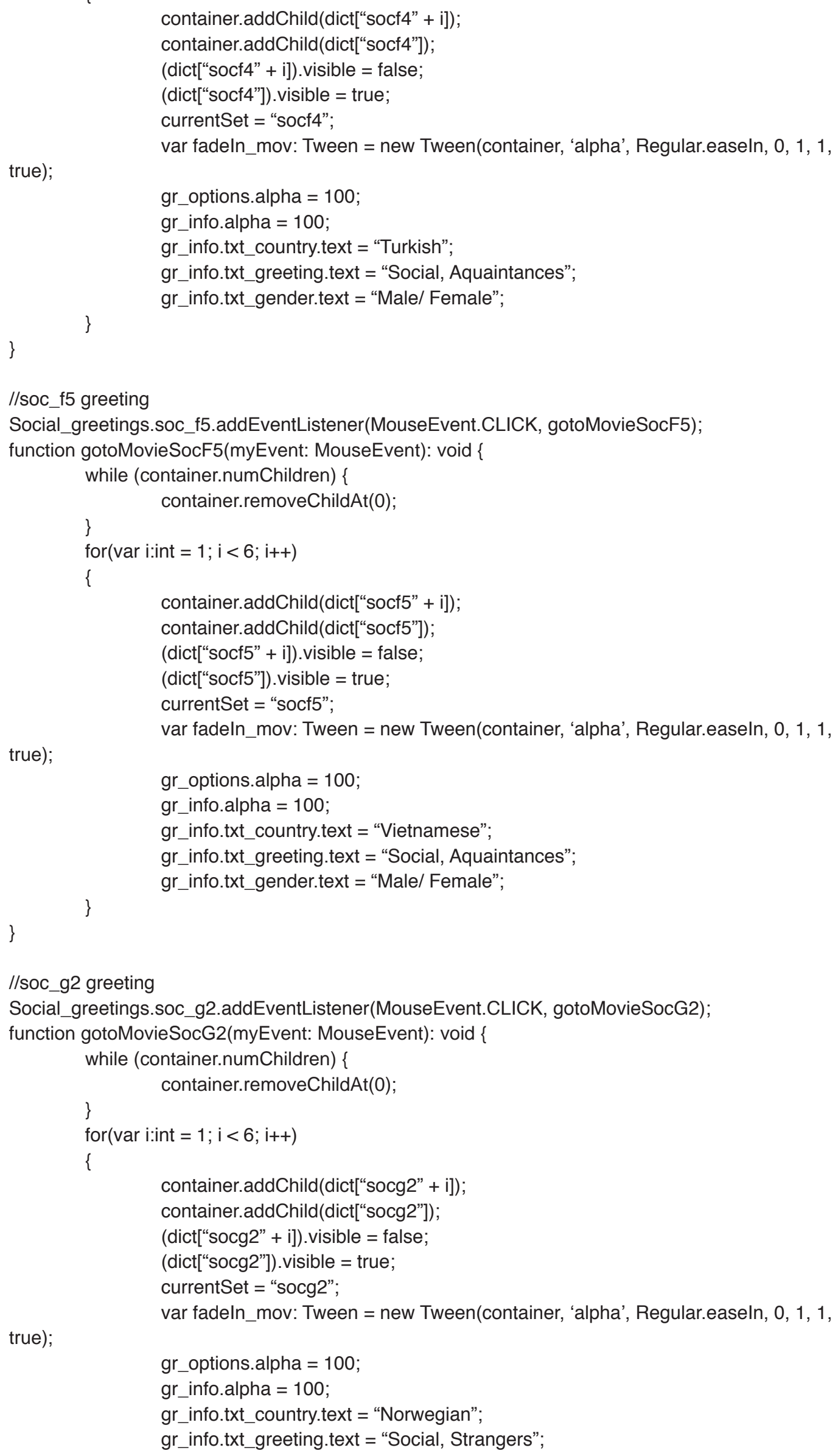




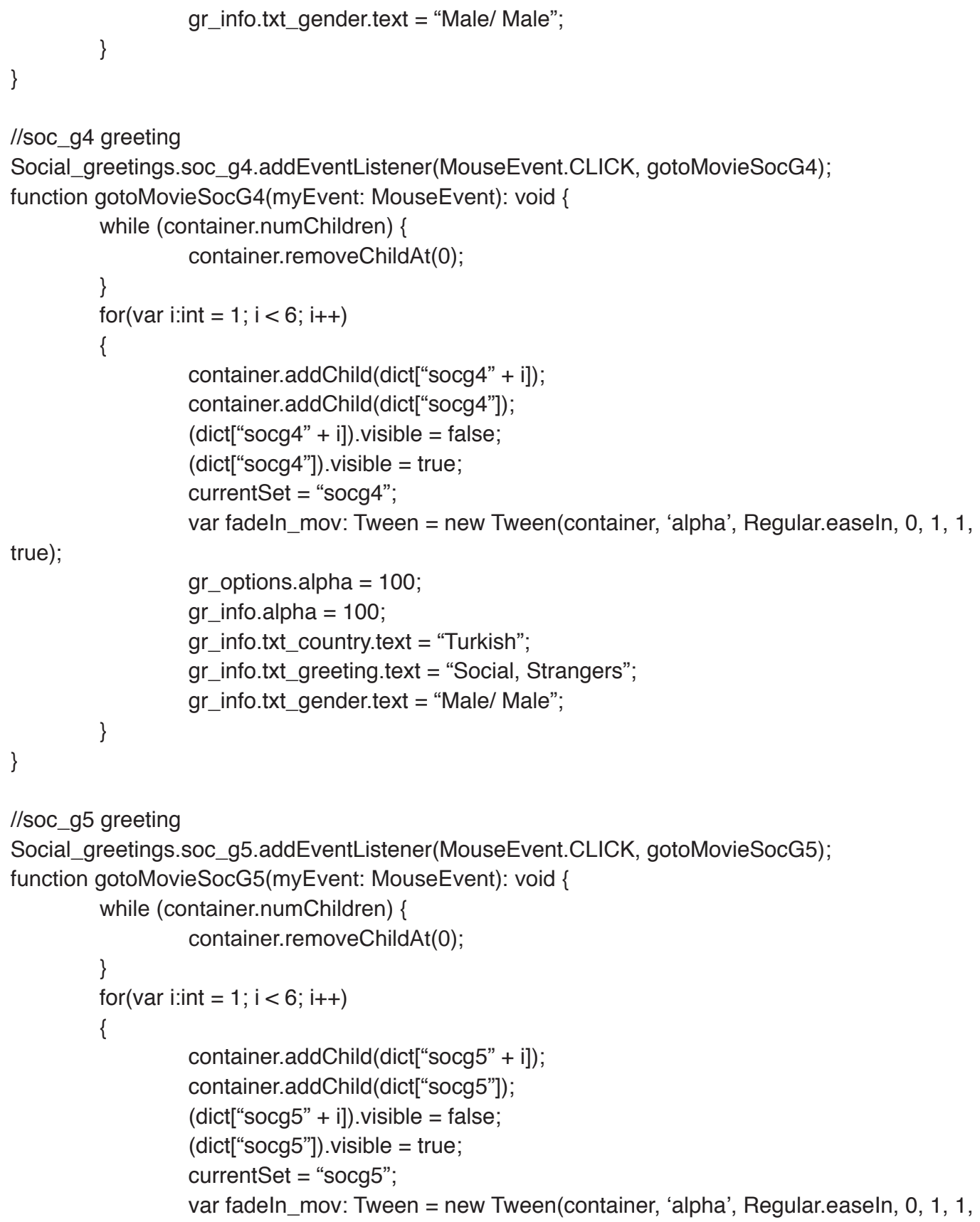




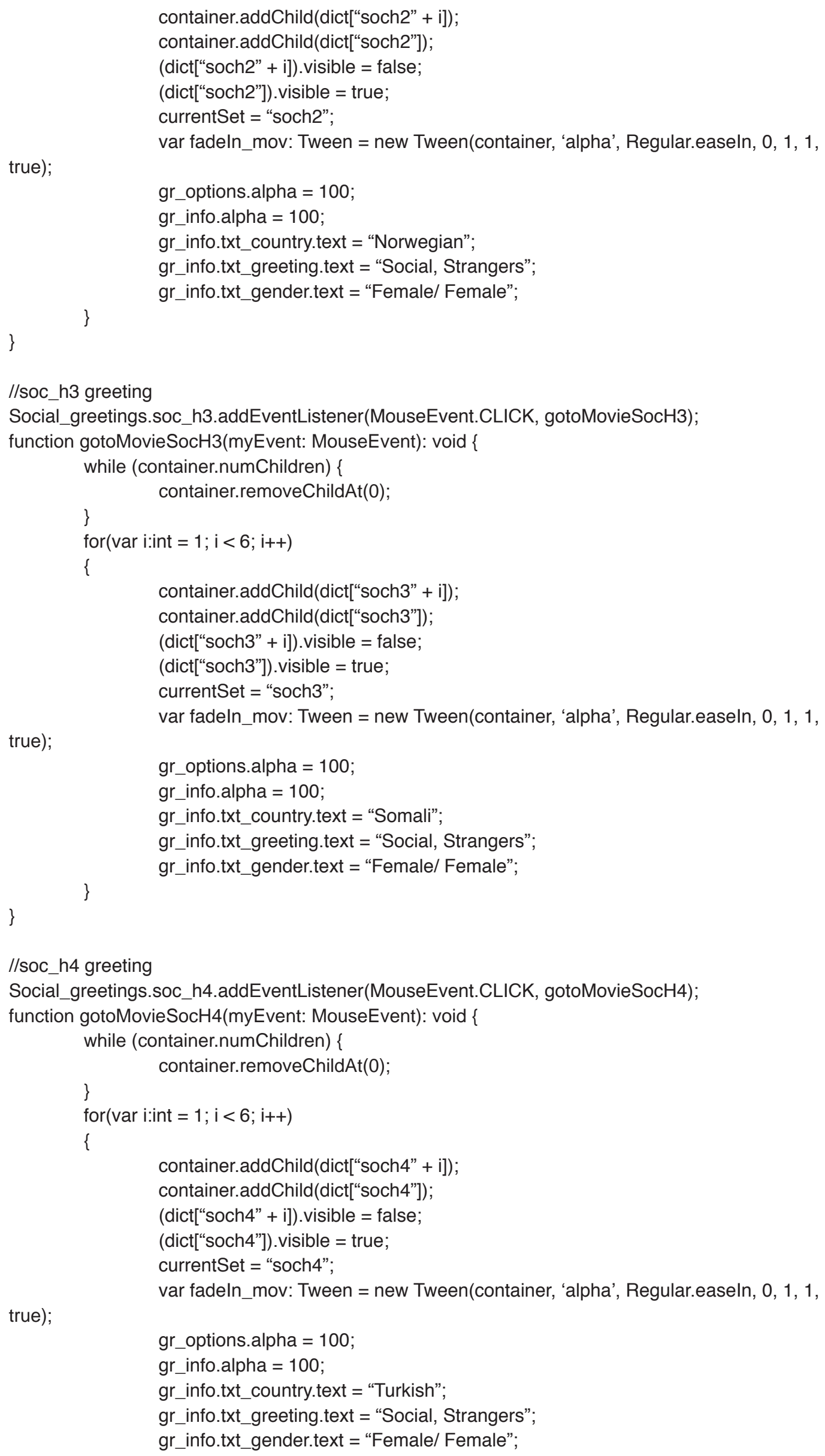




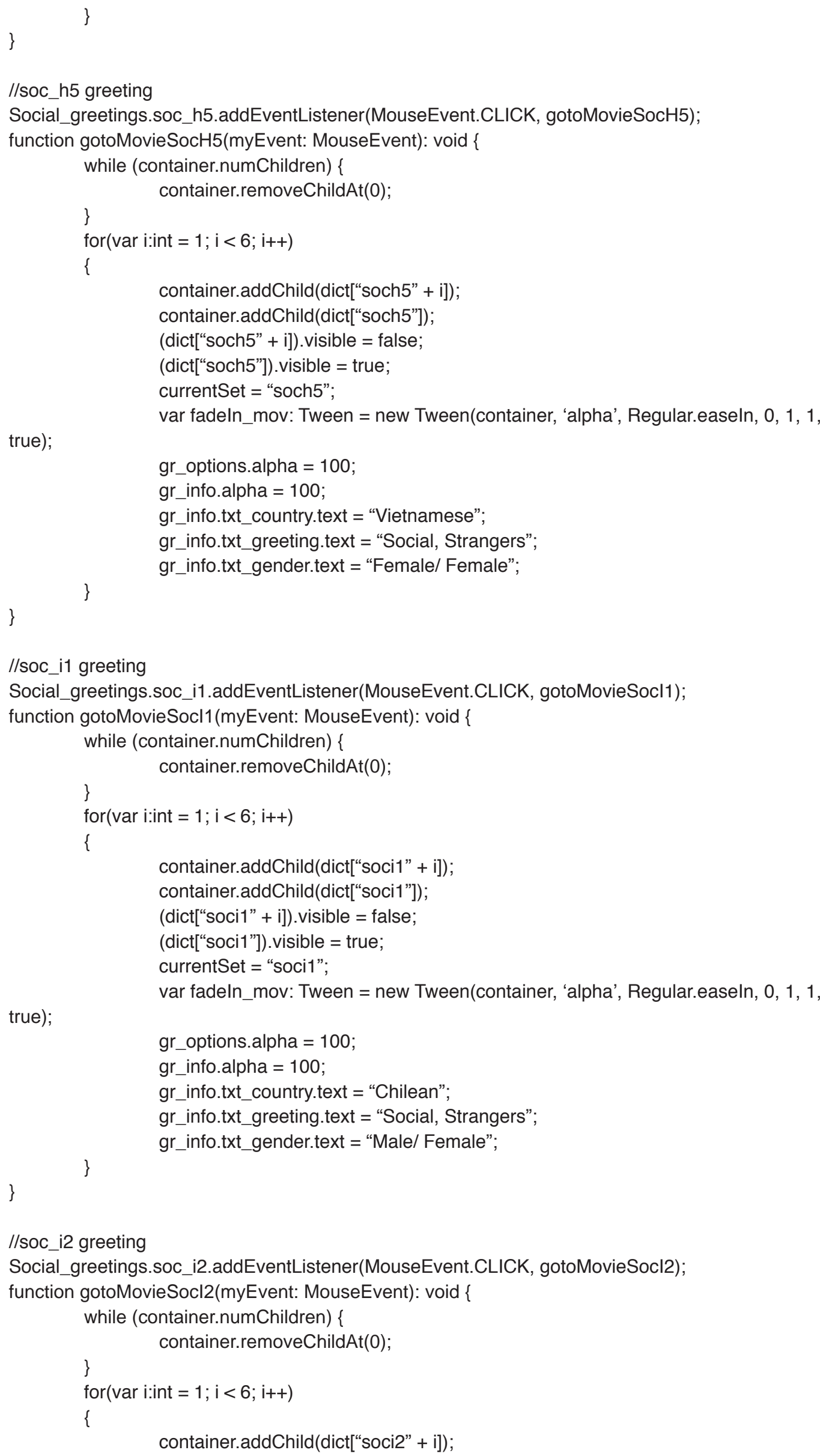




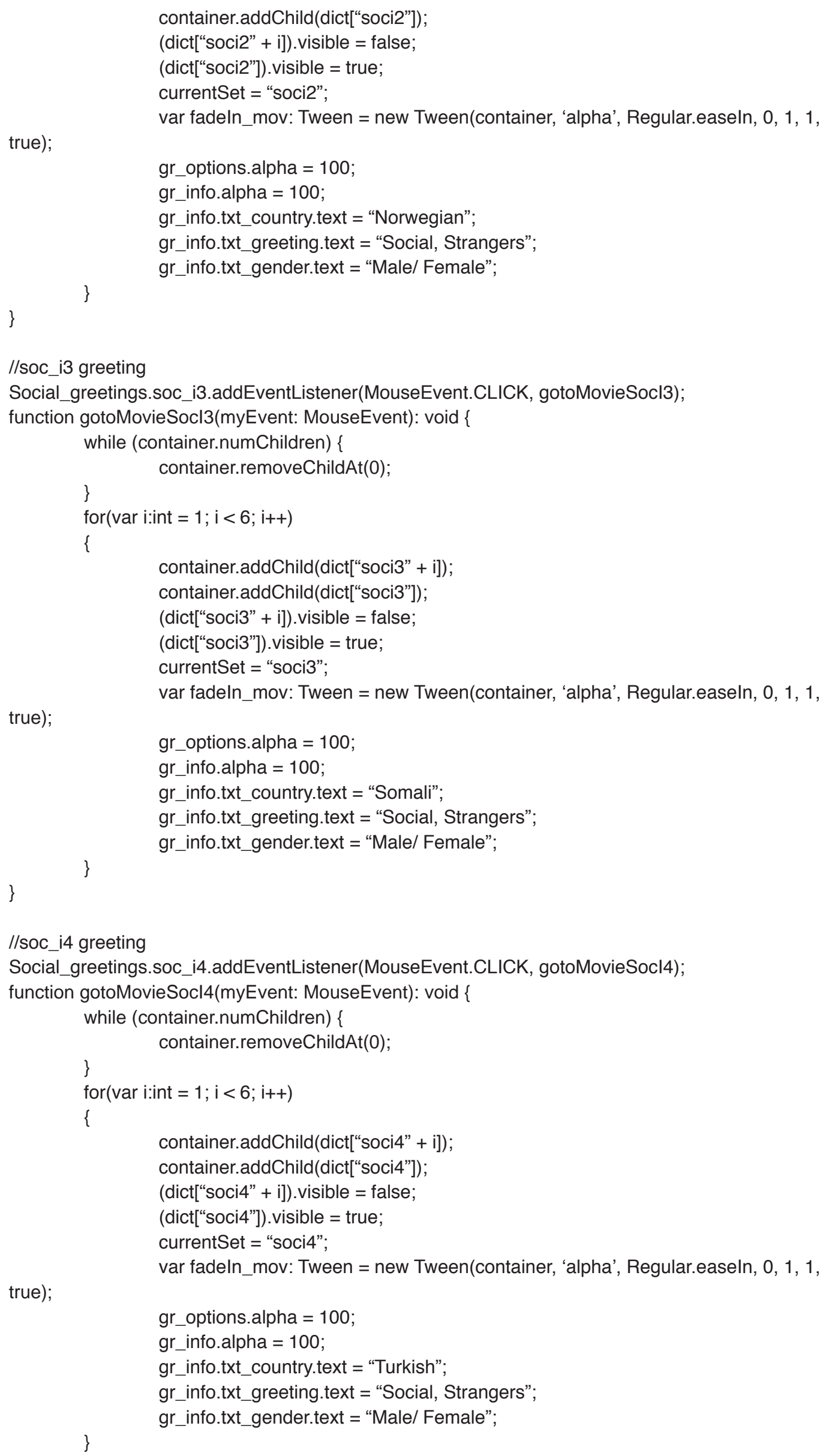


//soc_i5 greeting

Social_greetings.soc_i5.addEventListener(MouseEvent.CLICK, gotoMovieSocl5);

function gotoMovieSocl5(myEvent: MouseEvent): void \{

while (container.numChildren) \{ container.removeChildAt $(0)$ :

\} for (var i:int $=1 ; i<6 ; i++)$

\{

container.addChild(dict["soci5" + i]);

container.addChild(dict["soci5"]);

(dict["soci5" + i]).visible = false;

(dict["soci5"]).visible = true;

currentSet = "soci5";

var fadeln_mov: Tween = new Tween(container, 'alpha', Regular.easeln, 0, 1, 1,

true);

gr_options.alpha = 100;

gr_info.alpha $=100$;

gr_info.txt_country.text = "Vietnamese";

gr_info.txt_greeting.text = "Social, Strangers";

gr_info.txt_gender.text = "Male/ Female";

\}

\}

$/ / /^{\star \star \star \star \star \star \star \star \star \star \star \star \star \star \star \star \star \star \star \star \star \star \star \star \star \star ~}$

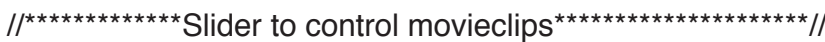

///*var sl:Slider = new Slider(); /

$/ /$ sl. width $=500$;

I//*sl.move(150, 450); */

//sl.alpha $=0$

//trace(cross_c1_sem.totalFrames);

//sl.maximum = cross_f1_sem.totalFrames;

//sl. liveDragging = true;

//sl. minimum = 1;

//sl. liveDragging = true;

//sl.addEventListener(Event.CHANGE, moveAlong);

//sl.addEventListener(Event.CHANGE,mcHandler);

//function mcHandler(e:Event)\{

// trace("aSlider.value: "+sl.value);

// cross_f1_sem.gotoAndStop(sl.value);

// cross_f1_sem.gotoAndStop(sl.value);

$/ /\}$

//function moveAlong(evt:Event):void \{

// cross_f1_sem.gotoAndStop(sl.value);

// cross_f1_sem.gotoAndStop(sl.value);

$/ /\}$

//addEventListener('enterFrame',enterFrame);

//function enterFrame(evt)\{

//sl.value = cross_f1_sem.currentFrame;

//\} 


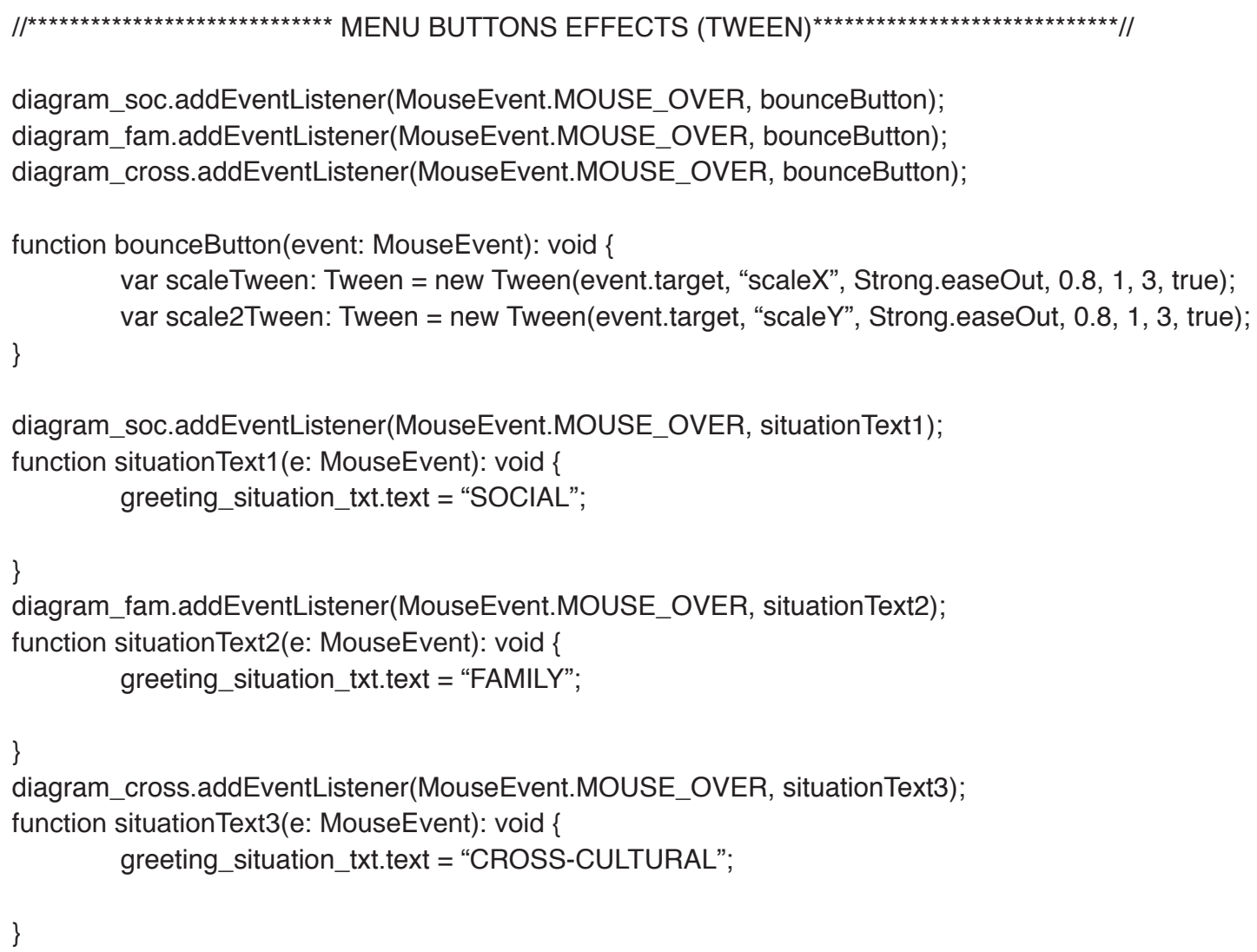


Cross_cultural_greetings.cross_b2.addEventListener(MouseEvent.MOUSE_OVER, highlightButton_ cross2);

Cross_cultural_greetings.cross_c2.addEventListener(MouseEvent.MOUSE_OVER, highlightButton_ cross2);

Cross_cultural_greetings.cross_d2.addEventListener(MouseEvent.MOUSE_OVER, highlightButton_ cross2);

Cross_cultural_greetings.cross_e2.addEventListener(MouseEvent.MOUSE_OVER, highlightButton_ cross2);

function highlightButton_cross2(event: MouseEvent): void \{

Cross_cultural_greetings.cross_2.gotoAndStop(“down_state”);

\}

Cross_cultural_greetings.cross_a2.addEventListener(MouseEvent.MOUSE_OUT, unhighlightButton_ cross2);

Cross_cultural_greetings.cross_b2.addEventListener(MouseEvent.MOUSE_OUT, unhighlightButton_ cross2);

Cross_cultural_greetings.cross_c2.addEventListener(MouseEvent.MOUSE_OUT, unhighlightButton_ cross2);

Cross_cultural_greetings.cross_d2.addEventListener(MouseEvent.MOUSE_OUT, unhighlightButton_ cross2);

Cross_cultural_greetings.cross_e2.addEventListener(MouseEvent.MOUSE_OUT, unhighlightButton_ cross2);

function unhighlightButton_cross2(event: MouseEvent): void \{

Cross_cultural_greetings.cross_2.gotoAndStop("up_state");

\}

//highlight \& unhighlight cross3 button//

Cross_cultural_greetings.cross_a3.addEventListener(MouseEvent.MOUSE_OVER, highlightButton_ cross 3 );

Cross_cultural_greetings.cross_b3.addEventListener(MouseEvent.MOUSE_OVER, highlightButton_ cross3);

Cross_cultural_greetings.cross_c3.addEventListener(MouseEvent.MOUSE_OVER, highlightButton_ cross3);

Cross_cultural_greetings.cross_d3.addEventListener(MouseEvent.MOUSE_OVER, highlightButton_ cross3);

Cross_cultural_greetings.cross_e3.addEventListener(MouseEvent.MOUSE_OVER, highlightButton_ cross3);

function highlightButton_cross3(event: MouseEvent): void \{

Cross_cultural_greetings.cross_3.gotoAndStop(“down_state");

\}

Cross_cultural_greetings.cross_a3.addEventListener(MouseEvent.MOUSE_OUT, unhighlightButton_ cross3);

Cross_cultural_greetings.cross_b3.addEventListener(MouseEvent.MOUSE_OUT, unhighlightButton_ cross3);

Cross_cultural_greetings.cross_c3.addEventListener(MouseEvent.MOUSE_OUT, unhighlightButton_ cross3);

Cross_cultural_greetings.cross_d3.addEventListener(MouseEvent.MOUSE_OUT, unhighlightButton_ cross3);

Cross_cultural_greetings.cross_e3.addEventListener(MouseEvent.MOUSE_OUT, unhighlightButton_ cross3);

function unhighlightButton_cross3(event: MouseEvent): void \{

Cross_cultural_greetings.cross_3.gotoAndStop("up_state");

\} 
//highlight \& unhighlight cross4 button//

Cross_cultural_greetings.cross_b4.addEventListener(MouseEvent.MOUSE_OVER, highlightButton_ cross4);

Cross_cultural_greetings.cross_c4.addEventListener(MouseEvent.MOUSE_OVER, highlightButton_ cross4);

function highlightButton_cross4(event: MouseEvent): void \{

Cross_cultural_greetings.cross_4.gotoAndStop("down_state");

\}

Cross_cultural_greetings.cross_b4.addEventListener(MouseEvent.MOUSE_OUT, unhighlightButton_ cross4);

Cross_cultural_greetings.cross_c4.addEventListener(MouseEvent.MOUSE_OUT, unhighlightButton_ cross 4$)$;

function unhighlightButton_cross4(event: MouseEvent): void \{

Cross_cultural_greetings.cross_4.gotoAndStop("up_state");

\}

//highlight \& unhighlight cross5 button//

Cross_cultural_greetings.cross_c5.addEventListener(MouseEvent.MOUSE_OVER, highlightButton_ cross5);

Cross_cultural_greetings.cross_f5.addEventListener(MouseEvent.MOUSE_OVER, highlightButton_ cross5);

function highlightButton_cross5(event: MouseEvent): void \{

Cross_cultural_greetings.cross_5.gotoAndStop("down_state");

\}

Cross_cultural_greetings.cross_c5.addEventListener(MouseEvent.MOUSE_OUT, unhighlightButton_ cross5);

Cross_cultural_greetings.cross_f5.addEventListener(MouseEvent.MOUSE_OUT, unhighlightButton_ cross5);

function unhighlightButton_cross5(event: MouseEvent): void \{

Cross_cultural_greetings.cross_5.gotoAndStop("up_state");

\}

//highlight \& unhighlight cross6 button//

Cross_cultural_greetings.cross_c6.addEventListener(MouseEvent.MOUSE_OVER, highlightButton_ cross6);

Cross_cultural_greetings.cross_f6.addEventListener(MouseEvent.MOUSE_OVER, highlightButton_ cross6);

function highlightButton_cross6(event: MouseEvent): void \{

Cross_cultural_greetings.cross_6.gotoAndStop(“down_state");

\}

Cross_cultural_greetings.cross_c6.addEventListener(MouseEvent.MOUSE_OUT, unhighlightButton_ cross6);

Cross_cultural_greetings.cross_f6.addEventListener(MouseEvent.MOUSE_OUT, unhighlightButton_ cross6);

function unhighlightButton_cross6(event: MouseEvent): void \{

Cross_cultural_greetings.cross_6.gotoAndStop("up_state");

\}

//highlight \& unhighlight crossa button//

Cross_cultural_greetings.cross_a1.addEventListener(MouseEvent.MOUSE_OVER, highlightButton_ crossa); 
Cross_cultural_greetings.cross_a2.addEventListener(MouseEvent.MOUSE_OVER, highlightButton_ crossa);

Cross_cultural_greetings.cross_a3.addEventListener(MouseEvent.MOUSE_OVER, highlightButton_ crossa);

function highlightButton_crossa(event: MouseEvent): void \{

Cross_cultural_greetings.cross_a.gotoAndStop(“down_state");

\}

Cross_cultural_greetings.cross_a1.addEventListener(MouseEvent.MOUSE_OUT, unhighlightButton_ crossa);

Cross_cultural_greetings.cross_a2.addEventListener(MouseEvent.MOUSE_OUT, unhighlightButton_ crossa);

Cross_cultural_greetings.cross_a3.addEventListener(MouseEvent.MOUSE_OUT, unhighlightButton_ crossa);

function unhighlightButton_crossa(event: MouseEvent): void \{

Cross_cultural_greetings.cross_a.gotoAndStop("up_state");

\}

//highlight \& unhighlight crossb button//

Cross_cultural_greetings.cross_b2.addEventListener(MouseEvent.MOUSE_OVER, highlightButton_ crossb);

Cross_cultural_greetings.cross_b3.addEventListener(MouseEvent.MOUSE_OVER, highlightButton_ crossb);

Cross_cultural_greetings.cross_b4.addEventListener(MouseEvent.MOUSE_OVER, highlightButton_ crossb);

function highlightButton_crossb(event: MouseEvent): void \{

Cross_cultural_greetings.cross_b.gotoAndStop(“down_state");

\}

Cross_cultural_greetings.cross_b2.addEventListener(MouseEvent.MOUSE_OUT, unhighlightButton_ crossb);

Cross_cultural_greetings.cross_b3.addEventListener(MouseEvent.MOUSE_OUT, unhighlightButton_ crossb);

Cross_cultural_greetings.cross_b4.addEventListener(MouseEvent.MOUSE_OUT, unhighlightButton_ crossb);

function unhighlightButton_crossb(event: MouseEvent): void \{

Cross_cultural_greetings.cross_b.gotoAndStop("up_state");

\}

//highlight \& unhighlight crossc button//

Cross_cultural_greetings.cross_c1.addEventListener(MouseEvent.MOUSE_OVER, highlightButton_ crossc):

Cross_cultural_greetings.cross_c2.addEventListener(MouseEvent.MOUSE_OVER, highlightButton_ crossc);

Cross_cultural_greetings.cross_c3.addEventListener(MouseEvent.MOUSE_OVER, highlightButton_ crossc);

Cross_cultural_greetings.cross_c4.addEventListener(MouseEvent.MOUSE_OVER, highlightButton_ crossc);

Cross_cultural_greetings.cross_c5.addEventListener(MouseEvent.MOUSE_OVER, highlightButton_ crossc);

Cross_cultural_greetings.cross_c6.addEventListener(MouseEvent.MOUSE_OVER, highlightButton_ crossc); 
function highlightButton_crossc(event: MouseEvent): void \{

Cross_cultural_greetings.cross_c.gotoAndStop("down_state");

\}

Cross_cultural_greetings.cross_c1.addEventListener(MouseEvent.MOUSE_OUT, unhighlightButton_ crossc);

Cross_cultural_greetings.cross_c2.addEventListener(MouseEvent.MOUSE_OUT, unhighlightButton_ crossc);

Cross_cultural_greetings.cross_c3.addEventListener(MouseEvent.MOUSE_OUT, unhighlightButton_ crossc);

Cross_cultural_greetings.cross_c4.addEventListener(MouseEvent.MOUSE_OUT, unhighlightButton_ crossc);

Cross_cultural_greetings.cross_c5.addEventListener(MouseEvent.MOUSE_OUT, unhighlightButton_ crossc);

Cross_cultural_greetings.cross_c6.addEventListener(MouseEvent.MOUSE_OUT, unhighlightButton_ crossc);

function unhighlightButton_crossc(event: MouseEvent): void \{

Cross_cultural_greetings.cross_c.gotoAndStop("up_state");

\}

//highlight \& unhighlight crossd button//

Cross_cultural_greetings.cross_d1.addEventListener(MouseEvent.MOUSE_OVER, highlightButton_ crossd);

Cross_cultural_greetings.cross_d2.addEventListener(MouseEvent.MOUSE_OVER, highlightButton_ crossd);

Cross_cultural_greetings.cross_d3.addEventListener(MouseEvent.MOUSE_OVER, highlightButton_ crossd);

function highlightButton_crossd(event: MouseEvent): void \{

Cross_cultural_greetings.cross_d.gotoAndStop(“down_state");

\}

Cross_cultural_greetings.cross_d1.addEventListener(MouseEvent.MOUSE_OUT, unhighlightButton_ crossd);

Cross_cultural_greetings.cross_d2.addEventListener(MouseEvent.MOUSE_OUT, unhighlightButton_ crossd);

Cross_cultural_greetings.cross_d3.addEventListener(MouseEvent.MOUSE_OUT, unhighlightButton_ crossd);

function unhighlightButton_crossd(event: MouseEvent): void \{

Cross_cultural_greetings.cross_d.gotoAndStop("up_state");

\}

//highlight \& unhighlight crosse button//

Cross_cultural_greetings.cross_e2.addEventListener(MouseEvent.MOUSE_OVER, highlightButton_ crosse);

Cross_cultural_greetings.cross_e3.addEventListener(MouseEvent.MOUSE_OVER, highlightButton_ crosse);

function highlightButton_crosse(event: MouseEvent): void \{

Cross_cultural_greetings.cross_e.gotoAndStop(“down_state");

\}

Cross_cultural_greetings.cross_e2.addEventListener(MouseEvent.MOUSE_OUT, unhighlightButton_ crosse);

Cross_cultural_greetings.cross_e3.addEventListener(MouseEvent.MOUSE_OUT, unhighlightButton_ crosse);

function unhighlightButton_crosse(event: MouseEvent): void \{ 
Cross_cultural_greetings.cross_e.gotoAndStop(“up_state”);

\}

//highlight \& unhighlight crossf button//

Cross_cultural_greetings.cross_f1.addEventListener(MouseEvent.MOUSE_OVER, highlightButton_ crossf);

Cross_cultural_greetings.cross_f5.addEventListener(MouseEvent.MOUSE_OVER, highlightButton_ crossf);

Cross_cultural_greetings.cross_f6.addEventListener(MouseEvent.MOUSE_OVER, highlightButton_ crossf);

function highlightButton_crossf(event: MouseEvent): void \{

Cross_cultural_greetings.cross_f.gotoAndStop("down_state");

\}

Cross_cultural_greetings.cross_f1.addEventListener(MouseEvent.MOUSE_OUT, unhighlightButton_ crossf);

Cross_cultural_greetings.cross_f5.addEventListener(MouseEvent.MOUSE_OUT, unhighlightButton_ crossf);

Cross_cultural_greetings.cross_f6.addEventListener(MouseEvent.MOUSE_OUT, unhighlightButton_ crossf);

function unhighlightButton_crossf(event: MouseEvent): void \{

Cross_cultural_greetings.cross_f.gotoAndStop("up_state”);

\}

\section{//FAMILY BUTTONS//}

//highlight \& unhighlight fama button//

Family_greetings.fam_a1.addEventListener(MouseEvent.MOUSE_OVER, highlightButton_fama); Family_greetings.fam_a2.addEventListener(MouseEvent.MOUSE_OVER, highlightButton_fama); Family_greetings.fam_a3.addEventListener(MouseEvent.MOUSE_OVER, highlightButton_fama); Family_greetings.fam_a4.addEventListener(MouseEvent.MOUSE_OVER, highlightButton_fama); Family_greetings.fam_a5.addEventListener(MouseEvent.MOUSE_OVER, highlightButton_fama);

function highlightButton_fama(event: MouseEvent): void \{

Family_greetings.fam_a.gotoAndStop(“down_state"); \}

Family_greetings.fam_a1.addEventListener(MouseEvent.MOUSE_OUT, unhighlightButton_fama); Family_greetings.fam_a2.addEventListener(MouseEvent.MOUSE_OUT, unhighlightButton_fama); Family_greetings.fam_a3.addEventListener(MouseEvent.MOUSE_OUT, unhighlightButton_fama); Family_greetings.fam_a4.addEventListener(MouseEvent.MOUSE_OUT, unhighlightButton_fama); Family_greetings.fam_a5.addEventListener(MouseEvent.MOUSE_OUT, unhighlightButton_fama);

function unhighlightButton_fama(event: MouseEvent): void \{

Family_greetings.fam_a.gotoAndStop(“up_state”); \}

//highlight \& unhighlight famb button//

Family_greetings.fam_b2.addEventListener(MouseEvent.MOUSE_OVER, highlightButton_famb); Family_greetings.fam_b5.addEventListener(MouseEvent.MOUSE_OVER, highlightButton_famb);

function highlightButton_famb(event: MouseEvent): void \{

Family_greetings.fam_b.gotoAndStop(“down_state");

\}

Family_greetings.fam_b2.addEventListener(MouseEvent.MOUSE_OUT, unhighlightButton_famb); 
Family_greetings.fam_b5.addEventListener(MouseEvent.MOUSE_OUT, unhighlightButton_famb);

function unhighlightButton_famb(event: MouseEvent): void \{

Family_greetings.fam_b.gotoAndStop(“up_state”);

\}

//highlight \& unhighlight famc button//

Family_greetings.fam_c2.addEventListener(MouseEvent.MOUSE_OVER, highlightButton_famc); Family_greetings.fam_c4.addEventListener(MouseEvent.MOUSE_OVER, highlightButton_famc); Family_greetings.fam_c5.addEventListener(MouseEvent.MOUSE_OVER, highlightButton_famc); function highlightButton_famc(event: MouseEvent): void \{

Family_greetings.fam_c.gotoAndStop(“down_state"); \}

Family_greetings.fam_c2.addEventListener(MouseEvent.MOUSE_OUT, unhighlightButton_famc); Family_greetings.fam_c4.addEventListener(MouseEvent.MOUSE_OUT, unhighlightButton_famc); Family_greetings.fam_c5.addEventListener(MouseEvent.MOUSE_OUT, unhighlightButton_famc); function unhighlightButton_famc(event: MouseEvent): void \{

Family_greetings.fam_c.gotoAndStop(“up_state");

\}

//highlight \& unhighlight famd button//

Family_greetings.fam_d1.addEventListener(MouseEvent.MOUSE_OVER, highlightButton_famd); Family_greetings.fam_d2.addEventListener(MouseEvent.MOUSE_OVER, highlightButton_famd); Family_greetings.fam_d5.addEventListener(MouseEvent.MOUSE_OVER, highlightButton_famd); function highlightButton_famd(event: MouseEvent): void \{

Family_greetings.fam_d.gotoAndStop(“down_state”);

\}

Family_greetings.fam_d1.addEventListener(MouseEvent.MOUSE_OUT, unhighlightButton_famd); Family_greetings.fam_d2.addEventListener(MouseEvent.MOUSE_OUT, unhighlightButton_famd); Family_greetings.fam_d5.addEventListener(MouseEvent.MOUSE_OUT, unhighlightButton_famd); function unhighlightButton_famd(event: MouseEvent): void \{

Family_greetings.fam_d.gotoAndStop(“up_state"); \}

//highlight \& unhighlight fame button//

Family_greetings.fam_e1.addEventListener(MouseEvent.MOUSE_OVER, highlightButton_fame); Family_greetings.fam_e2.addEventListener(MouseEvent.MOUSE_OVER, highlightButton_fame); Family_greetings.fam_e3.addEventListener(MouseEvent.MOUSE_OVER, highlightButton_fame); Family_greetings.fam_e4.addEventListener(MouseEvent.MOUSE_OVER, highlightButton_fame); Family_greetings.fam_e5.addEventListener(MouseEvent.MOUSE_OVER, highlightButton_fame);

function highlightButton_fame(event: MouseEvent): void \{

Family_greetings.fam_e.gotoAndStop(“down_state");

\}

Family_greetings.fam_e1.addEventListener(MouseEvent.MOUSE_OUT, unhighlightButton_fame); Family_greetings.fam_e2.addEventListener(MouseEvent.MOUSE_OUT, unhighlightButton_fame); Family_greetings.fam_e3.addEventListener(MouseEvent.MOUSE_OUT, unhighlightButton_fame); Family_greetings.fam_e4.addEventListener(MouseEvent.MOUSE_OUT, unhighlightButton_fame); Family_greetings.fam_e5.addEventListener(MouseEvent.MOUSE_OUT, unhighlightButton_fame);

function unhighlightButton_fame(event: MouseEvent): void \{ Family_greetings.fam_e.gotoAndStop("up_state"); 
//highlight \& unhighlight famf button//

Family_greetings.fam_f2.addEventListener(MouseEvent.MOUSE_OVER, highlightButton_famf); Family_greetings.fam_f5.addEventListener(MouseEvent.MOUSE_OVER, highlightButton_famf); function highlightButton_famf(event: MouseEvent): void \{

Family_greetings.fam_f.gotoAndStop("down_state"); \}

Family_greetings.fam_f2.addEventListener(MouseEvent.MOUSE_OUT, unhighlightButton_famf); Family_greetings.fam_f5.addEventListener(MouseEvent.MOUSE_OUT, unhighlightButton_famf); function unhighlightButton_famf(event: MouseEvent): void \{

Family_greetings.fam_f.gotoAndStop("up_state");

\}

//highlight \& unhighlight famg button//

Family_greetings.fam_g2.addEventListener(MouseEvent.MOUSE_OVER, highlightButton_famg); Family_greetings.fam_g4.addEventListener(MouseEvent.MOUSE_OVER, highlightButton_famg); Family_greetings.fam_g5.addEventListener(MouseEvent.MOUSE_OVER, highlightButton_famg);

function highlightButton_famg(event: MouseEvent): void \{

Family_greetings.fam_g.gotoAndStop(“down_state”);

\}

Family_greetings.fam_g2.addEventListener(MouseEvent.MOUSE_OUT, unhighlightButton_famg); Family_greetings.fam_g4.addEventListener(MouseEvent.MOUSE_OUT, unhighlightButton_famg); Family_greetings.fam_g5.addEventListener(MouseEvent.MOUSE_OUT, unhighlightButton_famg);

function unhighlightButton_famg(event: MouseEvent): void \{

Family_greetings.fam_g.gotoAndStop("up_state"); \}

//highlight \& unhighlight famh button//

Family_greetings.fam_h1.addEventListener(MouseEvent.MOUSE_OVER, highlightButton_famh); Family_greetings.fam_h2.addEventListener(MouseEvent.MOUSE_OVER, highlightButton_famh); Family_greetings.fam_h5.addEventListener(MouseEvent.MOUSE_OVER, highlightButton_famh); function highlightButton_famh(event: MouseEvent): void \{

Family_greetings.fam_h.gotoAndStop(“down_state"); \}

Family_greetings.fam_h1.addEventListener(MouseEvent.MOUSE_OUT, unhighlightButton_famh); Family_greetings.fam_h2.addEventListener(MouseEvent.MOUSE_OUT, unhighlightButton_famh); Family_greetings.fam_h5.addEventListener(MouseEvent.MOUSE_OUT, unhighlightButton_famh); function unhighlightButton_famh(event: MouseEvent): void \{

Family_greetings.fam_h.gotoAndStop(“up_state”);

\}

//highlight \& unhighlight fam1 button//

Family_greetings.fam_a1.addEventListener(MouseEvent.MOUSE_OVER, highlightButton_fam1); Family_greetings.fam_d1.addEventListener(MouseEvent.MOUSE_OVER, highlightButton_fam1); Family_greetings.fam_e1.addEventListener(MouseEvent.MOUSE_OVER, highlightButton_fam1); Family_greetings.fam_h1.addEventListener(MouseEvent.MOUSE_OVER, highlightButton_fam1);

function highlightButton_fam1(event: MouseEvent): void \{

Family_greetings.fam_1.gotoAndStop(“down_state");

\}

Family_greetings.fam_a1.addEventListener(MouseEvent.MOUSE_OUT, unhighlightButton_fam1); Family_greetings.fam_d1.addEventListener(MouseEvent.MOUSE_OUT, unhighlightButton_fam1); 
Family_greetings.fam_e1.addEventListener(MouseEvent.MOUSE_OUT, unhighlightButton_fam1); Family_greetings.fam_h1.addEventListener(MouseEvent.MOUSE_OUT, unhighlightButton_fam1);

function unhighlightButton_fam1(event: MouseEvent): void \{

Family_greetings.fam_1.gotoAndStop(“up_state”);

\}

//highlight \& unhighlight fam2 button//

Family_greetings.fam_a2.addEventListener(MouseEvent.MOUSE_OVER, highlightButton_fam2); Family_greetings.fam_b2.addEventListener(MouseEvent.MOUSE_OVER, highlightButton_fam2); Family_greetings.fam_c2.addEventListener(MouseEvent.MOUSE_OVER, highlightButton_fam2); Family_greetings.fam_d2.addEventListener(MouseEvent.MOUSE_OVER, highlightButton_fam2); Family_greetings.fam_e2.addEventListener(MouseEvent.MOUSE_OVER, highlightButton_fam2); Family_greetings.fam_f2.addEventListener(MouseEvent.MOUSE_OVER, highlightButton_fam2); Family_greetings.fam_g2.addEventListener(MouseEvent.MOUSE_OVER, highlightButton_fam2); Family_greetings.fam_h2.addEventListener(MouseEvent.MOUSE_OVER, highlightButton_fam2);

function highlightButton_fam2(event: MouseEvent): void \{

Family_greetings.fam_2.gotoAndStop(“down_state"); \}

Family_greetings.fam_a2.addEventListener(MouseEvent.MOUSE_OUT, unhighlightButton_fam2); Family_greetings.fam_b2.addEventListener(MouseEvent.MOUSE_OUT, unhighlightButton_fam2); Family_greetings.fam_c2.addEventListener(MouseEvent.MOUSE_OUT, unhighlightButton_fam2); Family_greetings.fam_d2.addEventListener(MouseEvent.MOUSE_OUT, unhighlightButton_fam2); Family_greetings.fam_e2.addEventListener(MouseEvent.MOUSE_OUT, unhighlightButton_fam2); Family_greetings.fam_f2.addEventListener(MouseEvent.MOUSE_OUT, unhighlightButton_fam2); Family_greetings.fam_g2.addEventListener(MouseEvent.MOUSE_OUT, unhighlightButton_fam2); Family_greetings.fam_h2.addEventListener(MouseEvent.MOUSE_OUT, unhighlightButton_fam2);

function unhighlightButton_fam2(event: MouseEvent): void \{ Family_greetings.fam_2.gotoAndStop(“up_state”); \}

//highlight \& unhighlight fam3 button//

Family_greetings.fam_a3.addEventListener(MouseEvent.MOUSE_OVER, highlightButton_fam3); Family_greetings.fam_e3.addEventListener(MouseEvent.MOUSE_OVER, highlightButton_fam3);

function highlightButton_fam3(event: MouseEvent): void \{

Family_greetings.fam_3.gotoAndStop(“down_state"); \}

Family_greetings.fam_a3.addEventListener(MouseEvent.MOUSE_OUT, unhighlightButton_fam3); Family_greetings.fam_e3.addEventListener(MouseEvent.MOUSE_OUT, unhighlightButton_fam3);

function unhighlightButton_fam3(event: MouseEvent): void \{

Family_greetings.fam_3.gotoAndStop(“up_state”);

\}

//highlight \& unhighlight fam4 button//

Family_greetings.fam_a4.addEventListener(MouseEvent.MOUSE_OVER, highlightButton_fam4); Family_greetings.fam_c4.addEventListener(MouseEvent.MOUSE_OVER, highlightButton_fam4); Family_greetings.fam_e4.addEventListener(MouseEvent.MOUSE_OVER, highlightButton_fam4); Family_greetings.fam_g4.addEventListener(MouseEvent.MOUSE_OVER, highlightButton_fam4);

function highlightButton_fam4(event: MouseEvent): void \{ 
Family_greetings.fam_4.gotoAndStop(“down_state”);

\}

Family_greetings.fam_a4.addEventListener(MouseEvent.MOUSE_OUT, unhighlightButton_fam4); Family_greetings.fam_c4.addEventListener(MouseEvent.MOUSE_OUT, unhighlightButton_fam4); Family_greetings.fam_e4.addEventListener(MouseEvent.MOUSE_OUT, unhighlightButton_fam4); Family_greetings.fam_g4.addEventListener(MouseEvent.MOUSE_OUT, unhighlightButton_fam4);

function unhighlightButton_fam4(event: MouseEvent): void \{

Family_greetings.fam_4.gotoAndStop(“up_state”);

\}

//highlight \& unhighlight fam5 button//

Family_greetings.fam_a5.addEventListener(MouseEvent.MOUSE_OVER, highlightButton_fam5); Family_greetings.fam_b5.addEventListener(MouseEvent.MOUSE_OVER, highlightButton_fam5); Family_greetings.fam_c5.addEventListener(MouseEvent.MOUSE_OVER, highlightButton_fam5); Family_greetings.fam_d5.addEventListener(MouseEvent.MOUSE_OVER, highlightButton_fam5); Family_greetings.fam_e5.addEventListener(MouseEvent.MOUSE_OVER, highlightButton_fam5); Family_greetings.fam_f5.addEventListener(MouseEvent.MOUSE_OVER, highlightButton_fam5); Family_greetings.fam_g5.addEventListener(MouseEvent.MOUSE_OVER, highlightButton_fam5); Family_greetings.fam_h5.addEventListener(MouseEvent.MOUSE_OVER, highlightButton_fam5);

function highlightButton_fam5(event: MouseEvent): void \{

Family_greetings.fam_5.gotoAndStop(“down_state”); \}

Family_greetings.fam_a5.addEventListener(MouseEvent.MOUSE_OUT, unhighlightButton_fam5); Family_greetings.fam_b5.addEventListener(MouseEvent.MOUSE_OUT, unhighlightButton_fam5); Family_greetings.fam_c5.addEventListener(MouseEvent.MOUSE_OUT, unhighlightButton_fam5); Family_greetings.fam_d5.addEventListener(MouseEvent.MOUSE_OUT, unhighlightButton_fam5); Family_greetings.fam_e5.addEventListener(MouseEvent.MOUSE_OUT, unhighlightButton_fam5); Family_greetings.fam_f5.addEventListener(MouseEvent.MOUSE_OUT, unhighlightButton_fam5); Family_greetings.fam_g5.addEventListener(MouseEvent.MOUSE_OUT, unhighlightButton_fam5); Family_greetings.fam_h5.addEventListener(MouseEvent.MOUSE_OUT, unhighlightButton_fam5);

function unhighlightButton_fam5(event: MouseEvent): void \{

Family_greetings.fam_5.gotoAndStop("up_state");

\}

//SOCIAL BUTTONS//

//highlight \& unhighlight soca button//

Social_greetings.soc_a2.addEventListener(MouseEvent.MOUSE_OVER, highlightButton_soca); Social_greetings.soc_a4.addEventListener(MouseEvent.MOUSE_OVER, highlightButton_soca); Social_greetings.soc_a5.addEventListener(MouseEvent.MOUSE_OVER, highlightButton_soca);

function highlightButton_soca(event: MouseEvent): void \{

Social_greetings.soc_a.gotoAndStop("down_state"); \}

Social_greetings.soc_a2.addEventListener(MouseEvent.MOUSE_OUT, unhighlightButton_soca); Social_greetings.soc_a4.addEventListener(MouseEvent.MOUSE_OUT, unhighlightButton_soca); Social_greetings.soc_a5.addEventListener(MouseEvent.MOUSE_OUT, unhighlightButton_soca);

function unhighlightButton_soca(event: MouseEvent): void \{ Social_greetings.soc_a.gotoAndStop("up_state"); 
//highlight \& unhighlight socb button//

Social_greetings.soc_b2.addEventListener(MouseEvent.MOUSE_OVER, highlightButton_socb); Social_greetings.soc_b3.addEventListener(MouseEvent.MOUSE_OVER, highlightButton_socb); Social_greetings.soc_b4.addEventListener(MouseEvent.MOUSE_OVER, highlightButton_socb); Social_greetings.soc_b5.addEventListener(MouseEvent.MOUSE_OVER, highlightButton_socb);

function highlightButton_socb(event: MouseEvent): void \{ Social_greetings.soc_b.gotoAndStop(“down_state”); \}

Social_greetings.soc_b2.addEventListener(MouseEvent.MOUSE_OUT, unhighlightButton_socb); Social_greetings.soc_b3.addEventListener(MouseEvent.MOUSE_OUT, unhighlightButton_socb); Social_greetings.soc_b4.addEventListener(MouseEvent.MOUSE_OUT, unhighlightButton_socb); Social_greetings.soc_b5.addEventListener(MouseEvent.MOUSE_OUT, unhighlightButton_socb);

function unhighlightButton_socb(event: MouseEvent): void \{ Social_greetings.soc_b.gotoAndStop("up_state"); \}

//highlight \& unhighlight socc button//

Social_greetings.soc_c1.addEventListener(MouseEvent.MOUSE_OVER, highlightButton_socc); Social_greetings.soc_c2.addEventListener(MouseEvent.MOUSE_OVER, highlightButton_socc); Social_greetings.soc_c3.addEventListener(MouseEvent.MOUSE_OVER, highlightButton_socc); Social_greetings.soc_c4.addEventListener(MouseEvent.MOUSE_OVER, highlightButton_socc); Social_greetings.soc_c5.addEventListener(MouseEvent.MOUSE_OVER, highlightButton_socc);

function highlightButton_socc(event: MouseEvent): void \{ Social_greetings.soc_c.gotoAndStop(“down_state"); \}

Social_greetings.soc_c1.addEventListener(MouseEvent.MOUSE_OUT, unhighlightButton_socc); Social_greetings.soc_c2.addEventListener(MouseEvent.MOUSE_OUT, unhighlightButton_socc); Social_greetings.soc_c3.addEventListener(MouseEvent.MOUSE_OUT, unhighlightButton_socc); Social_greetings.soc_c4.addEventListener(MouseEvent.MOUSE_OUT, unhighlightButton_socc); Social_greetings.soc_c5.addEventListener(MouseEvent.MOUSE_OUT, unhighlightButton_socc);

function unhighlightButton_socc(event: MouseEvent): void \{ Social_greetings.soc_c.gotoAndStop("up_state"); \}

//highlight \& unhighlight socd button//

Social_greetings.soc_d2.addEventListener(MouseEvent.MOUSE_OVER, highlightButton_socd); Social_greetings.soc_d4.addEventListener(MouseEvent.MOUSE_OVER, highlightButton_socd); Social_greetings.soc_d5.addEventListener(MouseEvent.MOUSE_OVER, highlightButton_socd);

function highlightButton_socd(event: MouseEvent): void \{

Social_greetings.soc_d.gotoAndStop(“down_state"); \}

Social_greetings.soc_d2.addEventListener(MouseEvent.MOUSE_OUT, unhighlightButton_socd); Social_greetings.soc_d4.addEventListener(MouseEvent.MOUSE_OUT, unhighlightButton_socd); Social_greetings.soc_d5.addEventListener(MouseEvent.MOUSE_OUT, unhighlightButton_socd);

function unhighlightButton_socd(event: MouseEvent): void \{ 
Social_greetings.soc_d.gotoAndStop("up_state");

\}

//highlight \& unhighlight soce button//

Social_greetings.soc_e2.addEventListener(MouseEvent.MOUSE_OVER, highlightButton_soce); Social_greetings.soc_e4.addEventListener(MouseEvent.MOUSE_OVER, highlightButton_soce); Social_greetings.soc_e5.addEventListener(MouseEvent.MOUSE_OVER, highlightButton_soce);

function highlightButton_soce(event: MouseEvent): void \{

Social_greetings.soc_e.gotoAndStop("down_state"); \}

Social_greetings.soc_e2.addEventListener(MouseEvent.MOUSE_OUT, unhighlightButton_soce); Social_greetings.soc_e4.addEventListener(MouseEvent.MOUSE_OUT, unhighlightButton_soce); Social_greetings.soc_e5.addEventListener(MouseEvent.MOUSE_OUT, unhighlightButton_soce);

function unhighlightButton_soce(event: MouseEvent): void \{ Social_greetings.soc_e.gotoAndStop("up_state"); \}

//highlight \& unhighlight socf button//

Social_greetings.soc_f1.addEventListener(MouseEvent.MOUSE_OVER, highlightButton_socf); Social_greetings.soc_f2.addEventListener(MouseEvent.MOUSE_OVER, highlightButton_socf); Social_greetings.soc_f4.addEventListener(MouseEvent.MOUSE_OVER, highlightButton_socf); Social_greetings.soc_f5.addEventListener(MouseEvent.MOUSE_OVER, highlightButton_socf);

function highlightButton_socf(event: MouseEvent): void \{

Social_greetings.soc_f.gotoAndStop(“down_state"); \}

Social_greetings.soc_f1.addEventListener(MouseEvent.MOUSE_OUT, unhighlightButton_socf); Social_greetings.soc_f2.addEventListener(MouseEvent.MOUSE_OUT, unhighlightButton_socf); Social_greetings.soc_f4.addEventListener(MouseEvent.MOUSE_OUT, unhighlightButton_socf); Social_greetings.soc_f5.addEventListener(MouseEvent.MOUSE_OUT, unhighlightButton_socf);

function unhighlightButton_socf(event: MouseEvent): void \{ Social_greetings.soc_f.gotoAndStop("up_state"); \}

//highlight \& unhighlight socg button//

Social_greetings.soc_g2.addEventListener(MouseEvent.MOUSE_OVER, highlightButton_socg); Social_greetings.soc_g4.addEventListener(MouseEvent.MOUSE_OVER, highlightButton_socg); Social_greetings.soc_g5.addEventListener(MouseEvent.MOUSE_OVER, highlightButton_socg);

function highlightButton_socg(event: MouseEvent): void \{ Social_greetings.soc_g.gotoAndStop(“down_state"); \}

Social_greetings.soc_g2.addEventListener(MouseEvent.MOUSE_OUT, unhighlightButton_socg); Social_greetings.soc_g4.addEventListener(MouseEvent.MOUSE_OUT, unhighlightButton_socg); Social_greetings.soc_g5.addEventListener(MouseEvent.MOUSE_OUT, unhighlightButton_socg);

function unhighlightButton_socg(event: MouseEvent): void \{ Social_greetings.soc_g.gotoAndStop("up_state"); \}

//highlight \& unhighlight soch button// 
Social_greetings.soc_h2.addEventListener(MouseEvent.MOUSE_OVER, highlightButton_soch); Social_greetings.soc_h3.addEventListener(MouseEvent.MOUSE_OVER, highlightButton_soch); Social_greetings.soc_h4.addEventListener(MouseEvent.MOUSE_OVER, highlightButton_soch); Social_greetings.soc_h5.addEventListener(MouseEvent.MOUSE_OVER, highlightButton_soch);

function highlightButton_soch(event: MouseEvent): void \{ Social_greetings.soc_h.gotoAndStop("down_state"); \}

Social_greetings.soc_h2.addEventListener(MouseEvent.MOUSE_OUT, unhighlightButton_soch); Social_greetings.soc_h3.addEventListener(MouseEvent.MOUSE_OUT, unhighlightButton_soch); Social_greetings.soc_h4.addEventListener(MouseEvent.MOUSE_OUT, unhighlightButton_soch); Social_greetings.soc_h5.addEventListener(MouseEvent.MOUSE_OUT, unhighlightButton_soch);

function unhighlightButton_soch(event: MouseEvent): void \{ Social_greetings.soc_h.gotoAndStop("up_state"); \}

//highlight \& unhighlight soci button//

Social_greetings.soc_i1.addEventListener(MouseEvent.MOUSE_OVER, highlightButton_soci); Social_greetings.soc_i3.addEventListener(MouseEvent.MOUSE_OVER, highlightButton_soci); Social_greetings.Soc_i2.addEventListener(MouseEvent.MOUSE_OVER, highlightButton_soci); Social_greetings.soc_i4.addEventListener(MouseEvent.MOUSE_OVER, highlightButton_soci); Social_greetings.soc_i5.addEventListener(MouseEvent.MOUSE_OVER, highlightButton_soci);

function highlightButton_soci(event: MouseEvent): void \{

Social_greetings.soc_i.gotoAndStop("down_state");

\}

Social_greetings.soc_i2.addEventListener(MouseEvent.MOUSE_OUT, unhighlightButton_soci); Social_greetings.soc_i3.addEventListener(MouseEvent.MOUSE_OUT, unhighlightButton_soci); Social_greetings.soc_i4.addEventListener(MouseEvent.MOUSE_OUT, unhighlightButton_soci); Social_greetings.soc_i5.addEventListener(MouseEvent.MOUSE_OUT, unhighlightButton_soci);

function unhighlightButton_soci(event: MouseEvent): void \{ Social_greetings.soc_i.gotoAndStop("up_state"); \}

//highlight \& unhighlight soc1 button// Social_greetings.soc_c1.addEventListener(MouseEvent.MOUSE_OVER, highlightButton_soc1); Social_greetings.soc_f1.addEventListener(MouseEvent.MOUSE_OVER, highlightButton_soc1); Social_greetings.soc_i1.addEventListener(MouseEvent.MOUSE_OVER, highlightButton_soc1);

function highlightButton_soc1(event: MouseEvent): void \{ Social_greetings.soc_1.gotoAndStop("down_state"); \}

Social_greetings.soc_c1.addEventListener(MouseEvent.MOUSE_OUT, unhighlightButton_soc1); Social_greetings.soc_f1.addEventListener(MouseEvent.MOUSE_OUT, unhighlightButton_soc1); Social_greetings.soc_i1.addEventListener(MouseEvent.MOUSE_OUT, unhighlightButton_soc1);

function unhighlightButton_soc1(event: MouseEvent): void \{ Social_greetings.soc_1.gotoAndStop("up_state"); \}

//highlight \& unhighlight soc2 button// 
Social_greetings.soc_a2.addEventListener(MouseEvent.MOUSE_OVER, highlightButton_soc2); Social_greetings.soc_b2.addEventListener(MouseEvent.MOUSE_OVER, highlightButton_soc2); Social_greetings.soc_c2.addEventListener(MouseEvent.MOUSE_OVER, highlightButton_soc2); Social_greetings.soc_d2.addEventListener(MouseEvent.MOUSE_OVER, highlightButton_soc2); Social_greetings.soc_e2.addEventListener(MouseEvent.MOUSE_OVER, highlightButton_soc2); Social_greetings.soc_f2.addEventListener(MouseEvent.MOUSE_OVER, highlightButton_soc2); Social_greetings.soc_g2.addEventListener(MouseEvent.MOUSE_OVER, highlightButton_soc2); Social_greetings.soc_h2.addEventListener(MouseEvent.MOUSE_OVER, highlightButton_soc2); Social_greetings.soc_i2.addEventListener(MouseEvent.MOUSE_OVER, highlightButton_soc2);

function highlightButton_soc2(event: MouseEvent): void \{

Social_greetings.soc_2.gotoAndStop("down_state"); \}

Social_greetings.soc_a2.addEventListener(MouseEvent.MOUSE_OUT, unhighlightButton_soc2); Social_greetings.soc_b2.addEventListener(MouseEvent.MOUSE_OUT, unhighlightButton_soc2); Social_greetings.soc_c2.addEventListener(MouseEvent.MOUSE_OUT, unhighlightButton_soc2); Social_greetings.soc_d2.addEventListener(MouseEvent.MOUSE_OUT, unhighlightButton_soc2); Social_greetings.soc_e2.addEventListener(MouseEvent.MOUSE_OUT, unhighlightButton_soc2); Social_greetings.soc_f2.addEventListener(MouseEvent.MOUSE_OUT, unhighlightButton_soc2); Social_greetings.soc_g2.addEventListener(MouseEvent.MOUSE_OUT, unhighlightButton_soc2); Social_greetings.soc_h2.addEventListener(MouseEvent.MOUSE_OUT, unhighlightButton_soc2); Social_greetings.soc_i2.addEventListener(MouseEvent.MOUSE_OUT, unhighlightButton_soc2);

function unhighlightButton_soc2(event: MouseEvent): void \{

Social_greetings.soc_2.gotoAndStop(“up_state"); \}

//highlight \& unhighlight soc3 button//

Social_greetings.soc_b3.addEventListener(MouseEvent.MOUSE_OVER, highlightButton_soc3); Social_greetings.soc_c3.addEventListener(MouseEvent.MOUSE_OVER, highlightButton_soc3); Social_greetings.soc_h3.addEventListener(MouseEvent.MOUSE_OVER, highlightButton_soc3); Social_greetings.soc_i3.addEventListener(MouseEvent.MOUSE_OVER, highlightButton_soc3);

function highlightButton_soc3(event: MouseEvent): void \{ Social_greetings.soc_3.gotoAndStop("down_state"); \}

Social_greetings.soc_b3.addEventListener(MouseEvent.MOUSE_OUT, unhighlightButton_soc3); Social_greetings.soc_c3.addEventListener(MouseEvent.MOUSE_OUT, unhighlightButton_soc3); Social_greetings.soc_h3.addEventListener(MouseEvent.MOUSE_OUT, unhighlightButton_soc3); Social_greetings.soc_i3.addEventListener(MouseEvent.MOUSE_OUT, unhighlightButton_soc3);

function unhighlightButton_soc3(event: MouseEvent): void \{

Social_greetings.soc_3.gotoAndStop("up_state"); \}

//highlight \& unhighlight soc4 button//

Social_greetings.soc_a4.addEventListener(MouseEvent.MOUSE_OVER, highlightButton_soc4); Social_greetings.soc_b4.addEventListener(MouseEvent.MOUSE_OVER, highlightButton_soc4); Social_greetings.soc_c4.addEventListener(MouseEvent.MOUSE_OVER, highlightButton_soc4); Social_greetings.soc_d4.addEventListener(MouseEvent.MOUSE_OVER, highlightButton_soc4); Social_greetings.soc_e4.addEventListener(MouseEvent.MOUSE_OVER, highlightButton_soc4); Social_greetings.soc_f4.addEventListener(MouseEvent.MOUSE_OVER, highlightButton_soc4); Social_greetings.soc_g4.addEventListener(MouseEvent.MOUSE_OVER, highlightButton_soc4); Social_greetings.soc_h4.addEventListener(MouseEvent.MOUSE_OVER, highlightButton_soc4); Social_greetings.soc_i4.addEventListener(MouseEvent.MOUSE_OVER, highlightButton_soc4); 
function highlightButton_soc4(event: MouseEvent): void \{

Social_greetings.soc_4.gotoAndStop("down_state"); \}

Social_greetings.soc_a4.addEventListener(MouseEvent.MOUSE_OUT, unhighlightButton_soc4); Social_greetings.soc_b4.addEventListener(MouseEvent.MOUSE_OUT, unhighlightButton_soc4); Social_greetings.soc_c4.addEventListener(MouseEvent.MOUSE_OUT, unhighlightButton_soc4); Social_greetings.soc_d4.addEventListener(MouseEvent.MOUSE_OUT, unhighlightButton_soc4); Social_greetings.soc_e4.addEventListener(MouseEvent.MOUSE_OUT, unhighlightButton_soc4); Social_greetings.soc_f4.addEventListener(MouseEvent.MOUSE_OUT, unhighlightButton_soc4); Social_greetings.soc_g4.addEventListener(MouseEvent.MOUSE_OUT, unhighlightButton_soc4); Social_greetings.soc_h4.addEventListener(MouseEvent.MOUSE_OUT, unhighlightButton_soc4); Social_greetings.soc_i4.addEventListener(MouseEvent.MOUSE_OUT, unhighlightButton_soc4);

function unhighlightButton_soc4(event: MouseEvent): void \{ Social_greetings.soc_4.gotoAndStop("up_state"); \}

//highlight \& unhighlight soc5 button//

Social_greetings.soc_a5.addEventListener(MouseEvent.MOUSE_OVER, highlightButton_soc5); Social_greetings.soc_b5.addEventListener(MouseEvent.MOUSE_OVER, highlightButton_soc5); Social_greetings.soc_c5.addEventListener(MouseEvent.MOUSE_OVER, highlightButton_soc5); Social_greetings.soc_d5.addEventListener(MouseEvent.MOUSE_OVER, highlightButton_soc5); Social_greetings.soc_e5.addEventListener(MouseEvent.MOUSE_OVER, highlightButton_soc5); Social_greetings.soc_f5.addEventListener(MouseEvent.MOUSE_OVER, highlightButton_soc5); Social_greetings.soc_g5.addEventListener(MouseEvent.MOUSE_OVER, highlightButton_soc5); Social_greetings.soc_h5.addEventListener(MouseEvent.MOUSE_OVER, highlightButton_soc5); Social_greetings.soc_i5.addEventListener(MouseEvent.MOUSE_OVER, highlightButton_soc5);

function highlightButton_soc5(event: MouseEvent): void \{ Social_greetings.soc_5.gotoAndStop(“down_state"); \}

Social_greetings.soc_a5.addEventListener(MouseEvent.MOUSE_OUT, unhighlightButton_soc5); Social_greetings.soc_b5.addEventListener(MouseEvent.MOUSE_OUT, unhighlightButton_soc5); Social_greetings.soc_c5.addEventListener(MouseEvent.MOUSE_OUT, unhighlightButton_soc5); Social_greetings.soc_d5.addEventListener(MouseEvent.MOUSE_OUT, unhighlightButton_soc5); Social_greetings.soc_e5.addEventListener(MouseEvent.MOUSE_OUT, unhighlightButton_soc5); Social_greetings.soc_f5.addEventListener(MouseEvent.MOUSE_OUT, unhighlightButton_soc5); Social_greetings.soc_g5.addEventListener(MouseEvent.MOUSE_OUT, unhighlightButton_soc5); Social_greetings.soc_h5.addEventListener(MouseEvent.MOUSE_OUT, unhighlightButton_soc5); Social_greetings.soc_i5.addEventListener(MouseEvent.MOUSE_OUT, unhighlightButton_soc5);

function unhighlightButton_soc5(event: MouseEvent): void \{ Social_greetings.soc_5.gotoAndStop("up_state"); 